\title{
Surface-Referenced Current Meter Measurements
}

by

\author{
Markku Juhani Santala \\ B.S., University of Massachusetts, Amherst (1985) \\ S.M., Massachusetts Institute of Technology (1987) \\ Submitted in partial fulfillment of the \\ requirements for the degree of \\ Doctor of Philosophy

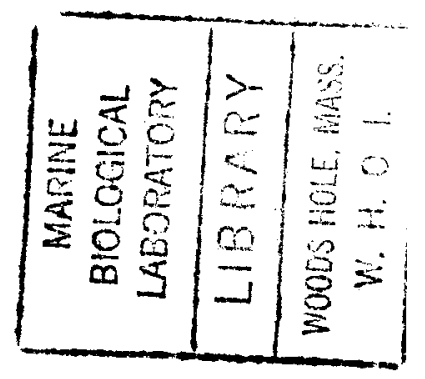 \\ at the
}
MASSACHUSETTS INSTITUTE OF TECHNOLOGY
and the

\section{WOODS HOLE OCEANOGRAPHIC INSTITUTION}

September 1991

(C) Markku J. Santala, 1991

The author hereby grants to MIT permission to reproduce and to distribute copies of this thesis document in whole or in part.

Signature of Author

Joint Program in Oceanographic Engineering

Massachusetts Institute of Technology

Woods Hole Oceanographic Institution

September, 1991

Certified by

Albert J. Williams, III

Senior Scientišt, Woods Hole Oceanographic Institution Thesis Supervisor

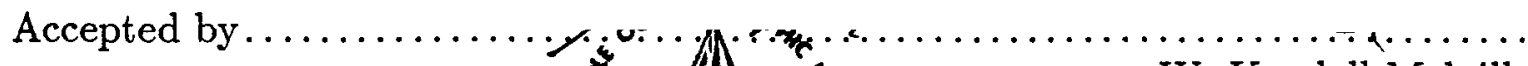




\title{
Surface-Referenced Current Meter Measurements
}

\author{
by \\ Markku Juhani Santala \\ Submitted to the Massachusetts Institute of Technology- \\ Woods Hole Oceanographic Institution \\ Joint Program in Oceanographic Engineering \\ on September, 1991, in partial fulfillment of the \\ requirements for the degree of \\ Doctor of Philosophy
}

\begin{abstract}
A general discussion of possible techniques for observation of near-surface currents indicates that the surface-following frame of reference will provide several advantages over the Eulerian or Lagrangian frames. One problem with surface-following measurements is the biasing effects of the waves. A technique for making unbiased measurements is developed. This technique requires that both the sensor velocity and the fluid velocity be measured. A sensor platform, the Surface Acoustic Shear Sensor (SASS), which makes the required measurements is described.

The processing scheme for interpreting the measurements from the SASS is described at length. The data that SASS has obtained from two deployments in the Shelf MIxed Layer Experiment (SMILE) is presented. This data shows clearly that the biasing effects of waves can not, in general, be ignored. In the summary of the data we find suprisingly little shear in the downwind direction in the top $4 m$ of the watercolumn. In the crosswind direction observed, observed shear seems to be indicative of an across shelf pressure gradient and intense near-surface mixing.
\end{abstract}

Thesis Supervisor: Albert J. Williams, III

Title: Senior Scientist, Woods Hole Oceanographic Institution 


\section{Acknowledgments}

This thesis is dedicated to the memory of my father, Teuvo Juhani Santala. Twenty-two years after him, I too receive my doctorate from M.I.T.

I would like to thank the members of my thesis committee; Arthur B. Baggeroer, Robert C. Beardsley, Eugene A. Terray, Robert A. Weller and Albert J. Williams, III; for serving on my committee. Particular thanks must go to Albert J. (Sandy) Williams, III and Eugene A. (Gene) Terray. Through Sandy's guidance I received a master degree in 1985, and then was introduced into experimental oceanography with the construction and deployment of the Surface Acoustic Shear Sensor (SASS). Many of the ideas presented in this thesis came to fruition after discussions with Gene; his willingness to freely exchange ideas has been a large factor in the growth of my scientific capability.

The work on the SASS buoy was a multiperson effort. Besides the overall direction of the project by Sandy, a great deal of the reason the SASS made it into the ocean were the efforts of Chris Dunn and Ellyn Montgomery. Steven Lentz and Carol Alessi were both helpful in making the $\mathrm{C} 3$ mooring data accessible and understandable to me.

Mark Grosenbaugh has been both a good friend, counselor and tennis partner. Andy Trivett and Judy White are two people who, throughout my years at Woods Hole, have helped to preserve my sanity through their gracious friendship.

I thank Deep-Sea Research for granting permission reprint and paraphase portions of Santala and Terray (1991) (found in Chapter 3). Financial support for my work was from NSF grant OCE-87-16937. 


\section{Contents}

1 INTRODUCTION 7

2 MEASUREMENT TYPES 10

2.1 Lagrangian Measurements . . . . . . . . . . . . . . . . 10

2.2 Eulerian Measurements . . . . . . . . . . . . . . 16

2.3 Surface Referenced . . . . . . . . . . . . . . . . . . 21

3 WAVE BIAS $\quad 25$

3.1 Measurements from a moving sensor . . . . . . . . . . . . 25

3.2 Estimation of the bias ........................... 31

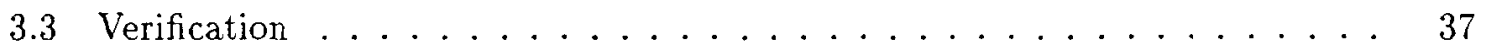

4 THE SURFACE ACOUSTIC SHEAR SENSOR (SASS) 42

4.1 Design of SASS . . . . . . . . . . . . . . . . . 42

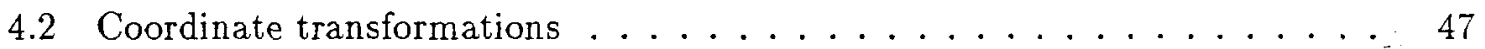

4.3 Estimation of directional spectra ................ 56

5 MAKING MEASUREMENTS FROM THE SASS

5.1 Deployment history . . . . . . . . . . . . . . . . 64

5.2 Processing strategy . . . . . . . . . . . . . . 68

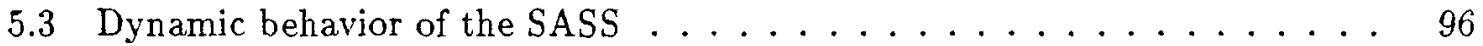

5.4 Errors . . . . . . . . . . . . . . . . . . 103

6 RESULTS $\quad 126$ 
6.1 The $\mathrm{C} 3$ mooring $\ldots \ldots \ldots \ldots \ldots \ldots \ldots$

6.2 The data ............................. 130

7 DISCUSSION $\quad 210$

7.1 Summarizing the data ........................ 210

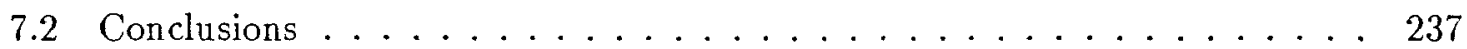

$\begin{array}{ll}\text { A } \mu \text {-FILTER INTEGRALS } & 239\end{array}$

B PARAMETERIZATION OF DRAG 242

C BASS FLOW DISTURBANCE 245

D WAVE DECAY 253

E COMPASS SENSITIVITY 262

F VERTICAL VELOCITIES $\quad 272$ 


\section{Chapter 1}

\section{INTRODUCTION}

In this thesis I will examine the nature of the mean shear current in the upper $20 \mathrm{~m}$ of the ocean. To a great extent the viewpoint taken will be observational. But, we will find that an appropriate observation can not be made without a broad theoretical understanding of the dynamics that govern the near-surface environment. Chapter 2 will be entirely devoted to a discussion on which frame of reference is the most appropriate (and practical) to make our measurements. We will find that a surface-following frame of reference provides some distinct advantages over the others proposed. In Chapter 3 an extended study of the wave bias - one of the outstanding problems associated with making upper-ocean current measurements - is undertaken. It is the fact that the measurements to be made are near a moving boundary that requires that our observation technique be discussed so carefully.

The dynamic problem that the current measurements will address directly is that of the form and size of the wind-induced shear current. The measurements will be time averaged over periods on the order of thirty minutes to obtain mean current profiles (the thirty minutes averaging was selected so as to be long enough to average out the wave signals but short enough so as to obtain a stationary sample of the current). $\mathrm{Wu}(1969,1975,1983)$ has emphasized some very practical consequences of the mean drift currents set up by the wind stress; the dispersion of man-made pollutants in the ocean and the hydrodynamic loading of offshore structures. Beyond these obvious direct implications, there exists a variety of diverse problems which will benefit from a more accurate understanding of near-surface 
currents. For example, the efficiency of the air-sea momentum exchange, which is deduced from the shear current profile, is an important input parameter for the modeling of large scale flows in both the oceans and atmosphere. On a smaller scale, the mixing processes which govern the momentum transfer also transport small marine organisms and thus effect biological productivity.

Studies of the near-surface currents have been made many times in both the laboratory and the ocean. With the SASS (Surface Acoustic Shear Sensor), a newly developed instrument platform discussed in Chapter 4 , we will be able to make unbiased measurements of the currents at distances as small as $1 m$ away from the ocean's surface. The SASS essentially consists of a vertical array of acoustic current meters and a motion sensing package. In addition to making measurements of the current, we are able to estimate the directional spectrum of the wavefield from the sensors mounted on the SASS. In the final analysis, the wave measurements provided invaluable information in the data interpretation. Not only is this data used to estimate the wave bias, it is used to interpret the instrument performance and to provide complementary environmental data to the current records. The SASS was deployed off the coast of northern California as part of SMILE (Self MIxed Layer Experiment). The other component of the SMILE program of interest here is a current meter mooring which was named the $\mathrm{C} 3$ mooring. This mooring provides windspeed and current meter data which will be essential in the final interpretation of the SASS data. That we need so much information besides just the near-surface current records to understand the shear current in the upper ocean is not a surprise when we consider dynamics which give rise to the shear current.

The driving force behind the near-surface shear current is the wind. Momentum is transferred from the wind to the water either by pressure forces or through viscous "skin-friction" forces. The momentum that is transferred through pressure forces must undoubtedly go into wave generation (Stewart, 1961). Through wave breaking (Melville and Rapp, 1985; Mitsuyasu, 1985), dissipation of waves (Csanady, 1984) or some other mechanism, some of the wave momentum may be transferred to the shear current. Momentum transferred 
by skin-friction may enter directly into the shear current or may enter the shear current indirectly via highly dissipative wavelets (Csanady, 1985; Okuda, 1982).

Whatever the input path of momentum from the air into the current may be, it then diffuses downward into the the deeper ocean. It is widely believed that the diffusion of momentum down from the surface is well described by the same dynamics which govern the boundary layer flow relative to a flat plate. In this view, the shear stress in the fluid is assumed to be constant. Coupled with the assumption that there exists an eddy viscosity which varies linearly from the boundary, one arrives at the the familiar "log-law of the wall". Csanady (1984) succinctly states the assumptions under which this may be done and supports his conjecture with a compilation of field data from various investigators. Because the log-layer has become something of a paradigm for near-surface momentum transfer, and Csanady's 1984 paper seems to well summarize why this is so, I will frequently reference this paper.

Not all measurements agree with the log-layer model. For instance, Kitaigorodskii et al (1983) have found dissipation rates in the near-surface which are inconsistent with a constant stress (log-law) model. Gordon (1970) has argued that if Langmuir circulation is present then the helical trajectories which define this flow pattern will act as a very efficient mechanism by which momentum can be transferred to the deeper flow. In his review of Langmuir circulation, Pollard (1977) notes that the theoretical understanding of the mechanisms which give rise to Langmuir circulation are still poorly understood. He concludes, nonetheless, that if Langmuir circulation is present, it will totally dominate the downward flux of horizontal momentum.

The data obtained from the SASS and C3 buoys is plotted out file-by-file in Chapter 6 . When the results are summarized in Chapter 7 we will find that at great depths (deeper than $10 \mathrm{~m}$ ) a region reasonably well described as a constant stress layer may exist. It is concluded that the form of the shear current in the upper $10 \mathrm{~m}$ must be a consequence of intense near-surface mixing. 


\section{Chapter 2}

\section{MEASUREMENT TYPES}

\subsection{Lagrangian Measurements}

The Lagrangian frame of reference is one in which we study the trajectories of an individual fluid particle. In a sense, this is the natural frame of reference in which to study mass transport. It is not, perhaps, the best frame in which to understand the physics of air-sea momentum exchange. Consider that we can roughly break the total mass transport into three contributions (not necessarily independent contributions!): (1) the wind-induced shear flow, (2) the wave-induced flow (Stokes' drift), and (3) the other contributions. Other contributions being pressure gradients, the coriolis force, bottom friction effects etc. Lagrangian techniques measure all three contributions. Theoretical problems arise with the Lagrangian techniques when we seek to understand the physics governing the flow. Because to do this it is necessary to understand what the relative contributions from each component are.

This is one reason why Lagrangian techniques are well suited to the laboratory. In the laboratory the factors giving rise to each parameter can, in theory, be independently varied ("other contributions" always being a problem). In fact, in the laboratory the wave-induced drift current can, in general, be ignored. This is because the waves generated in tanks are generally so small (due to fetch limitations) that the wave contributions are usually much smaller than the those due to the wind. For instance, $\mathrm{Wu}(1975)$ found that in his $22 \mathrm{~m}$ long 
wave tank that the Stokes' transport at the surface was only $10 \%$ of the total transport (wind speeds varied from $2 \mathrm{~m} / \mathrm{s}$ to $14 \mathrm{~m} / \mathrm{s}$ ). In subsequent analysis he therefore ignores wave drift. However, he does estimate that for fetches on the oceanic scale that the wave-induced transport will exceed the wind-induced transport. In Figure 2-1 I have reproduced the drift contributions for a fully developed sea where the $10 \mathrm{~m}$ windspeed, $U_{10}$, is $10 \mathrm{~m} / \mathrm{s}$ using Wu's expressions. It is important to recognize that the estimates do not include nonlocally generated swell.

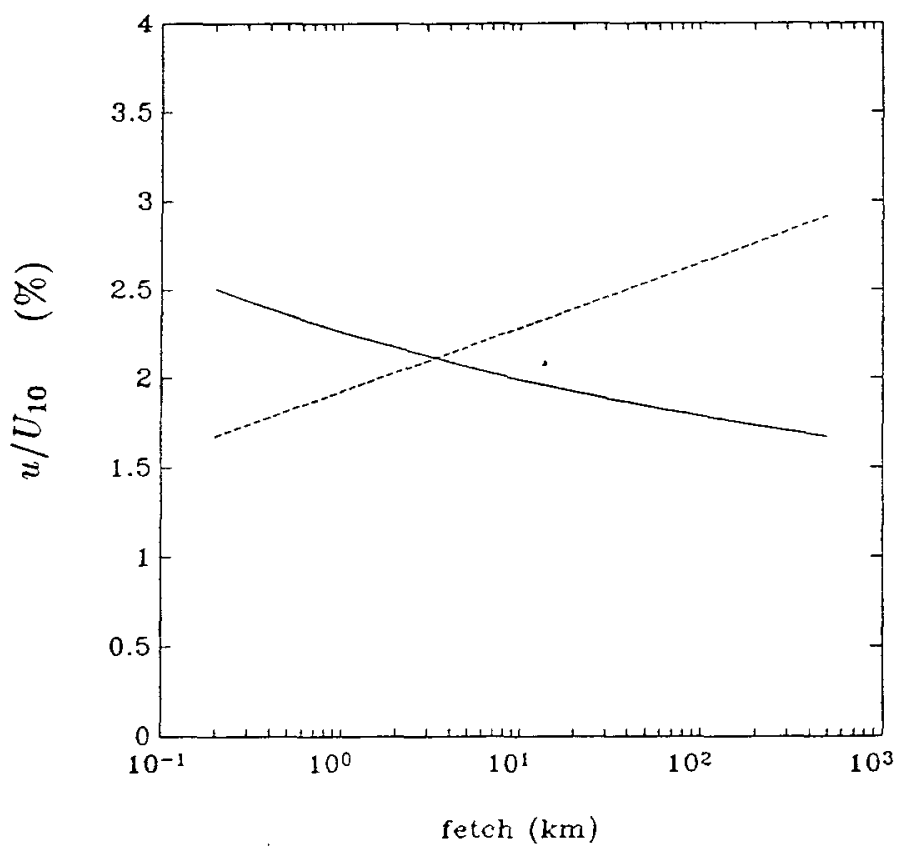

Figure 2-1: Wind-induced (-) and wave-induced (- - ) contributions to surface drift as a function of fetch for $U_{10}=10 \mathrm{~m} / \mathrm{s}$. Calculated using equations from $\mathrm{Wu}(1975)$.

Of course, if it is truly the air-sea momentum exchange that is of concern to us then the magnitude of the current at the surface is of little relevance. It is the magnitude of the mean near-surface shear that indicates the flux of momentum. In a deep water setting, it is usually only the wind-induced and wave-induced contributions that lead to depth dependent flows near the air-sea interface. Ideally, we would attempt to develop a plot similar to Figure 2-1 for the magnitude of the shear instead of the magnitude of the current. Here 
we'll settle for a bit less. The more modest goal set here is to determine whether or not it is possible to ignore wave-induced drift in interpreting Lagrangian measurements.

To examine the relative importance of the wave-induced drift we will again look at conditions expected with a windspeed of $U_{10}=10 \mathrm{~m} / \mathrm{s}$. If atmospheric conditions are neutral then a reasonable drag coefficient might be $C_{D}=1.2 \times 10^{-3}$. This implies that the air friction velocity $u_{*_{a}}$ is $34.6 \mathrm{~cm} / \mathrm{s}$. If the stress in the fluid were equal to the stress in the air we would have that the water friction velocity $u_{*_{w}}$ would be equal to $0.0355 u_{*_{a}}$. But, because some of the wind stress is supported by wave drag, the shear in the fluid is less than that in the air and we have that:

$$
\rho_{w} u_{*_{w}}^{2}<\rho_{a} u_{*_{a}}^{2}
$$

Let's just stick with Wu's numbers and say $u_{*_{w}}=0.0241 u_{*_{a}}=0.83 \mathrm{~cm} / \mathrm{s}$. If we assume that the logarithmic profile does indeed describe near-surface flow then we assume a profile of the form

$$
\frac{u(z)}{u_{*_{w}}}=\frac{1}{\kappa} \log \left(\frac{z}{r}\right)+8.5,
$$

and the slope of the velocity defect is determined solely by the friction velocity $u_{*_{w}}$. We assume the Von Karman's constant $\kappa$ is constant and has a value of 0.4. The roughness scale $r$ only affects the profile's offset. We'll choose a value of $r$ typical for modest windspeeds and let $r=100 \mathrm{~cm}$. The velocity defect for the log-profile with these parameters is shown as the solid line in Figure 2-2. On February 27, 1989, the SASS recorded data in conditions of neutral stability and $U_{10}=10.2 \mathrm{~m} / \mathrm{s}$ (SASS file o1). The Stokes' drift for that case was computed from the measured directional wave spectrum. The combined theoretical wind-induced and measured wave-induced current profile is plotted in Figure 2-2 as a dotted line. The Stokes' drift calculation was cut-off at wave frequencies of $1 \mathrm{~Hz}$, the highest frequency to which the SASS estimated the spectra. The message should be clear; if true Lagrangian drifters were released on February 27, then according to our computations, the greater portion of the shear measured would be due to wave effects and not wind effects. For our purposes then, wave effects are not negligible. In the past, some investigators have chosen to ignore wave effects. It may be that in fetch-limited or young wavefields, that most 
of the wind-stress is supported by the shear current and that the wave-induced current is in fact negligible. Here we only show that this is: (a) not always the case and (b) will probably never be the case with the SMILE data, where large, nonlocally generated swell was always present.

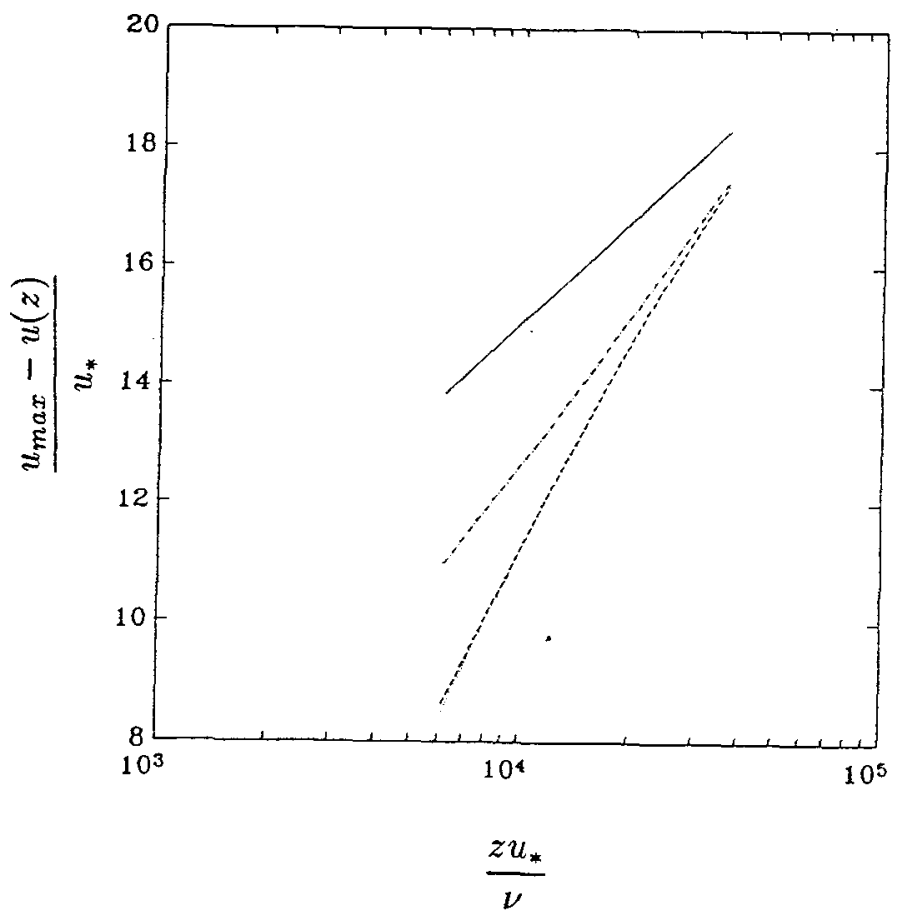

Figure 2-2: Surface defect velocity using a theoretical form for the wind-induced current $(-)$. When the total drift current is predicted $(\cdots)$ the shear is approximately twice as great. The total drift was computed from actual SASS measurements of waves (file 01) when the windspeed was $10 \mathrm{~m} / \mathrm{s}$. The sensitivity to high frequency waves was gauged by cutting off the Stokes' drift computation at $0.50 \mathrm{~Hz}(-$ - ) and at $0.25 \mathrm{~Hz}(-\cdots .$.$) . Scaling the$ distance using the molecular viscosity $\nu$ is the traditional wall-layer approach.

The cut-off frequency to which the Stokes' drift should be calculated is anything but a resolved issue. In Figure 2-2 the wave-induced drift was computed for three different cut-off frequencies; $1.0 \mathrm{~Hz}, 0.5 \mathrm{~Hz}$ and $0.25 \mathrm{~Hz}$ (wavelengths longer than $160 \mathrm{~cm}, 620 \mathrm{~cm}$ and $2500 \mathrm{~cm}$, respectively). Note, however, that because of the scaling of the problem, the computation cut off at $0.5 \mathrm{~Hz}$ gives about the same result as the computation done out to $1.0 \mathrm{~Hz}$. This is because the shallowest depth shown on the plot corresponds to $100 \mathrm{~cm}$. Since the wave spectrum falls off at higher frequency and the shorter waves decay rapidly with depth, the 
waves of frequency $0.5 \mathrm{~Hz}$ to $1.0 \mathrm{~Hz}$ make almost no contribution to the wave drift at depths $100 \mathrm{~cm}$ or more. The waves of frequency $0.25 \mathrm{~Hz}$ to $0.5 \mathrm{~Hz}$ definitely make a contribution. But, even considering only waves of frequency below $0.25 \mathrm{~Hz}$, the shear measured by a Lagrangian drifter would still be approximately twice the wind-induced shear. Though there is some dispute as to where to cut off the Stokes' calculation, most investigators would probably not argue that there is a wave-induced contribution from waves up to frequency $0.5 \mathrm{~Hz}$. What is in doubt is whether or not the types of drifters routinely deployed would measure their contributions.

The near-surface environment is often well mixed. With no density gradient, it becomes necessary to use drogue-type drifters. In this case there is a small surface float to which a drogue is attached via a line. The drogue may take different forms but the area of the drogue is usually large compared to the surface float. This geometry is necessary to ensure that the drifter follows the fluid at depth rather than the fluid at the surface. There are several consequences to using this type of drifter. First of all, if the drogue is set to a fixed depth, then the follower is not Lagrangian in that fluid particles do not remain at a constant depth beneath the air-sea interface. Csanady (1984) talks about wind loading and the effects of wave breaking on floats with surface expression. One thing that generally remains ignored, is that these types of floats have non-negligible size. This is true particularly when one considers the length scale from float to drogue. We usually assume that drifters measure both the wind- and wave-induced drift. If the motions of the drogue are not correlated to the higher frequency motions of the fluid then it will not measure the Stokes drift at those frequencies. Therefore, even if waves of frequency $0.25 \mathrm{~Hz}$ to $0.5 \mathrm{~Hz}$ do contribute to the Stokes' drift, if all the factors leading to float "slipage" cause the drifter motion to be imperfectly correlated with the fluid at higher frequencies, the drifter will not measure the wave drift at those frequencies. Suppose that a float is constructed so that it follows waves of frequency $0.25 \mathrm{~Hz}$ or lower perfectly but above this frequency, the motions are essentially uncorrelated. Then, the measured drift would be given by the $0.25 \mathrm{~Hz}$ cut-off curve of Figure 2-2 irrespective of how much the higher frequency components contribute. There are then two distinct questions. First, "To what frequency do waves contribute to 
the Stokes' drift ?" And second, "To what frequency does a drifter measure Stokes' drift ?" The second question implies that drifters need to be carefully calibrated. Calibrations of drifters for near-surface floats are crude. Both Niiler et al (1987) and Geyer(1989) have attempted to calibrate drougued drifters. However, these drifters were typically much larger than those we would find useful in the upper $5 \mathrm{~m}$ of the ocean. In fact, Niiler's approach was to try to mechanically decouple the drogue from the wave motion and hence to attempt to avoid measuring the Stokes' drift at all.

There is also the question of what the drifter is following. When releasing the drifters, if the floats diverge, then as the distance between floats becomes larger there arises the question of if the results from the two drifters can be compared. Spatial convergence may be a problem of equal difficulty. Drifters probably seek regions of convergence (Richman et al (1987)). Convergence zones due to Langmuir cells can have wildly different flow characteristics than that of the surrounding fluid field. If floats are released and converge into a down-welling zone then the resulting drift measurements are probably not representative of the overall flow field characteristics.

Deployment of drifters can also be troublesome. Tracking of drifters in a laboratory environment is simplified by the two dimensional nature of the flow and the ease with which subsurface drifters can be spotted in a glass-walled tank. Further, conditions can always be repeated so that multiple runs can be averaged over. These luxuries are not available to the field experimenter. Tracking schemes can be somewhat elaborate (see Churchill and Csanady(1983)). Tracking is usually limited to a relatively small number of buoys.

So, while the drifter approach remains popular due to the relative ease with which an experiment can be conducted, an accurate drifter study involves much more than throwing some floats over the side of a boat. Perhaps most difficult is interpretation of drifter results. Churchill and Csanady(1983) measured, in low wind-speed and high swell cases, anomalously large shears. This they attributed to wave effects. The real question we must now keep in the back of our minds is that, since most field observations of log-layer results 
have been performed with drifters, to what extent are of those results measurements of the wind-induced shear current and to what extent are those results measurements of the depth varying wave-induced drift.

\subsection{Eulerian Measurements}

Eulerian measurements are observations of time series made at fixed points in space. Therefore, sensor motions are not a concern. There do arise, however, many other measurement issues in this frame of reference - especially when making measurements in the upper ocean. The most important issues are: deployment problems, flow disturbance, and advection of the flow field.

Clearly, fixing a probe in a laboratory tow tank is a trivial problem. What is not so clear is how one is to fix probes in the deep ocean. In intermediate depths, lake measurements have been made by Donelan(1978) and others in Lake Ontario at the CCIW tower in water of $12 \mathrm{~m}$ depth. Cavaleri and Zecchetto(1987) have made tower measurements in $16 \mathrm{~m}$ of water; their tower was located in the Adriatic Sea. Shonting $(1968,1967)$ made measurements in $20 \mathrm{~m}$ of water in Buzzards Bay, Massachusetts. Other measurements have undoubtedly been made in these relatively shallow, $O(10 \mathrm{~m})$, depths. There is, however, a decided problem in erecting a stable platform in deep water. The SMILE study was to be made in $90 \mathrm{~m}$ of water and, hence, it was impracticable to build a tower specifically for this application. If choice of site is unimportant to a study then fixed platforms can be found in waters much deeper than $90 \mathrm{~m}$; e.g., the Bullwinkle oil production platform, owned by Shell Oil, is located in the Gulf of Mexico in $412 \mathrm{~m}$ of water. Oil platforms are not, of course, designed with a view towards making oceanographic measurements. The flow disturbance in the vicinity of these platforms makes their potential use of limited value.

The flow disturbance problem is the first concern with tower based measurements. A tower for oceanographic measurements must be strong enough to suffer the loading of the wave environment and at the same time must have as little structural elements as possible 
to minimize the flow disturbance. In normal situations this compromise of design cannot be fully achieved. Usually, an investigator making tower based measurements mounts his instruments such that they are minimally disturbed by wake effects in prevailing conditions. In some cases, as conditions change, instruments might be moved or steered so that they are not downstream of any wake generating members. Even in such cases, there is cause for caution.

The first concern is that in a wave environment that the orbital velocities will, even when sensors are placed downstream from structural elements, pull the wake back across sensors during the return flow. In a typical deployment from some of the smaller towers, $O(10 \mathrm{~m})$ depth, it would not be unusual to experience $30 \mathrm{~cm} / \mathrm{s} \mathrm{rms}$ velocities and wavefields with peak periods of 3 seconds. In such a case, the rms excursion of the wave velocity is about $29 \mathrm{~cm}$. Clearly, there is no difficulty in using booms to mount instruments outside this advection range even under more harsh' conditions. There will still be the wake of the instrument itself to contend with. The instrument wake problem is troublesome but is not peculiar to the Eulerian measurement frame.

In measurements of currents from towers it is predominately the wake effects which are of concern to us. In studying wave effects from towers, the problem of reflected waves should also be considered. The potential function for a wavefield, $\Phi$, in the vicinity of an obstruction can be written as the sum of an incident potential, $\Phi^{I}$, and a scattered potential, $\Phi^{S}$, as

$$
\Phi=\Phi^{I}+\Phi^{S}
$$

In radial coordinates, the potential for a monochromatic wave may be expressed as

$$
\Phi^{I}=\Phi_{\circ}(z, t) e^{\imath k r \cos \theta}
$$

In addition to satisfying the free surface kinematic and dynamic boundary conditions and the bottom boundary conditions, if there is a cylindrical obstruction in the fluid then, the total potential, $\Phi$, must satisfy

$$
\frac{\partial \Phi}{\partial r}=0
$$


on the cylinder surface $r=b$. Moreover, the scattered wave must satisfy the Sommerfield radiation condition

$$
\lim _{r \rightarrow \infty} r^{\frac{1}{2}}\left(\frac{\partial \Phi^{S}}{\partial r}-\imath k \Phi^{S}\right) \rightarrow 0 .
$$

When we solve for the scattered potential, subject to the above constraints we find a scattered potential of the form

$$
\Phi^{S}=\frac{\pi}{2}(k b)^{2} \Phi_{o}(z, t)\left[-\frac{\imath}{2} H_{0}(k r)-H_{1}(k r) \cos \theta\right]+O\left[(k b)^{3} \Phi_{o}\right]
$$

where the $H$ 's are Hankel functions. The most important features to note of the scattered potential are the scaling of the potential, $(k b)^{2}$, and that the scattered waves propagate in all directions (though there is an azimuthal dependence). Further note that the scattered potential falls off on the length scale of the wave, (i.e. $\left.\Phi^{S}=\Phi^{S}(k r)\right)$. This is in contrast to flow separation effects, whose decay is usually scaled by the diameter of the flow obstruction (intensity of disturbance $\sim \frac{r}{b}$ ). The consequence is that the scattering effect is felt even at relatively large distances from the tower. It is true that the scattered potential may be minimized by reducing the radius of the tower support $b$ but consider that even though the scattered potential is scaled by $(b k)^{2}$, the waves will interact with the first order incident waves. Calling the incident wave amplitude $a$, and the radian frequency $\sigma$, the velocities of the incident wave scales as $a \sigma$. The velocities of the radiated potential scales as $a b^{2} \sigma k^{2}$. For $50 \mathrm{~cm}$ amplitude waves with radian frequency $\sigma=1 \mathrm{rad} / \mathrm{s}$ scattering off a cylinder of radius $b=50 \mathrm{~cm}$, the Reynolds stress $\tau$ due to the resulting standing wave components is $\tau=\frac{1}{2} \rho u w^{*} \sim \rho a^{2} b^{2} \sigma k^{2}=O\left(1 N / m^{2}\right)$. These waves are much smaller than those we looked at in the last section. Even so, for the wind speed examined $(10 \mathrm{~m} / \mathrm{s})$, we expected a wind-induced shear stress, $\tau=\rho u_{*_{w}}^{2}$, of only $0.07 \mathrm{~N} / \mathrm{m}^{2}$. This effect may therefore entirely mask the wind-induced shear stress when measurements are made via a velocity correlation.

In higher windspeed conditions $(12 \mathrm{~m} / \mathrm{s}$ to $17 \mathrm{~m} / \mathrm{s})$ than those we have just discussed, Cavaleri and Zechetto (1987) used a theoretical model to predict a wind stress of approximately $0.3 \mathrm{~N} / \mathrm{m}^{2}$. They measured shear stresses in the fluid, using velocity correlations, as large as $30 \mathrm{~N} / \mathrm{m}^{2}$. The authors considered several sources of error but did not consider wave scattering from the tower. Using a computer model, and inputting a spectrum of waves, I 
found that stresses as large as $3 \mathrm{~N} / \mathrm{m}^{2}$ could be explained solely in terms of waves scattered from the single tower leg closest to the sensors. Obviously, inclusion of the entire support structure and a more accurate wave model would lead to different results. The point is, whether or not Cavaleri and Zechetto have found some unknown physical mechanism, as they seem to claim; it is doubtful that stresses of order $0.3 \mathrm{~N} / \mathrm{m}^{2}$ could be measured using velocity correlations so near a wave scatterer without carefully considering these effects.

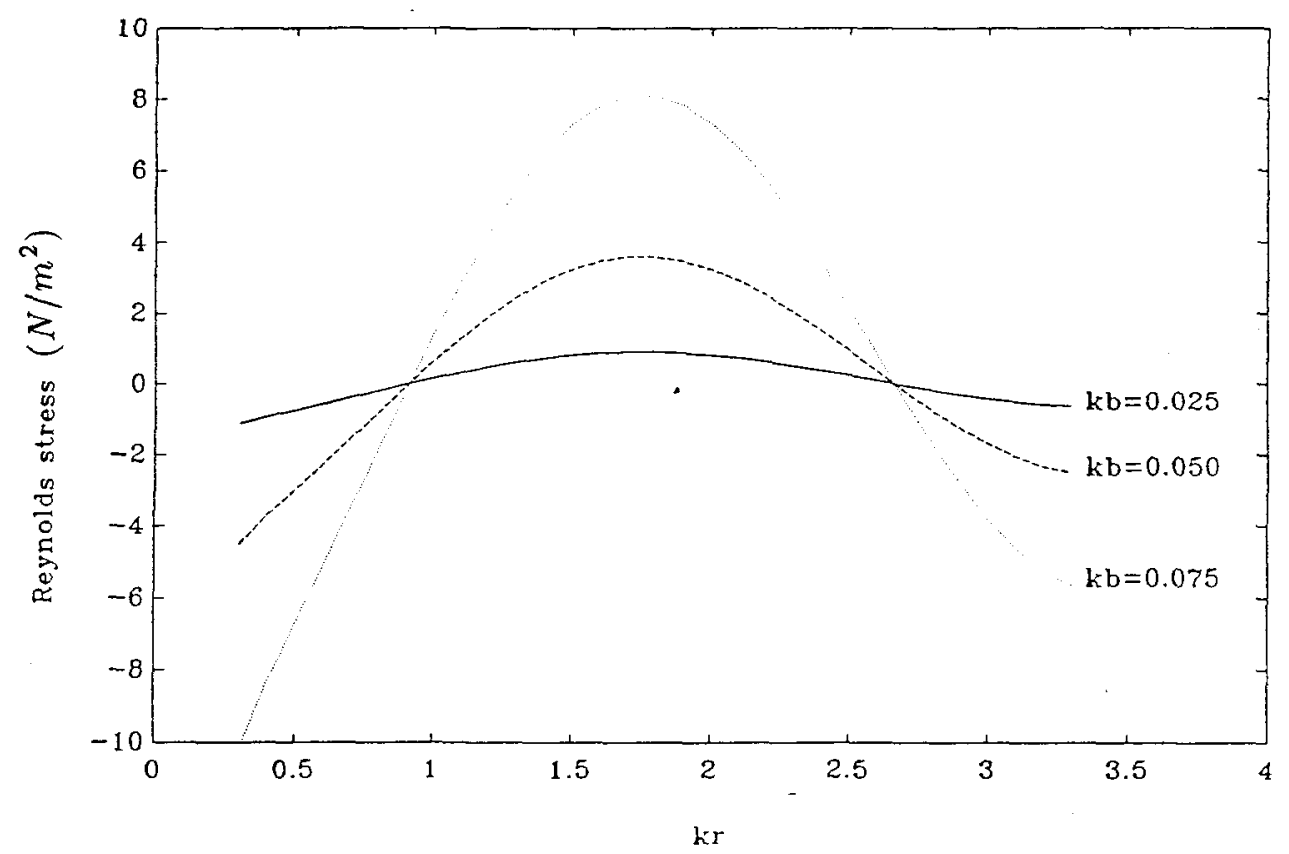

Figure 2-3: Reynolds stress due to a single monochromatic wave scattered from a circular cylinder. The incident wave amplitude is $1 \mathrm{~m}$, the radian frequency is $1 \mathrm{rad} / \mathrm{s}, r$ is the upwave radial distance from the scatterer and $k$ is the wavenumber. The result is plotted for three different cylinder radii, $b$.

Figure 2-3 shows the effect of wave scattering from a single wave as a function of radial distance from a tower. Only the upwave direction (the direction sensors would be steered to avoid flow separation effects) is shown. Obviously, the field experimenter must worry about a spectrum of incident waves. For a more detailed discussion on the derivation of scattered potential, Mei(1989) provides an excellent discussion. 
The last issue is that of the appropriateness of making an Eulerian measurement near the air-sea interface. The standard log-law model for wind-induced shear (Csanady, 1984) presumes that the free-surface is analogous to the wall in the classic wall-flow type experiment. In his paper, one of the central questions Csanady tries to answer is that of what to call the wall. Though subsequent investigators have refined the theory, Van Dorn's (1953) conclusion that most of the drag on the wind over water is due to shorter waves is still generally held to be true. It is supposed that the wind stress is supported by the shorter waves and longer waves grow through nonlinear wave-wave interactions. The growth of the shear current may be due directly to "skin-friction" and also due to the vorticity of the smaller waves. The longer waves, however, are nearly irrotational and, Csanady asserts, are dynamically unimportant with respect to the shear current except in that the shear current is advected by the orbital motions of the long waves. The wind-induced shear current is, from this point-of-view, the steady component of the current relative to the surface that is defined by the longer waves (Csanady argues that waves of wavelength $1 \mathrm{~m}$ or greater could be considered "long"). How does this affect near-surface Eulerian measurements? In small fetch situations the effect of shear flow advection is minimal because the energy in longer waves is such that the advection is small compared to the decay scale of the shear current. For the type of waves we encounter at the SMILE site the boundary layer advection would be serious.

To make an Eulerian measurement, our shallowest sensors should be located beneath the troughs of the waves to avoid exiting the water. The significant waveheights $\bar{H}_{\frac{1}{3}}$ measured by the SASS during its deployment varied from $1.6 \mathrm{~m}$ to $3.0 \mathrm{~m}$. The waveheight spectra tended to be heavily weighted by swell. Let's examine the implications of wave advection by considering a wavefield where $\bar{H}_{\frac{1}{3}}=2 \mathrm{~m}$. Here, our shallowest sensors would have to be located more than a meter below the mean free-surface to avoid exiting the water. Suppose we put our shallowest sensor $2 m$ below the mean free-surface. The boundary layer would be advected $\pm 1 \mathrm{~m}$ relative to this sensor. If the windspeed is $10 \mathrm{~m} / \mathrm{s}$ and we again assume that $u_{*_{w}}=0.83 \mathrm{~cm} / \mathrm{s}$, then the gradient of the wind-induced shear current would be given 
by

$$
\frac{\partial u}{\partial z}=\frac{u_{*_{w}}}{\kappa z}=\frac{2.075 \mathrm{~cm} / \mathrm{s}}{z}
$$

At a distance of $2 m$ from the boundary we expect to find a shear of about $0.0104 \mathrm{~s}^{-1}$. As the boundary layer advects up and down, we expect our sensor to measure shears which vary from $0.0069 s^{-1}$ to $0.0208 s^{-1}$. Hence, the variation in shear is about $30 \%$ greater than the mean shear we expect to measure. It seems logical that to improve our resolution, we need to make our measurements from sensors which follow the surface. Moreover, in a surfacefollowing mode, sensors may be placed very close to the boundary with little danger of exiting the water. This type of quasi-Lagrangian surface-following measurement, typically made from a buoy will be introduced in the following section.

Before moving on to discuss quasi-Lagrangian measurements, however, we mention a special case of what might be aptly termed a quasi-Eulerian measurement. In the case of the research vessel FLIP, the notable spar buoy, measurements may be made which are nearly Eulerian. Even with its enormous size $(\mathrm{draft} \approx 91 \mathrm{~m})$ the FLIP does move. But, with pitch and roll resonant frequencies of $0.021 \mathrm{~Hz}$ and a heave resonance of $0.037 \mathrm{~Hz}$ (Rudnick, 1967), we don't expect the coupling between the wave and buoy motions to be strong. Though the FLIP does respond to low frequency motions, it can be steered (see Weller, 1985 ) to keep current meters upstream of the hull wake. Furthermore, the fact that FLIP drifts with the large scale flows would further reduce flow disturbance effects (our earlier remarks about scattered waves need be considered; FLIP's hull diameter is $3.8 \mathrm{~m}$ at the surface and gradually increases to $6.1 \mathrm{~m}$ ). A FLIP type platform would not, in any case, have been appropriate at the SMILE site, only about $5 \mathrm{~km}$ from shore, in a strong current environment and having a water depth of only $90 \mathrm{~m}$.

\section{3 $\quad$ Surface Referenced}

Usually, when solving a fluid mechanics problem, we attempt to find our solution using either a Lagrangian or an Eulerian approach. Each approach has its advantages for studying 
near-surface dynamics. The Lagrangian measurement is a measure of total transport and is a conceptually easy experiment to design. For the Eulerian measurement wave effects are not an issue and, in tower-based measurements, the experimenter usually has the equipment overhead (e.g., power supplies and data storage capabilities) necessary to make extensive measurements.

But, as we discussed in the last two sections, each approach has its problems too. It is difficult to make a truly Lagrangian measurment near the air-sea interface. Also, the tracking problems associated with Lagrangian measurements make collection of meaningful amounts of data difficult. The Eulerian approach is often not possible in deep water. And, even if a deep water tower can be found or constructed, there remains the problem of the boundary layer advecting past the sensor.

The surface-following sensor might be seen as a compromise between the Eulerian and Lagrangian approaches. Our archtypical surface-following probe is a current meter hung beneath a buoy. Using this approach is consistent with the idea that the near-surface boundary layer is advected with the longer waves of the sea surface (Csanady, 1984). While making a boundary layer measurement it makes sense to use the boundary as a reference point. Buoy systems are relatively easy to deploy and can be left unattended.

The surface-referenced measurement is not a panacea. One of the better known difficulties with this approach is the fact that the measurements are biased in the downwave direction. Pollard (1973) developed a theory to explain the "wave bias" in terms of linearized potential theory. Here I'll repeat the simplest form of the argument Pollard gave as an introduction to the wave bias.

For a monochromatic wave, the surface elevation $\eta$ and horizontal velocity $u$ may be written as

$$
\begin{aligned}
& \eta=a \cos (k x-\sigma t), \\
& u=a \sigma \cos (k x-\sigma t) e^{k z} .
\end{aligned}
$$


If the buoy heaves with the surface, but does not move horizontally, then the motion of a sensor mounted a fixed distance $z_{0}$ beneath the waterline of the buoy is given by

$$
[X, Z]=\left[x_{o},-z_{o}+\eta\right]
$$

The horizontal velocity measured by the sensor is

$$
U=a \sigma \cos \left(k x_{0}-\sigma t\right) e^{k\left[-z_{0}+a \cos \left(k x_{0}-\sigma t\right)\right]}
$$

Expanding the exponential gives

$$
U=a \sigma \cos \left(k x_{0}-\sigma t\right) e^{-k z_{0}}\left[1+a k \cos \left(k x_{0}-\sigma t\right)+\cdots\right]
$$

and the resulting nonzero time average

$$
\bar{U}=\frac{1}{T} \int_{0}^{T} U d t=\frac{1}{2} a^{2} \sigma k e^{-k z_{0}}
$$

is the "wave bias." The wave bias for our surface following sensor is seen to have a form similar to the theoretical form of the Stokes' drift. In the next section we'll show that the derivation of the wave bias and the Stokes' drift can be simply related. Here, I'd like to show what type of effects this bias might have on the data. Figure 2-4 shows the expected shear current in a $10 \mathrm{~m} / \mathrm{s}$ wind (the conditions are assumed to be the same as those for Figure 2-2). Also shown are the indicated shear for a perfect wave-follower and the indicated shear when the bias is that measured by the SASS. 


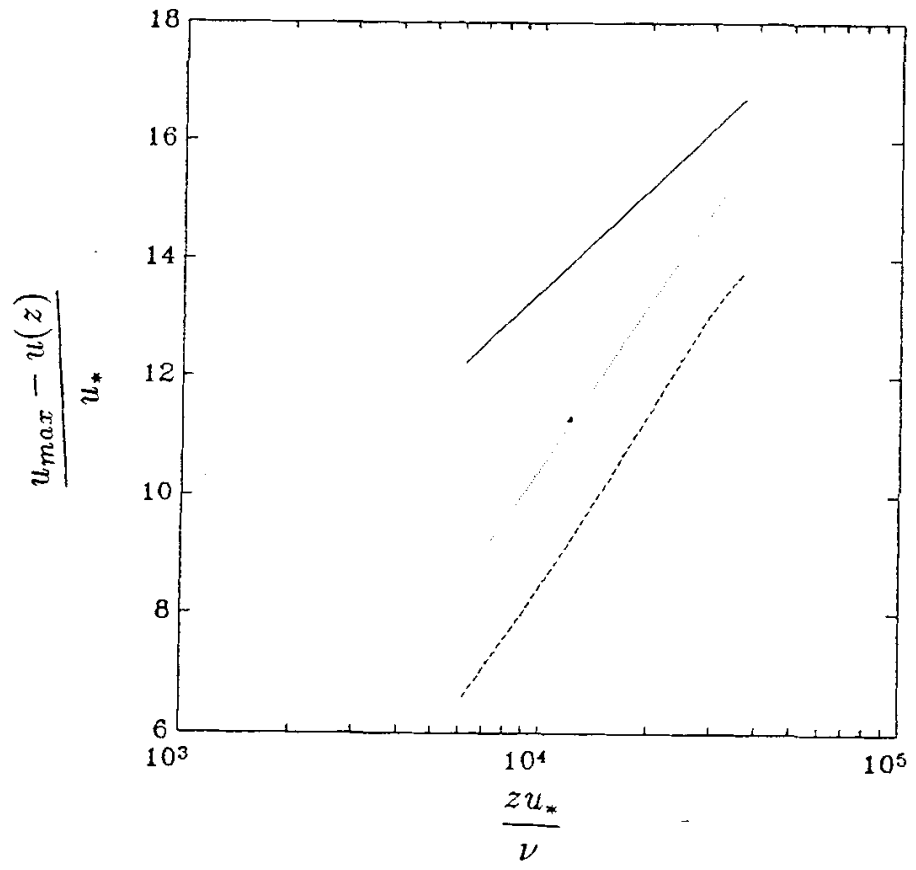

Figure 2-4: Surface defect velocity (-) using a theoretical form for the wind-ind uced current in a $10 \mathrm{~m} / \mathrm{s}$ wind. The shear indicated from a sensor which perfectly follows the vertical excursions of the surface $(\cdots)$ includes the wind-induced and a wave-induced contribution. The SASS follows both vertical and horizontal fluid displacements. If the shear were given by solid line we expect that SASS would overestimate $(--)$ the shear if the wave-induced portion of the measurement is not considered. 


\section{Chapter 3}

\section{WAVE BIAS}

\subsection{Measurements from a moving sensor}

In the last chapter we found that there were several advantages to making near-surface measurements in a surface-following frame. We also admitted current meters which move coherently with wave orbital velocities suffer from a downwave bias. This has long been recognized and yet is a problem which the oceanographic community has still not adequately addressed. We looked at the simplest form of Pollard's (1973) analytical model to show how the bias arises. In his paper Pollard concluded that:

If the directional spectrum is known, Kenyon's(1969) technique can be used to calculate that part of $\bar{u}$ caused by vertical motion. However, even if the horizontal motion of the current meter were measured it would be difficult if. not impossible to make an acceptable estimate of the error caused by horizontal motion.

Nonetheless, Santala and Terray(1991) developed a theory wherein the bias due to both vertical and horizontal motion could be estimated with reasonable accuracy. Before developing this theory in a form sophisticated enough to be applied to field data I'd like to look again at the different measurement types, again using a single monochromatic wave, but now using complex analysis. This will allow us to look at the consequences of sensor-fluid correlations in a compact way. 
Consider a two-dimensional monochromatic wave of amplitude $a$ and frequency $\sigma$. The potential function $\Phi$ for a deep water wave $\left(k=\sigma^{2} / g\right)$ may be written as:

$$
\Phi=-\imath a \frac{g}{\sigma} e^{\imath(k x-\sigma t)} e^{k z} .
$$

The surface elevation $\eta$ and the horizontal and vertical fluid velocities $(u, w)$ are given by

$$
\begin{aligned}
\eta(x, t) & =-\left.\frac{1}{g} \frac{\partial \Phi}{\partial t}\right|_{z=0}=a e^{\imath(k x-\sigma t)}, \\
u(x, z, t) & =\frac{\partial \Phi}{\partial x}=a \sigma e^{\imath(k x-\sigma t)} e^{k z} \\
w(x, z, t) & =\frac{\partial \Phi}{\partial z}=-\imath a \sigma e^{\imath(k x-\sigma t)} e^{k z}
\end{aligned}
$$

The sensor trajectories may be described as being comprised of a mean position $\left(x_{0},-z_{0}\right)$ and a time varying component $\left(x_{e}(t), z_{e}(t)\right)$ as

$$
[X, Z]=\left[x_{o}+x_{e}(t),-z_{o}+z_{e}(t)\right] .
$$

As the sensor traces out its time history it not only measures the time history of the field velocity but also samples its spatial variability. Substituting the trajectories (3.3) into the expressions for velocity (3.2) gives the expression for the measured velocity $(U, W)$ as a function of time (without loss of generality $x_{0}$ may be set to zero)

$$
\begin{aligned}
& U(t)=a \sigma e^{-\imath \sigma t} e^{-k z_{0}}\left[1-\imath k x_{e}+k z_{e}+\cdots\right]-\frac{d x_{e}}{d t}, \\
& W(t)=-\imath a \sigma e^{-\imath \sigma t} e^{-k z_{0}}\left[1-\imath k x_{\epsilon}+k z_{\epsilon}+\cdots\right]-\frac{d z_{e}}{d t} .
\end{aligned}
$$

In the above expression, the exponentials have been expanded into a power series with only those terms greater than $O\left(a^{3} \sigma k^{2}\right)$ being explicitly shown. If the response of the buoy is linear, then the time varying part of the sensor's trajectory may be written as

$$
x_{e}(t)=x_{m} e^{-\imath \sigma t}, \quad z_{e}(t)=z_{m} e^{-\imath \sigma t}
$$

with $x_{m}$ and $z_{m}$ being the complex coefficients describing the sensor motion. The time average of the horizontal and vertical velocity is then given by the real part of the following expressions:

$$
\begin{aligned}
\overline{U(t)} & =\frac{1}{2} a x_{m}^{*} \sigma e^{-k z_{\circ}}+\frac{1}{2} a z_{m}^{*} \sigma e^{-k z_{\circ}} \\
\overline{W(t)} & =\frac{2}{2} a x_{m}^{*} \sigma \epsilon^{-k z_{o}}+\frac{l}{2} a z_{m}^{*} \sigma e^{-k z_{\circ}}
\end{aligned}
$$


where * denotes a complex conjugate. The sensor motion has been assumed to average to zero. The preceeding expression, where we have yet to specify the form of the sensor motions, provides us with a compact way to examine measurement principles in general.

For instance, if the sensor is still, then the measurement is Eulerian and equation 3.7 reduces to

$$
\left(x_{m}, z_{m}\right)=(0,0) \Rightarrow \begin{aligned}
& \bar{U}_{\text {Eulerian }}=0 \\
& \bar{W}_{\text {Eulerian }}=0
\end{aligned}
$$

If a sensor does not drift, but to first order follows the particle trajectory at a certain depth, then we obtain (to first order) the Stokes' drift velocity

$$
\left(x_{m}, z_{m}\right)=\left(a e^{-k z_{0}}, a e^{-k z_{0}}\right) \Longrightarrow \begin{aligned}
& \bar{U}_{\text {Stokes }}=a a^{*} \sigma k e^{-2 k z_{0}} \\
& \bar{W}_{\text {Stokes }}=0
\end{aligned} .
$$

For the idealized case of a velocity sensor hanging from a buoy which is perfectly coupled to the surface, both in horizontal and vertical excursion,

$$
\left(x_{m}, z_{m}\right)=(a, a) \Longrightarrow \begin{aligned}
& \bar{U}_{\text {Pollard }}=a a^{*} \sigma k e^{-k z_{0}} \\
& \bar{W}_{\text {Pollard }}=0
\end{aligned} .
$$

In the case where the buoy response is not ideal but lags the wave excursions by a phase angle, $\psi$ (or a time delay of $t_{\text {lag }}=\frac{\psi}{\sigma}$ )

$$
\left(x_{m}, z_{m}\right)=\left(a e^{\imath \psi}, a e^{\imath \psi}\right) \Longrightarrow \begin{aligned}
& \bar{U}_{\text {lagged }}=a a^{*} \sigma k e^{-k z_{o}} \cos \psi \\
& \bar{W}_{\text {lagged }}=a a^{*} \sigma k e^{-k z_{o}} \sin \psi
\end{aligned} .
$$

The above case is most interesting because it shows that there can also be a bias in measuring vertical velocities.

What all the preceeding examples point out is that the final time-averaged output of a moving current meter is not only dependent on the velocity field in which the current meter is immersed, but is also dependent on the motion of the current meter itself. Furthermore, the size of this motion dependent mean is order $O\left(a^{2} \sigma k\right)$ and the apparent shear is order $O\left((a k)^{2} \sigma\right)$. These are the same order of magnitude as the expected current and shear in 
the wind-driven flow (Bye,1967; Wu,1975).

The only averaging procedure which does not measure the wave effects is the Eulerian. As was discussed in the last chapter, the Eulerian-type measurement is nearly impossible to realize and difficult to interpret in the near-surface environment in deep water. What we desire is to have an averaging procedure that will measure the wind-induced shear current and the mean flow without being biased by wave/motion effects. While it is possible to develop such a procedure, the derivation is not entirely straightforward. To pave the way for the full derivation, the concept of the unbiased estimator will be motivated by the use of a simple mathematical concept; that of a line integral in a potential field.

\section{line integration interpretation}

Since the waves are irrotational and periodic (in space), the line integral of the wave velocity, $\mathrm{u}(\mathrm{x})$, along any trajectory, at any instant of time vanishes

$$
\oint d \mathbf{x} \cdot \nabla \phi(\mathbf{x}) \equiv 0
$$

This integral can be regarded as an averaging procedure that results in zero for irrotational motions.

From a practical standpoint, equation 3.11 is of little use. It can be exploited if we recognize the time-space duality of water waves; i.e., the spatial variation of a wave may be sampled by a fixed observer who samples a wave as it passes (in time). Conceptually, this is most easily visualized for the case of a two-dimensional monochromatic wave, which is viewed in a frame of reference moving at the wave celerity, $c$, so that the wave profile is steady in time. Here, the trajectory of the sensor is described by the vector $\left(-c t+X(t), z_{0}+Z(t)\right)$. Equation 3.11 then becomes

$$
\frac{1}{T} \int_{0}^{T}\left\{U-\frac{1}{c} U \dot{X}-\frac{1}{c} W \dot{Z}\right\} d t=0
$$

where time derivatives have been indicated by dotting variables, $T$ is the wave period and, as above, $U(t)$ denotes the measured velocity. The case in which $\dot{X}(t) \ll c$ is depicted in 
Figure 3-1(a), where the dashed line shows the integration path. The lower "return path" is taken to be a line at some fixed depth below the mean water level. The integral along the return path is equivalent to an Eulerian measurement at that depth and, as such, will not measure a wave component. The net contribution over that segment will be due to the advection velocity, $c$, that is required when equation 3.11 is examined in the steady frame and will precisely cancel the advection component on the forward part of the path. In the case of infinite depth, the return path may be taken at $z=-\infty$.

a

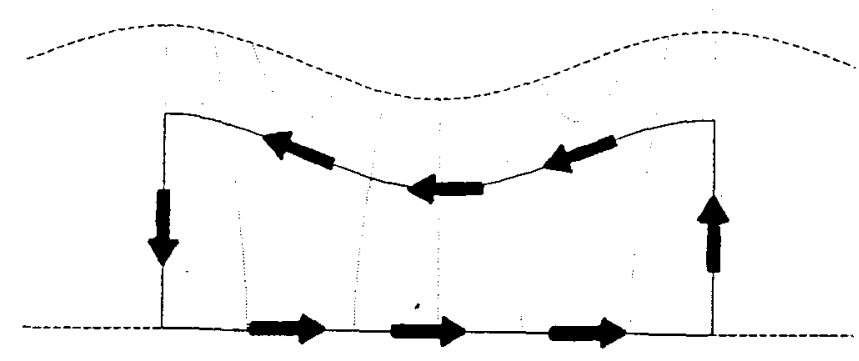

$\mathrm{b}$

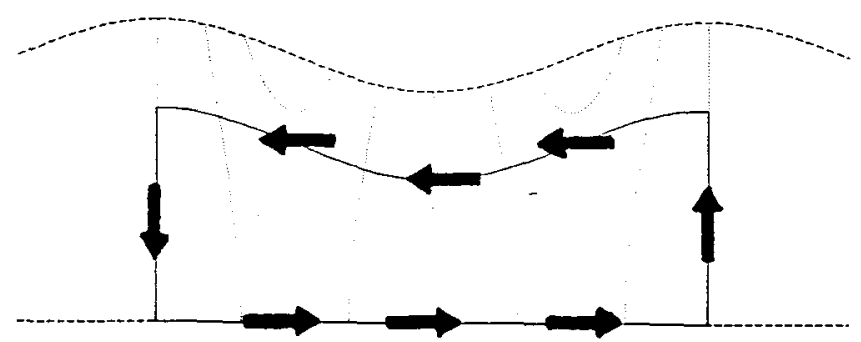

Figure 3-1: The line integral concept. In a frame of reference which travels at the wavespeed, the fluid velocities are steady. The dotted lines show indicate constant potential surfaces. Paths are shown which correspond to surface-following measurements. A line integral (a) through the wavefield will return a result of zero. In (b), the equation of a normal time average of horizontal velocity $\frac{1}{T} \int U d t$ has been transformed to this frame. Here, the differential distance vector $d \mathbf{x}$ does not lie along the path of integration and a zero wave contribution is not guaranteed.

Equation 3.12 defines a "trajectory average" of $U$, which will be denoted as $\widehat{U}$ (the notation is chosen in analogy to the conventional use of an overbar to indicate a simple 
time or space average). The first term in equation 3.12 reproduces the wave bias, while the remaining terms constitute "corrections" that estimate the bias. For the two-dimensional case just shown the cancellation is exact. When the definition of $\widehat{U}$ is generalized in the next section to three-dimensional, random waves we will find this is no longer true. With a spectrum of dispersive waves present, a steady reference frame does not exist. Still, it will be shown that while the cancellation is no longer exact, the bias remaining in $\widehat{U}$ is reduced by a factor of wave slope, ak, over that in $\bar{U}$.

While the corrections compensate for the wave contributions to the time average, they will not unduly affect the estimate of the nonwave contributions. When a rotational velocity $\left(U^{\prime}, W^{\prime}\right)$ is also present, the correction in equation 3.12 also includes terms such as $\overline{U^{\prime} \dot{X} / c}$ and $\overline{W^{\prime} \dot{Z} / c}$. Since $\dot{X}$ and $\dot{Z}$ are both of order of the wave orbital velocity $u$, the terms $\dot{X} / c$ and $\dot{Z} / c$ are at most $O(a k)$, although in general they are expected to be much smaller since it is likely that both $\dot{X}$ and $\dot{W}$ are poorly correlated with $\overline{U^{\prime}}$. So,

$$
\widehat{U} \approx \overline{U^{\prime}}+O\left(u(k a)^{2}\right)+O\left(k a \overline{U^{\prime}}\right)
$$

where $U$, the net observed velocity, is now the sum of irrotational (wave) and rotational components.

The line integral interpretation can also be used to illustrate the source of the wave bias. The time average of $U(t)$ is proportional to the integral of the horizontal velocity component $u(x, z, t)$ evaluated along the sensor trajectory. This situation (again in the steady-frame of reference) is shown in Figure 3-1(b). Since the differential distance elements do not lie along the path of integration, we cannot guarantee that this integral vanishes (actually performing the integral will, of course, yield the wave bias). 


\subsection{Estimation of the bias}

\section{general case}

When a spectrum of waves is present, it is not possible to find a reference frame in which the waves are steady and the development of the last section cannot be repeated exactly. Instead, it is assumed that equation 3.12 may be generalized to

$$
\widehat{V}_{\alpha} \equiv \frac{1}{T} \int_{0}^{T} d t\left[V_{\alpha}(t)-\left\{V_{i}(t) * \mu_{i}^{(\alpha)}(t)\right\} \dot{X}_{i}(t)\right]
$$

(repeated latin subscripts run from 1 to 3 and are summed). The unknown functions $\mu(t)$ replace the factors $1 / c$ appearing in equation 3.12. Since both the directional spreading and phase speed of the waves are frequency dependent, the $\mu$ 's are introduced as filters hence the convolution, denoted by * in equation 3.14 .

The problem is to find $\mu^{(\alpha)}$ 's that reduce the bias in $\widehat{V}_{\alpha}$ by an order of magnitude over that in $\bar{V}_{\alpha}$. Mathematically we express this as a requirement on the expected value of $\widehat{V}_{\alpha}$ as

$$
\left\langle\widehat{V}_{\alpha}\right\rangle=O\left(a^{3} \sigma k^{2}\right)
$$

On taking the expected value of equation 3.14, we then require that the following be satisfied to order $O\left(a^{3} \sigma k^{2}\right)$ :

$$
\frac{1}{T} \int_{0}^{T} d t\left\langle V_{\alpha}(t)\right\rangle=\frac{1}{T} \int_{0}^{T} d t\left\langle\left\{V_{i}(t) * \mu_{i}^{(\alpha)}(t)\right\} \dot{X}_{i}(t)\right\rangle
$$

Both the left- and right-hand sides of equation 3.16 can be evaluated using linear wave theory.

The output of a moving sensor is the relative velocity $V_{i}-\dot{X}_{i}$, where $V_{i}$ is related to the Eulerian velocity $v_{i}$ by

$$
V_{i}(t)=v_{i}(\mathrm{X}(t), t)
$$

In the following it is assumed that the additive contribution $\dot{X}_{i}$ of the sensor motion to the relative velocity has been subtracted and $V_{i}$ is referred to as the observed velocity. 
Expanding equation 3.17 to second order gives

$$
V_{i}(t)=v_{i}\left(\mathbf{X}^{(o)}, t\right)+\left(X_{j}(t)-X_{j}^{(o)}\right) \frac{\partial v_{i}\left(\mathbf{X}^{(0)}, t\right)}{\partial x_{j}}+\cdots
$$

where $\mathrm{X}^{(0)}$ denotes the average sensor position. The potential function of a directionally spread wavefield is conveniently described using the Fourier-Stieljes notation.

$$
\Phi=\int_{\sigma} \int_{\theta}-\imath d \mathbf{A}(\sigma, \theta) \frac{g}{\sigma} e^{\imath(\mathbf{k} \cdot \mathbf{x}-\sigma t)} e^{k z}
$$

With this notation, the expression for the vector velocity is

$$
\mathbf{v}=\nabla \Phi=\int_{\sigma} \int_{\theta} d \mathbf{A}(\sigma, \theta) \sigma\left\{\begin{array}{c}
\cos \theta \\
\sin \theta \\
-\imath
\end{array}\right\} e^{\imath(\mathbf{k} \cdot \mathbf{x}-\sigma t)} e^{k z}
$$

If we expand out the velocities, as we did for the two-dimensional case, and substitute in the sensor's trajectory components $\mathrm{X}$, we find that the measured velocity $\mathrm{V}$ is

$$
\mathrm{V}=\int_{\sigma} \int_{\theta} d \mathbf{A}(\sigma, \theta) \sigma\left\{\begin{array}{c}
\cos \theta \\
\sin \theta \\
-\imath
\end{array}\right\} e^{\imath\left(\mathrm{k} \cdot \mathrm{X}^{(0)}-\sigma t\right)} e^{-k z_{0}}\left[1+\imath k \cos \theta X_{1}+\imath k \sin \theta X_{2}+k X_{3}+\cdots\right]
$$

To first order, sensor and fluid velocities are related through the buoy transfer function $H_{i j}$. The transfer function below relates the sensors velocities to the fluid velocity at the surface.

$$
\hat{\dot{X}}_{i}(\sigma)=H_{i j}(\sigma) \hat{v}_{j}(\sigma, z=0)
$$

If we make the reasonable assumption that motion in a particular direction is predominately forced by fluid velocities in that direction (i.e. cross-coupled motions between orthogonal directions are relatively weak), the transfer function matrix is diagonal and the sensor trajectories and velocities are expressed as

$$
\mathbf{X}=\int_{\sigma} \int_{\theta} d \mathbf{A}(\sigma, \theta)\left\{\begin{array}{c}
\imath H_{11} \cos \theta \\
\imath H_{22} \sin \theta \\
H_{33}
\end{array}\right\} e^{-\imath \sigma t}
$$




$$
\dot{\mathbf{X}}=\int_{\sigma} \int_{\theta} d \mathbf{A}(\sigma, \theta) \sigma\left\{\begin{array}{c}
H_{11} \cos \theta \\
H_{22} \sin \theta \\
-\imath H_{33}
\end{array}\right\} e^{-\imath \sigma t}
$$

Substituting in the sensor trajectories of equation 3.23 into the expression for the measured velocity (equation 3.21) and taking the expected value of the time average gives a general result for the biased time average.

$$
\begin{aligned}
\langle\overline{\mathbf{V}}\rangle & =\frac{1}{2} \int_{\sigma} \int_{\theta} d \mathbf{A} d \mathbf{A}^{*} H_{11}^{*} \sigma k\left\{\begin{array}{c}
\cos ^{3} \theta \\
\sin ^{2} \theta \cos \theta \\
-\imath \cos ^{2} \theta
\end{array}\right\} e^{-k z_{o}} \\
& +\frac{1}{2} \int_{\sigma} \int_{\theta} d \mathbf{A} d \mathbf{A}^{*} H_{22}^{*} \sigma k\left\{\begin{array}{c}
\cos ^{2} \theta \sin \theta \\
\sin ^{3} \theta \\
-\imath \sin ^{2} \theta
\end{array}\right\} e^{-k z_{o}} \\
& +\frac{1}{2} \int_{\sigma} \int_{\theta} d \mathbf{A} d \mathbf{A}^{*} H_{33}^{*} \sigma k\left\{\begin{array}{c}
\cos \theta \\
\sin \theta \\
-\imath
\end{array}\right\} e^{-k z_{o}}
\end{aligned}
$$

The right hand side of equation 3.16 is our bias estimator and is rewritten in frequency space as

$$
\frac{1}{T} \int_{0}^{T} d t\left\langle\left[V_{i}(t) * \mu_{i}^{(\alpha)}(t)\right] \dot{X}_{i}(t)\right\rangle=\int_{\sigma}\left\langle d \hat{X}_{i}^{*}(\sigma) \hat{\mu}_{i}^{(\alpha)}(\sigma) d \hat{V}_{i}(\sigma)\right\rangle
$$

Using our first order expressions for $X_{i}$ and $U_{i}$ this becomes

$$
\frac{1}{2} \int_{\sigma} \int_{\theta} d \mathbf{A} d \mathbf{A}^{*} \sigma^{2}\left\{\hat{\mu}_{1}^{(\alpha)} H_{11}^{*} \cos ^{2} \theta+\hat{\mu}_{2}^{(\alpha)} H_{22}^{*} \sin ^{2} \theta+\hat{\mu}_{1}^{(\alpha)} H_{33}^{*}\right\} e^{-k z_{o}}
$$

To find the $\mu$ filters the $\alpha^{\text {th }}$ component of equation 3.25 is equated to equation 3.26 . The bias arises from the beating of like frequencies in sensor motion and fluid velocity. Since the $\mu$ 's are to model this process we will equate equations 3.25 and 3.26 on a frequency bin-by-frequency bin basis. In doing this, let's rewrite the wave coefficients, $d \mathbf{A}$, in terms of a waveheight spectrum, $S_{\eta \eta}(\sigma)$, and a directional spreading function, $f(\sigma, \theta)$, (normalized so that $\left.\int_{-\pi}^{\pi} f(\sigma, \theta) d \theta=1\right)$ as

$$
S_{\eta \eta}(\sigma) f(\sigma, \theta)=\frac{1}{2} \frac{\left\langle d \mathrm{~A} d \mathrm{~A}^{*}\right\rangle}{d \sigma d \theta}
$$


Then, for $\alpha=1$ we will find the $\mu$ 's from the following:

$$
\begin{gathered}
S_{\eta \eta}(\sigma) \sigma k e^{-k z_{\circ}} \int\left\{H_{11}^{*}(\sigma) f(\sigma, \theta) \cos ^{3} \theta+H_{22}^{*}(\sigma) f(\sigma, \theta) \cos ^{2} \theta \sin \theta+H_{33}^{*}(\sigma) f(\sigma, \theta) \cos \theta\right\} d \theta \\
=S_{\eta \eta}(\sigma) \sigma^{2} e^{-k z_{\circ}} \int\left\{H_{11}^{*}(\sigma) f(\sigma, \theta) \cos ^{2} \theta+H_{22}^{*}(\sigma) f(\sigma, \theta) \sin ^{2} \theta+H_{33}^{*}(\sigma) f(\sigma, \theta)\right\} d \theta
\end{gathered}
$$

The sensor transfer function can be completely removed from the solution by equating the above on a term-by-term basis. Solving in this way also allows the $i^{\text {th }}$ correction term of equation 3.14 to be interpreted as the bias originating from motion in the $i^{\text {th }}$ direction. If this is done, we find that the $\mu_{i}^{(1)}$ filters are given by:

$$
\begin{aligned}
\mu_{1}^{(1)} & =\frac{k}{\sigma} \frac{\int f(\sigma, \theta) \cos ^{3} \theta d \theta}{\int f(\sigma, \theta) \cos ^{2} \theta d \theta}, \\
\mu_{2}^{(1)} & =\frac{k}{\sigma} \frac{\int f(\sigma, \theta) \cos ^{2} \theta \sin \theta d \theta}{\int f(\sigma, \theta) \sin ^{2} \theta d \theta} \\
\mu_{3}^{(1)} & =\frac{k}{\sigma} \frac{\int f(\sigma, \theta) \cos \theta d \theta}{\int f(\sigma, \theta) d \theta} .
\end{aligned}
$$

Solving in the same way, the $\mu_{i}^{(2)}$ filters are found to be

$$
\begin{aligned}
\mu_{1}^{(2)} & =\frac{k}{\sigma} \frac{\int f(\sigma, \theta) \sin ^{2} \theta \cos \theta d \theta}{\int f(\sigma, \theta) \cos ^{2} \theta d \theta}, \\
\mu_{2}^{(2)} & =\frac{k}{\sigma} \frac{\int f(\sigma, \theta) \sin ^{3} \theta d \theta}{\int f(\sigma, \theta) \sin ^{2} \theta d \theta}, \\
\mu_{3}^{(2)} & =\frac{k}{\sigma} \frac{\int f(\sigma, \theta) \sin \theta d \theta}{\int f(\sigma, \theta) d \theta} .
\end{aligned}
$$

Finally, the filters for estimating the vertical bias $\mu_{i}^{(3)}$ are found to have the simple form

$$
\mu_{1}^{(3)}=\mu_{2}^{(3)}=\mu_{3}^{(3)}=-\frac{\imath}{c}
$$

If the spreading function $f(\sigma, \theta)$ is found using a pitch and roll type technique, only the first two trigonometric moments of $f(\sigma, \theta)$ are obtained directly from measurements (LonguetHiggins et al, 1963). This means that the above filters which require third moments of the spreading function will be somewhat dependent on the extrapolation used to form the final spreading function estimate. For the SASS data, the wave spreading is estimated using the maximum entropy method (see section 4.3). 
The derivation here was driven by the analogy with the two-dimensional line integral interpretation. Santala and Terray (1991) derive the same results in a more general way by starting with a three-dimensional Taylor series expansion. In that paper alternate forms of the bias estimator are also discussed.

\section{Limitations of the Theory}

The theory for reducing the bias has been based on small amplitude linearized potential theory. It further assumes that the directional spectrum can be accurately estimated. Let's consider the importance of these assumptions.

\section{steepness effects}

The assumption in the velocity relations of equations 3.19 and 3.20 is that the amplitude of the wave is infinitesimal. It is well known, however, that if surface tension effects are ignored, when the velocity potential is expanded out in a power series in slope, the leading term is $O\left(\frac{a g}{\sigma}\right)$ and the next highest order term is $O\left((a k)^{3} \frac{a g}{\sigma}\right)$ (Wehausen and Laitone, 1960). So, it is easy to see that even for relatively steep waves the nonlinear steepness effects are negligible.

In fact, if one notes the similarity between equation 3.18 and the expression for the wave mass transport velocity $V_{s}$, then it is reasonable to say that the error in using equation 3.18 to estimate the wave bias must be similar to the error in using first order linearized theory to predict the exact Stokes' drift. Longuet-Higgins(1987) computed the surface value of $V_{s}$ for a two-dimensional Stokes wavetrain and compared the exact result to the leading term, $O\left(a^{2} \sigma k\right)$, in the small amplitude expression. The two results are essentially indistinguishable for wave slopes $a k$ less than 0.3. Two things should be kept in mind. In the field, rms waveslopes are rarely much greater than 0.1 . Also, the decay with depth of the second term in the wave potential is much faster than that of the leading term and consequently even at small depths the error due to wave steepness will be small. 


\section{estimation of directional spreading}

The estimate of the bias is dependent on the directional spreading of the waves. Because error bounds are not well established for the estimation of directional spectra, the effect of errors in the estimation of the spreading function was examined by computer simulation.

As a model, the frequency-direction spectrum of DONELAN et al (1985) was used (hereafter referred to as the DHH model). Inputs to the spectral model are the wave-age $U / c$ and the peak frequency $\sigma_{p}$. For simulation results shown in this paper, $U / c=3.10$ and $\sigma_{p}=4.48 \mathrm{rad} / \mathrm{s}$ (these are the values for the field data analyzed in the next section). The directional spreading function is dependent on frequency and has the functional form

$$
f(\sigma, \theta)=\frac{1}{2} \beta \operatorname{sech}^{2}(\beta \theta),
$$

where $\beta$ is a frequency dependent directional-spreading parameter. According to the model, the value of $\beta$ is maximum at $\sigma=0.95 \sigma_{p}$, where $\beta=2.44$.

The numerical simulation itself was accomplished by creating wavefields from a finite number of sinusoids. The frequency spectrum was subdivided into $0.04 \mathrm{rad} / \mathrm{s}$ intervals and the angle of propagation was subdivided into $\pi / 51$ intervals over the range $\pm \pi / 2$. For all cases it was assumed that the buoy was a perfect wave-follower (i.e., $H_{11}=H_{22}=H_{33}=1$ and $\left.H_{i j}=0, i \neq j\right)$.

If we assume that the $3 d B$ width of the spreading function $f(\sigma, \theta)$ can be measured correctly to $\pm 50 \%$, then the estimate of the bias would be in error to $\pm 6 \%$, as is shown in figure $3-2$. The $\pm 50 \%$ is probably attainable with standard techniques, especially near the peak frequencies of wave energy. However, at frequencies above and below the spectral peak, where the signal-to-noise ratio begins to fall, even attaining such modest accuracy may be difficult. Fortuitously, the greatest portion of the bias, and hence correction, occurs due to motions at the spectral peak. If we allow the spectral form of the estimated bias to be called $\hat{V}^{\text {bias }}(\sigma)$, then

$$
\hat{V}^{\text {bias }}(\sigma)=\hat{\dot{X}}_{i}^{*}(\sigma) \hat{\mu}_{i}^{(\alpha)}(\sigma) \hat{V}_{i}(\sigma)
$$


Above the spectral peak waveheight spectra drop off as $\sigma^{-4}$. The spectra of orbital velocities must then fall off as $\sigma^{-2} e^{2 k z}$. Assuming that the motion of the sensor is coupled to the surface displacement, the cross-spectrum of sensor velocity and wave orbital velocity falls off as $\sigma^{-2} e^{k z}$. Therefore, above the spectral peak of the waves, the entire correction falls off as $\sigma^{-1} e^{k z}$. This implies that sensors extremely close to the surface would have corrections heavily weighted by the motions well above the spectral peak and thus would give inaccurate estimates. Figure 3-3 indicates how deep the sensors must be to give reliable results. The figure shows, for example, that for a wavefield with a peak frequency of $0.5 \mathrm{~Hz}$, a sensor at a mean depth of $50 \mathrm{~cm}$ will have $90 \%$ of the bias originating from waves of frequencies lower than $0.85 \mathrm{~Hz}$. The total contribution from waves above $0.85 \mathrm{~Hz}$ (wavelengths shorter than $2.1 \mathrm{~m}$ ) is only $10 \%$ of the bias. So, the decay of orbital velocities with depth acts as a lowpass filter and thereby lessens the problems that the shorter waves would create in our linearized solution. Because we assume in our model that sensor motion is correlated to even the highest frequency surface displacements, and thus overstate the contribution due to the high-frequencies, the curves shown in Figure 3-3 could be considered worst case bounds for most systems.

\subsection{Verification}

The theory developed for bias removal was tested using data taken from a tower in Lake Ontario. This tower is maintained specifically for research purposes by the Canada Center for Inland Waters (CCIW). As part of the WAVES '87 experiment, a two-axis acoustic velocimeter, shown in Figure 3-4, was mounted on the end of CCIW's wave-follower, with its acoustic axes aligned to measure the horizontal velocity of the fluid. Because the motion of the sensor is one-dimensional, we are provided with a relatively simple situation in which to evaluate the estimation procedure.

Data were collected at various depths in a wave-following mode with the velocity sensor maintained at a fixed distance beneath the water's surface. The vertical component of velocity at a given depth was not measured directly, but was calculated using linear theory to extrapolate from the surface value (the latter was computed from the vertical velocity of 


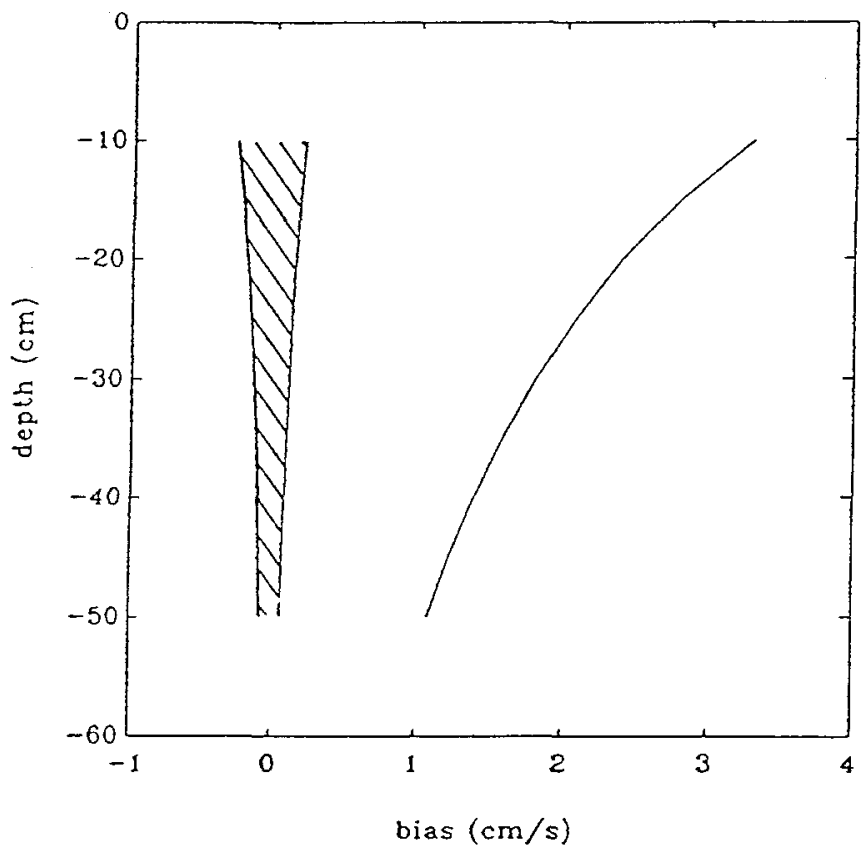

Figure 3-2: Effect of errors in estimating the directional wave spreading function. The bias (-) for a sensor free to move both vertically and horizontally. The shaded region indicates the range of $\widetilde{\mathrm{V}}$ when the $3 d B$ width is estimated correctly to within $\pm 50 \%$.

the sensor $\dot{Z}$ and the transfer function of the wave-follower).

In the one-dimensional case, the unbiased estimator reduces to

$$
\widehat{V}_{\alpha}=\frac{1}{T} \int_{0}^{T} d t\left[V_{\alpha}(t)-\left\{V(t)_{z} * \mu_{z}^{(\alpha)}(t)\right\} \dot{Z}(t)\right]
$$

and only a single filter $\mu_{z}^{(\alpha)}$ is required for each current direction. The directional spreading function necessary to compute $\mu$ was estimated from the measured velocities and the wave height using the maximum entropy method as outlined by Lygre and Krogstad (1986).

The data shown in Figure 3-5 were taken between 11:20 and 13:07 on 12 December 1987; during this time the wind was from the northwest $\left(283^{\circ}\right)$ at an average speed of approximately $7 \mathrm{~m} / \mathrm{s}$. The fetch in this direction is roughly $1.5 \mathrm{~km}$. Only those records where the average wind speed was between 6.8 and $7.2 \mathrm{~m} / \mathrm{s}$ and the mean wind direction was not outside a $6^{\circ}$ range were included (the possible exception is the Eulerian point, which was the last measurement made, and at which time wind speed and direction were not recorded). 


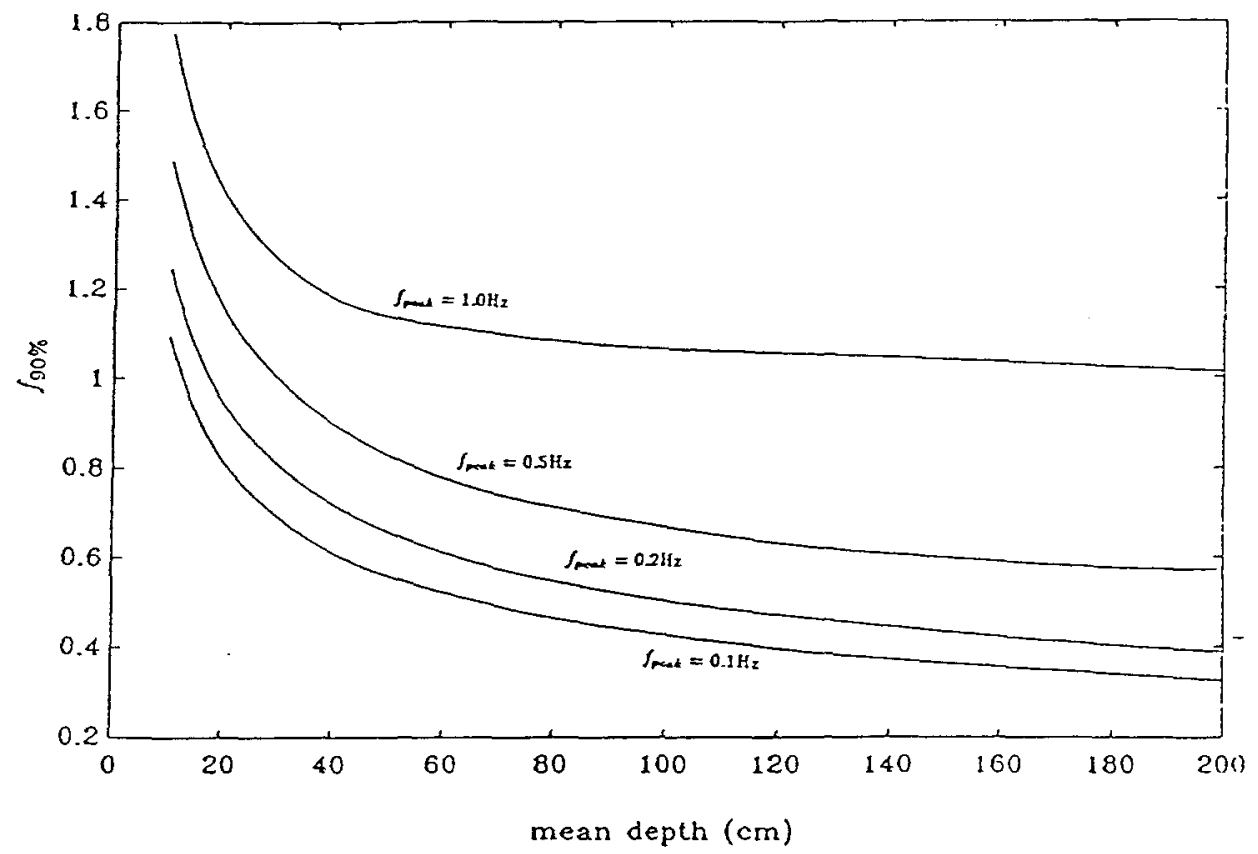

Figure 3-3: Bias $90 \%$ cutoff frequency. Each curve represents a DHH wave spectrum with $U / c=3.1$ and peak frequency as labelled. For a given sensor depth the height of the curve indicates the frequency below which $90 \%$ of the wave bias originates. Mathematically $f_{90 \%}$ is defined as: $\int_{0}^{f_{90 \%}} \hat{U}^{\text {bias }}(f) d f / \int_{0}^{\infty} \hat{U}^{\text {bias }}(f) d f=0.90$.

The waves had a peak period of $1.4 \mathrm{~s}$ and a significant height of $23 \mathrm{~cm}$. The data were sampled at $200 \mathrm{~Hz}$, lowpass filtered, and decimated to $12.5 \mathrm{~Hz}$. The resulting record at each depth was slightly over 5 minutes long and consisted of 3,968 samples. One record was taken with the wave-follower drive shut off. As expected, this Eulerian estimate of the velocity lies closer to the unbiased than to the biased estimate.

As a further check, the size of the correction made to each measurement is compared to the estimated bias using the computer model of the previous section. The sensor was only free to move in the vertical direction so the program was modified accordingly. For the simulation results, the bias was calculated for a perfect wave-follower and a DHH spectral form for the waves. The peak frequency and wave-age parameter $(U / c)$ of the simulated wavefield were matched to that of the field data to give an assumed spectral shape. When this was done we found that the integrated energy in the model was about $33 \%$ greater than for the field data. Therefore, the energy of the model spectrum was adjusted to match 


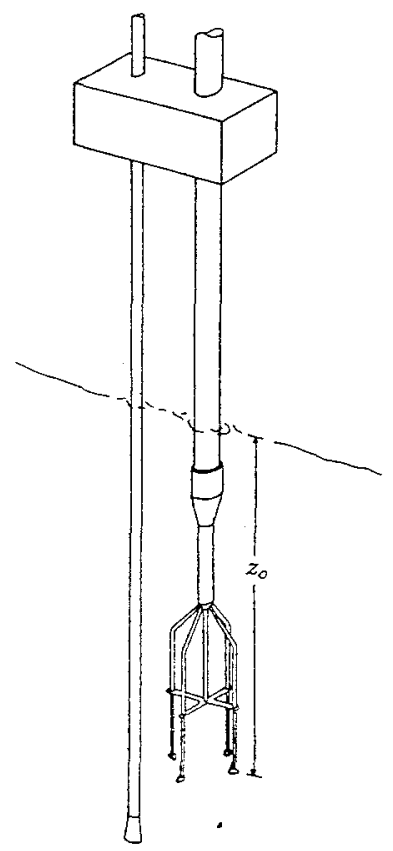

Figure 3-4: Acoustic velocimeter used in WAVES '87. The entire assembly shown, consisting of a wavestaff, and acoustic sensors (shown positioned $z_{0}$ below the surface), was mounted on a hydraulically-driven piston which kept the distance $z_{0}$ constant.

the conditions of the field data. Figure 3-6 compares the size of the corrections applied to the field data and the size of the corrections predicted by the model and, in spite of the extremely simplistic way in which the model was run, the agreement is at least qualitatively correct. 


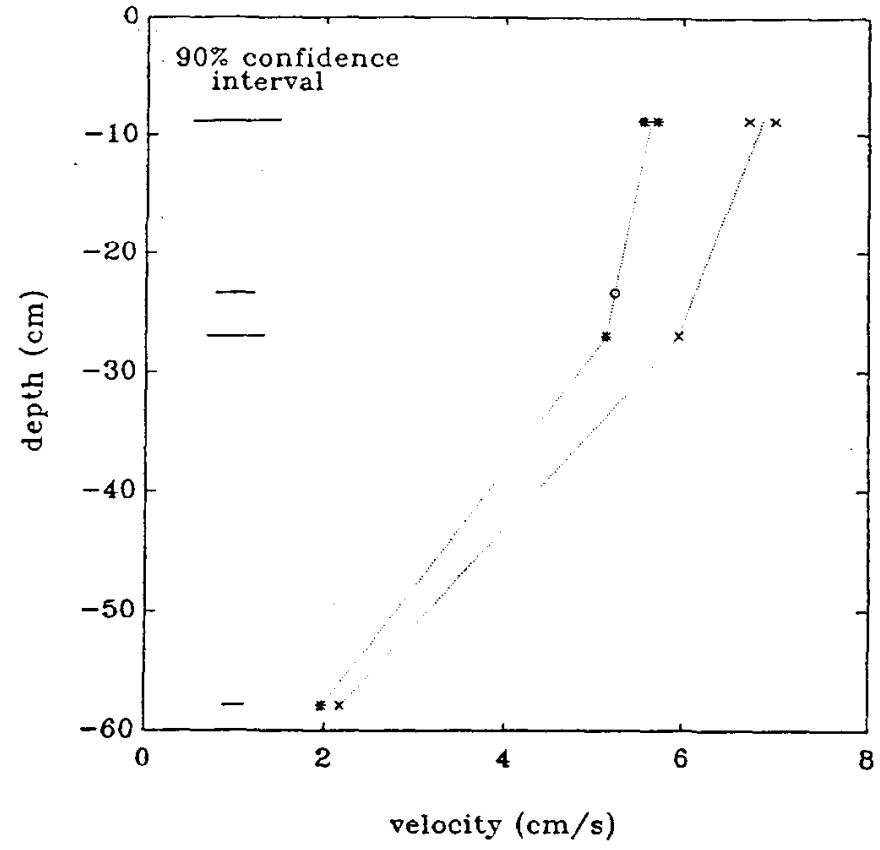

Figure 3-5: Magnitude of the time-averaged horizontal velocity. The *'s were computed using the unbiased estimator, confidence intervals for each depth are shown at the left. The $x$ 's were computed by directly time-averaging the horizontal components and have confidence intervals approximately $5 \%$ larger than those shown for the $*$ 's. o denotes an Eulerian measurement.

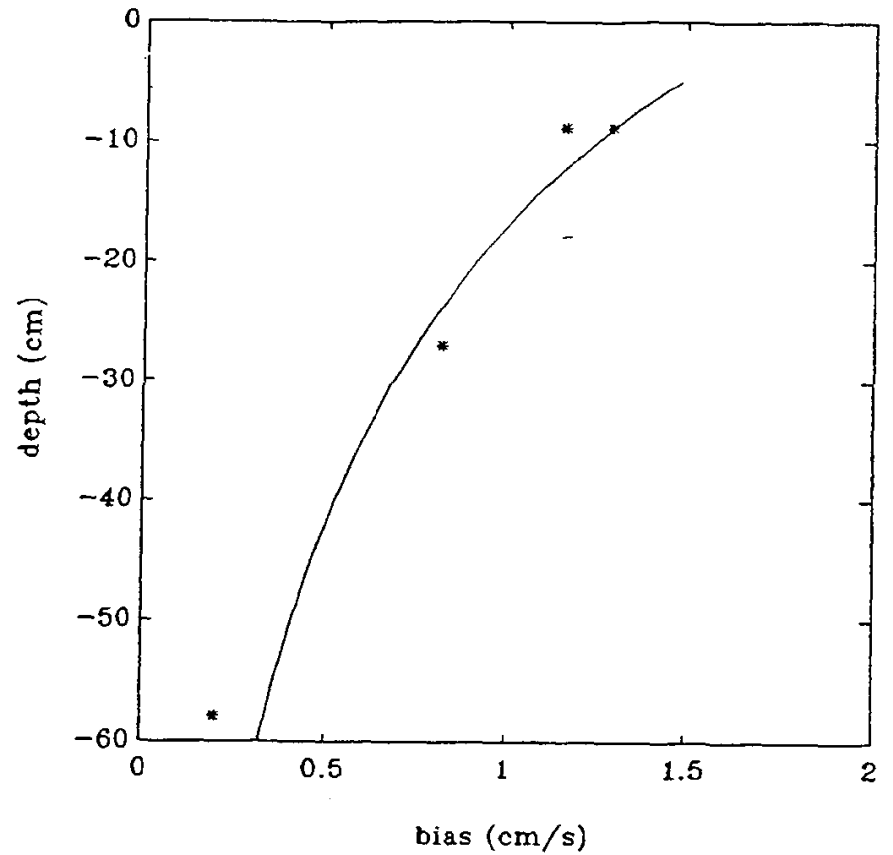

Figure 3-6: Bias computed from the field data $(*)$. The bias was also predicted from the computer model (-). The model was specialized here for the case where the sensor is free to move only up-and-down. 


\section{Chapter 4}

\section{THE SURFACE ACOUSTIC SHEAR SENSOR (SASS)}

\subsection{Design of SASS}

As mentioned previously, the SASS was designed to measure the velocities relative to the air-sea interface. The air-sea interface is an appropriate reference point for measurement to test if the wind-driven shear current is, as is commonly believed, similar to a wall-layer flow. By creating a buoy with a large waterplane area the dominant force governing the buoy's motion becomes the change in buoyancy with the relative motion of the free-surface. Traditionally, a large waterplane area buoy would be of a discus or similar type configuration. This classic type configuration was rejected for two reasons. First, as is noted by Collar et al (1983), velocity sensors beneath discus shaped buoys become trapped in the boundary layer beneath the buoy and give unreliable results. Secondly, the design called for a rigid array of sensors to be suspended beneath the buoy. To provide the necessary rigidity either large structural members would have to be used or a truss would have to be built. Since a premium was placed on minimizing flow obstruction it was decided that the current meter array would be supported by a truss. The design of SASS, shown in Figure $4-1$, is driven by both these requirements. By separating the buoyancy elements, the flow disturbance of the buoyancy itself moved away from the sensing volumes and the current meter array could be supported by a structure composed of relatively slender members. 


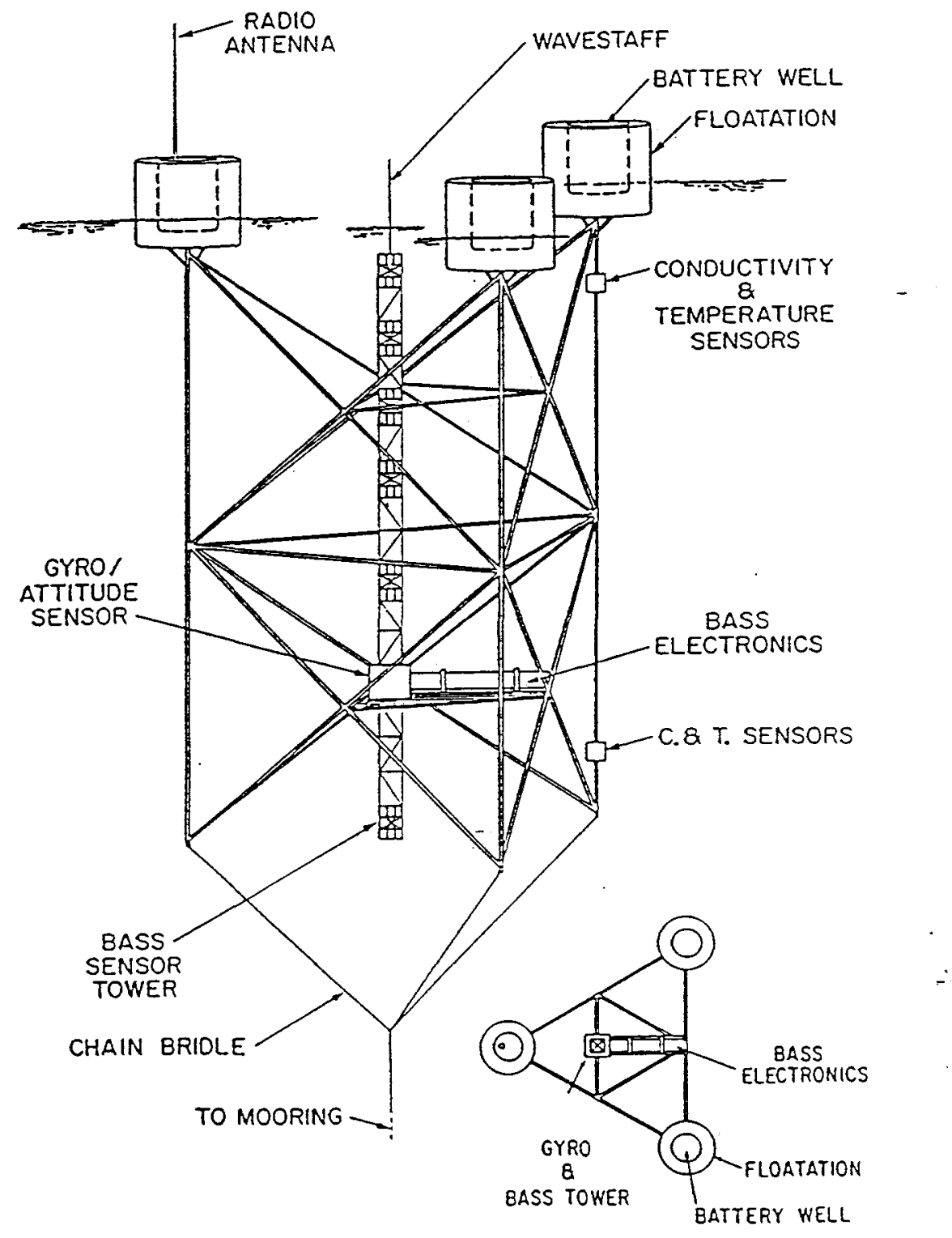

Figure 4-1: The Surface Acoustic Shear Sensor (SASS) 
Each of the SASS's three floats is $84 \mathrm{~cm}$ high. With a combined waterplane area of $2.0 \mathrm{~m}^{2}$ only $28 \mathrm{~cm}$ of the floats need be submerged to support the $5.5 k N$ (wet) buoy. This leaves $11 k N$ of excess buoyancy. The shallowness of draft and distance of separation (in plan view SASS is an equilateral triangle $3.7 \mathrm{~m}$ on a side) was an effort to minimize flow disturbance. The truss, constructed of 2 inch-OD aluminum tubing, connects the buoyancy elements and provides a rigid structure to which the current meter array could be attached and through which mooring forces could be transmitted.

Velocities relative to the instrument are measured with the BASS acoustic current meter (Williams et al, 1987). This current meter measures the velocity component along four acoustic axes. A schematic of a BASS current meter sensing "pod" is shown in Figure 4-2 The four axes are resolved, with some redundancy, into an orthogonal triplet relative to the sensor. Sensing volumes were located at each of the six following depths (in $\mathrm{cm}$ ): 111, 166, $251,311,391$ and 585 . With a sampling volume $\mathrm{O}(15 \mathrm{~cm})$ in length, and a high sampling rate, this current meter allows us to obtain an accurate time history of the fluid velocities in an instrument based frame of reference. The structure supporting the acoustic transducers is of stainless steel construction. The design rational here is the same as that for the overall SASS design; maximal rigidity with minimal flow disturbance is achieved by a truss.

A two axis gyroscope, manufactured by Colnbrook (Figure 4-3), provided a stabilized platform on which accelerometers were mounted to measure translational motions:- Two capacitive type potentiometers measured the tilt of the buoy by referencing the gyro platform. The yaw rotations were measured by a compass inside the BASS pressure case. Having measured all six degrees of freedom of the instrument's motion allows us to not only rotate the relative velocities into an inertial frame of reference, but also allows us to add in the instrument velocity. Thus, a time series of the fluid velocity (at the point of the sensor) may be obtained.

Of course, with the horizontal scale of SASS being $3.7 m$, it is expected that SASS will 


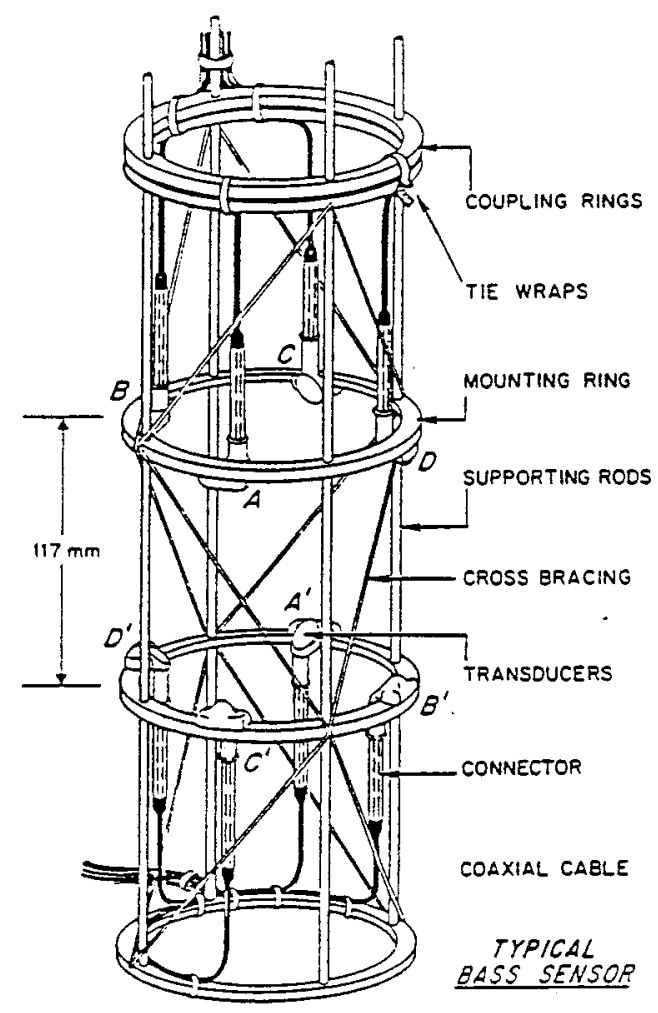

Figure 4-2: A typical BASS sensing "pod". Acoustic travel-time measurements are made along four axes.

only follow waves longer than $7.4 \mathrm{~m}$. The motion of the shorter waves (periods of $2.2 \mathrm{sec}$ and less) will be essentially uncorrelated to the buoy motion. In order to measure how well SASS follows the water's surface, a wavestaff was mounted directly above the sensor column.

The thermal gradient in the water column was measured by mounting small metal-clad thermistors directly adjacent to each BASS sensing volume. Though the small size of these sensors causes little flow disturbance they do not provide an extremely accurate absolute measurement of temperature. To this end, Sea-Bird thermistors were mounted at the same elevation as the top and bottom metal-clad thermistors (but far off to the side as these sensors are quite large). Each Sea-Bird thermistor was accompanied by a conductivity sensor.

The SASS was operated so that all channels were sampled at $4 \mathrm{~Hz}$. The BASS was op- 


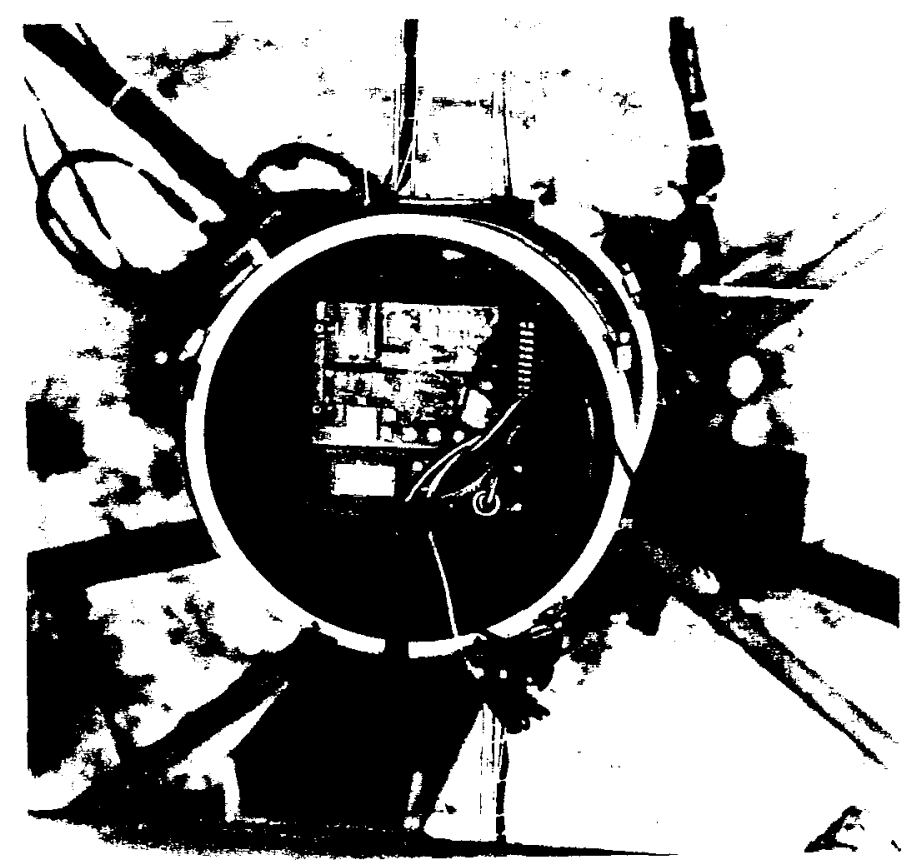

Figure 4-3: The gyro inside its pressure case (open) being prepared for deployment. The circuit mounted on the gyro is the TATTLETALE IV computer and FET switching circuits used for control of the gyro system.

erated at a $20 \mathrm{~Hz}$ sample rate and a block average of five samples was done by the current meter electronics. In fact, all of the data channels were fed into the BASS computer for formatting prior to transmission or recording.

Each record was 89 bytes long so that in the typical hour long files it was necessary to store 1.25 Mbytes of data. Data could be stored in one of two ways; it could be transmitted to ship or shore via a Clegg FM transceiver or it could be stored on an optical disk which was housed inside one of the battery wells. When not transmitting data the transciever was in a listening mode. A menu of twelve command options controlling SASS instrumentation could be selected by transmitting a DTMF signal to SASS. This signal was decoded and interpreted by a Tattletale IV computer which turned sensors on and off by a series of FET switches attached to its $\mathrm{I} / \mathrm{O}$ lines. 
The radio transmitter requires 24 Watts of power and the gyro 18 Watts. Recording data to optical disk provides hard-wired reliability to the data recovery but at the cost that the disk requires 20 percent more power than the radio. Power for the SASS was provided by Gell Cell batteries stored in the floats. The nominal capacity of each battery was $23 a m p$-hours at 12 volts. Two batteries connected in series were dedicated to providing the 24 volt input required for the BASS. The other 14 batteries were used in parallel to supply power to all of the other electronic devices aboard the SASS. The power budget is discussed in greater detail in Montgomery and Santala (1989). The conclusion reached there is that when derating of the batteries for temperature and reduced current compliance with discharge, that the fourteen Gell-Cell's provide, at most, enough power to transmit a total of 46 hours of data.

The SASS, in theory, could be used as a freely drifting instrument. For its application in SMILE, the SASS was moored. Figure $4-4$ shows the SASS mooring schematic.

\subsection{Coordinate transformations}

The current meters mounted onto the SASS measure velocities relative to the instrument. For purposes of interpretation we would like to be àble to relate all measured quantities to an inertial frame of reference. Since the SASS will both rotate and translate with respect to inertial coordinates, we must decompose and analyze both the rotational and translational velocities of the buoy.

The approach taken here will be to start from basic kinematic and dynamic equations and derive expressions that apply directly to the SASS instrument. The development of the final expressions is both straightforward and generalized and only trivial modifications would be necessary to derive expressions for similar systems (it is the sign convention of rotations and the "nesting" of the gimbals which require a specialized approach).

To begin, a set of coordinate axes is defined for the SASS system. The origin of the 


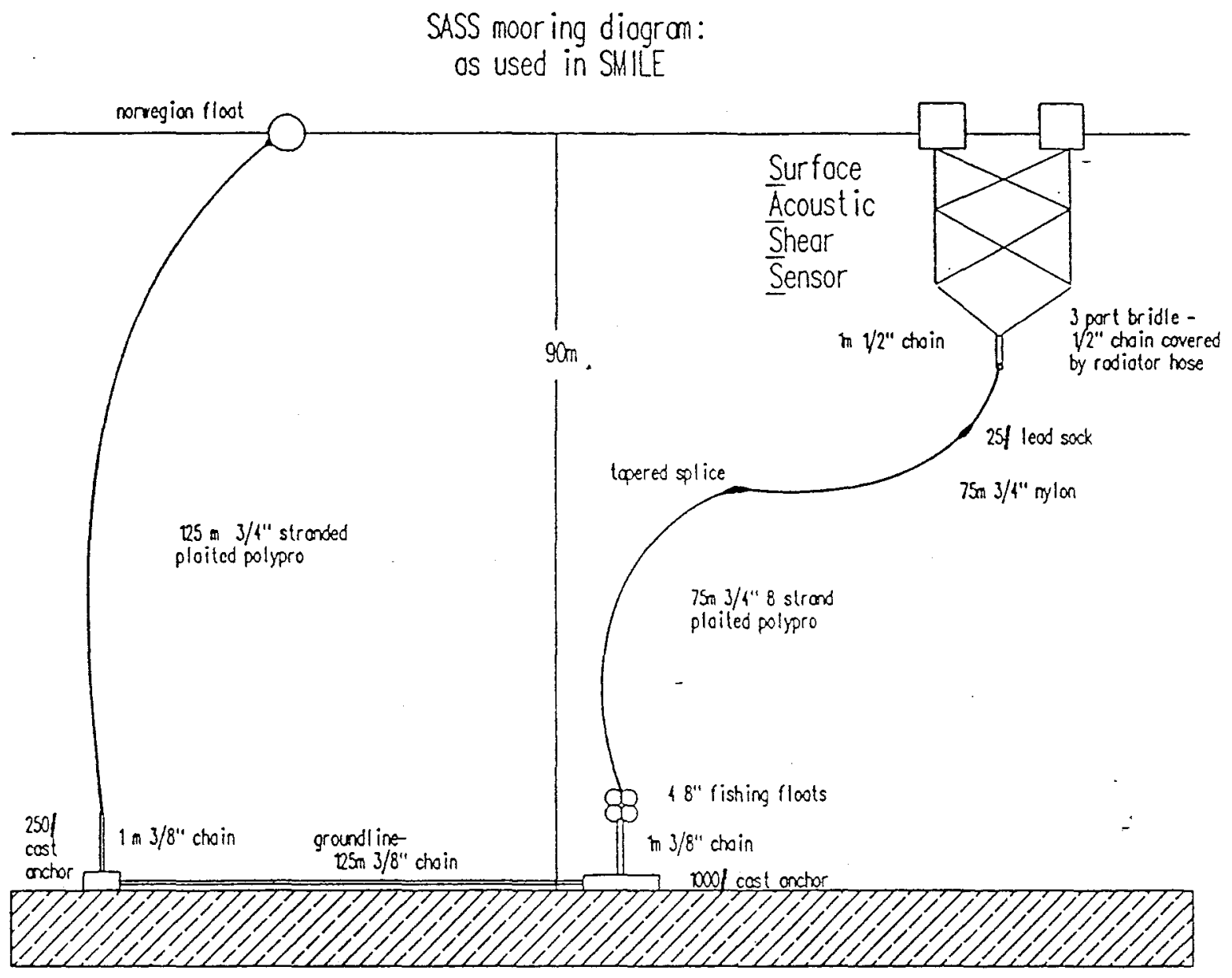

Figure 4-4: Schematic of the SASS mooring system for the SMILE deployments. 
instrument relative coordinates may be arbitrarily defined anywhere in fixed relation to the instrument. But, by choosing the origin carefully, we simplify the processing of the data. The definition of the coordinate axes is shown in Figure 4-5. As shown in the figure, the origin of the axes is chosen to be at the intersection of the pitch and roll axes of the gyroscope. In the development that follows this will be seen to be a "natural" choice. The gyroscope
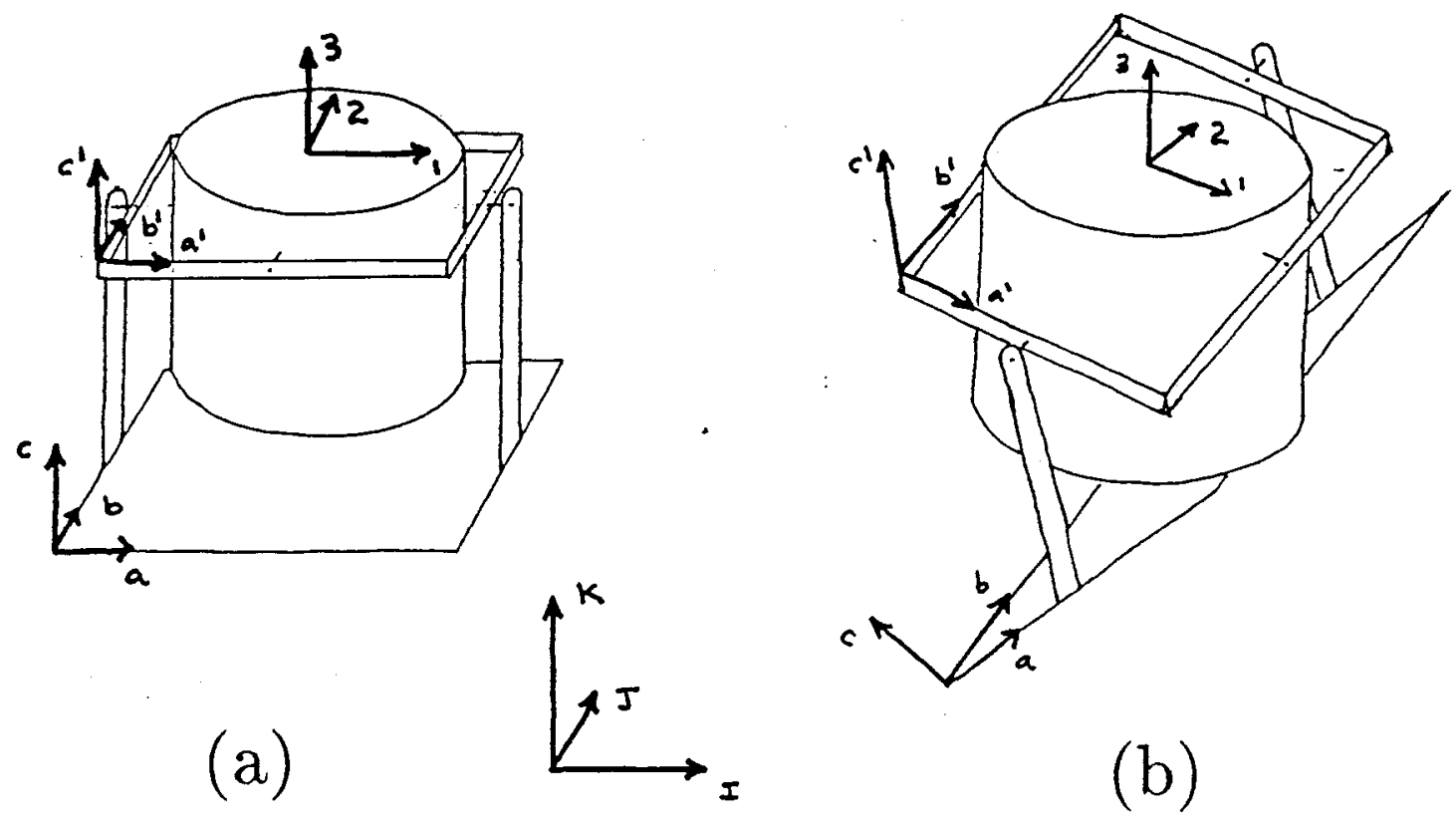

(b)

Figure 4-5: Definition of SASS coordinate axes: (a) gives the undisplaced gyro orientation while (b) shows the gyro under a general displacement. The $I-J-K$ axes are fixed in inertial space.

has two gimbals. Measurements are made between: the platform to which the gyro is fixed (instrument coordinates) and the outer gimbal, the outer gimbal and the inner gimbal (also called the stabilized platform), and the inner gimbal and inertial space. To be able to take the current meter measurements made in the instrument relative frame and transform them into the inertial frame, each of the motion measurements must be expressible in both of those frames. By defining a set of axes on each moving part of the gyro package we enable ourselves to mathematically express each measurement in any of the frames. 


\section{angular displacement}

The angular orientation of a solid body with respect to a given reference frame may be specified in a number of ways. With the defined set of coordinate axes on our body the problem is formally reduced to that of determining the angular position of two sets of coordinates with respect to one another.

Let $\mathbf{u}_{I}-\mathbf{u}_{J}-\mathbf{u}_{K}$ be a triad of unit vectors fixed in inertial space and $\mathbf{u}_{a}-\mathbf{u}_{b}-\mathbf{u}_{c}$ be a triad of unit vectors fixed to the body. The orientation of the $\mathbf{u}_{a}$-axis may be uniquely determined by specifying the cosine of the angle between the $\mathbf{u}_{a}$-axis and each of the three inertial axes

$$
\begin{aligned}
c_{a I} & =\cos (a, I) \\
c_{a J} & =\cos (a, J) \\
c_{a K} & =\cos (a, K) .
\end{aligned}
$$

If this is done for each axis, the angular position of the body is then defined at any instant of time. The result is conveniently expressed in matrix form as:

$$
\left\{\begin{array}{l}
\mathbf{u}_{a} \\
\mathbf{u}_{b} \\
\mathbf{u}_{c}
\end{array}\right\}=\left[\begin{array}{ccc}
c_{a I} & c_{a J} & c_{a K} \\
c_{b I} & c_{b J} & c_{b K} \\
c_{c I} & c_{c J} & c_{b K}
\end{array}\right]\left\{\begin{array}{l}
\mathbf{u}_{I} \\
\mathbf{u}_{J} \\
\mathbf{u}_{K}
\end{array}\right\} .
$$

The matrix of cosine angles is usually called the rotational translation matrix ( $r$-t matrix). In practice, the $r$-t matrix is not directly obtained from measurements. The task then, is to formulate the $r$ - $t$ matrix from the measurements we make.

The compass (ideally) measures the rotation of the stabilized platform with respect to inertial space. Because the compass angle $\theta_{Y}$ is positive when the stabilized platform turns in a left hand sense with respect to inertial coordinates

$$
\left\{\begin{array}{l}
\mathbf{u}_{1} \\
\mathbf{u}_{2} \\
\mathbf{u}_{3}
\end{array}\right\}=\left[\begin{array}{ccc}
\cos \theta_{Y} & -\sin \theta_{Y} & 0 \\
\sin \theta_{Y} & \cos \theta_{Y} & 0 \\
0 & 0 & 1
\end{array}\right]\left\{\begin{array}{c}
\mathbf{u}_{I} \\
\mathbf{u}_{J} \\
\mathbf{u}_{K}
\end{array}\right\}
$$


The roll potentiometer measures the relative angular motion between the outer gimbal $\mathbf{u}_{a^{\prime}}$ $\mathbf{u}_{b^{\prime}}-\mathbf{u}_{c^{\prime}}$ and the sensor coordinates $\mathbf{u}_{a^{-}}-\mathbf{u}_{b}-\mathbf{u}_{c}$, the roll angle $\theta_{R}$ is positive when the sensor coordinates rotate in a right hand sense with respect to the outer gimbal and thus

$$
\left\{\begin{array}{l}
\mathbf{u}_{a} \\
\mathbf{u}_{b} \\
\mathbf{u}_{c}
\end{array}\right\}=\left[\begin{array}{ccc}
1 & 0 & 0 \\
0 & \cos \theta_{R} & -\sin \theta_{R} \\
0 & \sin \theta_{R} & \cos \theta_{R}
\end{array}\right]\left\{\begin{array}{l}
\mathbf{u}_{a^{\prime}} \\
\mathbf{u}_{b^{\prime}} \\
\mathbf{u}_{c^{\prime}}
\end{array}\right\}
$$

The pitch potentiometer measures the angular displacement between the outer gimbal and the stabilized platform. In the SASS system, a right hand rotation of the outer gimbal with respect to the stabilized platform yields a positive output of pitch angle $\theta_{P}$ output and therefore

$$
\left\{\begin{array}{c}
\mathbf{u}_{a^{\prime}} \\
\mathbf{u}_{b^{\prime}} \\
\mathbf{u}_{c^{\prime}}
\end{array}\right\}=\left[\begin{array}{ccc}
\cos \theta_{P} & 0 & -\sin \theta_{P} \\
0 & 1 & 0 \\
\sin \theta_{P} & 0 & \cos \theta_{P}
\end{array}\right]\left\{\begin{array}{c}
\mathbf{u}_{1} \\
\mathbf{u}_{2} \\
\mathbf{u}_{2}
\end{array}\right\}
$$

By applying each of the r-t matrices above, hereafter denoted $\left[C_{\theta_{Y}}\right],\left[C_{\theta_{R}}\right]$ and $\left[C_{\theta_{P}}\right]$ respectively, in succession we can find the angular orientation of the sensor with respect to inertial coordinates

$$
\left\{\begin{array}{c}
\mathbf{u}_{a} \\
\mathbf{u}_{b} \\
\mathbf{u}_{c}
\end{array}\right\}=\left[C_{\theta_{R}}\right]\left[C_{\theta_{P}}\right]\left[C_{\theta_{Y}}\right]\left\{\begin{array}{c}
\mathbf{u}_{I} \\
\mathbf{u}_{J} \\
\mathbf{u}_{K}
\end{array}\right\}
$$

Recall that angular rotations are not vectors and are not commutative. Only the order of matrix multiplication specified above yields the appropriate result. The order is determined by the physical geometry of the sensors on the SASS buoy. The rotational translation matrix for the SASS will be defined as

$$
\left[C_{\theta}\right] \equiv\left[C_{\theta_{R}}\right]\left[C_{\theta_{P}}\right]\left[C_{\theta_{Y}}\right]
$$

and in terms of measurements we make $\left[C_{\theta}\right]$ is given by

$$
\left[\begin{array}{ccc}
\cos \theta_{P} \cos \theta_{Y} & -\cos \theta_{P} \sin \theta_{Y} & -\sin \theta_{P} \\
-\sin \theta_{R} \sin \theta_{P} \cos \theta_{Y}+\cos \theta_{R} \sin \theta_{Y} & \sin \theta_{R} \sin \theta_{P} \sin \theta_{Y}+\cos \theta_{R} \cos \theta_{Y} & -\sin \theta_{R} \cos \theta_{P} \\
\cos \theta_{R} \sin \theta_{P} \cos \theta_{Y}+\sin \theta_{R} \sin \theta_{Y} & -\cos \theta_{R} \sin \theta_{P} \sin \theta_{Y}+\sin \theta_{R} \cos \theta_{Y} & \cos \theta_{R} \cos \theta_{P}
\end{array}\right] .
$$




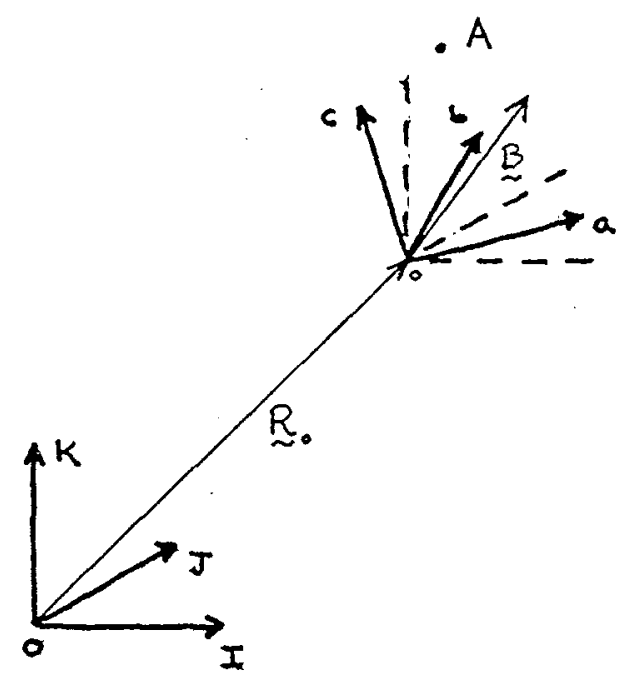

Figure 4-6: Coordinate system for transformation of velocities.

\section{linear displacement}

\section{general expressions}

Ultimately, our goal is to be able to describe the translational motion of all six velocity sensing pods. Let's begin, however, by considering the motion of a single point. In Figure 46 we have defined a set of inertial $O-I-J-K$ and moving $a-a-b-c$ coordinates. The velocity of point $A$ with respect to inertial space is

$$
\mathbf{v}_{A}=\frac{d \mathbf{R}_{0}}{d t}+\left(\frac{\partial \mathbf{r}}{\partial t}\right)_{r e l}+\Omega \times \mathbf{r}
$$

where $\mathbf{R}_{o}$ is the position vector of the moving axes with respect to the inertial axes, $\mathbf{r}$ is the position vector of point $A$ with respect to the moving axes, and $\Omega$ is the angular velocity of the moving axes. Differentiating the above expression shows the acceleration of point $A$ to be

$$
\mathbf{a}_{A}=\frac{d^{2} \mathbf{R}_{0}}{d t^{2}}+\mathbf{a}_{A r e l}+\dot{\Omega} \times \mathbf{r}+2 \Omega \times \mathbf{v}_{A r e l}+\Omega \times(\Omega \times \mathbf{r})
$$

\section{accelerometers}

To determine the acceleration of the SASS's coordinate origin we will utilize the output of the accelerometers. Of course, we must first understand what the accelerometer is measuring 
to do this. The accelerometers are mounted onto the stabilized platform of the gyro (i.e. they are fixed in frame o-1-2-3 of Figure 4-5). If we use the o-1-2-3 axes as our moving frame to define the acceleration of the accelerometers, then the relative velocity and acceleration terms in equation 4.10 vanish, and because the $\mathbf{u}_{1}-\mathbf{u}_{2}-\mathbf{u}_{3}$ triad only yaws with respect to the inertial axes, the angular velocity $\Omega$ is a function of $\theta_{Y}$ only. The acceleration of any point on the stabilized platform, then, is given by

$$
\mathbf{a}_{A_{i}}=\frac{d^{2} \mathbf{R}_{o}}{d t^{2}}+\left(\ddot{\theta}_{Y} r_{2}^{(i)}-\dot{\theta}_{Y}^{2} r_{1}^{(i)}\right) \mathbf{u}_{1}-\left(\ddot{\theta}_{Y} r_{1}^{(i)}+\dot{\theta}_{Y}^{2} r_{2}^{(i)}\right) \mathbf{u}_{2}
$$

with $r_{1}^{(i)}$ and $r_{2}^{(i)}$ being the component distances in the $\mathbf{u}_{1}$ and $\mathbf{u}_{2}$ directions to the $i^{\text {th }}$ point fixed on the stabilized platform ( $r_{3}$ does not appear because all points on a vertical axis have the same velocity on a body whose angular motion is pure yawing). The above expression gives the acceleration of any point on the stabilized platform. Accelerometers only measure the component of acceleration along:their sensitive axes. If we denote the output of the accelerometer whose axis is pointing in the $\mathbf{u}_{\mathbf{i}}$ (with $i=1,2,3$ ) direction as $a_{i}$ then the acceleration measured by each accelerometer is

$$
\begin{aligned}
& a_{1}=\mathbf{a}_{A_{3}} \cdot \mathbf{u}_{1}=\frac{d^{2} \mathbf{R}_{o}}{d t^{2}} \cdot \mathbf{u}_{1}+\left(\ddot{\theta}_{Y} r_{1}^{(1)}-\dot{\theta}_{Y}^{2} r_{1}^{(1)}\right) \\
& a_{2}=\mathbf{a}_{A_{2}} \cdot \mathbf{u}_{2}=\frac{d^{2} \mathbf{R}_{o}}{d t^{2}} \cdot \mathbf{u}_{2}-\left(\ddot{\theta}_{Y} r_{1}^{(2)}+\dot{\theta}_{Y}^{2} r_{1}^{(2)}\right) \\
& a_{3}=\mathbf{a}_{A_{3}} \cdot \mathbf{u}_{3}=\frac{d^{2} \mathbf{R}_{o}}{d t^{2}} \cdot \mathbf{u}_{3}-
\end{aligned}
$$

The accelerometers which output $a_{1}, a_{2}$ and $a_{3}$ are denoted the surge, sway and heave accelerometers, respectively. The expressions in equation 4.13 may be rearranged to find $\frac{d^{2} \mathbf{R}_{0}}{d t^{2}} \cdot \mathbf{u}_{(1,2,3)}$. Once this is done the linear acceleration of SASS's coordinate origin in inertial space is found by rotationally transforming $\frac{d^{2} \mathbf{R}_{0}}{d t^{2}} \cdot \mathbf{u}_{(1,2,3)}$ to $\frac{d^{2} \mathbf{R}_{0}}{d t^{2}} \cdot \mathbf{u}_{(I, J, K)}$ using $\left[C_{\theta_{Y}}\right]$. Explicitly, the acceleration, in terms of the accelerometer outputs, is

$$
\begin{aligned}
\frac{d^{2} \mathbf{R}_{\mathbf{o}}}{d t^{2}} & =\left\{a_{1} \cos \theta_{Y}+a_{2} \sin \theta_{Y}+\ddot{\theta}_{Y}\left(-r_{2}^{(1)} \cos \theta_{Y}+r_{1}^{(2)} \sin \theta_{Y}\right)+\dot{\theta}_{Y}^{2}\left(r_{1}^{(1)} \cos \theta_{Y}+r_{2}^{(2)} \sin \theta_{Y}\right)\right\} \mathbf{u}_{I} \\
& +\left\{a_{1} \sin \theta_{Y}+a_{2} \cos \theta_{Y}+\ddot{\theta}_{Y}\left(r_{2}^{(1)} \sin \theta_{Y}+r_{1}^{(2)} \cos \theta_{Y}\right)+\dot{\theta}_{Y}^{2}\left(-r_{1}^{(1)} \sin \theta_{Y}+r_{2}^{(2)} \cos \theta_{Y}\right)\right\} \mathbf{u}_{J} \\
& +\left\{a_{3}\right\} \mathbf{u}_{K} .
\end{aligned}
$$




\section{sensor velocities}

To describe the velocity $\mathrm{V}_{S_{i}}$ of a sensor at point $S_{i}$, it is convenient to again make use of the intermediate coordinate systems. In Figure 4-7 I show that the total velocity is composed of the translational velocity of the sensor axes' origin $\frac{d \mathbf{R}_{0}}{d t}$ and the velocity $\frac{d \mathbf{r}}{d t}$ relative to the origin,

$$
\mathrm{V}_{S_{i}}=\frac{d \mathbf{R}^{[i]}}{d t}=\frac{d \mathbf{R}_{o}}{d t}+\frac{d \mathbf{r}^{[i]}}{d t}
$$

Because the relative position of sensors are fixed with respect to the origin, the relative velocity vector only has an angular component and

$$
\mathrm{V}_{S_{i}}=\frac{d \mathbf{R}_{o}}{d t}+\omega \times \mathbf{r}^{[i]}
$$

where $\omega$ is the angular velocity of the SASS.

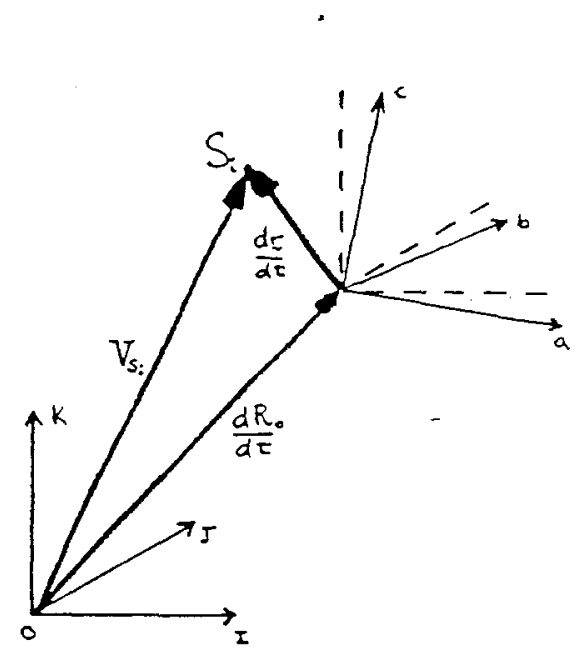

Figure 4-7: Composition of total velocity into coordinate and relative parts.

The angular velocity is defined by a right hand rule. Therefore, the angular velocities between intermediate coordinate systems defined in section 4.2 are

$$
\omega_{\theta_{Y}}=-\dot{\theta}_{Y^{\prime}} \mathbf{u}_{\mathbf{K}}, \quad \omega_{\theta_{P}}=-\dot{\theta}_{P} \mathbf{u}_{2}, \quad \omega_{\theta_{R}}=-\dot{\theta}_{R} \mathbf{u}_{\mathbf{a}^{\prime}} .
$$

Because angular velocities are vectors they may be added to find the total angular velocity 
of the SASS

$$
\omega=\omega_{\theta_{Y}}+\omega_{\theta_{P}}+\omega_{\theta_{R}}
$$

By performing the necessary $r-t$ matrix transformations to each of the terms in the above equation the angular velocity may be expressed in sensor coordinates as

$$
\begin{aligned}
\omega & =\left\{\dot{\theta}_{Y} \sin \theta_{P}-\dot{\theta}_{R}\right\} \mathbf{u}_{a} \\
& +\left\{\dot{\theta}_{P} \cos \theta_{R}+\dot{\theta}_{Y} \sin \theta_{R} \cos \theta_{P}\right\} \mathbf{u}_{b} \\
& +\left\{\dot{\theta}_{P} \sin \theta_{R}+\dot{\theta}_{Y} \cos \theta_{R} \cos \theta_{P}\right\} \mathbf{u}_{c} .
\end{aligned}
$$

The sensor coordinate representation is quite useful; as the sensor positions $\mathbf{r}^{[i]}$ are constants in the $o-a-b-c$ frame. However, as most of the presentation has been geared toward the inertial frame, the transformed result is given below:

$$
\begin{aligned}
\omega & =\left\{\dot{\theta}_{P} \sin \theta_{Y}-\dot{\theta}_{R} \cos \theta_{Y} \cos \theta_{P}\right\} \mathbf{u}_{I} \\
& +\left\{\dot{\theta}_{P} \cos \theta_{Y}-\dot{\theta}_{R} \sin \theta_{Y} \cos \theta_{P}\right\} \mathbf{u}_{J} \\
& +\left\{\dot{\theta}_{R} \sin \theta_{P}-\dot{\theta}_{Y}\right\} \mathbf{u}_{K}
\end{aligned}
$$

\section{field and sensor velocities}

The results of the preceeding sections allow us to find the vector velocities of all six BASS sensors as a function of time and to find the vector fluid velocities at each sensor as a function of time. The details of how this is done will be found in the Processing Strategy section. Nonetheless, it may be helpful to quickly outline the procedure here.

First, consider the sensor velocities. The acceleration of the SASS's coordinate origin is found from equation 4.13. These accelerations are integrated to find the velocity of the origin in inertial space. Each of the sensors is in pure rotation about the SASS coordinate axis origin. The angular velocity of the SASS, $\omega$, is found from equation 4.19 . The $\omega \times \mathbf{r}^{[i]}$ product is then formed for each sensor. After rotating the $\omega \times \mathbf{r}^{[i]}$ product into inertial coordinates using the inverse $\mathrm{r}$-t matrix $\left[C_{\theta}\right]^{-1}$, the total sensor velocity is formed as in equation 4.15 . 
The velocity relative to the instrument is measured by the BASS acoustic sensing pods. The measurements are rotated using $\left[C_{\theta}\right]^{-1}$ to obtain the fluid velocity relative to each sensor $\mathbf{v}_{r e l_{i}}$ in inertial coordinates. The two results may then be added to obtain the velocity of the fluid $V_{i}$ at the location of the $i^{\text {th }}$ sensor,

$$
V_{i}=V_{S_{i}}+v_{r e l_{i}}
$$

The sensor motions are small compared to the length scale of the waves and so, typically, one may take the above velocities to be the velocity at a point (when this is done we will address the errors that may be induced).

\subsection{Estimation of directional spectra}

Simply averaging the velocity $\mathrm{V}$ gives us a biased result due to the presence of waves. Earlier, we formed an unbiased estimator of the mean current $\widehat{V}$ which was written as

$$
\widehat{V}_{\alpha} \equiv \frac{1}{T} \int_{0}^{T} d t\left[V_{\alpha}(t)-\left\{V_{i}(t) * \mu_{i}^{(\alpha)}(t)\right\} \dot{X}_{i}(t)\right]
$$

The $\mu$ 's were filters that were made by performing several integrals of the form

$$
\int_{\theta} \cos ^{i} \theta \sin ^{j} \theta f(\sigma, \theta) d \theta
$$

where $f(\sigma, \theta)$ was the directional spreading function of the waves ( $i$ and $j$ are integers). The estimation of the directional wave spectrum is, then, central to our goal of making unbiased current meter measurements. But it is not only this kinematic effect of the waves with which we are concerned. The energetics of the wave field are important to understanding the dynamics on both the water and air side of the air-sea interface. The reader will not make many new discoveries here. The theory presented in this chapter represents an adaptation of other investigators work to the SASS.

The traditional way of estimating directional spectra from a surface following float is to use the pitching and rolling motions of the buoy as was done by Longuet-Higgins et al (1963). With SASS we find this is not the best way to proceed. 
With a horizontal length scale of $3.7 \mathrm{~m}$, it would not be reasonable to expect that the buoy could accurately follow waves considerably longer than twice this length. Further, pitch and roll estimates are heavily dependent on the transfer function of a buoy. SASS is a complex moored structure and prediction of a transfer function would be extremely difficult. Of course, with all the relative velocities and sensor velocities being measured a transfer function could be derived. Rather than take this circuitous path we can just use the field velocities of the fluid $\mathrm{V}^{(i)}(\mathbf{x}, t)$ instead of the heave, pitch and roll.

Barrick et al (1989) have demonstrated that not only will errors in the estimation of a pitch and roll buoy's transfer function degrade spectral estimates but so too will the nonlinearities of the wavefield. In correlating the components of $\mathbf{V}$ to estimate directional spectra we reduce the severity of both of these problems. The motion of SASS is measured and thus there is no need to estimate the transfer function. The Stokes' expansion of waves predicts that nonlinearities are two orders of magnitude smaller (when scaled by waveslope) in the velocity field than in the height/slope field (e.g., see Wehausen and Laitone, 1960). The estimate of velocity spectrum based on velocities rather than surface elevation will thus be far less sensitive to wave steepness effects.

The adaptation of the pitch and roll theory is straightforward to the use of velocities. As Longuet-Higgins et al (1963) did in their original development, I adopt the Fourier-Steiljes representation of the wavefield for this analysis and thereby will have a certain degree of parallelism with the well-known original document. In this representation the potential function for the wavefield may be written as

$$
\Phi \equiv \Re \iint-\imath \frac{g}{\sigma} e^{\imath(\mathbf{k} \cdot \mathbf{x}+\sigma t)} e^{k z} d \mathbf{A}(\sigma, \theta)
$$

where $\mathbf{x}=\left(x_{I}, x_{J}\right), \sigma^{2}=k g, \mathbf{k}=(k \cos \theta, k \sin \theta)$ and $z=x_{K}$. Note that the wavenumber vector points into the direction of wave origin and that $z$ is taken to be positive up.

From the above definition and sign convention we deduce that the surface elevation $\eta$ 
and field velocities $\left(V_{I}, V_{J}, V_{K}\right)$ are given by

$$
\eta=-\left.\frac{1}{g} \frac{\partial \Phi}{\partial t}\right|_{z=0}=\Re \iint-e^{\imath(\mathrm{k} \cdot \mathbf{x}+\sigma t)} d \mathbf{A}
$$

and

$$
\begin{gathered}
V_{I}=\frac{\partial \Phi}{\partial x_{I}}=\Re \iint \sigma \cos \theta e^{\imath(\mathbf{k} \cdot \mathbf{x}+\sigma t)} e^{k z} d \mathbf{A}, \\
V_{J}=\frac{\partial \Phi}{\partial x_{J}}=\Re \iint \sigma \sin \theta e^{\imath(\mathbf{k} \cdot \mathbf{x}+\sigma t)} e^{k z} d \mathbf{A}, \\
V_{K}=\frac{\partial \Phi}{\partial x_{K}}=\Re \iint-\imath \sigma e^{\imath(\mathbf{k} \cdot \mathbf{x}+\sigma t)} e^{k z} d \mathbf{A} .
\end{gathered}
$$

The directional spectrum $F(\sigma, \theta)$ can be expressed as a product of the surface elevation power spectral density $S(\sigma)$ and a directional spreading function $f(\sigma, \theta)$ (where the spreading function is normalized such that $\int_{-\pi}^{\pi} f(\sigma, \theta) d \theta=1$ ),

$$
F(\sigma, \theta)=S(\sigma) \dot{f}(\sigma, \theta)=\frac{\frac{1}{2} d \mathbf{A} d \mathbf{A}^{*}}{d \sigma d \theta}
$$

The first step in the estimation process is to form the co- and quad-spectra of the fluid velocities according to the following definition

$$
\hat{V}_{i} \hat{V}_{j}^{*} \equiv C_{i j}-2 Q_{i j}
$$

where $\hat{V}_{i}$ is the Fourier transform of $V_{i}$. If the motions of the sensor making the measurement are small compared to the wavelength of the waves, we can ignore the $\mathbf{k} \cdot \mathbf{x}$ in the exponential of equation 4.26 when finding the theoretical form of equation 4.28 . The needed co- and quad-spectra are:

$$
\begin{aligned}
& C_{11}(\sigma)=S(\sigma) \sigma^{2} e^{2 k z} \int \cos ^{2} \theta f(\sigma, \theta) d \theta \\
& C_{21}(\sigma)=S(\sigma) \sigma^{2} e^{2 k z} \int \cos \theta \sin \theta f(\sigma, \theta) d \theta \\
& C_{22}(\sigma)=S(\sigma) \sigma^{2} e^{2 k z} \int \sin ^{2} \theta f(\sigma, \theta) d \theta \\
& Q_{31}(\sigma)=S(\sigma) \sigma^{2} e^{2 k z} \int \cos \theta f(\sigma, \theta) d \theta \\
& Q_{32}(\sigma)=S(\sigma) \sigma^{2} e^{2 k z} \int \sin \theta f(\sigma, \theta) d \theta \\
& C_{33}(\sigma)=S(\sigma) \sigma^{2} e^{2 k z} \int f(\sigma, \theta) d \theta=S(\sigma) \sigma^{2} e^{2 k z}
\end{aligned}
$$


The spreading function is defined by the transform pair

$$
\begin{aligned}
& f(\sigma, \theta)=\sum_{n=-\infty}^{\infty} \hat{c}(\sigma, n) e^{-\imath \theta n}, \\
& \hat{c}(\sigma, n)=\frac{1}{2 \pi} \int_{-\pi}^{\pi} f(\sigma, \theta) e^{\imath \theta n} d \theta .
\end{aligned}
$$

Comparing the expressions in equation 4.30 to the expansion for the spreading function $f(\sigma, \theta)$ above, it is seen that the correlation coefficients are directly proportional to the various co- and quad-spectra. For instance, it is clear that the first lag may be written as

$$
\hat{c}(\sigma, 1)=\frac{1}{2 \pi} \frac{Q_{31}(\sigma)}{C_{33}(\sigma)}+\imath \frac{1}{2 \pi} \frac{Q_{32}(\sigma)}{C_{33}(\sigma)} .
$$

Longuet-Higgins et al (1963) formed all of their coefficients in an equivalent manner. This approach is entirely correct mathematically, but, forming the correlation coefficients as Long (1980) did reduces the errors in coefficient estimation from miscalibration of sensors. These estimates of the $\hat{c}$ correlation coefficients may be written as:

$$
\hat{c}_{0}=\frac{1}{2 \pi}, \quad \hat{c}_{1}=\hat{d}_{1}+\imath \hat{d}_{2}, \quad \hat{c}_{2}=\hat{d}_{3}+\imath \hat{d}_{4},
$$

with

$$
\begin{array}{ll}
\hat{d}_{1}=\frac{Q_{31}}{2 \pi \sqrt{C_{33}\left(C_{11}+C_{22}\right)}}, & \hat{d}_{3}=\frac{C_{11}-C_{22}}{2 \pi\left(C_{11}+C_{22}\right)}, \\
\hat{d}_{2}=\frac{Q_{32}}{2 \pi \sqrt{C_{33}\left(C_{11}+C_{22}\right)}}, & \hat{d}_{4}=\frac{C_{21}}{\pi\left(C_{11}+C_{22}\right)} .
\end{array}
$$

Since $f(\sigma, \theta)$ is real we have that $\hat{c}(\sigma, n)=\hat{c}^{*}(\sigma, n)$. The spreading function $f(\dot{\sigma}, \theta)$ in expanded form is

$$
f(\sigma, \theta)=\cdots+\hat{c}_{2}^{*} e^{\imath 2 \theta}+\hat{c}_{1}^{*} e^{\imath \theta}+\hat{c}_{0}+\hat{c}_{1} e^{-\imath \theta}+\hat{c}_{2} e^{-\imath 2 \theta}+\cdots
$$

The original Longuet-Higgins et al(1963) estimate of $f(\sigma, \theta)$ is just a windowed version of the above:

$$
f_{L H}(\sigma, \theta)=\frac{1}{6} \hat{c}_{2}^{*} e^{\imath 2 \theta}+\frac{2}{3} \hat{c}_{1}^{*} e^{\imath \theta}+\hat{c}_{0}+\frac{2}{3} \hat{c}_{1} e^{-\imath \theta}+\frac{1}{6} \hat{c}_{2} e^{-\imath 2 \theta}
$$

The window was applied because if the Fourier series in equation 4.34 is simply truncated there exists the possibility of the directional spectrum estimate being negative in some regions. Of course, windowing has the effect of reducing the resolution. With only five 
correlation coefficients to begin with, and the application of a wide window implied in equation 4.35 , we do not expect a high resolution estimate of the wave spreading.

While no one has yet developed a technique for measuring the higher Fourier coefficients to improve the resolution of the estimates, many investigators have tried to use modelling techniques to obtain better resolution with those parameters that are measured. The idea is that rather than assume that all non-observed correlation coefficients are zero, as truncating and/or windowing the Fourier series does, that the non-observed correlation coefficients should in some way be estimated.

\section{Maximum Entropy Method}

One such effort was presented by Lygre and Krogstad (1986). They claimed that a high resolution, minimally biased solution could be attained by using the maximum entropy $\operatorname{method}(\mathrm{MEM})$.

Consistent with our transform pair, the general MEM representation as an all-pole model is given by

$$
f(\sigma, \theta)=\frac{\vartheta^{2}}{\left|1+\sum_{k=1}^{P} \bar{a}_{k} e^{-\imath k \theta}\right|^{2}},
$$

where $P$ is the number of poles in the model. Because we only estimate two lags (besides the zeroeth) of the complex correlation sequence, our model will be a two-pole model: The MEM parameters are estimated from the Yule-Walker equations (see, for example, Kay and Marple, 1981). We find it convenient to write the order-two Yule-Walker equations in a form which includes $\vartheta$,

$$
\left[\begin{array}{ccc}
\hat{c}_{0} & \hat{c}_{1}^{*} & \hat{c}_{2}^{*} \\
\hat{c}_{1} & \hat{c}_{0} & \hat{c}_{1}^{*} \\
\hat{c}_{2} & \hat{c}_{1} & \hat{c}_{0}
\end{array}\right]\left\{\begin{array}{c}
1 \\
a_{1} \\
a_{2}
\end{array}\right\}=\left\{\begin{array}{c}
\vartheta^{2} \\
0 \\
0
\end{array}\right\} .
$$

Expressions for $a_{1}, a_{2}$, and $\vartheta^{2}$ may be derived by inverting equation 4.37 ,

$$
a_{1}=\frac{c_{1}^{*} c_{2}-c_{1} c_{0}}{c_{0}^{2}-c_{1}^{*} c_{1}}
$$




$$
\begin{aligned}
& a_{2}=-\frac{c_{2}+c_{1} a_{1}}{c_{0}}, \\
& \vartheta^{2}=c_{0}+c_{1}^{*} a_{1}+c_{2}^{*} a_{2} .
\end{aligned}
$$

These variables may then be combined in the expression below to arrive at the two-pole estimate of $f(\sigma, \theta)$,

$$
f(\sigma, \theta)=\frac{\vartheta^{2}}{\left|1+a_{1} e^{-i \theta}+a_{2} e^{-i 2 \theta}\right|^{2}} .
$$

Its interesting to note that because of the fact that the MEM model is an all-pole representation, that there's no need to perform the integrals of the form we desire (equation 4.22) by discrete approximation. The exact value of the integrals may be obtained algebraically using residue calculus (see Appendix A).

\section{motion errors}

Since the errors in making a mean current meter measurement have been discussed in so much detail, it seems that we should also address the motion issue as it applies to the estimation of the directional spectra. In deriving the expressions for the co- and quad-spectra of equation 4.28 we have assumed that measurements are made at a fixed location. This is not the case for most pitch and roll measurements; nor is it the case with the SASS. As we shall see, however, the errors in this case are not nearly so serious as they are for measurement of the current.

Now we address the fact that the SASS is a moving sensor. The pitch and roll theory assumes measurements are made at a fixed point $\mathbf{X}_{o}$,

$$
\mathrm{V}_{m}(\mathrm{X}(t) ; t) \approx \mathrm{V}\left(\mathrm{X}_{\circ} ; t\right)
$$

(subscript $m$ denotes measured velocity). Using potential theory expressions for the orbital velocities, we see that to $O(A k \mathrm{~V})$

$$
\mathrm{V}_{m}(t)=\mathrm{V}\left(\mathrm{X}_{o} ; t\right)+\left[2 k(t) * \int_{\theta} \cos \theta \mathrm{V}(\theta, t) d \theta\right] \cdot x_{I}(t)
$$




$$
+\left[\imath k(t) * \int_{\theta} \sin \theta \mathrm{V}(\theta, t) d \theta\right] \cdot x_{J}(t)+[k(t) * \mathrm{~V}(t)] \cdot x_{K}(t)
$$

with $\imath=\sqrt{-1}, k$ being the wavenumber, and $\theta$ being the angle in the horizontal $(I-J)$ plane (we take $K$ to be upwards). $V_{m_{I}}, V_{m_{J}}, V_{m_{K}}$ are the field velocities measured in the inertial coordinates $I, J, K$. The $*$ indicates convolution. The cross-spectra $S_{V_{i} V_{j}}=C_{V_{i} V_{j}}-{ }_{\imath} Q_{V_{i} V_{j}}$ are needed to estimate the directional spectra of the wavefield. If we denote radian frequency by $\sigma$, then we may express that the spectrum measured by the moving sensor will equal the spectrum at the mean location plus a "modulation spectrum" as

$$
S_{V_{m_{i}} V_{m_{j}}}(\sigma)=S_{V_{i} V_{j}}(\sigma)+S_{M O D_{i j}}(\sigma)
$$

From equation 4.42 we find $S_{M O D_{i}}$, to be

$$
\begin{aligned}
S_{M O D_{i j}} & =\left\{k^{2}(\sigma) S_{V_{i} V_{j}}(\sigma) \int_{\theta} \cos ^{2} \theta f(\sigma, \theta) d \theta\right\} * S_{X_{I} X_{I}}(\sigma) \\
& +\left\{k^{2}(\sigma) S_{V_{i} V_{j}}(\sigma) \int_{\theta} \sin ^{2} \theta f(\sigma, \theta) d \theta\right\} * S_{X_{J} X_{J}}(\sigma) \\
& +\left\{k^{2}(\sigma) S_{V_{i} V_{j}}(\sigma)\right\} * S_{X_{K} X_{K}}(\sigma) \\
& +\left\{k^{2}(\sigma) S_{V_{i} V_{j}}(\sigma) \int_{\theta} \cos \theta \sin \theta f(\sigma, \theta) d \theta\right\} * 2 \Re\left(S_{X_{I} X_{J}}(\sigma)\right) \\
& +\left\{k^{2}(\sigma) S_{V_{i} V_{j}}(\sigma) \int_{\theta} \cos \theta f(\sigma, \theta) d \theta\right\} * 2 \Im\left(S_{X_{K} X_{I}}(\sigma)\right) \\
& +\left\{k^{2}(\sigma) S_{V_{i} V_{j}}(\sigma) \int_{\theta} \sin \theta f(\sigma, \theta) d \theta\right\} * 2 \Im\left(S_{X_{K} X_{J}}(\sigma)\right) .
\end{aligned}
$$

The wave spreading function $f(\sigma, \theta)$ is normalized so that $\int_{\theta} f(\sigma, \theta) d \theta=1 . \Re$ and $\Im$ indicate where the real or imaginary part of the cross-spectrum is to be taken. Let's consider the form of equation 4.44. Each term is the cross-spectrum of the velocities to be estimated, times the wavenumber squared, convolved with a sensor displacement spectrum. Sensor motions should be the same order of magnitude as the wave orbital excursions. In this case it is clear that $S_{M O D} \sim(A k)^{2} S_{V_{i}} V_{j}$. Because the modulated energy is scaled by the waveslope squared this effect is usually ignored. However, since the spectra are convolved, we expect that the peaks in the modulation spectrum will be located at the sums and differences of the peaks of the fluid velocity spectra and motion spectra. With wave spectra typically being narrowband, this allows for the possibility that the energy is modulated into bands where $k^{2}\left(\sigma_{\text {peakV }}\right) S_{V_{i} V_{j}}\left(\sigma_{\text {peakV }}\right) S_{X_{a} X_{b}}\left(\sigma_{\text {peak }}\right)$ is non-negligible. 
So, the energy in the error spectrum is scaled by the waveslope squared and thus is negligible. It is only in certain spectral bands that the error may be significant. A more detailed discussion of this type of error is given in Santala and Williams (1990). 


\section{Chapter 5}

\section{MAKING MEASUREMENTS FROM THE SASS}

\subsection{Deployment history}

The SASS has been deployed, as part of the Shelf MIxed Layer Experiment (SMILE) program, in two different time intervals. The first deployment occurred from November 28 , 1988 to December 3, 1988. The second deployment occurred from February 24, 1989 to March 3, 1989. As part of the Shelf MIxed Layer Experiment (SMILE), the SASS was only one component of a multi-instrument experiment. The large scale shelf dynamics was sampled during SMILE by ship and by having buoys deployed at several sites. The SMILE experiment, on the grand scale, will not be discussed here. Figure 5-1 shows the. sites where SMILE instruments were deployed. The SASS was deployed at the C3 site for both deployments. The other component of SMILE that will bear directly on the work here is the buoy designated the "C3 buoy". The C3 buoy was a discus buoy instrumented with current meters and atmospheric sensors. The C3 buoy and the SASS were deployed as close as practicable (about $500 \mathrm{~m}$ apart) so that the data from the two buoys could be used for comparison and supplementation to one another. The C3 buoy, its sensors, and how its outputs were interpreted in comparison with the SASS are discussed in chapter 6. 


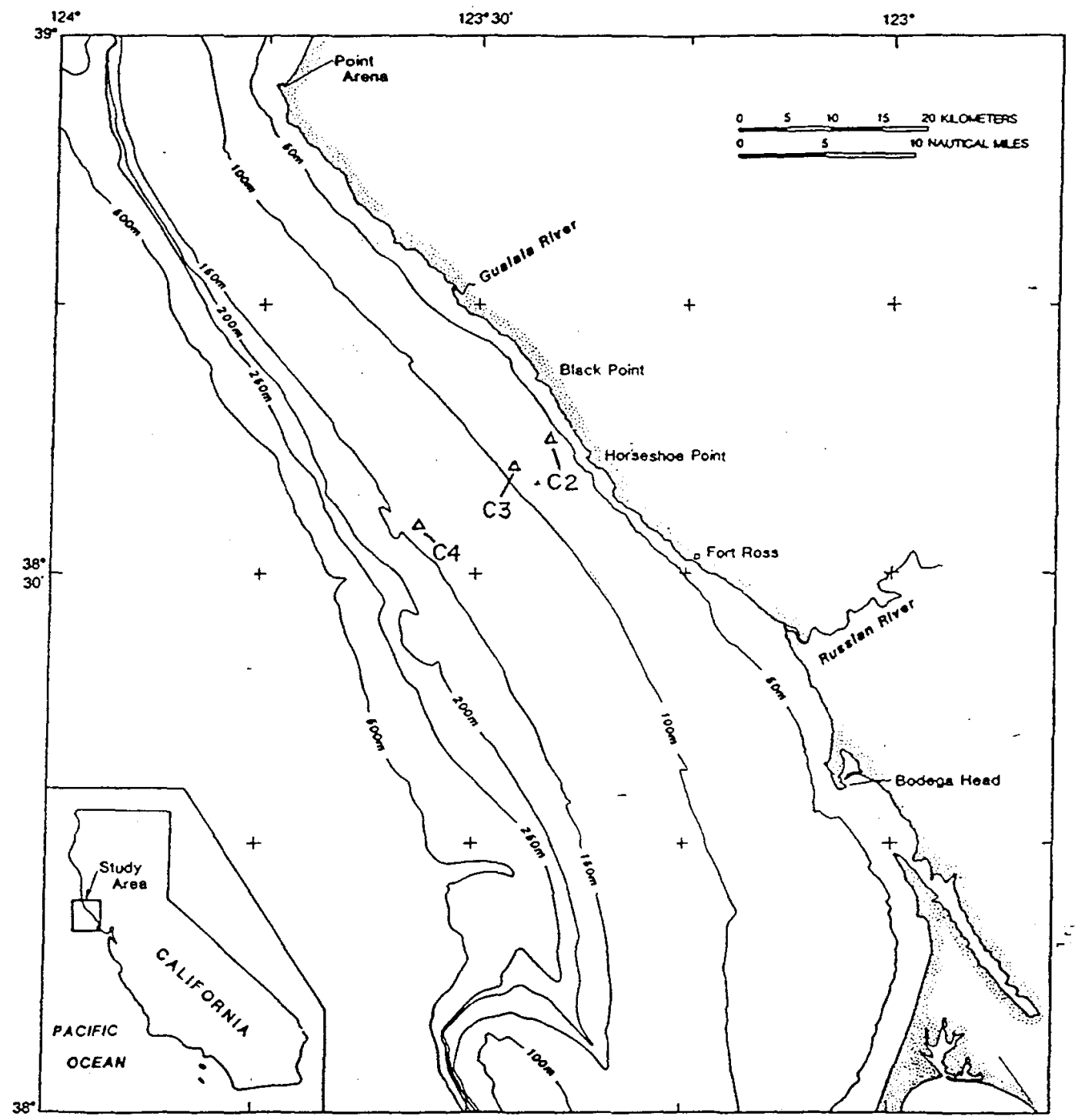

Figure 5-1: Site of SMILE deployments. The SASS was deployed at the C3 site. 


\section{the first deployment}

After its initial construction at Woods Hole, the SASS was disassembled and sent to Sausalito, CA. In Sausalito, the SASS was reconstructed and routine testing was performed on the instrument prior to loading the buoy onto the $R / V$ Wecoma (the ship which transported the SASS to its deployment site). Part of the testing regiment was to perform "bagged zeros". The scale factor of the BASS current meter is set by the speed of sound in water, the offset depends on the way the cables are run from transducers to the BASS computer. This presumably happens because the capacitance of the cables changes when the cables are flexed into new configurations. In the bagged zero, the BASS sensing pods are wrapped in plastic - guaranteeing zero net velocity - and a "zero file" is recorded. The bagged zero off the dock at Saulsilito was the first time the SASS was tested in the water. All tests were successful and the SASS was then transported to the site and initially deployed at November 28, 1988 at 07:50 (GMT). The conditions were extremely calm but to test the system we immediately commanded SASS to take data. This data was examined on board the Wecoma and seemed to indicate that everything worked perfectly. The report by Montgomery and Santala (1989) contains the entire cruise logs. The cruise log contains only rough estimates of wind (ship's anemometer) and wave (visual estimation) conditions. That information has now been superceded by the measurements made by the SMILE instruments. The position of the initial deployment was $38^{\circ} 38.88^{\prime} \mathrm{N}, 123^{\circ} 29.32^{\prime} \mathrm{W}$. The SASS successfully recorded data, upon command, only until December 3, 1988. The cause of failure was an electrical grounding problem which caused the batteries to discharge. The SASS was recovered after radio contact with the buoy could no longer be made. Once recovered, the wiring was fixed, the batteries recharged and SASS was, on December 4 , redeployed at almost the same position $\left(38^{\circ} 38.93^{\prime} \mathrm{N}, 123^{\circ} 29.38^{\prime} \mathrm{W}\right)$. The SASS again was recording data until its deployment window ended. On December 9, 1988 the SASS was recovered and returned to Sausalito where it stayed on the dock until the next deployment. 


\section{the second deployment}

A few minor improvements were made to the SASS in the time between the two deployments. The most notable change was the addition of the LOPACS optical disc recording system. This system was added as an alternate data recovery system to the radio.

Prior to its deployment there were serious questions regarding the survivability of the SASS. In the field, the SASS only suffered some minor structural damage (weld cracks). The SASS did suffer some major structural damage after deployment when being moved by forklift to its interdeployment storage area. New SASS members were constructed at Woods Hole during the intercruise hiatus and the SASS was restored to full health prior to its second deployment.

The buoy was deployed for its second time on February 24, 1989. The location $\left(38^{\circ}\right.$ $38.83^{\prime} N, 123^{\circ} 29.27^{\prime} W$ ) was again at the C3 site. The SASS recorded data successfully until February 29, 1989; when the batteries ran out. We were unable to recover the SASS until March 3. During this time when the SASS was "dead-in-the-water" there was a rather severe storm.

In Montgomery and Santala (1989), it was noted that the SASS may have experienced two failure modes on this deployment. At the time that report was written the performance of the SASS could not be evaluated from the data. Now that the data from the SASS has been fully analyzed, this situation is updated. First, the pitch potentiometer was found, upon return to Woods Hole, to have a highly nonlinear calibration (the precruise calibration was almost perfectly linear). It is believed that this damage may have occurred during return shipping (the gyro was improperly packaged for its final return trip to Woods Hole). Damage may also have occurred after the batteries ran out. The gyro, when not in use, is clamped (caged) by four bars. It may be that when the voltage levels dropped

that the caging mechanism was not triggered properly (the mechanism itself will work on extremely low voltages). If that was the case, then the gyro platform would have spent four days slamming around wildly. There is no evidence of the gyro losing its calibration 
while deployed. All evidence seems to indicate that one of the two reasons above explains the damage. The second failure mode of the SASS was structural. The SASS frame lost a structural member (one of the two inch $O-D$ aluminum tubes) due to the failure of a fastener. Once this occurred, the support of the BASS current meter tower was much weaker, and the BASS tower failed structurally. Due to the dramatic mode of failure, we can be certain that this failure did not occur during the interval when data was being recorded. Whether the final collapse of the BASS tower occurred immediately when the SASS lost its member, or whether the BASS tower collapse occurred once the additional stresses of the recovery procedure were applied still can not be determined. Nevertheless, the conclusion on the spring deployment, after data analysis, is that the SASS operated normally during the time data was being taken.

Wind conditions were one of the most important considerations driving our decision of when to record data. Figures 5-2 and 5-3 show the wind speed measured by the C3 buoy during the days when the SASS was actually recording data.

\subsection{Processing strategy}

In section 4.2 the coordinate transformations by which measurements in the instrument relative frame are transformed into the inertial frame were derived. The question remains as to what are the precise treatments of the data to perform these steps. Data are recorded as digital counts of a noisy signal and therefore care must be taken to ensure that the transformations of section 4.2 are not only mathematically correct but also can be implemented in such a way that measurement noise is not unduly amplified during processing.

Outlined here will be the entire processing strategy associated with SASS. Throughout the discussion we may take a look at various SASS files. For the sake of continuity two files will be closely examined; files $f$ and $q$. During file $f$, from the fall deployment, the $10 \mathrm{~m}$ windspeed was $5.7 \mathrm{~m} / \mathrm{s}$. The windspeed for file $q$ was almost twice as high $\left(U_{10}=10.2 \mathrm{~m} / \mathrm{s}\right)$. Note that, in the jargon used here, a record is a complete set of measurements made at a 

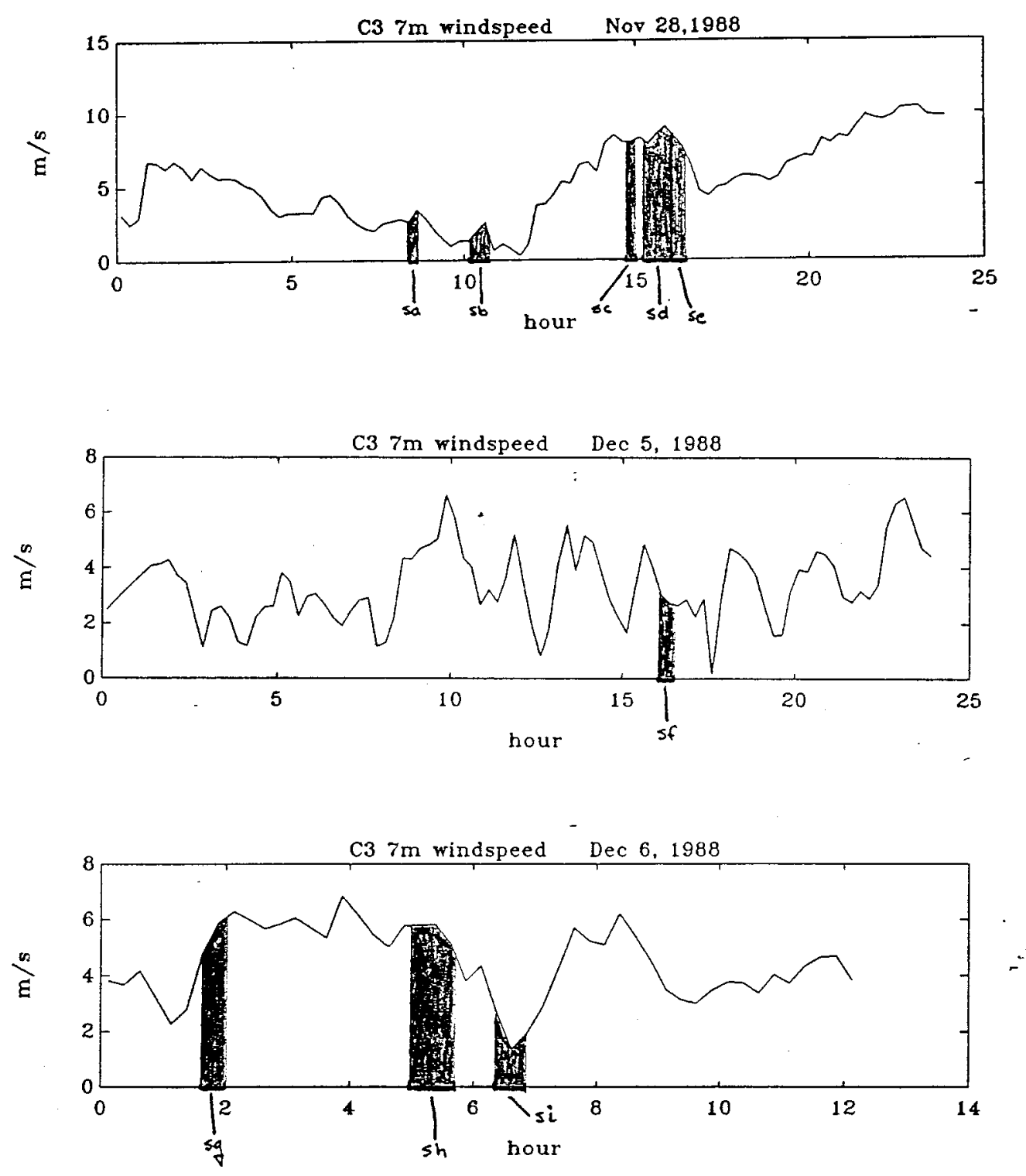

Figure 5-2: Wind speed and measured at the C3 buoy during the fall 1988 deployment. Intervals when the SASS was actually recording data are shaded. 

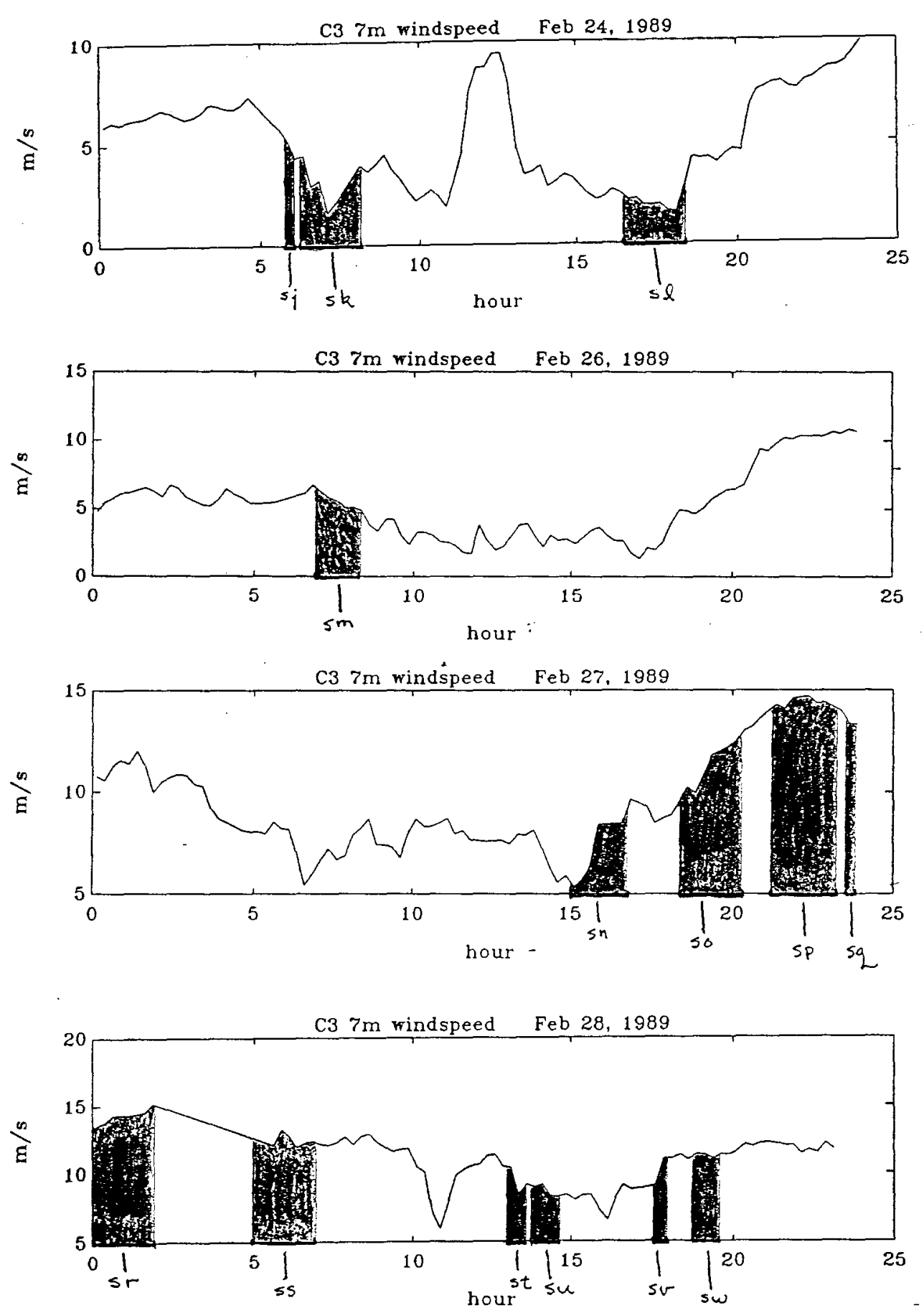

Figure 5-3: Wind speed and measured at the C3 buoy during the spring 1989 deployment. Intervals when the SASS was actually recording data are shaded. 
discrete interval in time. A file is made up of a sequence of records.

We begin our discussion of how the data was processed by starting right at the instrument, looking at the "processing" that occurred in the field. All the measurements made were sent to the BASS computer and digitized. The format of the output is given in Table 5-1. After each variable in the table, the number in brackets \{\} specifies the length of

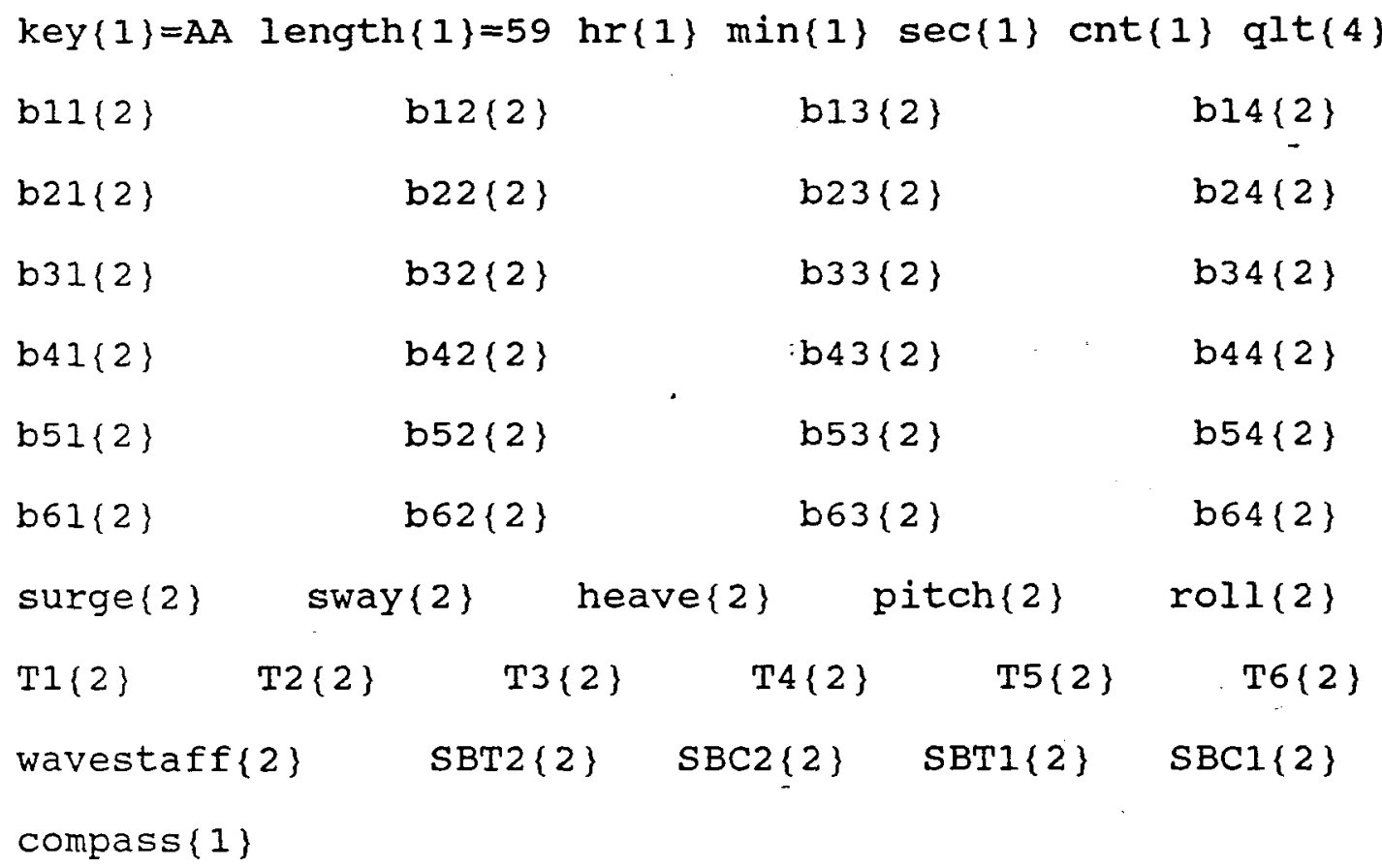

Table 5-1: SASS data format. The record begins with timing information. The $b_{i j}$ 's are the measurements made from the BASS. Following this is gyro data. $T 1$ to $T 6$ are metal-clad thermistors. Then the wavestaff and Sea-Bird sensor output are listed. The last piece of data in the record is the compass. The numbers in brackets indicate the length of each variable in bytes.

the variable in bytes. The length variable is the length of a complete record in bytes (the sum of all the numbers in brackets) represented in hexidecimal format. The beginning of each record sent is the same, namely $A A 59$. This string, common to each record, allows the data to be synchronized when unpacking the data. Following these synchronization bytes is the time stamp in hours, minutes, seconds, and a sample counter (which cycles from 0 to 
255). The quality word is a check on the received signal of the current meter.

The actual data follows this record synchronization and time information. Each of the B1's is the output of a BASS sensing axis. For each sensing pod there are four axes. These axes are labelled A,B,C and D according to the convention of Figure 5-4. Shortly, I will discuss how these axes are combined into vector velocities. BASS data is followed in the stream by the digitized gyro package data (the digitization actually is done by the BASS computer). The metal-clad thermistors are $\mathrm{T} 1$ to $\mathrm{T} 6$. Then comes the wavestaff data and the Sea-Bird temperature and conductivity data. The last variable in the record is the compass output.

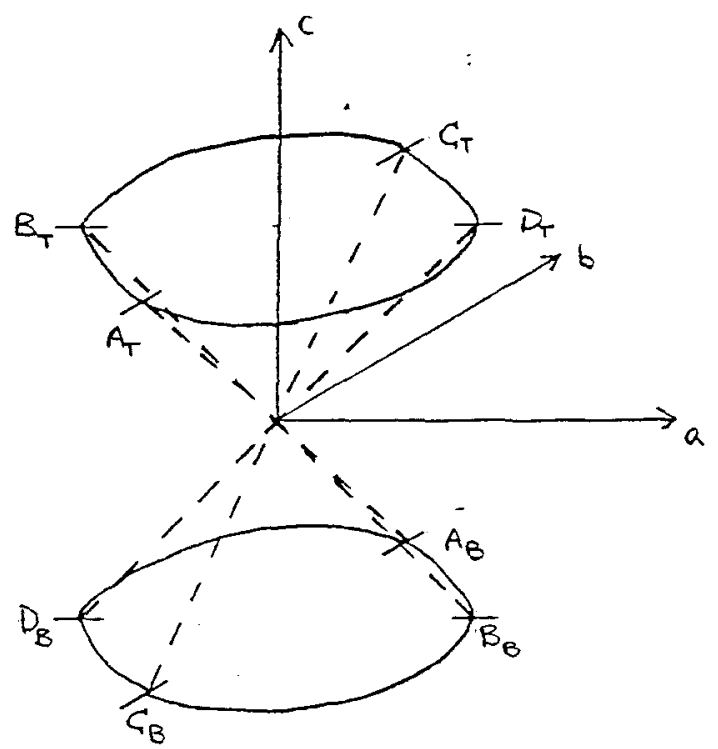

Figure 5-4: A schematic showing the axes labels of BASS. The $o-a-b-c$ coordinate system is also drawn in.

The actual details of the record structure may seem superfluous, but as I will now discuss, actually can be important to the data interpretation process. I will also point out why the above data structure is not "optimal". As noted earlier, the electronics which converted the Sea-Bird outputs malfunctioned, therefore I will no longer discuss those sensors. 


\section{dropout and data corruption: the first concern}

All of the data in the fall ' 88 deployment, and a great deal of the data in the spring ' 89 deployment, was sent by FM transceiver and recorded by a personal computer remote from the buoy. The data were sent and, of course, not all were received. The dropout rate of the radio link depended on several factors. Most important of the variables were: the transmitted power of the buoy's radio (which depended on how well the radio was tuned and the voltage level of the batteries), the weather conditions and the location of the receiver (which was usually located on the $R / V$ WECOMA during large scale hydrographic surveys). In addition to data transmission loss, there were several other sources of data corruption. Let's examine the possible causes of dropout and data corruption for each of the aforementioned variables.

The time information is generated inside the BASS computer, therefore there are no sources of error here besides transmission error. The counter helps to identify the fractional time of the samples within any integer second. This information was generated by the BASS computer program (not read off a clock). An error in the program caused the counter to occasionally take on false values. In cases where there was isolated dropout, the correct fractional time of the sample could be inferred from the other samples. However, in some cases, where two or three samples in a particular integer second were missing, the incorrect counter information made it impossible to correctly place the time of the record.

The BASS acoustic current meter has polarized transducers. In about $50 \%$ of the transducers the polarization was reversed. When the signal strength is sufficient, this effect does not adversely affect the final measurement. When signal strength is weak, the receive transducer misses the first acoustic pulse sent and synchronizes on a later pulse. This phenomenon is easily recognizable in the time series. It is manifested, always, as a quick up then down spike in the record. It was the invariant nature of this error, and the fact that the presence of the feature was uncorrelated between different axes measuring the same 
sample volume, that made it apparent that this error was not a physical flow characteristic but rather an instrument error. Figure 5-5 shows a time series of a segment of data that was affected by this phenomenon. Different pods were affected to different extents. Table 5-2 gives statistics for how bad the pod axes were for files $f$ and $v$. Note that pod 4 axes $A$ and $C$ are particularly bad.

For other reasons too, the received acoustic signal might be too weak for proper detection to occur. When this was the case the BASS computer would discard that record. The velocity axes were sampled at $20 \mathrm{H} z$. That means that for each record there were five samples of data taken. The output that was loaded into the data stream was the average of the good samples taken. In cases where no good sample was taken, the output was pushed to full scale (making the location of such points obvious).

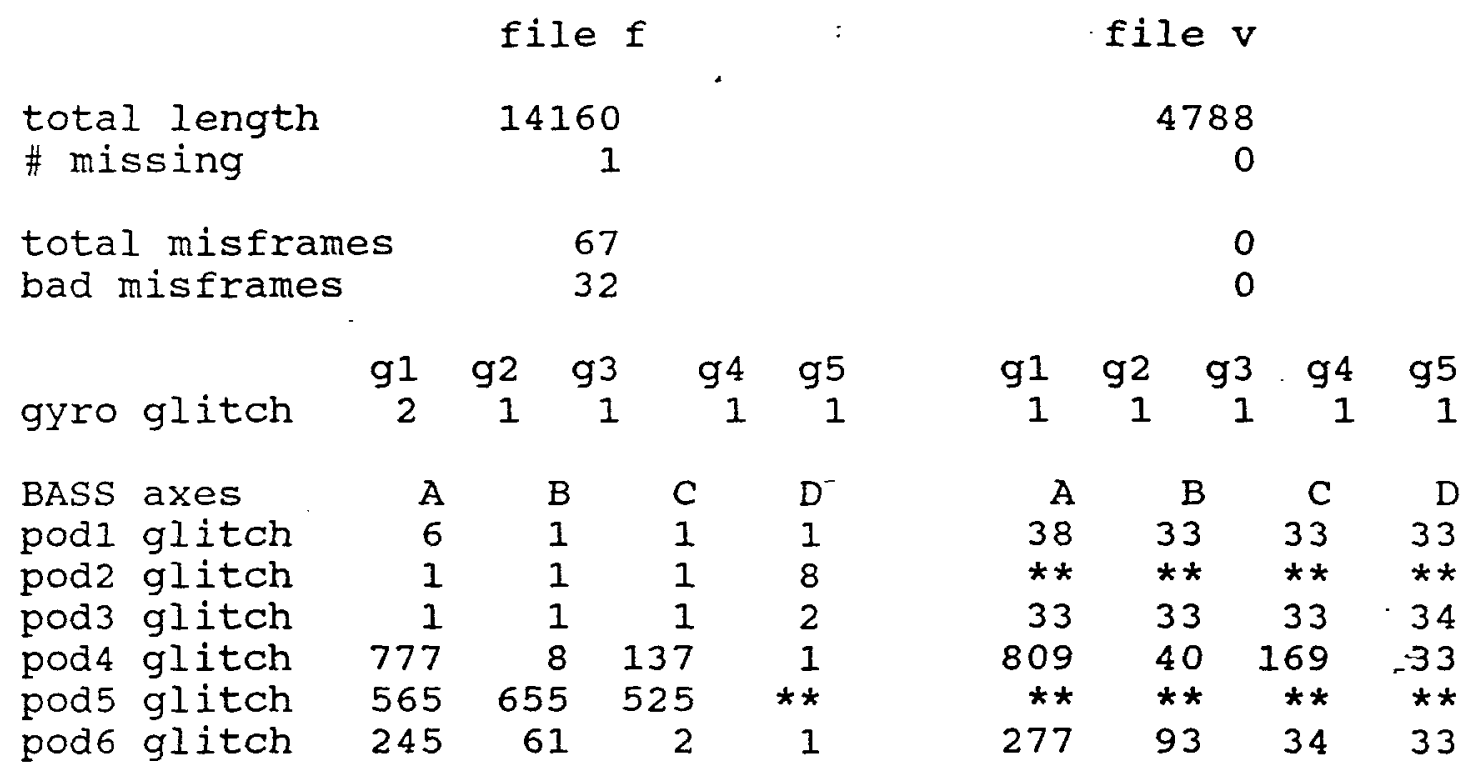

Table 5-2: Number of bad points in the time series of raw data channels for files $f$ and $v$. The total length gives the number of records that should be present based on the clock information. Next, the number of missing records in each file is given. Misframes are records where not all 91 bytes were received. Bad misframes are records where the desynchronization affected the gyro and BASS data. "Glitches" are bad data on a channel caused by reasons other than a bad misframe. Example: BASS pod 4 axis-C has 137 glitches, there were 32 bad misframes, and one record was missing entirely; this means that 170 (of the 14160) samples has to be interpolated from other points. 

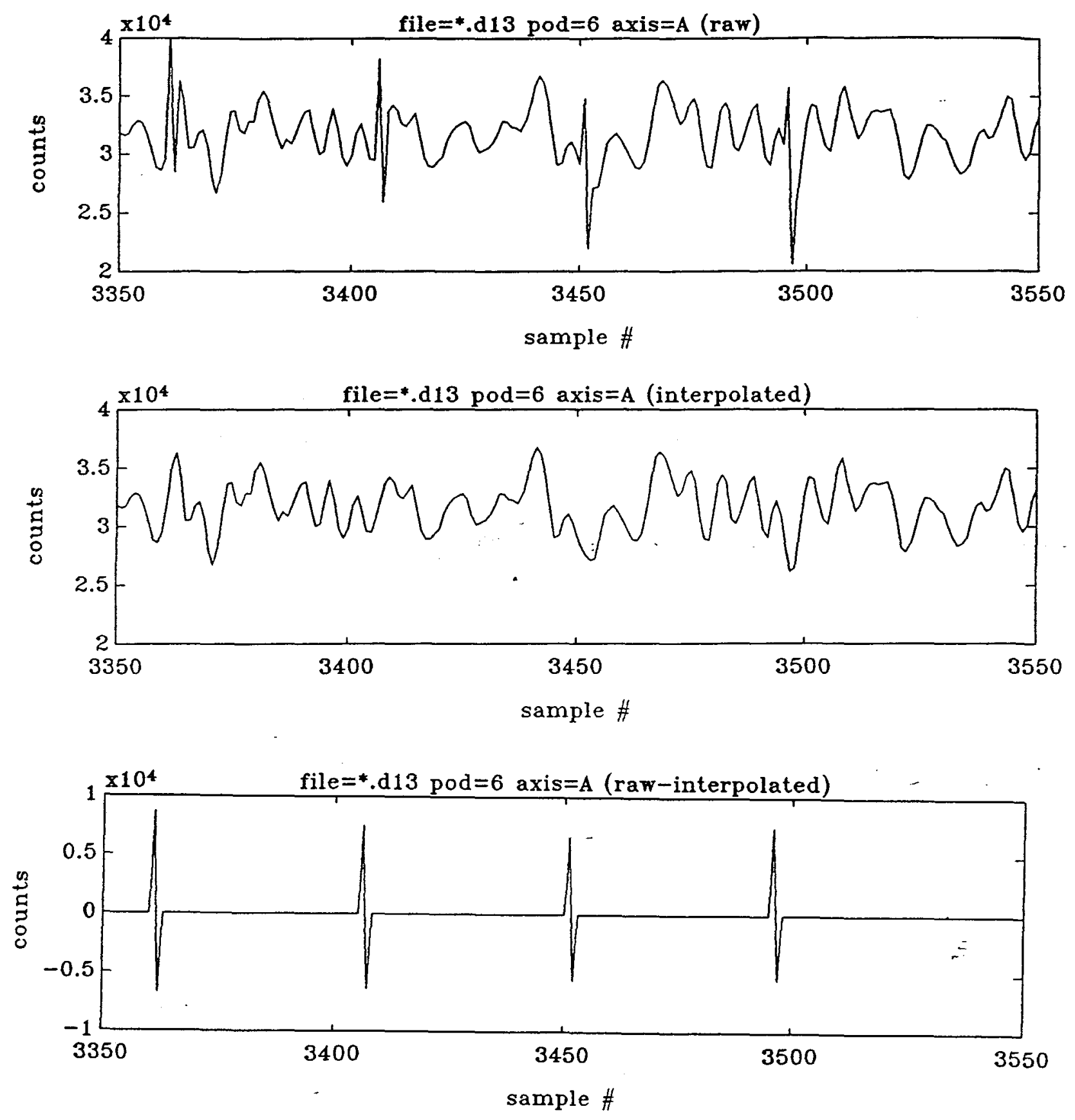

Figure 5-5: Example of data corruption by reversed transducers and dropout. Shows a piece of file $f$, pod 6 , axis-A. The "glitches" due to transducer reversal are seen corrupting the top plot. The second plot shows the interpolated version of the sequence. The final plot is the difference of the original and interpolated sequence. 

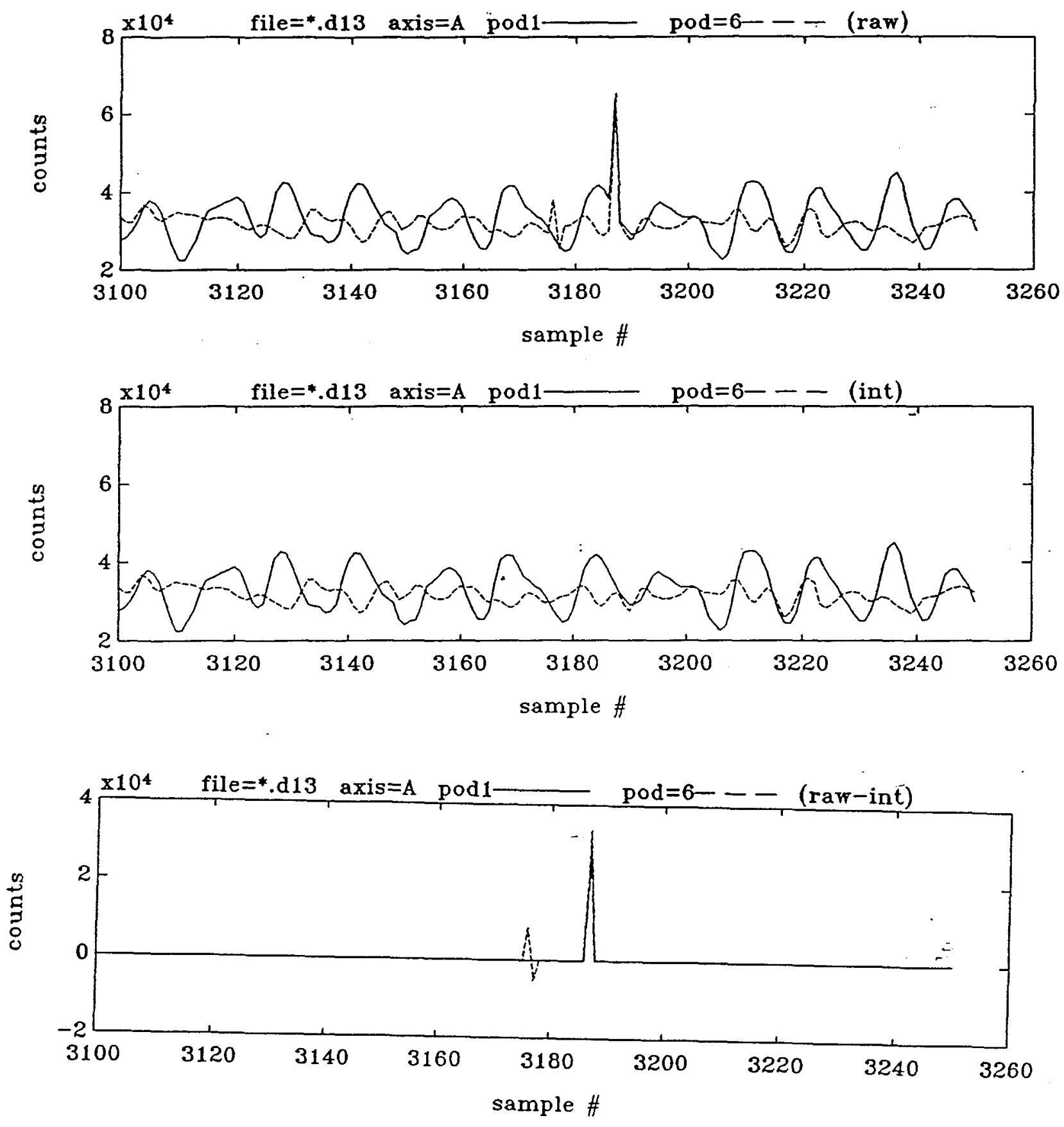

Figure 5-6: Shows two types of errors. At about sample 3175 we again see a transducer error in pod 6 . In the region of sample 3190 we see evidence of a misframed record. There is data but the bytes are corrupted on every channel (only axis-A of pods 1 and 6 are shown). 
Some of the BASS sensors used on SASS were 10 or more years old. For the most part, the sensors performed well. Problems with some of the cables did, however, cause us to lose data from particular pod axes. For the fall ' 88 deployment, axis-D on pod 5 recorded no data. With three axes left, there was still enough information to form a vector measurement. For the spring ' 89 deployment another axis went bad on pod 5 and two axes failed on pod 2. With only two functioning axes, pods 2 and 5 could not be resolved into vectors and hence provided no useful data for the spring deployment.

The gyro, metal-clad thermistor and compass data were free of spurious samples except an extremely rare spike, which was probably due to a bit being corrupted in transmission. The overall dropout from transmission at times was so bad that data had to just be thrown out. This, to a large extent, is why even though 2200 minutes of data were logged (see Montgomery and Santala, 1989), only 1433 minutes were finally analyzed.

\section{Consequences of dropout and corruption}

Sometimes, an entire record was lost in transmission. More frequently, only part of a record was lost. During data transmission a byte can become corrupted for a variety of reasons. Whatever the cause of the corruption, as long as a byte was transmitted, the final record length will be the same. This means that each byte in the record can be identified in the record from its position in the record, even if it does follow a corrupted byte. When dropout occurs, i.e. a byte is not received, the position of the bytes at the end of the record is shifted. There was no practicable way to resynchronize the bytes following a dropout in the record. The information at the beginning of the record may still be used. This argues for putting the most valuable data, or data which requires a high sample rate, early in the record.

Most important to SASS, and most rapidly varying in time, are the motion variables. This means BASS velocity measurements, the gyro outputs and the compass data. Note that the compass data is last in the record! This means that the compass experienced the highest corruption rate of any variable. Mercifully though, SASS's motions in yaw were 
dominated by low frequency motion. The $4 \mathrm{~Hz}$ sample rate constituted a great deal of oversampling and hence, the effect was not fatal.

Also, a very large percentage of the data sent was thermistor data. The time scales of variation in the thermistor data were extremely long (temperature was all but constant for most records). Perhaps what would have been preferable would have been to send only one temperature or conductivty word per record. This would have cut the amount of data to be sent by a considerable amount, lowered the necessary BAUD rate of transmission and probably have increased the throughput rate. The cost to the thermistor data would have been a sample rate of $0.4 \mathrm{~Hz}$ instead of $4 \mathrm{~Hz}$. This approach would have precluding our finding rapid variations in temperature, had they existed. For future experiments we must decide whether lower data transmission rates and less severe data storage requirements are preferable to highly sampled temperature data.

\section{time lines}

The first step in processing the data was to find the time at which each sample was taken and to find where there were missing samples. This was also the point where data sets were evaluated and discarded if the dropout rate was unacceptably large. Records taken that were shorter than 10 minutes long were not considered. At this preliminary stage we also noted that for some of the recorded files, battery power was sufficient to transmit but not to maintain proper voltage levels on the instrumentation. Therefore this "data" was" void of information and was thrown out.

\section{First level of processing}

\section{wild points}

Any part of the data stream could be corrupted in transmission or recording. The philosophy here was to only throw away samples that were obviously bad. Every motion channel was

plotted out and visually inspected before and after processing. Data were evaluated on the 
basis of the minimum value of the first difference of each sample with its adjacent samples. Samples where this minimum value were anomalously high were identified as "wild points". After wild points were located on each channel, they were removed and interpolated over using a cubic spline interpolator.

\section{motion data}

Once bad points were smoothed over, each of the instrument motion variables were converted from digital counts to voltage levels and thence to physical units. The proper calibrations for all the motion channels were obtained prior to the deployments. The gyro calibration was accomplished by putting the gyro on a tilting rotary table (a platform used by-machinists which can be tilted to within fractions of a minute). The potentiometers were calibrated first; the gyro was turned on so that the inner platform was stabilized and the table was tilted to a known orientation. Voltage output levels:were recorded against the imposed tilts to obtain the potentiometer calibrations.

With potentiometer scale factors in hand, the accelerometers were then calibrated. This was done with the gyro motor off and the gyro caged. In this way, when the table was tilted the central platform would tip to a known extent. The potentiometer calibration must be known so that the exact accelerometer orientation was known (because the exact caging position can vary and was never exactly "zero"). Accelerometers tilted thusly had their sensitive axes aligned with the local gravity vector to a varying extent and thus sensed different apparent accelerations. Output levels were recorded against the computed appärent acceleration to obtain the accelerometer calibrations.

The mechanical VACM compass used on the SASS needed no calibration. It was a seven-bit inverted gray code compass and the output could simply be interpreted. 


\section{BASS axes}

The BASS acoustic current meter measures the velocities along four axes. These velocities are, naturally, measured relative to the instrument. The ultimate goal is to combine the BASS axes with the motion sensing axes to derive a total velocity vector. To do this we must first combine the four axes into a triplet of orthogonal components. As a prelude to doing this, let's review the theory of travel time acoustic velocimetry.

Let's derive the results for a moving (see Figure 5-7) sensor. The travel time for sound from $T$ to $B, T_{T B}$, and the travel time for sound from $B$ to $T, T_{B T}$ are

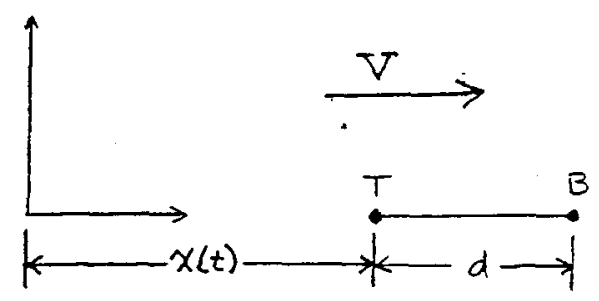

Figure 5-7: Theory of travel time measurement. A one dimensional case of an acoustic transducer moving through a moving fluid. The acoustic axis, $\bar{T} \bar{B}$, has a path length, $d$, . and is at time-varying position $x(t)$. The velocity of the surrounding fluid is $V$.

$$
T_{T B}=\frac{D_{T B}}{V_{T B}}=\frac{d+\Delta x}{c+V}, \quad T_{B T}=\frac{D_{B T}}{V_{B T}}=\frac{d-\Delta x}{c-V},
$$

and the differential travel time $\xi$ is then

$$
\xi=\frac{d-\Delta x}{c-V}-\frac{d+\Delta x}{c+V}=\frac{2 V d-2 c \Delta x}{c^{2}-V^{2}} \approx \frac{2 V d-2 c \Delta x}{c^{2}},
$$

where $\Delta x$ is the motion of the sensor over the time that the measurement is made. If the sensor motion is approximately constant over the measurement time, then $\Delta x=\frac{\dot{x} d}{c}$ and

$$
\frac{c^{2} \xi}{2 d}=V-\dot{x}=v_{r e l}
$$

(This is a pretty good assumption. The measurement time is only $100 \mu \mathrm{sec}$. With accelerations in the wavefield scaling as $a \sigma^{2}$ and velocities as $a \sigma$ the ratio of the change in velocity 
during a sample interval to velocity is $\sigma \Delta t \ll 1$.) The BASS electronics (Williams et al, $1987)$ convert the arrival time difference into a voltage. The relative velocity along any axis is

$$
v_{r e l}=s \frac{c^{2} \xi}{2 d}
$$

where $s$ is an appropriate scale factor. We note here that the measurement of relative velocity depends on the speed of sound squared. At a later time we discuss how errors in our estimate of $c$ will affect our final estimate of the relative velocity. Now we'll proceed to combine the four axes into an estimate of the vector velocity relative to the instrument.

Figure 5-8 shows the sign convention of the axes and how the $o-a-b-c$ axes have been defined in relation to them. From the geometry of that figure we see that the axes may be combined to form vector velocity components as

$$
\begin{aligned}
& v_{a}=\gamma(B-D) \\
& v_{b}=\gamma(A-C) \\
& v_{c}=-\gamma \frac{(A+B+C+D)}{2}
\end{aligned}
$$

if all four axes are used in the estimate $\left(\gamma=\frac{s}{\sqrt{2}}\right)$. The estimate of $v_{c}$ in the previous equation uses four BASS axes. By using all four axes we presumably get the "best estimate". Note though, that it is also possible to make two separate estimates of the $v_{c}$ velocity as

$$
v_{c_{1}}=-\gamma(B+D) \quad v_{c_{2}}=-\gamma(A+C) .
$$

These two separate estimates allow us some opportunity to evaluate the current meter performance. Traditionally, the estimate of BASS velocities is made as shown in equation 5.6.

In some cases, an axis may not be working or, we may choose not to use a particular axis in one of our estimates. In that case we may still form an estimate of the velocities. If the A-axis is not to be used, the estimate becomes

$$
v_{a}=\gamma(B-D), \quad v_{c}=-\gamma(B+D), \quad v_{b}=-2 C \gamma-v_{c}
$$

If the estimate is to be made without the B-axis,

$$
v_{b}=\gamma(A-C), \quad v_{c}=-\gamma(A+C), \quad v_{a}=-2 D \gamma-v_{c} .
$$



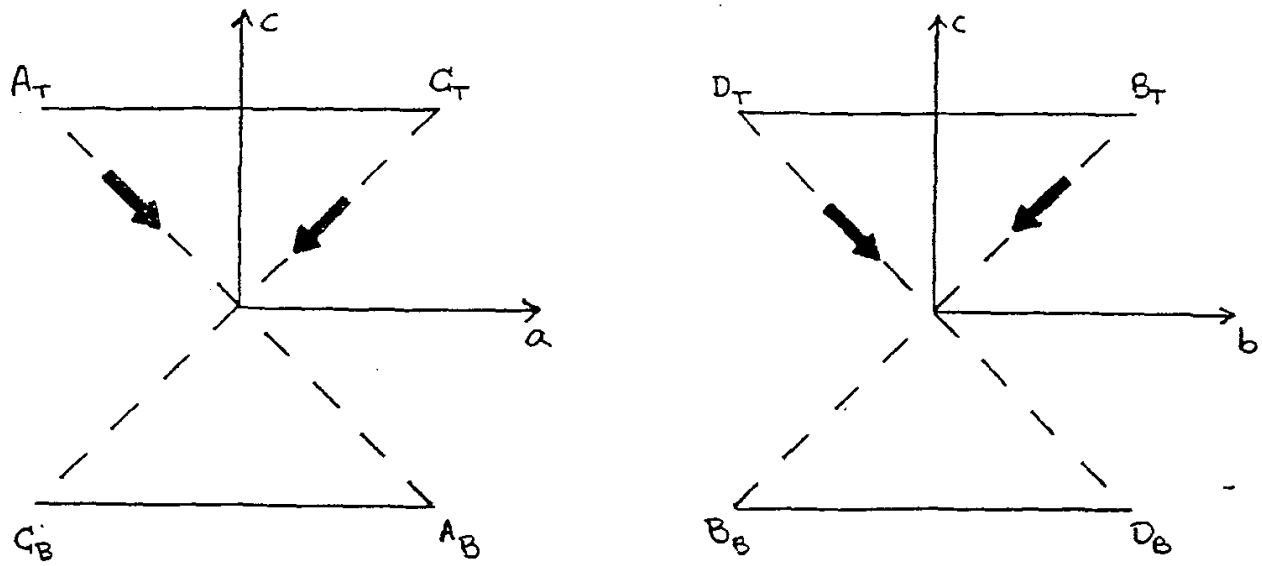

Figure 5-8: Projections of the BASS axes in the $o-a-c$ and $o-b-c$ planes. The bold arrows indicate the relative flow direction that yields positive output

If the estimate is to be made without the $\mathrm{C}$-axis,

$$
v_{a}=\gamma(B-D), \quad v_{c}=-\gamma(B+D), \quad v_{b}=2 A \gamma+v_{c}
$$

And finally, if the estimate is to be made without the $\mathrm{D}$-axis,

$$
v_{b}=\gamma(A-C), \quad v_{c}=-\gamma(A+C), \quad v_{a}=2 B \gamma+v_{c}
$$

We find the expressions to be of use even when all four acoustic axes were functioning properly.

The BASS axes are constructed so as to cause minimal flow disturbance. Williams (personal communication) has found, in tow tank calibrations, however, that if the flow direction is aligned to within $20^{\circ}$ to $30^{\circ}$ of a BASS axis, then that axis undermeasures the velocity due to the transducer wake. Upon first processing the data, all four axes were used to create the estimate of the velocity as in equation 5.6. It was found in the result that the two estimates $v_{c_{1}}$ and $v_{c_{2}}$ were not in good agreement. Furthermore, the error velocity $v_{\mathrm{c}_{1}}-v_{\mathrm{c}_{2}}$, tended to be episodic rather than a continuous noisy signal. This seemed 
to indicate that perhaps axis "shading" was occurring.

The correction of axis shading is quite simple. The methodology is to make an initial estimate of the relative velocity using equation 5.6. The alignment of the velocity vector is then checked against the physical orientation of the BASS axes. The unit vectors describing the orientation of the BASS axes are

$$
\begin{array}{ll}
\mathbf{u}_{A}=\frac{\mathbf{u}_{b}-\mathbf{u}_{c}}{\sqrt{2}} & \mathbf{u}_{C}=-\frac{\mathbf{u}_{b}+\mathbf{u}_{c}}{\sqrt{2}} \\
\mathbf{u}_{B}=\frac{\mathbf{u}_{a}-\mathbf{u}_{c}}{\sqrt{2}} & \mathbf{u}_{D}=-\frac{\mathbf{u}_{a}+\mathbf{u}_{c}}{\sqrt{2}} .
\end{array}
$$

The size of the angle between the flow direction and any BASS sensor axis can $^{-}$then be found by taking the dot product between the axis unit vector in question and the relative velocity vector. An axis is considered "shaded" when the angle is less than a certain level (or equivalently, we eliminate the axis from consideration when the cosine of the angle is too great). If

$$
\left|\frac{\mathrm{v} \cdot \mathrm{u}_{A}}{\sqrt{\mathrm{v} \cdot \mathrm{v}}}\right| \geq \cos \varphi
$$

then the $A$-axis is considered "shaded" and the estimate of the velocity is made from the $B, C$ and $D$ axes (as in equation 5.7). All other axes may be treated similarly. The cutoff angle $\varphi$ used in the SASS processing was $30^{\circ}$.

\section{Processing to inertial frame}

At this stage we have continuous and scaled measurements of the gyro data and the current meter data. The processing, from this point on, consists of taking these measurements and transforming them into the inertial frame of reference. The groundwork for this task was laid in the Coordinate Transformations section of the thesis. The equations of that section can not, however, be applied mindlessly.

The processing occurred in several stages. Figure 5-9 provides a block diagram of processing to the inertial variables. In the first stage the measurements were filtered. There were two reasons for doing this. First, to eliminate noise from spectral bands where we 
know there was no signal present. And second, since the transformation equations require the first or second derivative of some of the variables we measured we find it necessary to apply differentiating filters. In the second step, the transformation of all variables into the inertial frame of reference occurred. Then the inertial accelerations of SASS's coordinate origin were integrated to find the velocity. In the final stage, the different contributions to the velocity of each sensor were summed. The relative velocity was added to the sensor velocity to find the total fluid velocity at the (time-varying) sensor location. In what follows, each stage of the processing is outlined in detail.

\section{stage 1 : preliminary filtering justification of filter forms}

As shown in Figure 5-9, there are five different filters applied at the first stage of processing. There are three different types of filters; lowpass, lowpass differentiating and lowpass double differentiating. All of the filters are lowpass; i.e., they eliminate the high frequency part of the signal. The reason for doing this is justified both by the nature of the signal measured and by the processing to occur in later stages.

All of the variables measured (ignoring, in this section, the thermistors) are strong functions of the wavefield. This is reflected in the spectra of each of the variables. The peak frequencies tend to be in the $0.1 \mathrm{~Hz}$ to $0.3 \mathrm{~Hz}$ range. The spectral energy falls off relatively rapidly with increasing frequency. The Nyquist frequency for the SASS data was $2 \mathrm{~Hz}$. The noise sources for each channel were different, but we expect that, in the higher frequency bands, the signal to noise ratio is quite low. In the band of frequencies from $1 \mathrm{~Hz}$ to $2 \mathrm{~Hz}$, the signal to noise ratio is probably one or less. If all subsequent processing were linear, there would still be no strong reason to lowpass filter the data (at least not at this point), because the noisy frequency components at the high frequencies would not affect the lower frequency signals. However, the next stage of processing is the coordinate transformation section. The coordinate transformations require us to multiply different variables by each other and by trigonometric functions of one another. Since a multiplication in the 


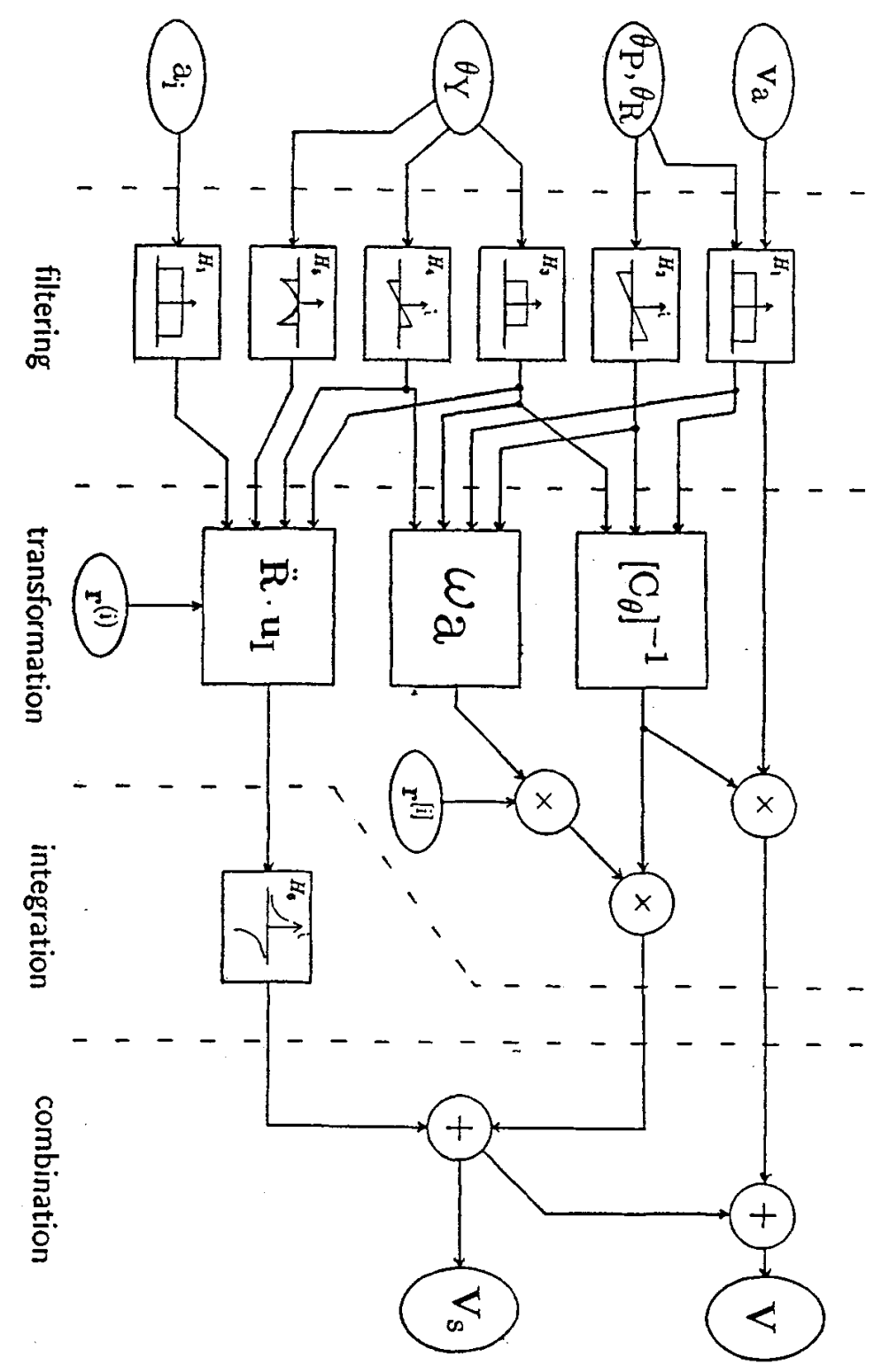

Figure 5-9: Block diagram of processing stages from interpolated data to inertial frame velocities. 
time domain implies a convolution in the frequency domain, it is clear that the noise at high frequencies will modulate the energetic signals in the lower frequency bands. For this reason, all channels, except the compass, had a cut-off frequency of $1.25 \mathrm{~Hz}$ in the first stage.

The compass measures the yaw angle of the buoy. Undeniably, the yaw angle is a function of the wave dynamics. The forcing of the yaw direction is, however, fundamentally different than the forcing for surge, sway, heave, roll and pitch. For the heave, roll and pitch the major forcing terms are the buoyancy change due to the surface slope. Temporal variations of the pressure forces govern the surging and swaying. For all these motions the mooring line tension is also an important forcing/restoring term. For the yaw angle there is forcing due to pressure forces, but to cause the buoy to yaw there must be large spatial gradients in the pressure field. Because the length scale of the SASS is much smaller than that of the large waves, this type of forcing is probably minimal. Drag forces undoubtedly play a large part in the yawing of the SASS. The forcing from the mooring is expected to be at low frequencies because in yaw it is the torsional (not tension) modes of the mooring line which affect the motion. The consequence of all this is that we expect the energy in the compass channel to be located at lower frequencies than that of other channels. This would allow us to use a lower cut-off frequency for the compass. After visually inspecting the spectra of all the raw compass data, a cut-off frequency of $0.6 \mathrm{~Hz}$ was selected for the compass data.

The lower cut-off frequency for the compass is important because the compass measurement is noisier at the high frequencies. The error in the gyro variables are totally dominated by errors in the stabilization of the gyro platform. These errors, because of the enormous angular momentum of the gyro, tend to be at low frequencies and at the frequencies where forcing will occur (in the waveband). At high frequencies (above $0.6 \mathrm{~Hz}$ ), the compass error seems to be dominated by quantization error. At lower frequencies there are several subtle issues which arise with with regards to the performance of the compass, these will discussed in section 5.4 . 
Modelling the quantization error sequence of a random variable (Oppenhiem and Schaffer, 1975) as (1) a stationary process, (2) being uncorrelated with the exact sequence, (3) a white-noise process and (4) having a uniform probability distribution over the range of quantization error, we can perform the following analysis. Shown in figure 5-10(a) is the assumed probability distribution for the error in a sequence due to its being quantized to a resolution $\Delta$. The compass used on the SASS was a seven-bit device so that $\Delta=\frac{2 \pi}{128}$ rad. The mean and variance of the error sequence are predicted from the probability distribution to be

$$
\begin{aligned}
m_{e} & =\int_{-\frac{\Delta}{2}}^{\frac{\Delta}{2}} e(n) p_{e}(e) d e=0 \\
\sigma_{e}^{2} & =\int_{-\frac{\Delta}{2}}^{\frac{\Delta}{2}}\left(e(n)-m_{e}(n)\right)^{2} p_{e}(e) d e=\frac{\Delta^{2}}{12}=2.01 \times 10^{-4} r_{a d}
\end{aligned}
$$

Figure 5-10(b) shows this predicted error energy (assumed to be white) and compares it to the noise floor for both files $f$ and $v$.
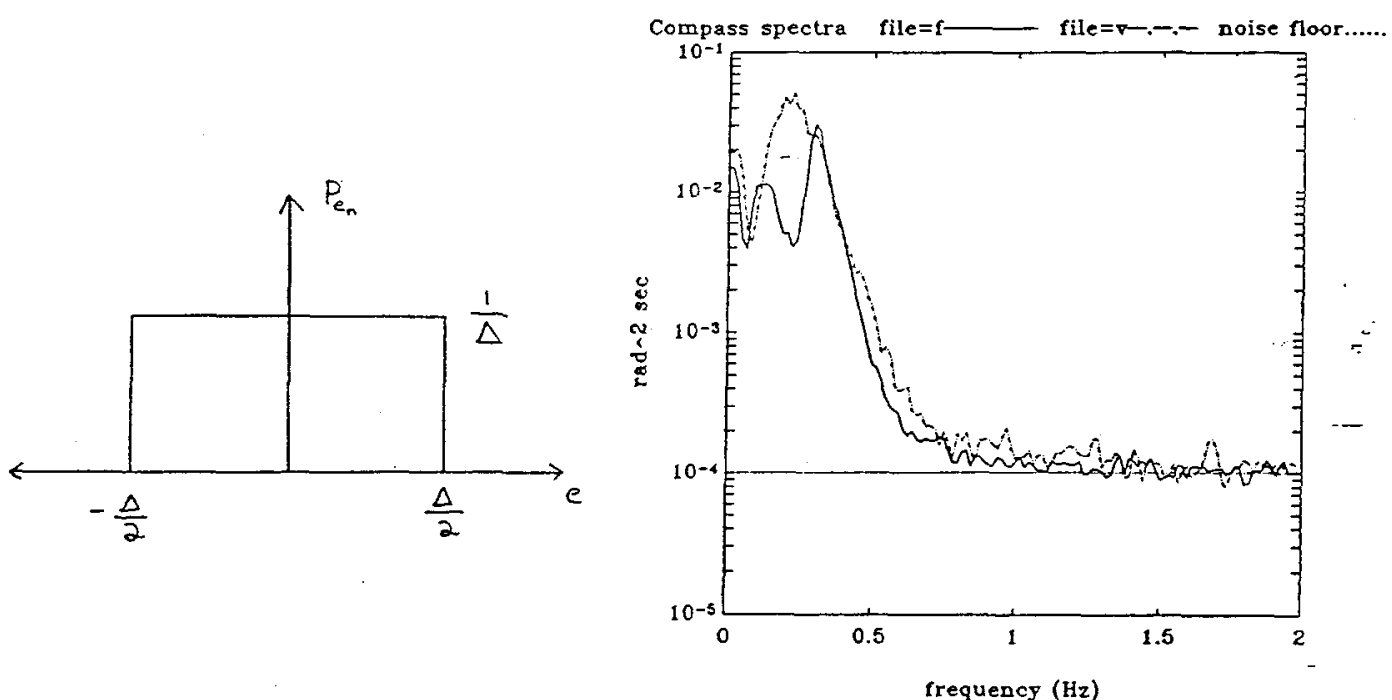

Figure 5-10: (a) The probability distribution assumed for quantizing a variable to level $\Delta$. (b) Spectrum of compass data for file $f$ and $v$ and predicted noise floor. 


\section{design of the filters}

For this stage of filtering there were three major criterion by which our filter would be judged. First, there should be perfectly linear phase. Second; pass-band characteristics are much more important than stop-band characteristics. Finally, the stop-band floor should be as low as possible.

The requirement of no phase shift was considered paramount. Relative phase shifts might seriously affect the cross-correlations we perform between different variables, necessary both for our derivation of the directional spectra and the wave bias. Also, due to the mooring forces, the SASS sometimes experienced significant surging motion at frequencies higher than where wave energy was present. In these cases, the relative motion is exactly the opposite of the sensor motion. If there is an introduced shift in the relative velocities or the sensor velocities, the cancellation between the sensor and relative velocities will not be exact. This will lead to a spurious "wave velocity" being indicated due to the improper cancellation. Because the time synchronization will be easier, we specify that the time lag of the filter be zero. These requirements can all be met by designing a symmetric, non-causal, finite impulse response (FIR) filter. All of the filters applied to the SASS data were FIR filters.

A technique which allows us, in a quasi-experimental way, to design FIR filters with good pass-band characteristics is the windowing approach. Using this design technique, the exact frequency response desired is specified. The theoretical impulse response is fourd via an inverse Fourier transform. Because our specified filters are band limited, the theoretical impulse responses derived will be infinite. The finite impulse response will be a windowed version of the infinite response. A survey of windowing techniques by Harris (1978) helped to guide the selection of an appropriate window for our application.

Perhaps even more important than window type is the length of the window 'we apply to the data. Longer windows will more nearly approximate the infinite (perfect) impulse response. Fortunately, our data sets were quite long and thus we had the luxury of using 
relatively long filters. The length chosen for the windows was 257 samples $(M=257)$. If we denote the length of the data sequence to be filtered as $N$ then the resulting filtered sequence will be of length $N+M-1$. The actual processing was done in the frequency domain, but, if we recall the form of the convolution sum, which may be written as

$$
y(n)=\sum_{m=-\frac{M-1}{2}}^{\frac{M-1}{2}} h(m) x(n-m), \quad n=0,1, \cdots, N-1
$$

then we see that for $n<\frac{M-1}{2}$ and $n>(N-1)-\frac{M-1}{2}$ that the filter $h(m)$ and the input sequence $x(m)$ do not fully overlap. These transient samples in the filtering process were thrown away. Thus, the final filtered sequence $y_{f}$ is smaller by $M-1$ samples than the sequence output by the filter $y(n)$ so that

$$
y_{f}=y\left(n^{\prime}\right) ; \quad n^{\prime}=\frac{M-1}{2}, \frac{M-1}{2}+1, \cdots,(N-1)-\left(\frac{M-1}{2}\right) .
$$

The loss of $M-1=256$ samples means that the length of our files was only reduced by 64 seconds.

With the goals of our filter design set we may proceed with the actual design. The filter designs are sketched in Figure 5-11 (an arbitrary gain factor of $A$ is allowed in each filter). The ideal impulse response $h(n)$ of any of the filters can be found from

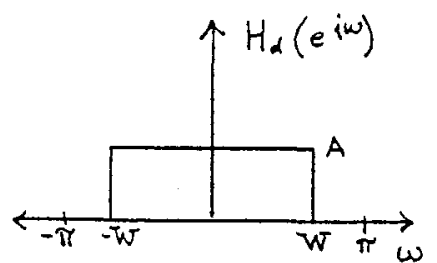

(a) LOWPASS

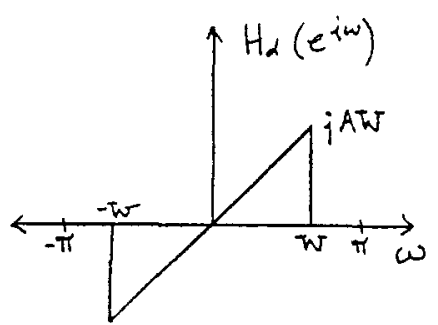

(b) LoWPASS DIFFERENTIATOR

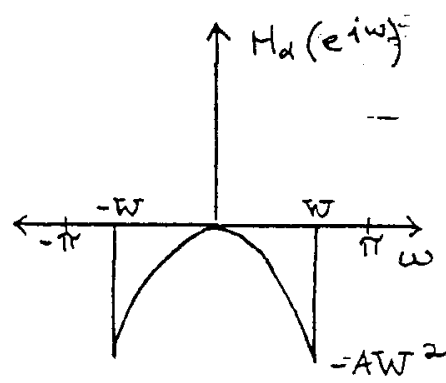

(C) LOWPASS DOUBLE
DIFFERENTIATOTR

Figure 5-11: (a) The lowpass filter, (b) the lowpass differentiating filter, (c) the lowpass double differentiating filter. 


$$
h(n)=\frac{1}{2 \pi} \int_{-\pi}^{\pi} H_{d}\left(e^{\imath \omega}\right) e^{\imath \omega n} d \omega .
$$

For the lowpass filter

$$
H_{d}\left(e^{\imath \omega}\right)= \begin{cases}A & \text { if }|\omega|<W \\ 0 & \text { otherwise }\end{cases}
$$

which results in an impulse response of

$$
\begin{array}{ll}
h(n)=\frac{A \sin W n}{\pi n}, & -\infty<n<\infty, \\
h(0)=\frac{A W}{\pi}, &
\end{array}
$$

when the integral of equation 5.16 is applied. The zeroeth sample of all the filters derived will be given explicitly even though each can be found by applying l'Hôpital's rule. The differentiating filter has an ideal frequency response of

$$
H_{d}\left(e^{\imath \omega}\right)= \begin{cases}\imath \omega A & \text { if }|\omega|<W \\ 0 & \text { otherwise }\end{cases}
$$

and a corresponding impulse response of

$$
\begin{aligned}
h(n) & =\frac{A}{\pi}\left(\frac{W n \cos W n-\sin W n}{n^{2}}\right), \quad-\infty<n<\infty, \\
h(0) & \doteq 0 .
\end{aligned}
$$

Finally the lowpass double-differentiator has a specified frequency response of

$$
H_{d}\left(e^{\imath \omega}\right)= \begin{cases}-\omega^{2} A & \text { if }|\omega|<W \\ 0 & \text { otherwise }\end{cases}
$$

and an impulse response of

$$
\begin{aligned}
& h(n)=\frac{A}{\pi}\left(\frac{\left(2-W^{2} n^{2}\right) \sin W n-2 W n \cos W n}{n^{3}}\right), \quad-\infty<n<\infty, \\
& h(0)=-\frac{A W^{3}}{3 \pi} .
\end{aligned}
$$

Windows that were considered for application to the impulse response were: (a) the cosine-squared, (b) the cosine $e^{4}$, (c) the Kaiser and (d) the Blackman. The $\cos ^{4}$ and Blackman seemed to give far superior results for the pass-band. The $\cos ^{4}$ had slightly larger 
ripple at the high-frequency portion of the pass-band, but, because the $\cos ^{4}$ had the best response in the range where we expect the most energy to be and because the stop-band floor is lower than that of the Blackman, the $\cos ^{4}$ window of length 257 samples was applied to all of the impulse responses.

The bandwidth $W$ in the preceeding section is the nominal bandwidth. There were slight adjustments made to find the exact width desired. Figure 5-12 shows the implemented magnitude response of the filters. The bandwidth specified for the compass filters was $W=0.675 \mathrm{~Hz}$. For the filters applied to the gyro data and to the BASS data $W=1.325 \mathrm{~Hz}$. The filters are all symmetric FIR filters and therefore the phase is guaranteed to be linear. The filters and filtering programs were all extensively tested with synthetic data. The differentiating filters, of course, create new forms of the variables that we measured. The effects of the lowpass filtering are not too noticeable:except for the case of the compass data where, as we have already seen, the seven bit digitization leads to a relatively high noise floor (incidentally, the BASS data and the gyro data was all digitized to sixteen bits). The effects of the lowpass filtering on the compass data are shown in Figure 5-13.

\section{stage 2 : coordinate transformations}

At this stage of the processing the equations of the Coordinate Transformation section are applied. Central to transformation process is the rotational transformation matrix ( $\mathrm{r}-\mathrm{t}$ matrix). The first step in the transformation process, then, is to form the $\mathrm{r}$ - $\mathrm{t}$ matrix $\left[C_{\theta}\right]$ according to equation 4.8. The velocity of the fluid relative to the instrument may then be expressed in inertial coordinates by applying the inverse $r$-t matrix to the relative velocities.

To form the fluid velocities we also need to derive the sensor velocities. We consider each sensor as a point. A point does not rotate. The translational velocity of each point, however, is composed of the translation of the SASS coordinate origin and the rotational velocity of each point about the origin. The first step in finding the sensor velocity due to rotation is to find the angular velocity of the buoy. It is most convenient to form the 

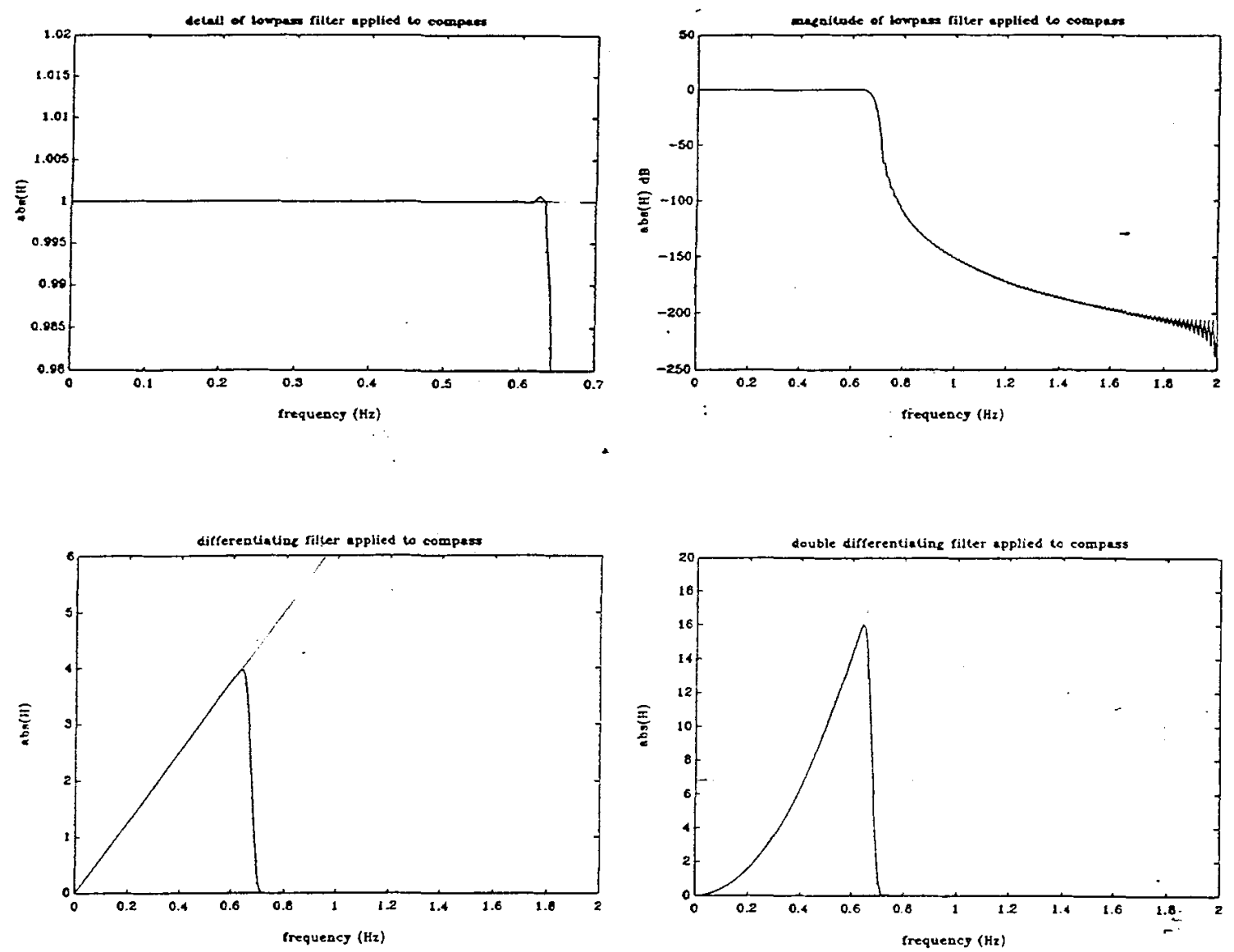

Figure 5-12: Filters for the compass data (a) detail of pass-band for lowpass filter (b) overall response for lowpass filter (c) response of lowpass differentiator (d) double differentiating filter. The filters applied to the other data differed only in their cut off frequency.- 


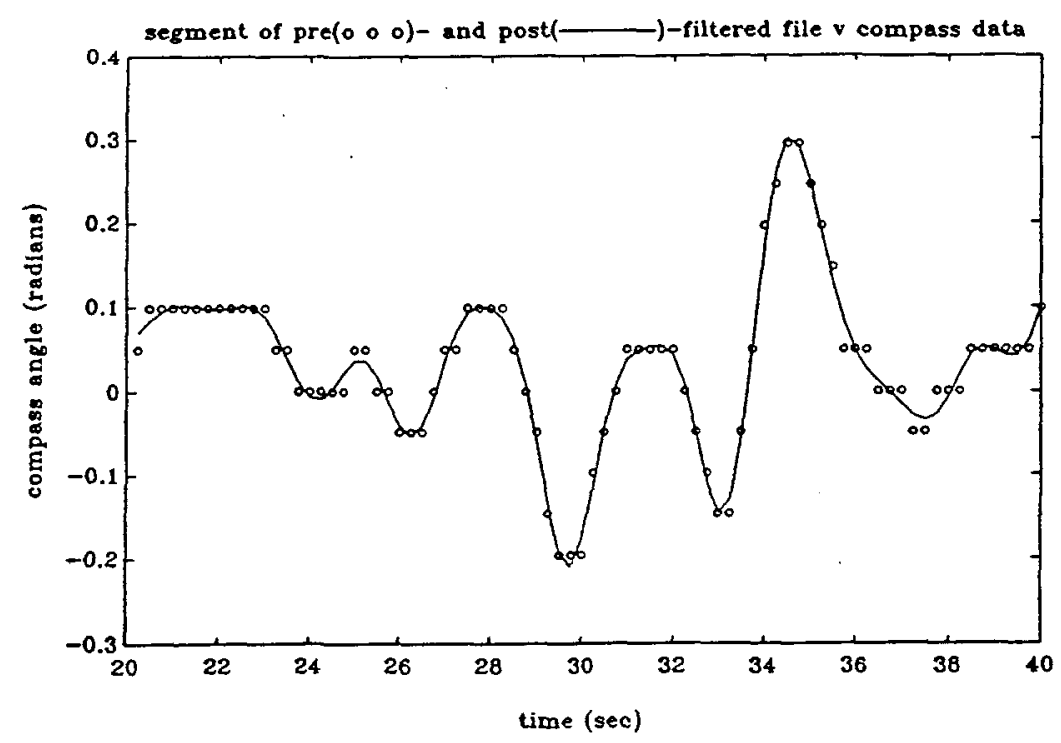

Figure 5-13: Pre- and post-lowpass filtered compass data

angular velocities in the instrument frame of reference (by using equation 4.19). In this way the $\omega \times \mathbf{r}^{[i]}$ product can be formed directly (since $\mathbf{r}^{[i]}$ is the physical position of the $i^{\text {th }}$ sensor relative to the SASS's origin). Once the cross-product is formed, it too is transformed into the inertial frame using the r-t matrix.

Determining the translational velocity requires two steps. First, the accelerations of the coordinate origin are found in the inertial frame. As is indicated in equation 4.13 the acceleration is a function of the measured acceleration, the yawing of the buoy and the position of surge and sway accelerometer sensing elements. During a second stage of the filtering, the acceleration of the SASS is integrated into a velocity.

\section{stage 3 : integration}

The acceleration of the coordinate origin $\ddot{\mathbf{R}}_{0}$ must now be integrated. The philosophy here will be the same as that of the previous filtering stage. We again will use an FIR filter to ensure that the integrated data is not shifted with respect to the other channels. The design procedure here will be slightly different. The reason for this is that the desired filter 
response is not easily integrable. The response of an ideal integrating filter is sketched in Figure 5-14 below. The problem, as shown in the figure, is the singular nature of the filter
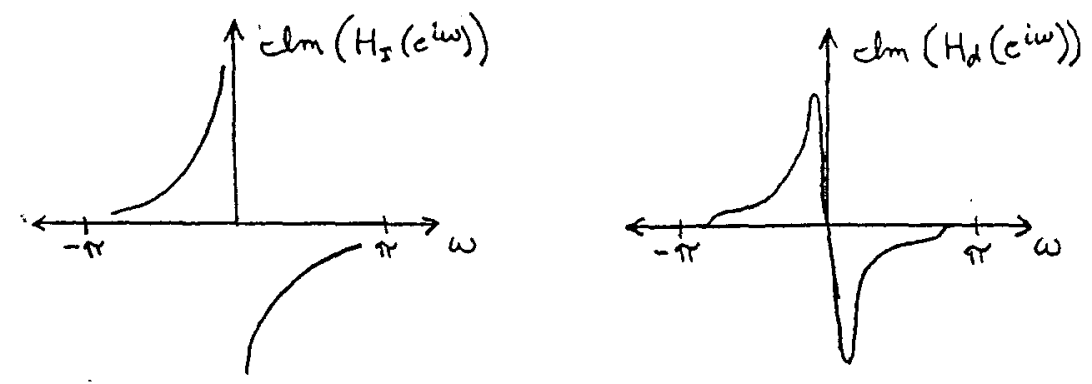

Figure 5-14: Sketch of an integrating filter; (a) ideal response and, (b) implemented response near the origin. Not only does this make the windowing method impractical, but the form of the filter will greatly amplify the low frequency signals. Below the wave-band frequencies the errors due to gyro stabilization will be much greater than the actual accelerations. Therefore, we'd like to have a stop-band near zero frequency. There are now conflicting interests. We want to integrate all of the wave energy and we want to attenuate the noise signal at low frequency. To do this we have to carefully pick the frequency band where the integrator will be rolled off. Waves with periods of 15 seconds $(f=0.067 \mathrm{~Hz})$ are-expected to be present in our data. However, choosing too narrow a transition band will cause the impulse response to "ring". After much trial and error design, it was decided to make the low-frequency cut-off at $f=0.038 \mathrm{~Hz}$.

Here, the frequency sampling method of FIR filter design was used. The idea in this approach (e.g., see Oppenheim and Schafer, 1975) is to specify the desired response of the samples in the discrete Fourier representation of the filter as

$$
\tilde{H}(k)=H_{d}\left(e^{\imath(2 \pi / N) k}\right) \quad k=0,1, \cdots, N-1 .
$$

The impulse response is found by taking the discrete Fourier transform of the sampled filter. The response is truncated to the desired length and the final design is the Fourier transform of the resulting sequence. With this method, better pass-band performance can be obtained 
by allowing a transition band between the pass- and stop-bands. There do exist techniques to optimize the selection of transition band points. These were not used here. On the philosophy that a smoother transition causes less ringing in the impulse response than a quick one we used the following method: define as wide a transition band as possible and then use a cubic spline interpolator to find the transition samples. This method may not be optimal but it was very simple, the cubic spline does provide a minimum curvature fit in the transition region, and the final design proved to be acceptable (see figure 5-15). The

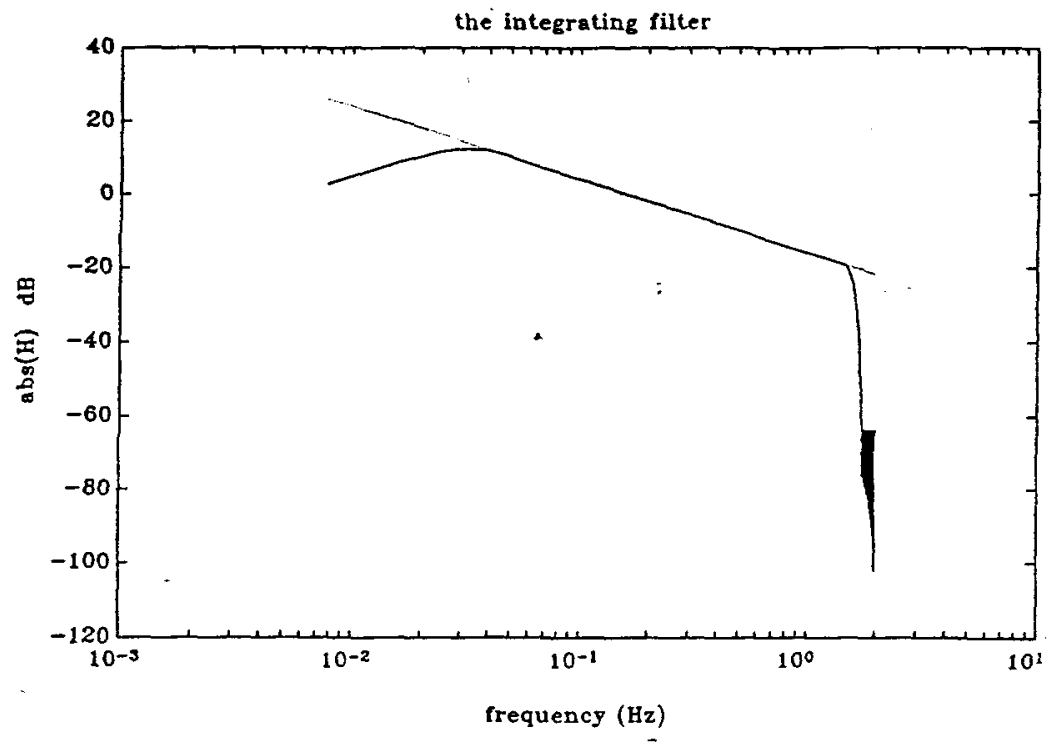

Figure 5-15: The actual filter response versus the perfect integrator

length of impulse response used here was again 257 samples. This again leads to the final sequence being 256 samples shorter than the original sequence. Only the accelerations need be filtered at this stage. The other channels were synchronized by discarding their first and last 128 samples.

\section{stage 4 : combining the terms}

At this point we have transferred all the measurements into the inertial frame. All that remains to be done is to combine the different variables into the desired form. First, the sensor velocities are formed by adding the contributions from SASS's translations and 
rotations as

$$
\mathrm{V}_{S_{i}}=\frac{d \mathbf{R}_{o}}{d t}+\mathbf{V}_{r t_{i}}
$$

where $V_{r t_{i}}$ is the cross-product $\omega \times \mathbf{r}^{[i]}$ that we formed in the second stage of processing. Finally, the velocity of the fluid at the sensor location is found by adding the sensor and relative velocities

$$
\mathrm{V}_{i}=\mathrm{V}_{S_{i}}+\mathrm{v}_{r_{e} l_{i}}
$$

Of course, since we filtered out the very low frequency component contributions to the sensor velocities, the mean of the total velocity is the same as the mean for the relative velocity. With a watch circle of $250 \mathrm{~m}$ in diameter it is theoretically possible that the SASS could experience some large drift velocities. For instance, if the SASS drifted $250 m$ over a twenty minute period, the unmeasured drift velocity would be $20 \mathrm{~cm} / \mathrm{s}$. However, because the current and wind conditions over the duration of the records tended to be quite constant in both magnitude and position, we expect, that the mean drift velocity of the SASS was usually $1 \mathrm{~cm} / \mathrm{s}$ or less.

From the fluid velocities, we can find the directional spectra of the waves as was outlined in section 4.3. With the fluid and sensor velocities we can find the unbiased estimates of the surface current as was outlined in section 3.2. In the next section we examine the effects of noise on the processing scheme that's just been ouflined.

\subsection{Dynamic behavior of the SASS}

\section{expectations}

With a complete processing package we can begin to look at how the SASS behaves in the wavefield. We do this as a prelude to our data and error analysis because the interpretation of our data, and estimation of errors, will be strongly dependent on the SASS's motion. Again, in this section we'll closely examine files $f$ and $q$, and extend our observations to the other files. 
Before looking at those files, let's consider what we expect to see in the SASS data. The SASS, as has been mentioned previously, has a very large waterplane area. Thus, we expect that the relative vertical motion between the floats and the surface to be small. The nominal draft of the SASS floats was $28 \mathrm{~cm}$. The top sensor was at a nominal depth of $110.8 \mathrm{~cm}$. With only $83 \mathrm{~cm}$ separating the top float from the top velocity sensor, we anticipate that the top velocity sensor will also tend to move with the surface. So, in spite of the fact that the vertical component of fluid velocity is greatest at the surface, we expect to see small relative vertical velocities being measured by the top sensor. Because the SASS has a rigid array of velocity sensors, the vertical motion of the bottom sensor is expected to be about the same as that of the top sensor. The fluid velocities, however, decay with depth. Since the sensor motions do not decay, it must be that we will observe the largest relative velocities in the vertical direction at the bottom sensor.

The case of the horizontal motion is the one, perhaps, that we find more interesting. It is a bit more difficult to guess intuitively what will happen in this case. The reason being that the mooring force will play a large part in the overall dynamics. We will let observation, therefore, be our guide. Nevertheless, we still can say we expect the same type of phenomenon to occur for the horizontal velocities as for the vertical velocities (although not for the same reasons). The pressure and drag forces on the structure and floats will tend to "try" to get the array to follow the fluid. For a short, unmoored spar-like array we might expect that the relative horizontal motion would be more-or-less uniform with depth. The SASS, however, has its flotation spread over a relatively large area (the distance-from any flotation element center to another being $366 \mathrm{~cm}$ ). Because of this, the surface slope will cause the SASS to tilt. This tilting, being forced from the surface will cause little slippage at small radii (sensors nearer the surface) and large slippage at larger radii (deeper sensors). The horizontal component of the mooring force (applied at the bottom of the frame) will tend to retard the buoy's motion as it tries to follow the water's trajectories. Again, the fact that the SASS can tip will favor the top sensor. The lower part of the buoy will be held in place by the mooring forces but, the buoy can tip to allow the upper part of the buoy to follow the fluid. It seems then, that both surface forcing and mooring forces conspire 
to cause less relative motion at the top sensors and greater motion at the bottom sensors. Now, we will take a look and see if our observations bear out these suppositions.

\section{observations}

Because the final analysis is to be done in an inertial frame, the observations at this point will be looked at in the inertial frame. As an attempt to look at the horizontal velocities in a dynamically significant way, I've rotated the horizontal velocities into a downwave/crosswave coordinate system. To simplify the plots, only three velocity sensors are plotted out per plot. The velocity sensors chosen were pods 6,3 and 1 with nominal depths of $110.8 \mathrm{~cm}$, $311.1 \mathrm{~cm}$ and $584.6 \mathrm{~cm}$, respectively.

From Figure 5-16 (a-c) we see that the velocities decay as expected. The directional spreading of the wave spectra is relatively harrow so that the downwave spectrum (b) is nearly the same as the vertical spectrum (a), and comparatively little energy is present in the cross-wave spectrum(c). The vertical motions of all three sensors are so nearly alike that the plots are indistinguishable (Figure 5-16 (d)). In the downwave direction the sensor motion spectrum (e) for pod 6 is almost the same as the fluid velocity spectrum (b), but, . for sensing pod 1 the motion spectrum is much greater than the fluid velocity spectrum. Clearly, the predominate forcing of the motion at the bottom of the SASS is not by the local fluid velocities. It must be, as we intuited above, that the surface slope and mooring forces are playing a significant role. The relative velocities are shown last (Figure 5-16 (g-i) $)$. At low frequencies, the buoy is a good follower and the velocities measured are small. The buoy is best coupled to the fluid motion near the surface. As a consequence, the relative velocities measured at pod 6 are the smallest and the velocities at pod 1 are the greatest. This effect is most notable in the down-wave velocity $(h)$.

Figure 5-17 repeats the above exercise for file $q$. The fluid velocities again decay as expected. Note how much greater the spectral levels are here than in Figure 5-16. For this strongly wind driven case we can deduce that the buoy must have been tipping quite 

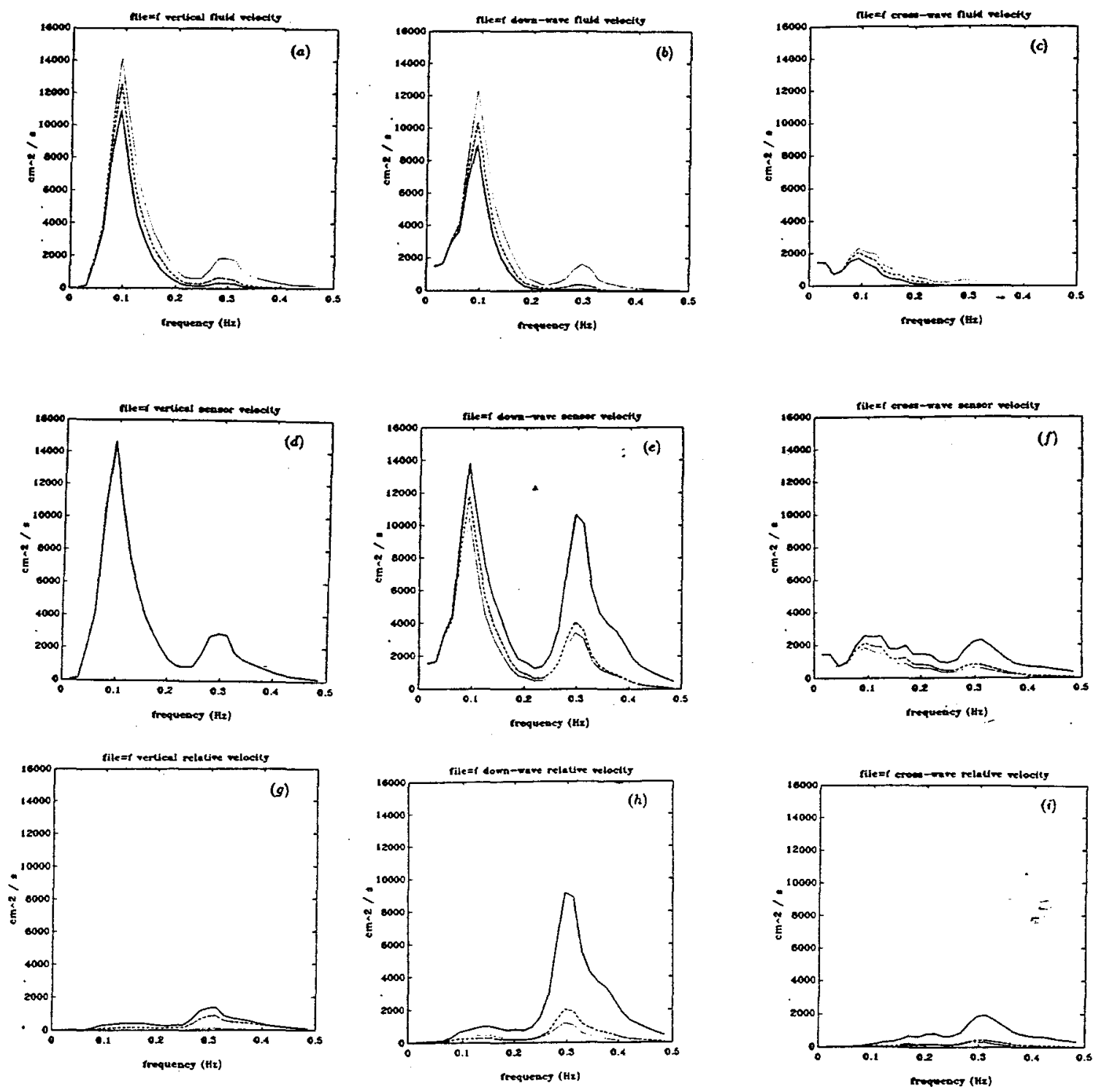

Figure 5-16: Spectra of motion variables for file $f$, sensor 1 (-) at a depth of $585 \mathrm{~cm}$, sensor $3(. .$.$) at a depth of 311 \mathrm{~cm}$ and sensor $6(\cdots)$ at a depth of $111 \mathrm{~cm}$. (a) Vertical fluid velocity (b) down-wave fluid velocity (c) cross-wave fluid velocity (d) vertical sensor velocity (e) down-wave sensor velocity (f) cross-wave sensor velocity (g) vertical: relative velocity $(\mathrm{h})$ down-wave relative velocity (i) cross-wave relative velocity 
a bit more than in the previous case because the vertical velocity of the sensors is not the same for each sensor (d). In this case, however, the downwave component of sensor velocity (e) is greatest for pod 6. Undoubtedly, the mooring force has more tension here and is thereby suppressing the motion at the bottom sensor. The overall result is similar for the relative velocities. The top sensing volume, pod 6 , is best coupled to its local fluid velocity and therefore measures the smallest relative velocity. With increasing depth the relative velocities increase (in spite of the fact that the fluid velocities decrease with depth).

\section{low frequency response}

Throughout the thesis, I've talked about the errors in stabilization of the gyro platform at low frequencies. Consider typical accelerations of the buoy (when the relative motion between the uppermost sensing volume and the fluid $\mathrm{v}_{r e l}$ was small compared to the motion of the sensor $\mathrm{V}_{S}$, so that for estimates in this section we can use $\mathrm{V}$ and $\mathrm{V}_{S}$ interchangeably). In file $f$, the rms surface elevation was $58 \mathrm{~cm}$ and the peak frequency was $0.1 \mathrm{~Hz}$. The typical accelerations of the buoy may be approximated as $\eta_{r m s} \sigma_{\text {peak }}^{2}=20 \mathrm{~cm} / \mathrm{s}^{2}=0.02 g$ (where $g$ is the acceleration due to gravity). If there is a small error angle in stabilization $\varepsilon(t)$, the error in the horizontal acceleration of the buoy $\ddot{X}_{\text {err }}$ and the vertical acceleration of the buoy $\ddot{Z}_{\text {err }}$ may be approximated solely as a function of $g$ and $\varepsilon(t)$ as

$$
\begin{aligned}
& \ddot{X}_{e r r} \approx g \sin \varepsilon(t) \approx g, \varepsilon(t) \\
& \ddot{Z}_{e r r} \approx g(1-\cos \varepsilon(t)) \approx g\left(\frac{\varepsilon^{2}(t)}{2 !}\right) .
\end{aligned}
$$

We define an energy ratio $u$ to be

$$
u \equiv \frac{C_{V_{I} V_{I}}+C_{V_{J} V_{J}}}{C_{V_{K} V_{K}}}
$$

where $C_{V_{I} V_{I}}$ and $C_{V_{J} V_{J}}$ are the autocospectra of orthogonal fluid velocity components in the horizontal plane and $C_{V_{K} V_{K}}$ is the autocospectra of the vertical fluid velocity. For deep water waves, the ratio $u$ should be equal to one over all frequencies. In the energetic wave band $(0.1 \mathrm{~Hz}$ to $0.25 \mathrm{~Hz})$ we can see in Figure $5-18$ that this is true for all six sensing 

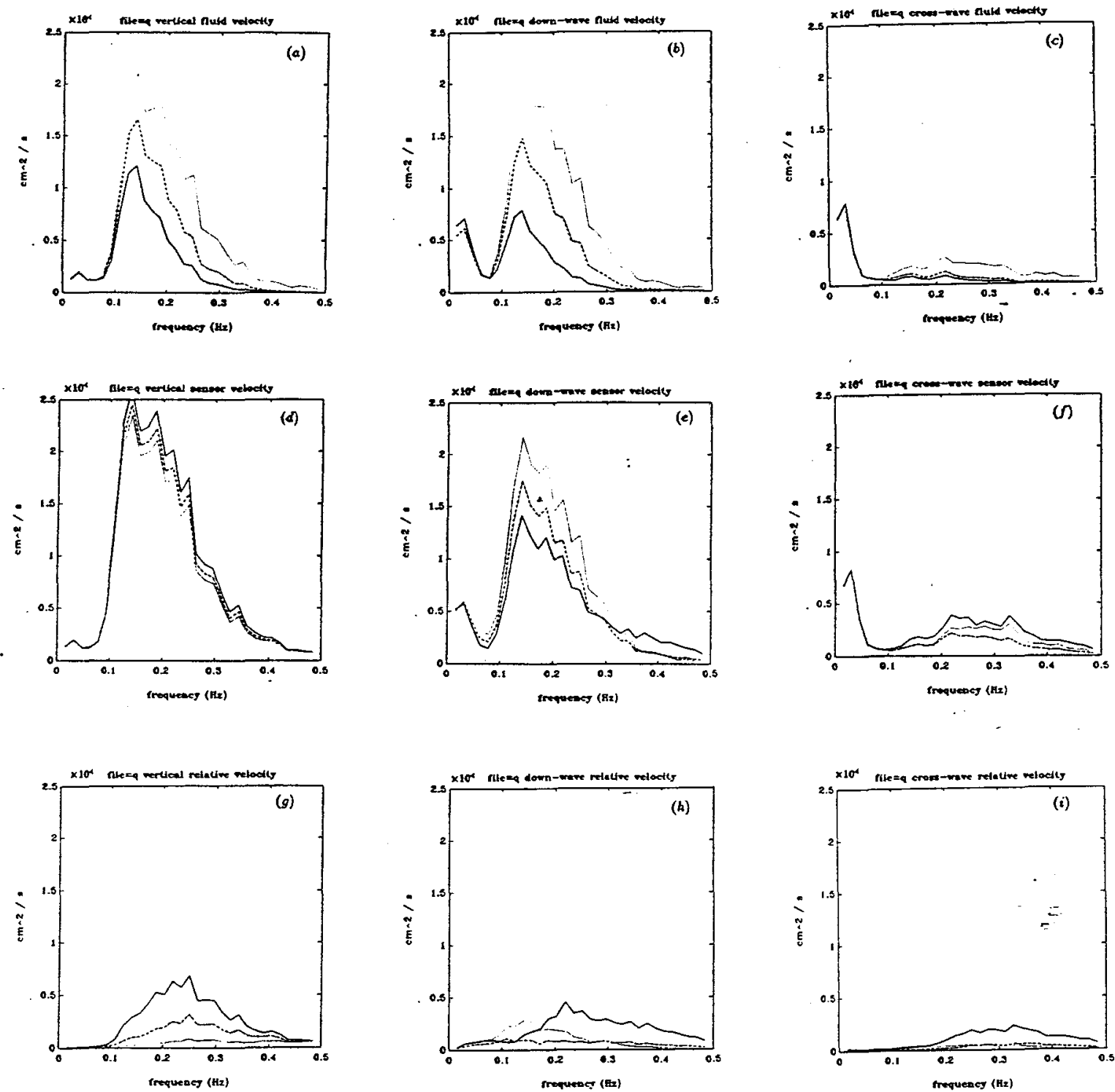

Figure 5-17: Spectra of motion variables for file $q$, sensor 1 (-) at a depth of $585 \mathrm{~cm}$, sensor $3(\cdots)$ at a depth of $311 \mathrm{~cm}$ and sensor $6(\cdots)$ at a depth of $111 \mathrm{~cm}$. (a) Vertical fluid velocity (b) down-wave fluid velocity (c) cross-wave fluid velocity (d) vertical sensor velocity (e) down-wave sensor velocity (f) cross-wave sensor velocity (g) vertical relative velocity (h) down-wave relative velocity (i) cross-wave relative velocity 


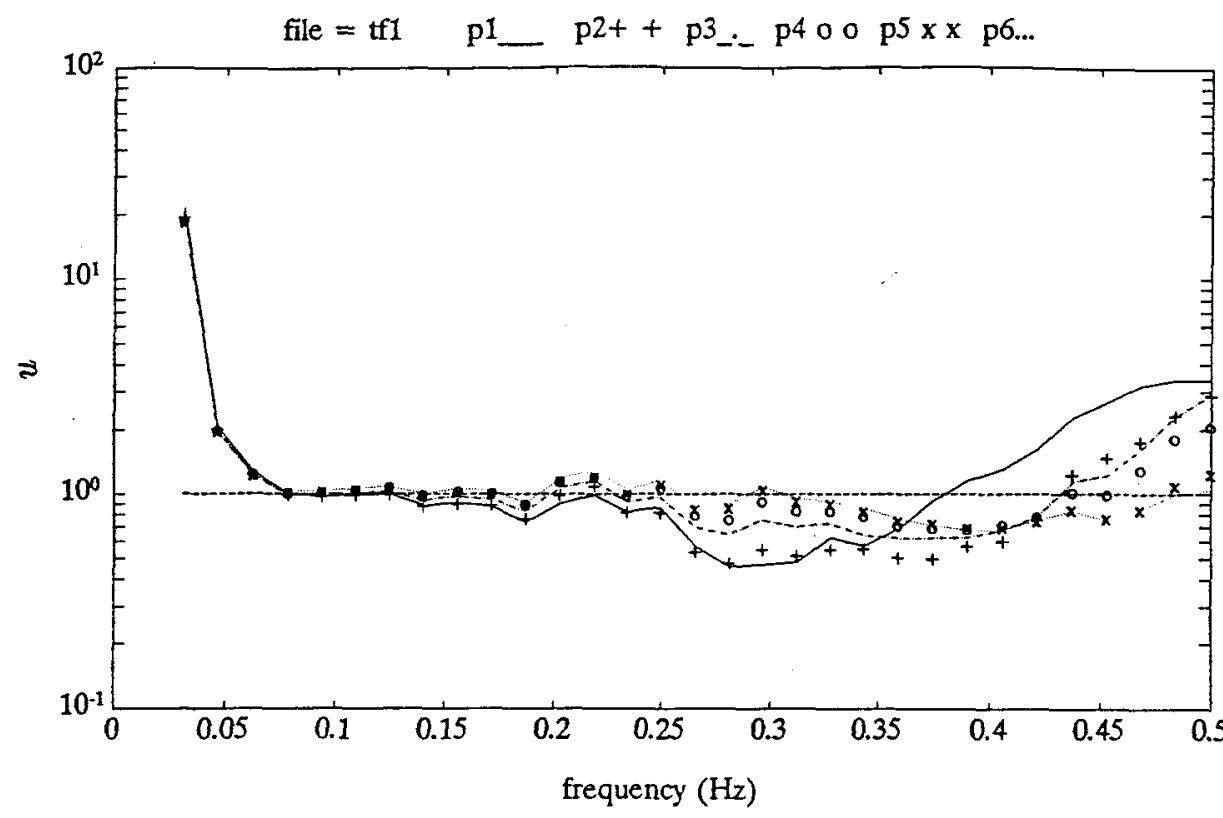

Figure 5-18: The ratio of energy $u$ is plotted for all six sensing pods during file $f$ is shown. The key to symbols is above the plot. The rapid increase of the ratio at low frequency is thought to be due to gyro stabilization error.

pods during file $f$. If we look at the energy in the Fourier coefficient at the three lowest non-zero frequency bins (central frequency is $0.03125 \mathrm{~Hz}$ ), we find the energy ratio $u$ to be 7.7. The sum $C_{V_{I} V_{I}}+C_{V_{J} V_{J}}=66\left(\frac{\mathrm{cm}}{\mathrm{s}}\right)^{2}$ and $C_{V_{K} V_{K}}=8.6\left(\frac{\mathrm{cm}}{\mathrm{s}}\right)^{2}$. Approximating the low frequency error of the gyro as a pure sinusoid at frequency $0.03125 \mathrm{~Hz}$ with an energy equal to the eccentricity in the ratio $u, 55\left(\frac{\mathrm{cm}}{\mathrm{s}}\right)^{2}$, we can estimate the magnitude of the error in stabilization from

$$
S_{\dot{X}_{\text {err }} \dot{X}_{e r r}}=\frac{g^{2}\left|\varepsilon\left(\sigma_{o}\right)\right|^{2}}{\sigma_{o}^{2}}
$$

and find that $|\varepsilon| \approx 0.00151 \mathrm{rad}\left(00^{\circ} 05^{\prime} 11^{\prime \prime}\right)$. This is consistent with the manufacturer's claim that the mean stabilization is accurate to within $7^{\prime}$.

An error of such magnitude is easily explained. The gyro maintains its alignment with the local gravity vector by averaging the output of spirit-level mercury switches which allow an electrical current to pass to solenoids which slowly torque the stabilized platform. The enormous angular momentum of the gyro, spinning at 10,000 rpm, causes the error angle to 
rapidly decrease with increasing frequency. It does not seem unreasonable that at frequencies in the range of $0.03 \mathrm{~Hz}$ that there be an error angle of $5^{\prime}$.

The anisotropy of the autocospectra at low frequency is well explained by the error angle argument given above. Previously, Barricket al (1989) have attempted to explain this aberrant behavior of $u$ at low frequency by the presence of small amounts of wave energy at frequencies below the spectral peak. Since the SASS was moored in $90 \mathrm{~m}$ of water, very low frequency waves will no longer have circular orbital trajectories but will have more energy in the horizontal excursion than in the vertical. So, if the SASS were measuring waves at extremely low frequency one would expect the ratio $u$ to increase with decreasing frequency. For intermediate depth the theoretical value of $u$ is given by

$$
u=\frac{C_{V_{I} V_{I}}+C_{V_{J} V_{J}}}{C_{V_{K} V_{K}}}=\left(\frac{1}{\tanh k(z+h)}\right)^{2}
$$

where $h$ is the water depth. For the case under consideration, to obtain a $u$ of 7 requires waves with a wavenumber $k=0.00447 \mathrm{~m}^{-1}$. Such a wave has a frequency of $0.020 \mathrm{~Hz}$. Beyond the fact that $u$ reaches 7 at a higher frequency than $0.020 \mathrm{~Hz}$, if a wave of frequency $0.020 \mathrm{~Hz}$ were to contribute energy of $66\left(\frac{\mathrm{cm}}{\mathrm{s}}\right)^{2}$ it would have to have an rms amplitude $O(4 m)$ (and here we have not even taken into account the fact that the frequencies under consideration were in the roll-off bands of the integrating filters!) Considering this, it seems that for the SASS, most of the deviation of $u$ from unity at low frequency is due to imperfect gyro stabilization.

\subsection{Errors}

\section{processing errors}

The first concern is that all of the processing steps outlined in the previous sections have been properly implemented. This is checked by synthesizing fake wavefield and motion data using theoretical forms for wave spectra and theoretical response functions. A complete set of transformation equations is developed so that the results are known in both the instrument relative and inertial frames of reference. The instrument relative fake data can then 
be entered into the processing routines and the results compared to the theoretically derived inertial frame results. Because some of the processing steps are nonlinear we are concerned that noise may bias our final results. To test to what extent this is true, we develop (guess) noise models for the input channels, add the noise to the theoretically developed input data, process, and once again compare to the theoretical output data. First, however, we use data with no noise to ensure that the processing routines work as expected in the ideal case.

The input wave spectrum used for the data creation program was that developed by Donelan et al (1985). The directional spectrum was approximated discretely with 113 frequency bins and 23 directional bins, making each time step the synthesis of 2599_Fourier components. With many channels of data to predict, this makes the simulation program extremely costly to run in terms of time. The buoy response was assumed to be described by a simple, single pole, lowpass filter. Filter coefficients and cut-off frequencies were adjusted so that the fake data was representative of the typical conditions we found in the field. The results of the simulation show that the processed output and theoretically derived output are in excellent agreement (Figure 5-19).

The real concern for the noise on the channels is that this noise will in some way be modulated down to d.c. and hence, bias our results. As we've already discussed, it was necessary to assume that the SASS had zero mean drift. The estimate of the current, then, comes entirely from the BASS velocity sensors. Here we seek to ensure that our processing does not affect the means of the BASS sensors. Errors in the motion sensing will be important when we consider the bias in shear due to the processing. If the noise adversely effects our estimates of rotational velocities, a spurious shear could be introduced into our results. The first step in doing the noise analysis is to estimate the noise levels that will be present on each channel. The static calibration provided us with scale factors but there was no extensive dynamic calibration. To estimate noise levels I have used a mix of specifications, theory, simple dynamic tests and intuition. In actuality, we find that there is no appreciable bias in either the depth averaged mean or in the mean shear estimates. 

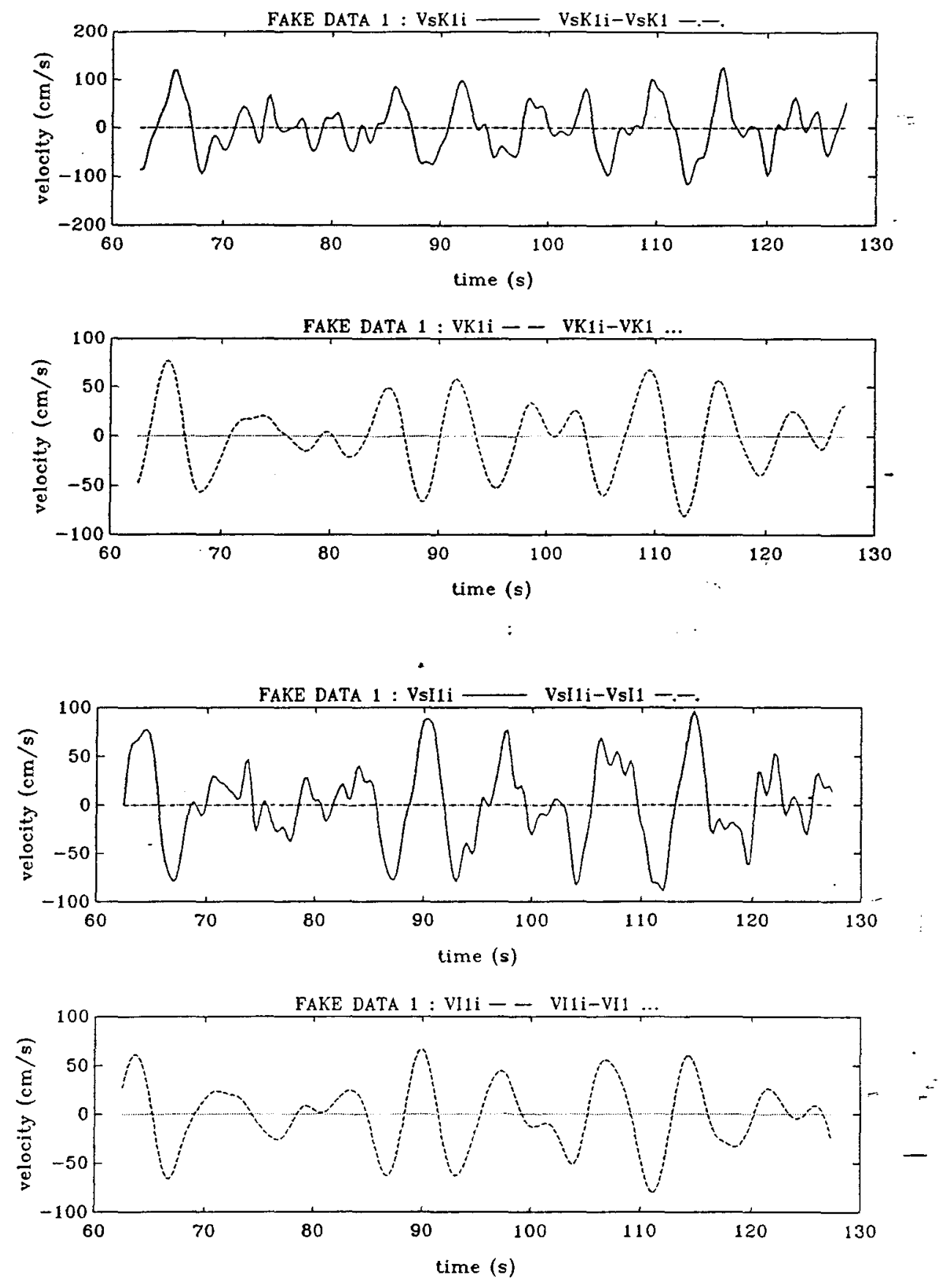

Figure 5-19: Comparison of processed fake data and theoretically derived fake data. (a) Sensor velocity in vertical direction (-) and error (-.--). (b) Fluid velocity in vertical direction (- - ) and error ( ..). (c) Sensor velocity in downwave direction (-) and error $(\cdots-\cdot)$. (d) Fluid velocity in downwave direction $(-$.$) and error (\cdots)$ 
The gyroscope's potentiometers are digitized by the BASS computer. Since the BASS digitizer is a 16-bit device and the full scale output of the potentiometers is $\pm 0.61 \mathrm{rad}$, the ideal resolution of this measurement would be $1.86 \times 10^{-5} \mathrm{rad}$ (recall that the compass resolution was only $\left.4.91 \times 10^{-2} \mathrm{rad}\right)$. Obviously, digitization error is not expected to be significant for the potentiometers. Indeed, the specification sheet for the potentiometers only claims a resolution of $2.03 \times 10^{-3} \mathrm{rad}$. This number provides the absolute noise floor for the potentiometers. There are, however, larger sources of error.

The potentiometers measure the angle of rotation of different gimbals. The two potentiometers together measure the angular displacement between the gyro case (fixed in the instrument relative frame) and the stabilized platform (which theoretically only yaws in inertial space). The major source of error in the potentiometer measurement is the stabilization error of the gyro. There are two factors: that cause the gyro to deviate from a constant angular orientation. First of all, the gyro is on a rotating planet and therefore, if there is no other forcing, the gyro will rotate due to the rotation of the earth. More importantly, if the gyro case is moving, the friction in the gimbals and the friction and springiness of the wires carrying power and signals to and from the stabilized platform will force the platform to deviate from its constant position. To force the gyro platform to maintain a fixed orientation (conveniently defined as colinear with the local gravity vector), two mercury switches are employed. These mercury switches are small spirit levels which, as they seek level, turn on and off power to solenoids which torque the stabilized platform. The feedback system is designed such that the stabilized platform maintains a nearly constant attitude. There are small deviations.

In a small survey of people who were familiar with gyros, the concensus was that such a gyro should be "good to" about $1^{\circ}$. The gyro specification sheet claims mean stabilization to be within $7^{\prime}$. From field data, Santala and Williams (1990) found that low frequency errors indicated that the mean stabilization errors were approximately in this range. Several pre-deployment dynamic tests were performed to give some idea of what the instantaneous errors might be. The problem with all the tests was that there was no way to know what 
the actual gyro motion was during the time it was being forced. The only referenceable position was the mean stabilization position. The procedure was, then, to force the gyro with some motion, and then to stop the motion as instantaneously as possible. If stabilization were perfect, the gyro output would be constant once the motion stopped. However, if the stabilized platform were at some other orientation than level when the gyro was finally held fixed, the output would change until the solenoids could torque the platform back to the mean stabilized position. In this way we could get samples of the instantaneous errors of the gyro. The problem is that in each test the shaking lasted for one to six minutes and only one point sample resulted. Further, with no available shaking device, the author found himself shaking the gyro (along with power supplies and batteries). Physical fatigue lead to a less than statistically significant amount of samples. Nonetheless, these tests do give us some idea of what to expect in terms of gyro performance.

In the first dynamic test, only translations in the horizontal plane took place. The gyro, the power supplies and the recording devices were loaded onto a small cart (a power cord was run down to the cart from the ceiling). A large area of floor space was cleared and then the cart was rolled around on the floor for anywhere from one to six minutes and then abruptly stopped. There were seven trial tests. The typical horizontal accelerations ranged from 0.2 to $0.3 \mathrm{~g}$. The dominant frequency of forcing ranged from 0.12 to $0.15 \mathrm{~Hz}$. The maximum observed stabilization error was $1.2^{\circ}$. The mean magnitude of potentiometer error for the seven trials was $0.26^{\circ}$.

For the second test, the gyro was mounted onto a board. Under the center of the board a small block was placed. The placement of the small block allowed the board to be tilted easily. If the gyro was tipped too far, the board would hit the counter and prevent further tipping. The peak tipping angle was adjusted for the different test runs by using different sized blocks. The peak tip angles varied, for eight trials, between $\pm 10^{\circ}$ to $\pm 18^{\circ}$. The peak periods of forcing were between 0.09 to $0.13 \mathrm{~Hz}$. The mean magnitude of the potentiometer error was $0.70^{\circ}$. The error seemed to be correlated with the amplitude of the tilts. In the field, rms tilts of $7^{\circ}$ would have to be considered extreme. Here we had $r m s$ tilts varying 
from $7^{\circ}$ to $12^{\circ}$.

A final test was performed by putting the gyro in a basket and swinging the basket. In the "reasonable" tests, the gyro errors appeared to be small. The goal of this test was to see how to induce large errors (i.e., we wanted to find the failure modes). The only motion "failure mode" was found to be rapid spinning of the gyro. The reason that the stabilized platform deviated from level in this case was because the apparent local gravity vector, as detected by the gyro, included the angular acceleration vector. In field deployments the buoy did not spin. In fact, during no file (usually 20 to 40 minutes long) did the buoy ever perform a single complete rotation. Hence, we are reasonably assured that the gyro did not experience this failure mode during deployment. Another error mode, by far the worst mode of failure, was when the gyro tilts beyond its maximum allowable tilt angle. In this case, the stabilized platform hits the gyro case. The force of contact torques the platform out of stabilization. As noted though, the tilts experienced in the field were mild compared to those induced in the lab and never approached an angle where this failure mode would have occurred. The violent nature of these tests do not make them suitable for inclusion in our development of noise bounds. For cases where the forcing was more gentle the errors were similar to those found in the previous tests.

Even when the gyro case does not move at all, there is still a small amount of error present (the mercury switches have finite resolution). Dynamic error seems to be a stronger function of tilting than of translation. The most probable cause of this is the friction of the gimbals and torquing of the power and signal wires. The error must be dominated by low frequency errors because it is extremely difficult to get the stabilized platform to move quickly under any circumstances. The precision of the error estimates presented here is not exceedingly great. However from these tests, and with these observations, we make the following model for the stabilization noise. The noise for the potentiometers will be created by filtering a Gaussian white noise signal with a single-pole lowpass filter which has a cut-off frequency of $0.04 \mathrm{~Hz}$. Hence, the assumed noise level above $0.04 \mathrm{~Hz}$ drops off by $6 d B$ per octave. The gain of the filter was adjusted so that the rms error signal was $0.6^{\circ}$ 
for the reference noise level case.

The accelerometers have inherent errors but, just as in the case of the potentiometers, these errors are dominated by imperfect stabilization. So the accelerometer errors were estimated using the stabilization error model given for the potentiometers. In fact, its important to realize that the errors of both the potentiometers and accelerometers are dominated by the stabilization error because in this case the errors are correlated.

By far the worst performer of the motion sensing instruments is the compass. It was not until only very recently that we have discovered just how poorly the compass really is. Fortunately, as we will see, the impact to our measurement of the mean current and the estimation of the bias is only minimally affected. This error will to a noticeable extent affect our description of the directional spreading of the waves.

As we saw previously, the high frequency noise of the compass is dominated by the digitization error. At lower frequency we originally felt that since the motions of the buoy at low frequency were expected to be mild, that the errors in the energetic part of yawing spectrum would be minimal. A recent report by Patch et al (1991), however, points to serious problems in the frequency response of the compass that was used on the SASS. Patch et al (1991) performed a detailed calibration of the frequency response of the VACM compass to yawing and found that the transfer function of the compass was given by:

$$
H_{c o m p}(\sigma)=\frac{c-a \sigma^{2}}{c+\imath b \sigma-a \sigma^{2}}
$$

This transfer function relates the actual yawing motion of the compass to the indicated output. The parameters $a, b$ and $c$ are typically about $126 \frac{\mathrm{mg} \cdot \mathrm{s}^{2}}{\mathrm{rad}}, 200 \frac{\mathrm{mg} \cdot \mathrm{s}}{\mathrm{rad}}$ and $126 \mathrm{mg}$, respectively. With these coefficients, the transfer function has a null at about $4.5 \mathrm{sec}$. Furthermore, in a simple test done here (Appendix E) it was shown that lateral accelerations can lead to noise in the compass measurement. This may explain the absence of any spectral gap, as would be expected from Patch's results, in the SASS compass data. 
Typical errors for the BASS pods are $4 \%$ of the total velocity (Trivett et al, 1991). The reference level for BASS pod measurement noise was chosen to be such that the rms error was $4 \%$ of the rms measured velocity. More will be said on the measurement capabilities of the BASS sensors in the next section.

The procedure to see if the processing technique induced a bias was to create a random wavefield, add noise and process. The energy levels of the signals were matched to meet those we typically found in the field. The compass was a bit special, at high frequency, the transfer function is approximately unity. At high frequency (above $0.6 \mathrm{~Hz}$ ), we did not measure any appreciable signal. This, as we also argued intuitively, is probably an accurate reflection of the high frequency motions of the buoy in yaw. At frequencies of about $0.22 \mathrm{~Hz}$, our measurements are probably quite corrupted by the poor frequency response of the compass. For purposes of evaluation, it was assumed that the integrated energy in the compass signal was the same as that for the pitching and rolling motions. This is almost certainly an absolute upper bound, especially since we are concentrating the energy in a narrower band of the spectrum. The results of 20 random realizations show that the bias in the currents was less than $0.2 \mathrm{~cm} / \mathrm{s}$ and, the bias in the shear was $2.3 \times 10^{-6} \mathrm{~s}^{-1}$. This means that even with the estimated noise levels the processing will induce negligible biases on our raw measurements.

It is worth noting that the test just described only included wave velocities. If there were a mean current and we were making poor compass measurements, we expect that we will slightly underestimate the mean current. Suppose that the buoy is yawing sinusoidily between the angles $\theta_{Y}= \pm 15^{\circ}$ at a frequency which is in the null of the compass response. The indicated yawing will be zero. If there is a mean current $V_{o}$ then in the fixed direction we believe the current is actually pointing, we will measure the velocity $v_{m}$, where

$$
v_{m}=V_{o} \cos \theta_{Y}, \quad \theta_{Y}=\frac{\pi}{12} \sin \sigma t
$$

Even for this extreme case, the error in the measured mean current is only $3 \%$,

$$
\frac{V_{o}-\overline{v_{m}}}{V_{o}} \approx \frac{\overline{\theta_{Y}}}{2}=0.034
$$


The place where the poor compass performance should become apparent is in the estimation of the directional spectra. Even here, though, the results are not too bad (Figure 5-20). In fact, if small amounts of gaussian white noise ( $\mathrm{SNR}=27 \mathrm{~dB}$ ) are added to the mathematically transformed data (so that we know this is not a transformation phenomenon), the two peaks of the directional spectrum for that case merge together into a single broad peak centered at $\theta=0$. Because the compass should perform well at low frequency, we will still get the mean propagation direction of the waves correct. This is the single most important factor in properly estimating the bias. The second thing we need to estimate the-bias are the moments of the directional spreading. In spite of the fact that the spreading functions of the noisy signals in Figure 5-20 tend to be more "peaky", the $\mu$ filters we use to estimate the bias tends to be underestimated due to the broader tails in the noisy estimates. In the notch band of the compass response, this error reaches $12 \%$. In the lower band, where the peak energy of the wave orbital velocity is located, the error is about $5 \%$. Since the very largest bias corrections are rarely above $5 \mathrm{~cm} / \mathrm{s}$, these errors are not expected to be too large. Also, in the conclusion section we attempt to bound the shear from above. Since we underestimate the $\mu$ 's, the bias will be underestimated and, consistent with the approach we will finally take, the estimated shears will represent upper bounds.

The discussion above is based almost entirely on the computer simulation. From these results we see that the resonant performance of the compass could noticeably affeet our results. This conclusion is based on the assumption, used in the computer model, that the yawing motions of the SASS are as energetic as the pitching and rolling motions. In section 5.3, however, it was argued that the yawing motions of the buoy could be expected to be less energetic than the pitching and rolling motions. In Appendix $\mathrm{E}$ we examine the sensitivity of our actual data to the compass signal. This is done by comparing the directional wave spectra and mean current profiles from normally analyzed data and fromdata where the cutoff frequency of the compass filters was reduced to $0.125 \mathrm{~Hz}$. As is seen in the appendix, both processing paths lead to almost identical results. This, along with 

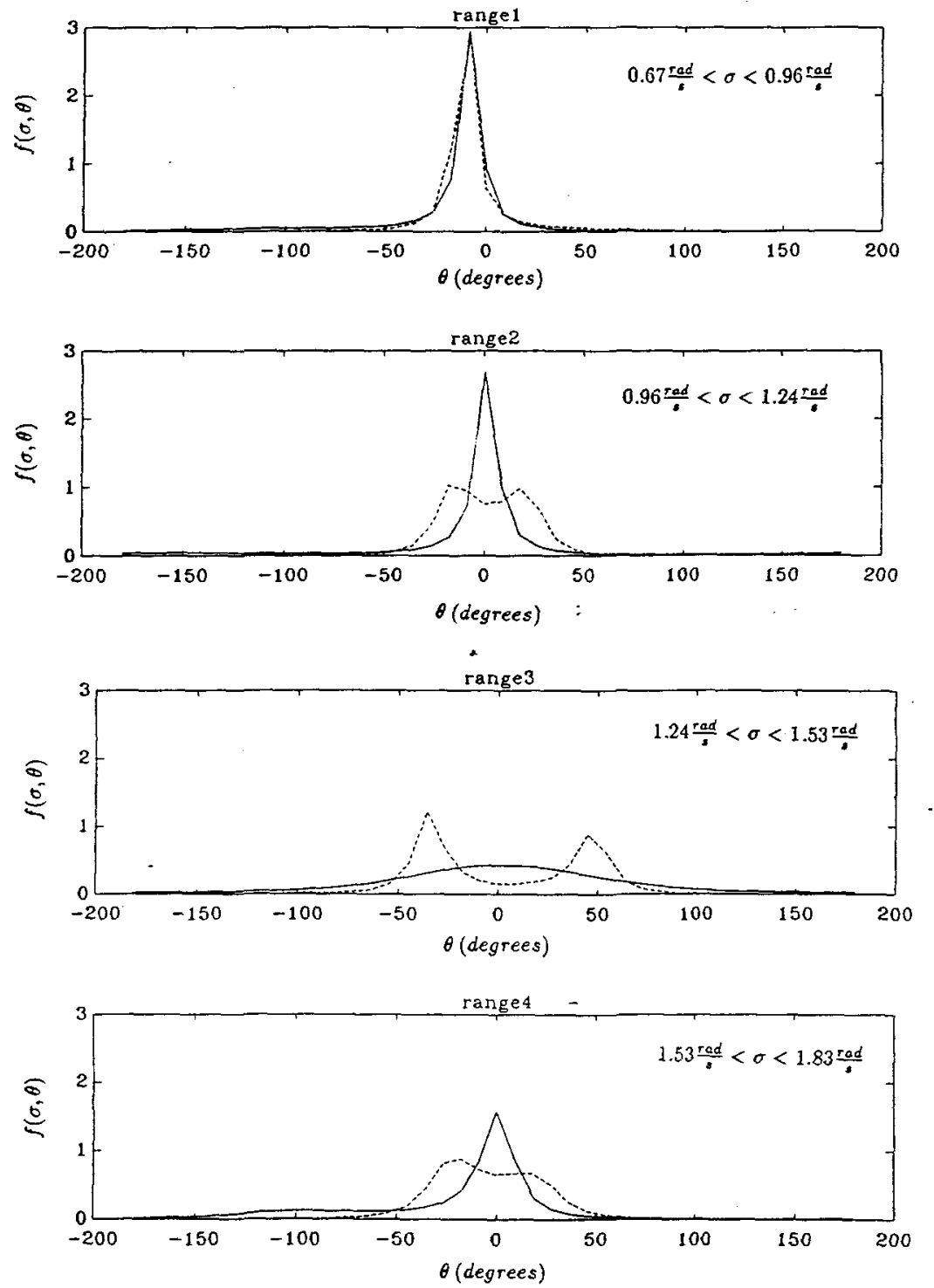

Figure 5-20: The directional spreading function of the wavefield $f(\sigma, \theta)$ estimated from one of the computer simulations for four frequency bands (as labelled) for the mathematically transformed data (-.) and for the data where noise levels have been estimated (-). The noisy data consistently exhibits stronger peaks in the downwave direction (the waves in the model were propagated from the direction $\theta=0$ ) but also have higher "tails" at large angles from the true propagation angle. The main factor in the mismatch between the results is the resonant null of the compass. 
the computer simulation results leads us to the following conclusions. First, if the actual yawing motions of the SASS were as strong as assumed in the computer model, the errors in estimating the bias would be about $10 \%$. The resulting estimates in the mean current would be less owing to the fact that the bias is only a fraction of the measured mean (as is shown in equations 5.28 and 5.29 the raw estimate of the mean should only be minimally affected by compass errors). In fact, it seems that the yaw motions of the SASS are not so intense as assumed in the model. The results of the appendix clearly show that whether or not yaw motions above $0.125 \mathrm{~Hz}$ are considered, the estimates of the directional spectra are nearly the same. Much of the energy observed in the compass signal above $0.125 \mathrm{~Hz}$ is most likely due to compass sensitivity to lateral accelerations. If this is the case, then we also have an explanation for why no spectral gap is observed in the compass records in the vicinity of $0.22 \mathrm{~Hz}$. Also, the filtering test described above was also repeated for frequencies lower than $0.125 \mathrm{~Hz}$ and the results were then found to be heavily dependent on the cutoff frequency (these results, being of no practical use, are not shown). So finally, we conclude that since the compass is expected to perform well at frequencies below $0.125 \mathrm{~Hz}$ that the results we have are only slightly affected by the resonant null at $0.22 \mathrm{~Hz}$ and the other sources of compass error we have discussed.

\section{flow disturbance}

Roughly speaking, the flow disturbance on the SASS can be thought of as coming from three sources.- There is the slender truss that directly supports the BASS acoustic transducers. About this is the SASS superstructure, constructed of 2 in $O-D$ aluminum tubing. On top of the SASS frame are the three floats, the largest bluff bodies in the SASS's construction. Let's consider each of these components in turn.

While developing the specifications for a proposed current meter, Trivett et al (1991)

performed a flow disturbance analysis on the BASS current meter. This analysis showed that, in steady flow, the BASS underestimated the fluid velocity by $4 \%$ due to the drag on the transducer support structure. This result was independent of flow velocity but did 
vary with BASS cage orientation. As the orientation of the cage is tipped to $20^{\circ}$, the drag induced error increased to about $8 \%$ (these are the results from Trivett et al (1991), more extensive though less accurate results are presented in Appendix C). In the case of SASS, the relative velocities measured by the BASS sensors is composed of both a steady and unsteady component. The mean drag, in such a case, can not be derived from the mean velocity. The mean drag of a symmetric object in purely oscillatory flow is zero. The drag on an object in a mean flow with a superposed oscillatory component is much greater than the drag on an object in steady flow with the same mean. The normal way of estimating form drag on an object is via a drag coefficient, $C_{D}$, as

$$
D=\frac{1}{2} \rho C_{D} A_{p} V|V|
$$

where $\rho$ is the fluid density and $A_{p}$ is the projected area of the object in the flowfield. Figure 5-21 shows how the net drag on an object in a mean flow is amplified as the rms velocity of a superposed oscillatory flow component is increased.

Figure 5-21 is the basis for the flow disturbance correction model we seek to create. It allows us to relate the steady flow calibration results to the unsteady flow data, if we can relate the velocity defect inside the cage to the drag on the cage. This will be done using a simple model based on momentum considerations. The BASS measurement is made just behind the supports which give rise to the velocity defect. Being in the near wake, we will be unable to estimate the functional form of the wake. Still, we will be able to compute an average velocity defect.

The basic physical idea is that there is a drag force exerted on the fluid by the support structure. The application of that force gives rise to a change in momentum in the fluid. If the free stream velocity $V_{o}$ is much greater than the velocity defect $v_{d}$ then the momentum deficit, see Batchelor (1967), can be modeled as the momentum of the free stream fluid $\rho V_{\circ}$ times an equivalent sink $Q$

$$
D=\rho V_{\circ} Q
$$

The volume defect represented by $Q$ can be amortized over the wake area to find the velocity 


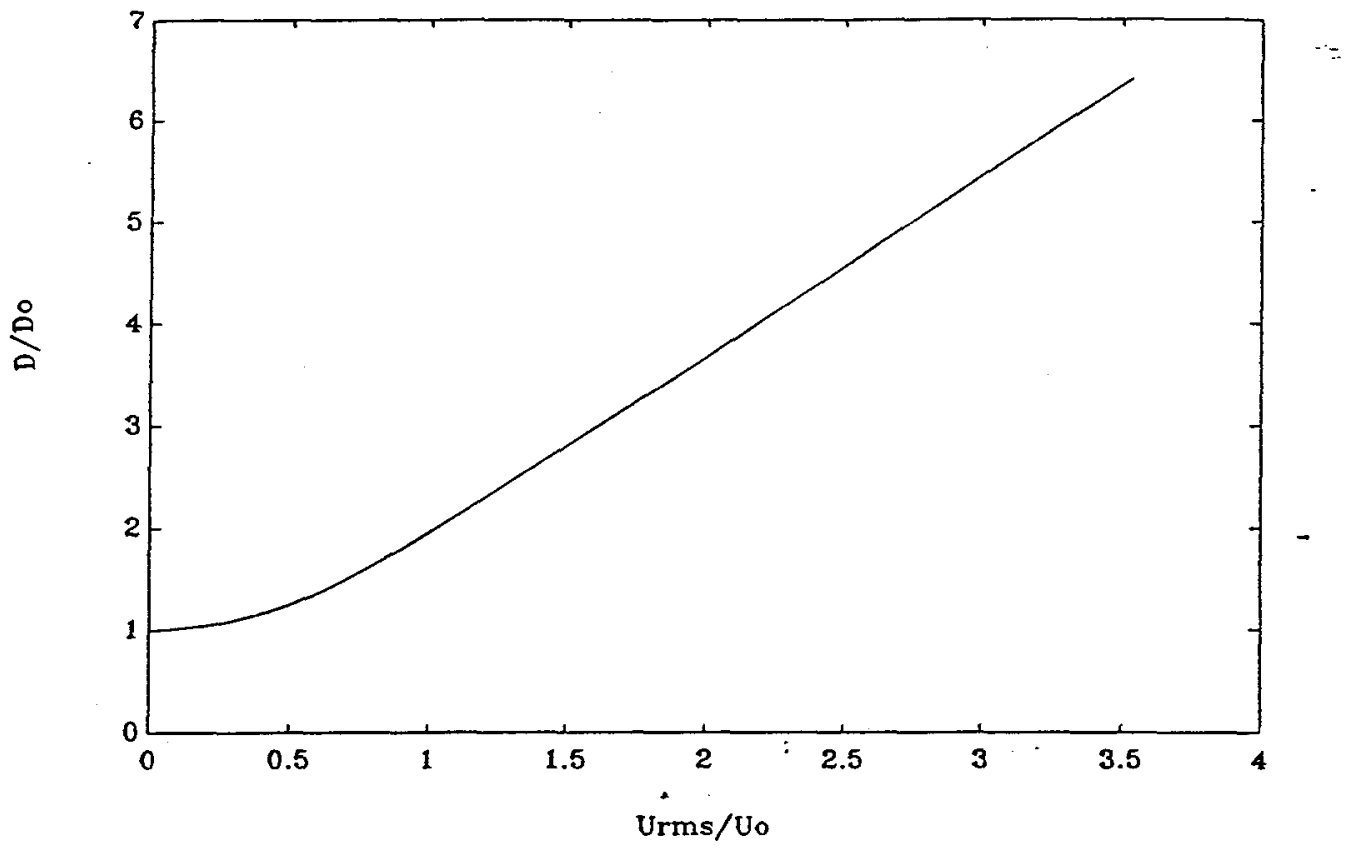

Figure 5-21: Amplification of drag due to a superposed oscillatory flow. Urms is the rms velocity of flow superposed on steady flow, $U o$. The actual drag, $D$, can be much greater than the drag, Do, in steady flow.

defect $v_{d}$

$$
v_{d}=\frac{Q}{A_{w a k e}}
$$

where we've assumed in the above that the wake is uniform. Batchelor shows that not only can the wake behind a still body in a moving flow be characterized in this way, but so too can the entrainment behind a moving body. This is not a surprising result, though the two frames differ dynamically, in ideal flow (and what we have here is really an ideal flow wake model) the kinematics of a body moving through a fluid with velocity $V_{o}$ and a body moving through a fluid with velocity $V_{0}$ are indistinguishable. In this model fluid is conserved. The equivalent sink $Q$ decelerates the fluid in the wake, and an equivalent source-term, of equal strength to the sink, accelerates the fluid outside the wake. The wake encompasses only a small region downstream of the body. The source term is radially distributed in all directions. Because the source term is amortized over a much larger area, the upstream 
effects are much smaller than the downstream effects. That the drag can be parameterized in terms of the freestream velocity is not entirely obvious. I have included a simple example in Appendix B which shows from first principles that this may be done (refer to Batchelor, 1967, for a more complete discussion).

The velocity defect behind our object in a combined steady and oscillating flow can be found by combining equations $5.30,5.31$ and 5.32 as

$$
v_{d}=\frac{\frac{1}{2} C_{D} A_{p} V|V|}{A_{\text {wake }} V_{o}}
$$

In certain engineering applications, the estimation of the projected area is one of the most simple that we might hope to encounter. However, considering the complex form of the BASS support structure, it can be seen that its not even certain which elements will cause wakes in the sampling volume and to what extent.: The near wake area is not well understood even for simple geometries. Even the drag coefficient of a cylinder will change in the presence of other cylinders.

Since none of the coefficients are easily estimated we seek to determine their value experimentally. The individual coefficients themselves are of no intrinsic value to us here so we'll just lump them all together into a new coefficient $\chi$, and then

$$
v_{d}=\chi \frac{V|V|}{V_{o}}
$$

If the coefficient $\chi$ can be determined from calibrations we avoid the unsavory task of estimating $C_{D}, A_{p}$ and $A_{w a k e}$. From the findings of Trivett et al (1991) we know that in steady flow, independent of the magnitude of the velocity, the defect velocity of the BASS current meter is $4 \%$ of the freestream.

$$
\frac{v_{d}}{V_{o}}=0.04
$$

This implies that $\chi$ is simply the fractional error (and a sign function)

$$
\chi=0.04 \frac{V_{o}}{\left|V_{o}\right|}
$$


Equation 5.34 provides the basis of the BASS pod error estimation. That equation is used iteratively to find the velocity defect at each pod. Equation 5.36 provides the calibration of the expression.

An alternate approach to estimating the drag would attempt to include the dependence of the BASS calibration on the relative angle of attack of the flow. Because this must be done in instrument coordinates, this approach essentially abandons the interpretation of the deficit velocity as outlined in equations 5.30 to 5.36 . If the flow disturbance is extremely localized, this instantaneous correction may have some justification. Because a large part of the flow dependence on elevation angle is undoubtedly due to the "shading" error, the three axis estimates should not be used at the the same time as an "angle of attack correction" is made. The information on the dependence of the flow error based on elevation angle is extremely limited; at $0^{\circ}$ (flow in vector in the $a-b$ plane in instrument coordinates) the error is $4 \%$, at $20^{\circ}$ the error increases to $8 \%$. Several different forms of calibration were tried to test the sensitivity of corrections to this type of dependence. The results did not greatly depart from those we find using the drag based approach though in general, the corrections here tended to be slightly larger (similar results could be obtained by adjusting the $4 \%$ coefficient in equation 5.36). The drag based approach was finally used because it seems that: (i) at low angles of attack, the $4 \%$ is probably about right, (ii) at higher angles of attack, flow disturbance is compensated for by using the minimally disturbed BASS axes (between elevation angles of $15^{\circ}$ and $75^{\circ}$ the shading correction is made on $76 \%$ of the possible relative flow directions), (iii) at angles of attack greater than $75^{\circ}$, the calibration is likely quite different than $4 \%$ but, the horizontal component of flow in these cases is small so that irrespective of how large the velocity is, the effect on the horizontal mean should be quite small, and $(i v)$ its not clear that making corrections to the instantaneous relative velocity is justified in a rotating frame of reference (in the drag approach we deal with the velocities that have been rotated to fixed inertial orientation). In fact, in the BASS calibration of Appendix $\mathrm{C}$, it is shown that in an unsteady calibration the best three-axis estimate coupled with the momentum defect model (the scheme applied to the SASS data) give far superior results than those obtained by applying the steady calibration results to 
the unsteady BASS data. The wave measurements of Appendix D further show that steady calibration results tend to overstate the errors of the BASS in unsteady flow.

The velocity defect due to the large aluminum superstructure which supports the BASS towers was also considered. Here again, the computation of the velocity defect is hampered by the difficulty is estimating a precise form for the wake. What was done in this case, was exactly what we shunned to do in the last case (since there's not too much hope of getting the SASS into a calibration tank); the projected area of all the members (including instrument pressure cases) was computed, a drag coefficient was estimated, and a wake area was estimated. The wake area was considered to be equal to the projected area of a cylinder circumscribing the SASS frame. Because the flow is three-dimensional, and the SASS structure is complex, it would be extremely difficult to predict "wake paths" from individual SASS frame elements. The wake was assumed to be uniform over the wake area. The SASS's frame is a relatively open structure. The maximum velocity defect estimates due to the frame were on order of $0.2 \mathrm{~cm} / \mathrm{s}$. Localized affects could be much greater but we assume that sensor locations were no worse than others and thus consider this error to be of negligible size.

The three floats which provide the bulk of the SASS's buoyancy were also investigated for flow disturbance. During the design of the SASS a great emphasis was placed on keeping the weight of the buoy to a minimum so that as little flotation as possible would be necessary. In the final design, the still water draft of the flotation elements was only $28 \mathrm{~cm}$. And even for the design current (a sheared flow with surface velocity equaling $50 \mathrm{~cm} / \mathrm{s}$ at the surface and $25 \mathrm{~cm} / \mathrm{s}$ at $90 \mathrm{~m}$ depth), the predicted mean draft only increased to $30 \mathrm{~cm}$. Even so, with a width of $91 \mathrm{~cm}$, there was concern that the floats would cause considerable flow disturbance. The problems of estimating the flow deficit due to the floats is even more difficult, conceptually, than those we encounter in treating the BASS cages or the SASS frame. Therefore we'll take the problem in steps, adding degrees of sophistication ass we go.

At the simplest level, we can study the flow effects of the floats in a mean current. 
Figure 5-22 shows a schematic of the float, the wake region and the relative location of the closest sensor. The basic approach will be ; calculate a drag force, find the equivalent sink

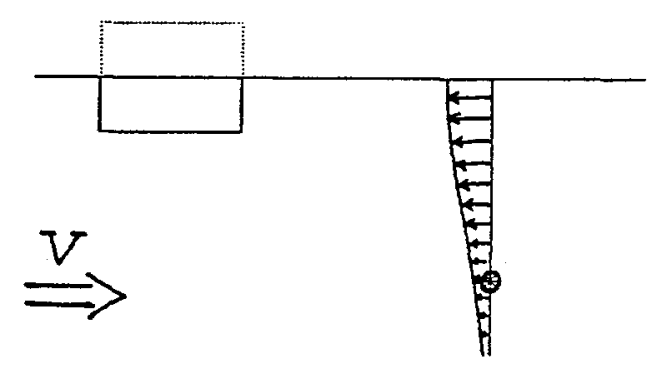

Figure 5-22: Schematic showing the relative positions of the upstream SASS float and the uppermost sensor. For computational purposes the float is viewed as a half-body in an infinite fluid.

term and amortize the deficit velocity over the wake region. The free-surface effects will be ignored. To simplify calculations we assume that the submerged part of the float represents . a half of a cylinder which is immersed in an infinite fluid. For this computation it becomes unavoidable to assume a functional form for the wake. A Gaussian form is often used to describe the far wake region. For our geometry we certainly are not in the far wake. But, it is probably safe to assume that the velocity deficit is greatest directly behind the-float and decreases in intensity with radial distance. Since the Gaussian is a convenient function which has these properties, we will use this form of wake function. (Note, if we assume that the near-field wake is an intense wake with a nearly uniform velocity deficit, then the extent of the wake necessary to explain the predicted drag force is such that the top sensor is not in the wake). The form of the velocity deficit in the wake is

$$
v_{d e f}(x, r)=v_{d e f_{0}}(x) e^{-\frac{r^{2}}{R(x)^{2}}}
$$

where $v_{\text {defo }}$ is the wake intensity at $r=0$ and will be a decreasing function of downstream distance $x$ from the float. The integrated deficit velocity at any downstream distance must 
equal the total sink intensity,

$$
v_{d e f_{o}}(x)=\frac{Q}{\pi R(x)^{2}} .
$$

We desire to bound the problem to see if the floats could indeed be causing flow disturbance. To do this, we want to know what the maximal value $v_{d e f}(x, r)$ can take at the radial distance $r_{s}$ and the downstream distance $x_{s}$ where the closest sensor lies. If a maximization is performed with respect to the parameter $R$ then we find that $R=r_{s}$. If we recall our previous relations relating $Q$ to the drag, we can express the velocity deficit as a function of the drag on the float

$$
v_{d e f}\left(x_{s}, r\right)=\frac{D}{\rho V_{o} \pi r_{s}} e^{-\frac{r^{2}}{r_{s}^{2}}}
$$

The ratio of the defect velocity at the top sensor to the total velocity, in terms of the geometry of our float, is then

$$
\frac{v_{d e f}\left(x_{s}, r_{s}\right)}{V_{o}}=\frac{C_{D} A_{p}}{2 \pi r_{s}^{2}} e^{-1}=0.018
$$

where $r_{s}=110.8 \mathrm{~cm}, A_{p}=5484 \mathrm{~cm}^{2}$ and $C_{D}=0.7$. The estimate of the drag coefficient is from Zdravkovich et al (1989). So, our worst case steady model leads to velocity defects of only $1.8 \%$. For a free stream velocity of $30 \mathrm{~cm} / \mathrm{s}$ this leads to an error of only $0.5 \mathrm{~cm} / \mathrm{s}$. Of course, as we begin to consider unsteady effects, this defect estimate will increase. However, in the most strongly forced cases, the ratio of the rms velocity relative to the float versus the mean current is just under two. A rough estimate of the resulting defect can be found by referring back to Figure 5-21. An amplification of the deficit velocity by three is appropriate for unsteady to steady ratios of two; thus we have a final estimate of $1.5 \mathrm{~cm} / \mathrm{s}$ for the final velocity defect. This still only represents $5 \%$ of the mean flow but can significantly alter the form of the shear profile near the surface.

Its always important to keep in mind the limitations of the models that we apply. To improve our models, we obviously start with what we believe to be our weakest assumption. The photograph in Figure 5-23 gives a clue as to what aspect of the model could best benefit from some refinement. In strongly forced cases, the draft of the upstream float is not well modeled as being constant. The drag on the float is a function of the projected area of the float and the velocity of the fluid past the float. If the draft of the buoy is not constant, then variable projected area will modulate the $V|V|$ term. If the float tends to be 


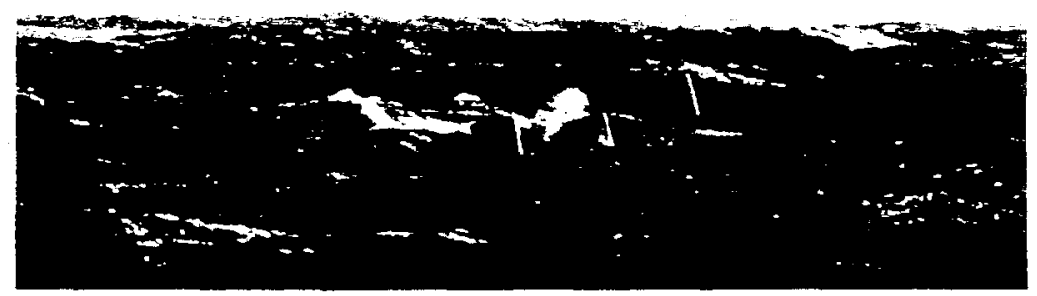

Figure 5-23: The SASS in high wind and waves.

more deeply immersed when a crest comes by than when a trough comes by, the estimate of the net drag could be significantly larger than that predicted by the constant draft model.

Estimation of the non-constant draft is not so simple as might be expected. The model provided by Figure 5-24 is the basis for our computations. Several simplifying assumptions are again made. The seas during the SASS deployment were characterized by narrow directional spreads. I took the limit of narrow directional spreading and assumed that all of the waves travelled in the mean downwave direction. In the spirit of computing an upper bound defect, the initial mean velocity estimate in this direction was taken to be the magnitude of the measured mean velocity vector (i.e., the current and waves were assumed to be travelling in the same direction). The height of the upwave float is equal to the surface elevation at the downwave float plus the gain in elevation due to tilting

$$
z_{u}=\eta_{d}+D \sin \xi
$$

The draft $T$ of the upwave float is estimated as

$$
T=T_{b}+\eta_{u}-z_{u}
$$

where $T_{b}$ is the draft of the downwave floats. Using a small angle approximation for $\sin \xi$, and realizing that when $T$ is negative that there is no projected area to oncoming flow, the 


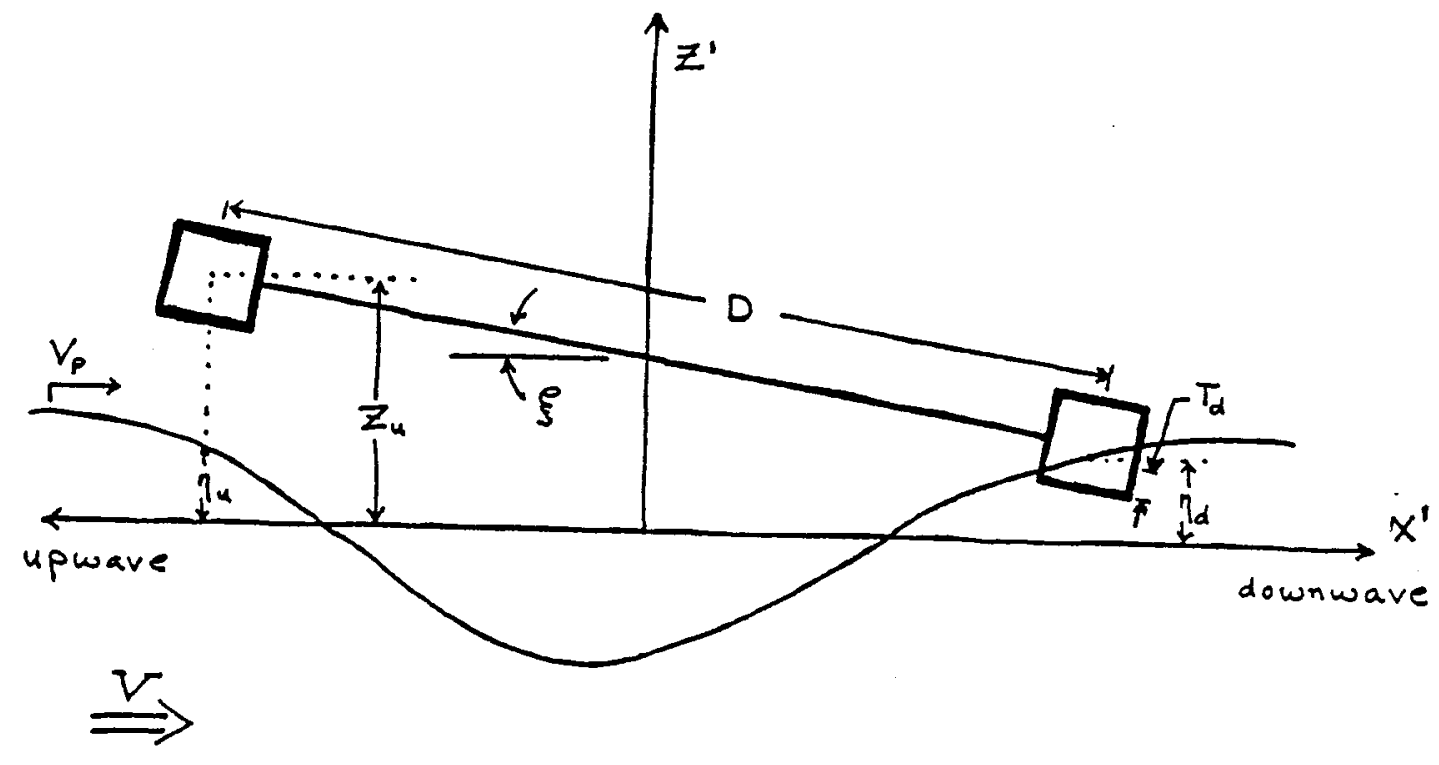

Figure 5-24: Model for predicting drag on floats when the draft is not constant.

final form of our draft estimate is

$$
T= \begin{cases}T_{b}+\Delta \eta-D \xi & \text { if } T_{b}+\Delta \eta-D \xi>0 \\ 0 & \text { if } T_{b}+\Delta \eta-D \xi \leq 0\end{cases}
$$

with $\Delta \eta=\eta_{u}-\eta_{d}$. The draft of the downwave floats must increase or decrease based on the relative immersion of the upstream float. The actual downwave component of tilt $\xi$ of the buoy can be found as

$$
\xi=\tan ^{-1} \frac{\mathbf{u}_{c} \cdot \mathbf{u}_{d w}}{\mathbf{u}_{c} \cdot \mathbf{u}_{K}}
$$

where $\mathbf{u}_{c}$ is the unit vector in instrument coordinates which is aligned along the BASS current meter array, $\mathbf{u}_{d w}$ is the unit vector pointing in the down-wave direction, and $\mathbf{u}_{K}$ is the unit vector pointing vertically in inertial coordinates. Assuming that all the waves propagate in one direction, the surface elevation at both the upstream and downstream float can be predicted from the measured wave velocity at the top sensor using the linearized theory for waves. A single filter can be formed to give the elevation difference

$$
\Delta \dot{\eta}(\sigma)=H_{\eta}(\sigma) \hat{v}\left(\sigma, x^{\prime}+\frac{D}{2}-x_{s}\right)
$$


where $x_{s}$ is the horizontal distance from the top sensor to the upwave float. The filter is given by

$$
H_{\Delta \eta}(\sigma)=\frac{\left\{1-e^{-\imath k D}\right\}}{\sigma} e^{\imath k x_{s}+k \frac{\tau_{s}}{r_{f}}}
$$

where $r_{f}$ is the distance from the surface to the centroid of float depth. Because the distance $D$ is a significant fraction of the wavelength of some of the waves we are estimating, using the filter above should give far better results than estimating $\Delta \eta$ by using the surface slope at a single point. In a similar manner, we need to predict the downwave velocity at the float. Again, we rely on the linear theory and use a filtering approach

$$
\hat{v}_{d w}\left(\sigma, x^{\prime}+\frac{D}{2}\right)=H_{v}(\sigma) \hat{v}_{d w}\left(\sigma, x^{\prime}+\frac{D}{2}-x_{s}\right)
$$

with

$$
H_{v}(\sigma)=e^{i k x_{s}+k \frac{r_{s}}{r_{f}}}
$$

Because the float is part of the rigid SASS structure, the float motions can be easily predicted from our sensor motion measurements. With all the needed variables estimated at the float, the drag estimates are made, and the final estimate of the velocity defect is iteratively arrived upon (the final estimate of "float drag" also included the projected area of the trusswork immediately below the floats).

The results of the error analysis are presented in the next chapter along with all of the other data. The principal result of the "variable depth of immersion model" is that the velocity defect increases in calm conditions due to the correlation of the fluctuations of draft and velocities. In strongly forced conditions, the velocity deficit actually decreases. This is because the mean tilt of the buoy in such conditions pulls the upwave float out of the water and greatly reduces the mean draft of the buoy. Indeed, for some files it is predicted that the upwave float is completely in the air more than $30 \%$ of the time.

\section{speed of sound}

Previously, we talked about errors due to the variation of the speed of sound in water. The BASS makes a travel time measurement and as we discussed, the final estimate of velocity 
depends on the speed of sound squared. The speed of sound in water is a function of the temperature and the salinity. Clay and Medwin (1977) give the relation

$$
c=1449.2+4.6 T-0.055 T^{2}+0.00029 T^{3}+(1.34-0.010 T)(S-35)+0.016 z,
$$

where $T$ is the water temperature in Celsius, $S$ is the salinity in parts per thousand, and $z$ is the depth in $m$. The variations of temperature and salinity that the SASS encountered would not lead to variations in sound much greater than $\pm 2.5 \mathrm{~m} / \mathrm{s}$. In spite of the fact that the calibration of the BASS is proportional to the speed of sound squared, these variations in sound speed will only lead to deviations in BASS calibration of $0.33 \%$. Because the predicted errors were so small, the BASS calibration was not varied from file to file.

A potentially more serious error is the variation in the sound speed that may occur due to the presence of bubbles. The BASS transducers transmit at a frequency of $1.75 \mathrm{MHz}$. Under the conditions of deployment during SMILE, a $2 \mu \mathrm{m}$ bubble will be resonant at this frequency. Unfortunately, bubble populations are only estimated down to radii of $10 \mu \mathrm{m}$ (Medwin and Breitz, 1989). The accuracy of measurements of such small bubbles is still hotly disputed (Wu and Hwang, 1991). Still, experience with high frequency acoustic mea-

surements seems to indicate that at the frequency that the BASS transmits at, the errors due to bubbles will be minimal (Clay and Medwin, 1977). Trivett(1991) has developed a circuit to add to the BASS computer which measures the one way travel time (not just the travel time difference as the BASS has traditionally done) and therefore, the speed of sound in water. Making such a modification in future near-surface deployments of the BASS would not only provide useful information on the upper-ocean environment, but would also help assess the current meter's performance in measuring velocities.

\section{statistical errors}

When estimating the mean of a time varying signal, the variance of the estimate can be computed from the following expression:

$$
\operatorname{Var}(\bar{V})=\frac{1}{T} \int_{-T}^{T}\left(1-\frac{|\tau|}{T}\right) C_{V V}(\tau) d \tau
$$


where $C_{U U}$ is the auto-covariance of $V(t)$. Because the mean velocities we measured were small compared to the orbital velocities we found, it was necessary to average data over relatively long time periods so that the variance of our estimate would be much.smaller than the mean velocities we sought to measure.

Our final statistical error bounds are not only a function of the averaging time, but also the energetics of the velocities we measured. The $90 \%$ confidence intervals were computed for pods 3 and 6 and are interpolated and extrapolated to the other pods. Because the wave energy varies with depth, we notice that the statistical error bounds will decrease with depth. Where the files were short (less than 20 minutes long), the $90 \%$ interval at the top pod did reach $\pm 1 \mathrm{~cm} / \mathrm{s}$. The statistical errors tended to be much smaller than the more systematic errors that we've discussed throughout this section. 


\section{Chapter 6}

\section{RESULTS}

\subsection{The C3 mooring}

In the last chapter the sources of error for the SASS were extensively reviewed. Here I'll talk a little about the C3 mooring. This mooring was another of the components of the SMILE program. Intercomparisons of the SASS data with the C3 data will provide a check on the theories that have been discussed throughout the entire thesis.

The C3 mooring was a $3 m$ discuss buoy that was moored about $500 m$ from the SASS. Hence, the SASS and the C3 will nominally be making measurements of the same fluid. The C3 mooring was equipped with both atmospheric and oceanic sensors. On the atmospheric side were sensors for wind speed and direction, air temperature, long and short wave radiation and humidity. Vector measuring current meters (VMCMs; see Weller and Davis (1980) for a description of the VMCM) were attached along the length of the C3 mooring line. The VMCM's examined herein were distributed from depths of 4 to 47 meters. The Scripps Institute of Oceanography had VMCMs mounted (at depths greater than $30 \mathrm{~m}$ ) on the C3 mooring but that data is not included. The VMCMs provided fifteen minute averaged data over a period of several months. All but a few of the VMCM's provided complete coverage across both deployments of the SASS. The depths of the VMCM current meter data will be given with each file analyzed. 


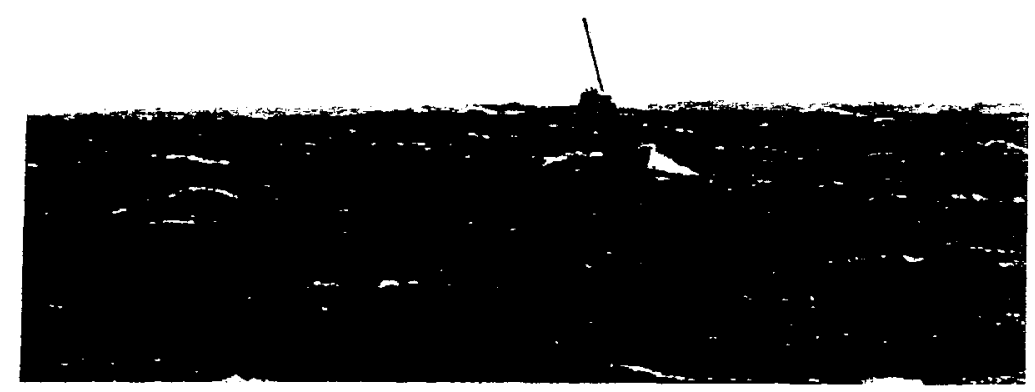

Figure 6-1: The C3 mooring in winds between 10 and $15 \mathrm{~m} / \mathrm{s}$

\section{Corrections to the $\mathrm{C3}$ measurements : the wave-bias revisited}

The VMCMs on the C3 mooring were equipped with compasses. No other motion measurements were made. Because the $\mathrm{C} 3$ buoy has a large surface expression we expect that it will follow the waves quite well. Watching the buoy on site seemed to confirm this notion. If a buoy is translating in a correlated way with the waves then we expect to measure a downwave bias. Previously, we assumed that measurements were made at a moving point but that they were made (by correcting for tilt) with a constant orientation. If measurements are not corrected for tilt, there can also be a bias arising from tilting motions correlated to the waves. This bias will also be estimated for the C3 mooring.

To estimate the biases we need to know the motions of the sensors. Because the motions were not measured we'll need to assume a transfer function. Fortunately, the C3 mooring design is dynamically simple enough so that we can probably do a good job bounding the errors with a fairly simple model. Figure 6-2 shows a schematic of the mooring design as well as the idealized model for the tipping of the buoy. The VMCM's are distributed in the upper $47 \mathrm{~m}$ of the mooring line. The mooring "line" in the top $70 \mathrm{~m}$ was $\frac{1}{2}$-inch chain. To keep the upper segment of the mooring taut, a 1500 pound depressor weight was inserted into the line. The weight, $20 \mathrm{~m}$ off the seafloor bottom, was attached to the bottom anchor via $50 \mathrm{~m}$ of $\frac{3}{4}$-inch chain. In the model shown below, the upper section of the mooring line 
is kept perfectly linear. The depressor weight maintains constant horizontal position due to the horizontal component of tension in the bottom segment of the mooring. The buoy is assumed to perfectly follow the orbital motions of the fluid at the surface.

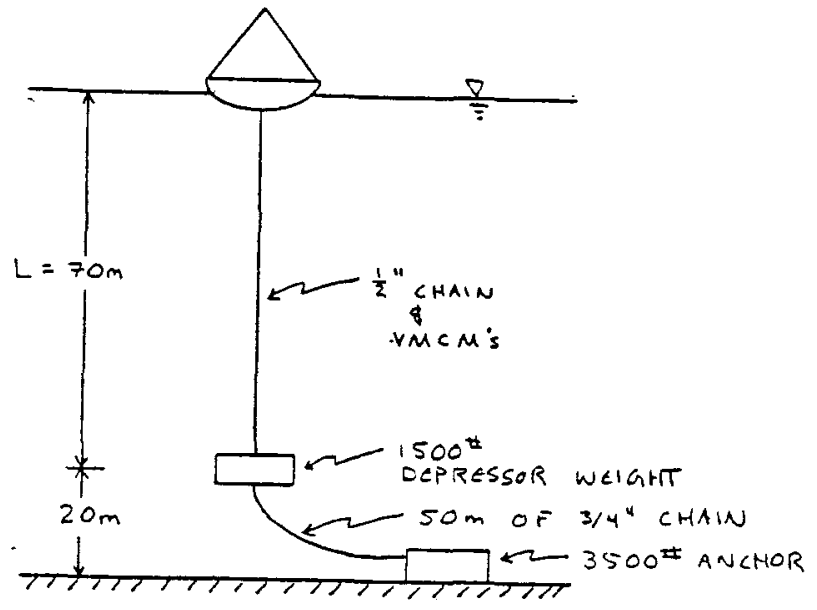

(a)

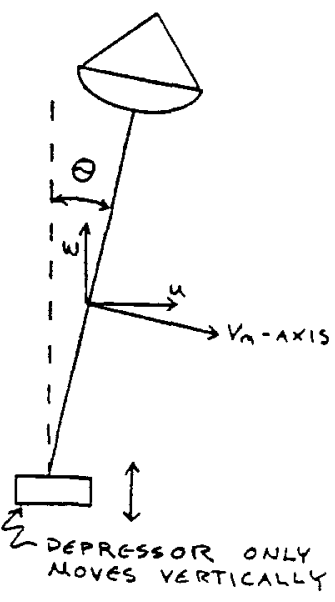

(b)

Figure 6-2: (a) A schematic of the C3 mooring (b) The segment of mooring where VMCMs are distributed is assumed to tilt rigidly.

The bias for the C3 mooring will now be estimated for a small amplitude monochromatic wave. We assume that the wave surface elevation $\eta$ and horizontal and vertical velocities $[u, w]$ are given by

$$
\begin{aligned}
\eta & =a \cos (k x-\sigma t), \\
u & =a \sigma \cos (k x-\sigma t) e^{k z} \\
w & =a \sigma \sin (k x-\sigma t) e^{k z}
\end{aligned}
$$

Without loss of generality we can take the mean horizontal position to be zero. If we do this, the first order description of motion for a sensor at depth $z=-z_{0}$ is

$$
[x, z]=\left[a \frac{L-z_{0}}{L} \sin \sigma t, a \cos \sigma t\right] \text {. }
$$

The measurement axis of the current meter is perpendicular to the mooring line so that the measured velocity $V_{m}$ is

$$
V_{m}=u \cos \theta-w \sin \theta \approx u-w \theta
$$


with the mooring line tilt $\theta$ being

$$
\theta \approx \frac{a \sin \sigma t}{L}
$$

If the expressions for horizontal and vertical velocity are inserted into equation 6.3 and the spatial dependences are expanded out, we arrive at the following expression for the measured velocity (where extensive use of the assumption that $a k \ll 1$ has been made)

$$
\begin{aligned}
V_{m} & \approx a \sigma \cos \sigma t e^{-k z_{o}}\{1+a k \cos \sigma t\} \\
& +a \sigma k \sin \sigma t e^{-k z_{\circ}}\left\{a \frac{L-z_{o}}{L} \sin \sigma t\right\} \\
& +a \sigma \sin \sigma t e^{-k z_{\circ}}\left\{a \frac{1}{L} \sin \sigma t\right\} .
\end{aligned}
$$

The first line of the expression is the first order wave velocity and the vertical motion modulation. The second line is the term which arises from coupling between the horizontal motion of the buoy and the horizontal gradient of the fluid velocity. The final line is the component of vertical fluid velocity which gets mixed into the measured velocity due to tipping. Taking the time average of the above expression leads to the upper bound for the bias

$$
\left.\bar{V}_{m}\right|_{\text {bound }} ^{\text {upper }} \approx \frac{1}{2} a^{2} \sigma k\left\{1+\frac{L-z_{0}}{L}\right\} e^{-k z_{\circ}}+\frac{1}{2} a^{2} \sigma \frac{1}{L} e^{-k z_{\circ}} .
$$

Its doubtful that the buoy perfectly follows the horizontal motions of the fluid. Therefore, as a lower bound for the C3 mooring bias we'll assume that the buoy's horizontal motions are totally uncorrelated with the fluid motion. Since a discus buoy can not be significantly decoupled from the longer waves we will still assume that the vertical motion of the buoy is perfectly coupled to the waves. Waves shorter than $10 \mathrm{~m}$ are sufficiently decayed at the depth of the top VMCM ( $4 m$ nominal depth) that their contribution to the bias is negligible independent of the extent to which the buoy's vertical motions are correlated to the surface displacement. The lower bound estimate of the bias is then

$$
\left.\bar{V}_{m}\right|_{\text {lower }} \approx \frac{1}{2} a^{2} \sigma k e^{-k z_{0}} .
$$

For the field data, the upper and lower bounds were calculated in a slightly more sophisticated fashion. The general assumptions were the same. The expressions for the bound were rederived for a directionally spread wave spectrum. These calculations are almost identical 
to those presented earlier and so are not repeated here. Because the buoy's diameter is $3 \mathrm{~m}$, I further assumed that there was no correlation of buoy motion with waves shorter than $6 \mathrm{~m}$.

\section{atmospheric data}

The stated purpose of the thesis was to examine the mean near-surface shear current. In this case we have to consider wind speed measurements as being something more than ancillary. The wind speed and direction data from the $7 \mathrm{~m}$ anemometer on the C3 buoy were used to predict the applied wind stress. Unfortunately, the air temperature sensors on the C3 buoy failed. The air-sea temperature difference at the $\mathrm{C} 3$ site was interpolated from the temperature differences at NDBC buoy's 13 and 14 (the buoys are located approximately 53 kilometers to the south and 143 kilometers to the north of the $\mathrm{C} 3$ site, respectively). The similarities between the temperature data at the two buoys for the times when SASS was sampling leads us to believe that, despite the large distance of separation between the NDBC buoys, they may have given a reasonably good indication of the atmospheric stability at the C3 site. The wind stress and $10 \mathrm{~m}$ wind speed were computed based on the $7 m$ wind speed measurements and air-sea temperature differences using a scheme presented by Large and Pond (1981). During the intervals when the SASS sampled, the atmospheric conditions tended to be neutrally stable or unstable. When the air friction velocity for each file was calculated just assuming neutral stability, the rms difference between the results and those obtained when considering the air-sea temperature differences was only $0.84 \%$ (the maximum difference of $2.7 \%$ was for files $p 3$ and $q 1$ ). Just the same, in the data presentation section, the friction velocities given do include the stability estimate arrived at from the temperature information.

\subsection{The data}

In all there were twenty-three recorded events where data was of analyzable quality. These events are labelled $a, b, c, \ldots, w$ according to the sequence in which they were recorded. The 
longer events were broken into shorter segments for analysis. I'll refer to the subdivided data sequences as files. If recording was of sufficient duration, it was broken into two or more files of forty minutes or longer. The shorter recordings were kept in tact as individual files. The file subdivision is a numerical label added onto the event label (e.g., the event $p$ is broken into files $p 1, p 2$ and $p 3)$. The total number of files is thirty-seven.

In this section, two pages will be dedicated to the presentation of each file. To make the presentation more efficient I will now explain all of the variables and nomenclature that will be used. The final results we seek are the information on the near-surface currents. This data would be of little use without knowing the wind and wave conditions. The furst page of data is entirely to background information of this type.

\section{first page : environmental parameters}

- date: Gives the month, day, and year on which the file was recorded.

- time: The start time of the processed file. Date and day are GMT. Time of day at the experiment site was GMT-8hrs.

- duration: The duration of the processed file.

- $U_{10}:$ The 10-meter wind speed.

- $\theta_{\tau}$ : The direction from which the wind was blowing.

- $\frac{z}{L}:$ The atmospheric stability parameter as defined in Large and Pond (19人1).

- $u_{*}$ : The wind friction velocity derived from the windspeed and the air-sea temperature difference as in Large and Pond (1981).

- $\bar{H}_{1 / 3}$ : The significant (or one-third highest) wave height. The spectrum of waveheight $S_{\eta \eta}(f)$ was predicted from the velocity at the uppermost BASS velocity sensor using a linear theory model for the waves. The significant waveheight is defined as

$$
\bar{H}_{1 / 3} \equiv 4\left\{\int_{0}^{\infty} S_{\eta \eta}(f) d f\right\}^{\frac{1}{2}}
$$


- $\theta_{w}$ : The direction the waves were coming from. This direction was defined as the direction of the computed Stokes' drift at the depth of the uppermost BASS sensor $(111 \mathrm{~cm})$.

- $T_{p}$ : The peak period of the waves. The peak frequency $f_{p}$ of the spectrum was found by maximizing $I$ below

$$
I=S_{\eta \eta}(f) w\left(f-f_{p}\right),
$$

where $w$ is a triangular window with a width of $0.078 \mathrm{~Hz}$. The resolution for this calculation was $0.016 \mathrm{~Hz}$. The peak period is the inverse of peak frequency.

- $\bar{T}:$ The average period of the waves. The average frequency of the waves (see Dewman, 1977) can be defined as

$$
\bar{f} \equiv\left\{\frac{\int_{0}^{\infty} f^{2} S_{\eta \eta}(f) d f}{\int_{0}^{\infty} S_{\eta \eta}(f) d f}\right\}^{\frac{1}{2}}
$$

The average period is the inverse of the average frequency.

- $\Delta_{\text {temp }}$ : The calibration mismatch between the SASS thermistors and the C3 thermistor data. The metal clad thermistors on the SASS should yield good temperature differences between one another. Their absolute calibration is not too accurate. The temperature difference between the lowest metal-clad thermistor (nominal depth of $585 \mathrm{~cm}$ ) and uppermost $\mathrm{C} 3$ thermistor (nominal depth of $700 \mathrm{~cm}$ ), $\Delta_{t e m p}$, is considered a calibration offset in the metal-clad thermistors.

There are also three plots on the first page.

- The directional wave spectrum. The directional wave spectrum of waves was computed as outlined earlier in the thesis. It is plotted out in contour plot form with a relative scale. The contours are levels

$$
\left[\begin{array}{lllll}
1 & 3 & 5 & 10 & 20
\end{array}\right]
$$

$d B$ down from the peak frequency.

- The waveheight spectrum. The waveheight spectrum $S_{\eta \eta}(f)$ is plotted out on a full logarithmic scale to allow comparison of the high frequency fall off with a $f^{-4}$ theoretical dependence (also plotted). 
- Stratification. The temperature and salinity data are plotted together. The offset, $\Delta_{t e m p}$, has been subtracted out of the BASS thermistor data.

\section{second page : velocities}

The second page of data is devoted to the current meter data. When the data from the SASS and C3 were originally plotted out against each other, uncorrected for bias and in NorthSouth/East-West coordinates, there was extremely poor agreement between the two sets of measurements. Plotting the data in the downwind/crosswind direction did not resolve the apparent miscalibration. When the data was plotted in the downwave/crosswave direction it was found that the agreement between measurements in the crosswave direction was very good, and the agreement between measurements in the downwave direction was very poor (the sign convention for these axes are given in Figure 6-3). The bias correction brought the two measurements into only slightly better agreement. There remained some very peculiar looking profiles measured by the SASS. It was conjectured that there was some error in the SASS measurements. The last section of chapter 5 dealt with estimates of these errors. If error estimates for the downwave component of the SASS measurements are added in, the agreement between the SASS and the C3 measurements seems to improve considerably (for most files). Because the corrections will be so important to our final conclusions, both the corrected and uncorrected data will be plotted. The different contributions of the corrections will be presented in tabular form.

The current meter data are presented for each instrument in order of increasing depth. The SASS data are presented in the top portion of the table and the C3 data in the böttom portion of the table. From left to right, the columns in the SASS portion of the table are:

- $z$ : Mean sensor depth in meters.

- $\bar{v}_{d w}$ : The raw, time averaged velocity in the downwave direction.

- $\Delta_{b i a s}$ : The correction added to $\bar{v}_{d w}$ to compensate for the correlation of the sensor motions with the wave orbital velocities.

- $\triangle_{B A S S}:$ The correction added to $\bar{v}_{d w}$ to compensate for flow disturbance errors from the BASS transducer support structure. 


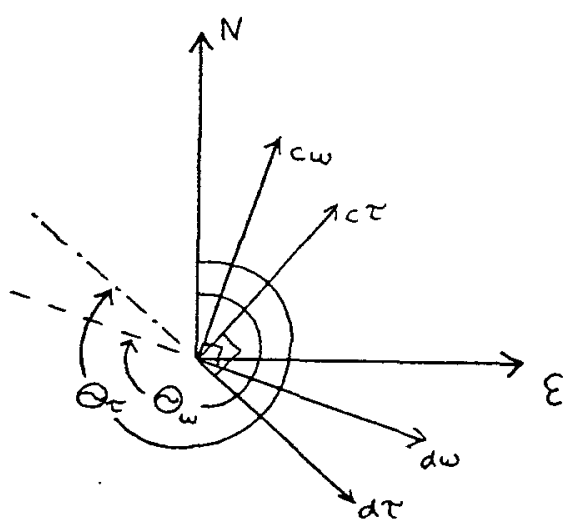

Figure 6-3: Sign convention for the different sets of axes. N-E are true North and true East. The label $d w$ and $c w$ refer to downwave and crosswave directions, while $d \tau$ and $c \tau$ are downwind and crosswind directions.

- $\Delta_{\text {float }}:$ The correction added to $\bar{v}_{d w}$ to compensate for flow disturbance from the flotation elements of the SASS.

- $\widehat{v}_{d w}$ : The estimate of the unbiased velocity in the downwave direction $\left(\widehat{v}_{d w}=\bar{v}_{d w}+\right.$ $\left.\Delta_{\text {bias }}+\Delta_{B A S S}+\Delta_{\text {float }}\right)$.

- $\bar{v}_{c w}$ : The time averaged velocity in the crosswave direction.

- $\widehat{v}_{c w}$ : The estimate of the unbiased velocity in the crosswave direction $\left(\widehat{v}_{d w}=\bar{v}_{c w}+\right.$ $\left.\triangle_{B A S S}\right)$

In the $\mathrm{C} 3$ portion of the table:

- $z$ : Nominal sensor depth in meters.

- $\bar{v}_{d w}$ : The raw, time averaged velocity in the downwave direction.

- $\Delta_{\text {bias }}^{\max }$ : The correction added to $\bar{v}_{d w}$ to compensate for the correlation of the sensormotions with the wave orbital velocities. Assumes that the C3 buoy perfectly follows the waves. 
- $\Delta_{b i a s}^{\min }:$ The correction added to $\bar{v}_{d w}$ to compensate for the correlation of the sensor motions with the wave orbital velocities. Assumes that the C3 buoy only follows the waves in vertical excursion.

- $\widehat{v}_{d w}^{\min }$ : The lower bound estimate of the unbiased velocity in the downwave direction $\left(\widehat{v}_{d w}=\bar{v}_{d w}+\Delta_{b i a s}^{\max }\right)$.

- $\widetilde{v}_{d w}^{m a x}$ : The upper bound estimate of the unbiased velocity in the downwave direction $\left(\widehat{v}_{d w}=\bar{v}_{d w}+\Delta_{b i a s}^{\min }\right)$.

- $\bar{v}_{d w}$ : The time averaged velocity in the crosswave direction.

There are two plots on the second page.

- The downwave velocity. Plotted here are the raw time averaged velocities (as points) as well as the corrected velocity profiles (in lines). For comparison, the predicted log-law shear in the downwave direction is plotted at a depth of 3 to 10 meters. The line plotted,

$$
-\frac{u_{*}}{\kappa} \log (z) \cos \left(\theta_{\tau}-\theta_{w}\right)
$$

has the slope of the predicted component of shear flow and is only for comparison of predicted slope. The curve is arbitrarily offset.

- The crosswave velocity. The time averaged component of the crosswave velocities are plotted. The predicted log-law shear in the crosswave direction is plotted. The line plotted,

$$
\frac{u_{*}}{\kappa} \log (z) \sin \left(\theta_{\tau}-\theta_{w}\right)
$$

has the slope of the predicted component of shear flow. As with the downwave component, the curve is arbitrarily offset. 


\section{file a1}

date $=$ November $28,1988 \quad$ time $=08-21-04.25 \quad$ duration $=00-15-16.75$

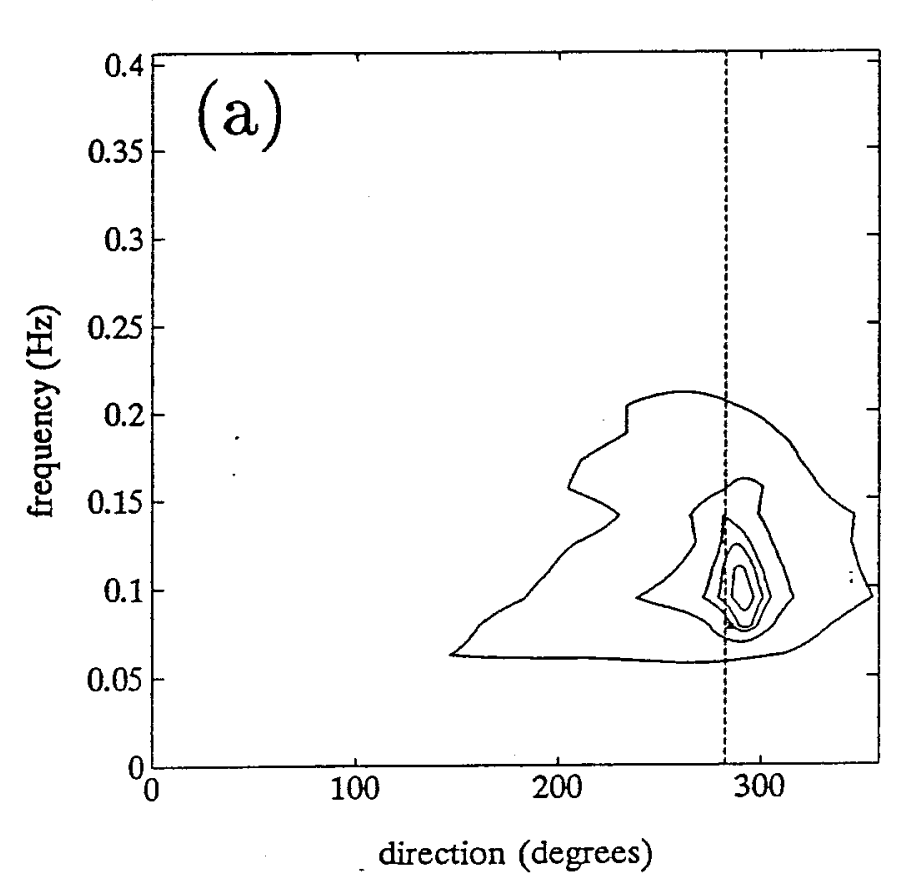

$$
\begin{aligned}
& \underline{\text { wind }} \quad \underline{\text { waves }} \\
& U_{10}=2.6 \mathrm{~m} / \mathrm{s} \quad \bar{H}_{1 / 3}=1.6 \mathrm{~m} \\
& \theta_{\tau}=283^{\circ} \quad \theta_{w}=279^{\circ} \\
& \frac{z}{L}=0.02 \quad T_{p}=10.7 \mathrm{sec} \\
& u_{* a}=8.2 \mathrm{~cm} / \mathrm{s} \quad \bar{T}=7.8 \mathrm{sec} \\
& \Delta_{\text {temp }}=-0.48 C
\end{aligned}
$$

(a) directional spectrum

(b) energy spectrum $\left(\cdots=f^{-4}\right)$

(c) $o=$ salinity, $x,+=$ temperature
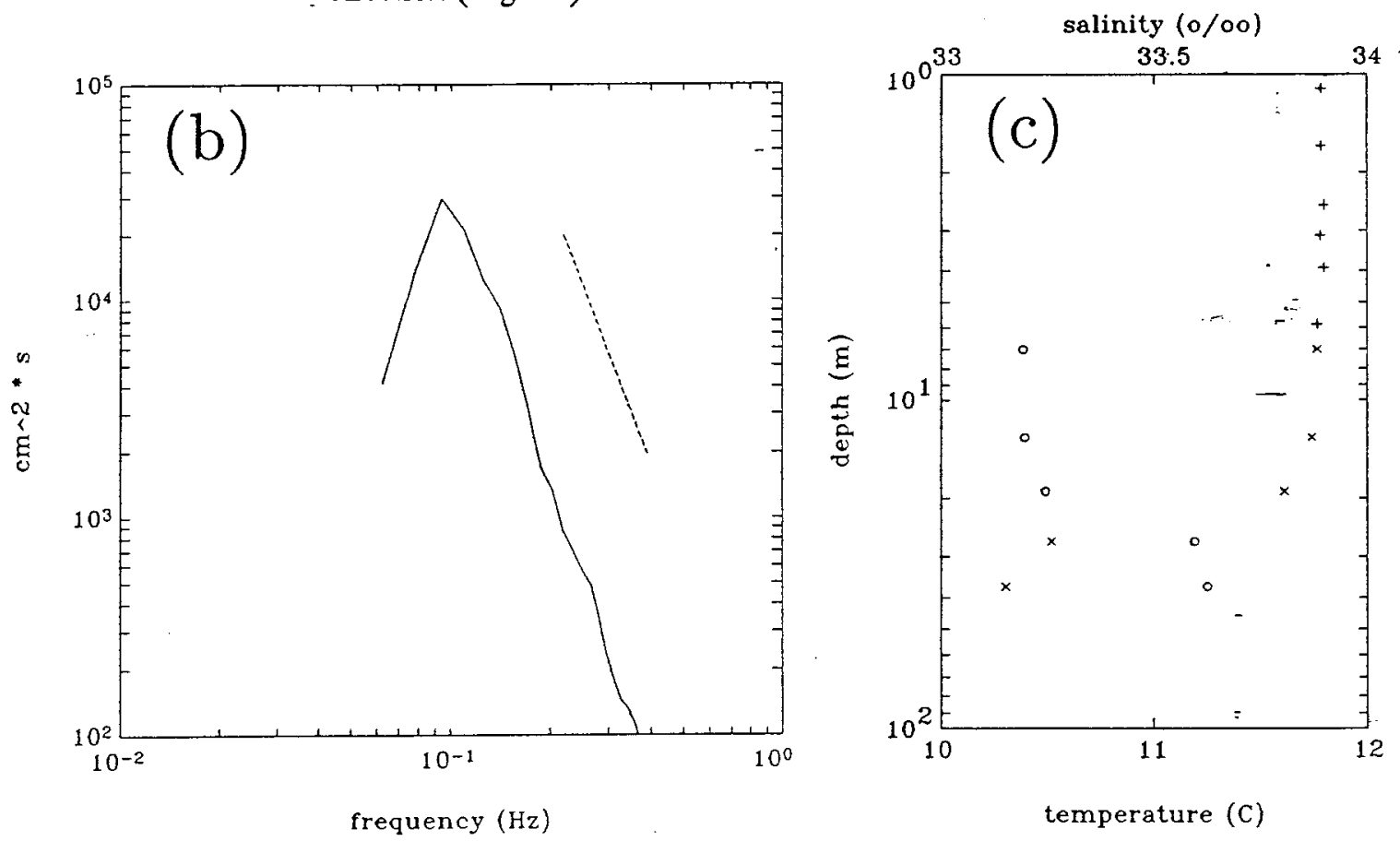


\section{file a1}

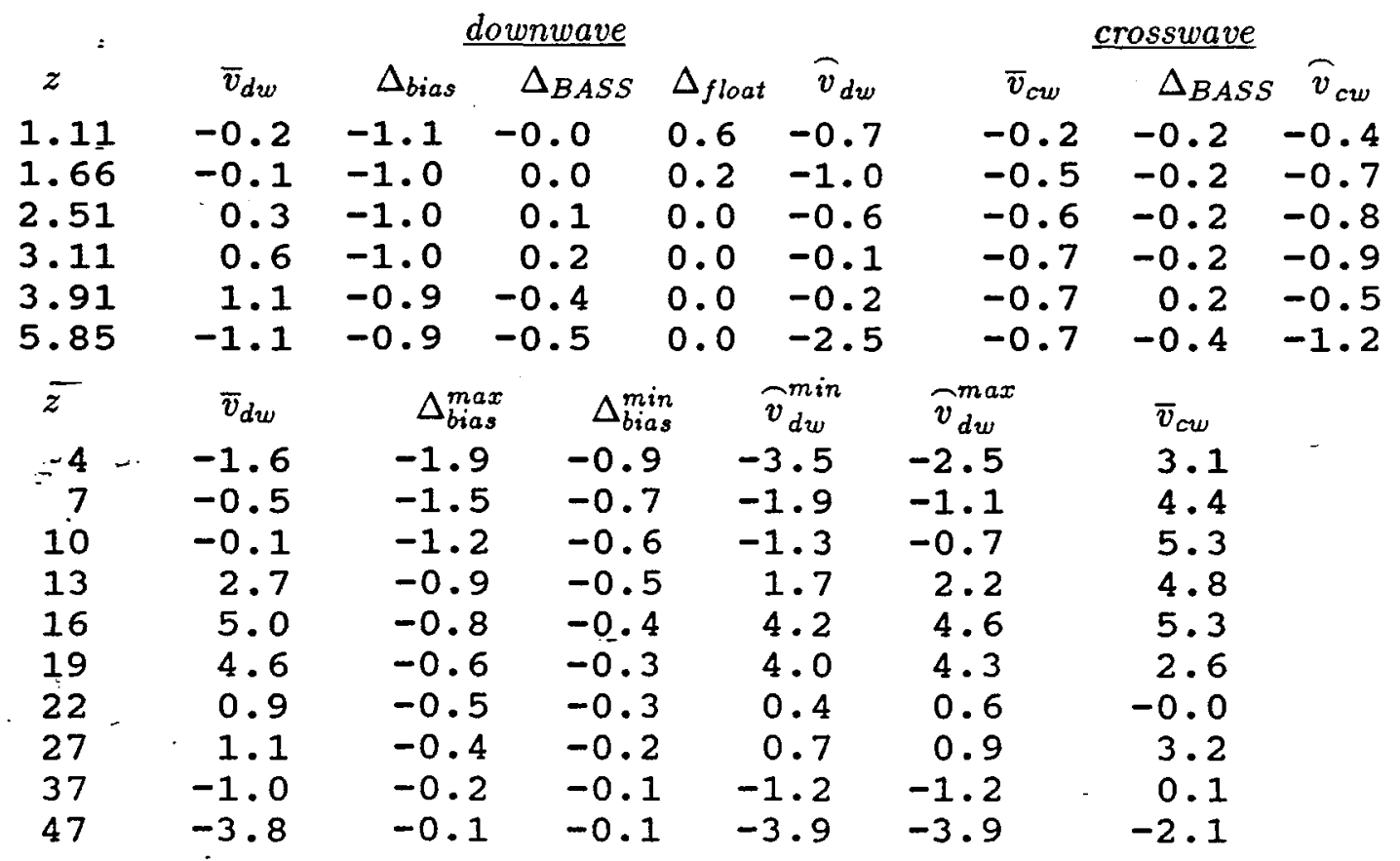

Below: SASS $=x($ raw $) \cdots($ unbiased $), \mathrm{C} 3=0($ raw $)-\cdots($ unbiased $)$, theoretical log-law shear $=-$
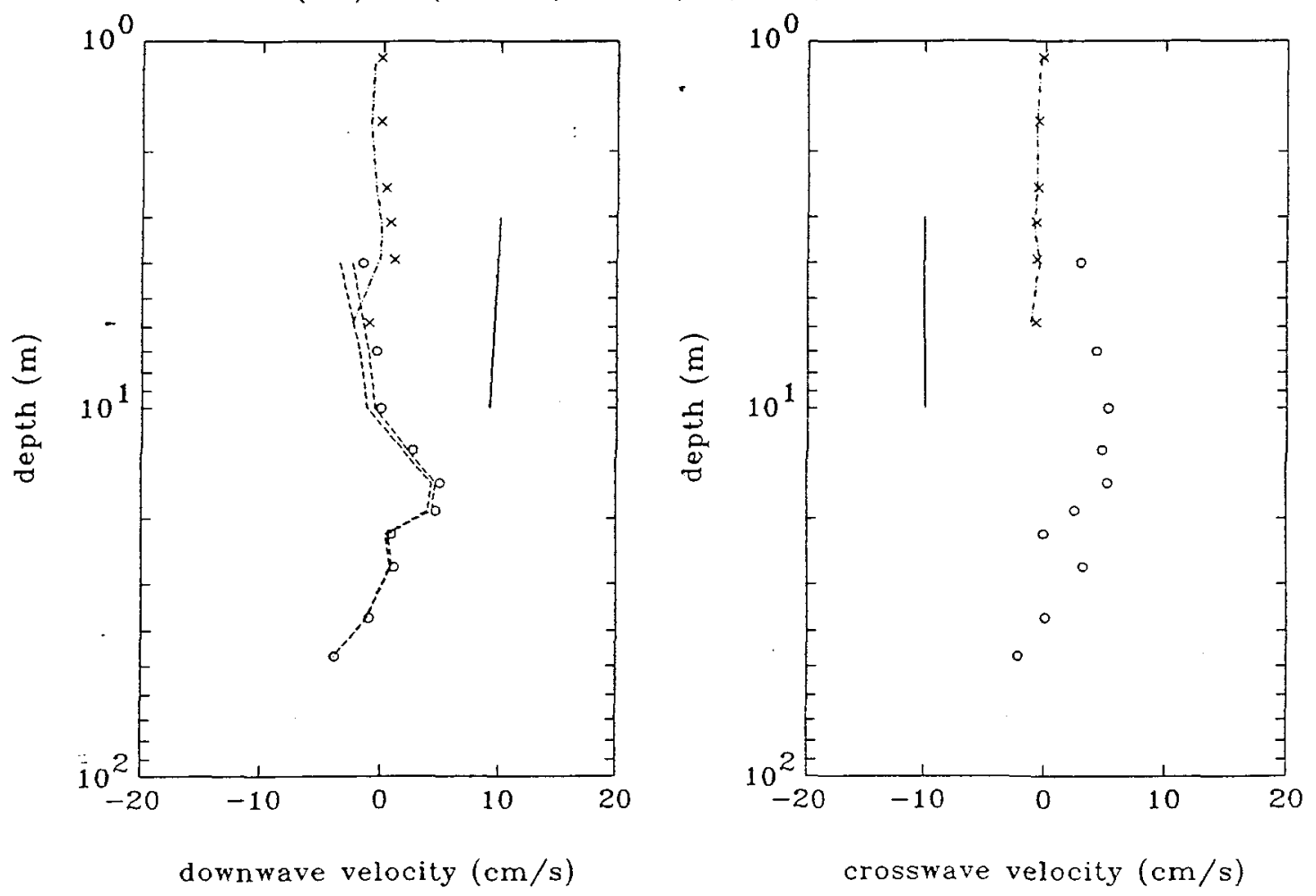


\section{file b1}

date $=$ November $28,1988 \quad$ time $=10-04-32.00 \quad$ duration $=00-38-40.75$

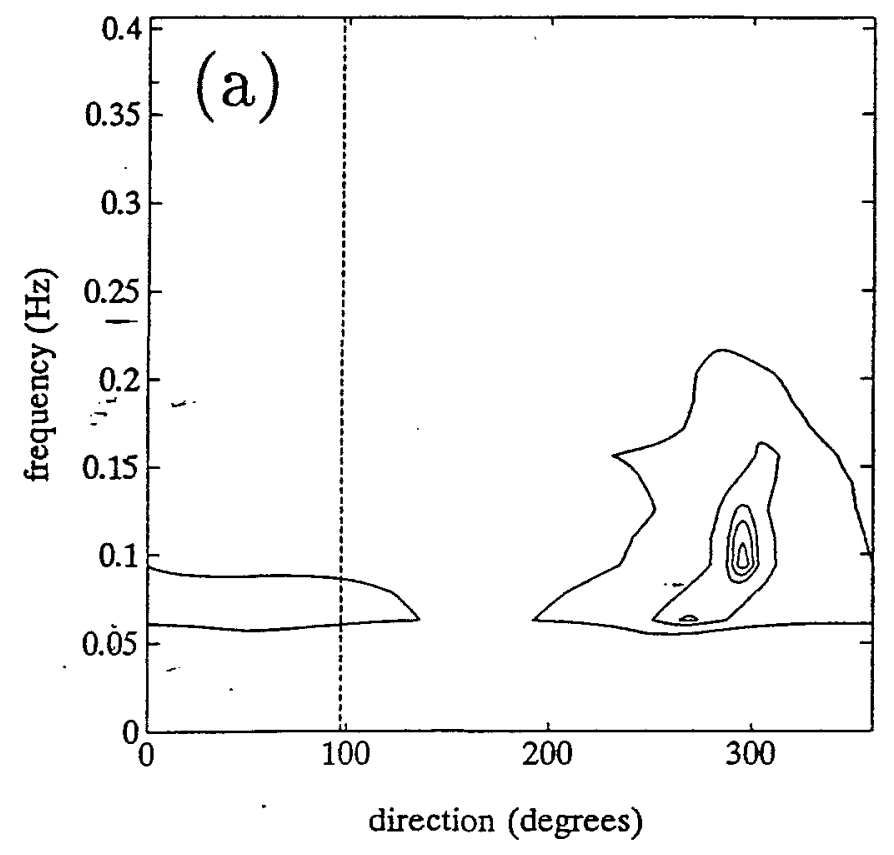

$$
\begin{array}{ll}
\text { wind } & \multicolumn{1}{c}{\text { waves }} \\
U_{10}=2.0 \mathrm{~m} / \mathrm{s} & \bar{H}_{1 / 3}=1.7 \mathrm{~m} \\
\theta_{\tau}=97^{\circ} & \theta_{w}=293^{\circ} \\
\frac{z}{L}=0.04 & T_{p}=10.7 \mathrm{sec} \\
u_{* a}=6.1 \mathrm{~cm} / \mathrm{s} & \bar{T}=8.2 \mathrm{sec} \\
\multicolumn{2}{c}{\Delta_{\text {temp }}=-0.44 \mathrm{C}}
\end{array}
$$

(a) directional spectrum $\left(--=\theta_{\tau}\right)$

(b) energy spectrum $\left(-\cdots=f^{-4}\right)$

(c) $o=$ salinity, $x,+=$ temperature
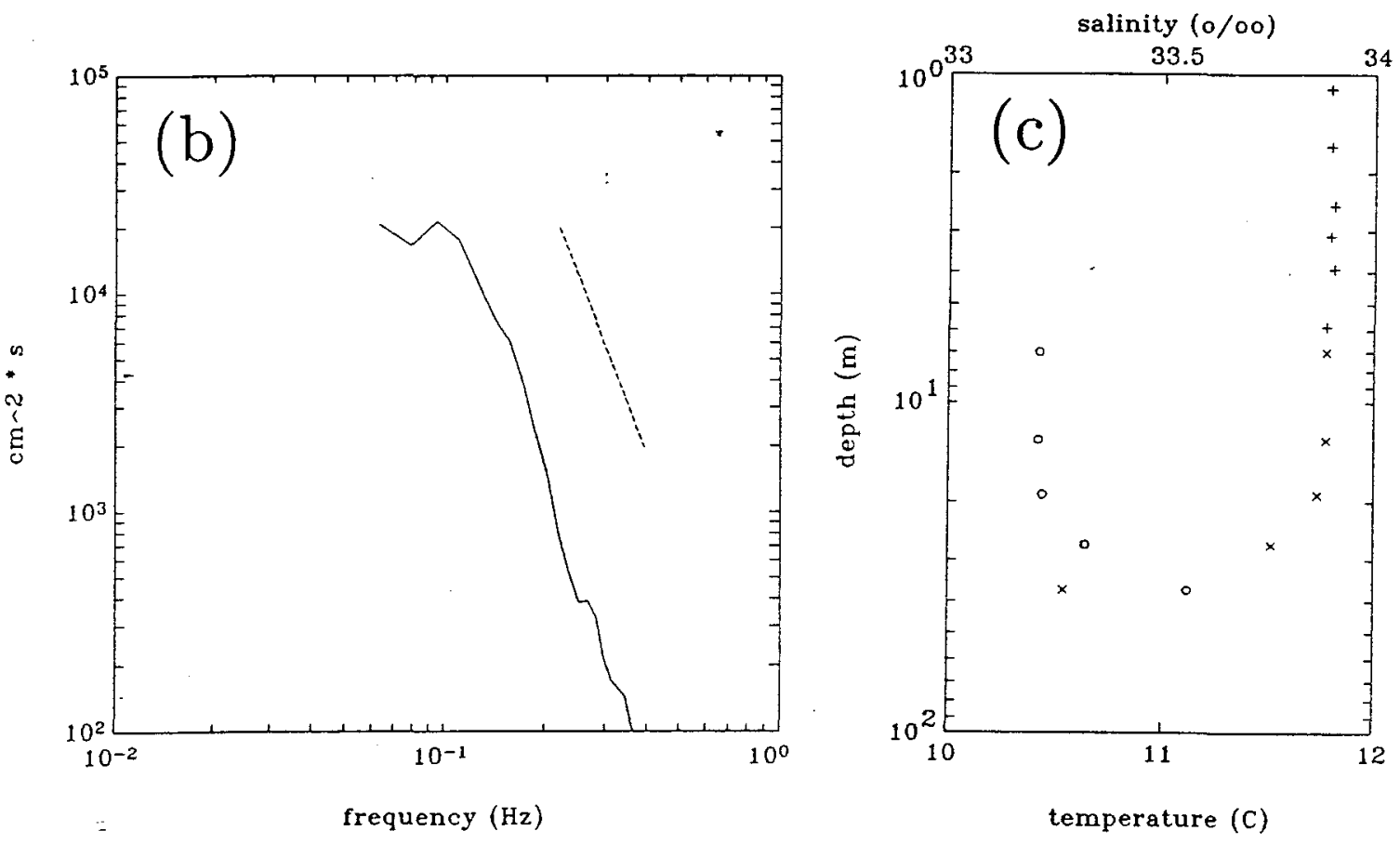


\section{file b1}

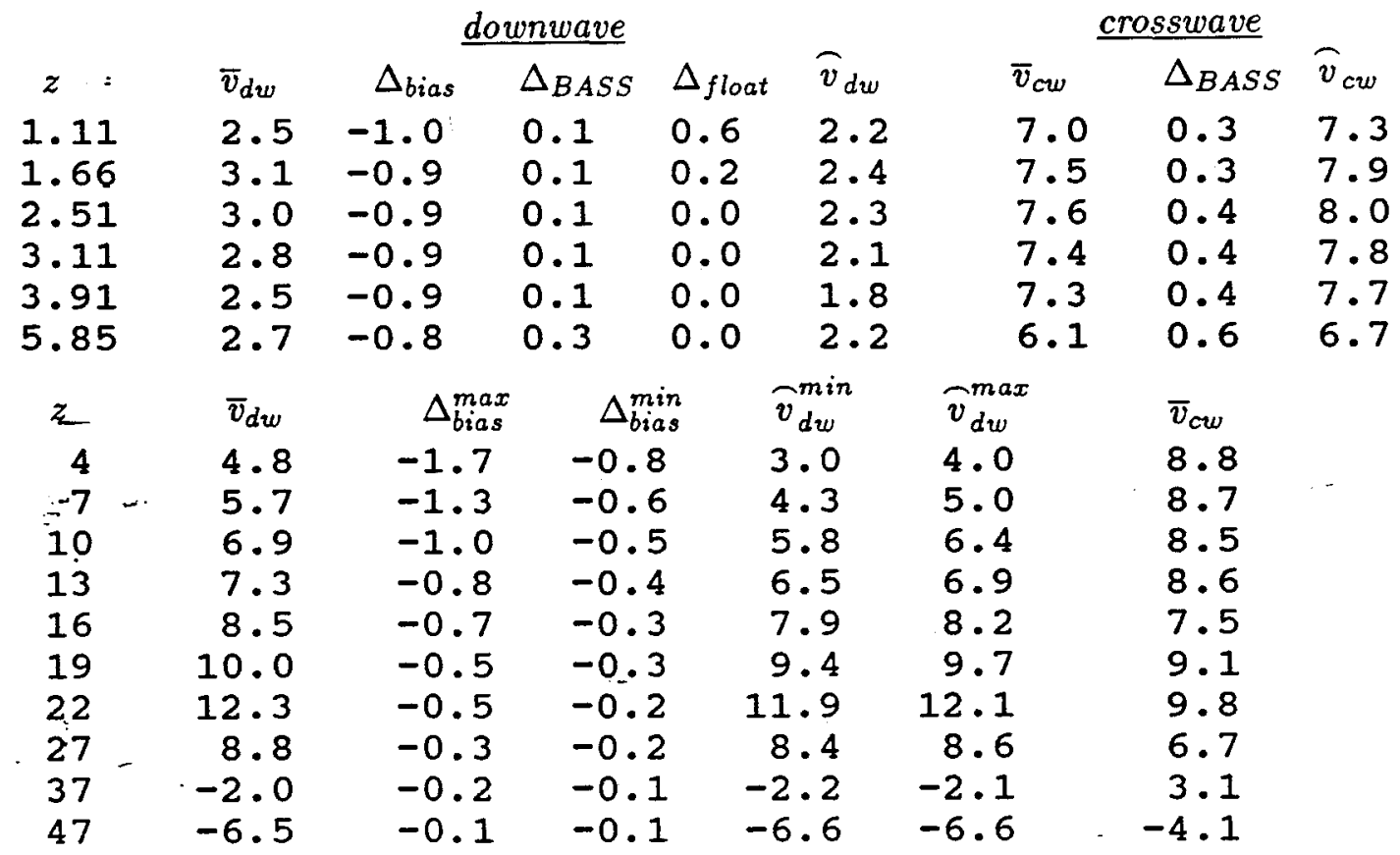

Below: SASS $=x($ raw $) \cdots($ unbiased $), \mathrm{C} 3=o($ raw $)--($ unbiased $)$, theoretical log-law shear $=-$
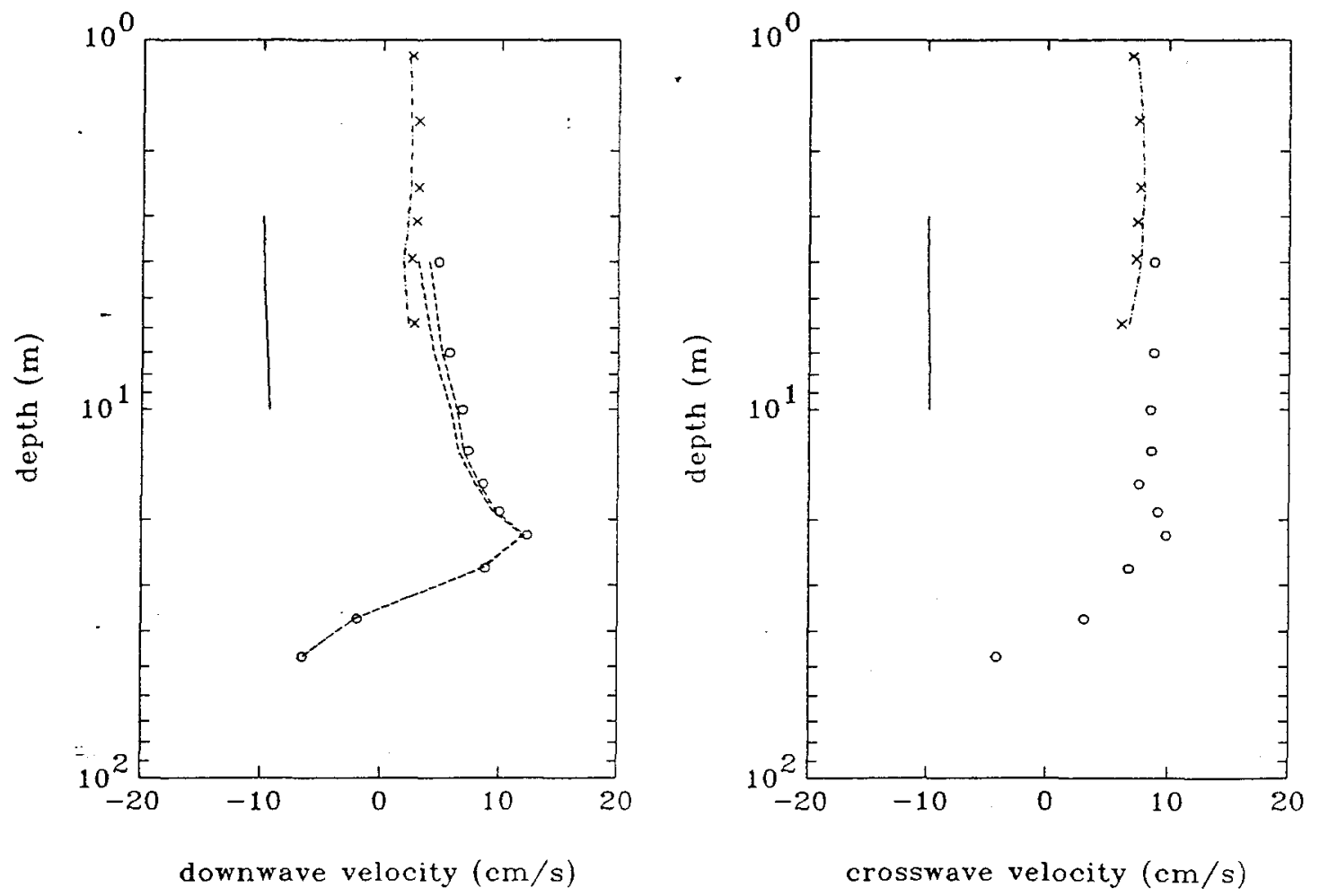


\section{file $\mathrm{c} 1$}

date $=$ November $28,1988 \quad$ time $=14-38-31.00 \quad$ duration $=00-21-24.75$

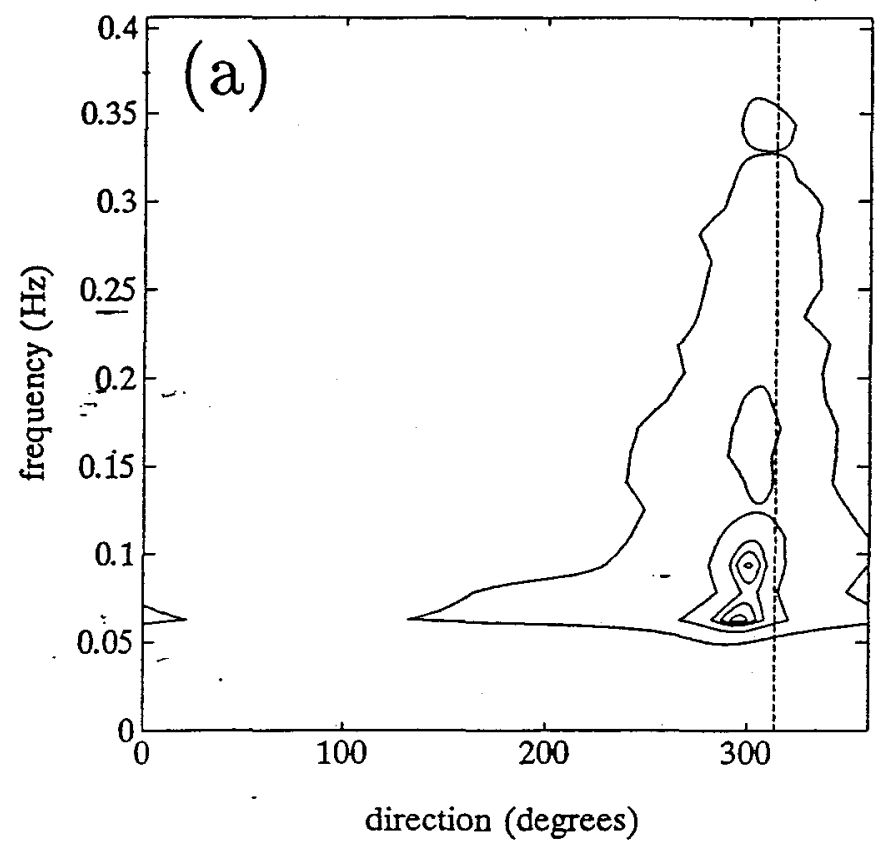

$$
\begin{array}{cc}
\text { wind } & \multicolumn{1}{c}{\text { waves }} \\
U_{10}=8.1 \mathrm{~m} / \mathrm{s} & \bar{H}_{1 / 3}=2.0 \mathrm{~m} \\
\theta_{\tau}=313^{\circ} & \theta_{w}=304^{\circ} \\
\frac{z}{L}=-0.02 & T_{p}=12.8 \mathrm{sec} \\
u_{*_{a}}=28.3 \mathrm{~cm} / \mathrm{s} & \bar{T}=6.1 \mathrm{sec} \\
\multicolumn{2}{c}{\Delta_{\text {temp }}=-0.38 \mathrm{C}}
\end{array}
$$

(a) directional spectrum $\left(---=\theta_{\tau}\right)$

(b) energy spectrum $\left(--=f^{-4}\right)$

(c) $\mathrm{o}=$ salinity, $\mathrm{x}, \mathrm{t}=$ temperature
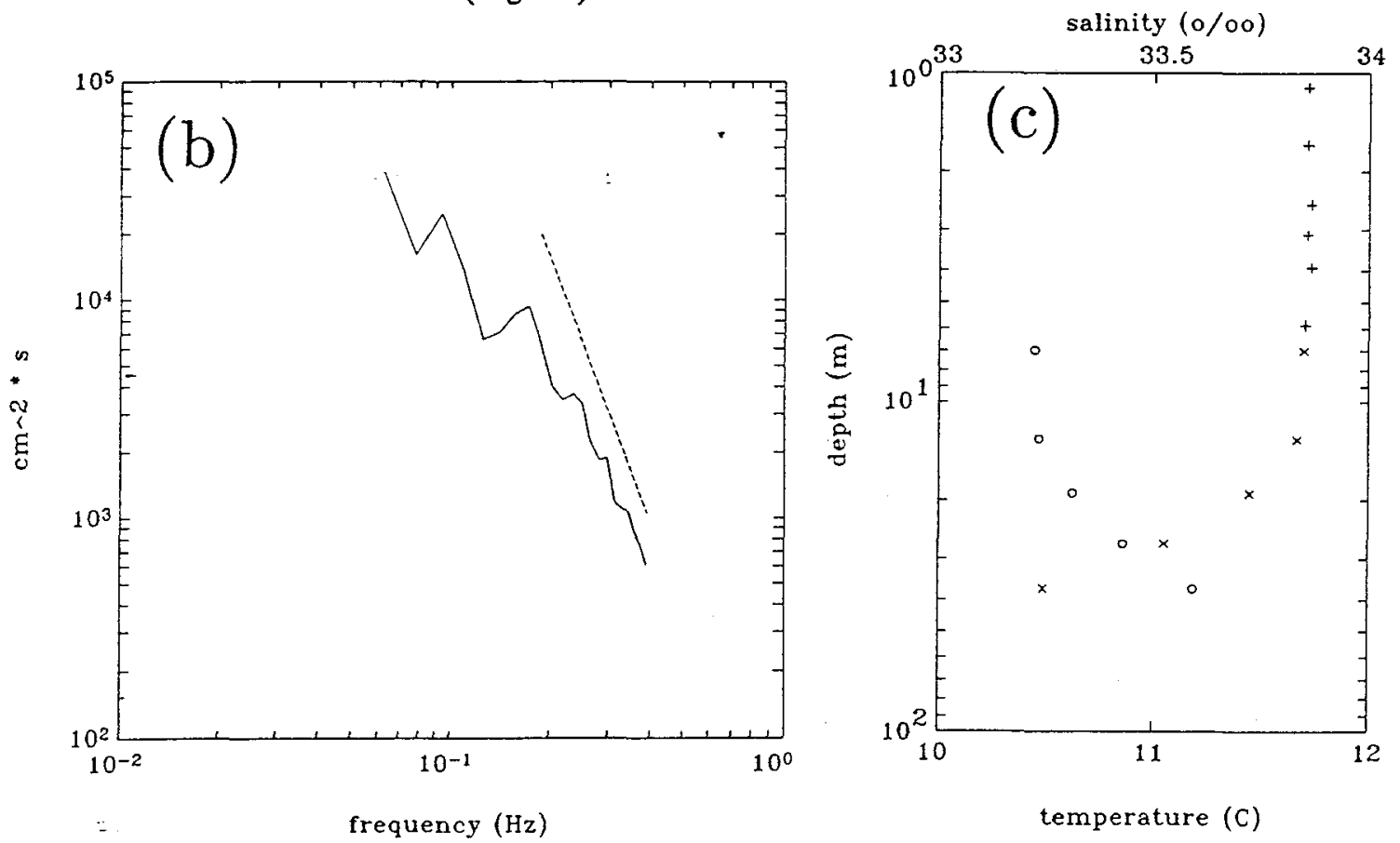


\section{file $\mathrm{c} 1$}

\begin{tabular}{|c|c|c|c|c|c|c|c|c|}
\hline \multirow[b]{2}{*}{$z=$} & \multicolumn{5}{|c|}{ downwave } & \multicolumn{3}{|c|}{ crosswave } \\
\hline & $\bar{v}_{d w}$ & $\Delta_{\text {bias }}$ & $\Delta_{B A S S}$ & $\Delta_{\text {float }}$ & $\widehat{v}_{d w}$ & $\bar{v}_{c w}$ & $\Delta_{B A S S}$ & $\widehat{v}_{c w}$ \\
\hline $\begin{array}{l}1.11 \\
1.66 \\
2.51 \\
3.11 \\
3.91 \\
5.85\end{array}$ & $\begin{array}{r}10.7 \\
13.4 \\
13.7 \\
14.2 \\
12.5 \\
8.0\end{array}$ & $\begin{array}{l}-3.3 \\
-3.0 \\
-2.5 \\
-2.4 \\
-2.2 \\
-1.7\end{array}$ & $\begin{array}{r}-0.7 \\
0.7 \\
0.8 \\
0.9 \\
1.0 \\
1.3\end{array}$ & $\begin{array}{l}1.4 \\
0.4 \\
0.0 \\
0.0 \\
0.0 \\
0.0\end{array}$ & $\begin{array}{r}8.1 \\
11.5 \\
11.9 \\
12.7 \\
11.3 \\
7.6\end{array}$ & $\begin{array}{l}-1.4 \\
-1.8 \\
-2.4 \\
-1.8 \\
-0.7 \\
-2.8\end{array}$ & $\begin{array}{r}0.1 \\
-0.1 \\
-0.1 \\
-0.1 \\
-0.1 \\
-0.5\end{array}$ & $\begin{array}{l}-1.3 \\
-1.9 \\
-2.5 \\
-2.0 \\
-0.8 \\
-3.3\end{array}$ \\
\hline$z$ & $\bar{v}_{d w}$ & $\Delta_{\text {bias }}^{\max }$ & $\Delta_{b i}^{m}$ & & $\widehat{v}_{d w}^{\min }$ & $\overbrace{v_{d w}^{\max }}^{\max }$ & $\bar{v}_{c w}$ & \\
\hline 4 & 14.8 & $-4 \cdot 1$ & -2. & & 0.7 & 12.8 & -2.7 & \\
\hline $\begin{array}{r}-7 \\
-10\end{array}$ & $\begin{array}{l}13.3 \\
11.6\end{array}$ & $\begin{array}{l}-2.5 \\
-1.7\end{array}$ & $\begin{array}{l}-1.2 \\
-0.8\end{array}$ & & $\begin{array}{l}0.8 \\
0.0\end{array}$ & $\begin{array}{l}12.1 \\
10.8\end{array}$ & $\begin{array}{l}-1.2 \\
-0.3\end{array}$ & - \\
\hline 13 & 10.1 & $-1 \cdot 2$ & -0.6 & & 8.9 & 9.5 & -0.2 & \\
\hline 16 & 9.8 & -0.9 & -0.5 & & 8.9 & 9.4 & -2.6 & \\
\hline 19 & 9.9 & -0.7 & -0.2 & & 9.2 & 9.5 & -5.0 & \\
\hline 22 & 5.7 & -0.6 & -0.3 & & 5.1 & 5.4 & -5.0 & \\
\hline 27 & -1.2 & -0.4 & -0.2 & & 1.6 & -1.5 & $-4 \cdot 3$ & \\
\hline 37 & -5.1 & -0.2 & -0.1 & & 5.3 & -5.2 & 4.1 & \\
\hline 47 & -6.4 & -0.1 & -0.1 & & 6.6 & -6.5 & 1.0 & \\
\hline
\end{tabular}

Below: SASS $=x$ (raw) $\cdots($ unbiased $), C 3=o($ raw $) \cdots($ unbiased $)$, theoretical log-law shear $=-$
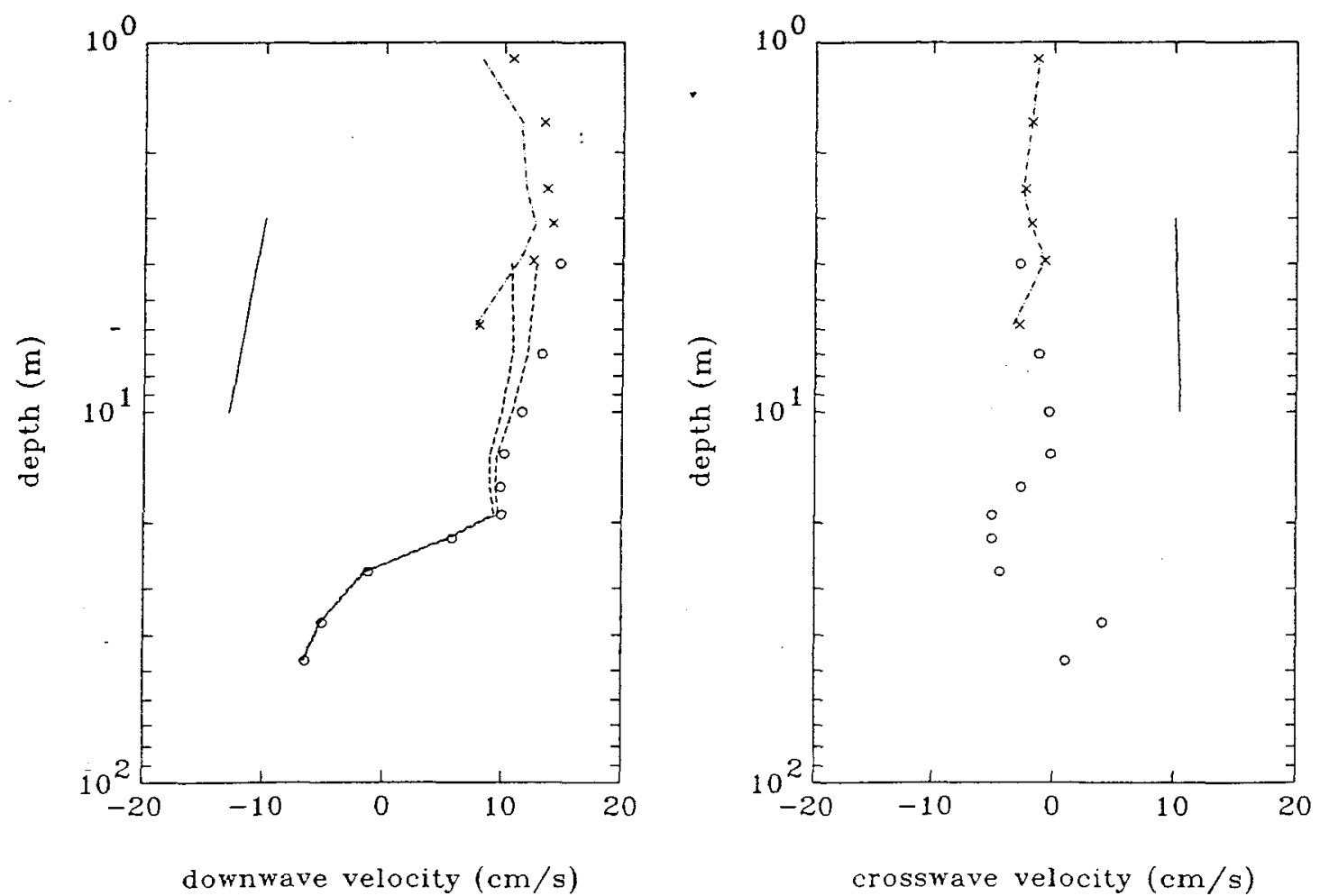


\section{file $\mathrm{d} 1$}

date $=$ November $28,1988 \quad$ time $=15-09-12.50 \quad$ duration $=00-51-14.25$

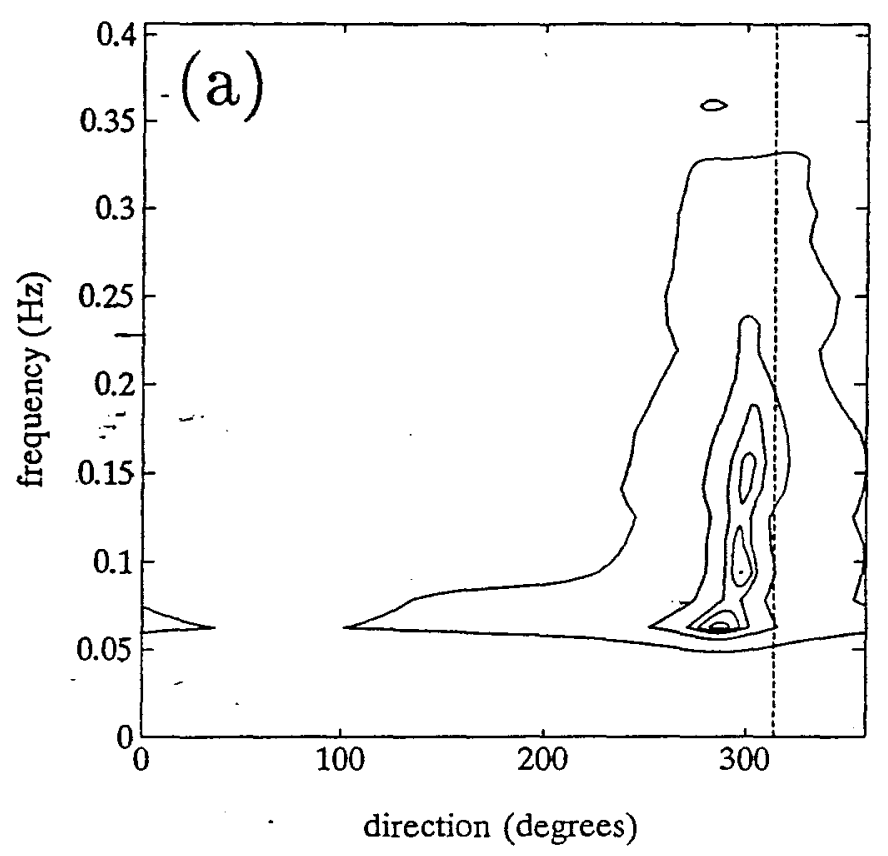

$$
\begin{array}{cc}
\underline{\text { wind }} & \multicolumn{1}{c}{\text { waves }} \\
U_{10}=8.6 \mathrm{~m} / \mathrm{s} & \bar{H}_{1 / 3}=2.2 \mathrm{~m} \\
\theta_{\tau}=314^{\circ} & \theta_{w}=300^{\circ} \\
\bar{z}=-0.02 & T_{p}=12.8 \mathrm{sec} \\
u_{*_{a}}=29.9 \mathrm{~cm} / \mathrm{s} & \bar{T}=5.9 \mathrm{sec} \\
\multicolumn{2}{c}{\Delta_{\text {temp }}=-0.41 \mathrm{C}}
\end{array}
$$

(a) directional spectrum $\left(--=\theta_{\tau}\right)$

(b) energy spectrum $\left(\cdots=f^{-4}\right)$

(c) $\mathrm{o}=$ salinity, $\mathrm{x},+=$ temperature
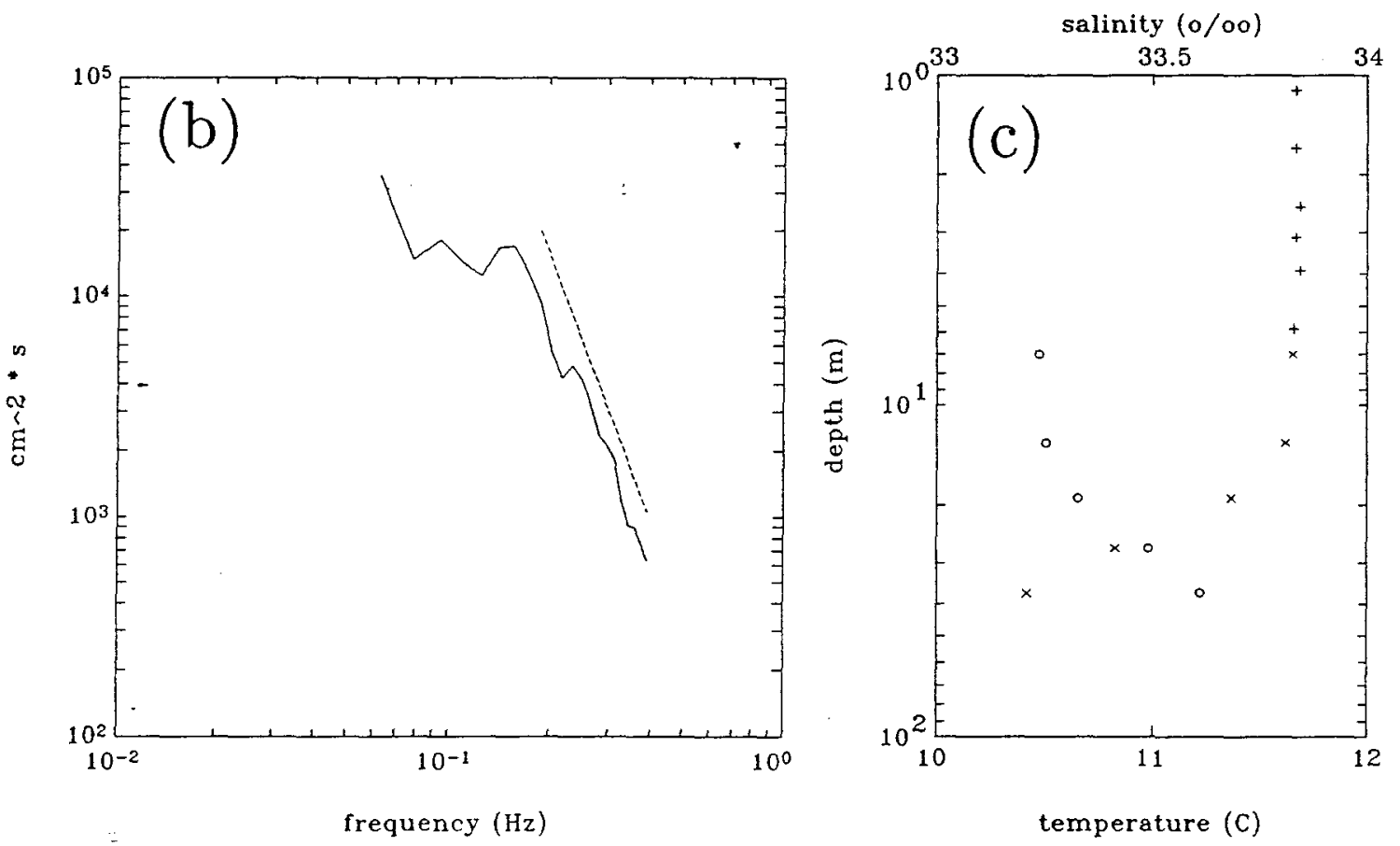


\section{file $\mathrm{d} 1$}

\begin{tabular}{|c|c|c|c|c|c|c|c|c|}
\hline \multirow[b]{3}{*}{$\begin{array}{c}z \\
1.11 \\
1.66 \\
2.51 \\
3.11 \\
3.91 \\
5.85\end{array}$} & \multicolumn{5}{|c|}{ downwave } & \multicolumn{3}{|c|}{ crosswave } \\
\hline & $\bar{v}_{d w}$ & $\Delta_{\text {bias }}$ & $\Delta_{B A S S}$ & $\Delta_{\text {float }}$ & $\widehat{v}_{d w}$ & $\bar{v}_{c w}$ & $\Delta_{B A S S}$ & $\widehat{v}_{c w}$ \\
\hline & $\begin{array}{r}9.6 \\
11.9 \\
11.8 \\
12.2 \\
10.6 \\
4.6\end{array}$ & $\begin{array}{l}-4.3 \\
-3.9 \\
-3.4 \\
-3.2 \\
-3.0 \\
-2.3\end{array}$ & $\begin{array}{r}-0.7 \\
0.7 \\
0.7 \\
0.8 \\
0.9 \\
-0.6\end{array}$ & $\begin{array}{l}1.5 \\
0.4 \\
0.0 \\
0.0 \\
0.0 \\
0.0\end{array}$ & $\begin{array}{l}6.0 \\
9.0 \\
9.1 \\
9.8 \\
8.5 \\
1.6\end{array}$ & $\begin{array}{l}-4.9 \\
-5.7 \\
-6.1 \\
-5.9 \\
-4.9 \\
-6.5\end{array}$ & $\begin{array}{r}0.3 \\
-0.3 \\
-0.3 \\
-0.4 \\
-0.4 \\
0.9\end{array}$ & $\begin{array}{l}-4.5 \\
-6.1 \\
-6.4 \\
-6.3 \\
-5.3 \\
-5.6\end{array}$ \\
\hline$-z$ & $\bar{v}_{d w}$ & $\Delta_{b i a}^{m o}$ & $\Delta_{b i}^{m}$ & & & $\widehat{v}_{d w}^{\max }$ & $\bar{v}_{c w}$ & \\
\hline $\begin{array}{r}4 \\
=7\end{array}$ & $\begin{array}{l}13.4 \\
12.9\end{array}$ & $\begin{array}{l}-5.7 \\
-3.5\end{array}$ & $\begin{array}{l}-2 . \\
-1 .\end{array}$ & & .7 & $\begin{array}{l}10.6 \\
11.2\end{array}$ & $\begin{array}{l}-7.5 \\
-5.9\end{array}$ & . \\
\hline 10 & 11.0 & $-2 \cdot 3$ & $-1 .:$ & & .7 & 9.8 & -5.8 & \\
\hline 13 & 8.6 & -1.6 & -0.8 & & .0 & 7.8 & -5.8 & \\
\hline 16 & 6.1 & -1.2 & -0.6 & & .9 & 5.5 & -8.4 & \\
\hline 19 & 5.6 & -0.9 & -0. & & .7 & 5.1 & -7.2 & \\
\hline 22 & 1.6 & -0.7 & -0. & & .9 & 1.2 & -6.0 & \\
\hline 27 & -3.6 & -0.5 & -0.3 & & & -3.8 & -2.5 & \\
\hline 37 & -5.0 & -0.2 & -0 & & & -5.2 & 2.9 & \\
\hline 47 & -6.8 & -0.1 & -0.1 & & & -6.9 & 3.3 & \\
\hline
\end{tabular}

Below: $\mathrm{SASS}=x(\mathrm{raw}) \cdots($ unbiased $), \mathrm{C} 3=o(\mathrm{raw}) \cdots($ unbiased $)$, theoretical log-law shear $=-$
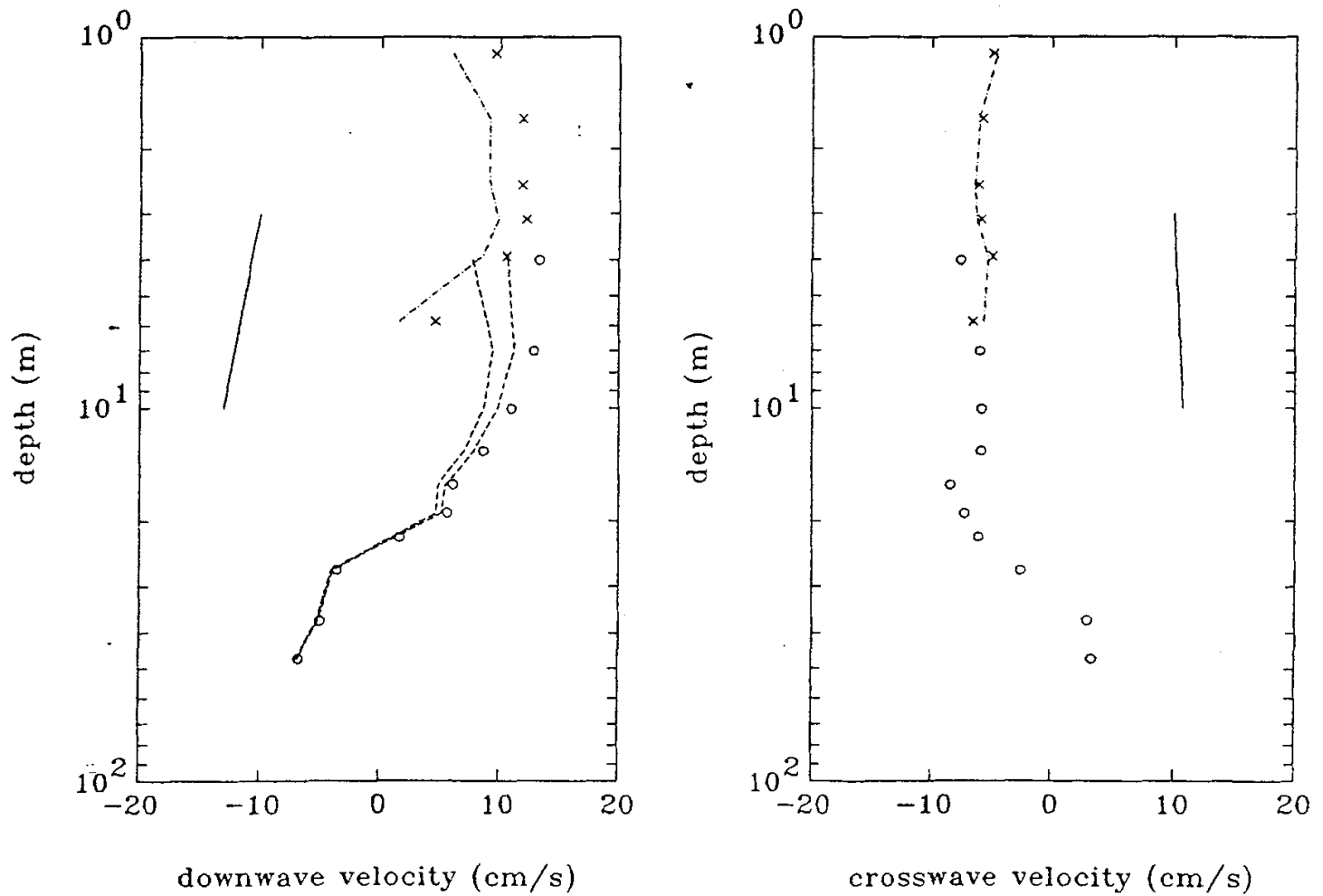


\section{file e1}

date $=$ November $28,1988 \quad$ time $=16-02-50.00 \quad$ duration $=00-26-01.50$

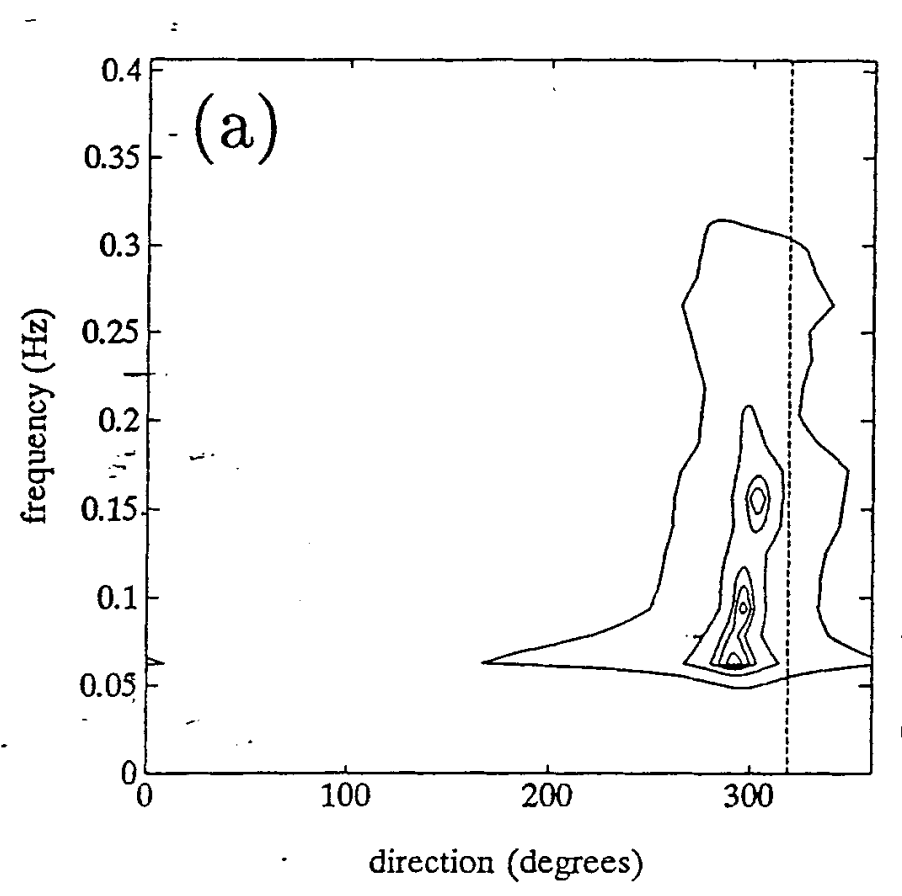

$$
\begin{array}{cc}
\text { wind } & \multicolumn{1}{c}{\text { waves }} \\
U_{10}=8.2 \mathrm{~m} / \mathrm{s} & \bar{H}_{1 / 3}=2.4 \mathrm{~m} \\
\theta_{\tau}=319^{\circ} & \theta_{w}=301^{\circ} \\
\frac{z}{L}=-0.02 & T_{p}=12.8 \mathrm{sec} \\
u_{*_{a}}=28.5 \mathrm{~cm} / \mathrm{s} & \bar{T}=6.3 \mathrm{sec} \\
\multicolumn{2}{c}{\Delta_{\text {temp }}=-0.41 \mathrm{C}}
\end{array}
$$

(a) directional spectrum $\left(-\cdots=\theta_{\tau}\right)$

(b) energy spectrum $\left(-\cdots=f^{-4}\right)$

(c) $0=$ salinity, $x,+=$ temperature
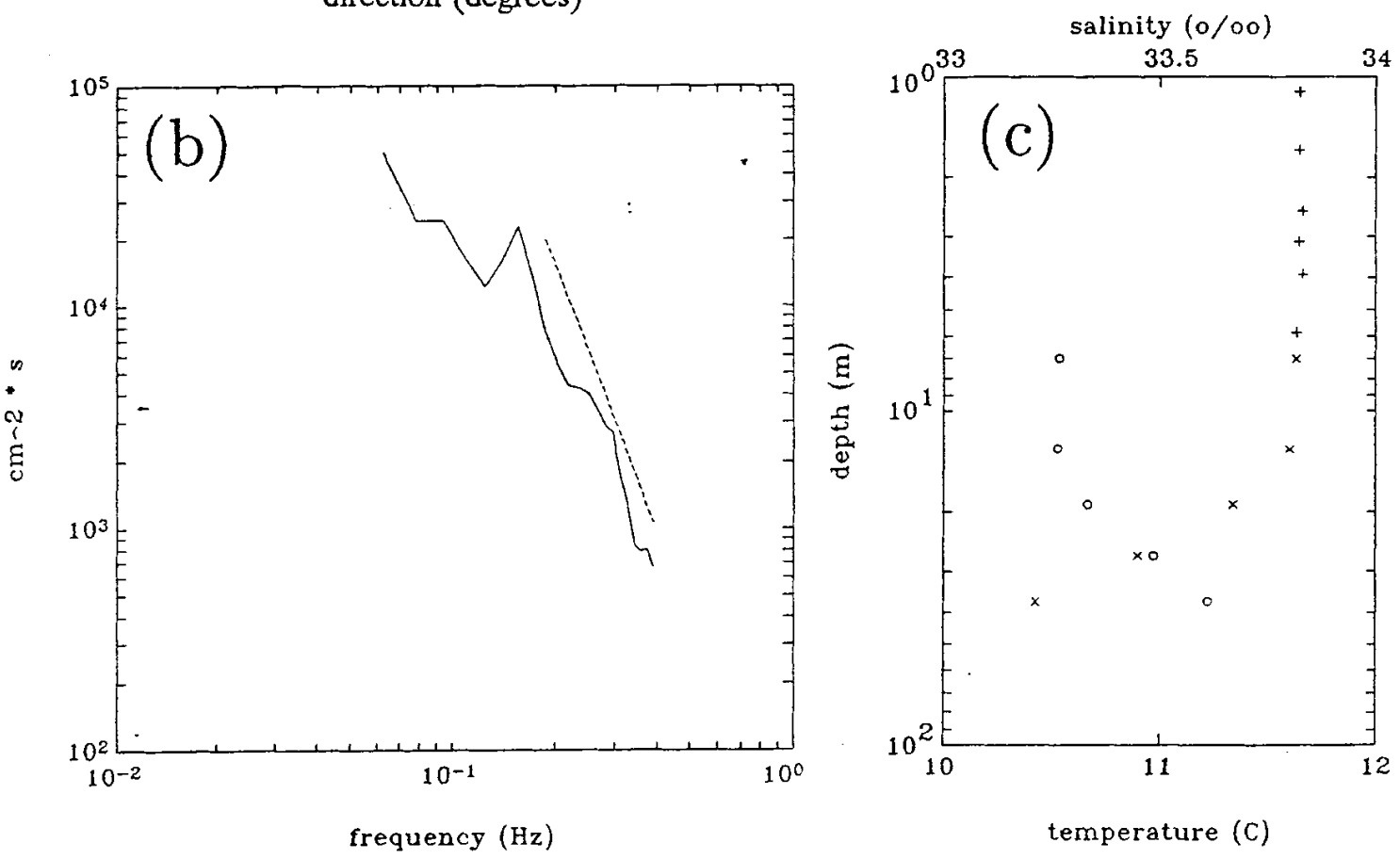


\section{file e1}

\begin{tabular}{|c|c|c|c|c|c|c|c|c|}
\hline & \multicolumn{5}{|c|}{ downwave } & \multicolumn{3}{|c|}{ crosswave } \\
\hline & $\bar{v}_{d w}$ & $\Delta_{\text {bias }}$ & $\triangle_{B A S S}$ & $\Delta_{\text {float }}$ & $\widehat{v}_{d w}$ & $\bar{v}_{c w}$ & $\overline{\Delta_{B A S S}}$ & $\widehat{v}_{c w}$ \\
\hline $\begin{array}{l}1.11 \\
1.66 \\
2.51 \\
3.11 \\
3.91 \\
5.85\end{array}$ & $\begin{array}{l}6.5 \\
8.4 \\
8.2 \\
9.4 \\
8.3 \\
1.5\end{array}$ & $\begin{array}{l}-4.5 \\
-4.1 \\
-3.6 \\
-3.4 \\
-3.2 \\
-2.6\end{array}$ & $\begin{array}{r}-0.5 \\
0.5 \\
0.5 \\
0.6 \\
0.7 \\
0.2\end{array}$ & $\begin{array}{l}1.4 \\
0.4 \\
0.0 \\
0.0 \\
0.0 \\
0.0\end{array}$ & $\begin{array}{r}2.9 \\
5.2 \\
5.1 \\
6.6 \\
5.8 \\
-1.0\end{array}$ & $\begin{array}{l}-5.9 \\
-7.4 \\
-7.5 \\
-7.7 \\
-7.3 \\
-8.0\end{array}$ & $\begin{array}{r}0.4 \\
-0.4 \\
-0.4 \\
-0.5 \\
-0.6 \\
-0.9\end{array}$ & $\begin{array}{l}-5.5 \\
-7.8 \\
-8.0 \\
-8.2 \\
-7.8 \\
-8.9\end{array}$ \\
\hline $\bar{z}$ & $\bar{v}_{d w}$ & $\Delta_{b i a s}^{m a}$ & $\Delta_{b i}^{m}$ & & $\widehat{v}_{d w}^{\min }$ & $\widetilde{v}_{d w}^{\max }$ & $\bar{v}_{c w}$ & \\
\hline $\begin{array}{r}4 \\
-47 \\
1.0 \\
13 \\
16 \\
19 \\
22 \\
27 \\
37 \\
47\end{array}$ & $\begin{array}{r}10.0 \\
9.9 \\
7.5 \\
5.2 \\
2.6 \\
1.5 \\
-0.8 \\
-5.1 \\
-4.9 \\
-6.2\end{array}$ & $\begin{array}{l}-6.1 \\
-3.8 \\
-2.6 \\
-1.9 \\
-1.4 \\
-1.1 \\
-0.9 \\
-0.6 \\
-0.3 \\
-0.2\end{array}$ & $\begin{array}{l}-2.5 \\
-1.5 \\
-1.5 \\
-0.5 \\
-0.2 \\
-0 . \\
-0.5 \\
-0.3 \\
-0.2 \\
-0.1\end{array}$ & & $\begin{array}{l}3.9 \\
6.1 \\
4.9 \\
3.3 \\
1.2 \\
0.4 \\
1.7 \\
5.7 \\
5.2 \\
6.4\end{array}$ & $\begin{array}{r}7.1 \\
8.1 \\
6.2 \\
4.3 \\
1.9 \\
1.0 \\
-1.2 \\
-5.4 \\
-5.1 \\
-6.4\end{array}$ & $\begin{array}{r}-11.0 \\
-9.3 \\
-8.2 \\
-7.4 \\
-8.2 \\
-7.6 \\
-5.6 \\
0.2 \\
3.5 \\
3.6\end{array}$ & \\
\hline
\end{tabular}

Below: SASS $=x$ (raw) -..- (unbiased), C3=o (raw) - - (unbiased), theoretical log-law shear $=-$
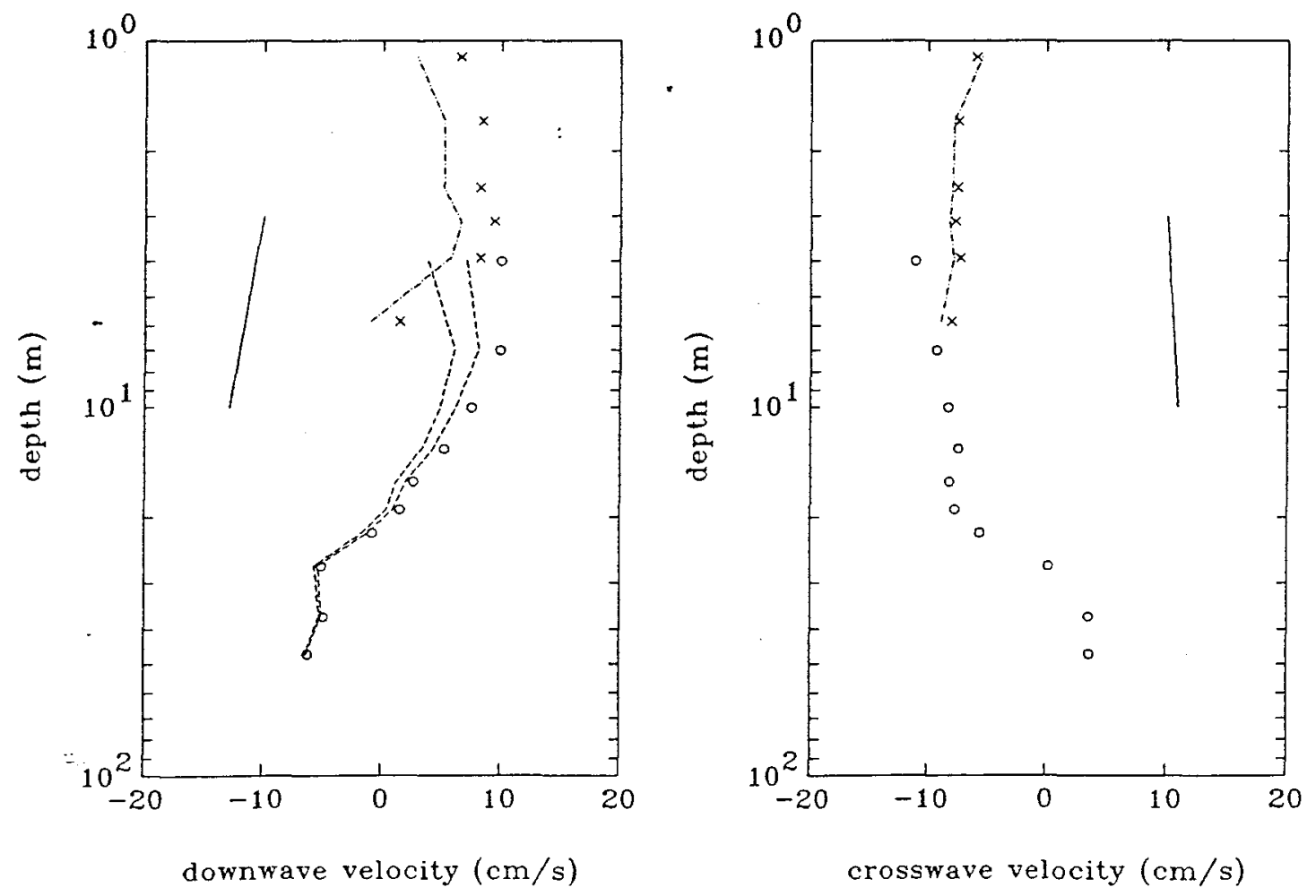


\section{file $\mathrm{f} 1$}

date $=$ December $5,1988 \quad$ time $=18-48-54.75 \quad$ duration $=00-56-52.00$

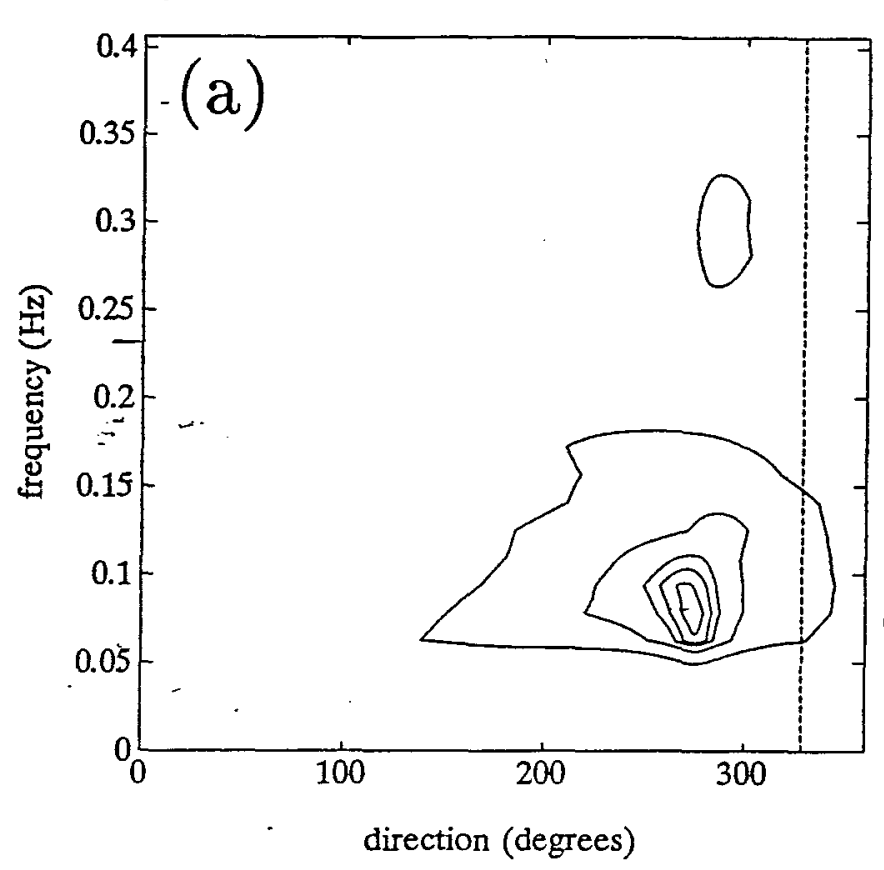

$$
\begin{array}{cc}
\underline{\text { wind }} & \multicolumn{1}{c}{\text { waves }} \\
U_{10}=2.4 \mathrm{~m} / \mathrm{s} & \bar{H}_{1 / 3}=2.1 \mathrm{~m} \\
\theta_{\tau}=329^{\circ} & \theta_{w}=275^{\circ} \\
\frac{z}{L}=-0.14 & T_{p}=10.7 \mathrm{sec} \\
u_{*_{a}}=7.5 \mathrm{~cm} / \mathrm{s} & \bar{T}=8.2 \mathrm{sec} \\
\Delta_{\text {temp }}=-0.36 \mathrm{C}
\end{array}
$$

(a) directional spectrum $\left(-\cdots=\theta_{\tau}\right)$

(b) energy spectrum $\left(---=f^{-4}\right)$

(c) $o=$ salinity, $x,+=$ temperature
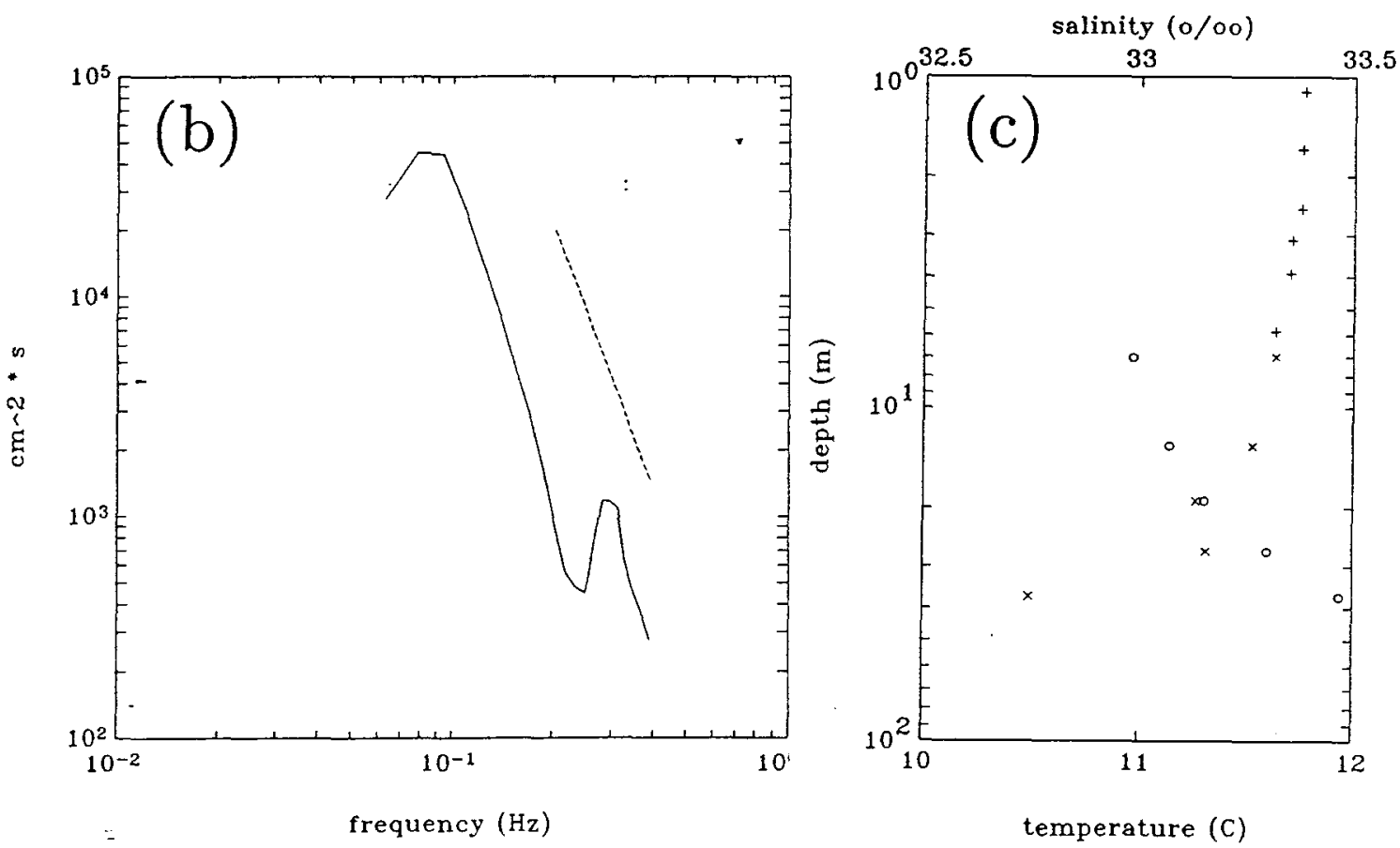


\section{file $\mathrm{f} 1$}

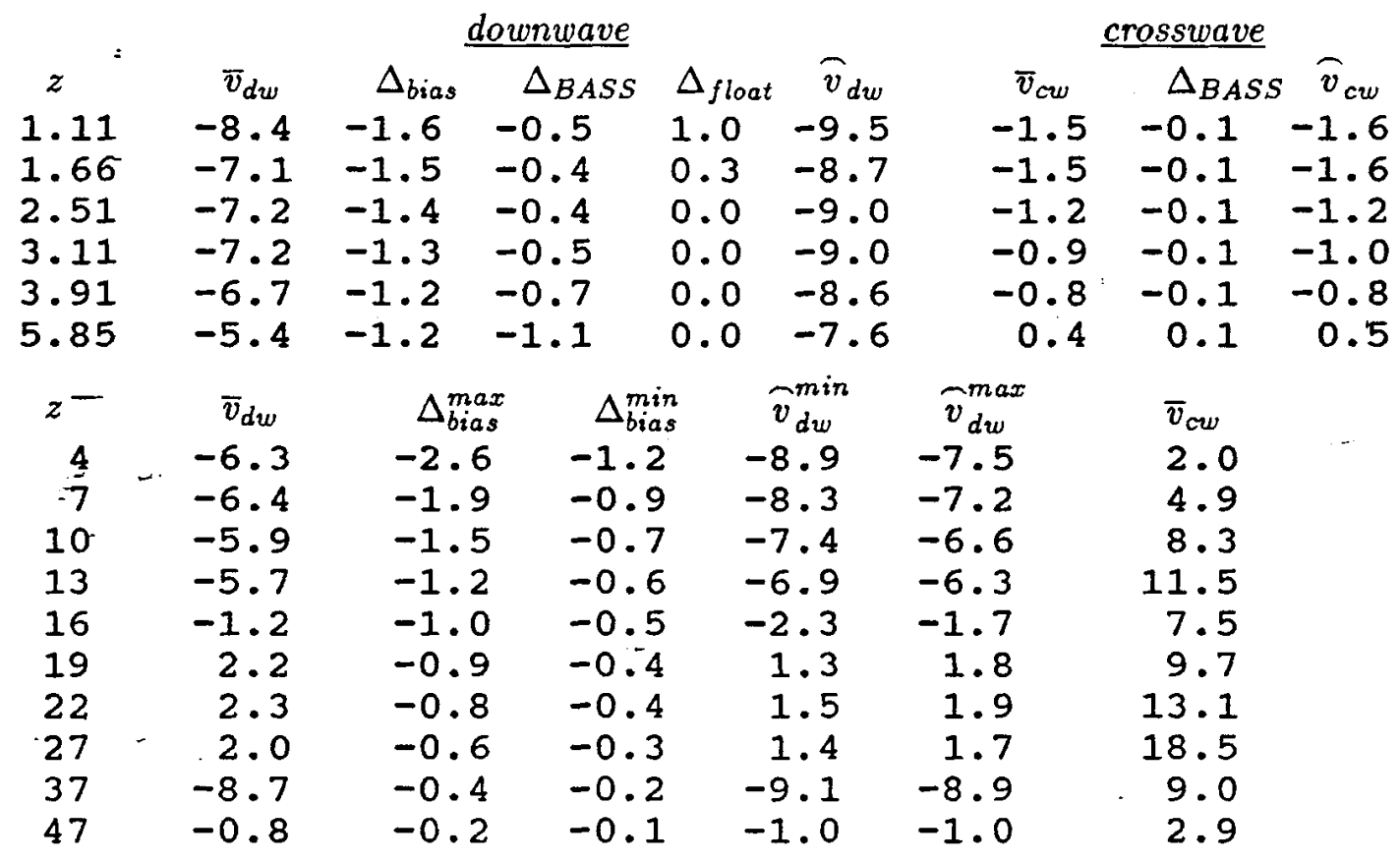

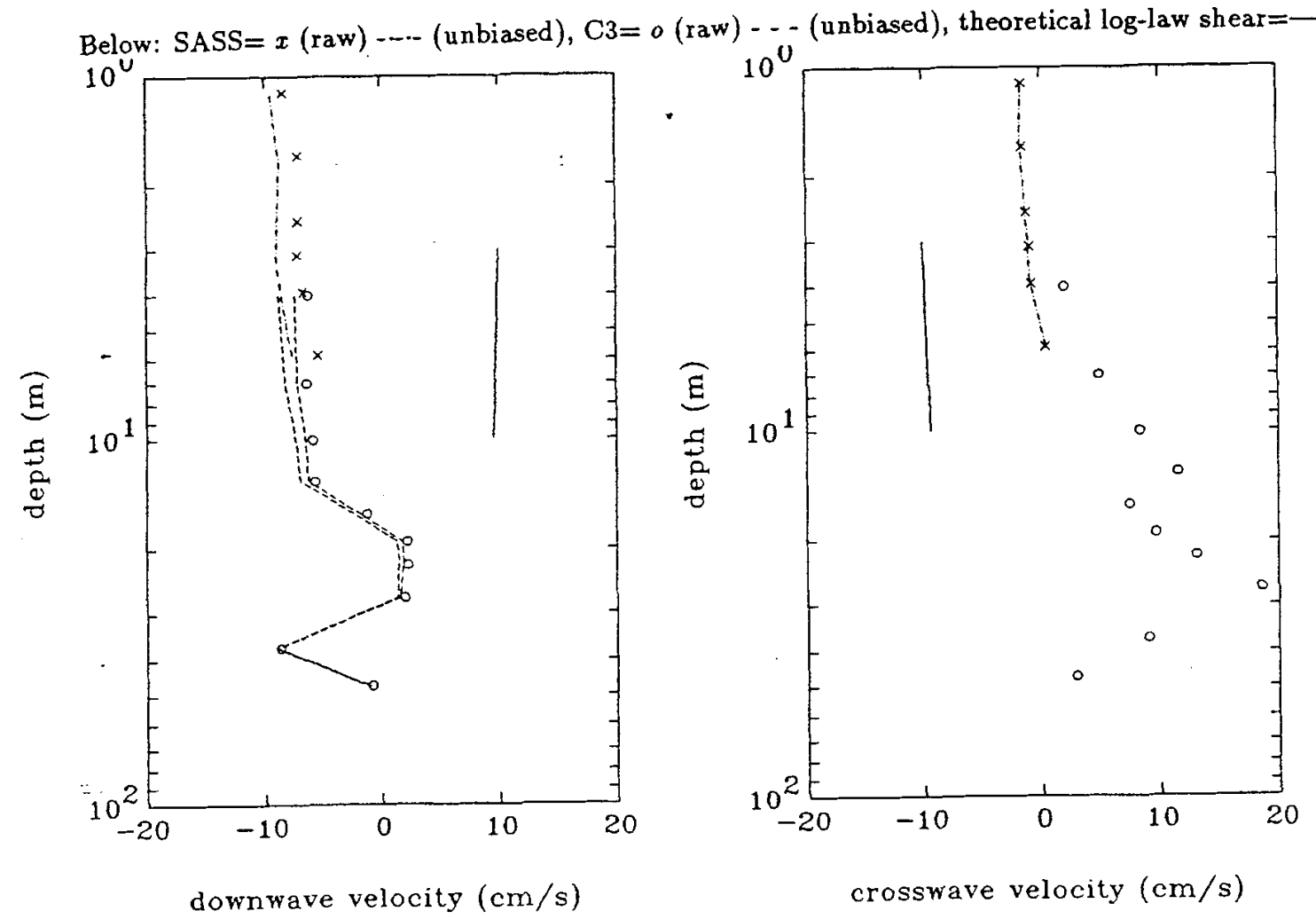




\section{file $\mathrm{g} 1$}

date $=$ December $6,1988 \quad$ time $=01-37-28.00 \quad$ duration $=00-21-10.00$

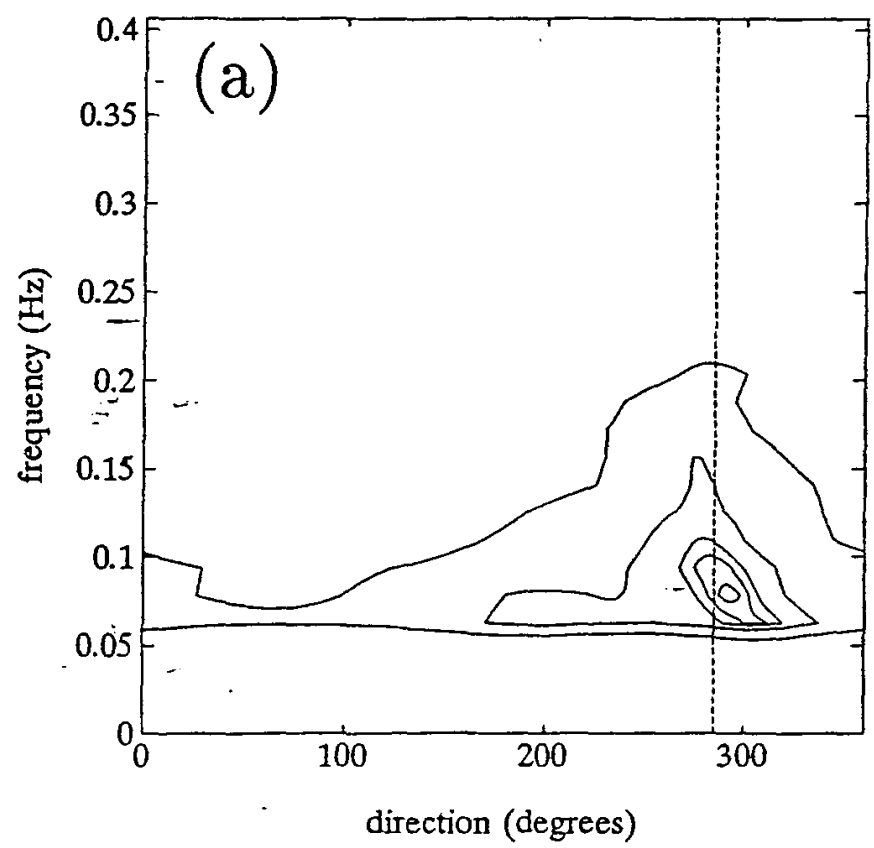

$$
\begin{array}{ll}
\underline{\text { wind }} & \multicolumn{1}{c}{\text { waves }} \\
U_{10}=5.4 \mathrm{~m} / \mathrm{s} & \bar{H}_{1 / 3}=2.4 \mathrm{~m} \\
\theta_{\tau}=285^{\circ} & \theta_{w}=283^{\circ} \\
\frac{z}{L}=-0.12 & T_{p}=12.8 \mathrm{sec} \\
u_{* a}=18.9 \mathrm{~cm} / \mathrm{s} & \bar{T}=8.8 \mathrm{sec} \\
\multicolumn{2}{c}{\Delta_{\text {temp }}=-0.45 \mathrm{C}}
\end{array}
$$

(a) directional spectrum

(b) energy spectrum $\left(--=f^{-4}\right)$

(c) $0=$ salinity, $x,+=$ temperature
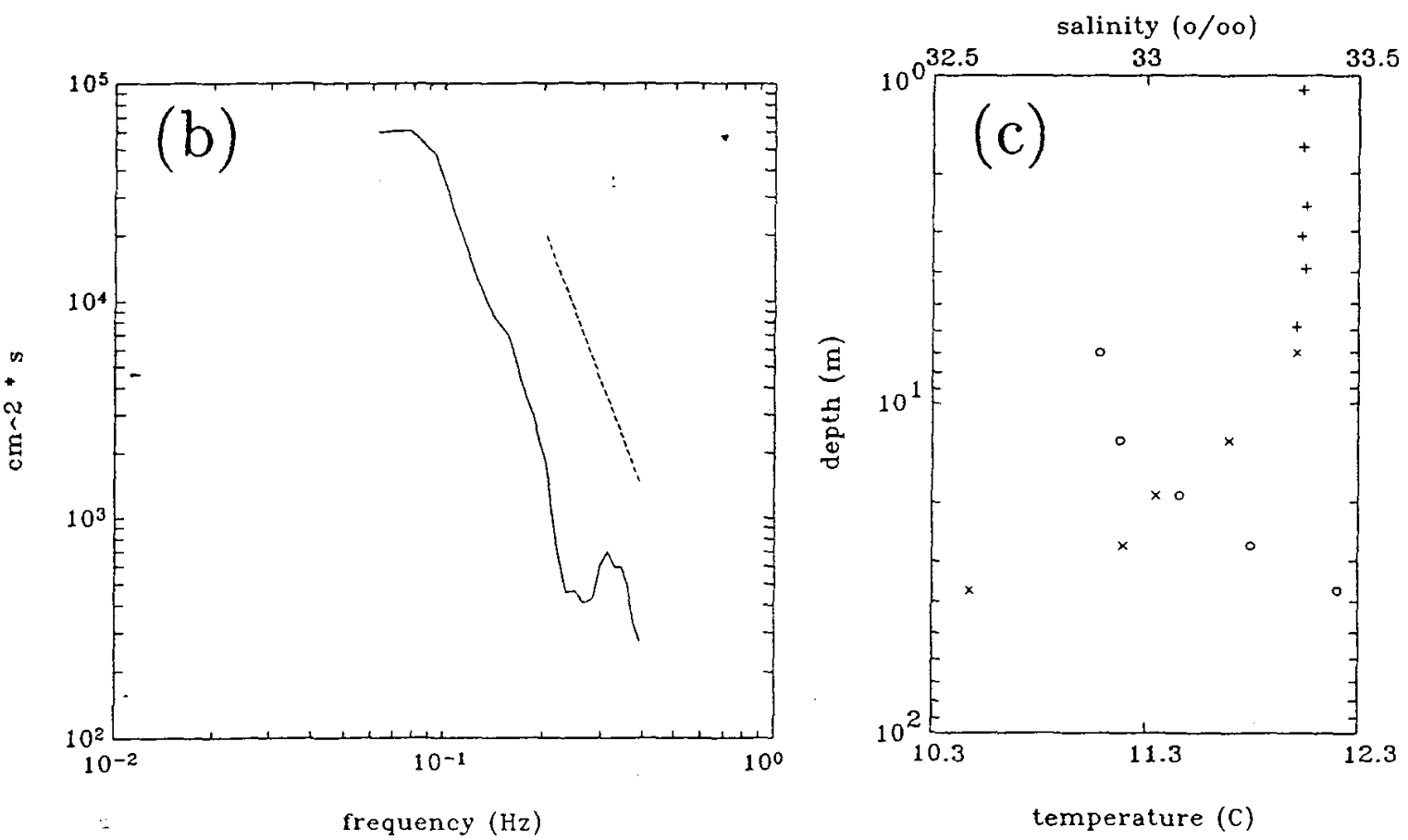
file $\mathrm{g} 1$

\begin{tabular}{ccccccccc} 
& \multicolumn{9}{c}{ downwave } & \multicolumn{3}{c}{ crosswave } \\
$z$ & $\bar{v}_{d w}$ & $\Delta_{\text {bias }}$ & $\Delta_{\text {BASS }}$ & $\Delta_{\text {float }}$ & $\widehat{v}_{d w}$ & $\bar{v}_{c w}$ & $\Delta_{B A S S}$ & $\widehat{v}_{c w}$ \\
1.11 & -1.8 & -1.5 & -0.1 & 1.0 & -2.4 & 4.3 & 0.3 & 4.6 \\
1.66 & -2.3 & -1.4 & -0.2 & 0.3 & -3.6 & 4.7 & 0.3 & 5.0 \\
2.51 & -3.2 & -1.3 & -0.2 & 0.0 & -4.7 & 5.5 & 0.4 & 5.8 \\
3.11 & -3.0 & -1.3 & -0.3 & 0.0 & -4.5 & 4.5 & 0.4 & 4.9 \\
3.91 & -3.6 & -1.2 & -0.4 & 0.0 & -5.2 & 4.9 & 0.5 & 5.4 \\
5.85 & -3.8 & -1.2 & -0.7 & 0.0 & -5.7 & 4.0 & 0.7 & 4.7 \\
7 & $\bar{v}_{d w}$ & $\Delta_{\text {bias }}^{\max }$ & $\Delta_{\text {bias }}^{\min }$ & $\widehat{v}_{d w}^{\min }$ & $\widehat{v}_{d w}^{\max }$ & $\bar{v}_{c w}$ & \\
4 & -5.6 & -2.6 & -1.2 & -8.3 & -6.8 & 6.7 & \\
-7 & -9.3 & -2.0 & -0.9 & -11.4 & -10.3 & 7.6 & \\
10 & -13.0 & -1.6 & -0.7 & -14.6 & -13.7 & 9.5 & \\
13 & -10.0 & -1.3 & -0.6 & -11.4 & -10.7 & 12.9 & \\
16 & -6.2 & -1.1 & -0.5 & -7.3 & -6.7 & 10.0 & \\
19 & -7.5 & -1.0 & -0.5 & -8.5 & -8.0 & 9.4 & \\
22 & -9.9 & -0.8 & -0.4 & -10.7 & -10.3 & 7.3 & \\
27 & -7.1 & -0.6 & -0.3 & -7.7 & -7.4 & 2.0 & \\
37 & -1.7 & -0.4 & -0.2 & -2.0 & -1.9 & 2.8 \\
47 & -5.0 & -0.2 & -0.2 & -5.2 & -5.1 & 8.2 &
\end{tabular}

Below: SASS $=x$ (raw) … (unbiased), C3=o(raw) - - (unbiased), theoretical log-law shear $=-$
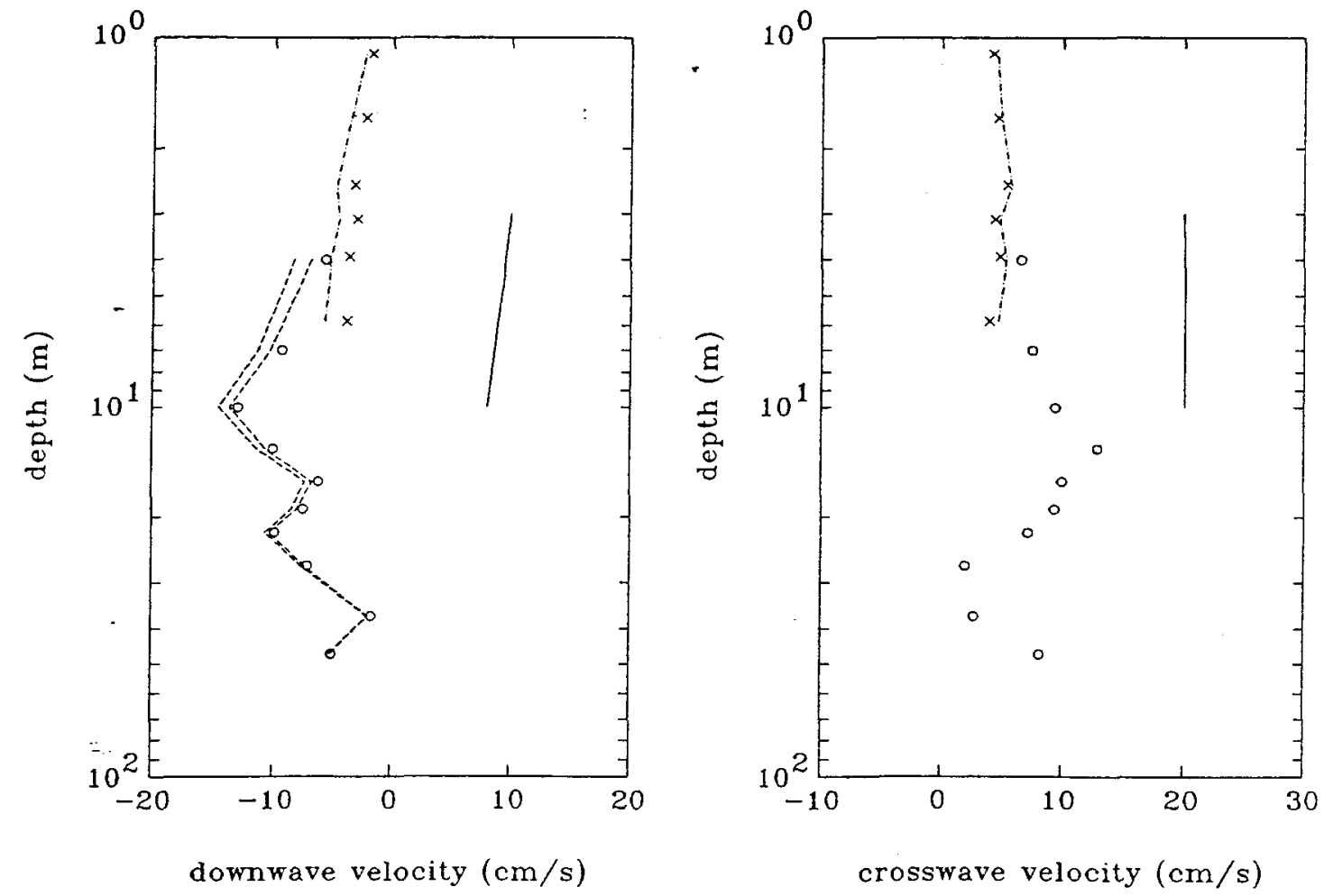


\section{file $\mathrm{h} 1$}

date $=$ December 6, $1988 \quad$ time $=04-58-19.00 \quad$ duration $=00-41-43.00$

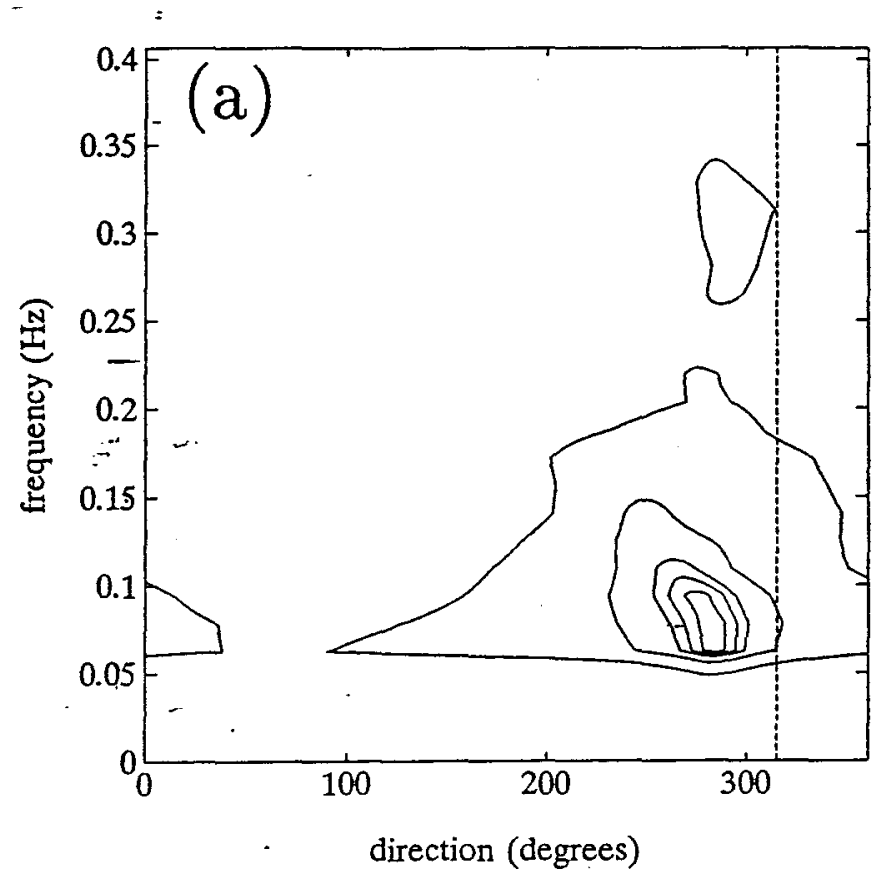

$$
\begin{array}{cc}
\underline{\text { wind }} & \multicolumn{1}{c}{\text { waves }} \\
U_{10}=5.7 \mathrm{~m} / \mathrm{s} & \bar{H}_{1 / 3}=2.2 \mathrm{~m} \\
\theta_{\tau}=315^{\circ} & \theta_{w}=280^{\circ} \\
\bar{L}=-0.10 & T_{p}=12.8 \mathrm{sec} \\
u_{* a}=19.9 \mathrm{~cm} / \mathrm{s} & \bar{T}=7.8 \mathrm{sec} \\
\multicolumn{2}{c}{\Delta_{\text {temp }}=-0.33 \mathrm{C}}
\end{array}
$$

(a) directional spectrum $\left(--=\theta_{\tau}\right)$

(b) energy spectrum $\left(--=f^{-4}\right)$

(c) $0=$ salinity, $x, t=$ temperature
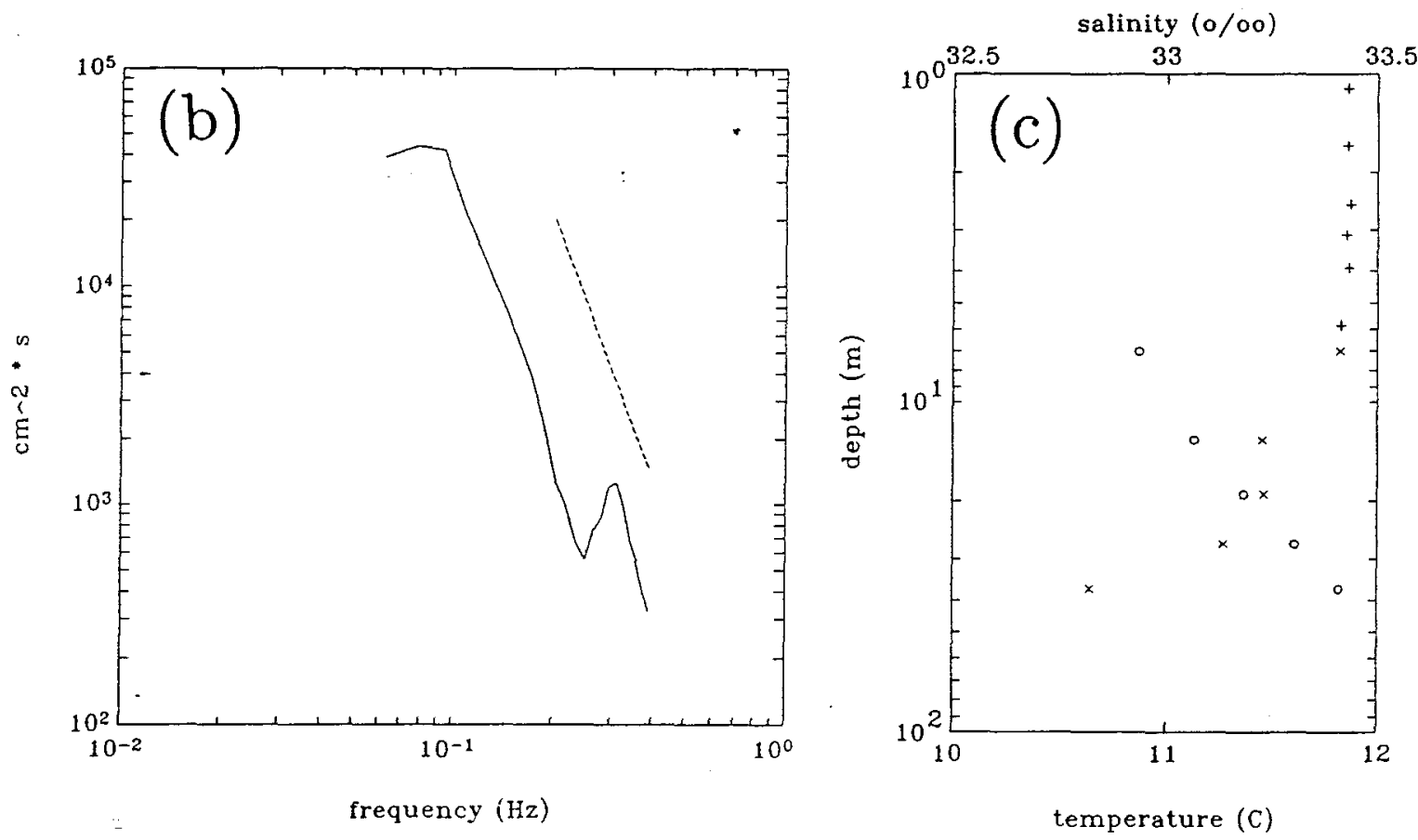


\section{file $\mathrm{h} 1$}

\begin{tabular}{|c|c|c|c|c|c|c|c|c|c|}
\hline \multirow[b]{2}{*}{$z^{=}$} & \multicolumn{6}{|c|}{ downwave } & \multicolumn{3}{|c|}{ crosswave } \\
\hline & $\bar{v}_{d w}$ & $\Delta_{\text {bias }}$ & & $B A S S$ & $\Delta_{\text {float }}$ & $\widehat{v}_{d w}$ & $\bar{v}_{c w}$ & $\Delta_{B A S S}$ & $\widehat{v}_{c w}$ \\
\hline 1.11 & 0.5 & -1.8 & -0 & & 1.0 & -0.4 & $-2 \cdot 2$ & 0.3 & -1.8 \\
\hline 1.6 & 1.0 & -1.7 & -0 & & 0.3 & -0.5 & -2 & 0.3 & $-2 \cdot 3$ \\
\hline 2.51 & 0.9 & -1.5 & -0 & & 0.0 & -0.8 & $-2 \cdot 4$ & 0.3 & -2.0 \\
\hline 3.11 & 1.2 & -1.5 & & & 0.0 & -0.4 & -2.8 & 0.4 & -2.4 \\
\hline 3.91 & 1.1 & -1.4 & & & 0.0 & -0.5 & $-2 \cdot 9$ & 0.5 & -2.4 \\
\hline 5.85 & -2.9 & -1.3 & & & 0.0 & -4.8 & -3.2 & -0.6 & -3.8 \\
\hline$z$ & $\bar{v}_{d w}$ & $\Delta_{b i c}^{m}$ & & $\Delta_{b i 0}^{m i}$ & & $v_{d w}^{\min }$ & $\widehat{v}_{d w}^{\max }$ & $\bar{v}_{c w}$ & \\
\hline 4 & -2.4 & -2.8 & & -1.3 & & 5.1 & $-3 \cdot 6$ & -3.8 & \\
\hline$=7-$ & $-4 \cdot 9$ & $-2 \cdot c$ & & -0.9 & & 5.9 & -5.8 & 0.1 & \\
\hline 10 & -5.8 & -1.5 & & -0.7 & & 7.4 & -6.5 & 4.2 & \\
\hline 13 & -5.5 & -1.3 & & -0.6 & & 5.7 & -6.1 & 7.7 & \\
\hline 16 & -4.8 & -1.1 & & -0.5 & & 5.9 & -5.3 & 10.9 & \\
\hline 19 & -9.2 & -0.5 & & -0.4 & -10 & 0.1 & -9.7 & 11.2 & \\
\hline 22 & -11.6 & -0.8 & & -0.4 & -12 & 2.4 & -12.0 & 7.3 & \\
\hline 27 & -9.8 & -0.6 & & -0.3 & -10 & 0.4 & -10.1 & 5.5 & \\
\hline 37 & -7.8 & -0.4 & & -0.2 & & 8.1 & -8.0 & -5.5 & \\
\hline 47 & 0.0 & -0.2 & & -0.1 & & 0.2 & -0.1 & 0.1 & \\
\hline
\end{tabular}

Below: SASS $=x$ (raw) -...- (unbiased), C3=o (raw) - - (unbiased), theoretical log-law shear $=$ -
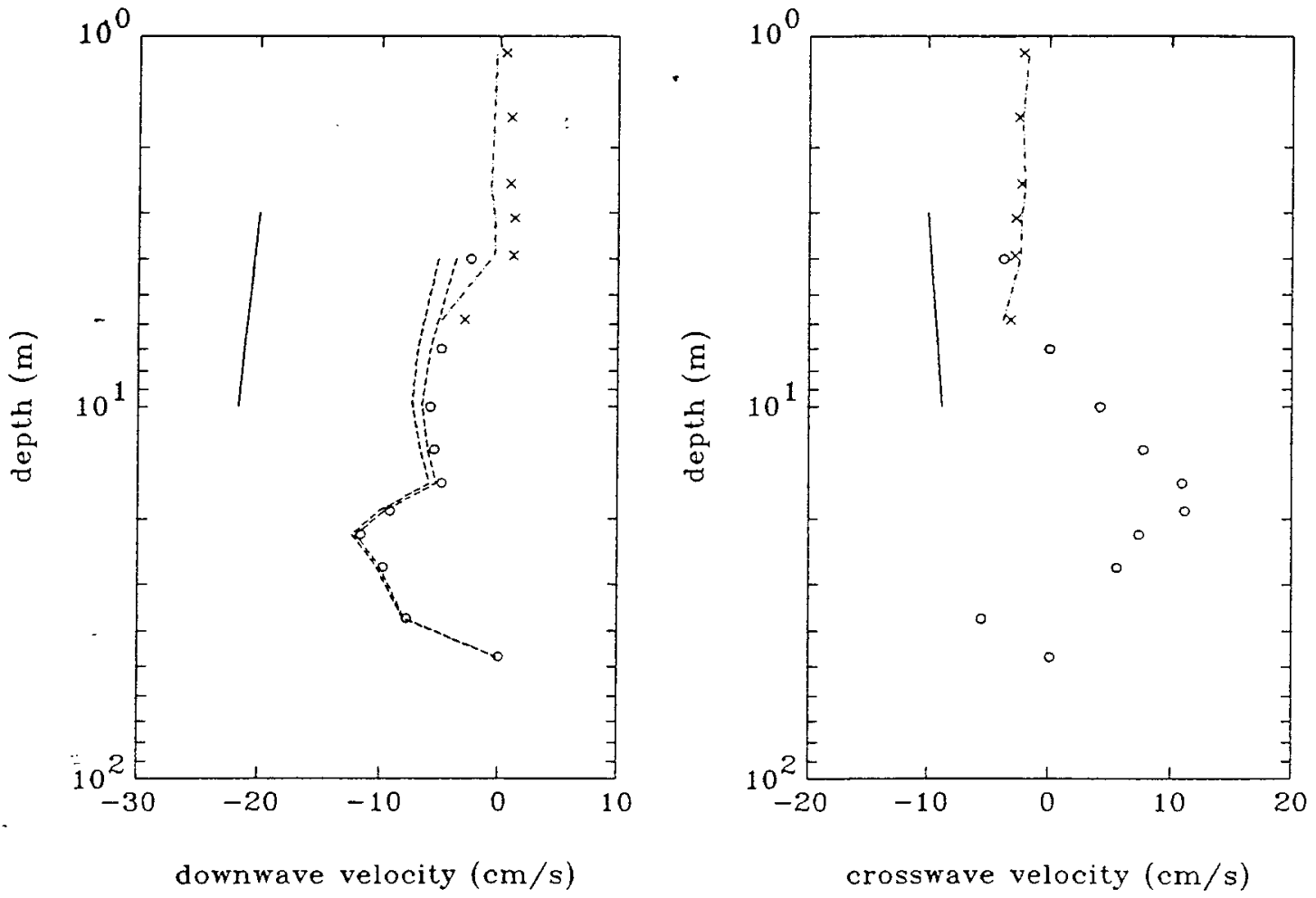


\section{file i1}

date $=$ December $6,1988 \quad$ time $=06-20-32.00 \quad$ duration $=00-32-21.50$

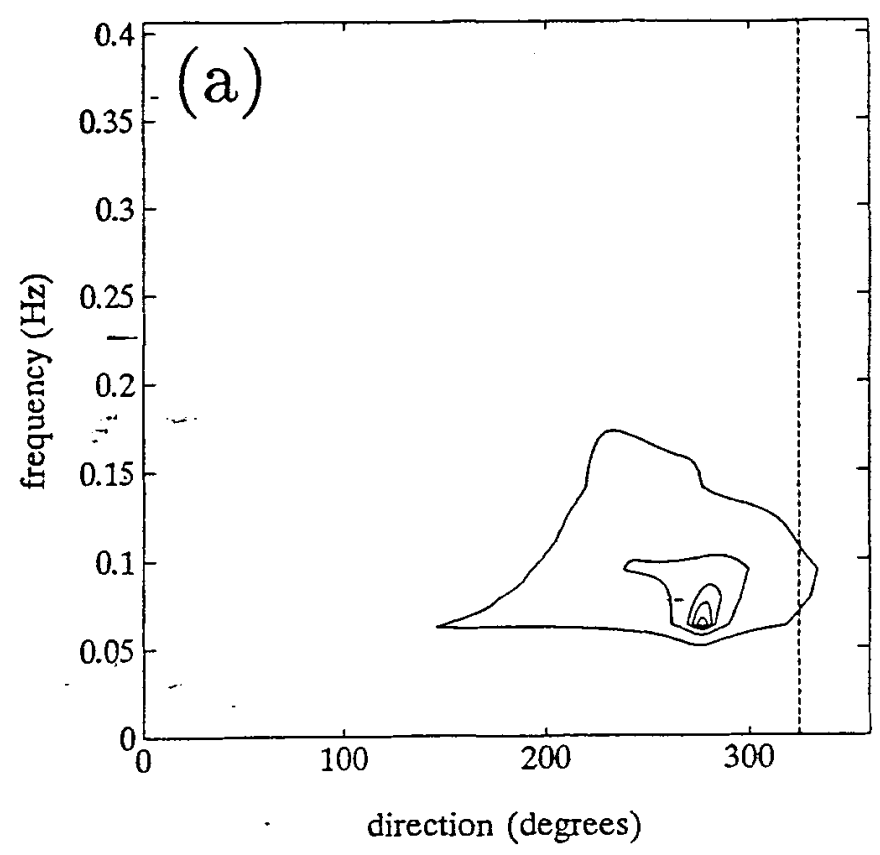

$$
\begin{array}{cc}
\underline{\text { wind }} & \multicolumn{1}{c}{\text { waves }} \\
U_{10}=2.0 \mathrm{~m} / \mathrm{s} & \bar{H}_{1 / 3}=2.2 \mathrm{~m} \\
\theta_{\tau}=325^{\circ} & \theta_{w}=276^{\circ} \\
\frac{z}{L}=-0.47 & T_{p}=12.8 \mathrm{sec} \\
u_{*_{a}}=6.3 \mathrm{~cm} / \mathrm{s} & \bar{T}=8.3 \mathrm{sec} \\
\multicolumn{2}{c}{\Delta_{\text {temp }}=-0.45 \mathrm{C}}
\end{array}
$$

(a) directional spectrum $\left(--=\theta_{\tau}\right)$

(b) energy spectrum $\left(-\cdots=f^{-4}\right)$

(c) $o=$ salinity, $x,+=$ temperature
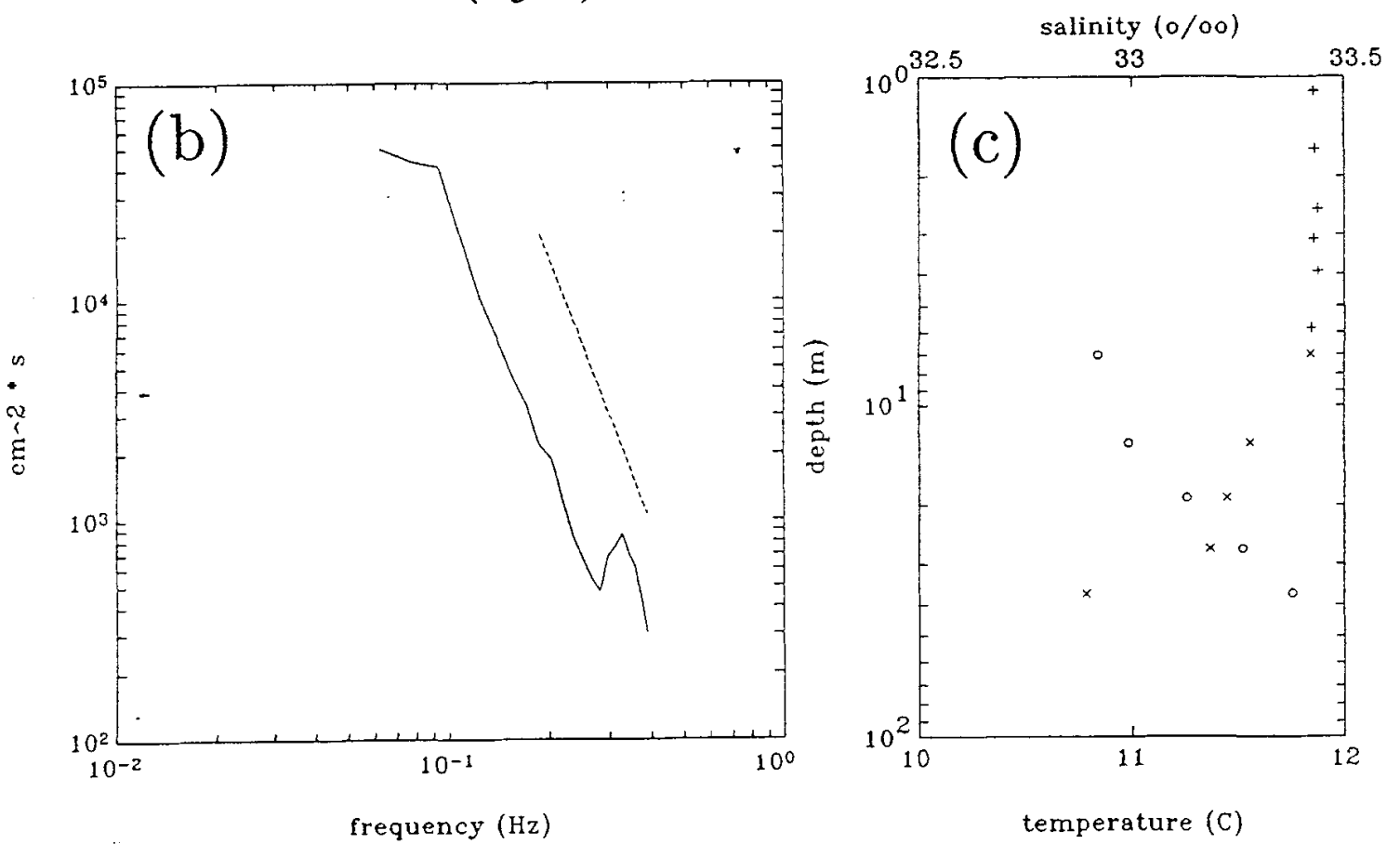


\section{file i1}

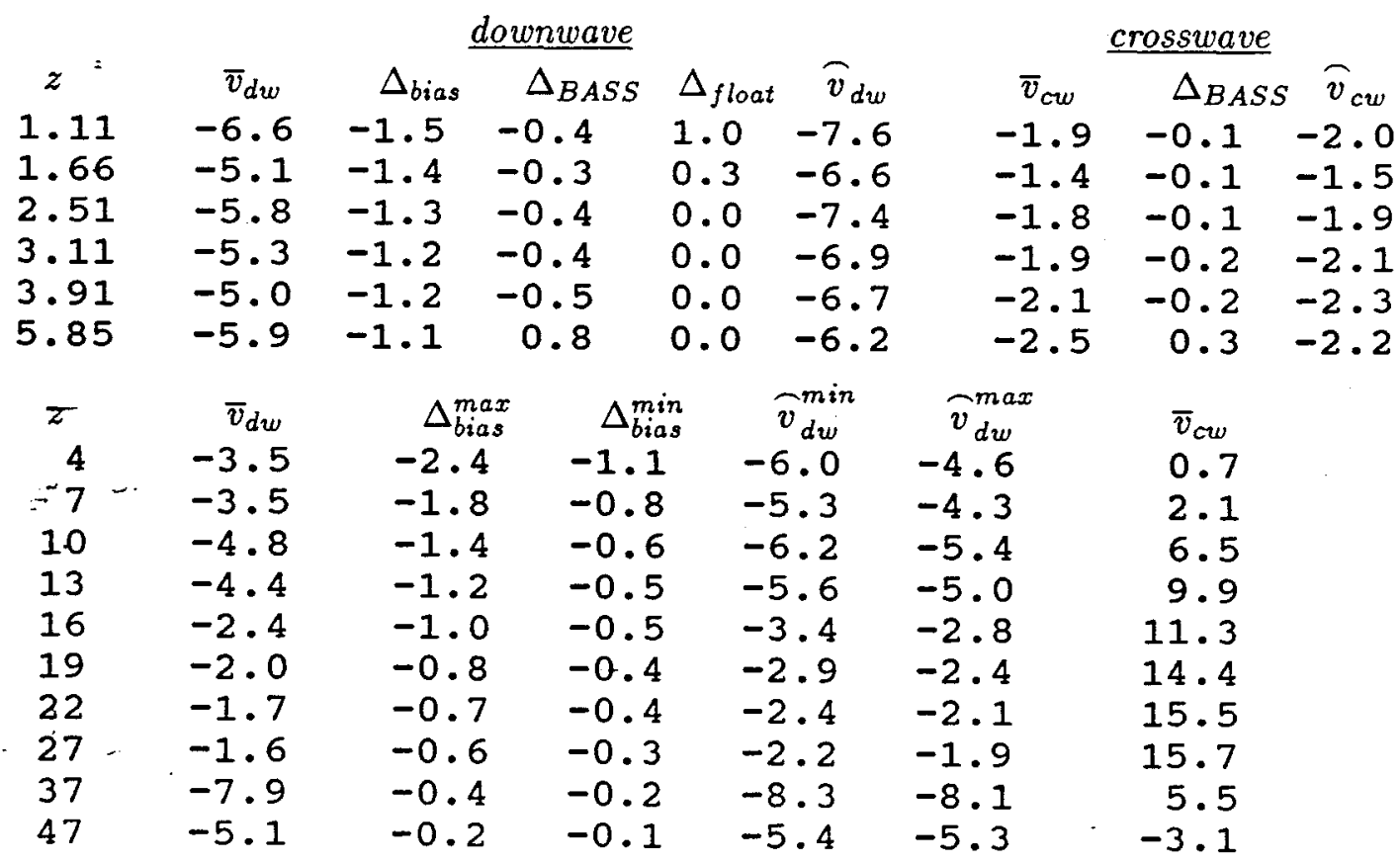

Below: $\mathrm{SASS}=x(\mathrm{raw}) \cdots($ unbiased $), \mathrm{C} 3=0(\mathrm{raw}) \cdots($ unbiased $)$, theoretical $\log -\mathrm{law}$ shear $=-$

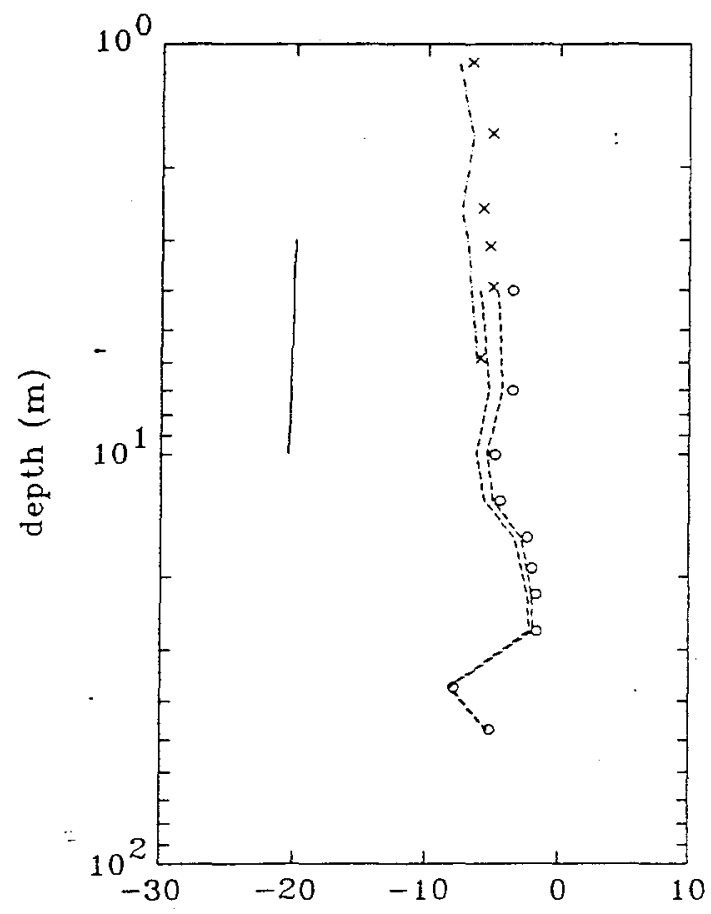

downwave velocity $(\mathrm{cm} / \mathrm{s})$

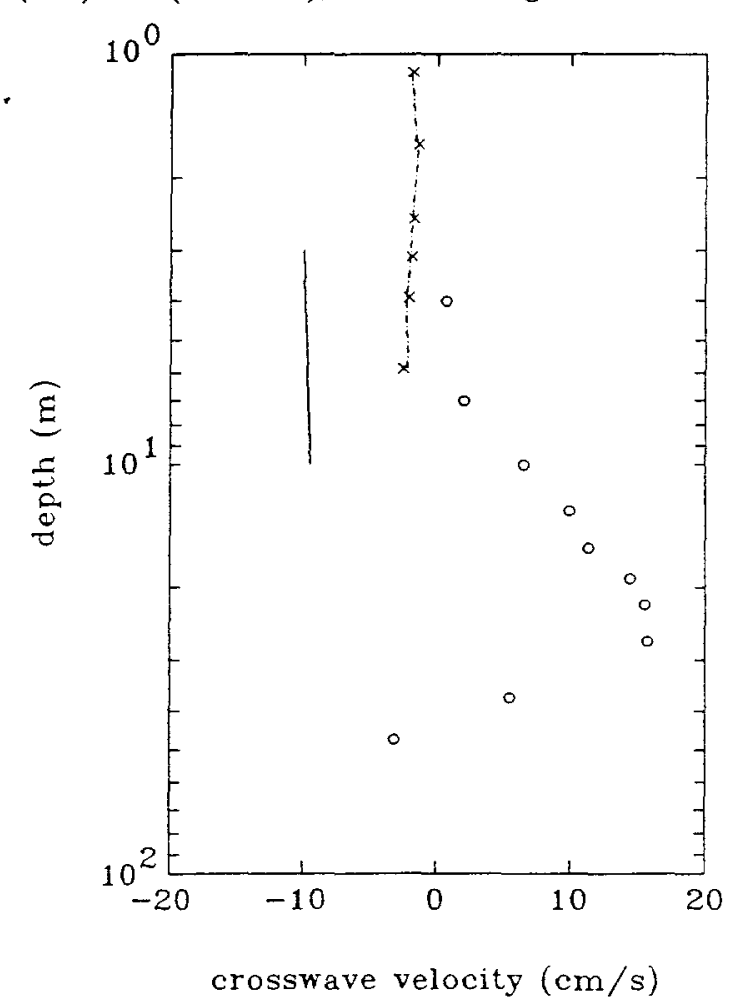




\section{file $\mathrm{j} 1$}

date $=$ February 24, $1989 \quad$ time $=05-48-44.00 \quad$ duration $=00-17-43.75$

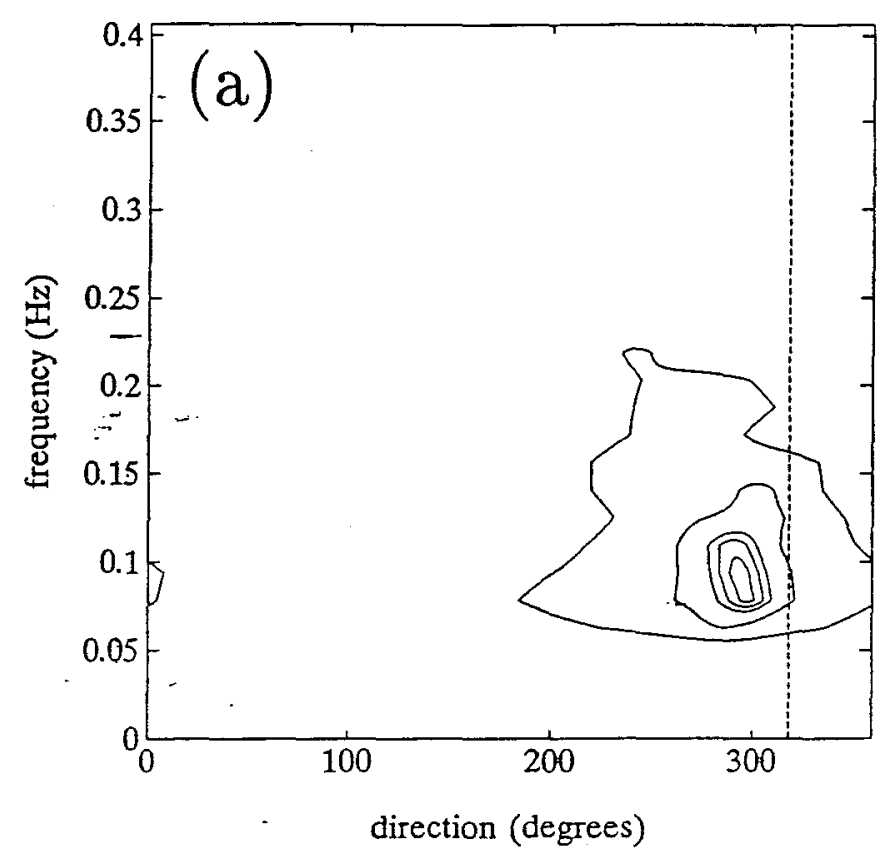

$$
\begin{array}{cc}
\text { wind } & \multicolumn{1}{c}{\text { waves }} \\
U_{10}=5.3 \mathrm{~m} / \mathrm{s} & \bar{H}_{1 / 3}=2.4 \mathrm{~m} \\
\theta_{\tau}=318^{\circ} & \theta_{w}=291^{\circ} \\
\frac{z}{L}=-0.12 & T_{p}=10.7 \mathrm{sec} \\
u_{*_{a}}=18.4 \mathrm{~cm} / \mathrm{s} & \bar{T}=7.7 \mathrm{sec} \\
\multicolumn{2}{c}{\Delta_{\text {temp }}=-0.36 \mathrm{C}}
\end{array}
$$

(a) directional spectrum $\left(-\cdots=\theta_{\tau}\right)$

(b) energy spectrum $\left(-\cdots=f^{-4}\right)$

(c) $0=$ salinity, $x,+=$ temperature
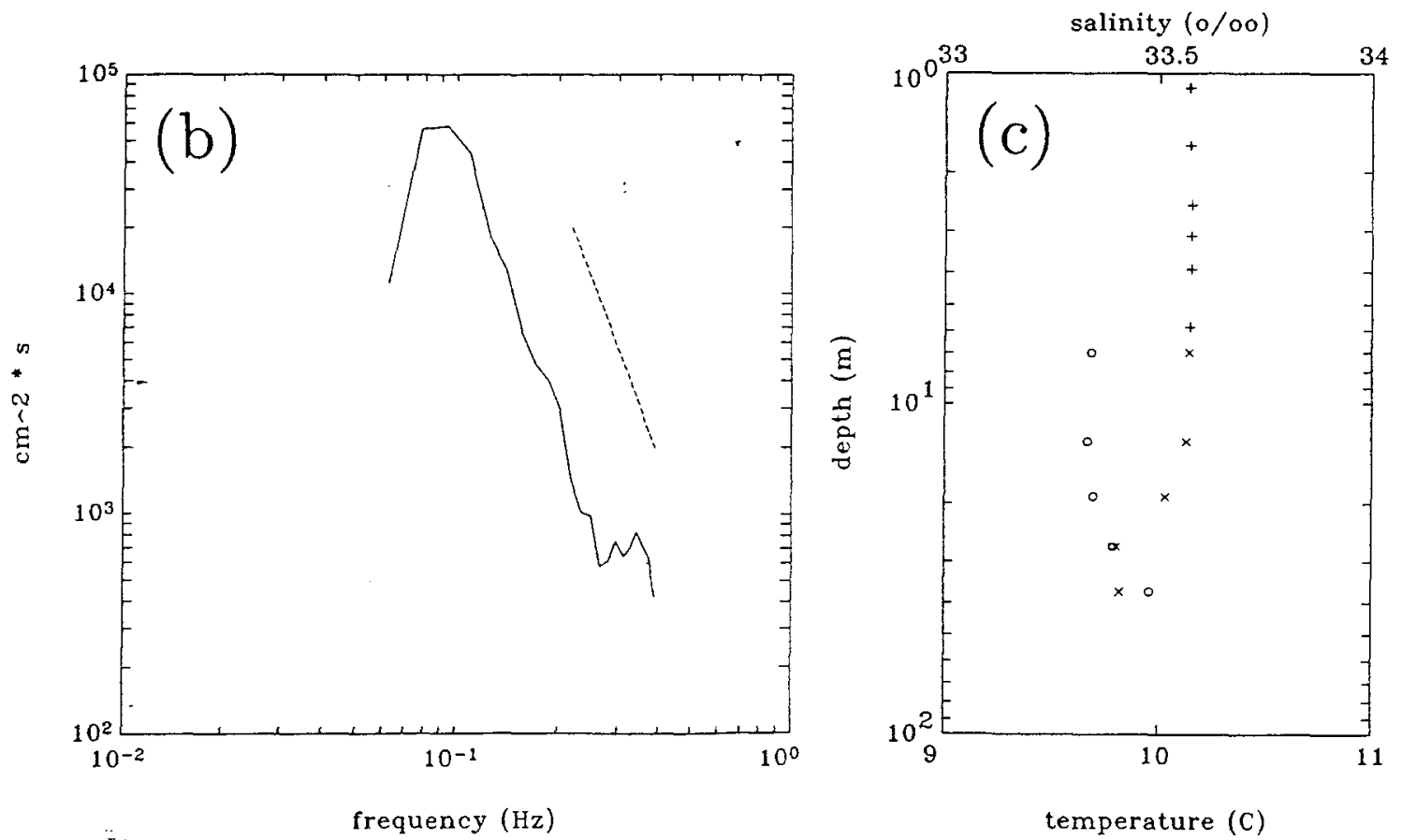


\section{file $\mathrm{j} 1$}

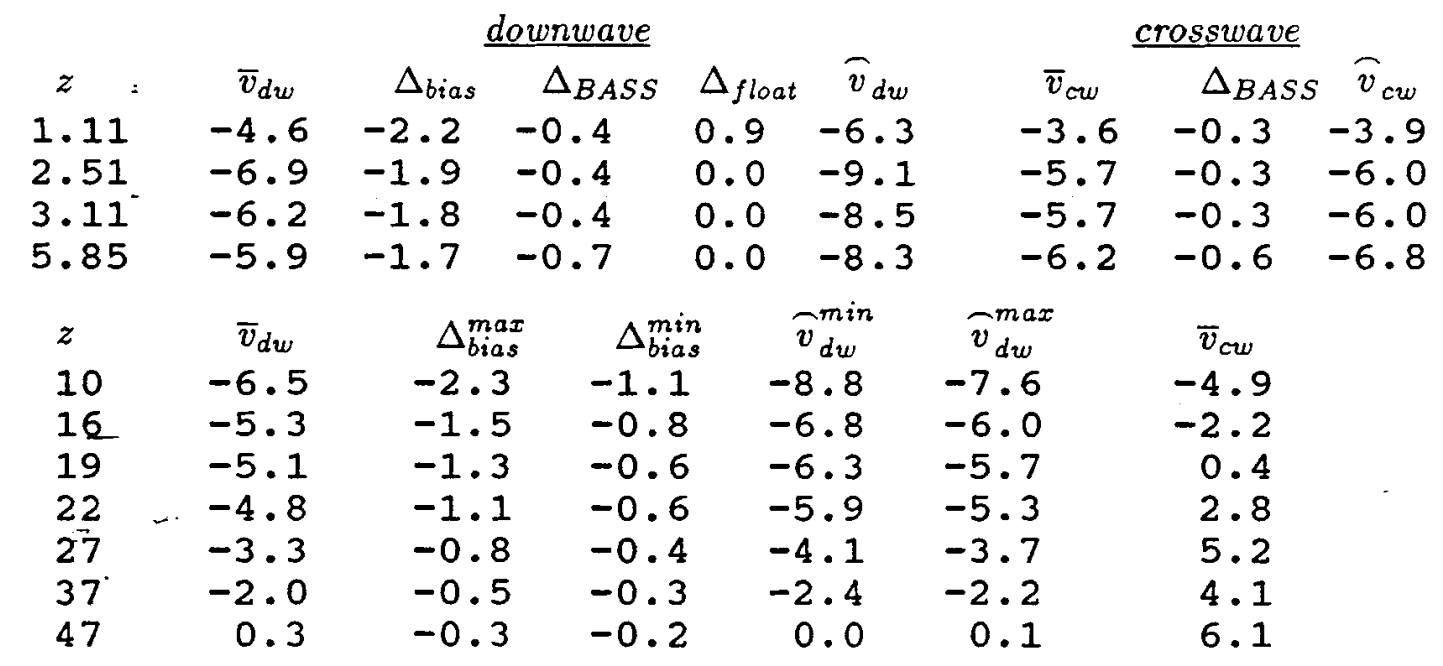

Below: $\mathrm{SASS}=x(\mathrm{raw}) \ldots($ unbiased $), \mathrm{C} 3=0(\mathrm{raw})-\cdots($ unbiased $)$, theoretical $\log -\mathrm{law}$ shear $=-$
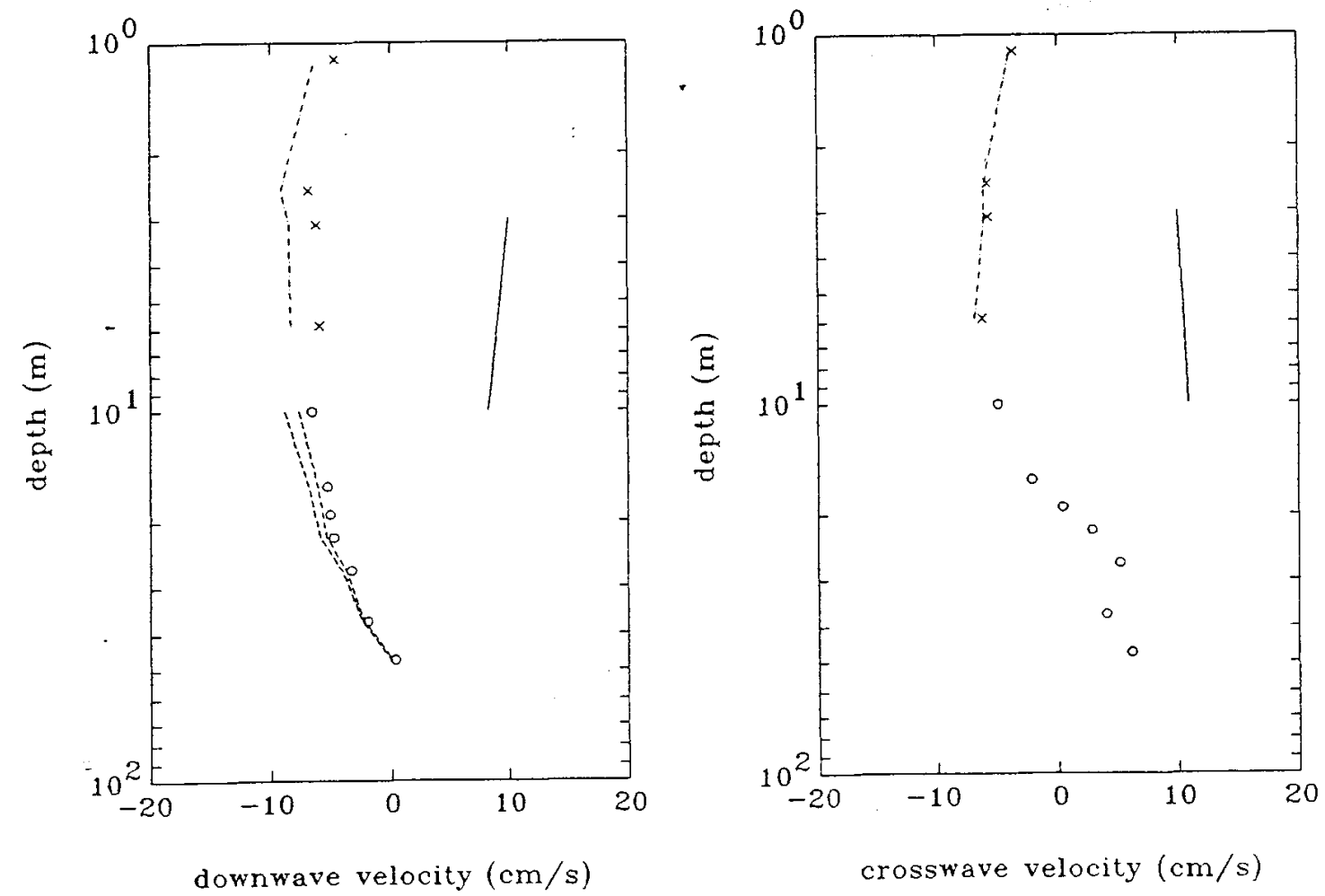


\section{file $\mathrm{k} 1$}

date $=$ February $24,1989 \quad$ time $=06-11-56.50 \quad$ duration $=00-39-17.75$

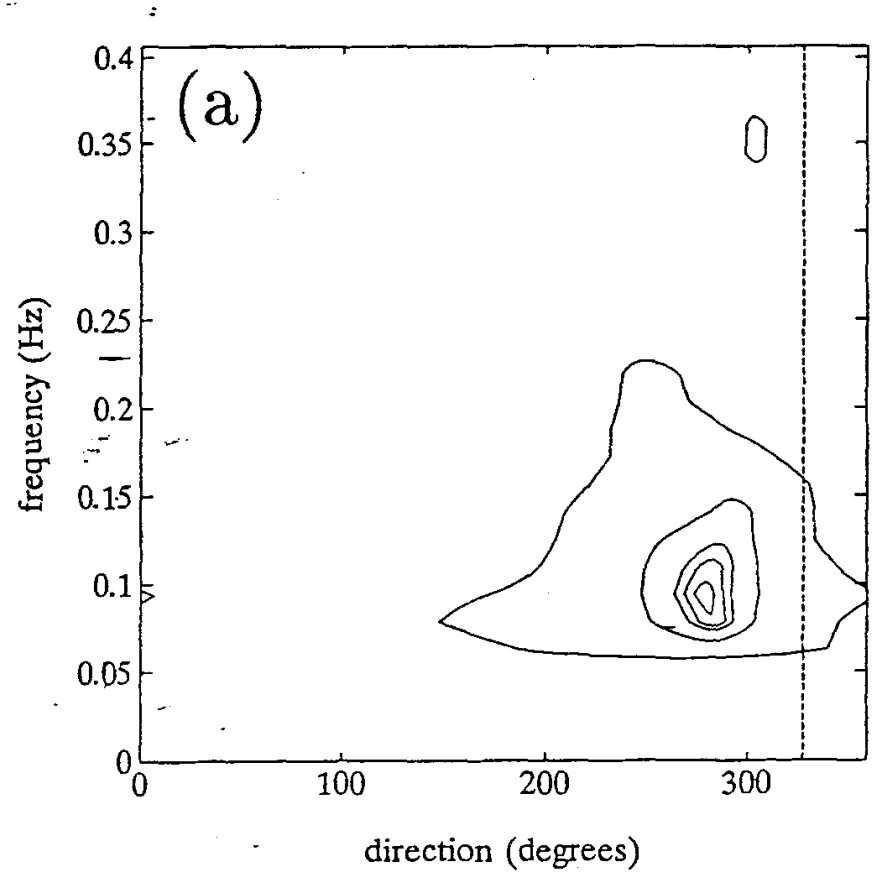

$$
\begin{array}{lc}
\multicolumn{1}{c}{\text { wind }} & \multicolumn{1}{c}{\text { waves }} \\
U_{10}=3.7 \mathrm{~m} / \mathrm{s} & \bar{H}_{1 / 3}=2.3 \mathrm{~m} \\
\theta_{\tau}=328^{\circ} & \theta_{w}=279^{\circ} \\
\frac{z}{L}=-0.29 & T_{p}=10.7 \mathrm{sec} \\
u_{*_{a}}=12.2 \mathrm{~cm} / \mathrm{s} & \bar{T}=7.5 \mathrm{sec} \\
\multicolumn{2}{c}{\Delta_{\text {temp }}=-0.35 \mathrm{C}}
\end{array}
$$

\section{(a) directional spectrum}

(b) energy spectrum $\left(-\cdots=f^{-4}\right)$

(c) $o=$ salinity, $x,+=$ temperature
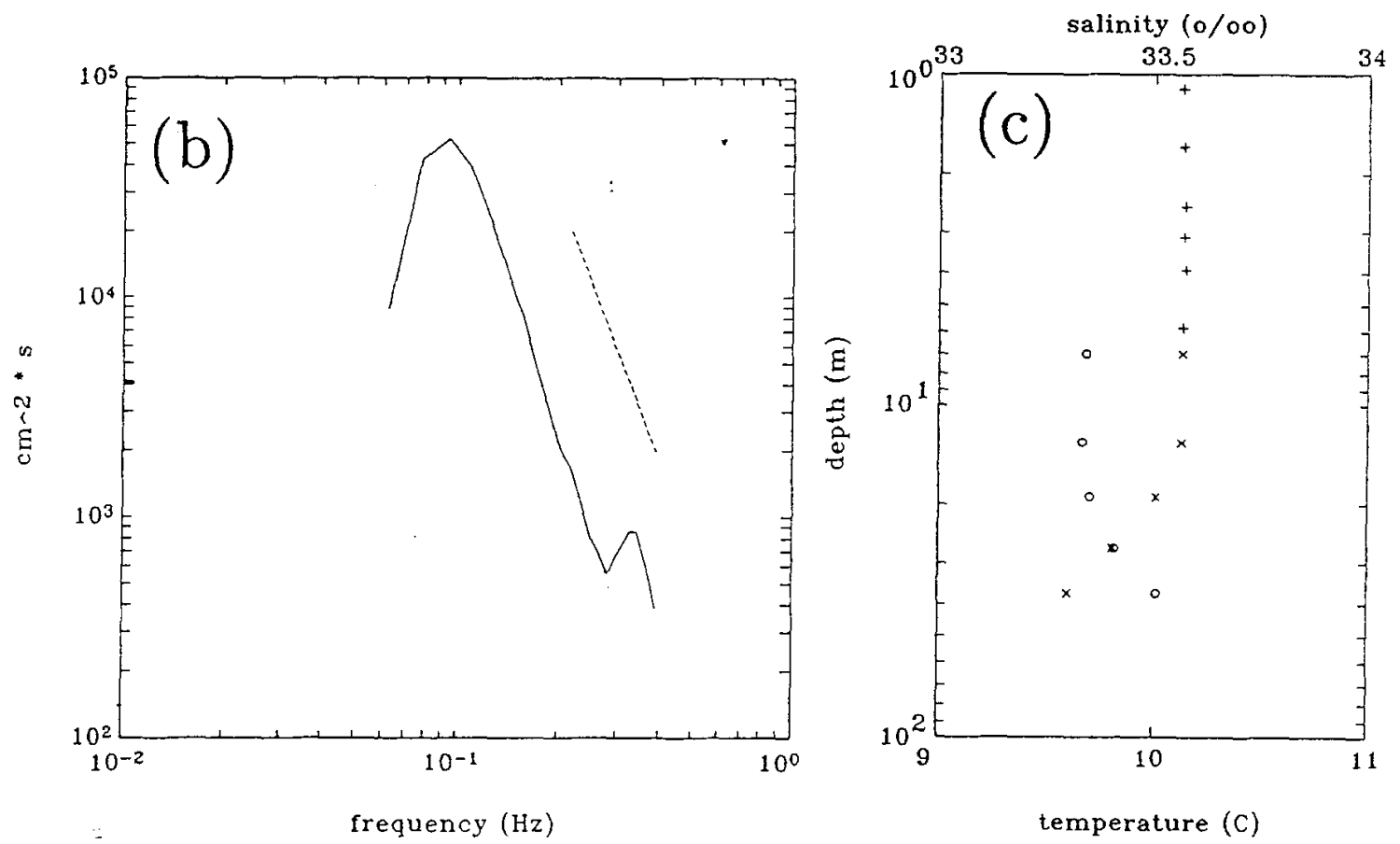


\section{file $\mathrm{k} 1$}

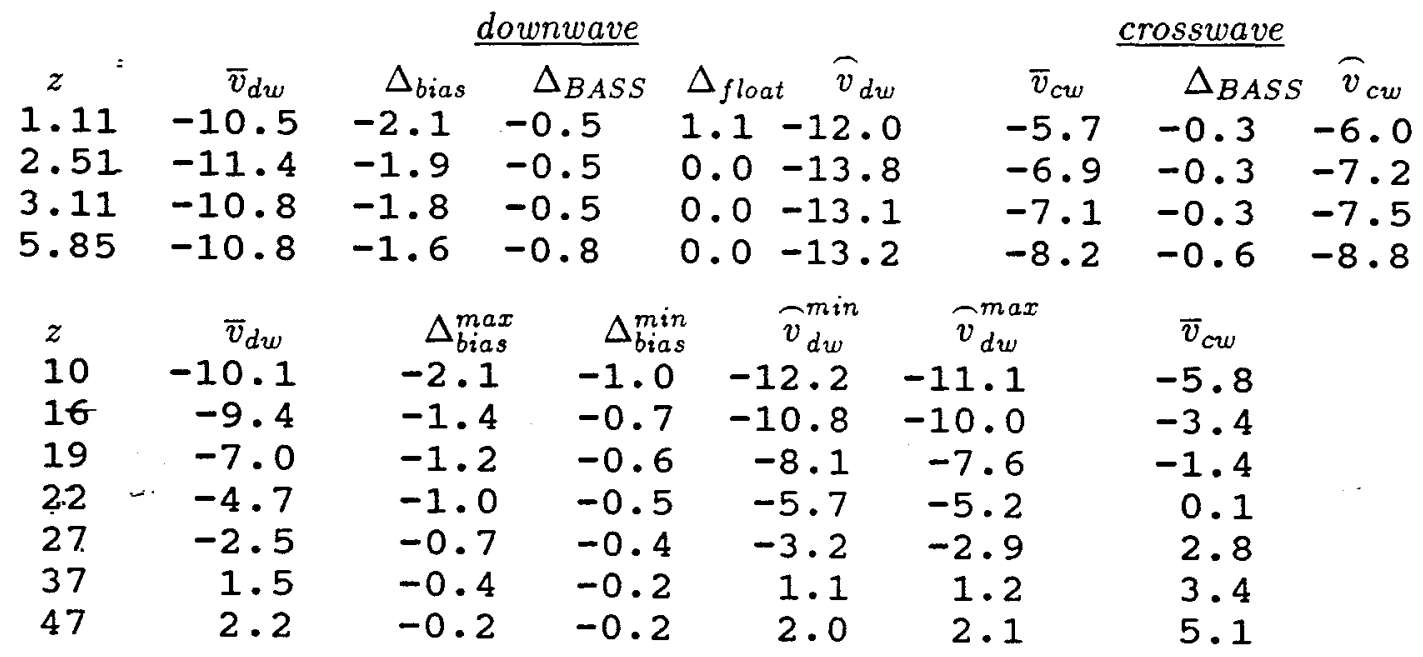

Below: $\mathrm{SASS}=x($ raw $) \cdots(-\cdots$ (unbiased), C3=o (raw) - - (unbiased), theoretical log-law shear $=-$
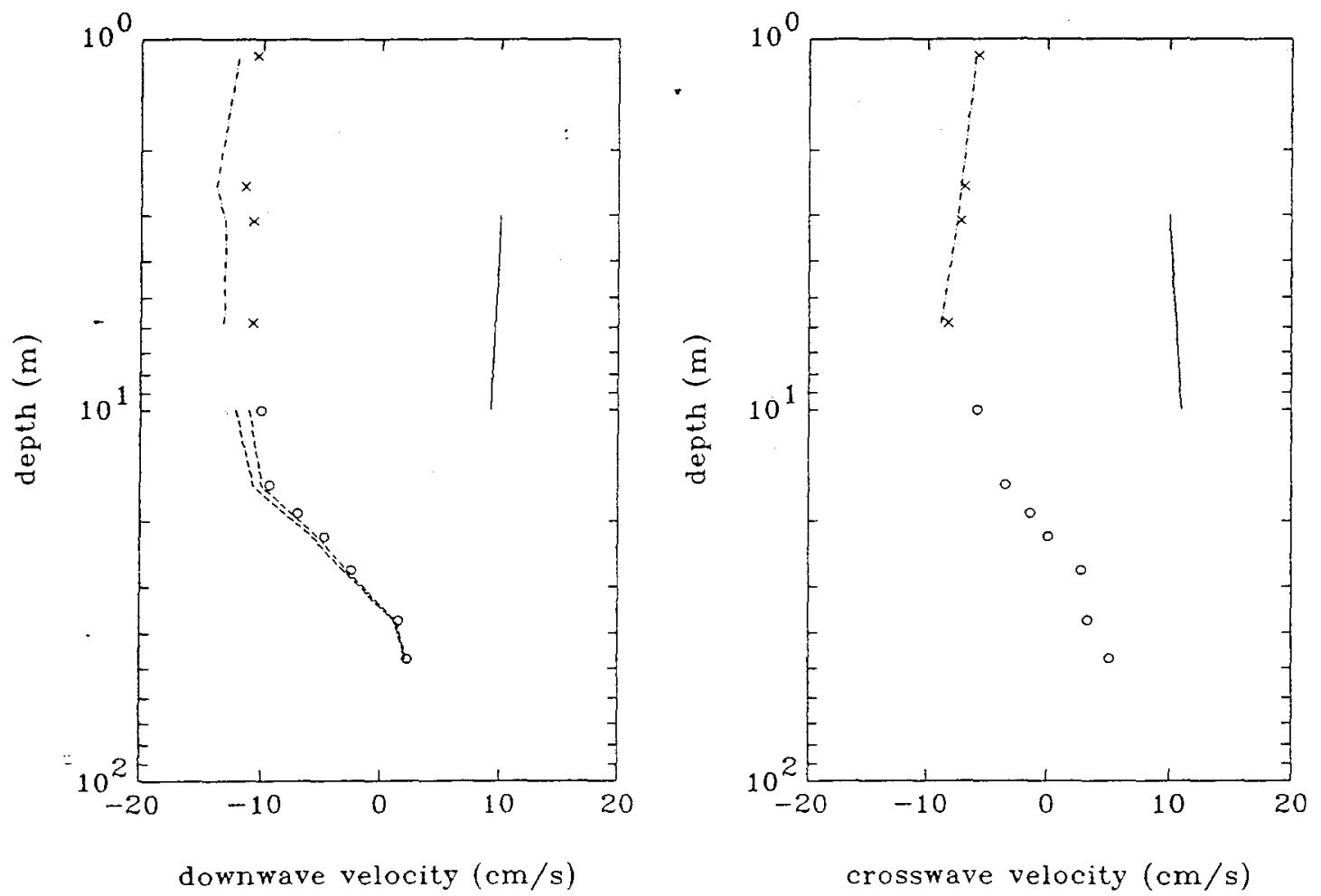


\section{file $\mathrm{k} 2$}

$$
\text { date }=\text { February } 24,1989 \quad \text { time }=06-51-14.25 \quad \text { duration }=00-39-17.50
$$

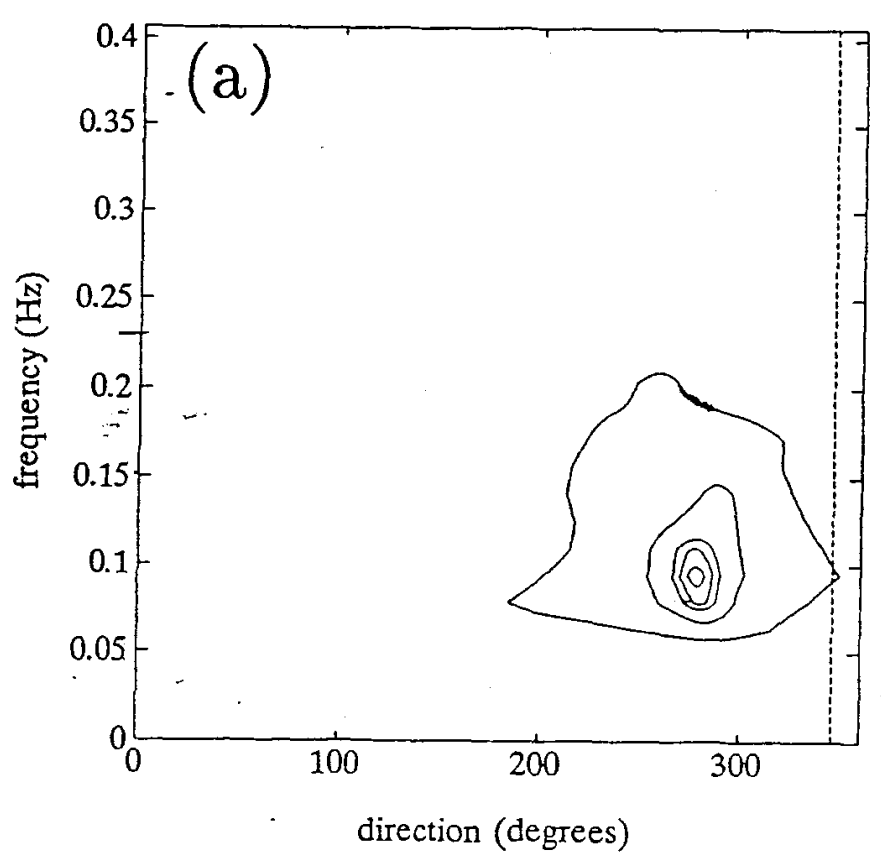

$$
\begin{array}{cc}
\underline{\text { wind }} & \multicolumn{1}{c}{\text { waves }} \\
U_{10}=2.3 \mathrm{~m} / \mathrm{s} & \bar{H}_{1 / 3}=2.3 \mathrm{~m} \\
\theta_{\tau}=346^{\circ} & \theta_{w}=278^{\circ} \\
\frac{z}{L}=-0.74 & T_{p}=10.7 \mathrm{sec} \\
u_{* a}=7.4 \mathrm{~cm} / \mathrm{s} & \bar{T}=7.8 \mathrm{sec} \\
\Delta_{\text {temp }}=-0.35 \mathrm{C}
\end{array}
$$

(a) directional spectrum

(b) energy spectrum $\left(-\cdots=f^{-4}\right)$

(c) $o=$ salinity, $x,+=$ temperature
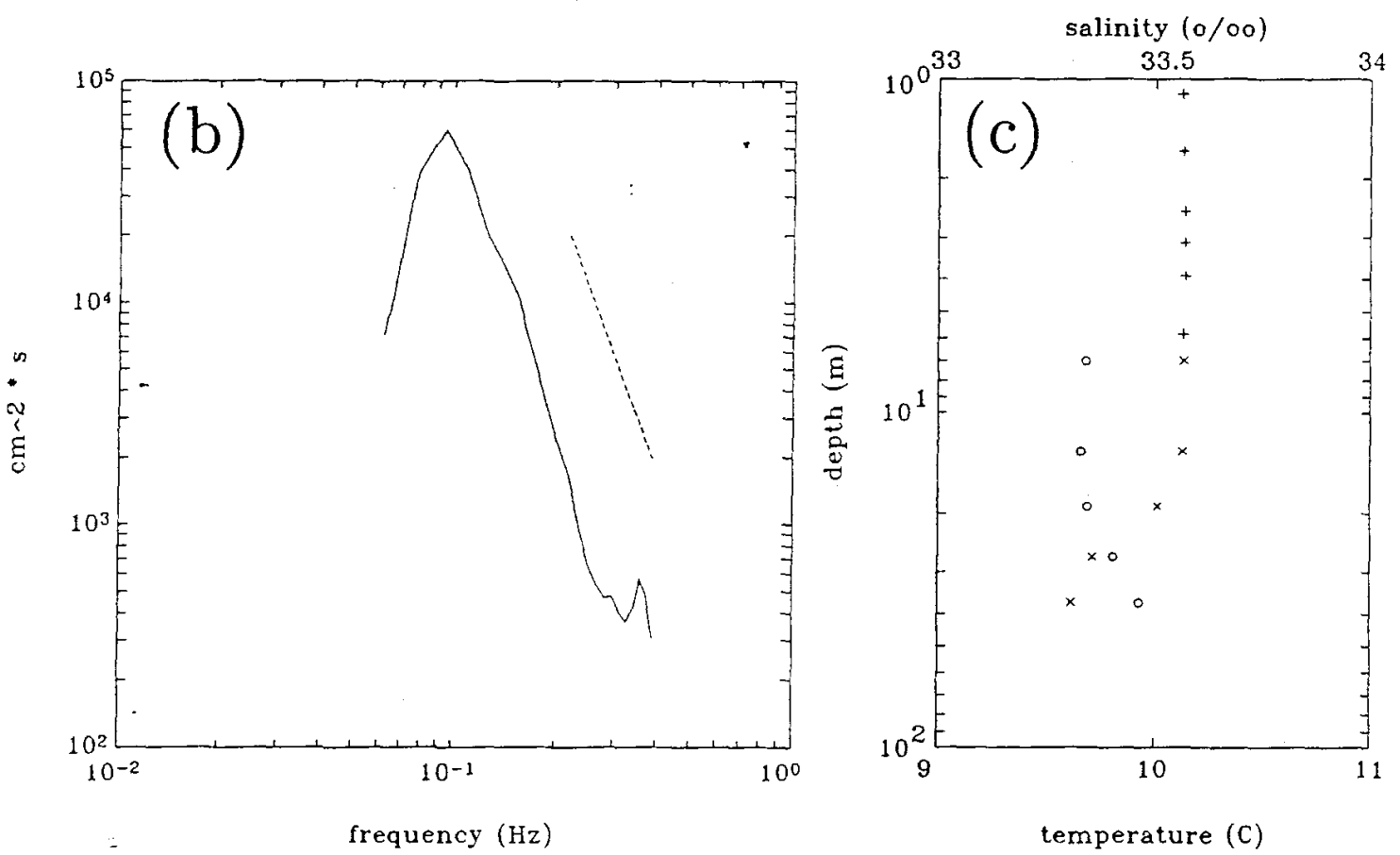


\section{file $\mathrm{k} 2$}

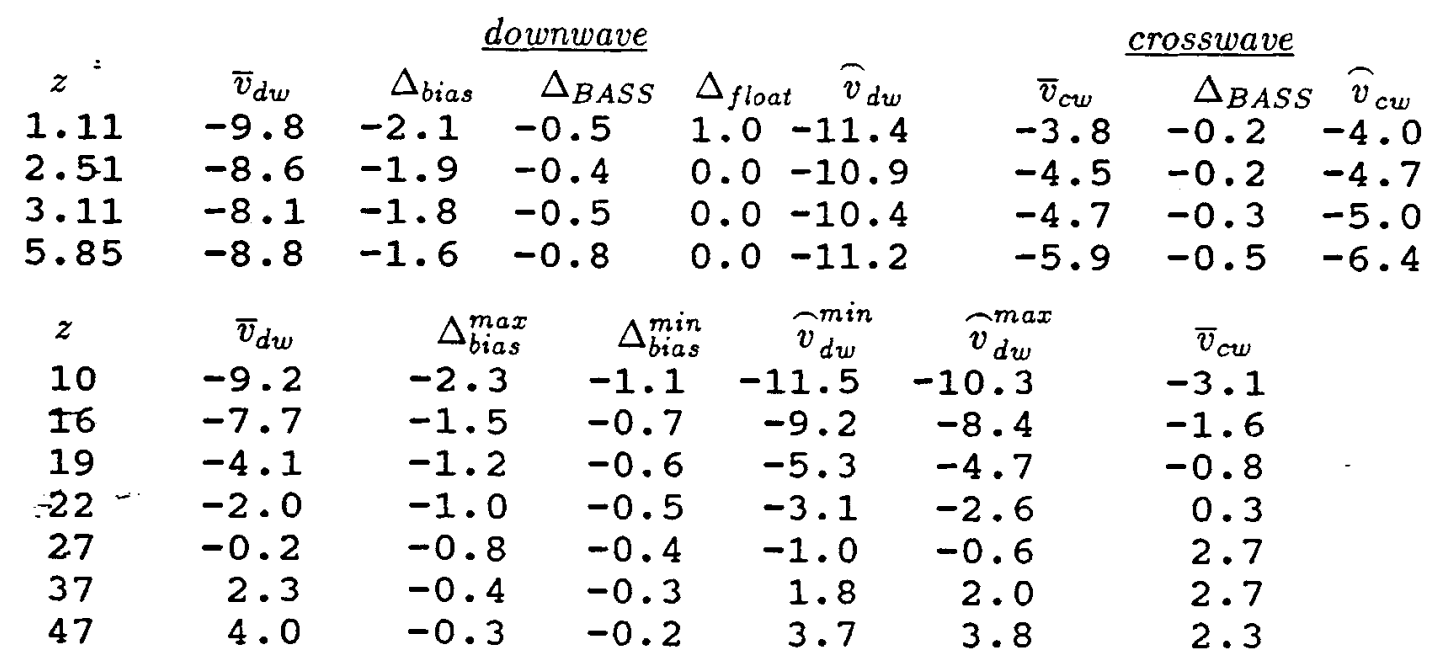

Below: $\mathrm{SASS}=x(\mathrm{raw}) \cdots($ unbiased $), \mathrm{C} 3=o(\mathrm{raw}) \cdots($ unbiased $)$, theoretical log-law shear $=-$
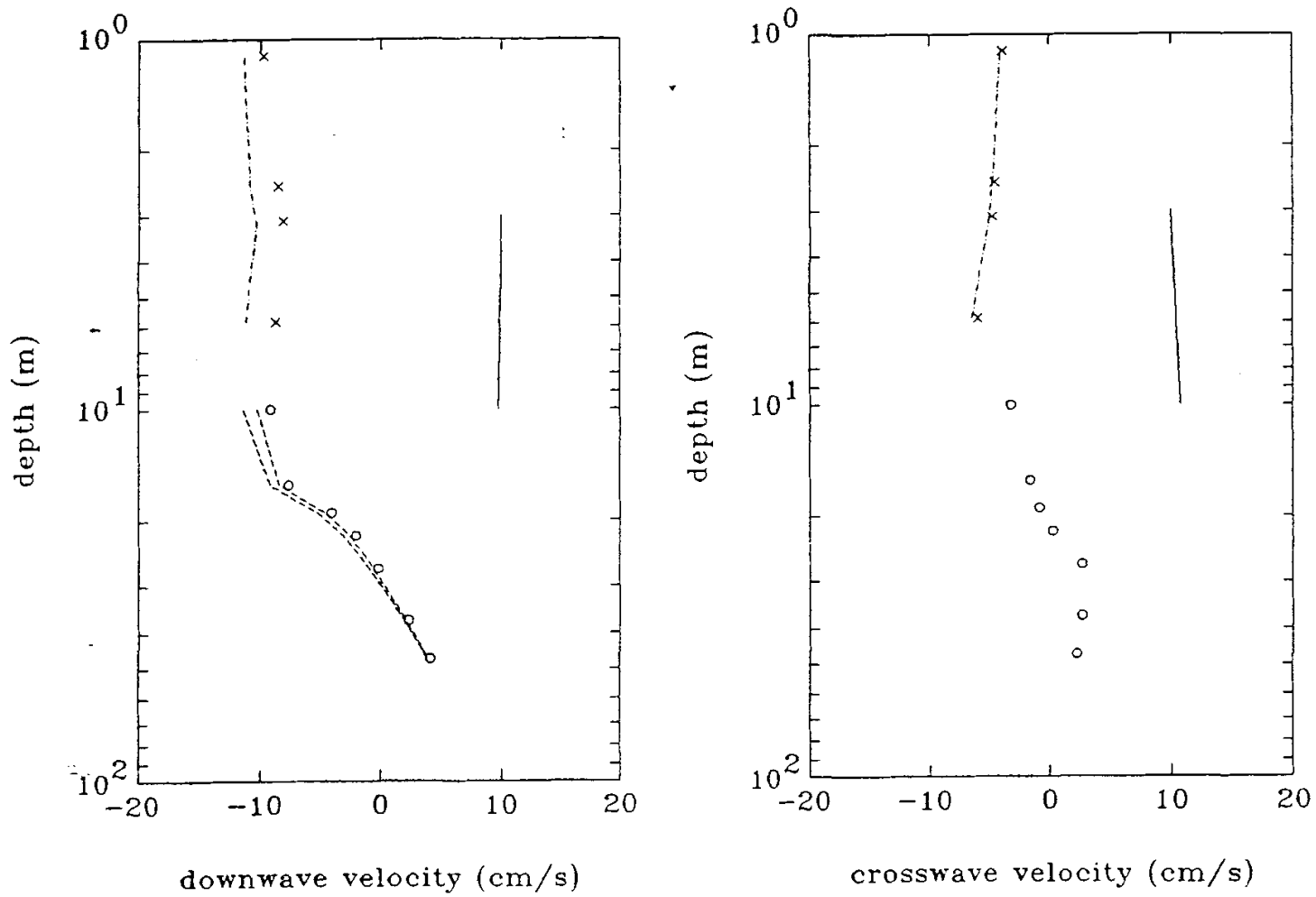


\section{file $\mathrm{k} 3$}

$$
\text { date }=\text { February } 24,1989 \quad \text { time }=07-30-31.75 \quad \text { duration }=00-39-17.50
$$

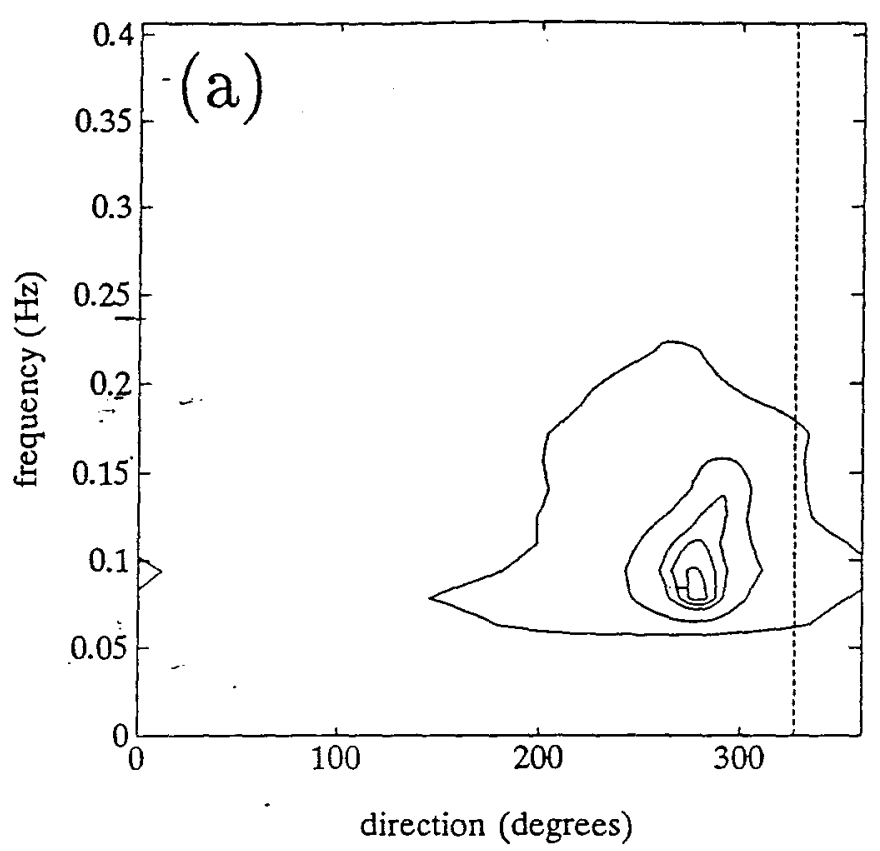

$$
\begin{array}{lc}
\multicolumn{1}{c}{\underline{\text { wind }}} & \underline{\text { waves }} \\
U_{10}=3.3 \mathrm{~m} / \mathrm{s} & \bar{H}_{1 / 3}=2.3 \mathrm{~m} \\
\theta_{\tau}=327^{\circ} & \theta_{w}=276^{\circ} \\
\frac{z}{L}=-0.36 & T_{p}=10.7 \mathrm{sec} \\
u_{*_{a}}=10.8 \mathrm{~cm} / \mathrm{s} & \bar{T}=7.9 \mathrm{sec} \\
\multicolumn{2}{c}{\Delta_{\text {temp }}=-0.35 \mathrm{C}}
\end{array}
$$

(a) directional spectrum $\left(-\cdots=\theta_{\tau}\right)$

(b) energy spectrum $\left(-\cdots=f^{-4}\right)$

(c) $\mathrm{o}=$ salinity, $\mathrm{x},+=$ temperature
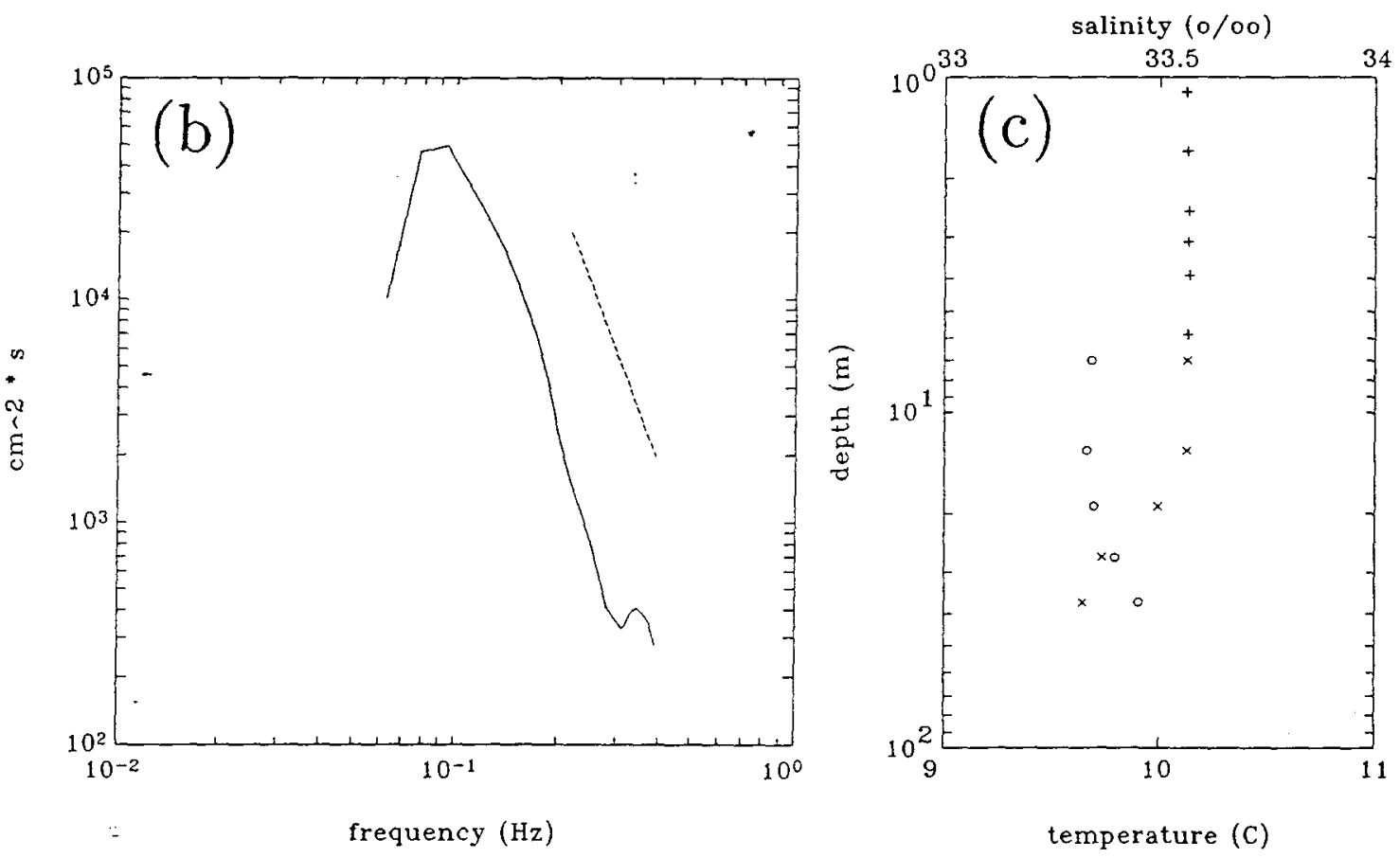


\section{file $\mathrm{k} 3$}

\begin{tabular}{|c|c|c|c|c|c|c|c|}
\hline \multirow[b]{2}{*}{$\begin{array}{c}z \\
1.11 \\
2.51 \\
3.11 \\
5.85\end{array}$} & \multicolumn{4}{|c|}{$\underline{\text { downwave }}$} & \multicolumn{3}{|c|}{ crosswave } \\
\hline & $\begin{array}{l}\bar{v}_{d w} \\
-5.7 \\
-7.8 \\
-6.9 \\
-7.3\end{array}$ & $\begin{aligned} & \Delta_{\text {bias }} \\
&-2.0-1.8 \\
&-1.8-1.8 \\
&-1.6\end{aligned}$ & $\begin{array}{rl} & \Delta_{B A S S} \\
-0.5 & 0.5 \\
-0.4 & -0.5 \\
- & 0.8\end{array}$ & $\begin{array}{lr}\Delta_{\text {float }} & \widehat{v}_{d w} \\
0.9 & -7.3 \\
0.0 & -10.1 \\
0.0 & -9.1 \\
0.0 & -9.8\end{array}$ & $\begin{array}{l}\bar{v}_{c w} \\
-1.3 \\
-2.5 \\
-2.7 \\
-3.8\end{array}$ & $\begin{aligned} & \Delta_{B A S S} \\
- & 0.1 \\
- & 0.1 \\
- & 0.2 \\
- & 0.4\end{aligned}$ & $\begin{aligned} & \widehat{v}_{c w} \\
&-1.4 \\
&-2.6 \\
&-2.9 \\
&-4.3\end{aligned}$ \\
\hline$z$ & $\bar{v}_{d w}$ & $\Delta_{b i a}^{m a}$ & $\Delta_{b i c}^{m}$ & $\widehat{v}_{d w}^{\min }$ & $\widetilde{v}_{d w}^{\max }$ & $\bar{v}_{c w}$ & \\
\hline $\begin{array}{l}10 \\
16 \\
19 \\
22 \\
27 \\
37 \\
47\end{array}$ & $\begin{array}{r}-6.4 \\
-5.4 \\
-1.6 \\
-0.8 \\
0.9 \\
1.7 \\
3.4\end{array}$ & $\begin{array}{l}-2.2 \\
-1.4 \\
-1.2 \\
-1.0 \\
-0.7 \\
-0.4 \\
-0.2\end{array}$ & $\begin{array}{l}-1.1 \\
-0.7 \\
-0.6 \\
-0.5 \\
-0.4 \\
-0.2 \\
-0.2\end{array}$ & $\begin{array}{r}-8.7 \\
-6.8 \\
-2.8 \\
-1.8 \\
0.2 \\
1.3 \\
3.12\end{array}$ & $\begin{array}{r}-7.5 \\
-6.1 \\
-2.2 \\
-1.3 \\
0.5 \\
1.4 \\
3.2\end{array}$ & $\begin{array}{r}-3.2 \\
-2.0 \\
-1.1 \\
0.4 \\
2.9 \\
2.2 \\
1.6\end{array}$ & - \\
\hline
\end{tabular}

Below: SASS $=x(\mathrm{raw})-\ldots$ (unbiased), C3=o(raw) - - (unbiased $),$ theoretical log-law shear $=-$
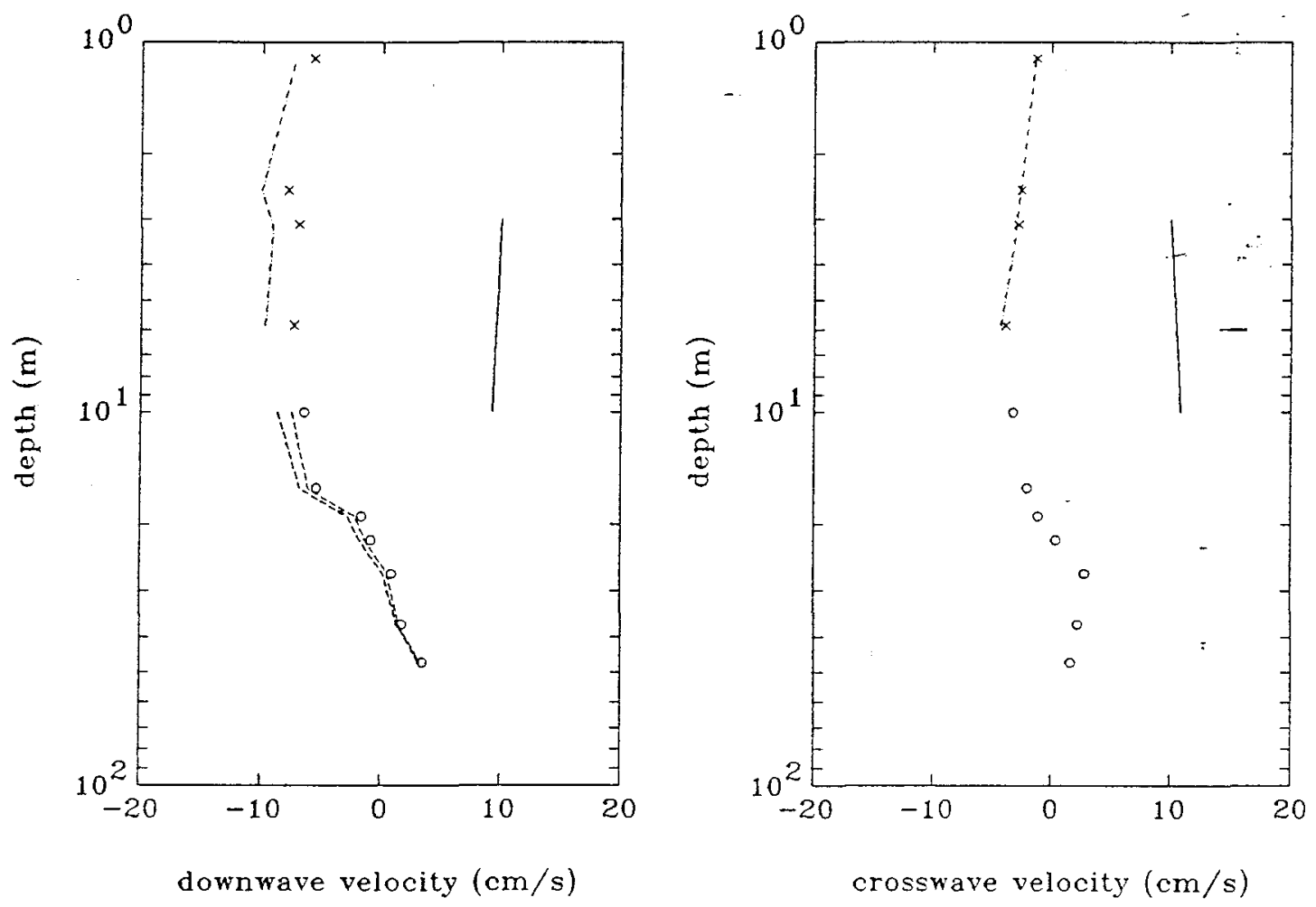


\section{file 11}

date $=$ February 24, $1989 \quad$ time $=16-23-44.25 \quad$ duration $=00-39-17.75$

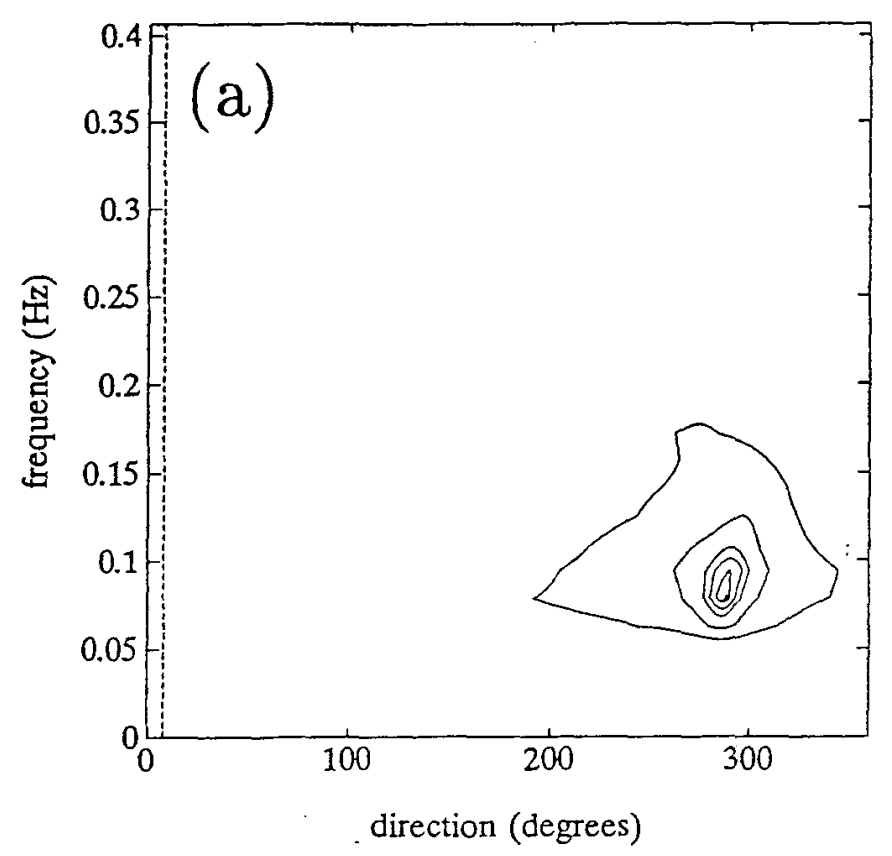

$$
\begin{array}{lc}
\underline{\text { wind }} & \multicolumn{1}{c}{\text { waves }} \\
U_{10}=2.3 \mathrm{~m} / \mathrm{s} & \bar{H}_{1 / 3}=2.6 \mathrm{~m} \\
\theta_{\tau}=8^{\circ} & \theta_{w}=286^{\circ} \\
\bar{L}=-0.51 & T_{p}=10.7 \mathrm{sec} \\
u_{*_{\alpha}}=7.1 \mathrm{~cm} / \mathrm{s} & \bar{T}=8.5 \mathrm{sec} \\
\multicolumn{2}{c}{\Delta_{\text {temp }}=-0.35 \mathrm{C}}
\end{array}
$$

(a) directional spectrum $\left(--=\theta_{\tau}\right)$

(b) energy spectrum $\left(-\cdots=f^{-4}\right)$

(c) $\mathrm{o}=$ salinity, $\mathrm{x},+=$ temperature
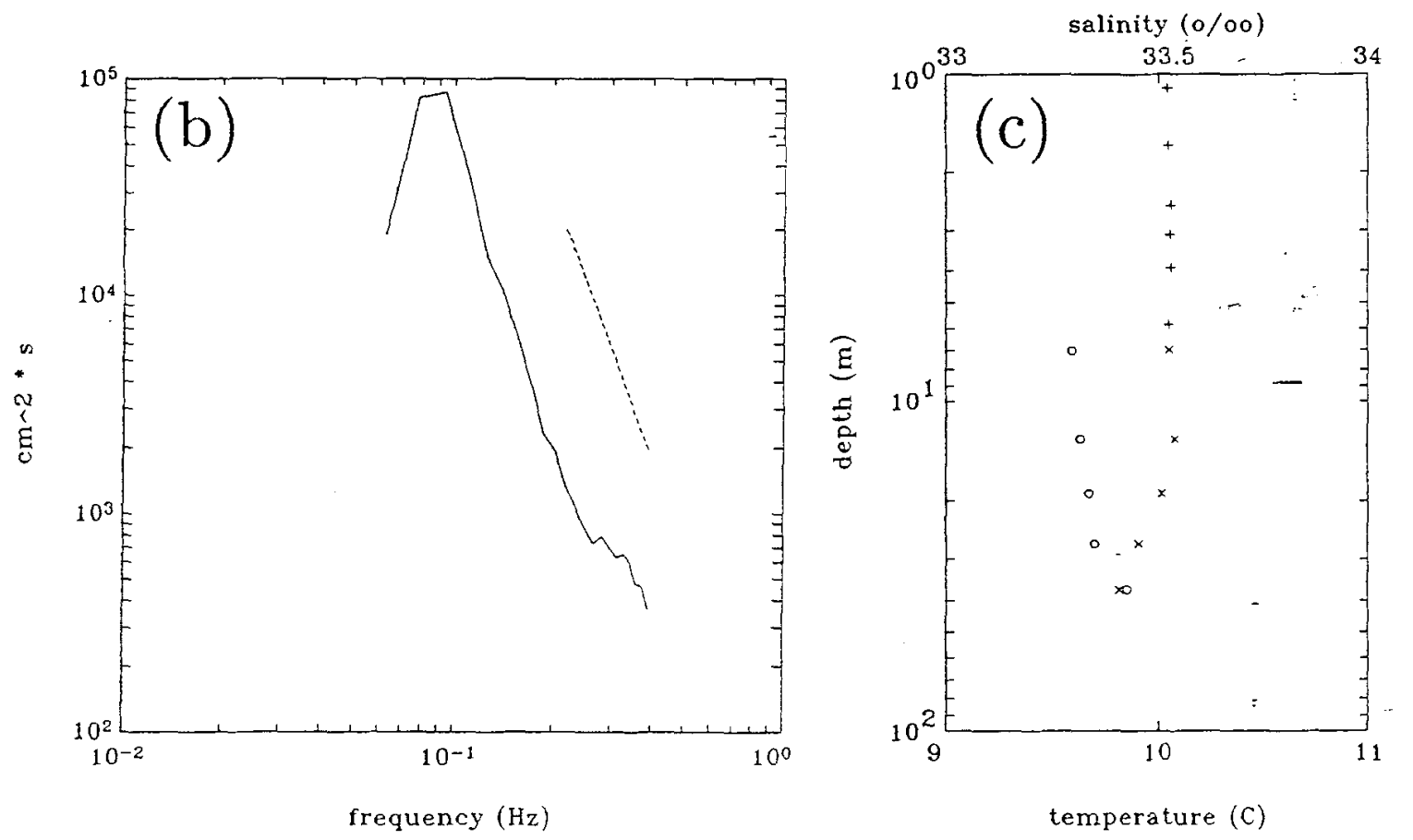


\section{file 11}

\begin{tabular}{|c|c|c|c|c|c|c|c|c|}
\hline \multirow[b]{2}{*}{$\begin{array}{c}z \\
1.11 \\
2.51 \\
3.11 \\
5.85\end{array}$} & \multicolumn{5}{|c|}{ downwave } & \multicolumn{3}{|c|}{ crosswave } \\
\hline & $\begin{array}{r}\bar{v}_{d w} \\
1.9 \\
1.6 \\
2.1 \\
-0.0\end{array}$ & $\begin{aligned} & \Delta_{\text {bias }} \\
&-2.2-2.0 \\
&-2.0-1.9 \\
&-1.7\end{aligned}$ & $\begin{array}{rl} & \Delta_{B A S S} \\
- & 0.4 \\
-0.3 & 0.3 \\
0.3 & 0.0\end{array}$ & $\begin{array}{l}\Delta_{\text {float }} \\
1.0 \\
0.0 \\
0.0 \\
0.0\end{array}$ & $\begin{array}{r}\widehat{v}_{d w} \\
0.3 \\
-0.6 \\
0.5 \\
-1.7\end{array}$ & $\begin{array}{l}\bar{v}_{c w} \\
-1.1 \\
-1.3 \\
-1.4 \\
-1.4\end{array}$ & $\begin{array}{l}\Delta_{B A S S} \\
0.2 \\
0.2 \\
-0.2 \\
-0.7\end{array}$ & $\begin{array}{r}\widehat{v}_{c w} \\
-0.9 \\
-1.1 \\
-1.7 \\
-2.1\end{array}$ \\
\hline $\begin{array}{l}z \\
10 \\
16 \\
19 \\
22 \\
27 \\
37 \\
47\end{array}$ & $\begin{array}{r}\bar{v}_{d w} \\
7.5 \\
10.9 \\
11.3 \\
9.5 \\
8.7 \\
7.2 \\
0.8\end{array}$ & $\begin{array}{r}\Delta_{b i o}^{m c} \\
-2.5 \\
-1.8 \\
-1.5 \\
-1.3 \\
-1.0 \\
-0.6 \\
-0.4\end{array}$ & $\begin{array}{l}\Delta_{b}^{n} \\
-1 . \\
-0 . \\
-0 . \\
-0 . \\
-0 . \\
-0 . \\
-0 .\end{array}$ & & $\begin{array}{l}\widehat{v}_{d w}^{\min } \\
5.0 \\
9.1 \\
9.8 \\
8.2 \\
7.7 \\
6.6 \\
0.4\end{array}$ & $\begin{array}{r}\widehat{v}_{d w}^{\max } \\
6.4 \\
10.0 \\
10.6 \\
8.8 \\
8.1 \\
6.8 \\
0.5\end{array}$ & $\begin{array}{l}\bar{v}_{c w} \\
-2.7 \\
-3.0 \\
-1.5 \\
-2.6 \\
-4.2 \\
-3.6 \\
-1.7\end{array}$ & - \\
\hline
\end{tabular}

Below: SASS $=x($ raw $) \cdots($ unbiased $), C 3=o($ raw $) \cdots($ unbiased $)$, theoretical log-law shear $=-\cdots$.
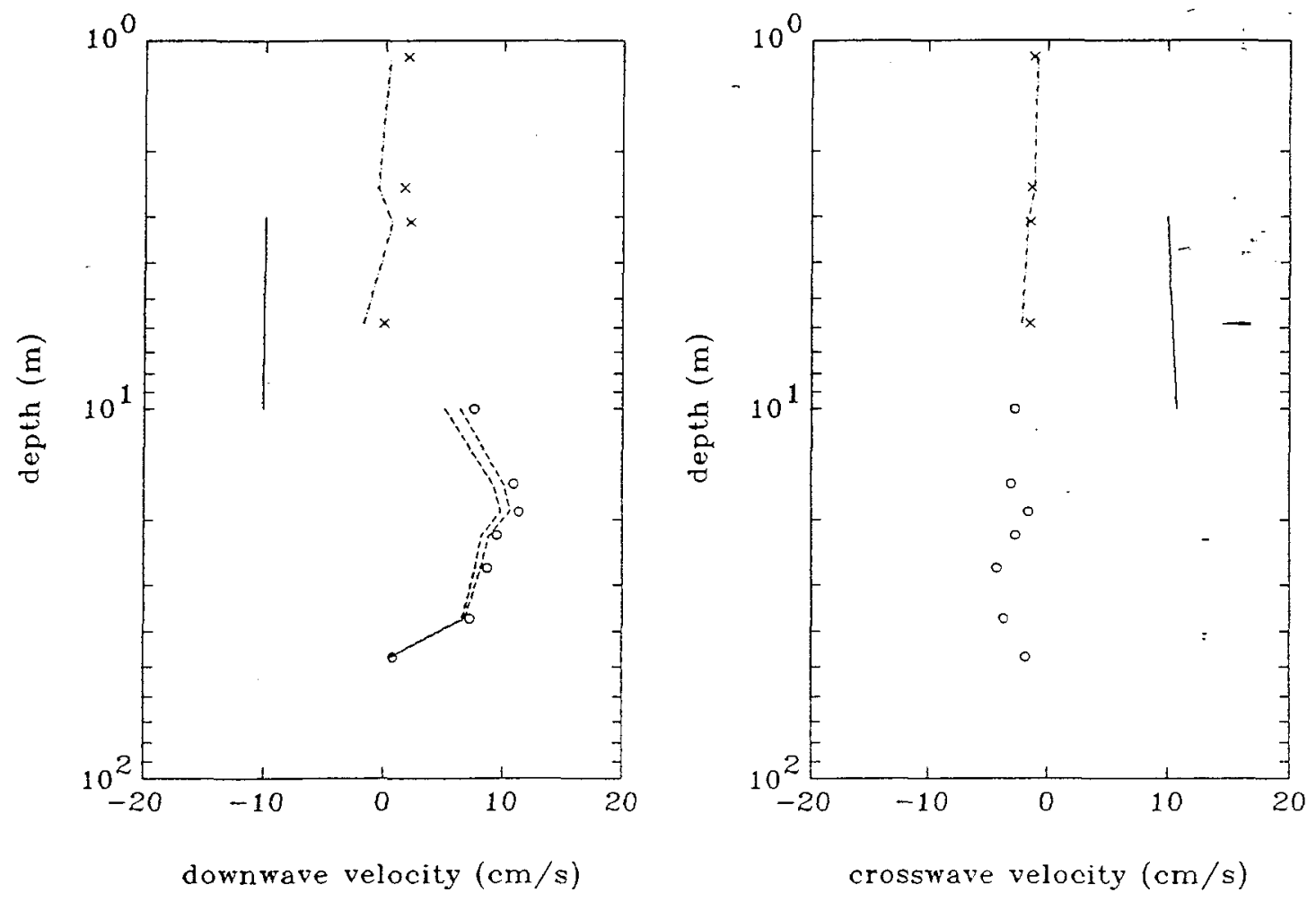


\section{file 12}

date $=$ February 24, $1989 \quad$ time $=17-03-02.00 \quad$ duration $=00-39-17.75$

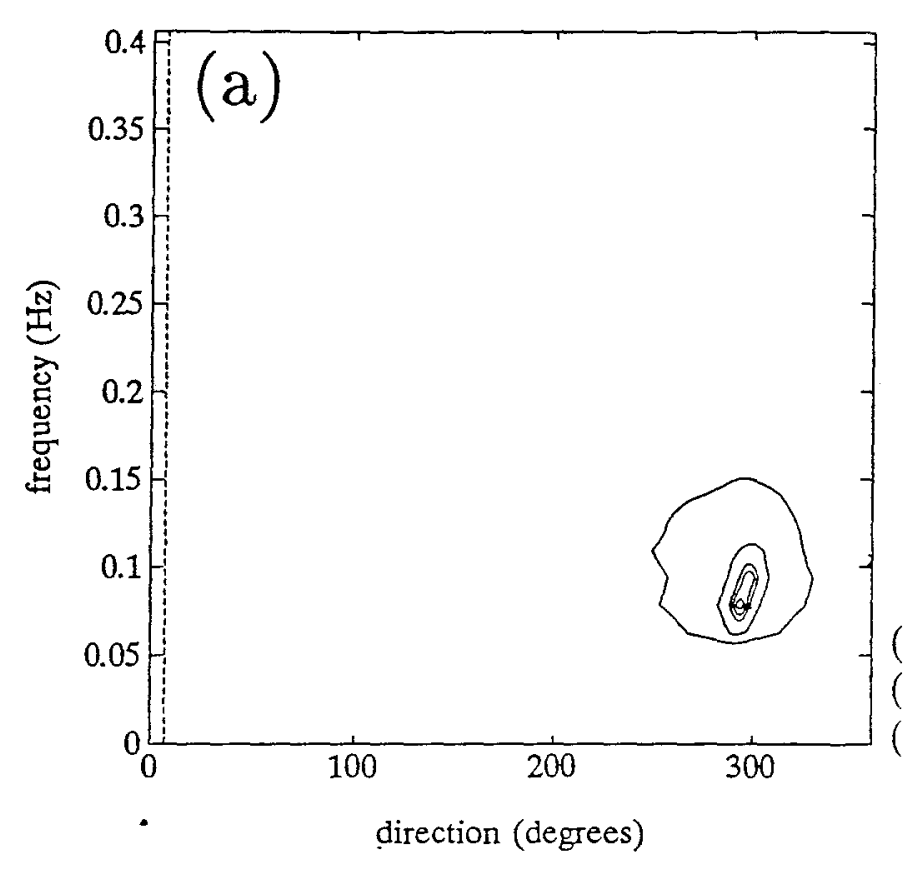

$$
\begin{array}{lc}
\text { wind } & \multicolumn{1}{c}{\text { waves }} \\
U_{10}=2.0 \mathrm{~m} / \mathrm{s} & \bar{H}_{1 / 3}=3.0 \mathrm{~m} \\
\theta_{\tau}=7^{\circ} & \theta_{w}=295^{\circ} \\
\frac{z}{L}=-0.68 & T_{p}=10.7 \mathrm{sec} \\
u_{* a}=6.1 \mathrm{~cm} / \mathrm{s} & \bar{T}=9.2 \mathrm{sec} \\
\Delta_{\text {temp }}=-0.37 \mathrm{C}
\end{array}
$$

(a) directional spectrum $\left(-\cdots=\theta_{\tau}\right)$

(b) energy spectrum $\left(-\cdots=f^{-4}\right)$

(c) $\mathrm{o}=$ salinity, $\mathrm{x},+=$ temperature
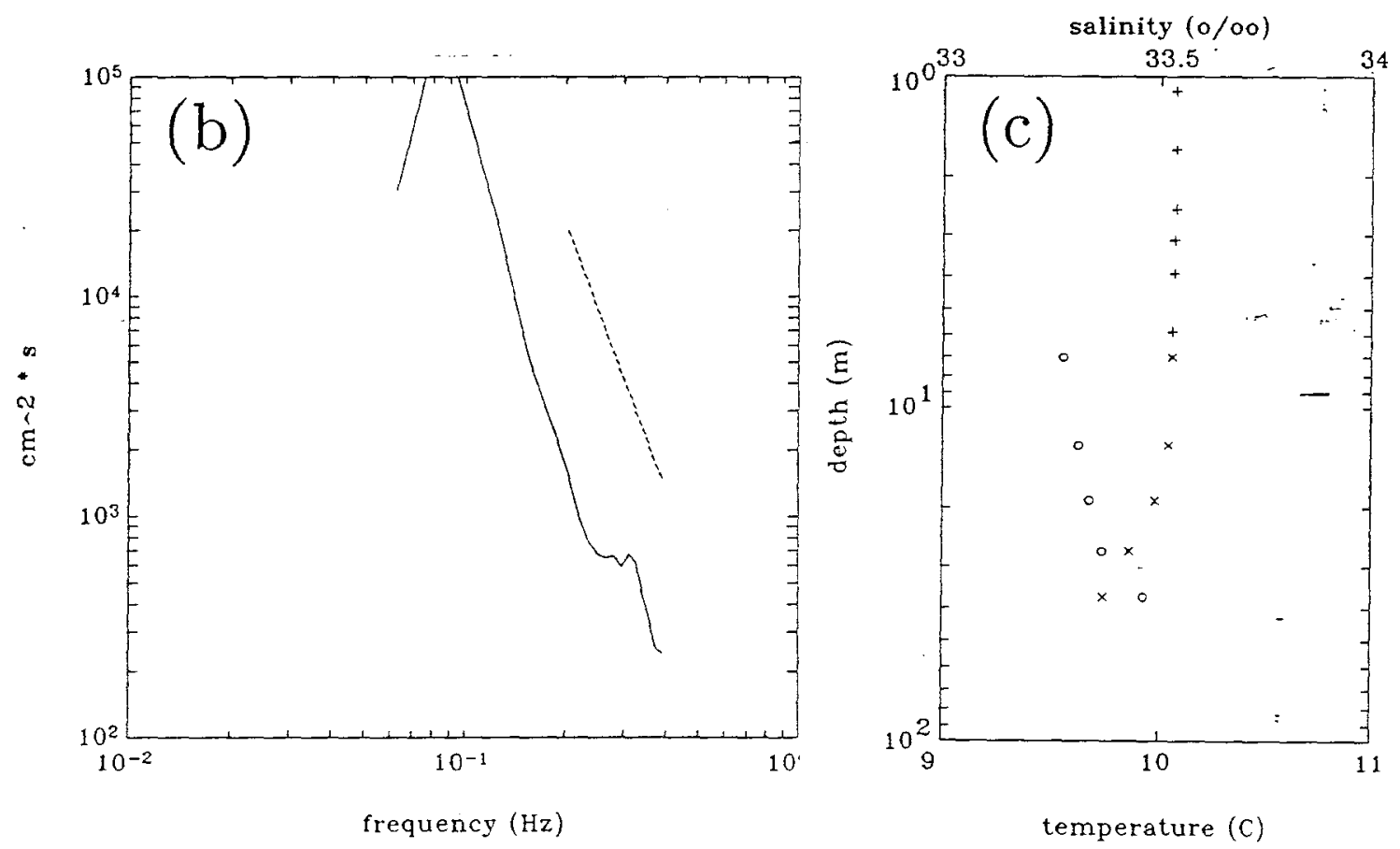


\section{file 12}

\begin{tabular}{|c|c|c|c|c|c|c|c|c|}
\hline \multirow[b]{2}{*}{$\begin{array}{l}z \\
1.11 \\
2.51 \\
3.11 \\
5.85\end{array}$} & \multicolumn{5}{|c|}{ downwave } & \multicolumn{3}{|c|}{ crosswave } \\
\hline & $\begin{array}{l}\bar{v}_{d w} \\
5.5 \\
8.2 \\
8.0 \\
4.2\end{array}$ & $\begin{aligned} & \Delta_{\text {bias }} \\
&-2.3-2.2 \\
&-2-2.1 \\
&-1.9\end{aligned}$ & $\begin{array}{l}\triangle_{B A S S} \\
0.5 \\
0.5 \\
0.5 \\
0.8\end{array}$ & $\begin{array}{l}\Delta_{\text {float }} \\
1.0 \\
0.0 \\
0.0 \\
0.0\end{array}$ & $\begin{array}{l}\widehat{v}_{d w} \\
4.6 \\
6.5 \\
6.4 \\
3.1\end{array}$ & $\begin{array}{c}\bar{v}_{c w} \\
-2.3 \\
-3.2 \\
-2.7 \\
-2.5\end{array}$ & $\begin{aligned} & \Delta_{B A S S} \\
&-0.2-0.2 \\
&-0.2-0.5 \\
&-0.5\end{aligned}$ & $\begin{array}{l}\widehat{v}_{c w} \\
-2.4 \\
-3.4 \\
-2.9 \\
-3.0\end{array}$ \\
\hline $\begin{array}{l}z \\
10 \\
16 \\
19 \\
22 \\
27 \\
37 \\
47\end{array}$ & $\begin{array}{r}\bar{v}_{d w} \\
7.3 \\
7.6 \\
7.8 \\
6.3 \\
5.3 \\
1.4 \\
-4.5\end{array}$ & $\begin{aligned} & \Delta_{\text {bias }}^{\max } \\
-3.1 & -1 \\
-2.3 & -1.9 \\
-1.7 & -1.3 \\
-0.8 & -0.5\end{aligned}$ & $\begin{array}{r}\Delta_{b i a}^{m i} \\
-1.4 \\
-1.1 \\
-1.0 \\
-0.8 \\
-0.7 \\
-0.5 \\
-0.3\end{array}$ & $\begin{array}{r}\widehat{v} \\
4 \\
5 \\
5 \\
4 \\
4 \\
0 \\
-5\end{array}$ & $\begin{array}{l}\min \\
d w \\
.2 \\
.3 \\
9 \\
.7 \\
7 \\
0 \\
6 \\
0\end{array}$ & $\begin{array}{c}\widehat{v}_{d w}^{\max } \\
5.9 \\
6.5 \\
6.9 \\
5.5 \\
4.6 \\
0.9 \\
-4.8\end{array}$ & $\begin{array}{c}\bar{v}_{c w} \\
-4.7 \\
-4.7 \\
-3.6 \\
-4.8 \\
-4.9 \\
-2.5 \\
-1.4\end{array}$ & - \\
\hline
\end{tabular}

Below: SASS $=x($ raw $) \cdots($ unbiased $), C 3=o($ raw $)--($ unbiased $)$, theoretical log-law shear $=-$
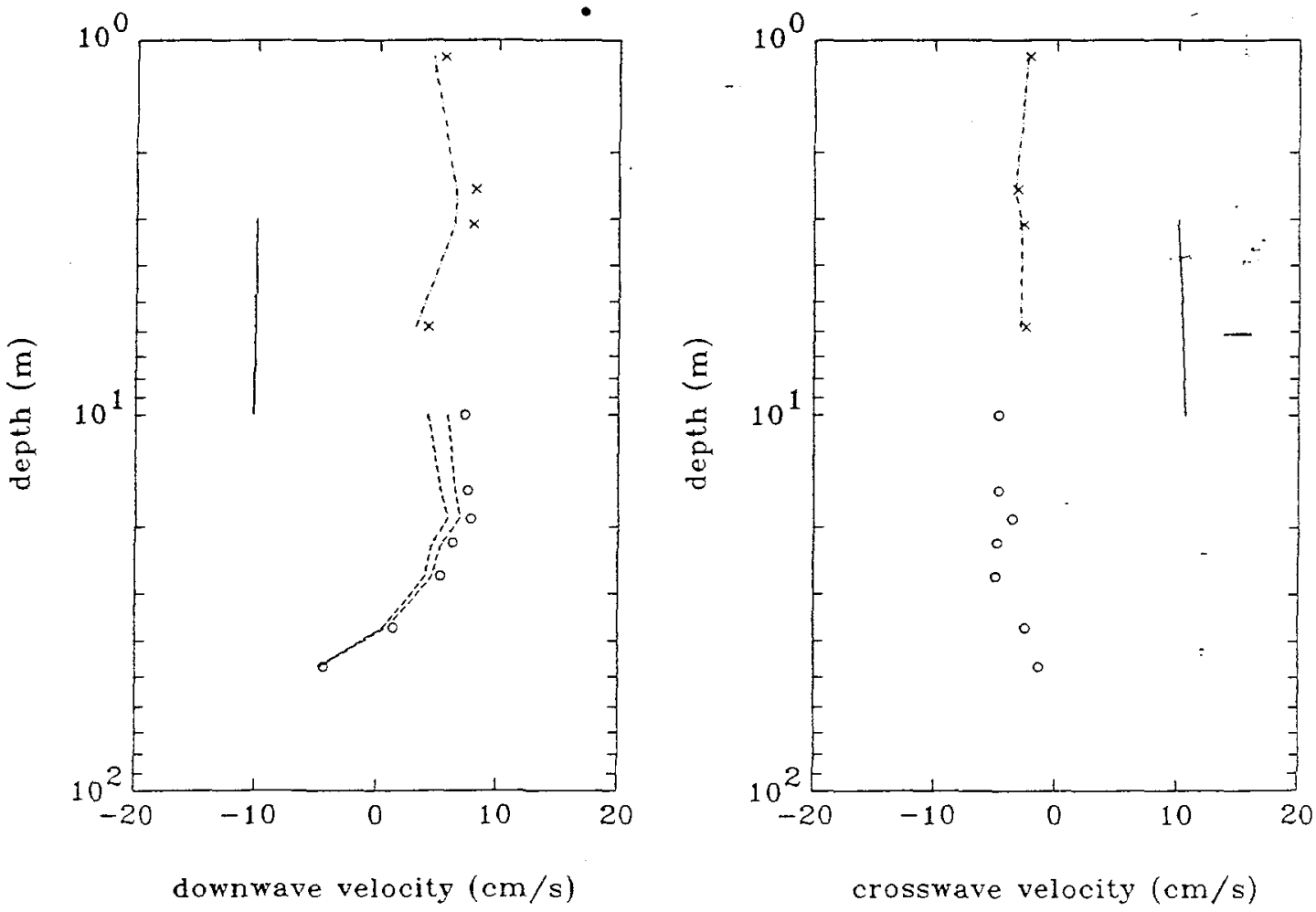


\section{file 13}

$$
\text { date }=\text { February } 24,1989 \quad \text { time }=17-42-19.75 \quad \text { duration }=00-39-17.50
$$

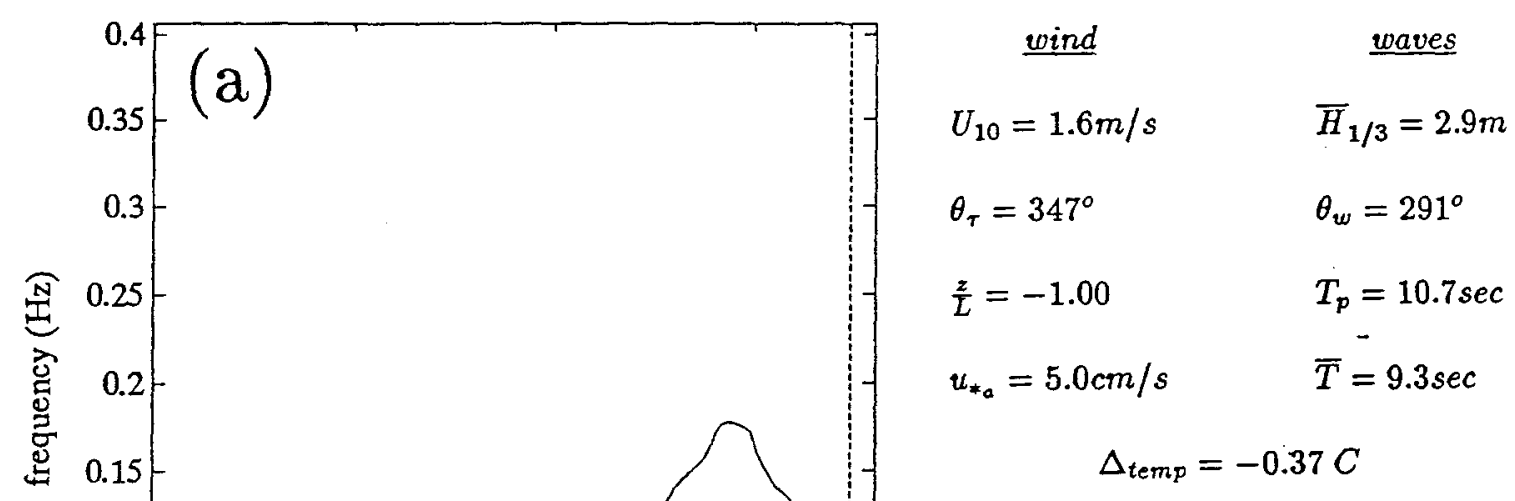

(a) directional spectrum

(b) energy spectrum $\left(-\cdots=f^{-4}\right)$

(c) $o=$ salinity, $x,+=$ temperature

direction (degrees)
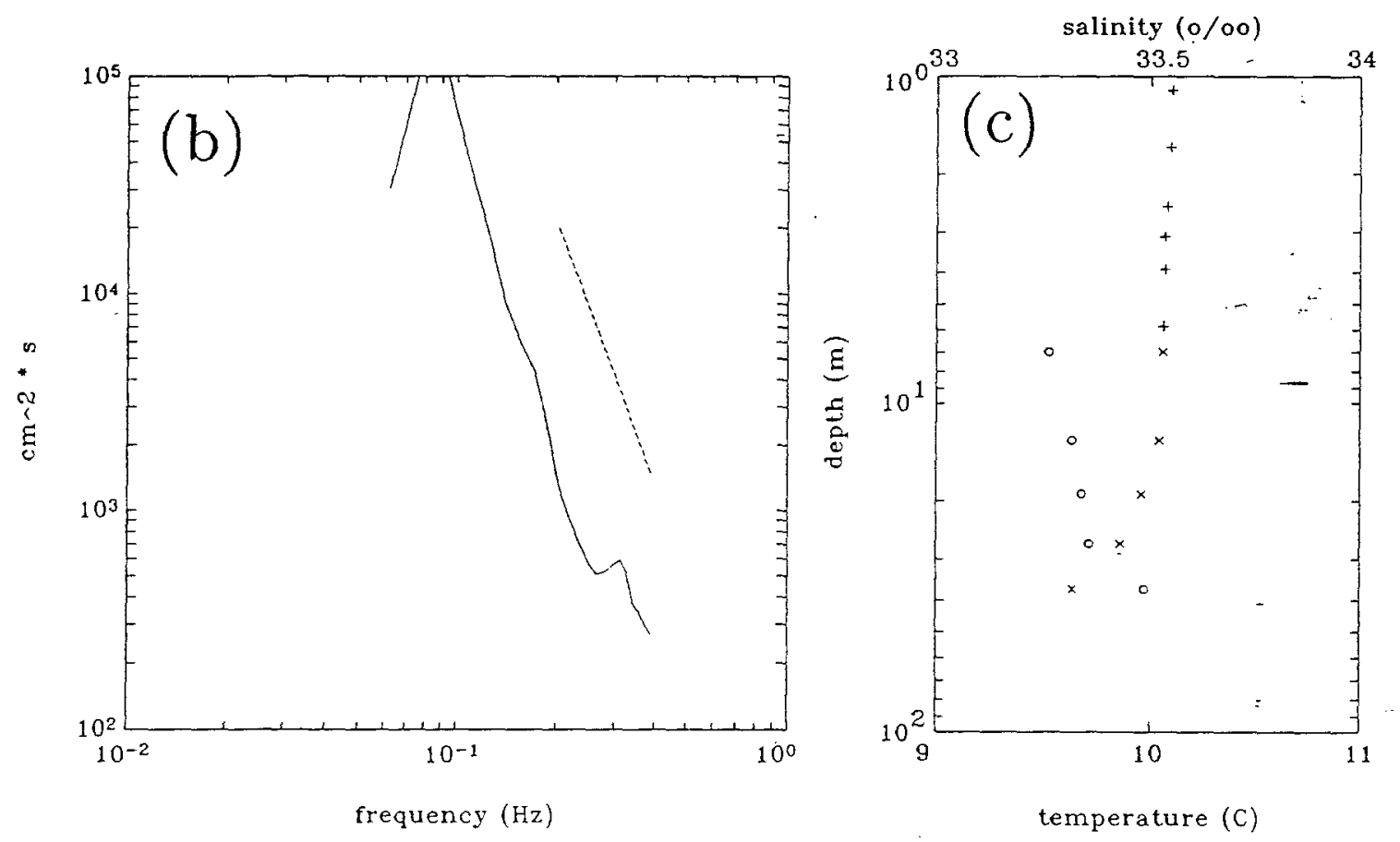


\section{file 13}

\begin{tabular}{ccccccccc} 
& \multicolumn{9}{c}{ downwave } & \multicolumn{3}{c}{ crosswave } \\
$z$ & $\bar{v}_{d w}$ & $\Delta_{\text {bias }}$ & $\Delta_{\text {BASS }}$ & $\Delta_{\text {float }}$ & $\bar{v}_{d w}$ & $\bar{v}_{c w}$ & $\Delta_{B A S S}$ & $\widehat{v}_{c w}$ \\
1.11 & 4.1 & -2.2 & 0.3 & 0.9 & 3.1 & -4.5 & -0.3 & -4.8 \\
2.51 & 5.8 & -2.1 & 0.3 & 0.0 & 4.1 & -5.7 & -0.3 & -6.0 \\
3.11 & 5.8 & -2.0 & 0.4 & 0.0 & 4.2 & -5.1 & -0.3 & -5.5 \\
5.85 & 3.2 & -1.8 & 0.5 & 0.0 & 1.9 & -4.2 & -0.7 & -4.8
\end{tabular}

$z \quad \bar{v}_{d w} \quad \Delta_{b i a s}^{\max } \quad \Delta_{b i a s}^{\min } \quad \widehat{v}_{d w}^{\min } \widehat{v}_{d w}^{\max } \quad \bar{v}_{c w}$

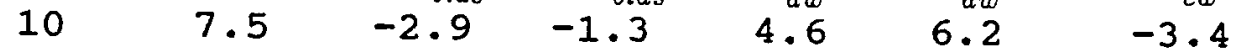

$16 \quad 6.2 \quad-2.1 \quad-1.0 \quad 4.1 \quad 5.2 \quad-1.9$

$\begin{array}{lllllll}19 & 6.4 & -1.8 & -0.9 & 4.6 & 5.5 & -2.0\end{array}$

$\begin{array}{lllllll}22 & 6.1 & -1.6 & -0.8 & 4.5 & 5.3 & -2.7\end{array}$

$\begin{array}{lllllll}27 & 4.7 & -1.2 & -0.7 & 3.5 & 4.0 & -4.6\end{array}$

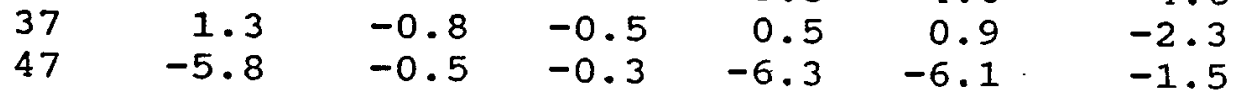

Below: $S A S S=x$ (raw) -..- (unbiased), C3=o(raw) - - (unbiased), theoretical log-law shear $=-$
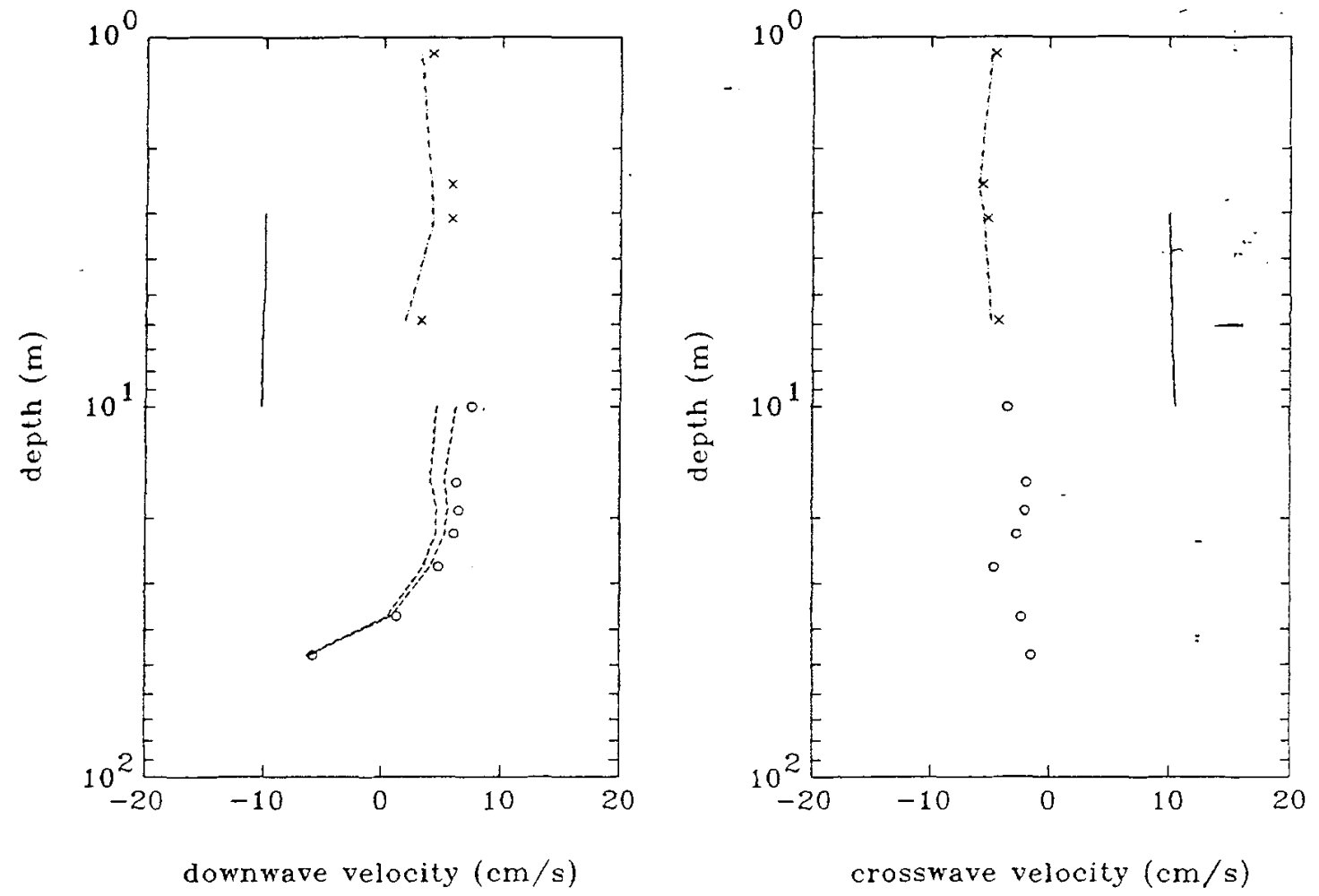


\section{file $m 1$}

date $=$ February $26,1989 \quad$ time $=06-55-08.75 \quad$ duration $=00-38-48.50$

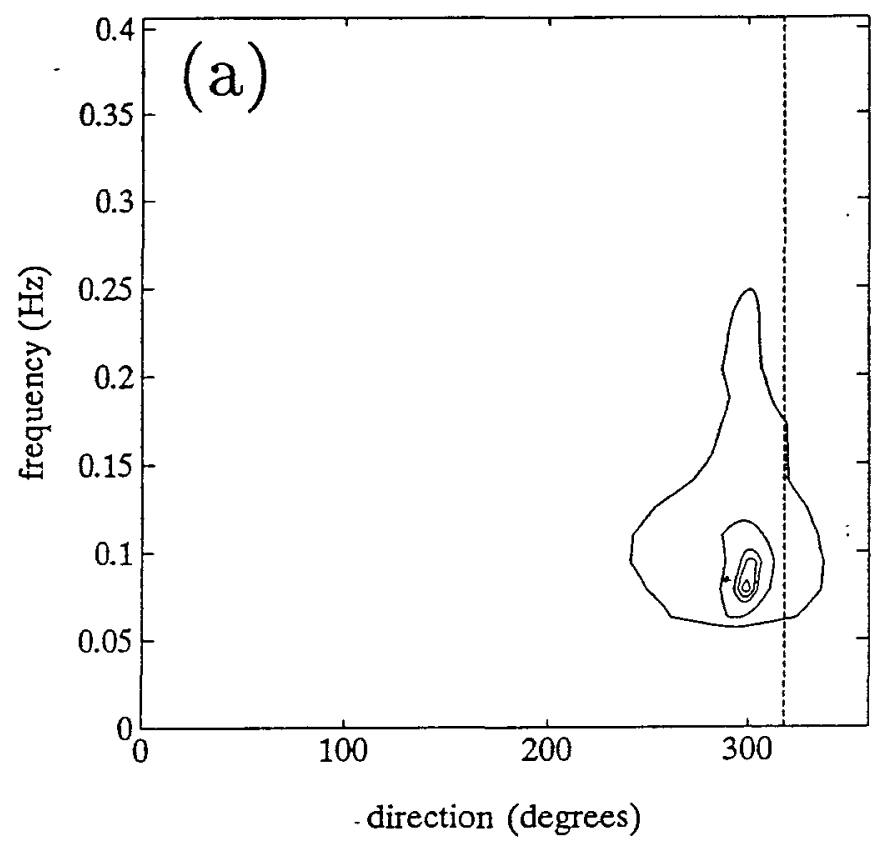

$$
\begin{array}{cc}
\underline{\text { wind }} & \multicolumn{1}{c}{\underline{\underline{\text { waves }}}} \\
U_{10}=6.1 \mathrm{~m} / \mathrm{s} & \bar{H}_{1 / 3}=2.3 \mathrm{~m} \\
\theta_{\tau}=317^{\circ} & \theta_{w}=298^{\circ} \\
\frac{z}{L}=-0.03 & T_{p}=10.7 \mathrm{sec} \\
u_{*_{a}}=21.2 \mathrm{~cm} / \mathrm{s} & \bar{T}=7.4 \mathrm{sec} \\
\multicolumn{2}{c}{\Delta_{\text {temp }}=-0.35 \mathrm{C}}
\end{array}
$$

(a) directional spectrum $\left(--=\theta_{\tau}\right)$

(b) energy spectrum $\left(-\cdots=f^{-4}\right)$

(c) $o=$ salinity, $x,+=$ temperature
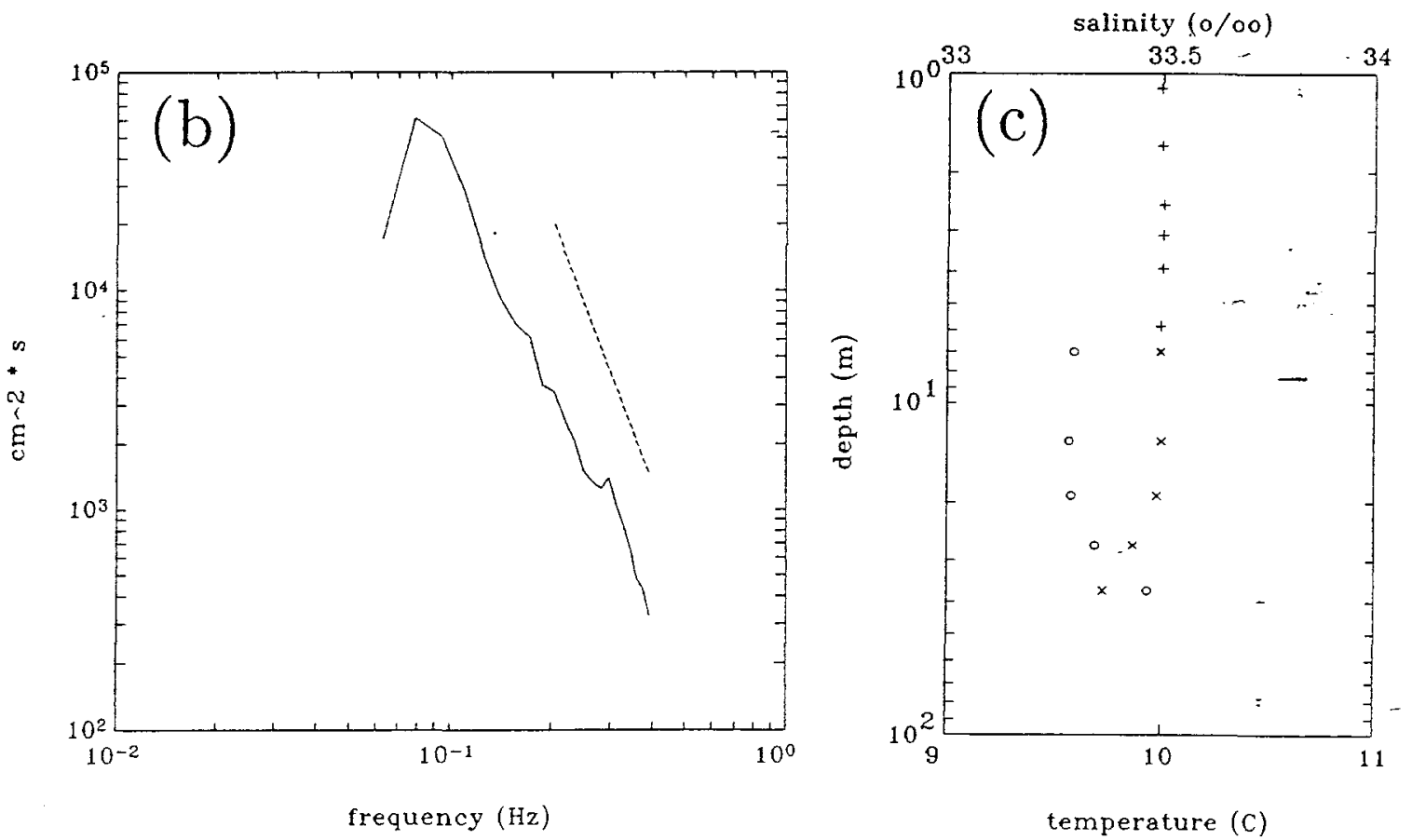


\section{file $\mathrm{m} 1$}

\begin{tabular}{|c|c|c|c|c|c|c|c|c|}
\hline \multirow{6}{*}{$\begin{array}{c}z \\
1.11 \\
2.51 \\
3.11 \\
5.85\end{array}$} & \multicolumn{5}{|c|}{ downwave } & \multicolumn{3}{|c|}{ crosswave } \\
\hline & $\bar{v}_{d w}$ & $\Delta_{\text {bias }}$ & $\Delta_{B A S S}$ & $\Delta_{\text {float }}$ & $\widehat{v}_{d w}$ & $\bar{v}_{c w}$ & $\Delta_{B A S S}$ & $\widehat{v}_{c w}$ \\
\hline & 2.4 & -2.7 & -0.2 & 1.1 & 0.6 & -5.2 & 0.4 & -4.8 \\
\hline & 3.8 & $-2 \cdot 2$ & 0.2 & 0.0 & 1.8 & -6.1 & -0.4 & -6.5 \\
\hline & 4.6 & $-2 \cdot 1$ & 0.3 & 0.0 & 2.7 & -6.2 & -0.4 & -6.7 \\
\hline & 1.1 & -1.8 & 0.1 & 0.0 & -0.6 & -6.2 & -0.7 & -6.9 \\
\hline$z$ & $\bar{v}_{d w}$ & $\Delta_{b i c}^{m}$ & $\Delta_{b_{1}}^{m}$ & & $\widehat{v}_{d w}^{\min }$ & $\widehat{v}_{d w}^{\max }$ & $\bar{v}_{c w}$ & \\
\hline 10 & 1.9 & -2.2 & -1 & & -0.3 & 0.8 & -6.0 & \\
\hline 16 & 1.1 & -1.4 & -0 & & -0.3 & 0.4 & -2.6 & - \\
\hline 19 & 1.4 & -1.2 & -0 & & 0.3 & 0.9 & 0.2 & \\
\hline 22 & 3.1 & -1.0 & -0 & & 2.2 & 2.6 & 1.2 & \\
\hline 27 & 7.8 & -0 & -0 & & 7.1 & 7.4 & 1.6 & \\
\hline 37 & 4.8 & -0 & -0 & & 4.4 & 4.6 & 5.1 & \\
\hline 47 & 5.8 & -0 & -0 & & 5.5 & 5.6 & 3.4 & \\
\hline
\end{tabular}

Below: $\mathrm{SASS}=x$ (raw) $\cdots$ (unbiased), C3=o (raw) - - (unbiased), theoretical $\log -\mathrm{law}$ shear $=-\cdots$
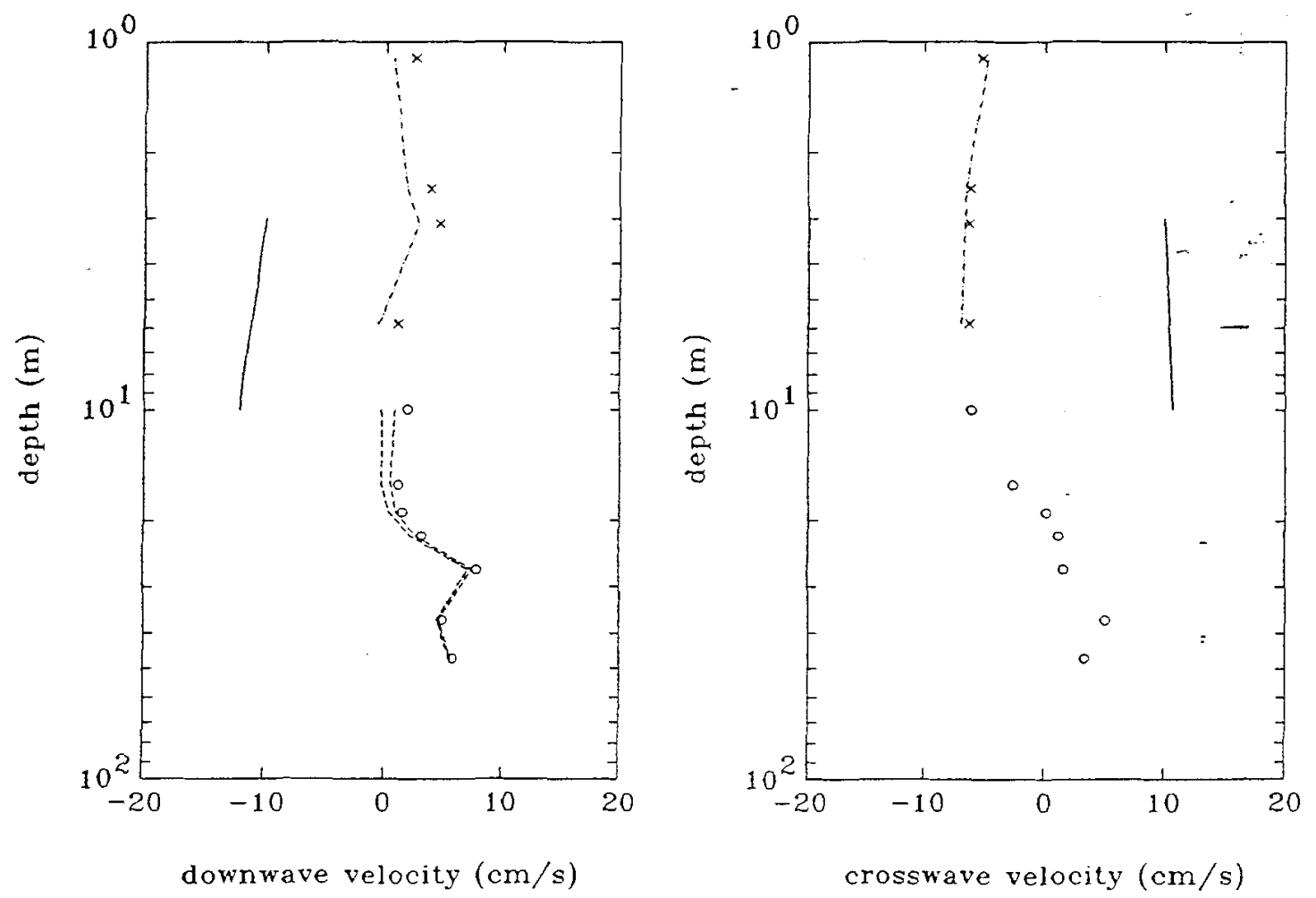


\section{file $\mathrm{m} 2$}

date $=$ February $26,1989 \quad$ time $=07-33-57.25 \quad$ duration $=00-38-48.50$
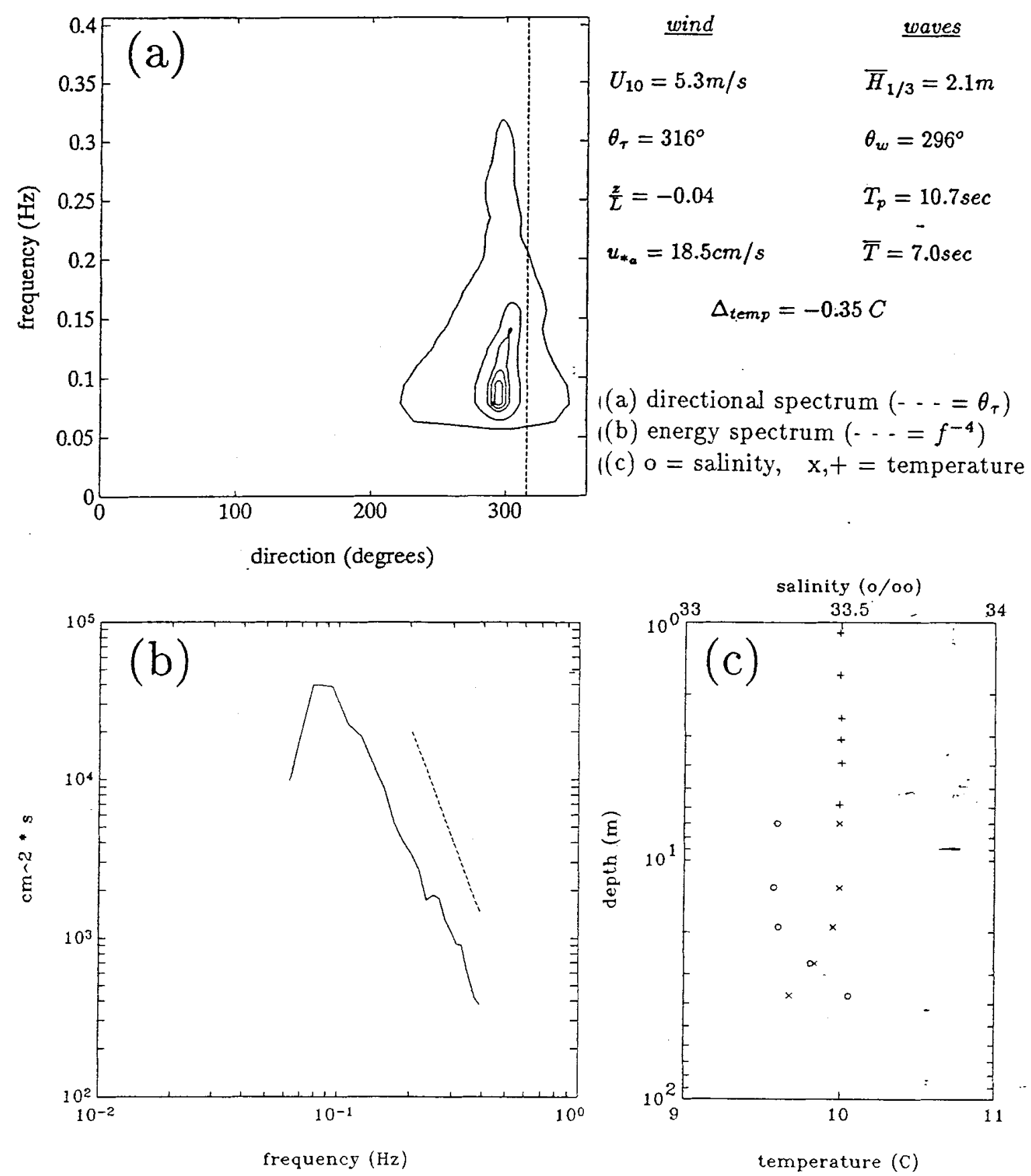


\section{file $\mathrm{m} 2$}

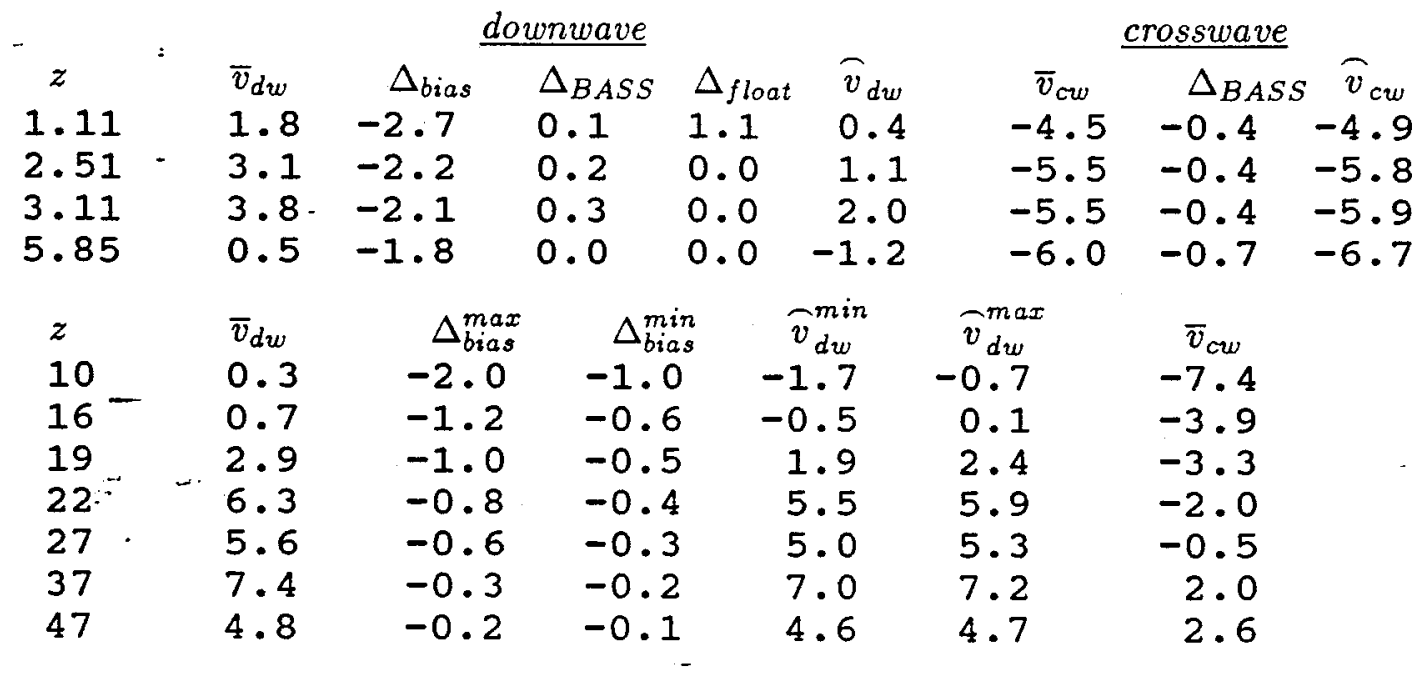

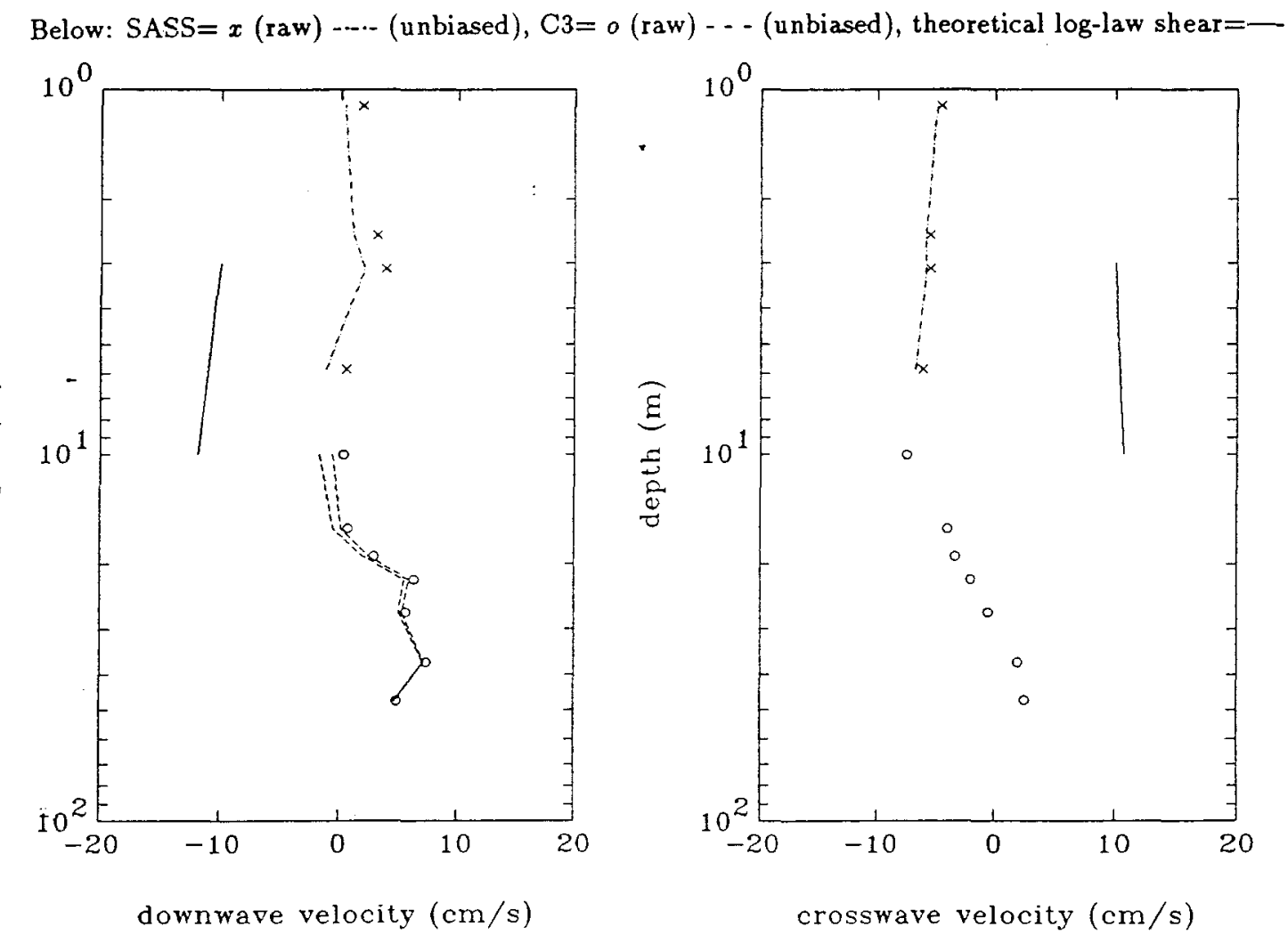




\section{file $\mathrm{n} 1$}

date $=$ February $27,1989 \quad$ time $=15-02-29.25 \quad$ duration $=00-51-04.75$

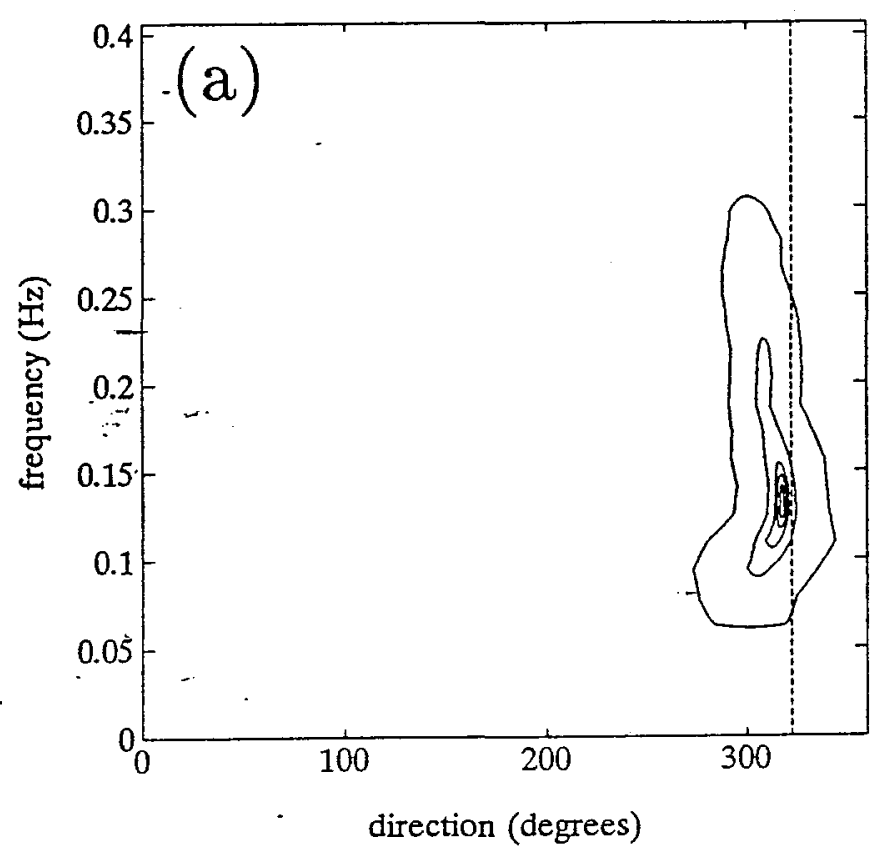

$$
\begin{array}{cc}
\underline{\text { wind }} & \multicolumn{1}{c}{\text { waves }} \\
U_{10}=6.5 \mathrm{~m} / \mathrm{s} & \bar{H}_{1 / 3}=1.8 \mathrm{~m} \\
\theta_{\tau}=322^{\circ} & \theta_{w}=307^{\circ} \\
\frac{z}{L}=-0.03 & T_{p}=8.0 \mathrm{sec} \\
u_{*_{a}}=22.7 \mathrm{~cm} / \mathrm{s} & \bar{T}=5.6 \mathrm{sec} \\
\multicolumn{2}{c}{\Delta_{\text {temp }}=-0.34 \mathrm{C}}
\end{array}
$$

(a) directional spectrum $\left(-\cdots=\theta_{\tau}\right)$

(b) energy spectrum $\left(---=f^{-4}\right)$

(c) $\mathrm{o}=$ salinity, $\mathrm{x},+=$ temperature
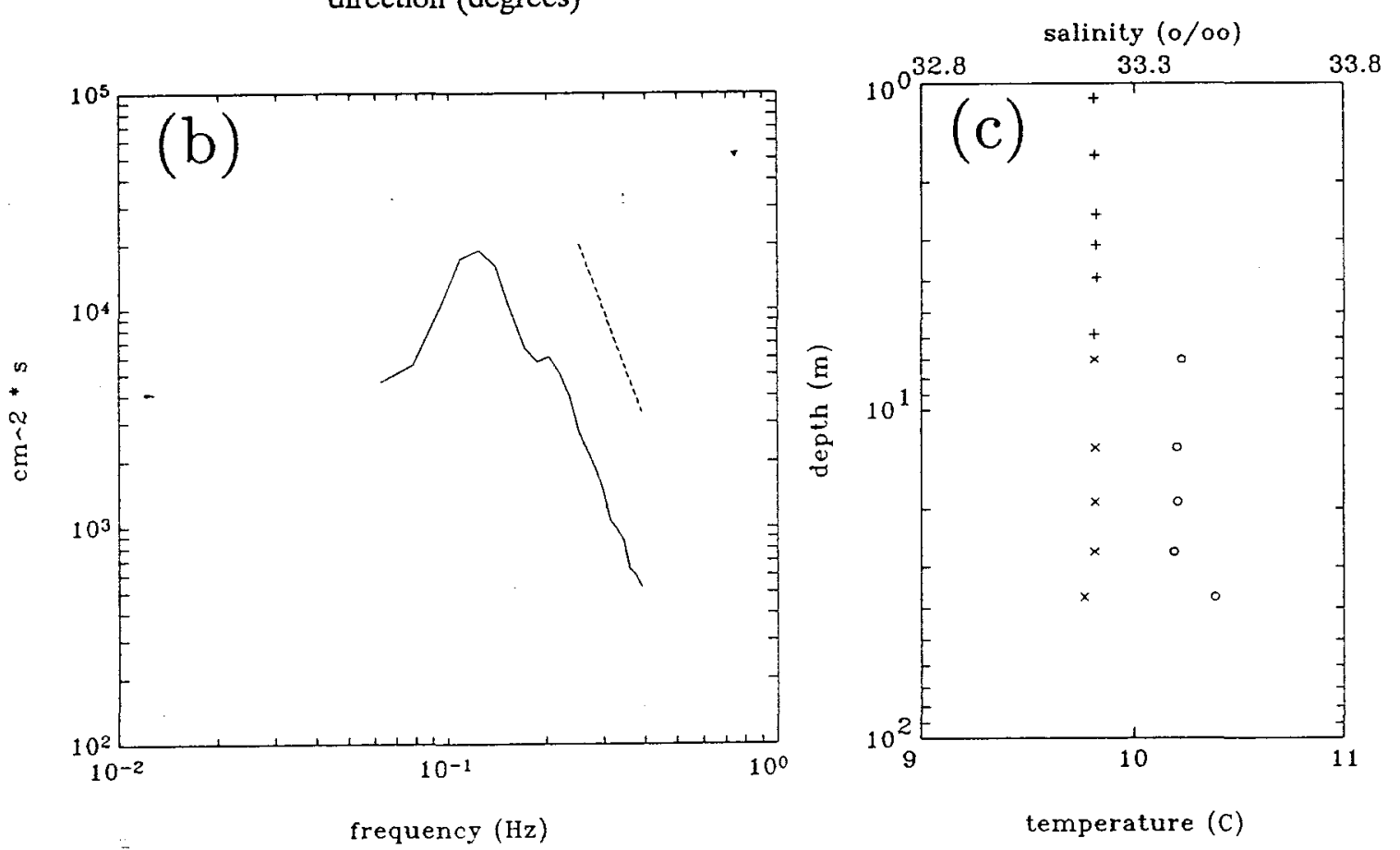


\section{file $\mathrm{n} 1$}

\begin{tabular}{|c|c|c|c|c|c|c|c|}
\hline$=$ & & & ownwave & & & & \\
\hline$z$ & $\bar{v}_{d w}$ & $\Delta_{\text {bias }}$ & $\triangle_{B A S S}$ & $\Delta_{\text {float }}$ & $\widehat{v}_{d w}$ & $\bar{v}_{c w}$ & \\
\hline $1 .-11$ & 9.8 & -3.4 & -0.7 & 1.3 & 7.1 & -4.9 & \\
\hline 2.51 & 13.0 & -2.7 & 0.7 & 0.0 & 11.0 & -5.2 & $-l$ \\
\hline 3.11 & 13.0 & -2.6 & 0.8 & 0.0 & 11.2 & -4.6 & -0 \\
\hline 5.85 & 8.3 & -1.9 & 1.0 & 0.0 & 7.3 & -5.1 & -0.6 \\
\hline
\end{tabular}

$\begin{array}{rrrrrrr}z & \bar{v}_{d w} & \Delta_{b i a s}^{\max } & \Delta_{b i a}^{\min } & \widehat{v}_{d w}^{\min } & \widehat{v}_{d w}^{\max } & \bar{v}_{c w} \\ -4 & 16.4 & -4.5 & -2.2 & 11.9 & 14.2 & -3.5 \\ 10 & 15.2 & -1.8 & -0.9 & 13.4 & 14.3 & -2.3 \\ -16 & 14.8 & -0.9 & -0.5 & 13.9 & 14.3 & -2.2 \\ 19 & 15.6 & -0.7 & -0.4 & 14.9 & 15.2 & -0.7 \\ 22 & 15.6 & -0.5 & -0.3 & 15.1 & 15.3 & -0.4 \\ 27 & 16.0 & -0.3 & -0.2 & 15.7 & 15.8 & -1.9 \\ 37 & 7.1 & -0.2 & -0.1 & 6.9 & 7.0 & 0.6 \\ 47 & -3.7 & -0.1 & -0.1 & -3.8 & -3.8 & -0.4\end{array}$

Below: $\mathrm{SASS}=x(\mathrm{raw}) \cdots($ unbiased $), \mathrm{C} 3=o(\mathrm{raw}) \ldots($ unbiased $)$, theoretical $\log -\mathrm{law}$ shear $=-$
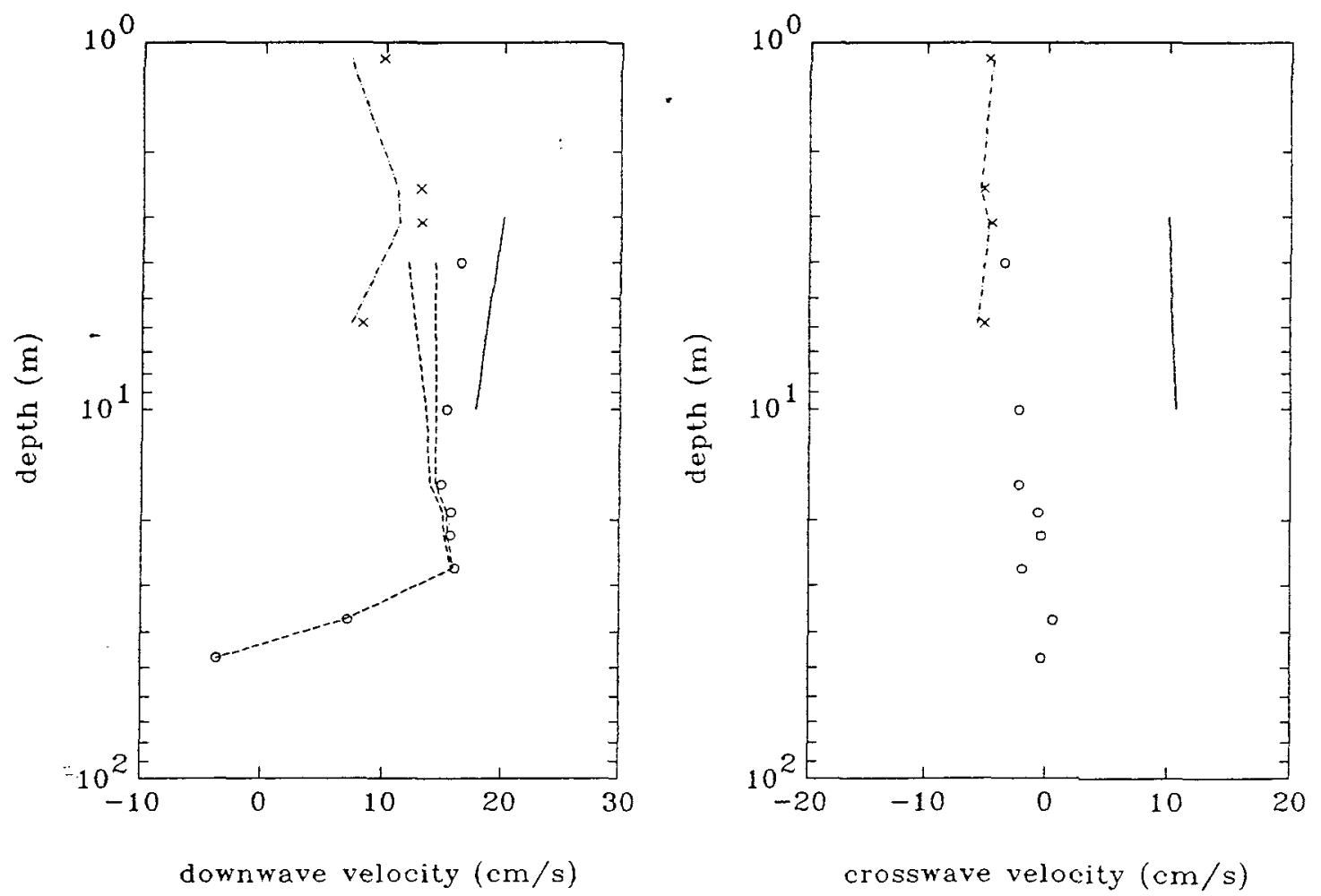


\section{file $\mathrm{n} 2$}

$$
\text { date }=\text { February 2.7, } 1989 \quad \text { time }=15-53-34.00 \quad \text { duration }=00-51-04.50
$$

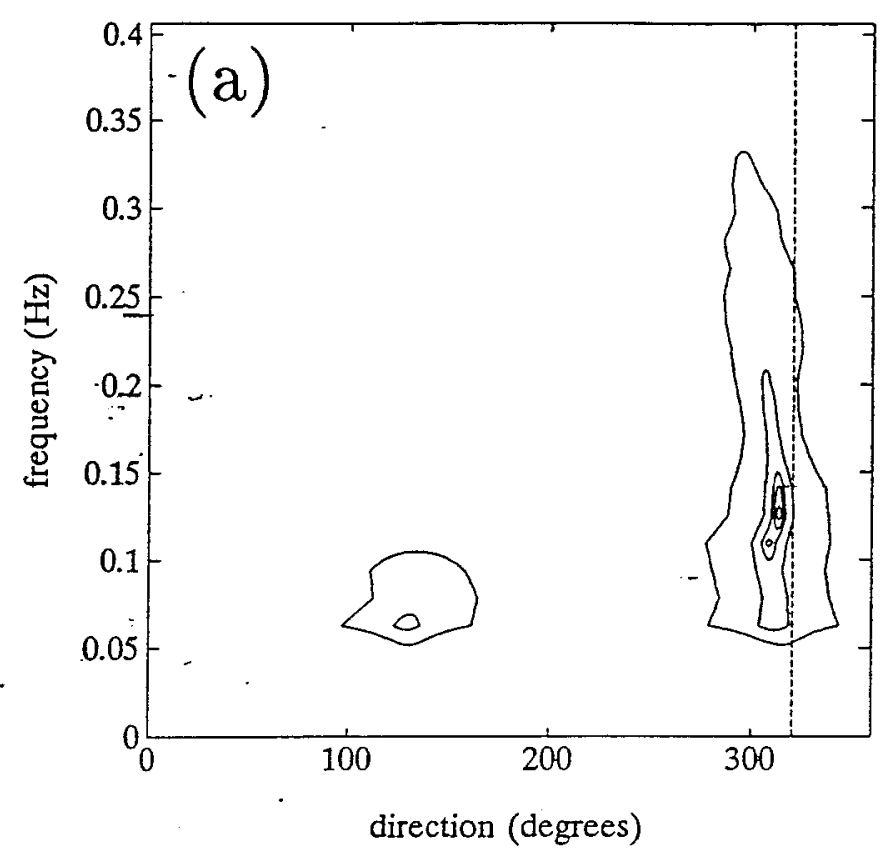

$$
\begin{array}{ll}
\underline{\text { wind }} & \multicolumn{1}{c}{\text { waves }} \\
U_{10}=8.6 \mathrm{~m} / \mathrm{s} & \bar{H}_{1 / 3}=2.1 \mathrm{~m} \\
\theta_{\tau}=320^{\circ} & \theta_{w}=305^{\circ} \\
\frac{z}{L}=-0.02 & T_{p}=9.1 \mathrm{sec} \\
u_{* a}=29.7 \mathrm{~cm} / \mathrm{s} & \bar{T}=6.1 \mathrm{sec} \\
\multicolumn{2}{c}{\Delta_{\text {temp }}=-0.33 \mathrm{C}}
\end{array}
$$

(a) directional spectrum

(b) energy spectrum $\left(--=f^{-4}\right)$

(c) $o=$ salinity, $x,+=$ temperature
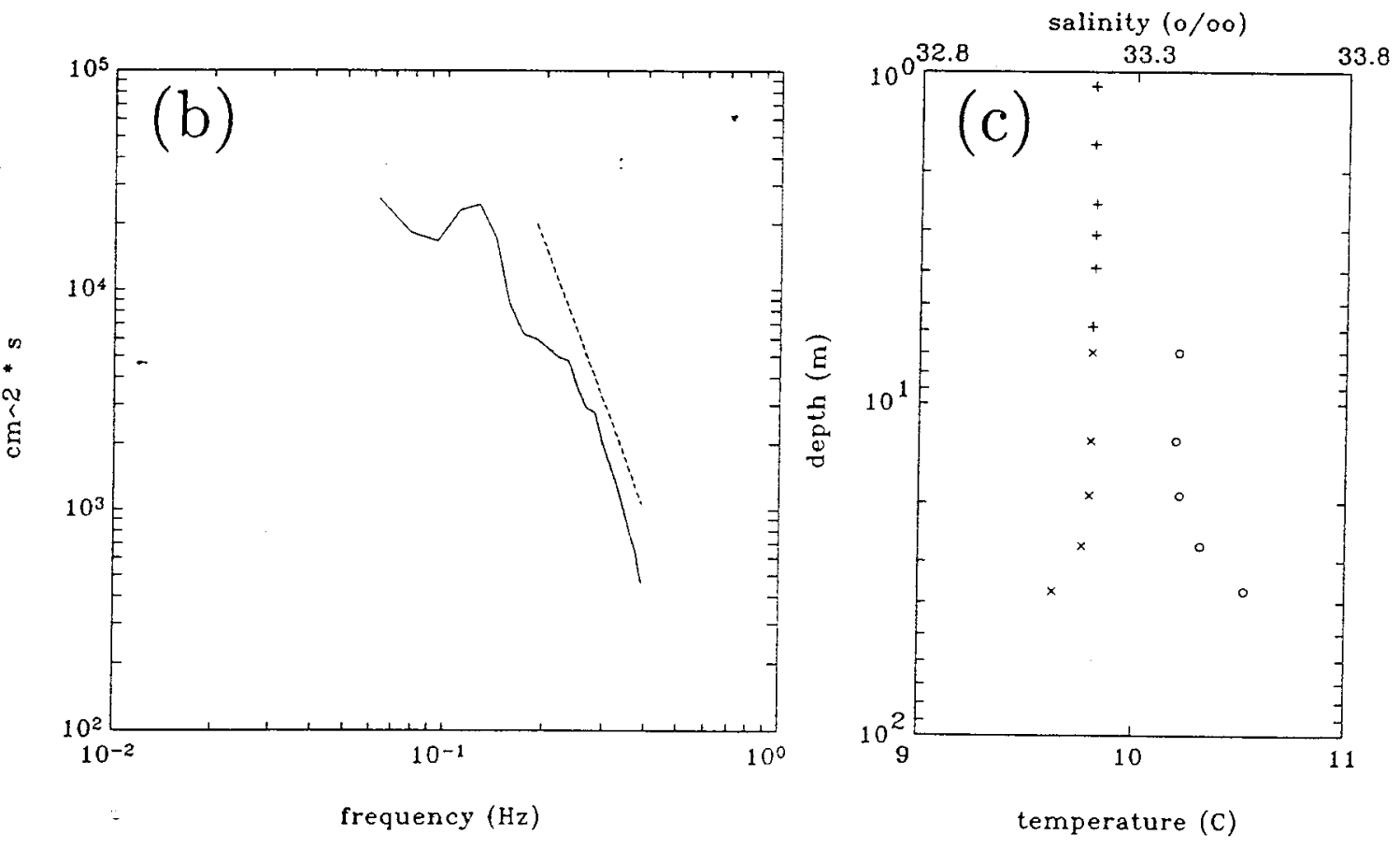


\section{file $n 2$}

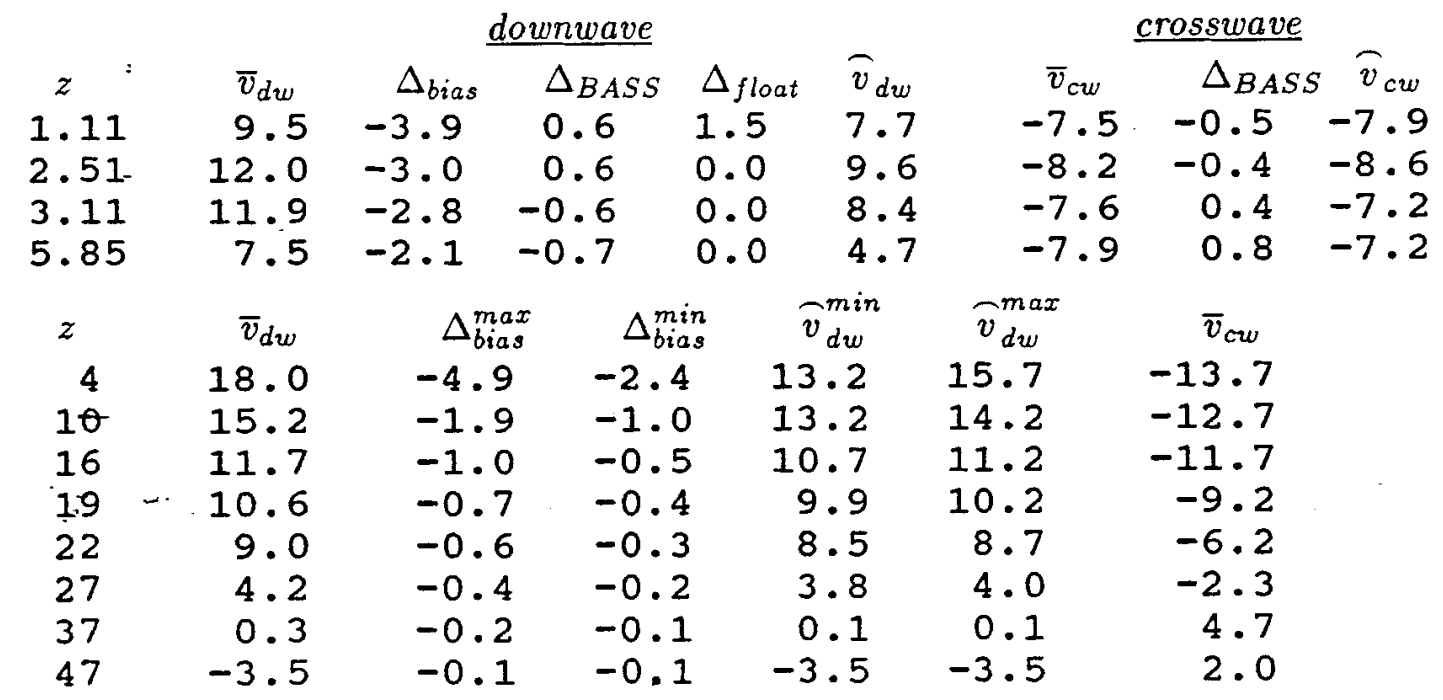

Below: SASS $=x($ raw $) \cdots($ unbiased $), C 3=o($ raw $) \cdots($ unbiased $)$, theoretical log-law shear $=-$
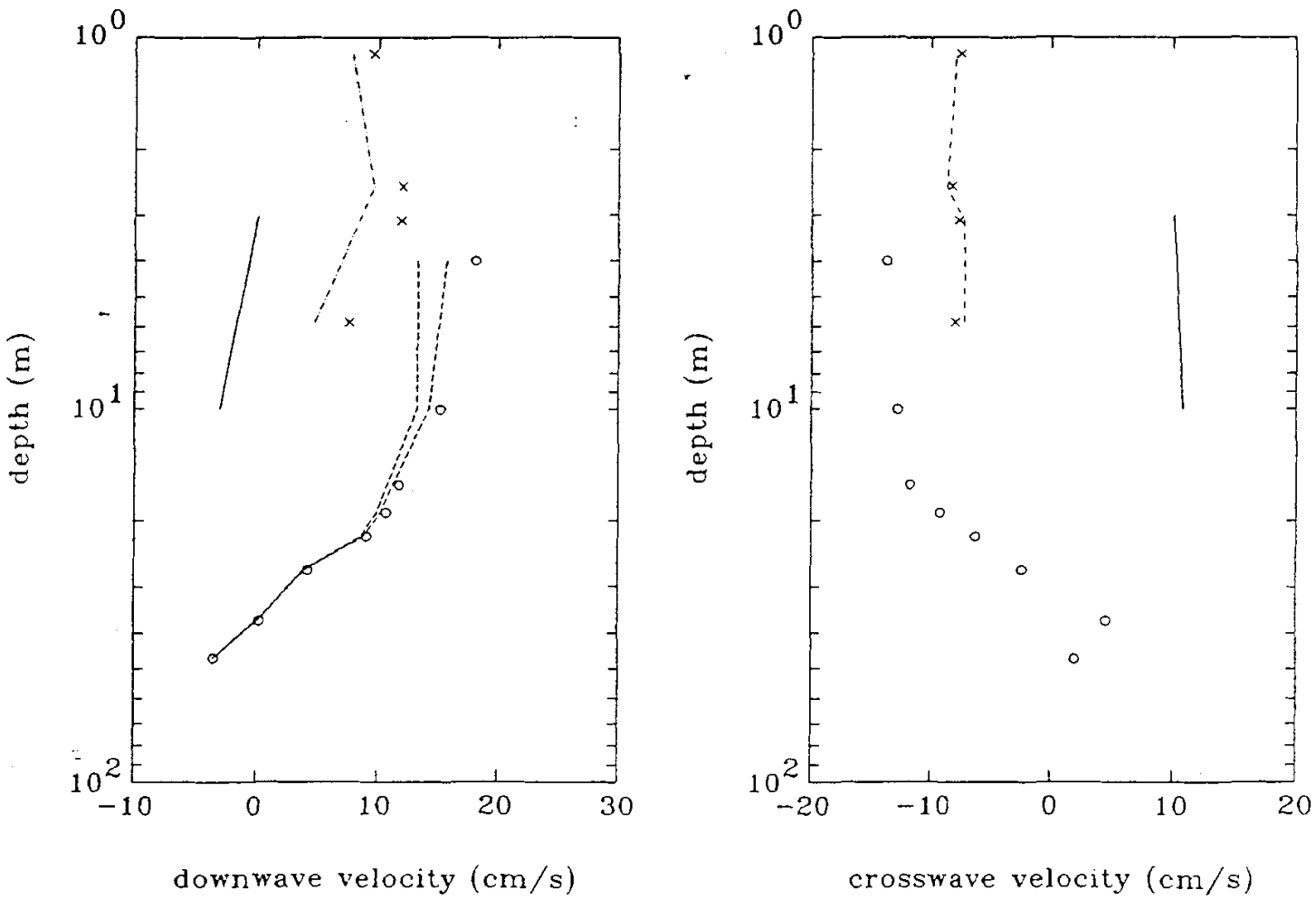


\section{file o1}

$$
\text { date }=\text { February } 27,1989 \quad \text { time }=18-21-33.75 \quad \text { duration }=00-39-13.25
$$

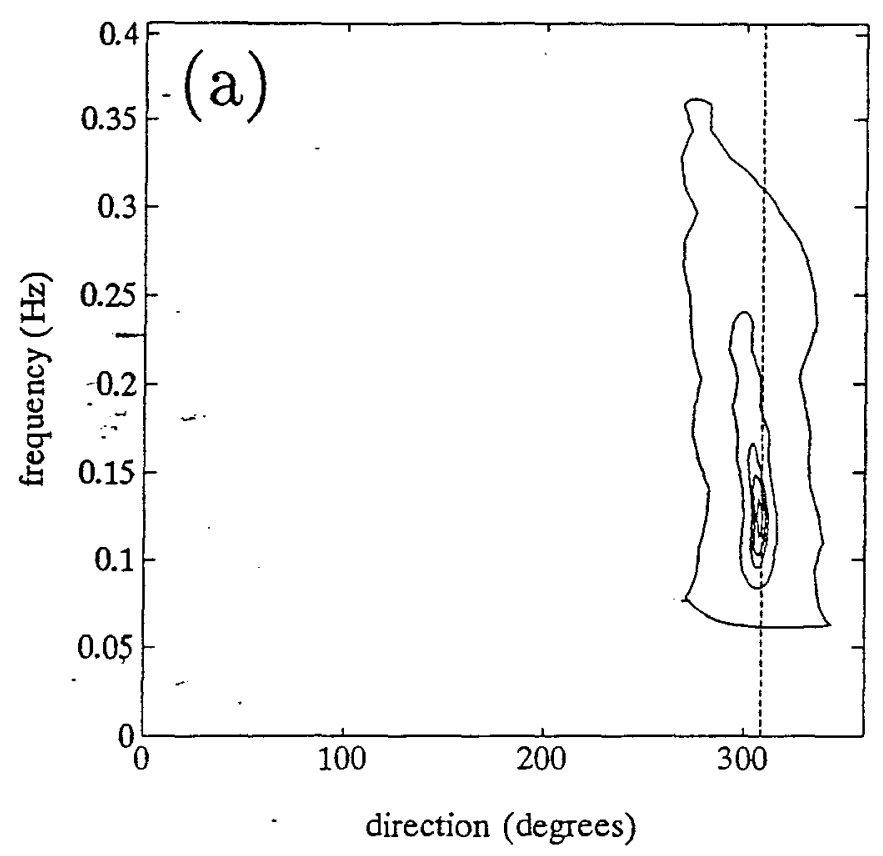

$$
\begin{array}{cc}
\underline{\text { wind }} & \multicolumn{1}{c}{\underline{\text { waves }}} \\
U_{10}=10.2 \mathrm{~m} / \mathrm{s} & \bar{H}_{1 / 3}=1.8 \mathrm{~m} \\
\theta_{\tau}=309^{\circ} & \theta_{w}=300^{\circ} \\
\underline{z}=0.00 & T_{p}=8.0 \mathrm{sec} \\
u_{*_{a}}=35.4 \mathrm{~cm} / \mathrm{s} & \bar{T}=5.1 \mathrm{sec} \\
\multicolumn{2}{c}{\Delta_{\text {temp }}=-0.34 \mathrm{C}}
\end{array}
$$

(a) directional spectrum $\left(-\cdots=\theta_{\tau}\right)$

(b) energy spectrum $\left(-\cdots=f^{-4}\right)$

(c) $\mathrm{o}=$ salinity, $\mathrm{x},+=$ temperature
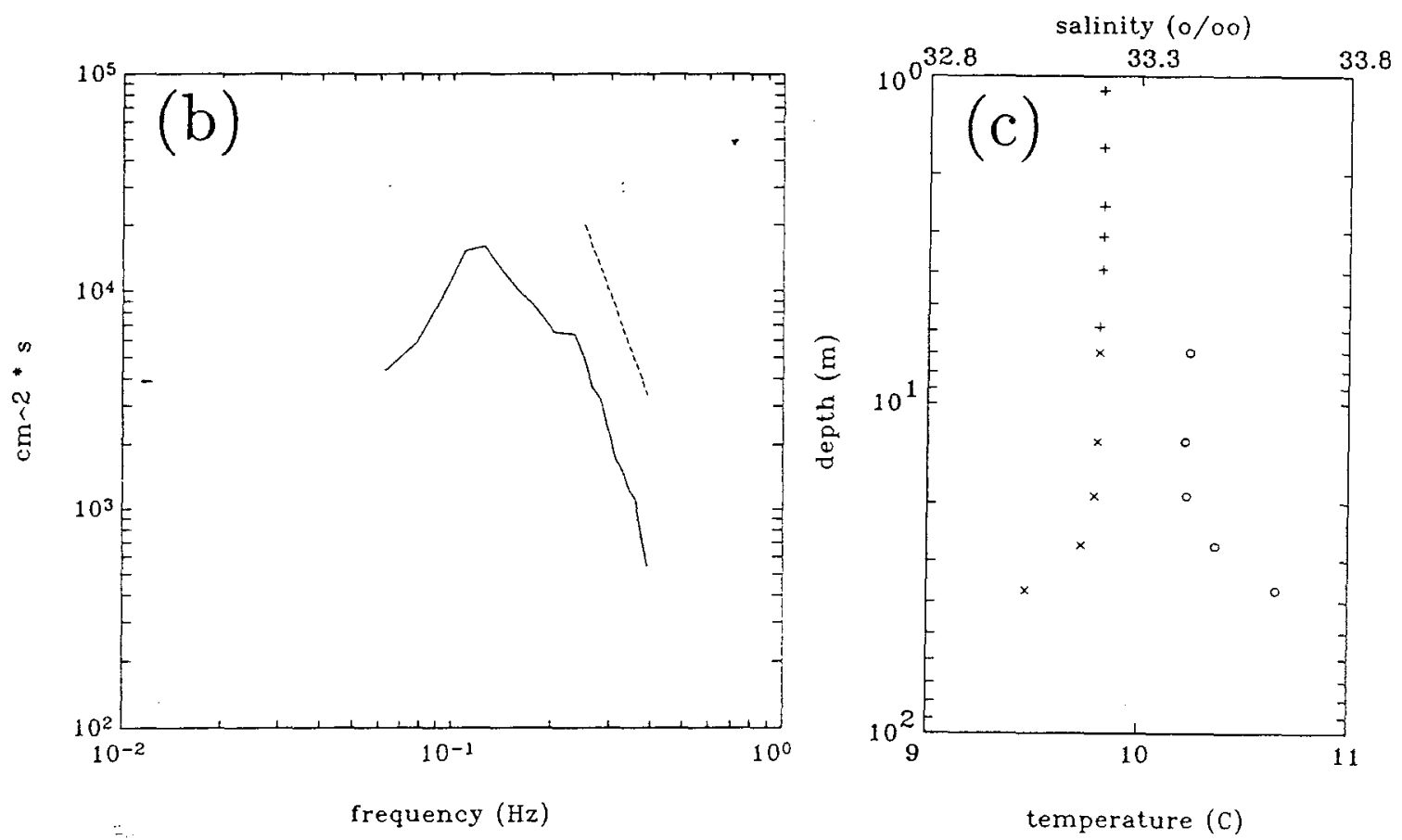


\section{file o1}

\begin{tabular}{|c|c|c|c|c|c|c|c|c|}
\hline \multirow{6}{*}{$\begin{array}{c}z \\
1.11 \\
2.51 \\
3.11 \\
5.85\end{array}$} & \multicolumn{5}{|c|}{ downwave } & \multicolumn{3}{|c|}{ crosswave } \\
\hline & $\bar{v}_{d w}$ & $\Delta_{\text {bias }}$ & $\Delta_{B A S S}$ & $\Delta_{\text {float }}$ & $\widehat{v}_{d w}$ & $\bar{v}_{c w}$ & $\triangle_{B A S S}$ & $S \widehat{v}_{c w}$ \\
\hline & 9.2 & -4.6 & 0.4 & 1.7 & 6.8 & -14.7 & $-0.7-$ & -15.4 \\
\hline & 10.1 & -3.5 & 0.5 & 0.0 & 7.1 & $-13 \cdot 6$ & $-0.6-$ & -14.2 \\
\hline & 10.5 & -3.3 & 0.5 & 0.0 & 7.7 & $-13 \cdot 3$ & $-0.6-$ & -14.0 \\
\hline & 8.5 & -2.4 & 0.6 & 0.0 & 6.6 & $-13 \cdot 2$ & -0.9 & -14.1 \\
\hline$z$ & $\bar{v}_{d w}$ & $\Delta_{b i a s}^{\max }$ & $\Delta_{b i}^{m}$ & & $\widehat{v}_{d w}^{m i n}$ & $\widehat{v}_{d w}^{\max }$ & $\bar{v}_{c w}$ & \\
\hline 4 & 14.2 & -5.6 & -2 . & & 8.6 & 11.5 & -15.7 & \\
\hline Io & 11.6 & -2.0 & -1 & & 9.6 & 10.6 & -13.4 & \\
\hline 16 & 7.4 & -0.9 & -0 & & 6.4 & 6.9 & -10.6 & \\
\hline$\because 19$ & 4.9 & -0.7 & -0. & & 4.2 & 4.6 & -8.8 & \\
\hline 22 & 5.1 & -0.5 & -0. & & 4.5 & 4.8 & -6.4 & \\
\hline 27 & 1.3 & -0.3 & -0. & & 1.0 & 1.1 & -0.8 & \\
\hline 37 & 5.2 & -0.2 & -0 & & 5.1 & 5.1 & 9.5 & \\
\hline 47 & 2.2 & -0.1 & -0 & & 2.1 & 2.2 & 8.6 & \\
\hline
\end{tabular}

Below: $\mathrm{SASS}=x(\mathrm{raw}) \cdots(-\cdot-($ unbiased $), \mathrm{C} 3=0(\mathrm{raw})-\cdots$ (unbiased), theoretical log-law shear $=-$
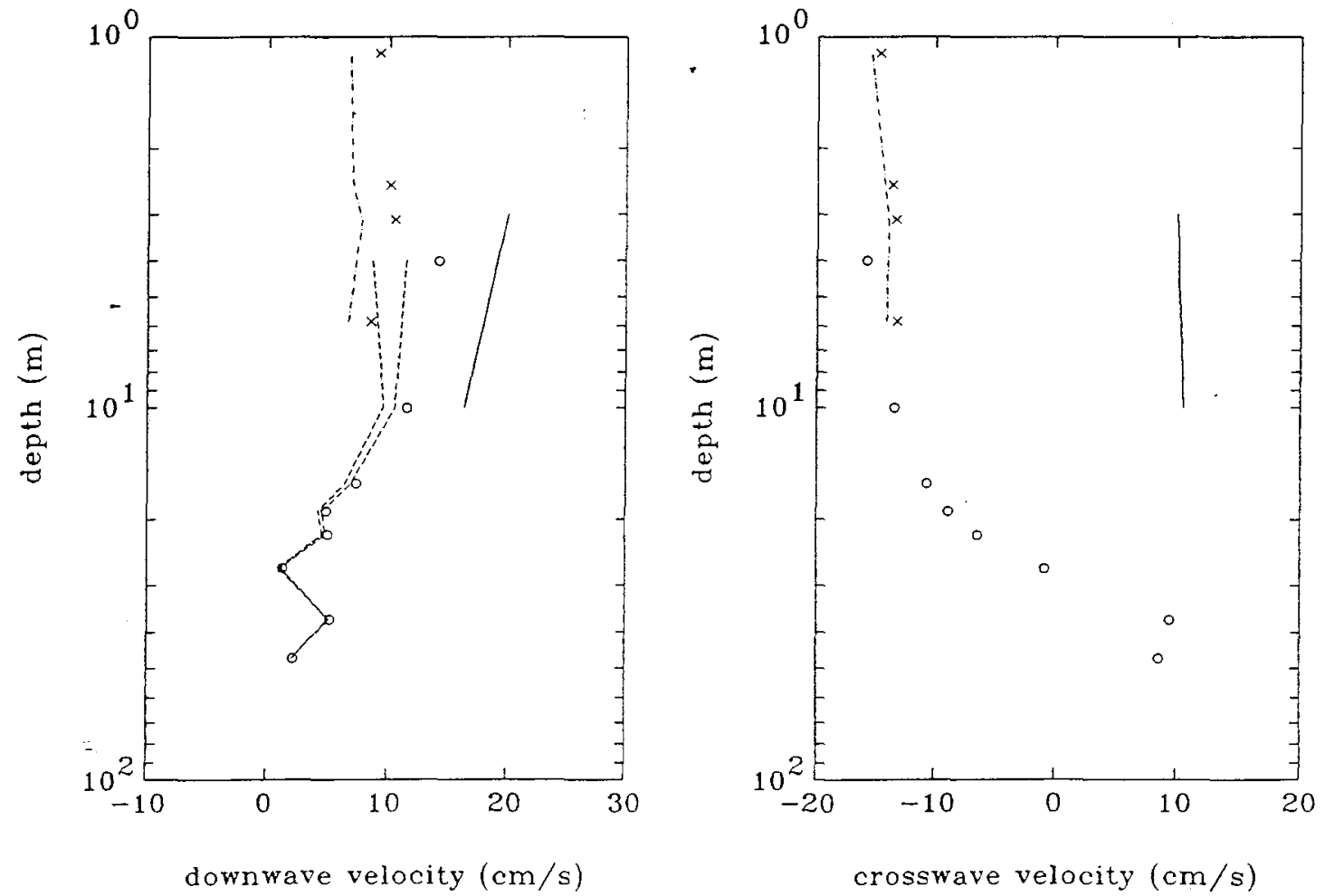


\section{file o2}

$$
\text { date }=\text { February } 27,1989 \quad \text { time }=19-00-47.00 \quad \text { duration }=00-39-13.25
$$

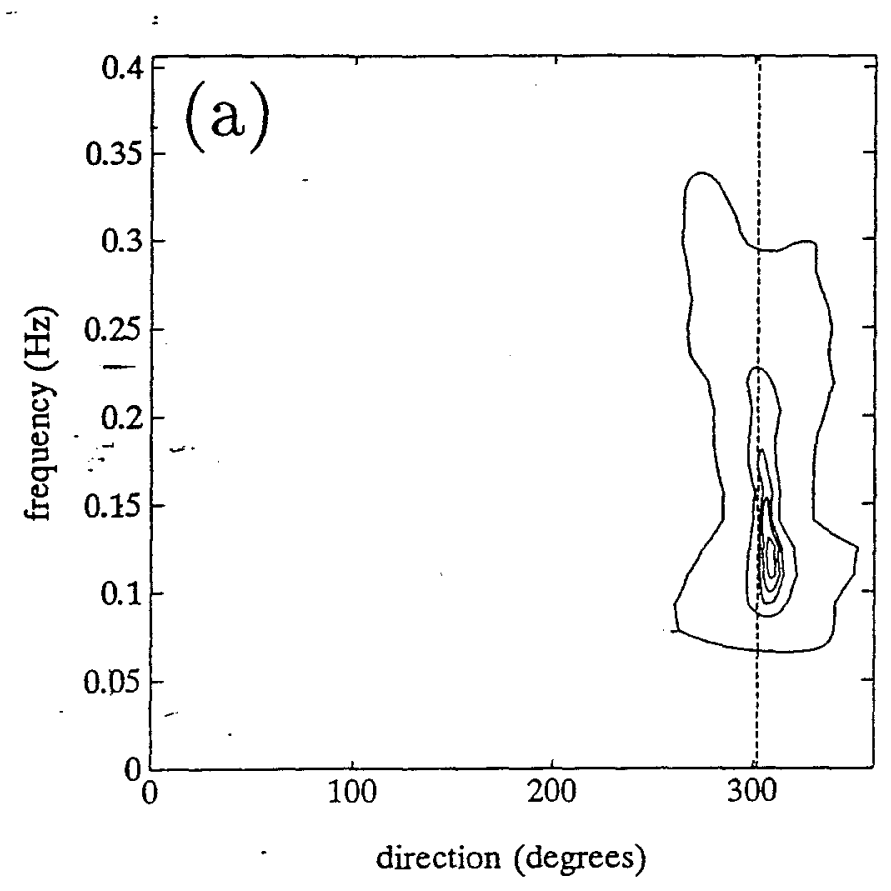

$$
\begin{array}{lc}
\text { wind } & \text { waves } \\
U_{10}=11.7 \mathrm{~m} / \mathrm{s} & \bar{H}_{1 / 3}=2.0 \mathrm{~m} \\
\theta_{\tau}=302^{\circ} & \theta_{w}=301^{\circ} \\
\frac{z}{L}=0.00 & T_{p}=8.0 \mathrm{sec} \\
u_{*_{a}}=41.4 \mathrm{~cm} / \mathrm{s} \quad & \widehat{T}=5.2 \mathrm{sec} \\
\Delta_{\text {temp }}=-0.35 \mathrm{C} \\
\\
\text { (a) directional spectrum } \\
\text { (b) energy spectrum }\left(--=f^{-4}\right) \\
\text { (c) o = salinity, } \mathrm{x},+=\text { temperature }
\end{array}
$$
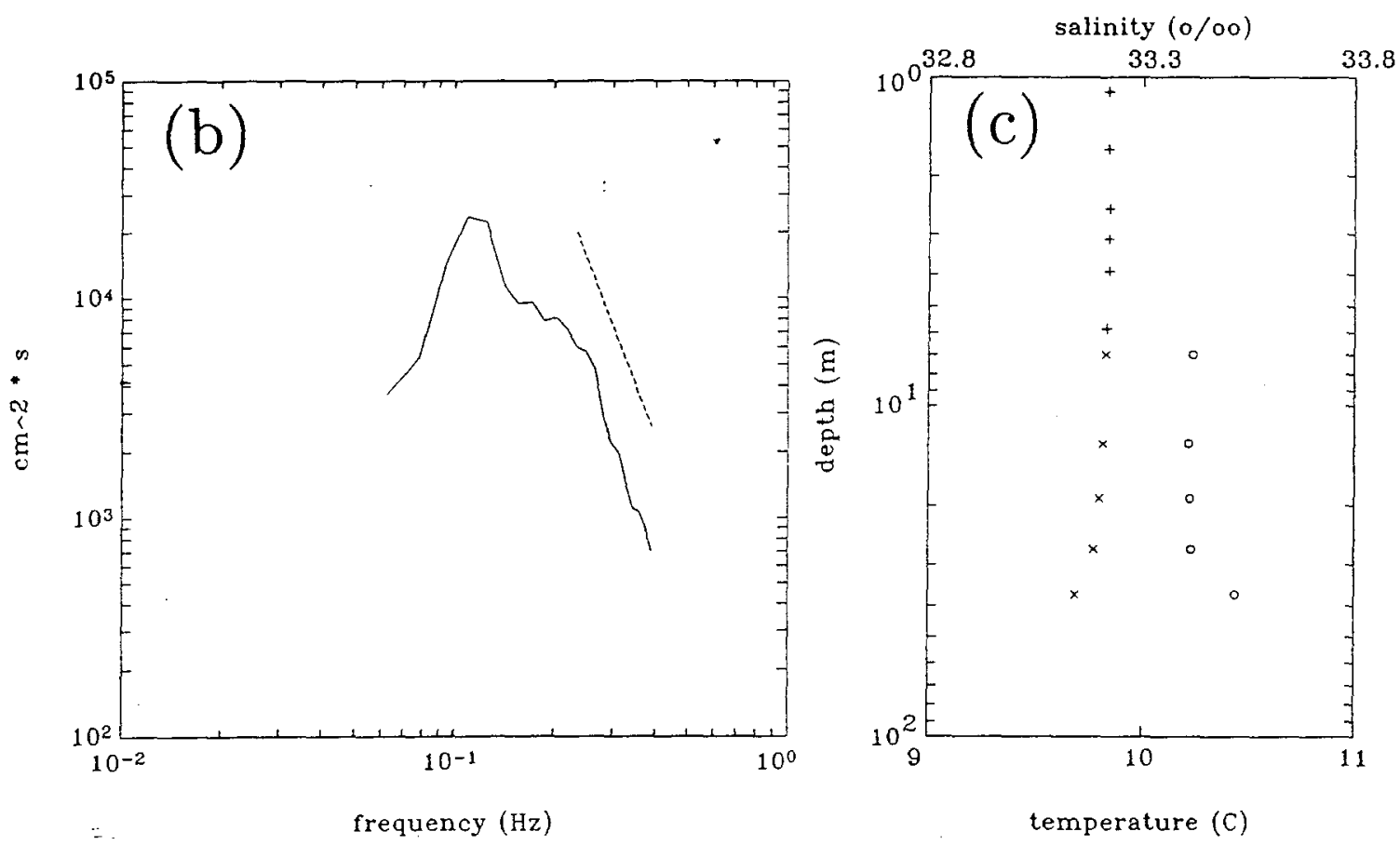


\section{file o2}

\begin{tabular}{|c|c|c|c|c|c|c|c|}
\hline \multirow[b]{2}{*}{$\begin{array}{c}z= \\
1.11 \\
2.51 \\
3.11 \\
5.85\end{array}$} & \multicolumn{5}{|c|}{ downwave } & \multicolumn{2}{|r|}{ crosswave } \\
\hline & $\begin{array}{l}\bar{v}_{d w} \\
8.6 \\
8.8 \\
9.4 \\
6.4\end{array}$ & $\begin{aligned} & \Delta_{\text {bias }} \\
&-5.1=3.9 \\
&-3.9-3.6 \\
&-2.7\end{aligned}$ & $\begin{array}{l}\triangle_{B A S S} \\
0.4 \\
0.4 \\
0.5 \\
0.4\end{array}$ & $\begin{array}{l}\Delta_{\text {float }} \\
1.7 \\
0.0 \\
0.0 \\
0.0\end{array}$ & $\begin{array}{l}\widehat{v}_{d w} \\
5.6 \\
5.3 \\
6.3 \\
4.2\end{array}$ & $\begin{array}{c}\bar{v}_{c w} \\
-13.5 \\
-12.1 \\
-12.3 \\
-12.9\end{array}$ & $\begin{aligned} & \Delta_{B A S} \\
-0.7 & -0.6 \\
- & 0.6 \\
- & 0.9\end{aligned}$ \\
\hline$z$ & $\bar{v}_{d w}$ & $\Delta_{\text {bias }}^{\max }$ & $\Delta_{b i a}^{m i}$ & & $\min$ & $\widehat{v}_{d w}^{m a x}$ & $\bar{v}_{c w}$ \\
\hline 4 & 18.6 & -6.2 & -3.0 & & & 15.5 & -10.1 \\
\hline 10 & 15.6 & $-2 \cdot 3$ & -1.2 & & & 14.5 & -8.6 \\
\hline 16 & 11.8 & -1.1 & -0.6 & & & 11.2 & -7.2 \\
\hline $\begin{array}{l}19 \\
22\end{array}$ & $\begin{array}{l}9.5 \\
8.6\end{array}$ & $\begin{array}{l}-0.8 \\
-0.6\end{array}$ & $\begin{array}{l}-0.4 \\
-0.3\end{array}$ & & $\begin{array}{l}.7 \\
.0\end{array}$ & $\begin{array}{l}9.0 \\
8.3\end{array}$ & $\begin{array}{l}-6.5 \\
-4.1\end{array}$ \\
\hline 27 & 6.0 & -0.4 & -0.2 & & .5 & 5.7 & -2.7 \\
\hline 37 & 3.7 & -0.2 & -0.1 & & .5 & 3.6 & 5.3 \\
\hline 47 & 2.7 & -0.1 & -0.1 & & .6 & 2.6 & 5.1 \\
\hline
\end{tabular}

Below: SASS $=x$ (raw) -..- (unbiased), C3=o(raw) - - (unbiased), theoretical log-law shear $=-$
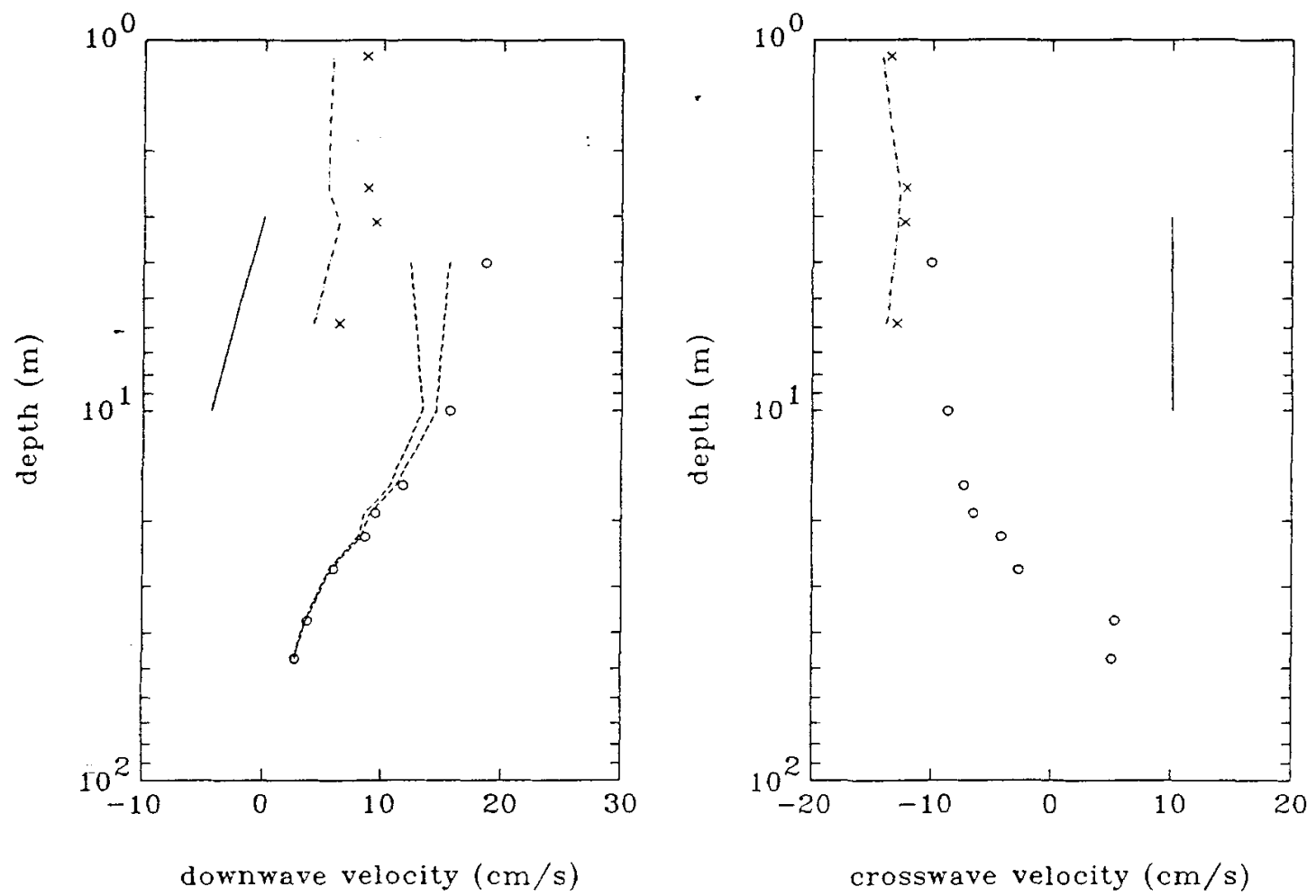


\section{file o3}

date $=$ February 27, $1989 \quad$ time $=19-40-00.25 \quad$ duration $=00-39-13.25$

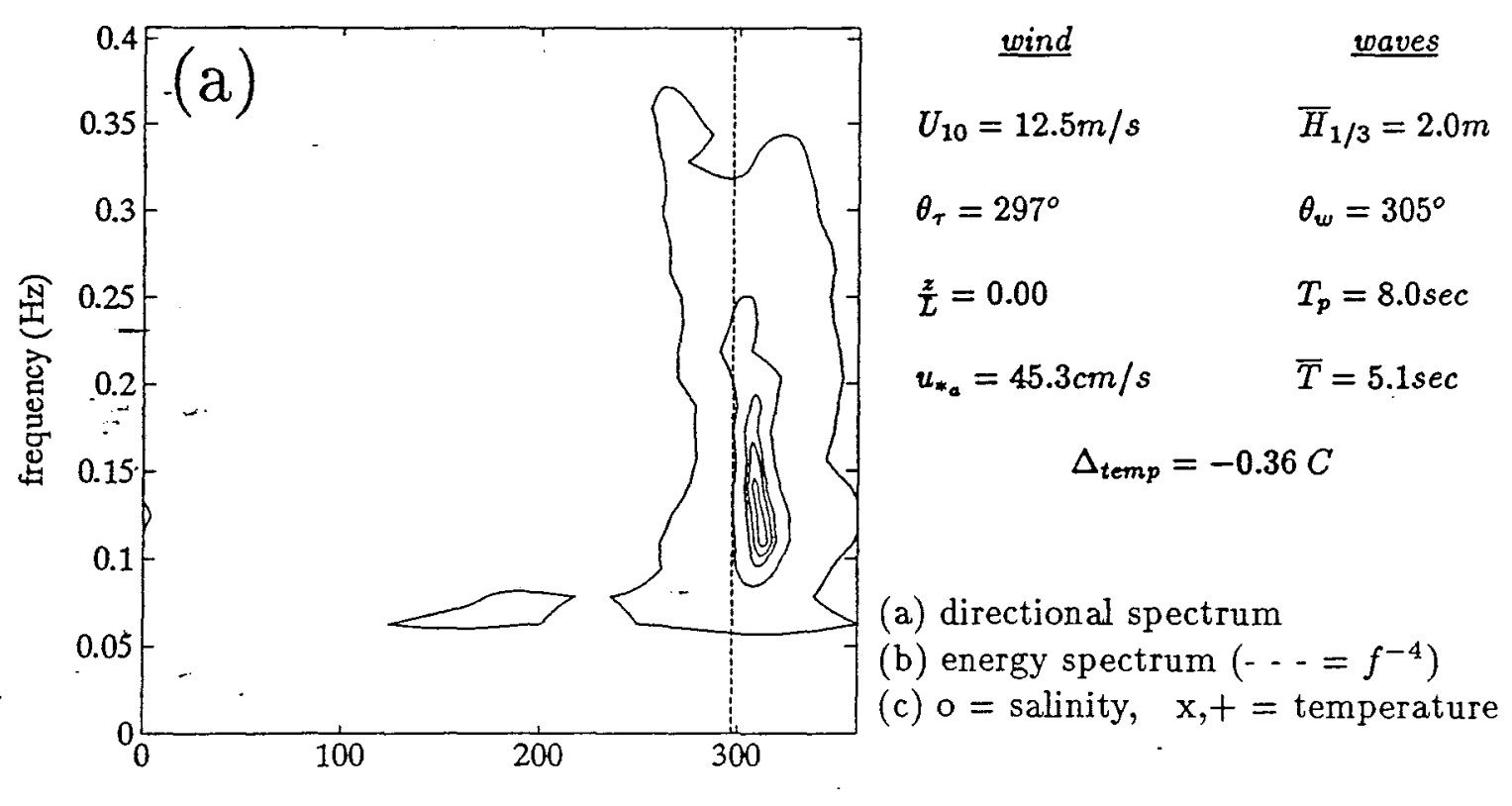

direction (degrees)
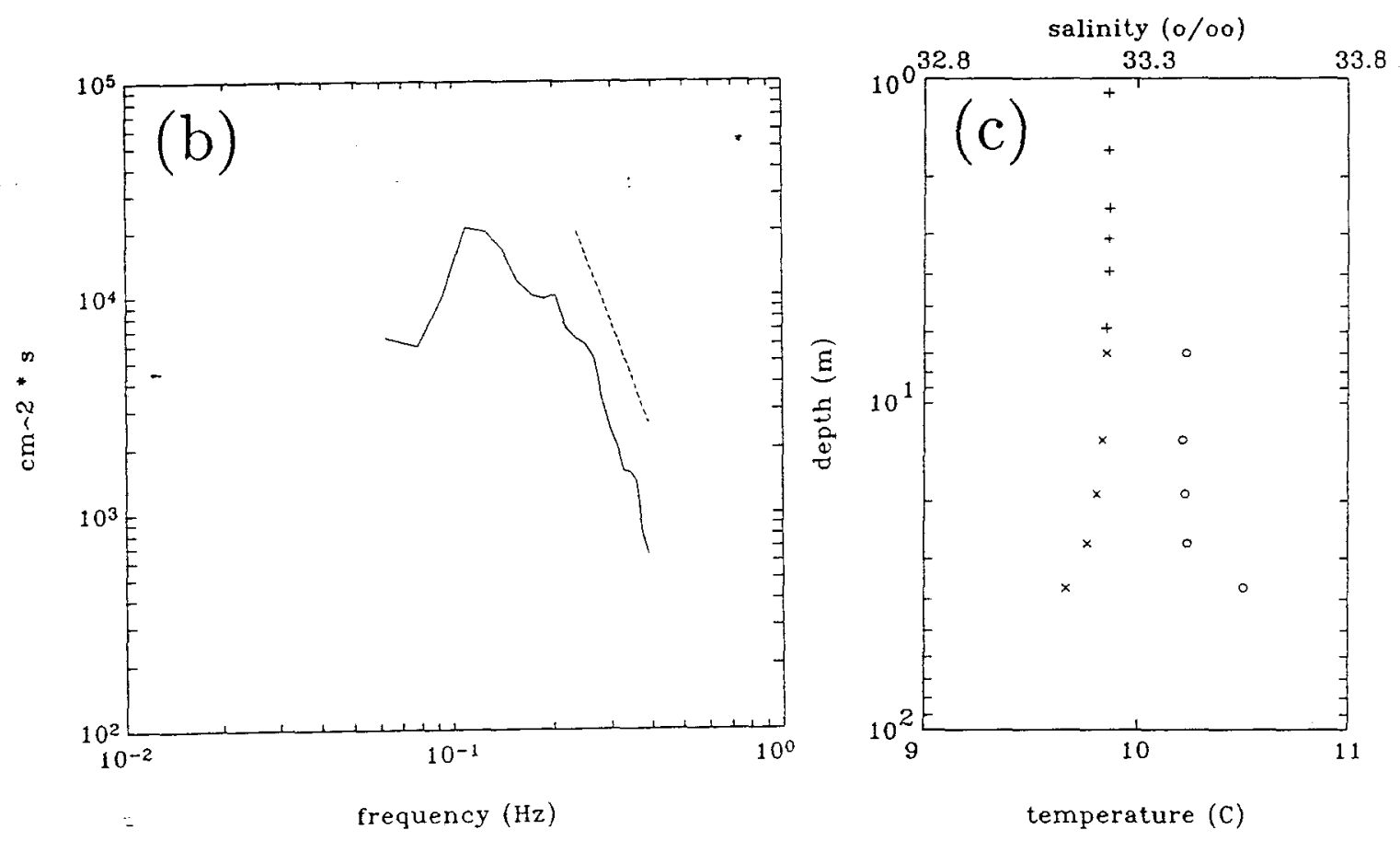


\section{file o3}

\begin{tabular}{|c|c|c|c|c|c|c|c|c|c|}
\hline \multirow{6}{*}{\multicolumn{2}{|c|}{$\begin{array}{c}z \\
1.11 \\
2.51 \\
3.11 \\
5.85\end{array}$}} & \multicolumn{5}{|c|}{ downwave } & \multicolumn{3}{|c|}{ crosswave } \\
\hline & & $\bar{v}_{d w}$ & $\Delta_{\text {bias }}$ & $\Delta_{B A S S}$ & $\Delta_{\text {float }}$ & $\widehat{v}_{d w}$ & \multirow{5}{*}{$\begin{array}{c}\bar{v}_{c w} \\
-8.8 \\
-8.4 \\
-8.0 \\
-7.4\end{array}$} & \multicolumn{2}{|l|}{$\Delta_{B A S S}$} \\
\hline & & 13.2 & -5.5 & -0.7 & 1.9 & 8.8 & & 0.5 & $-8 \cdot 3$ \\
\hline & & 14.0 & -4.1 & 0.7 & 0.0 & 10.6 & & -0.4 & -8.8 \\
\hline & & 14.1 & -3.9 & 0.7 & 0.0 & 11.0 & & -0.4 & -8.5 \\
\hline & & 8.1 & -2.7 & 0.8 & 0.0 & 6.3 & & -0.7 & -8.1 \\
\hline$z$ & & $\bar{v}_{d w}$ & $\Delta_{b i a}^{m a}$ & $\Delta_{b i c}^{m}$ & & $\widehat{v}_{d w}^{\min }$ & $\widehat{v}_{d w}^{\max }$ & $\bar{v}_{c w}$ & \\
\hline 4 & & 22.0 & -6.8 & -3.3 & & 5.3 & 18.7 & -8.9 & \\
\hline 10 & & 18.0 & -2.5 & -1.2 & & 5.5 & 16.7 & -7.5 & \\
\hline 16 & & 12.4 & -1.2 & -0.6 & & 1.3 & 11.8 & -6.4 & \\
\hline 1.9 & - & 9.5 & -0.9 & -0.5 & & 8.6 & 9.0 & -5.7 & \\
\hline 22 & & 8.4 & -0.6 & -0.4 & & 7.7 & 8.0 & -3.7 & \\
\hline 27 & & 4.8 & -0.4 & -0.2 & & 4.4 & 4.6 & -1.8 & \\
\hline 37 & & 4.6 & -0.2 & -0.1 & & 4.4 & 4.5 & 7.1 & \\
\hline 47 & & 2.7 & -0.1 & -0.1 & & 2.6 & 2.6 & 2.5 & \\
\hline
\end{tabular}

Below: $\mathrm{SASS}=x(\mathrm{raw}) \cdots($ unbiased $), C 3=\circ(\mathrm{raw})-\cdots($ unbiased $)$, theoretical log-law shear $=-$
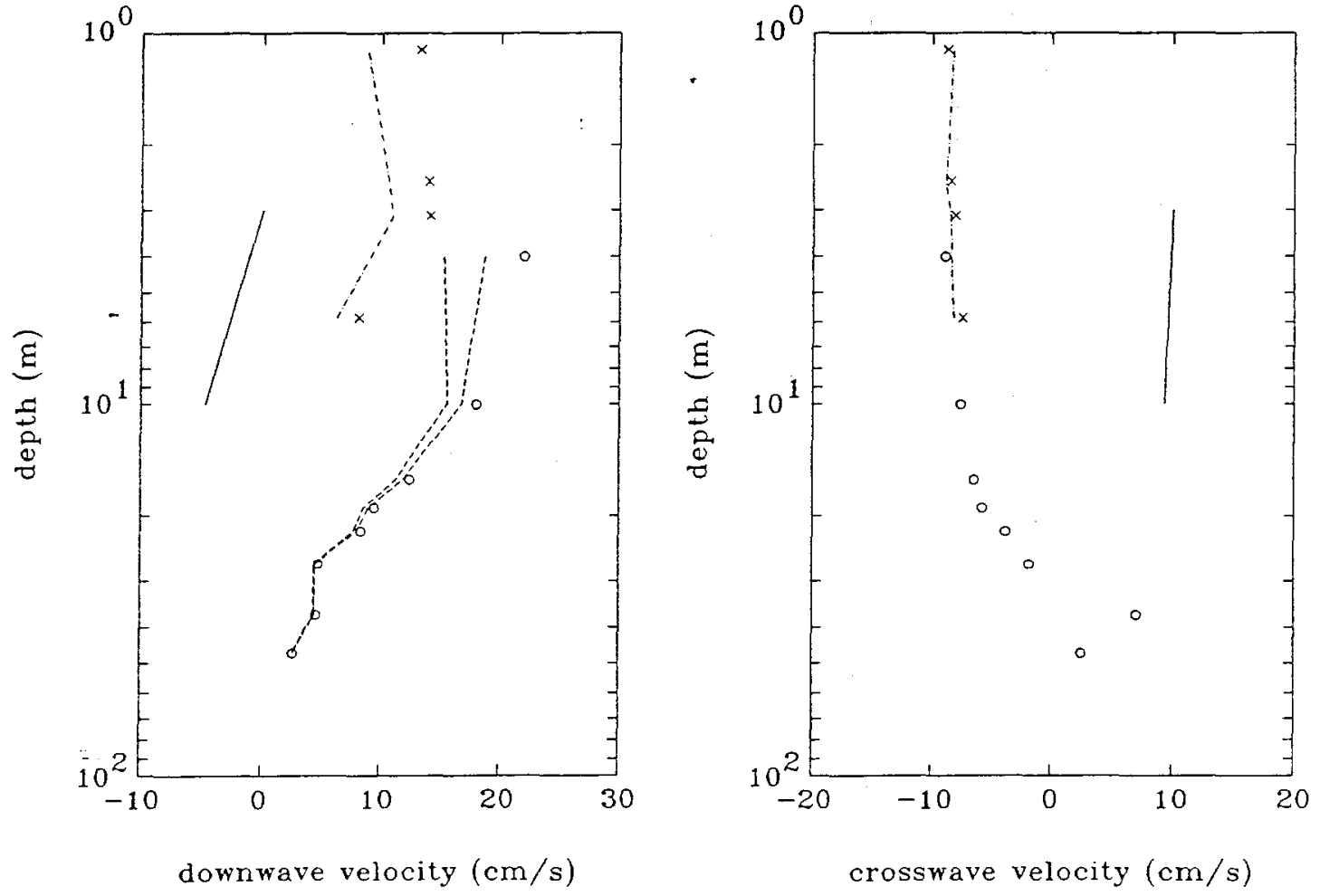


\section{file $\mathrm{p} 1$}

date $=$ February 27, $1989 \quad$ time $=21-12-16.75 \quad$ duration $=00-39-53.00$

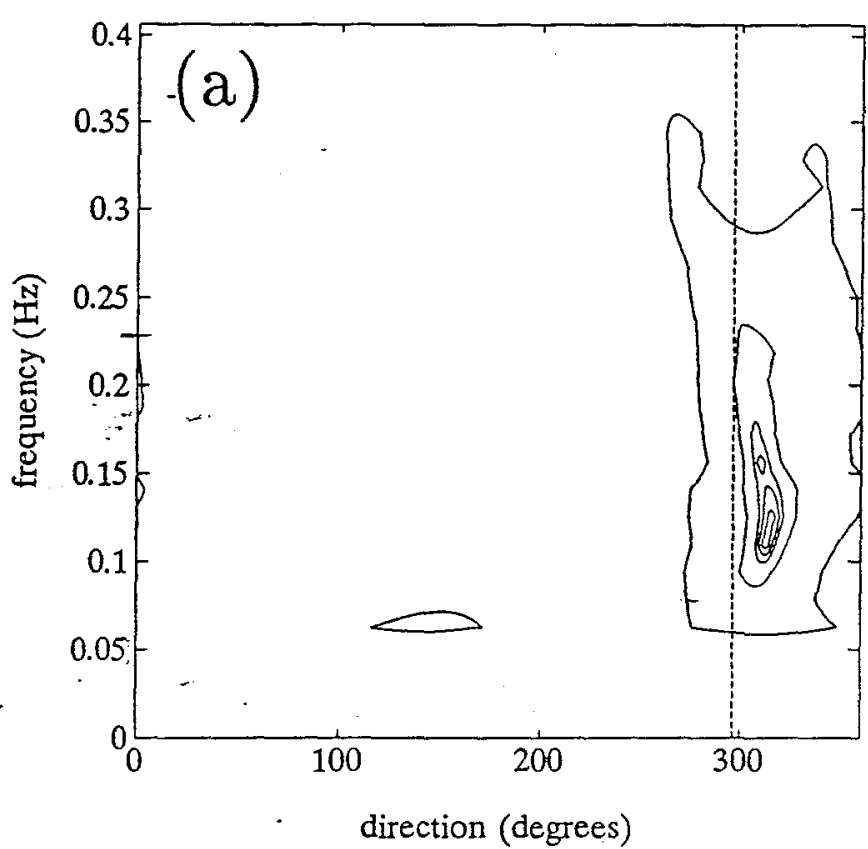

$$
\begin{array}{cc}
\underline{\text { wind }} & \underline{\text { waves }} \\
U_{10}=14.4 \mathrm{~m} / \mathrm{s} & \bar{H}_{1 / 3}=2.3 \mathrm{~m} \\
\theta_{\tau}=297^{\circ} & \theta_{w}=309^{\circ} \\
\frac{z}{L}=-0.01 & T_{p}=8.0 \mathrm{sec} \\
u_{*_{a}}=54.3 \mathrm{~cm} / \mathrm{s} & \bar{T}=5.3 \mathrm{sec} \\
\multicolumn{2}{c}{\Delta_{\text {temp }}=-0.35 \mathrm{C}}
\end{array}
$$

(a) directional spectrum $\left(--=\theta_{\tau}\right)$

(b) energy spectrum $\left(\cdots=f^{-4}\right)$

(c) $0=$ salinity, $x,+=$ temperature
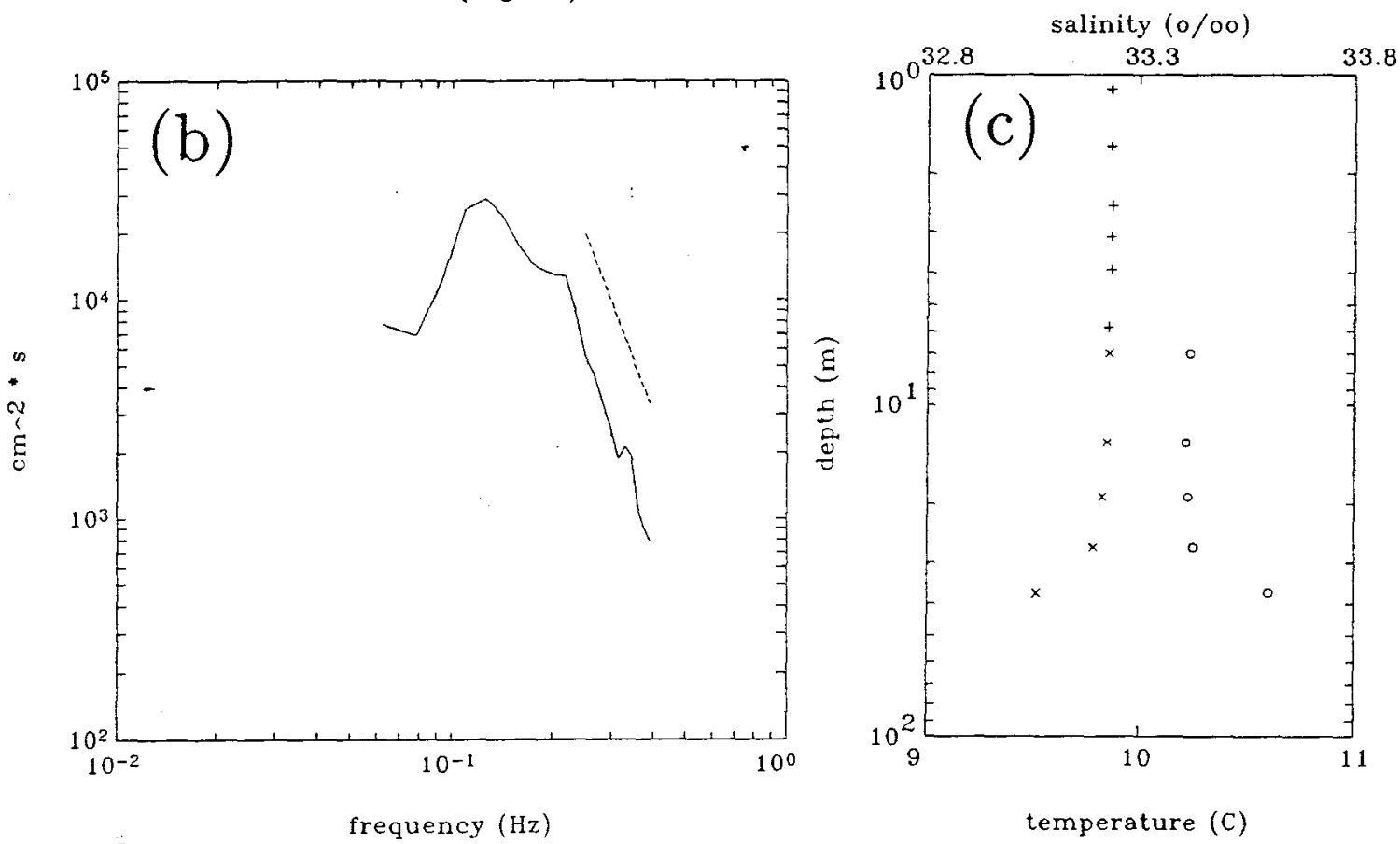


\section{file $\mathrm{p} 1$}

\begin{tabular}{|c|c|c|c|c|c|c|c|}
\hline \multirow[b]{3}{*}{$\begin{array}{c}z \\
1.11 \\
2.51 \\
3.11 \\
5.85\end{array}$} & \multicolumn{5}{|c|}{ downwave } & \multicolumn{2}{|c|}{ crosswave } \\
\hline & $\bar{v}_{d w}$ & $\Delta_{\text {bias }}$ & $\Delta_{B A S S}$ & $\Delta_{\text {float }}$ & $\widehat{v}_{d w}$ & $\bar{v}_{c w}$ & $\Delta_{B}$ \\
\hline & $\begin{array}{l}19.2 \\
19.7 \\
19.5 \\
16.1\end{array}$ & $\begin{array}{l}-6.8 \\
-5.1 \\
-4.8 \\
-3.3\end{array}$ & $\begin{array}{l}0.9 \\
0.9 \\
0.9 \\
1.0\end{array}$ & $\begin{array}{l}2.4 \\
0.0 \\
0.0 \\
0.0\end{array}$ & $\begin{array}{l}15.7 \\
15.5 \\
15.6 \\
13.7\end{array}$ & $\begin{array}{l}-13.1 \\
-12.5 \\
-12.0 \\
-11.3\end{array}$ & $\begin{array}{l}-0.6 \\
-0.6 \\
-0.5 \\
-0.7\end{array}$ \\
\hline$z$ & $\bar{v}_{d w}$ & $\Delta_{b i a s}^{\max }$ & $\Delta_{b i}^{m}$ & & $\widehat{v}_{d w}^{\min }$ & $\widehat{v}_{d w}^{\max }$ & $\bar{v}_{c w}$ \\
\hline $\begin{array}{r}4 \\
10\end{array}$ & $\begin{array}{l}20.2 \\
16.3\end{array}$ & $\begin{array}{l}-8.7 \\
-3.3\end{array}$ & $\begin{array}{l}-4 . \\
-1 .\end{array}$ & & $\begin{array}{l}1.5 \\
3.0\end{array}$ & $\begin{array}{l}16.0 \\
14.6\end{array}$ & $\begin{array}{l}-9.4 \\
-7.8\end{array}$ \\
\hline 16 & 11.5 & -1.6 & -0.8 & & 9.9 & 10.7 & -6.4 \\
\hline 19 & 9.3 & -1.2 & -0 & & 8.1 & 8.6 & -6.8 \\
\hline 22 & 7 . & -0.9 & -0.5 & & 6.7 & 7.1 & $-4 \cdot 3$ \\
\hline 27 & 4 . & -0.6 & -0.1 & & 4.3 & 4.5 & -2.6 \\
\hline 37 & 3.2 & -0.2 & -0.2 & & 3.0 & 3.1 & 1.4 \\
\hline 47 & -1.2 & -0.1 & -0. & & 1.4 & $-1 \cdot 3$ & 4.5 \\
\hline
\end{tabular}

Below: SASS $=x$ (raw) -... (unbiased), C3=o (raw) - - (unbiased), theoretical log-law shear $=-$
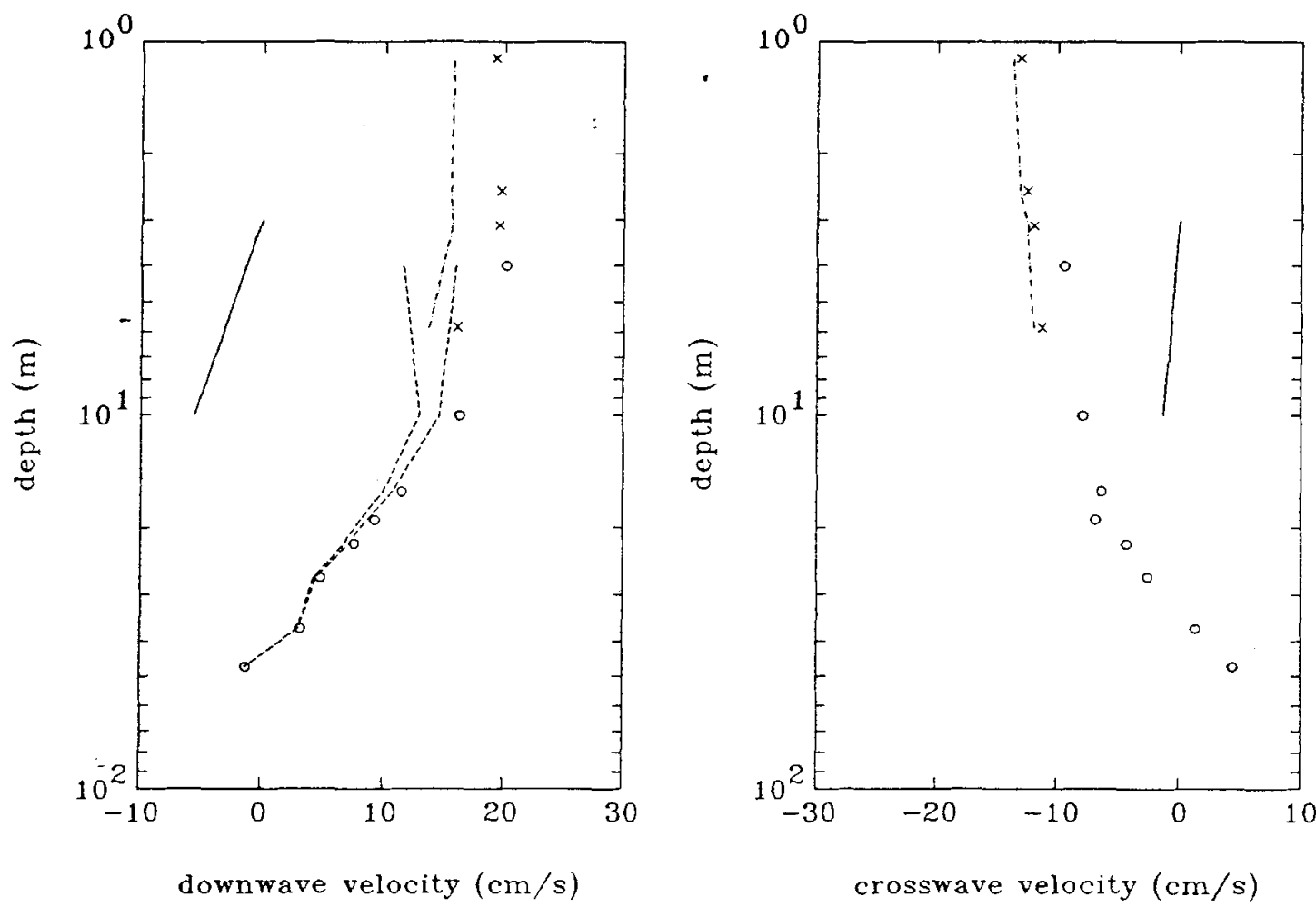


\section{file $\mathrm{p} 2$}

date $=$ February 27, $1989 \quad$ time $=21-52-09.75 \quad$ duration $=00-39-52.75$
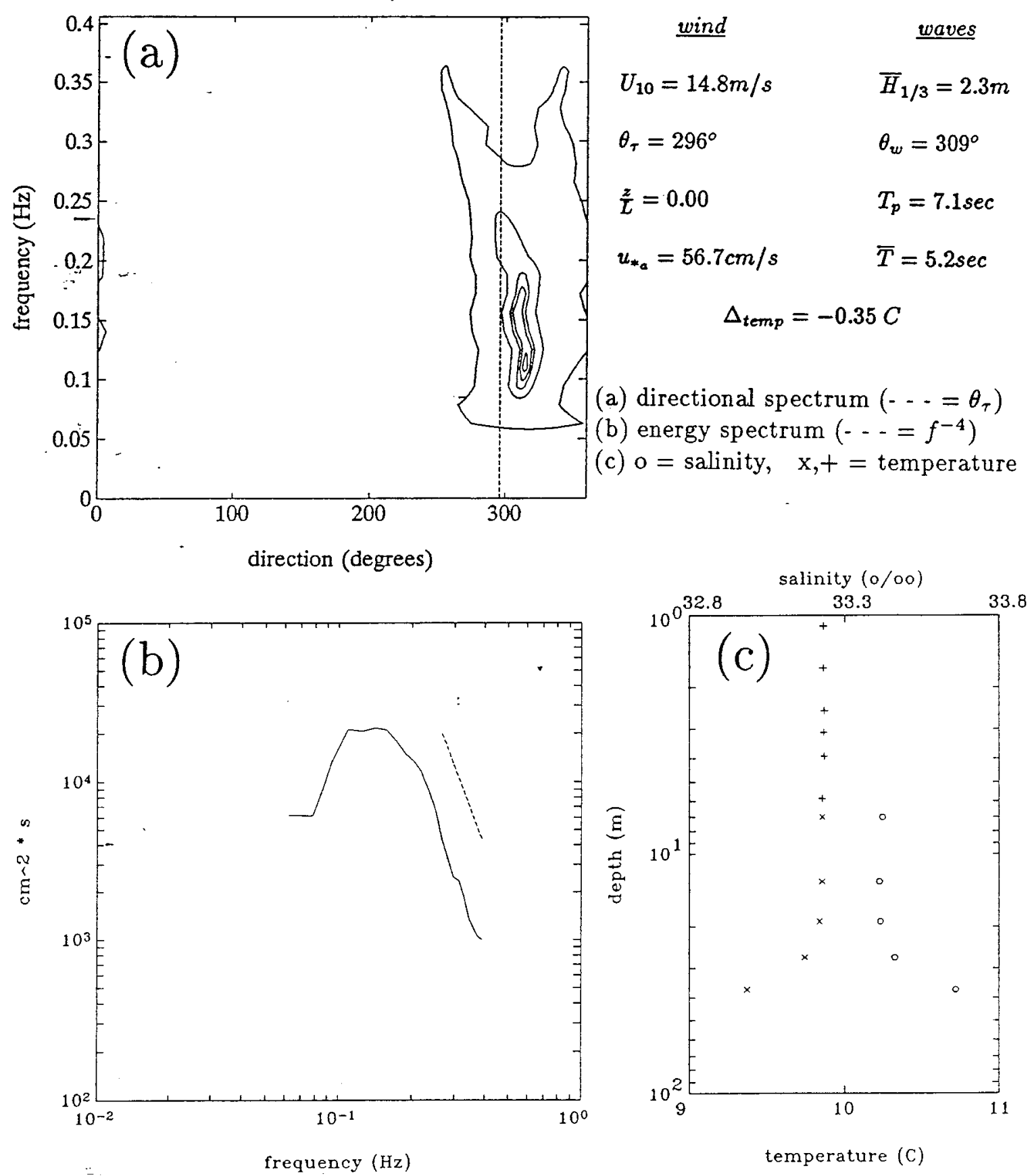


\section{file $\mathrm{p} 2$}

\begin{tabular}{cccccccc} 
& \multicolumn{9}{c}{$\frac{}{\text { downwave }}$} & \multicolumn{3}{c}{ crosswave } \\
1.11 & $\bar{v}_{d w}$ & $\Delta_{\text {bias }}$ & $\Delta_{\text {BASS }}$ & $\Delta_{\text {float }}$ & $\widehat{v}_{d w}$ & $\bar{v}_{c w}$ & $\Delta_{B A S S} \widehat{v}_{c w}$ \\
2.51 & 20.1 & -7.0 & 1.0 & 2.5 & 16.5 & -13.5 & $-0.6-14.1$ \\
3.11 & 20.1 & -5.2 & 0.9 & 0.0 & 15.8 & -12.7 & $-0.6-13.3$ \\
5.85 & 19.8 & -4.8 & 0.9 & 0.0 & 15.9 & -12.0 & $-0.5-12.6$ \\
5.9 & -3.3 & 1.0 & 0.0 & 14.6 & -12.3 & $-0.7-13.1$
\end{tabular}

$\begin{array}{lrrrrrr}z & \bar{v}_{d w} & \Delta_{\text {bias }}^{\max } & \Delta_{\text {bias }}^{\min } & \widehat{v}_{d w}^{\min } & \widehat{v}_{d w}^{\max } & \bar{v}_{c w} \\ 4 & 21.7 & -8.6 & -4.2 & 13.1 & 17.5 & -12.7 \\ 10- & 17.5 & -3.3 & -1.7 & 14.2 & 15.8 & -10.6 \\ 16 & 13.4 & -1.6 & -0.8 & 11.8 & 12.6 & -9.8 \\ 19 . & 11.3 & -1.1 & -0.6 & 10.1 & 10.7 & -10.7 \\ 22 & 9.0 & -0.8 & -0.5 & 8.2 & 8.5 & -7.8 \\ 27 & 6.5 & -0.5 & -0.3 & 6.0 & 6.2 & -4.4 \\ 37 & 2.3 & -0.2 & -0.1 & 2.1 & 2.2 & -1.0 \\ 47 & -1.8 & -0.1 & -0.1 & -1.9 & -1.9 & 6.5\end{array}$

Below: $\mathrm{SASS}=x$ (raw) -.-- (unbiased), C3=o (raw) - - (unbiased), theoretical log-law shear $=-$
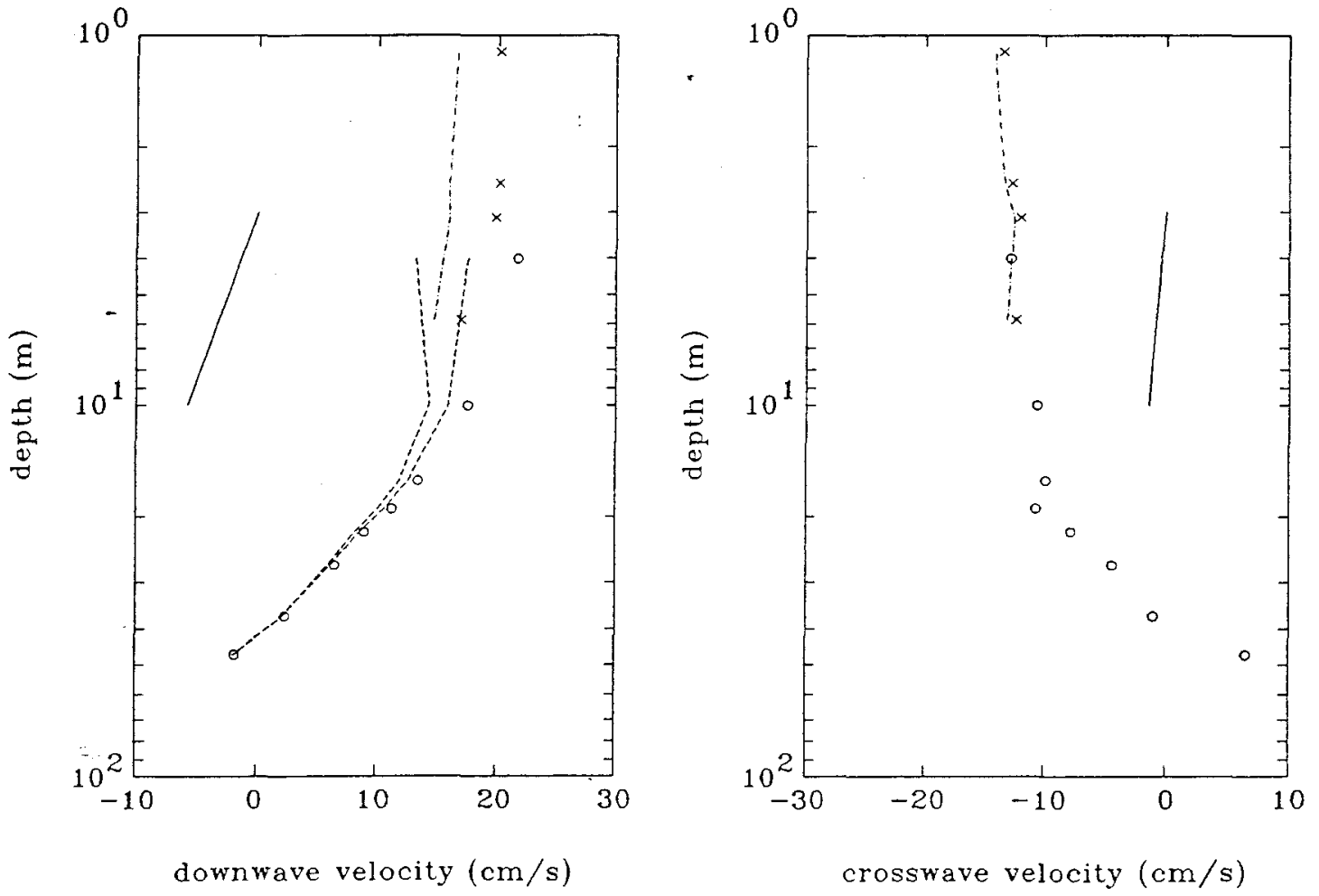


\section{file $\mathrm{p} 3$}

date $=$ February 27, $1989 \quad$ time $=22-32-02.50 \quad$ duration $=00-39-52.75$

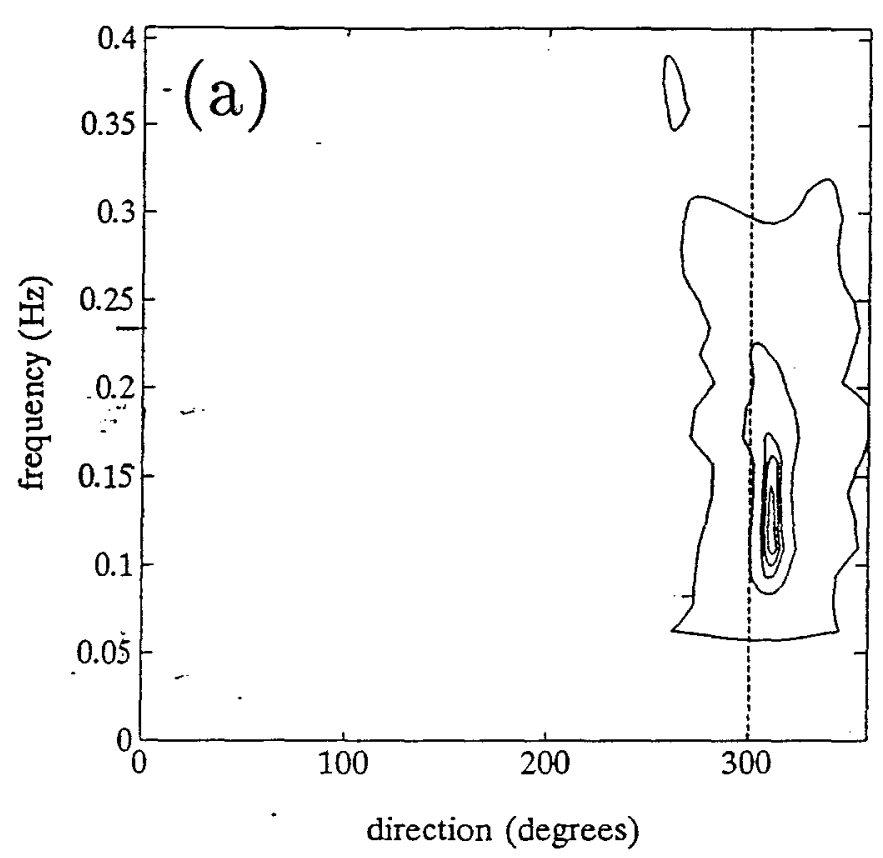

$$
\begin{array}{cc}
\underline{\text { wind }} & \multicolumn{1}{c}{\underline{\text { waves }}} \\
U_{10}=14.5 \mathrm{~m} / \mathrm{s} & \bar{H}_{1 / 3}=2.3 \mathrm{~m} \\
\theta_{\tau}=301^{\circ} & \theta_{w}=309^{\circ} \\
\bar{L}=0.00 & T_{p}=8.0 \mathrm{sec} \\
u_{*_{a}}=55.0 \mathrm{~cm} / \mathrm{s} & \bar{T}=5.3 \mathrm{sec} \\
\multicolumn{2}{c}{\Delta_{\text {temp }}=-0.35 \mathrm{C}}
\end{array}
$$

(a) directional spectrum

(b) energy spectrum $\left(---=f^{-4}\right)$

(c) $o=$ salinity, $x,+=$ temperature
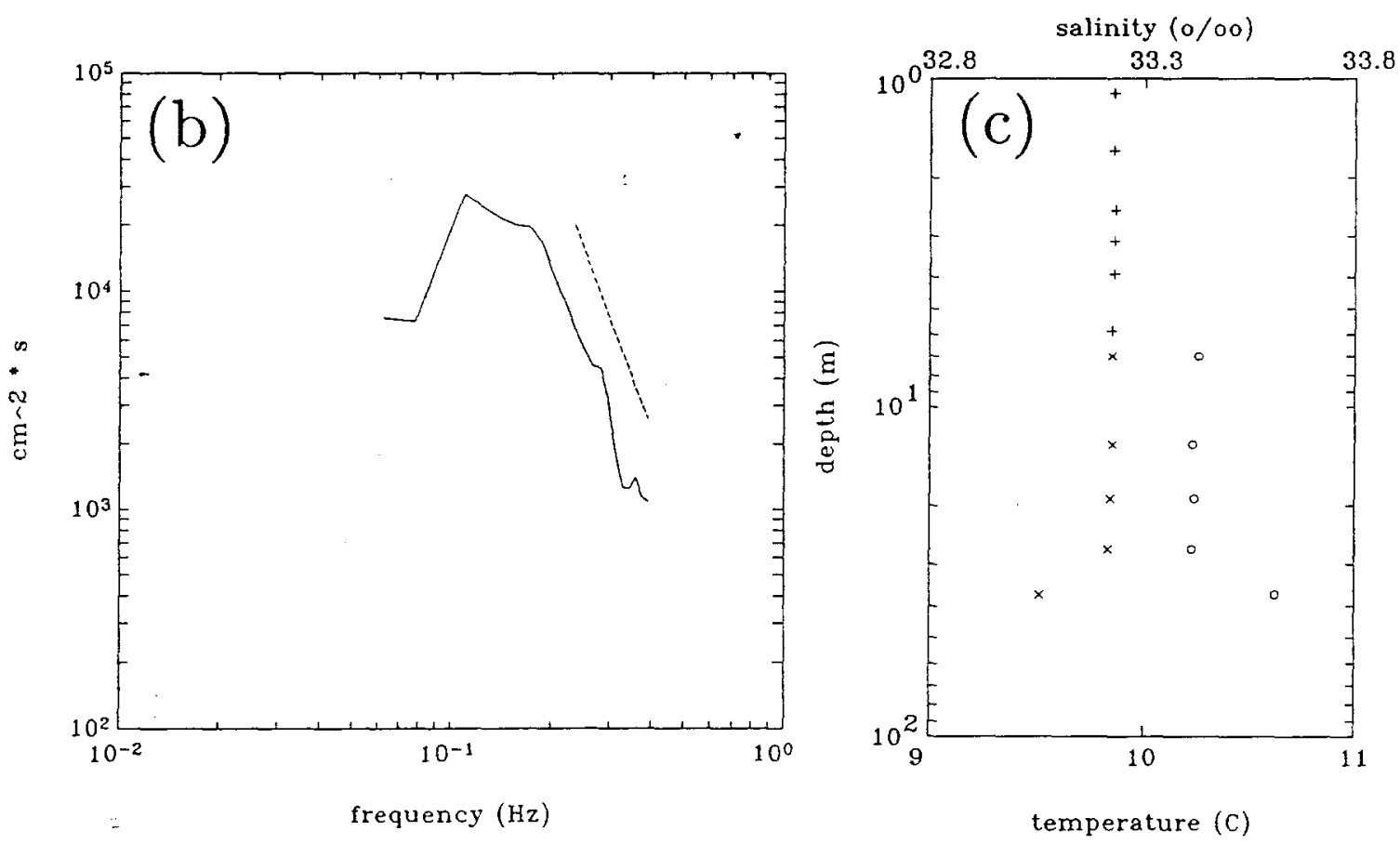


\section{file p3}

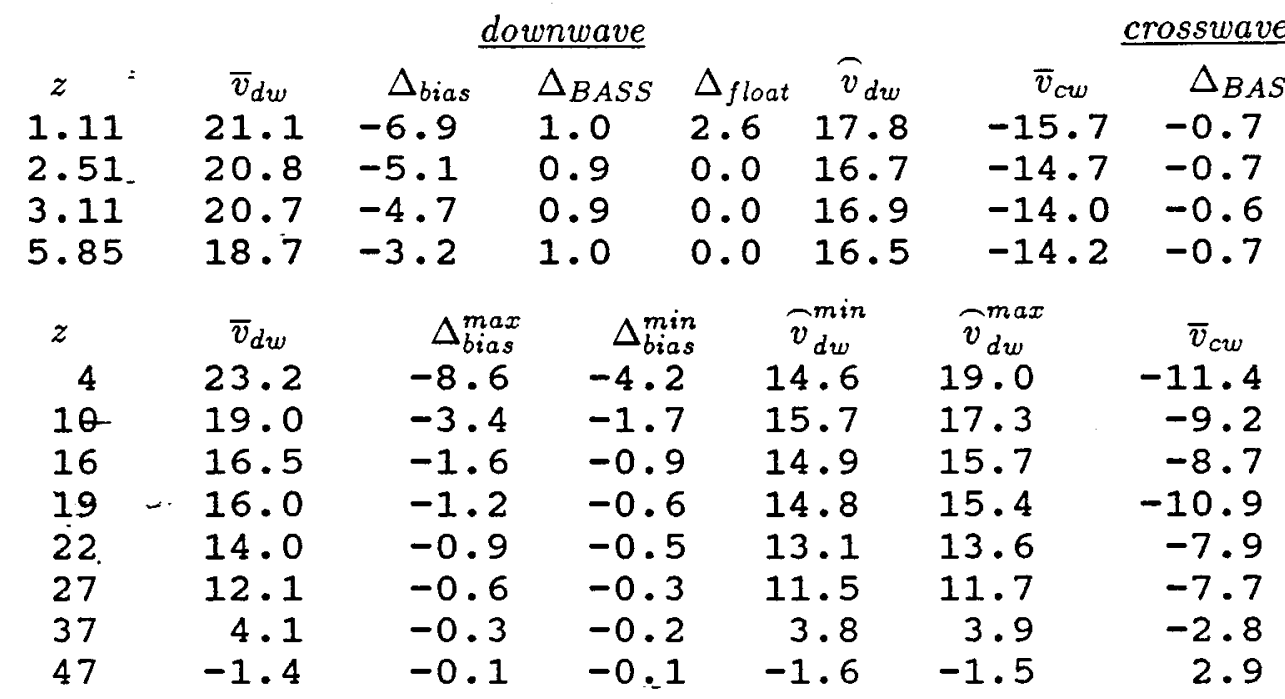

Below: SASS $=x$ (raw) -..- (unbiased), C3=o (raw) - - (unbiased), theoretical log-law shear $=-$
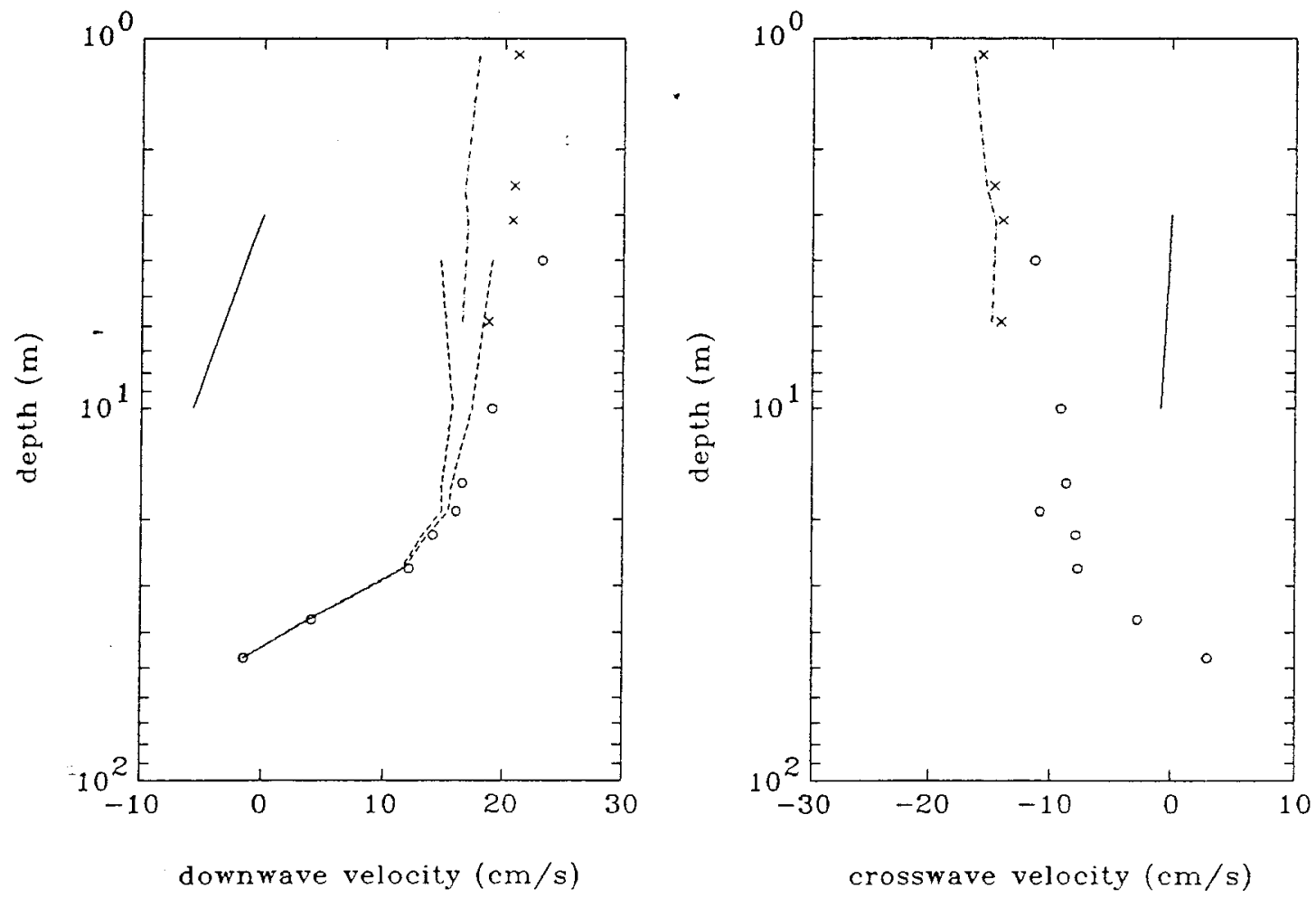


\section{file $\mathrm{q} 1$}

$$
\text { date }=\text { February } 27,1989 \quad \text { time }=23-32-30.50 \quad \text { duration }=00-17-49.00
$$

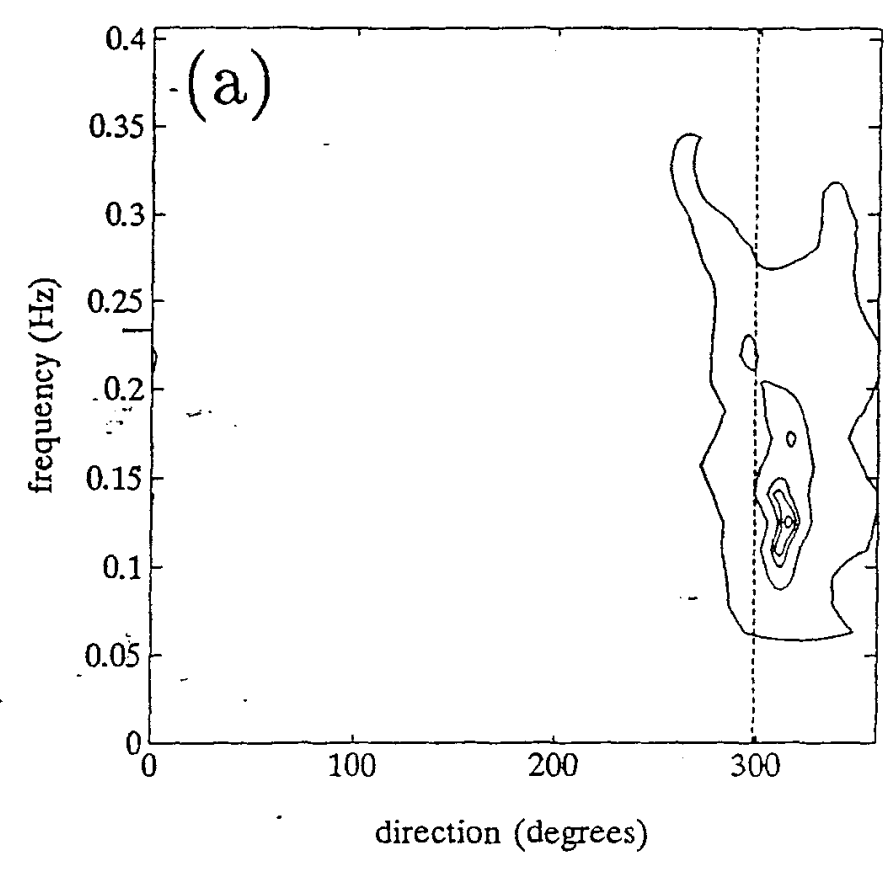

$$
\begin{array}{cc}
\underline{\text { wind }} & \multicolumn{1}{c}{\text { waves }} \\
U_{10}=13.5 \mathrm{~m} / \mathrm{s} & \bar{H}_{1 / 3}=2.5 \mathrm{~m} \\
\theta_{\tau}=298^{\circ} & \theta_{w}=310^{\circ} \\
\frac{z}{L}=-0.01 & T_{p}=8.0 \mathrm{sec} \\
u_{* a}=50.2 \mathrm{~cm} / \mathrm{s} & \bar{T}=5.4 \mathrm{sec} \\
\multicolumn{2}{c}{\Delta_{\text {temp }}=-0.35 \mathrm{C}}
\end{array}
$$

(a) directional spectrum

(b) energy spectrum $\left(-\cdots=f^{-4}\right)$

(c) $0=$ salinity, $x,+=$ temperature
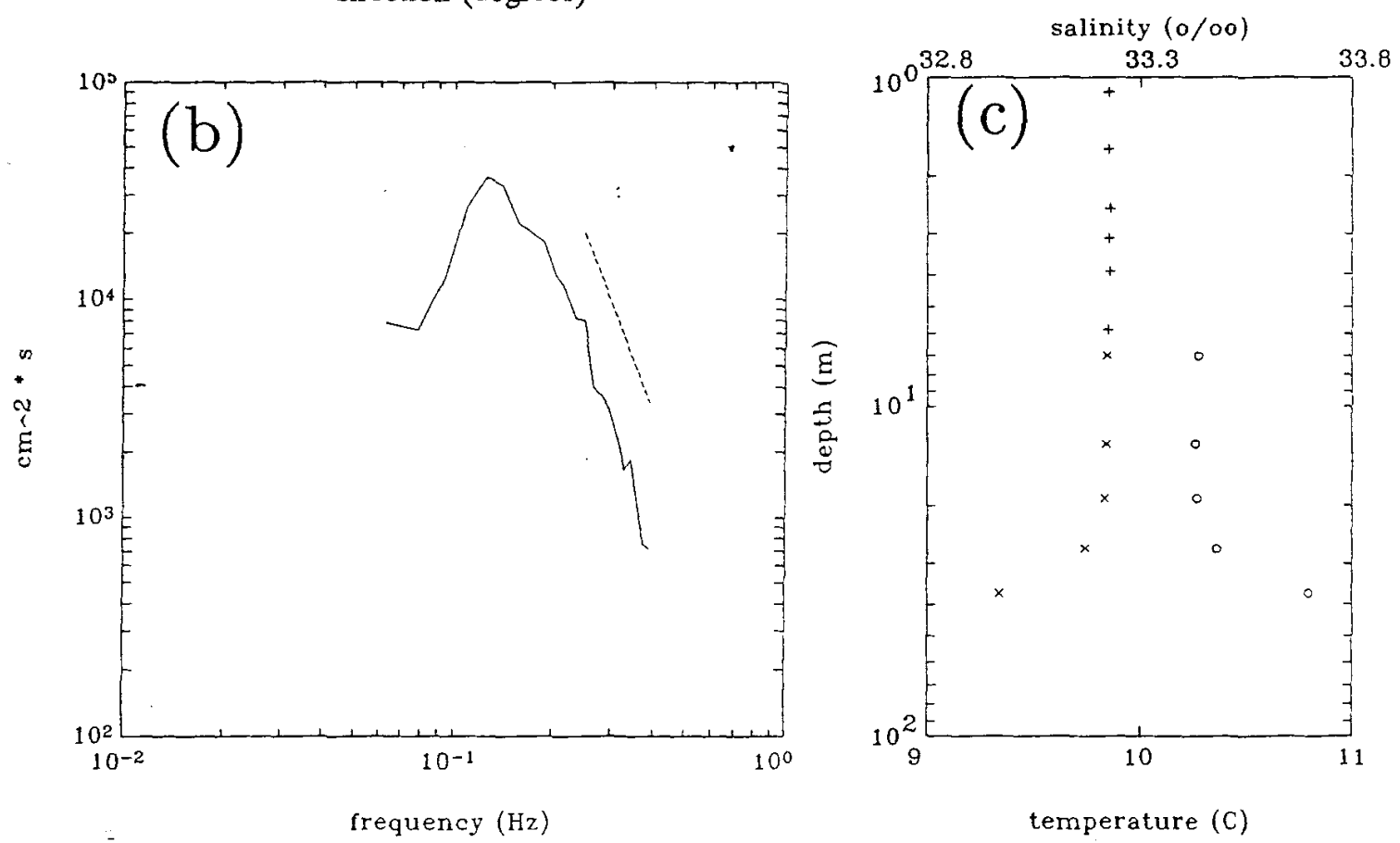


\section{file q1}

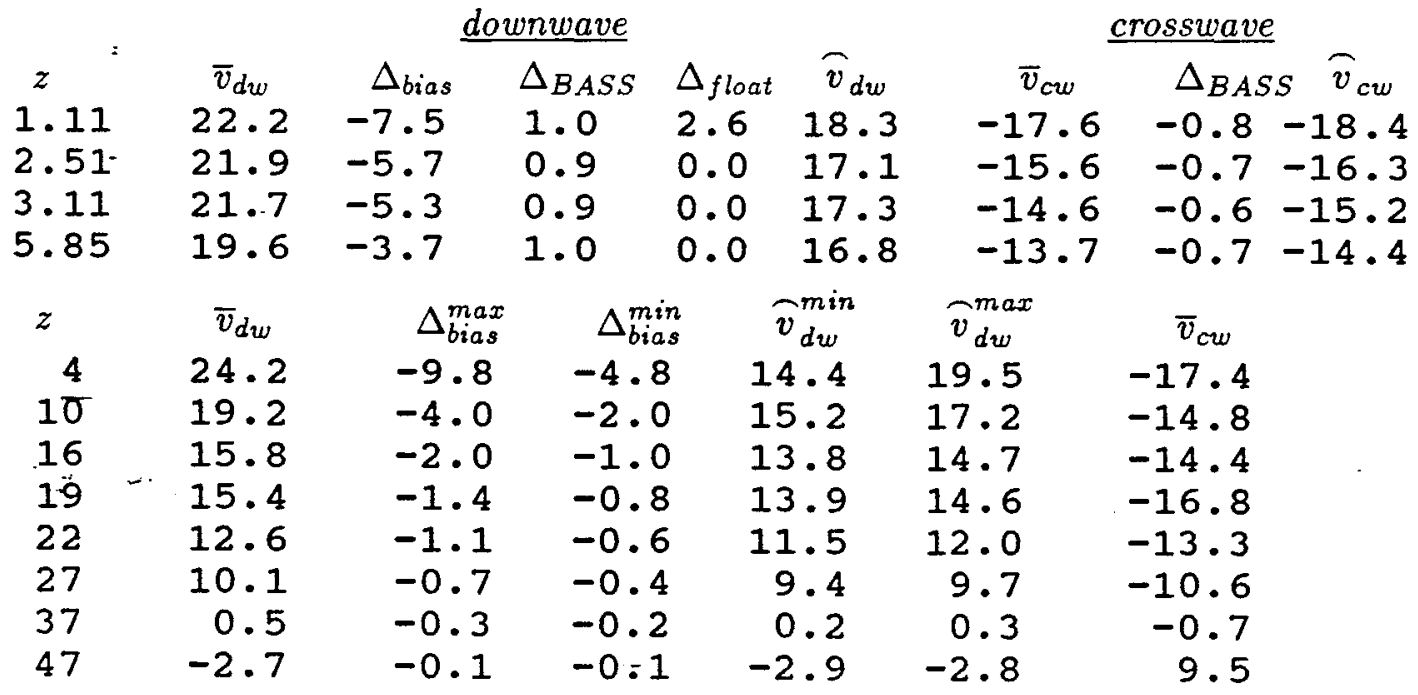

Below: $\mathrm{SASS}=x(\mathrm{raw}) \cdots($ ).-. (unbiased $), \mathrm{C} 3=o(\mathrm{raw}) \cdots($ unbiased $)$, theoretical log-law shear $=$ -
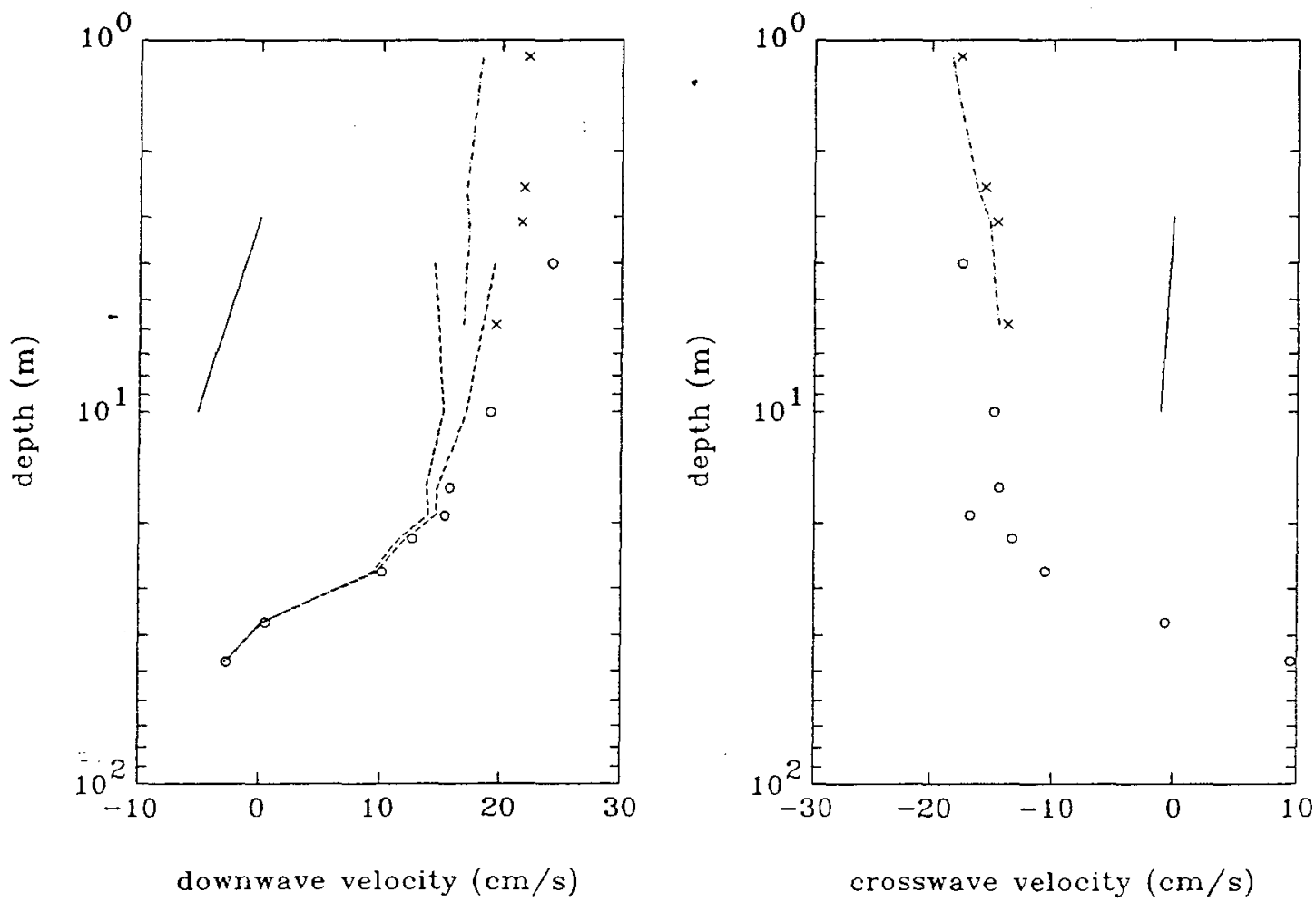


\section{file $r 1$}

date $=$ February 27, $1989 \quad$ time $=23-57-40.00 \quad$ duration $=00-39-17.00$

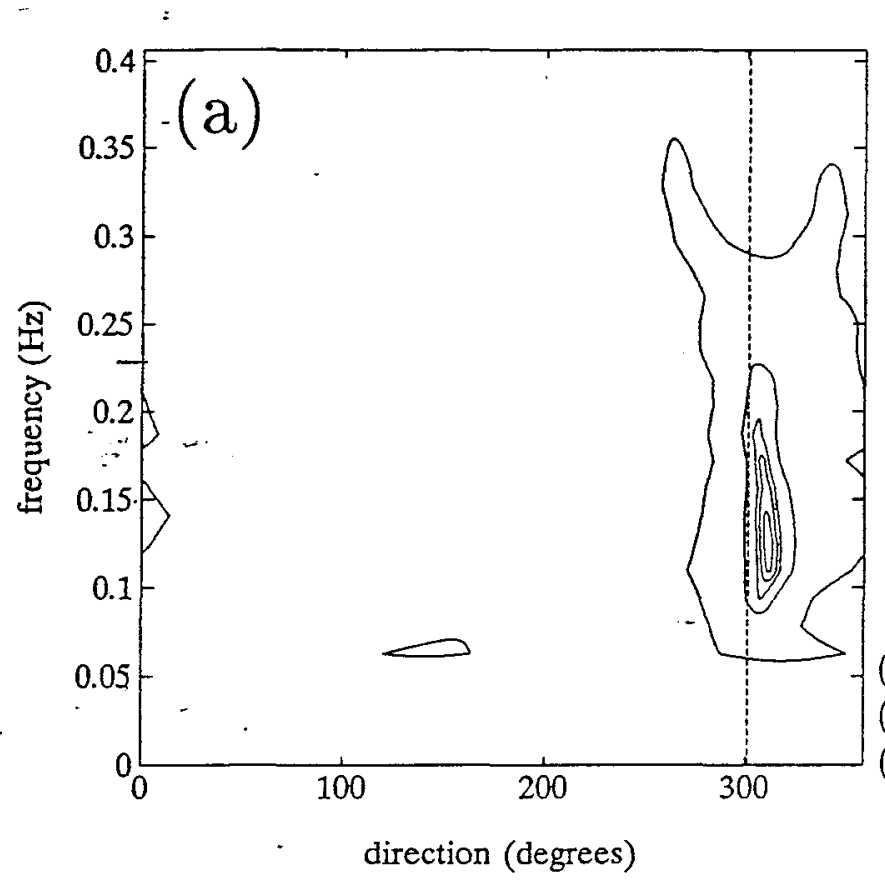

$$
\begin{array}{cc}
\underline{\text { wind }} & \multicolumn{1}{c}{\underline{\text { waves }}} \\
U_{10}=13.8 \mathrm{~m} / \mathrm{s} & \bar{H}_{1 / 3}=2.5 \mathrm{~m} \\
\theta_{\tau}=301^{\circ} & \theta_{w}=310^{\circ} \\
\frac{z}{L}=-0.01 & T_{p}=8.0 \mathrm{sec} \\
u_{*_{a}}=51.7 \mathrm{~cm} / \mathrm{s} & \bar{T}=5.4 \mathrm{sec} \\
\multicolumn{2}{c}{\Delta_{\text {temp }}=-0.35 \mathrm{C}}
\end{array}
$$

(a) directional spectrum

(b) energy spectrum $\left(-\cdots=f^{-4}\right)$

(c) $o=$ salinity, $x,+=$ temperature
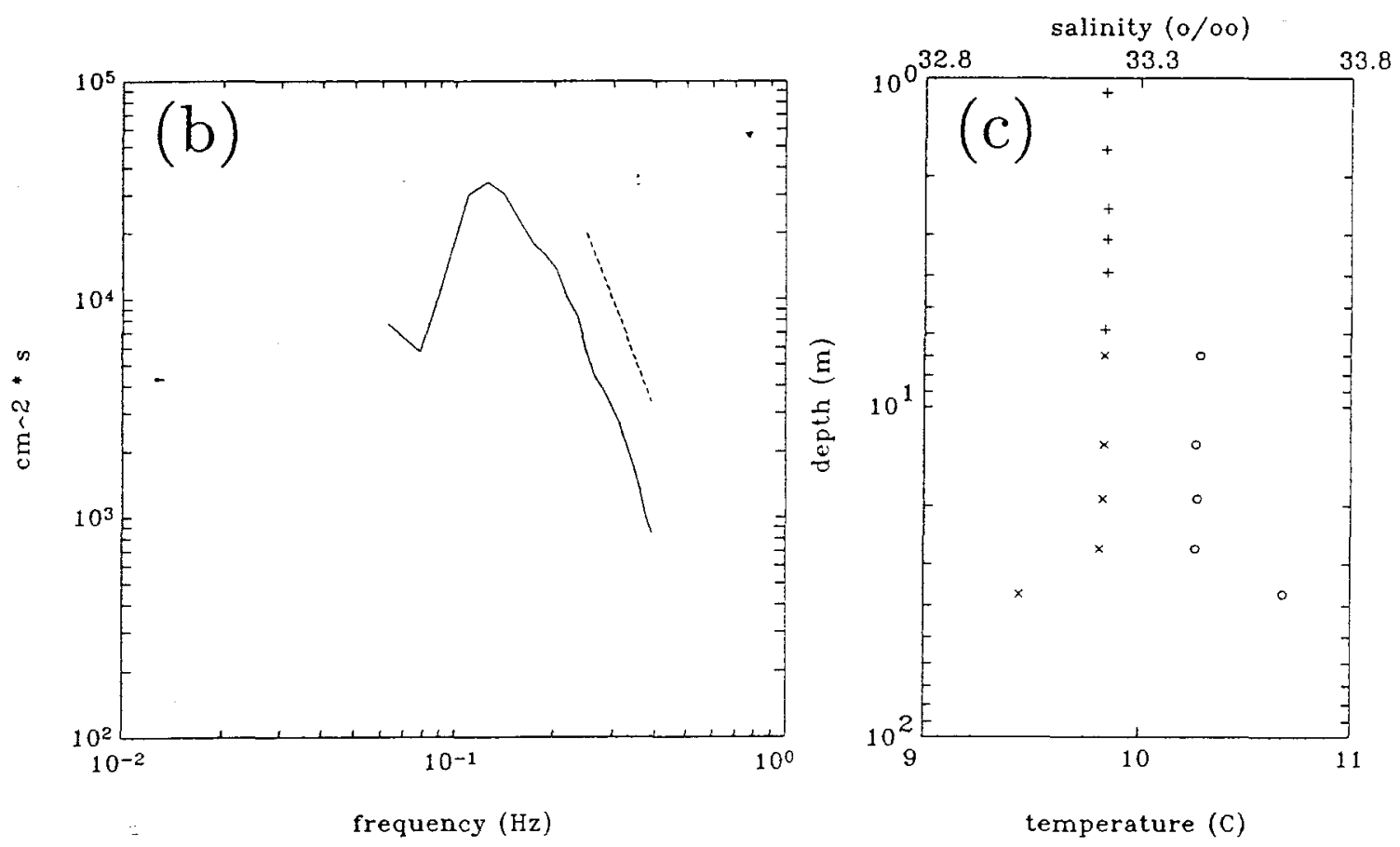


\section{file $\mathrm{r} 1$}

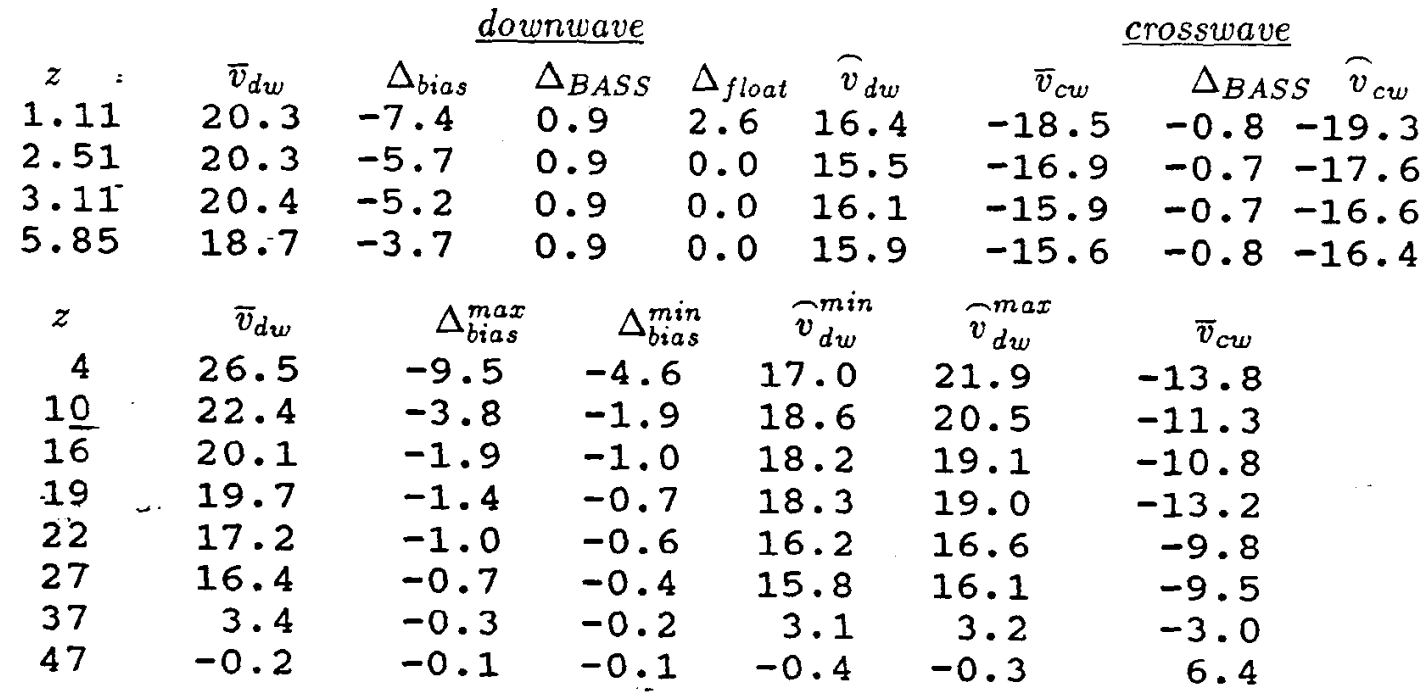

Below: SASS $=x$ (raw) -... (unbiased), $C 3=0$ (raw) - - (unbiased), theoretical log-law shear $=-$
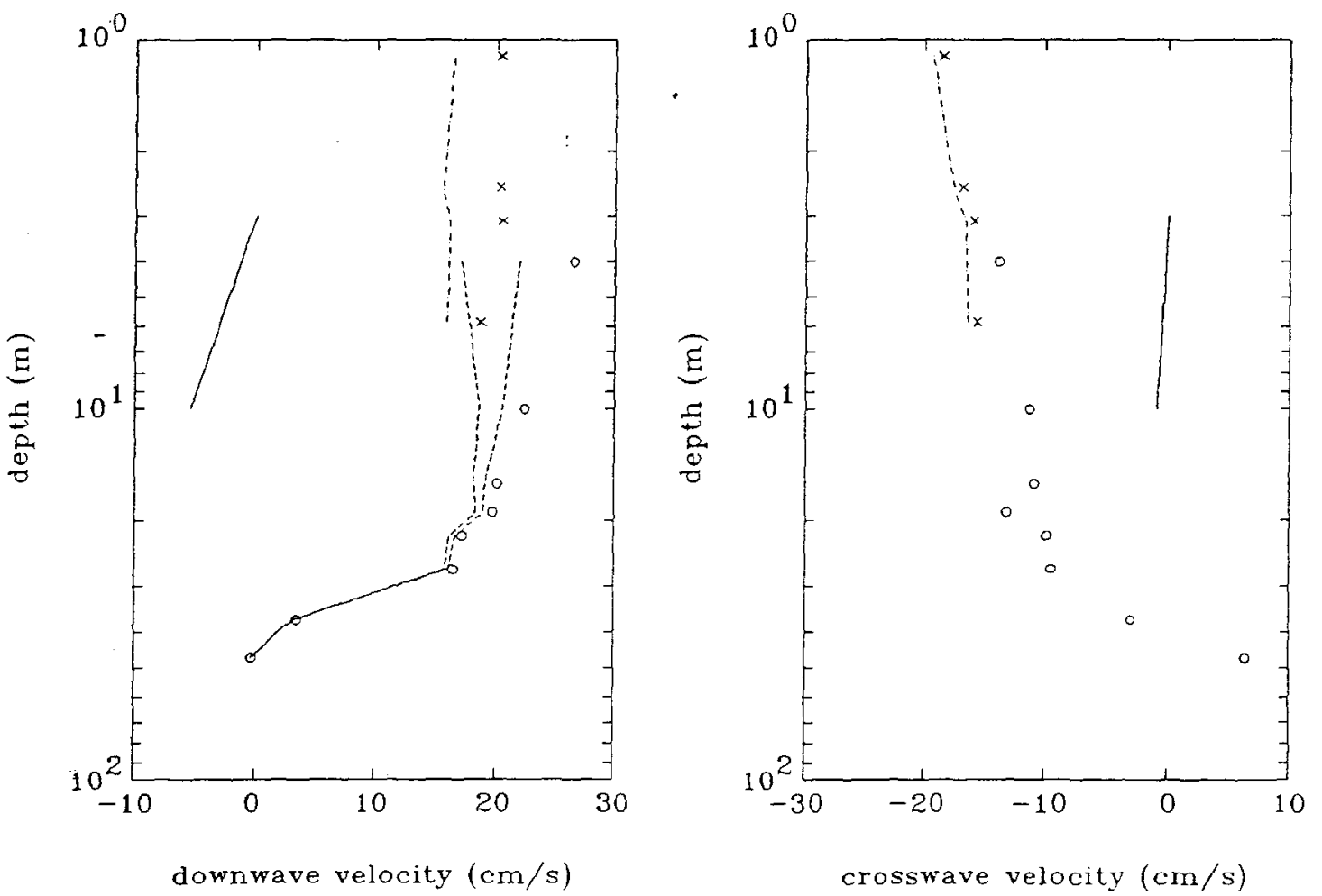


\section{file $r 2$}

$$
\text { date }=\text { February 28, } 1989 \quad \text { time }=00-36-57.00 \quad \text { duration }=00-39-17.00
$$

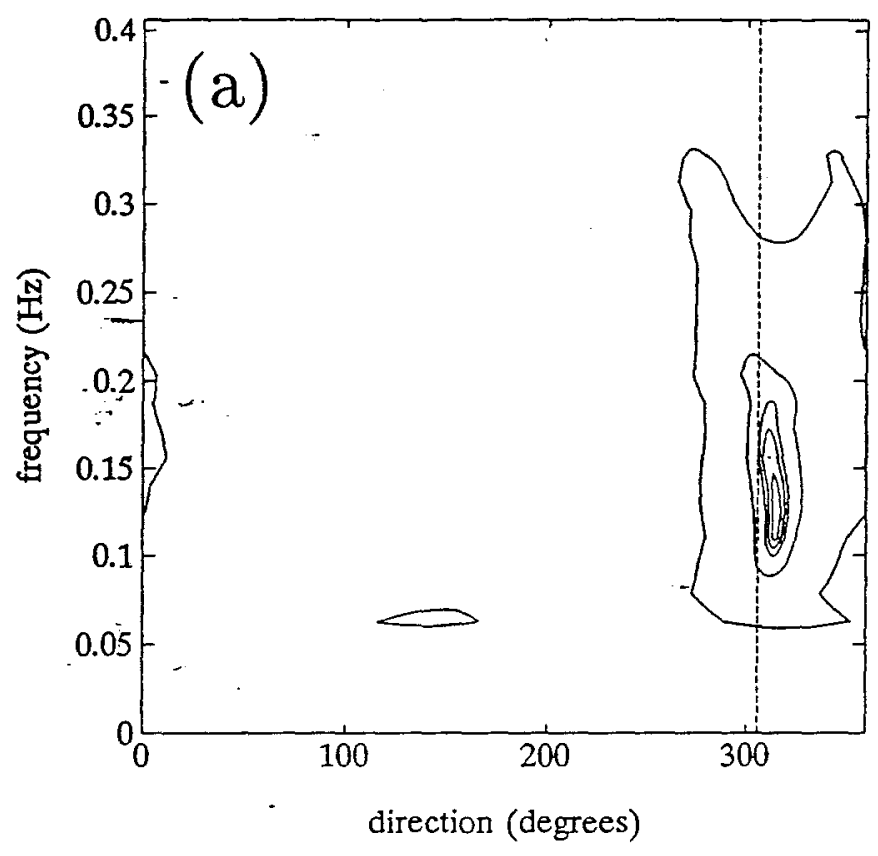

$$
\begin{array}{cc}
\underline{\text { wind }} & \multicolumn{1}{c}{\text { waves }} \\
U_{10}=14.5 \mathrm{~m} / \mathrm{s} & \bar{H}_{1 / 3}=2.4 \mathrm{~m} \\
\theta_{\tau}=306^{\circ} & \theta_{w}=313^{\circ} \\
\frac{z}{L}=-0.01 & T_{p}=7.1 \mathrm{sec} \\
u_{*_{a}}=55.1 \mathrm{~cm} / \mathrm{s} & \bar{T}=5.3 \mathrm{sec} \\
\multicolumn{2}{c}{\Delta_{\text {temp }}=-0.34 \mathrm{C}}
\end{array}
$$

(a) directional spectrum $\left(--=\theta_{\tau}\right)$

(b) energy spectrum $\left(-\cdots=f^{-4}\right)$

(c) $o=$ salinity, $x,+=$ temperature
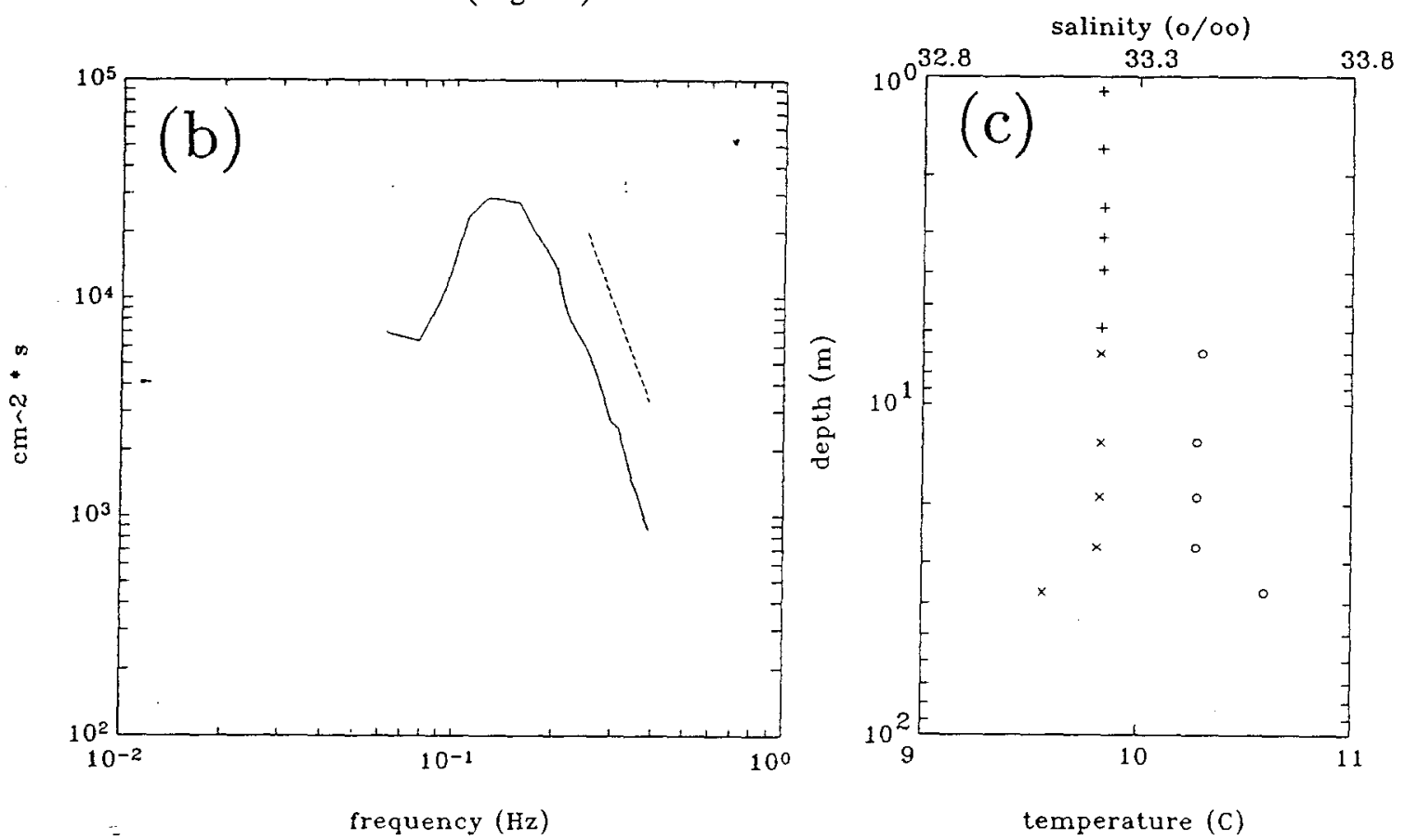


\section{file r2}

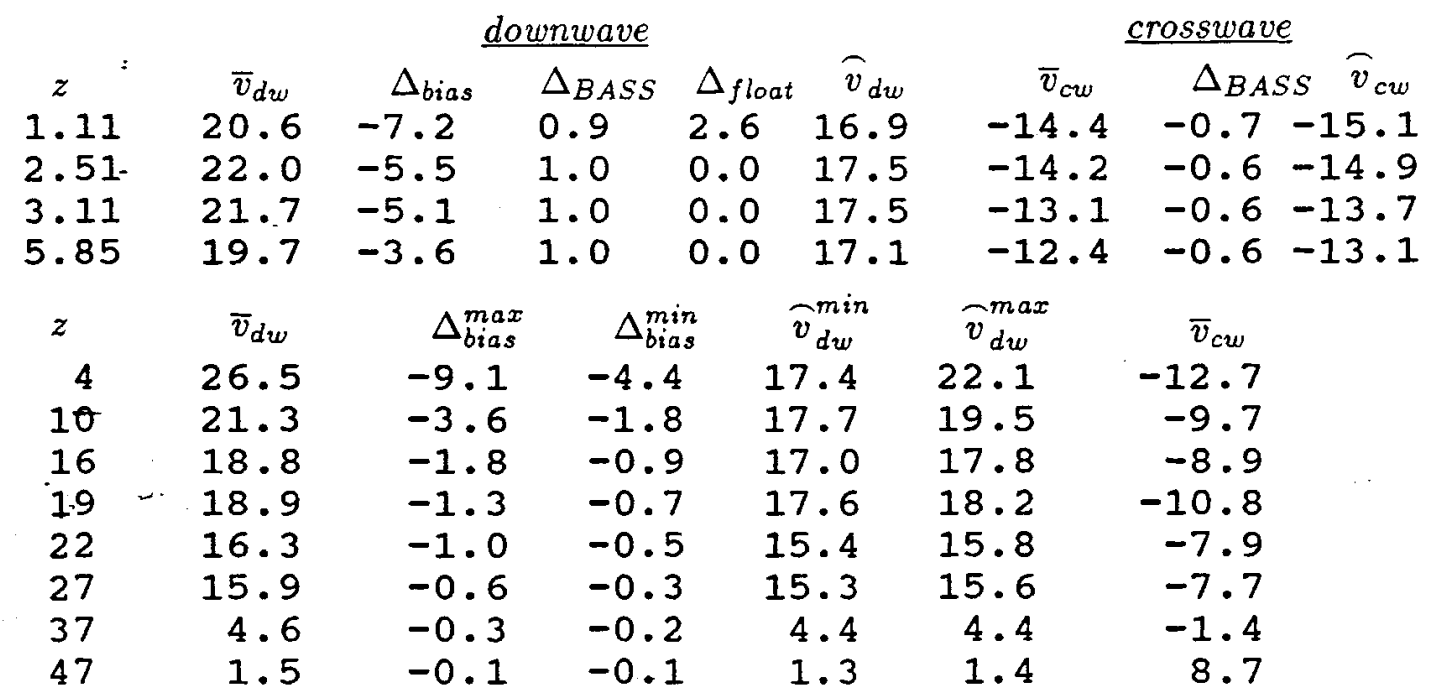

Below: $\mathrm{SASS}=x$ (raw) -...- (unbiased), $\mathrm{C} 3=0(\mathrm{raw}) \cdots($ unbiased $)$, theoretical log-law shear $=-$
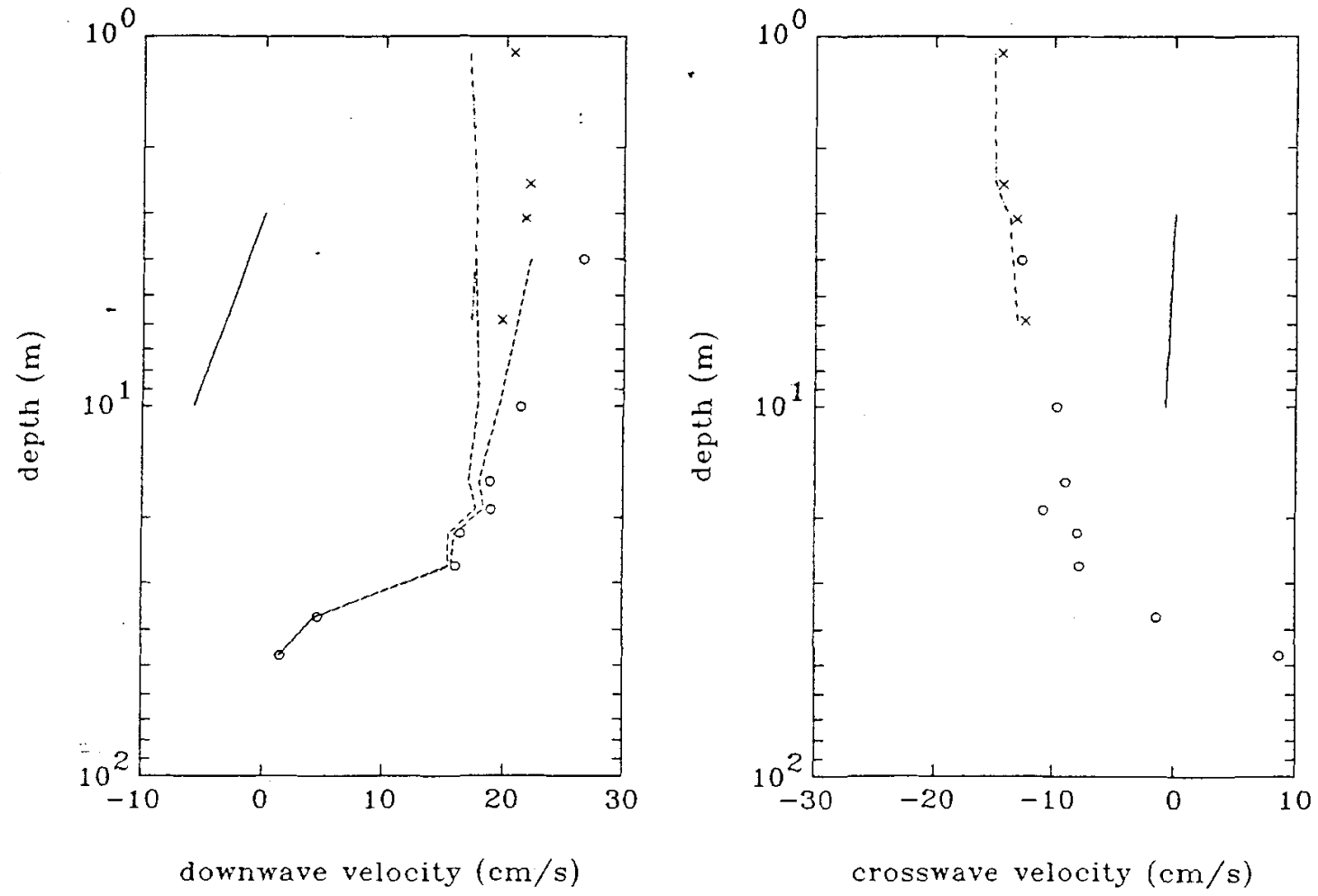


\section{file $r 3$}

date $=$ February $28,1989 \quad$ time $=00-01-16-14.00 \quad$ duration $=00-39-17.00$

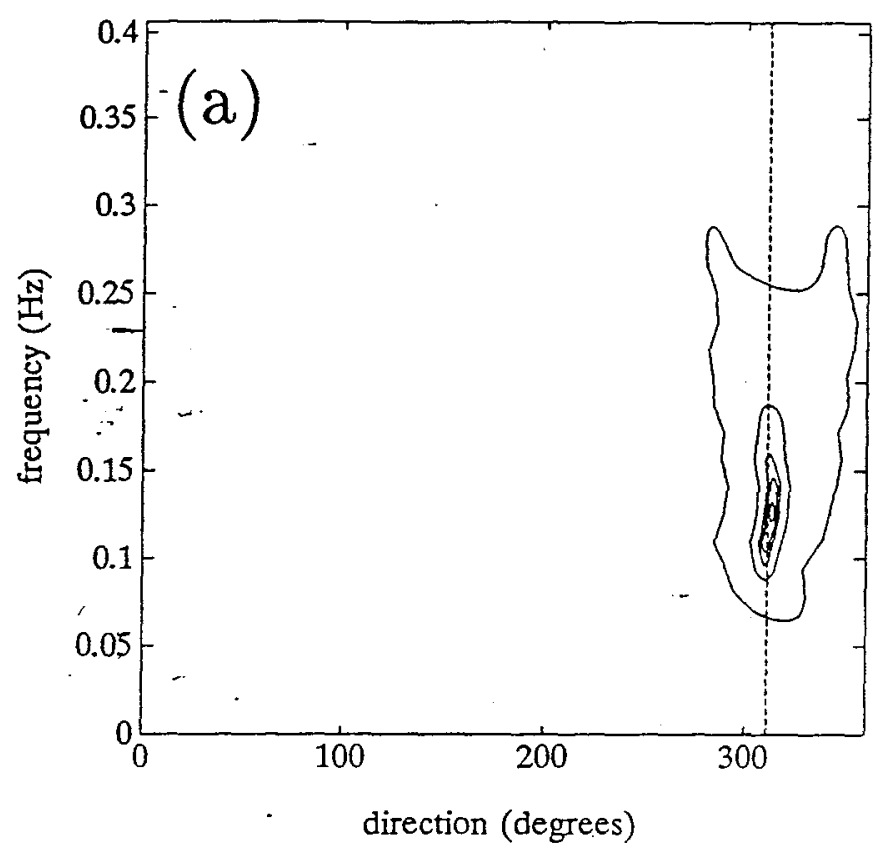

$$
\begin{array}{cc}
\underline{\text { wind }} & \underline{\text { waves }} \\
U_{10}=14.9 \mathrm{~m} / \mathrm{s} & \bar{H}_{1 / 3}=2.5 \mathrm{~m} \\
\theta_{\tau}=311^{\circ} & \theta_{w}=314^{\circ} \\
z=-0.01 & T_{p}=8.0 \mathrm{sec} \\
u_{*_{a}}=57.1 \mathrm{~cm} / \mathrm{s} & \bar{T}=5.6 \mathrm{sec} \\
\Delta_{\text {temp }}=-0.34 \mathrm{C}
\end{array}
$$

(a) directional spectrum

(b) energy spectrum $\left(\cdots=f^{-4}\right)$

(c) $o=$ salinity, $x,+=$ temperature
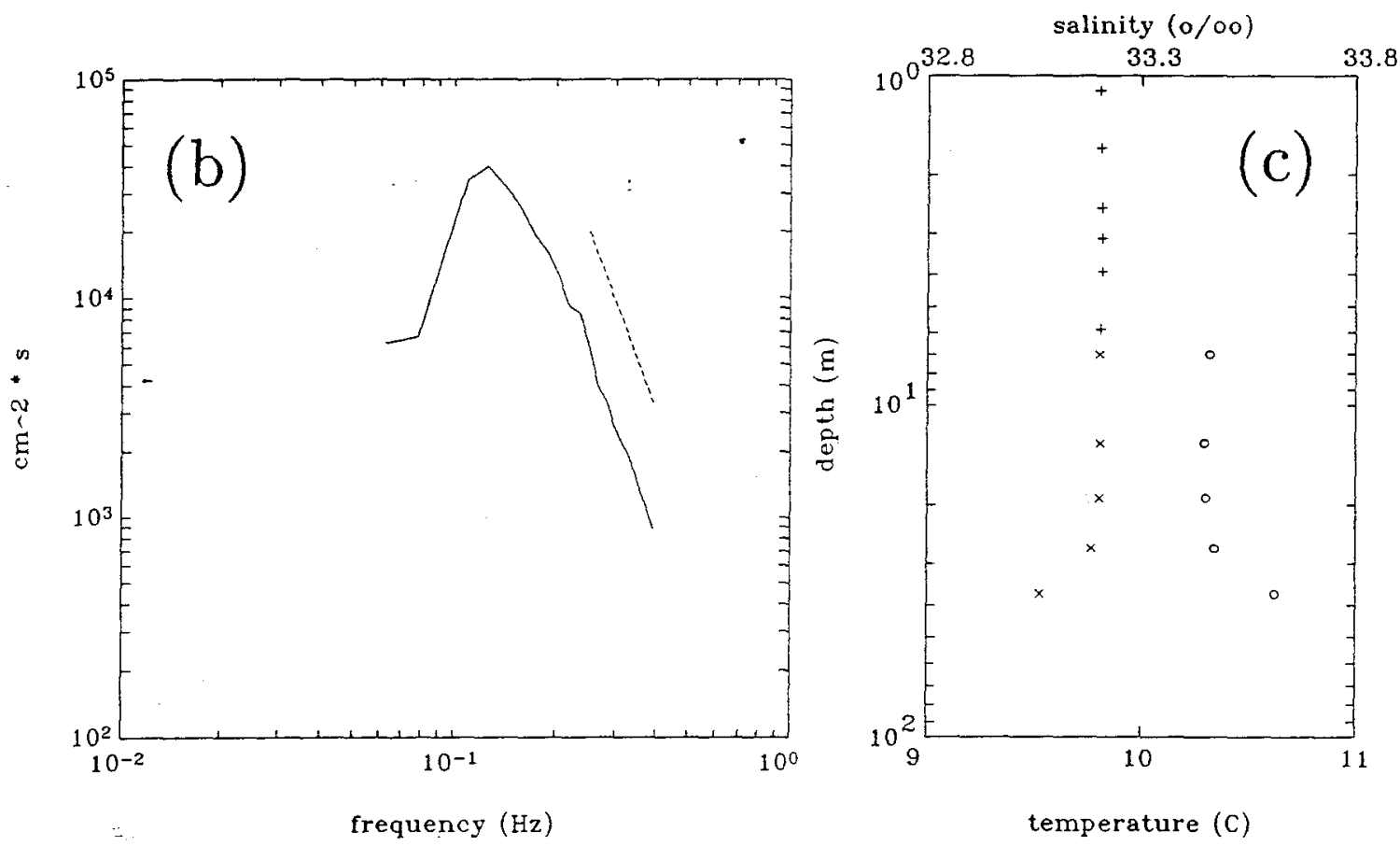


\section{file r3}

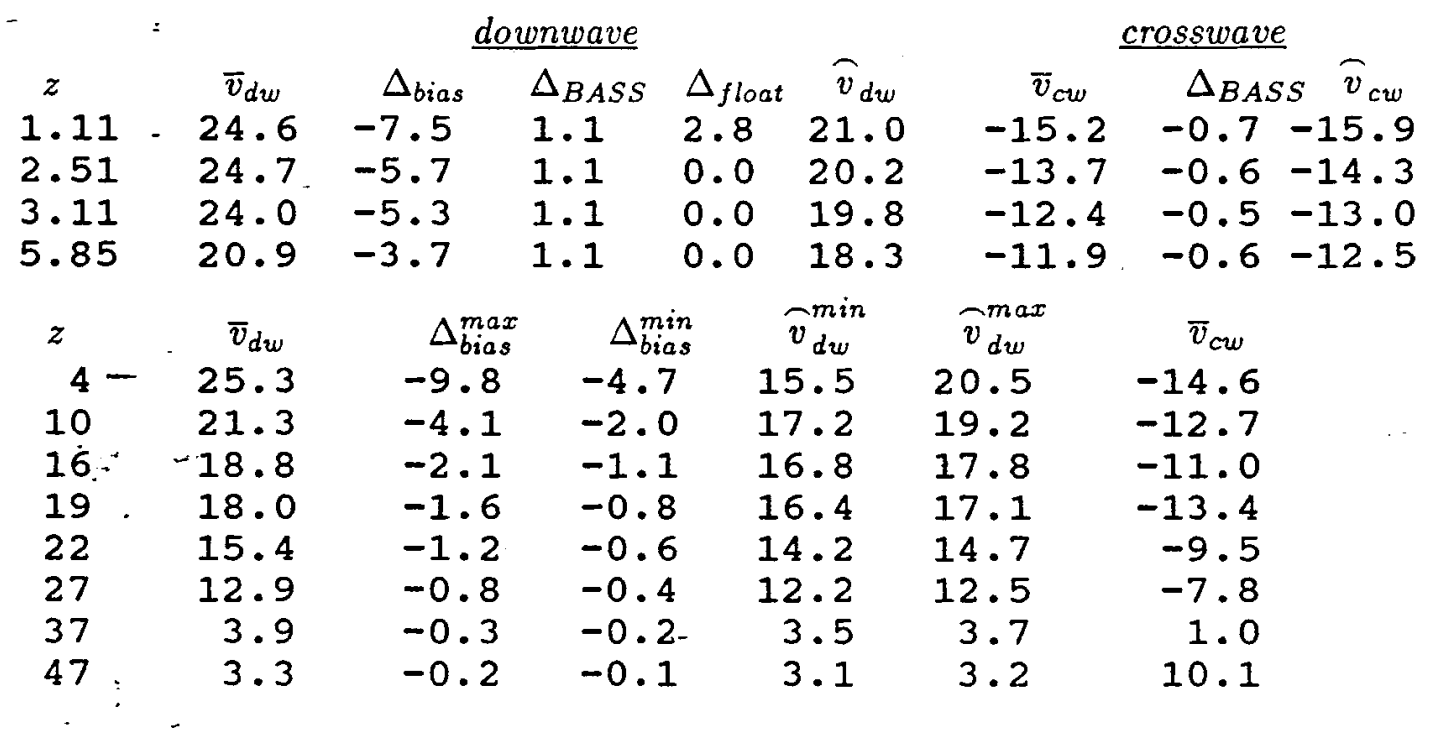

Below: SASS $=x$ (raw) $\cdots$ (unbiased), C3=o(raw) - (unbiased), theoretical log-law shear $=-$
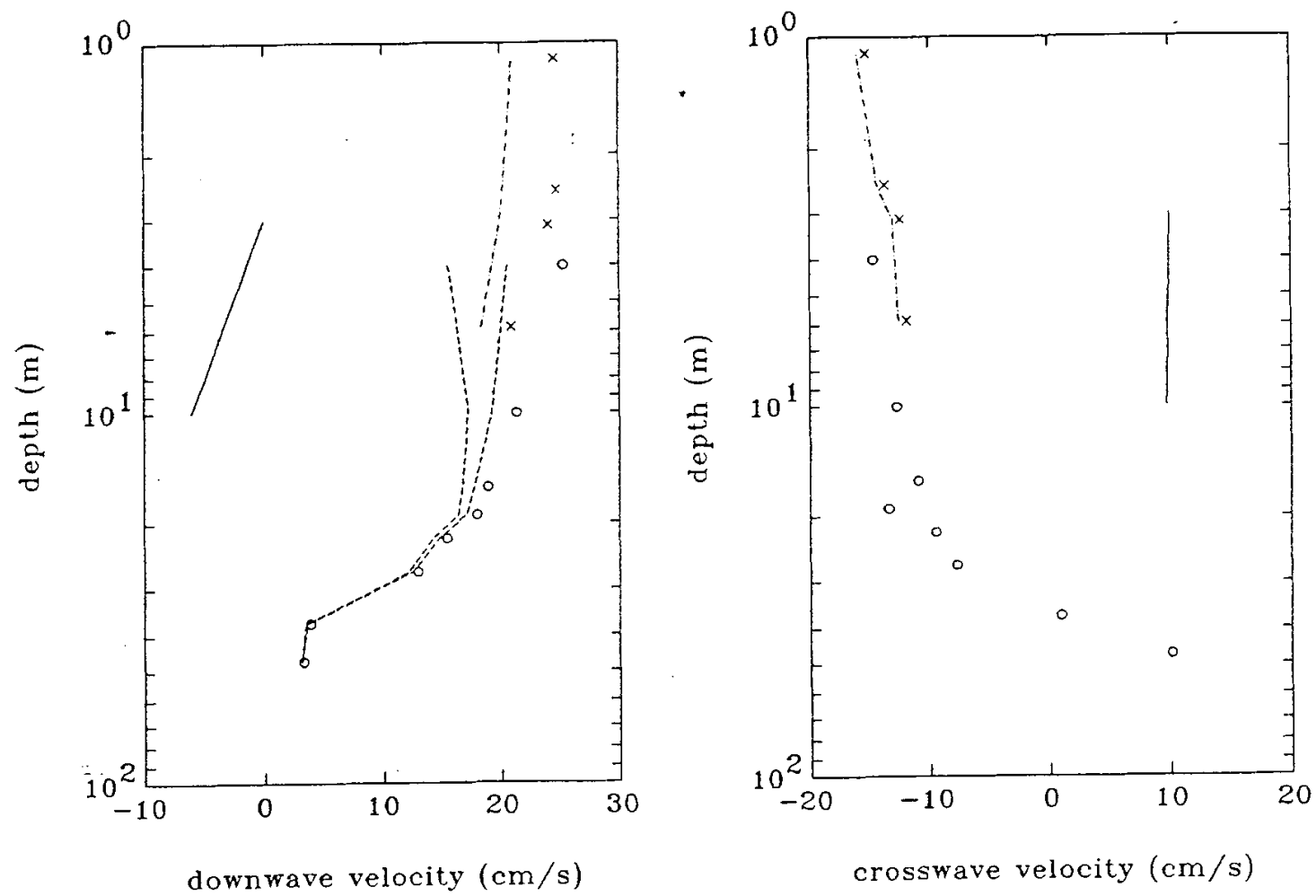


\section{file $\mathrm{s} 1$}

date $=$ February 28, $1989 \quad$ time $=04-57-24.00 \quad$ duration $=00-39-10.25$

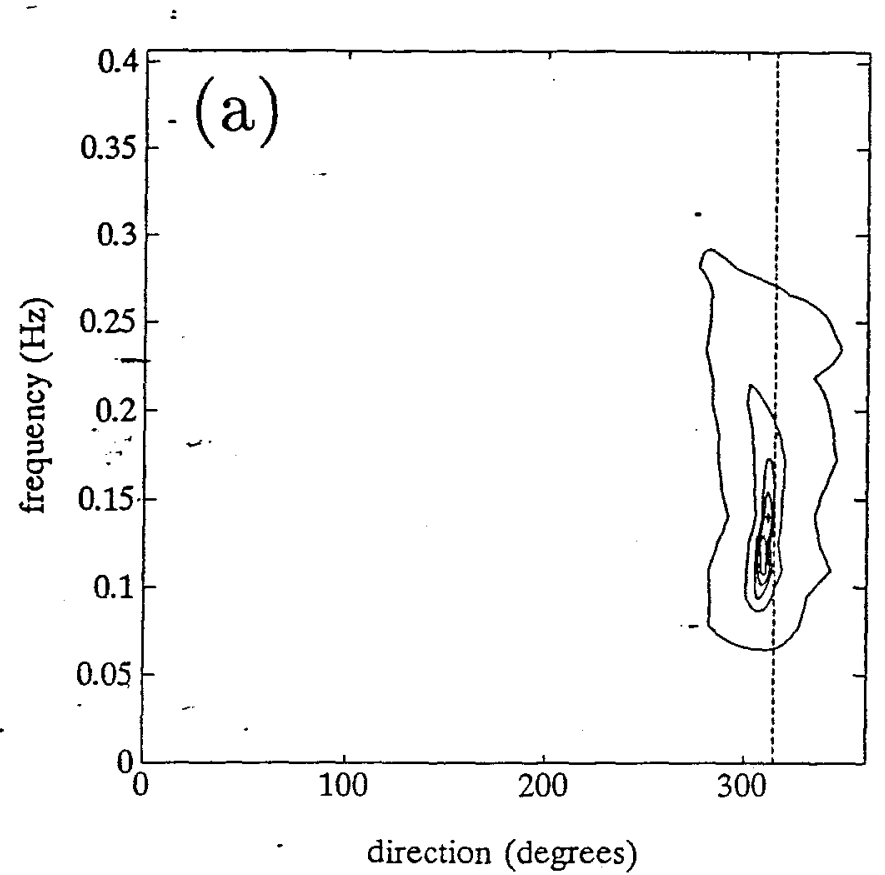

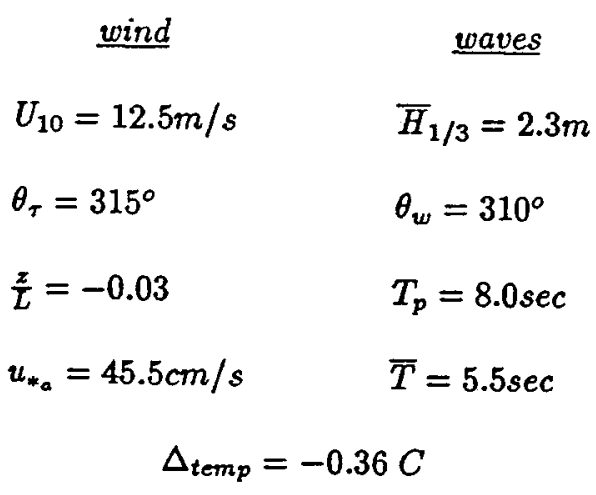

(a) directional spectrum $\left(--=\theta_{r}\right)$

(b) energy spectrum $\left(--=f^{-4}\right)$

(c) $0=$ salinity, $\mathrm{x},+=$ temperature
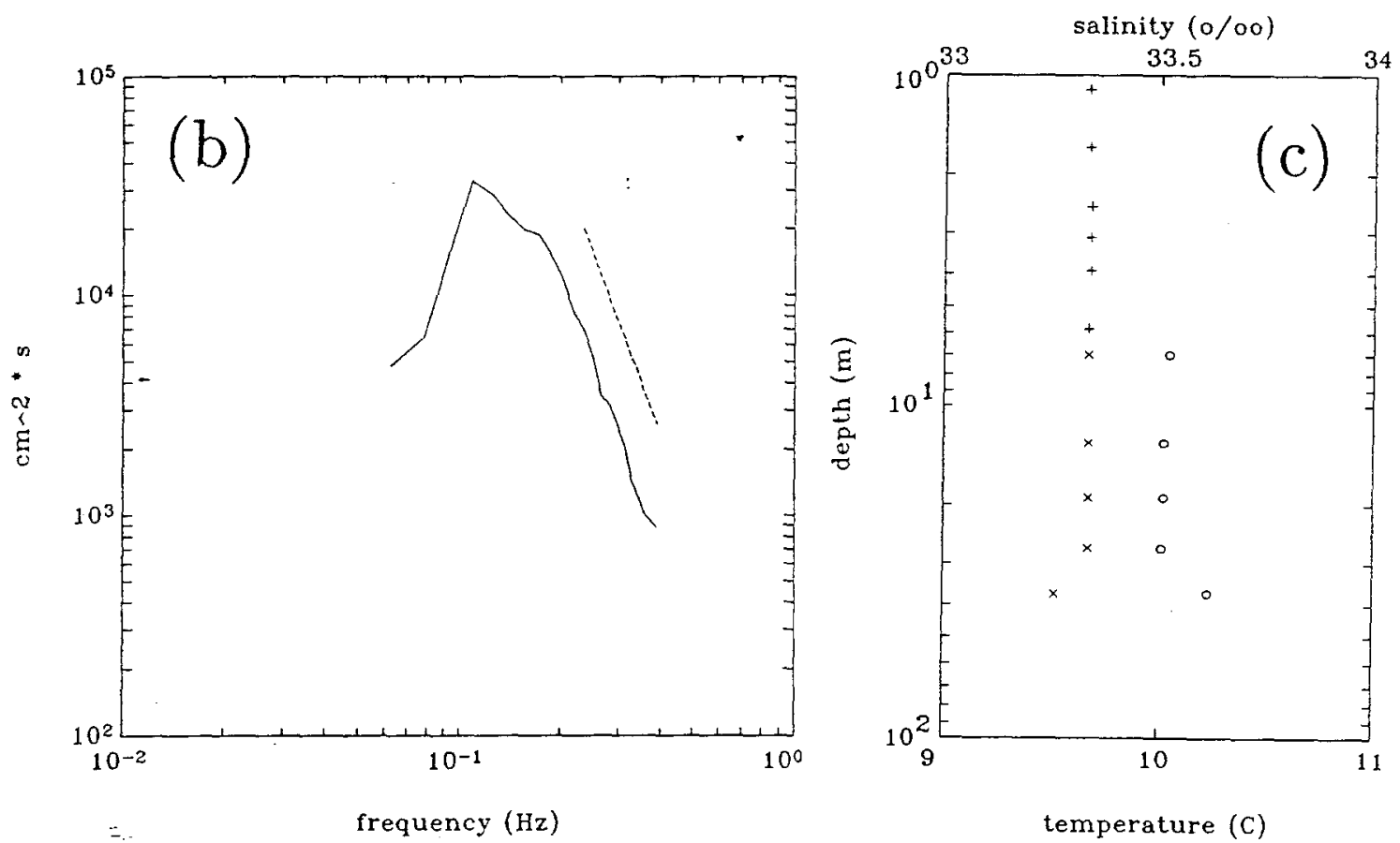


\section{file $\mathrm{s} 1$}

\begin{tabular}{|c|c|c|c|c|c|c|c|c|c|}
\hline \multirow{6}{*}{\multicolumn{2}{|c|}{$\begin{array}{c}z: \\
1.11 \\
2.51 \\
3.11 \\
5.85\end{array}$}} & \multicolumn{5}{|c|}{ downwave } & \multicolumn{3}{|c|}{ crosswave } \\
\hline & & $\bar{v}_{d w}$ & $\Delta_{\text {bias }}$ & $\Delta_{B A S S}$ & $\Delta_{\text {float }}$ & $\widehat{v}_{d w}$ & $\bar{v}_{c w}$ & $\Delta_{B A}$ & $S S \widehat{v}_{c w}$ \\
\hline & & 16.9 & -6.5 & 0.9 & 2.2 & 13.4 & -11.4 & -0.6 & -11.9 \\
\hline & & 17.8 & -5.0 & 0.8 & 0.0 & 13.7 & -10.2 & -0.5 & -10.7 \\
\hline & & 17.8 & -4.7 & 0.9 & 0.0 & 14.0 & -9.1 & -0.4 & \\
\hline & & 13.8 & $-3 \cdot 3$ & 1.0 & 0.0 & 11.4 & -8.9 & -0.6 & \\
\hline$z$ & & $\bar{v}_{d w}$ & $\Delta_{\text {bias }}^{\max }$ & $\Delta_{b i a}^{m i}$ & & $\widehat{v}_{d w}^{\min }$ & $\overbrace{v_{d w}^{\max }}$ & $\bar{v}_{c w}$ & \\
\hline 4 & & 22.8 & $-8 \cdot 4$ & -4.1 & & 14.3 & 18.7 & $-17 \cdot 5$ & \\
\hline $1 \theta$ & & 18.1 & -3.5 & -1.7 & & 14.6 & 16.3 & -16.2 & \\
\hline 16 & & 14.9 & -1.7 & -0.9 & & 13.2 & 14.0 & -15.4 & \\
\hline 19 & . & 14.3 & -1.3 & -0.7 & & 13.0 & 13.6 & -16.9 & \\
\hline 22 & & 13.1 & -1.0 & -0.5 & & 12.1 & 12.5 & -14.0 & \\
\hline 27 & & 13.2 & -0.6 & -0.4 & & 2.6 & 12.8 & -12.8 & \\
\hline 37 & & 9.8 & -0.3 & -0.2 & & 9.5 & 9.6 & 0.9 & \\
\hline 47 & & 11.0 & -0.1 & -0.1 & & 10.9 & 10.9 & 11.2 & \\
\hline
\end{tabular}

Below: $\mathrm{SASS}=x$ (raw) -.- (unbiased), C3=o(raw) - - (unbiased), theoretical log-law shear $=-$
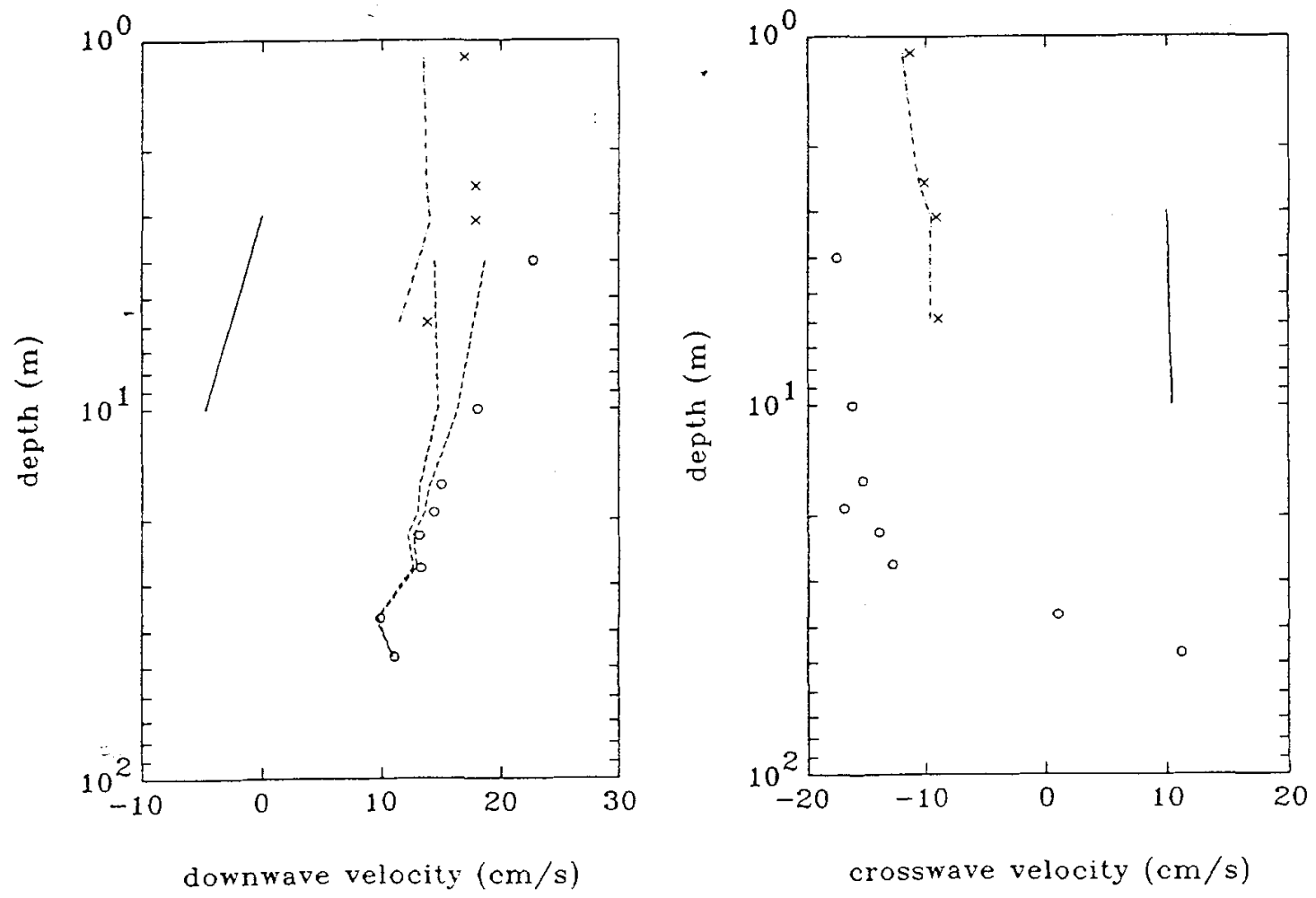


\section{file s2}

$$
\text { date }=\text { February 28, } 1989 \quad \text { time }=05-36-34.25 \quad \text { duration }=00-39-10.00
$$

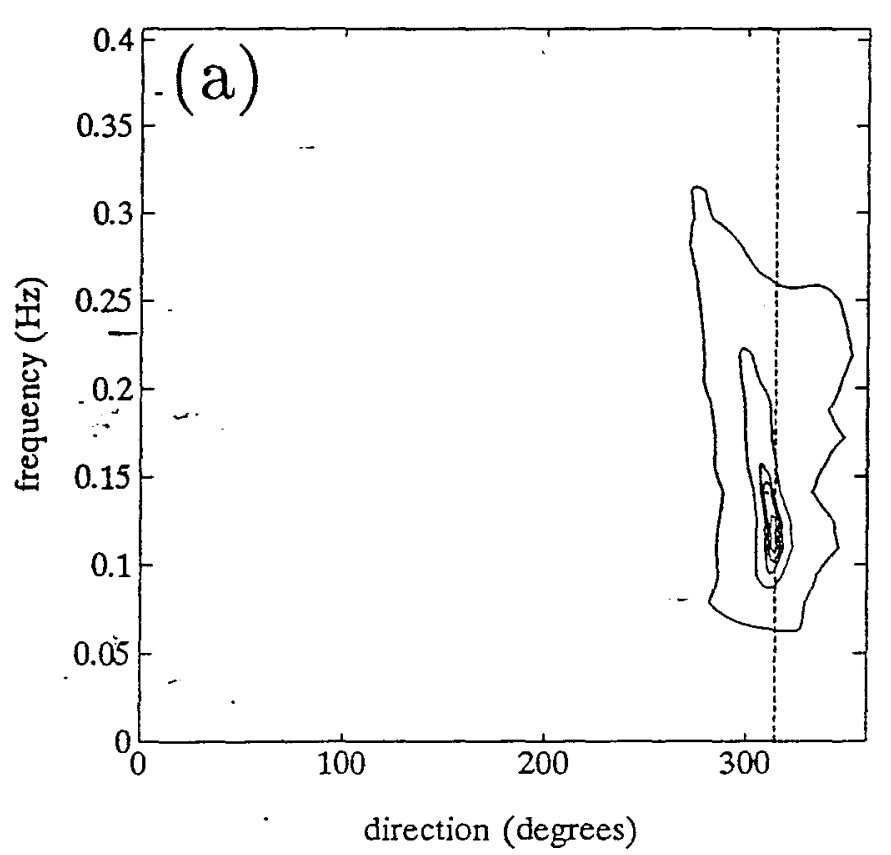

$$
\begin{array}{cc}
\text { wind } & \multicolumn{1}{c}{\text { waves }} \\
U_{10}=12.9 \mathrm{~m} / \mathrm{s} & \bar{H}_{1 / 3}=2.3 \mathrm{~m} \\
\theta_{\tau}=314^{\circ} & \theta_{w}=309^{\circ} \\
\frac{z}{L}=-0.02 & T_{p}=8.0 \mathrm{sec} \\
u_{*_{\alpha}}=47.0 \mathrm{~cm} / \mathrm{s} & \bar{T}=5.4 \mathrm{sec} \\
\multicolumn{2}{c}{\Delta_{\text {temp }}=-0.37 \mathrm{C}}
\end{array}
$$

(a) directional spectrum $\left(--=\theta_{\tau}\right)$

(b) energy spectrum $\left(-.=f^{-4}\right)$

(c) $o=$ salinity, $x,+=$ temperature
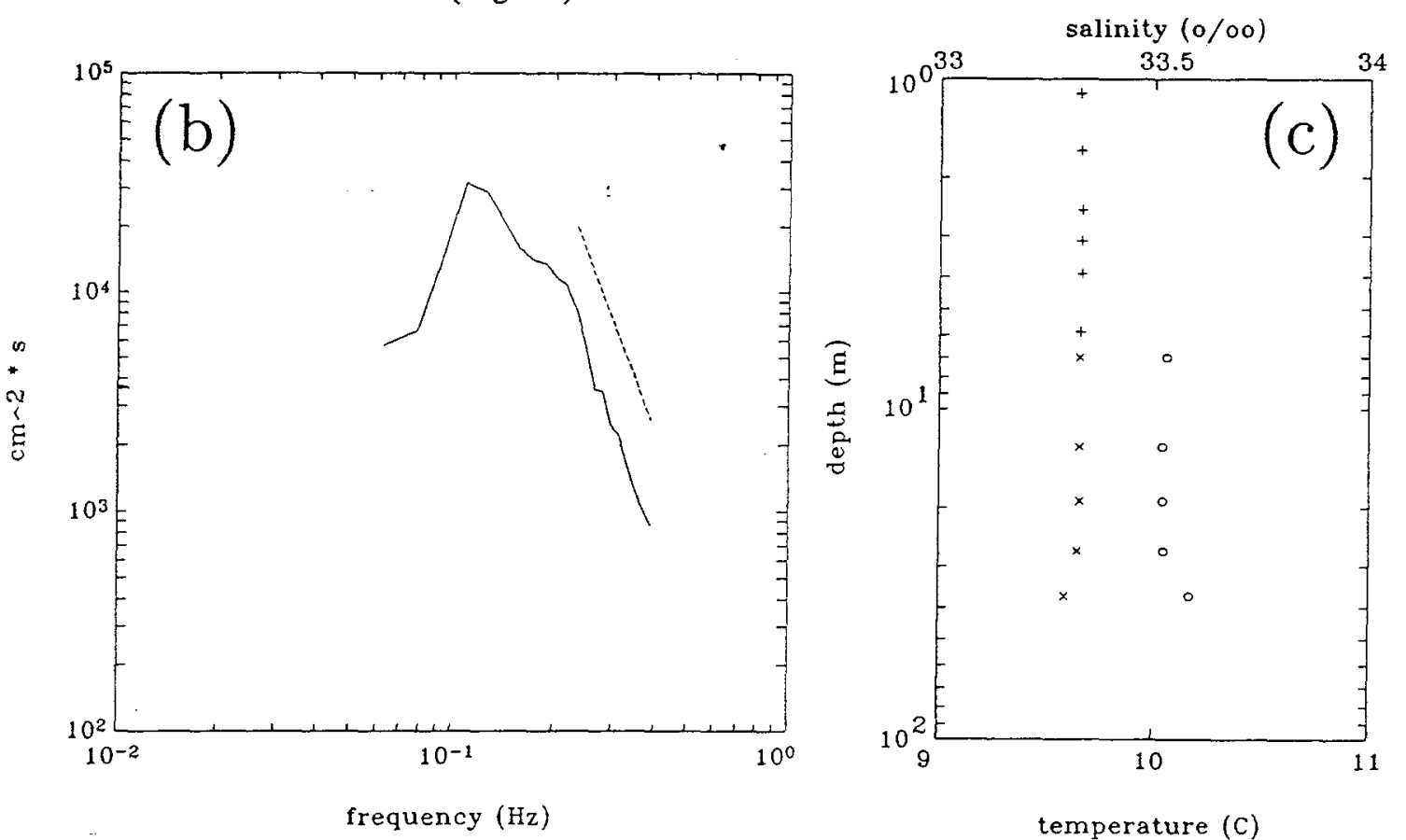


\section{file s2}

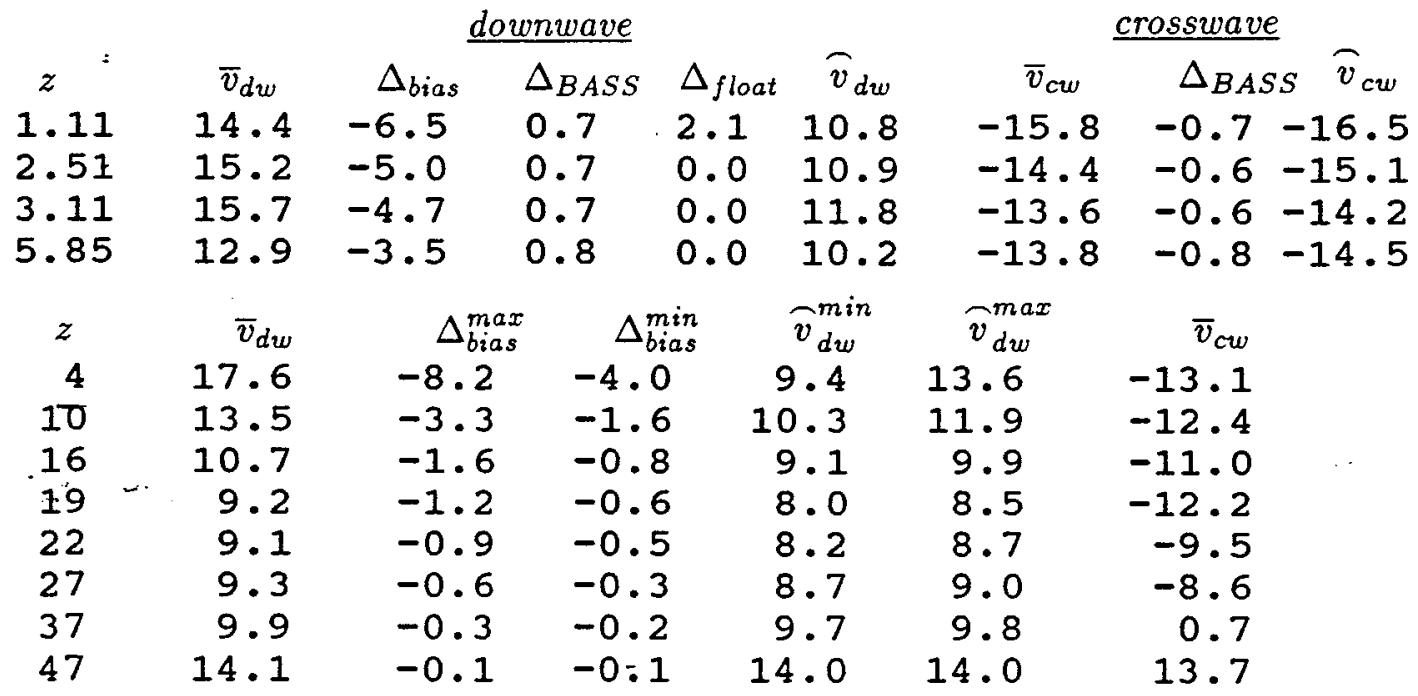

Below: SASS $=x$ (raw) -... (unbiased), C3=o(raw) - - (unbiased), theoretical log-law shear $=-$
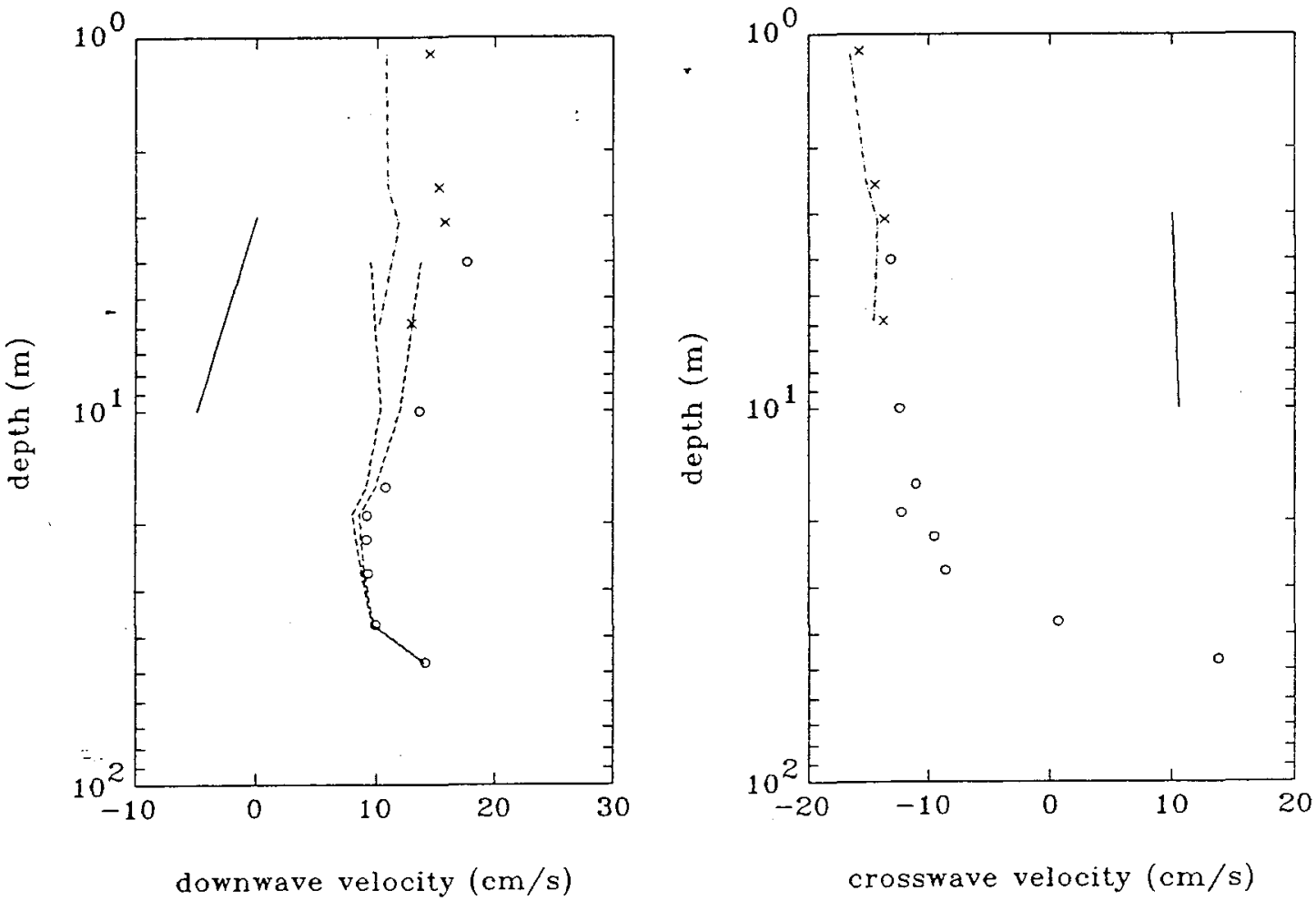
file $\mathrm{s} 3$

date $=$ February $28,1989 \quad$ time $=06-15-44.25 \quad$ duration $=00-39-10.00$

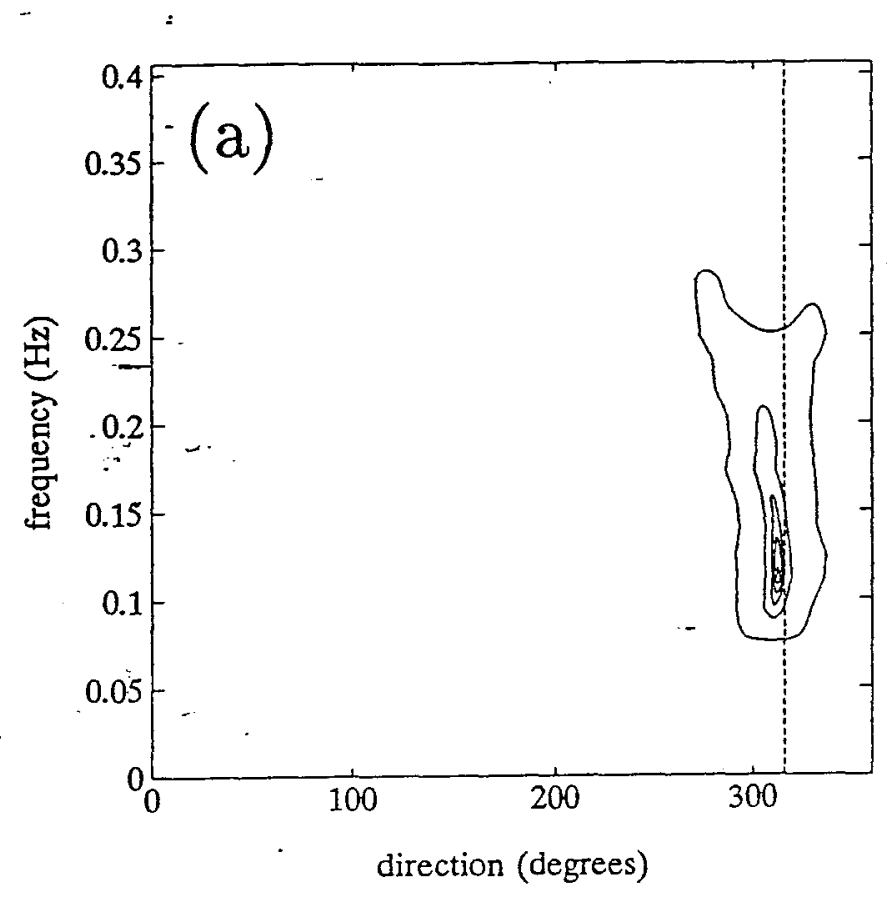

$$
\begin{array}{ll}
\text { wind } & \multicolumn{1}{c}{\text { waves }} \\
U_{10}=12.3 \mathrm{~m} / \mathrm{s} & \bar{H}_{1 / 3}=2.4 \mathrm{~m} \\
\theta_{\tau}=316^{\circ} & \theta_{w}=307^{\circ} \\
\frac{z}{L}=-0.03 & T_{p}=8.0 \mathrm{sec} \\
u_{* a}=44.5 \mathrm{~cm} / \mathrm{s} & \bar{T}=5.5 \mathrm{sec} \\
\multicolumn{2}{c}{\Delta_{\text {temp }}=-0.35 \mathrm{C}}
\end{array}
$$

(a) directional spectrum $\left(-\cdots=\theta_{\tau}\right)$

(b) energy spectrum $\left(-\cdots=f^{-4}\right)$

(c) $\mathrm{o}=$ salinity, $\mathrm{x},+=$ temperature
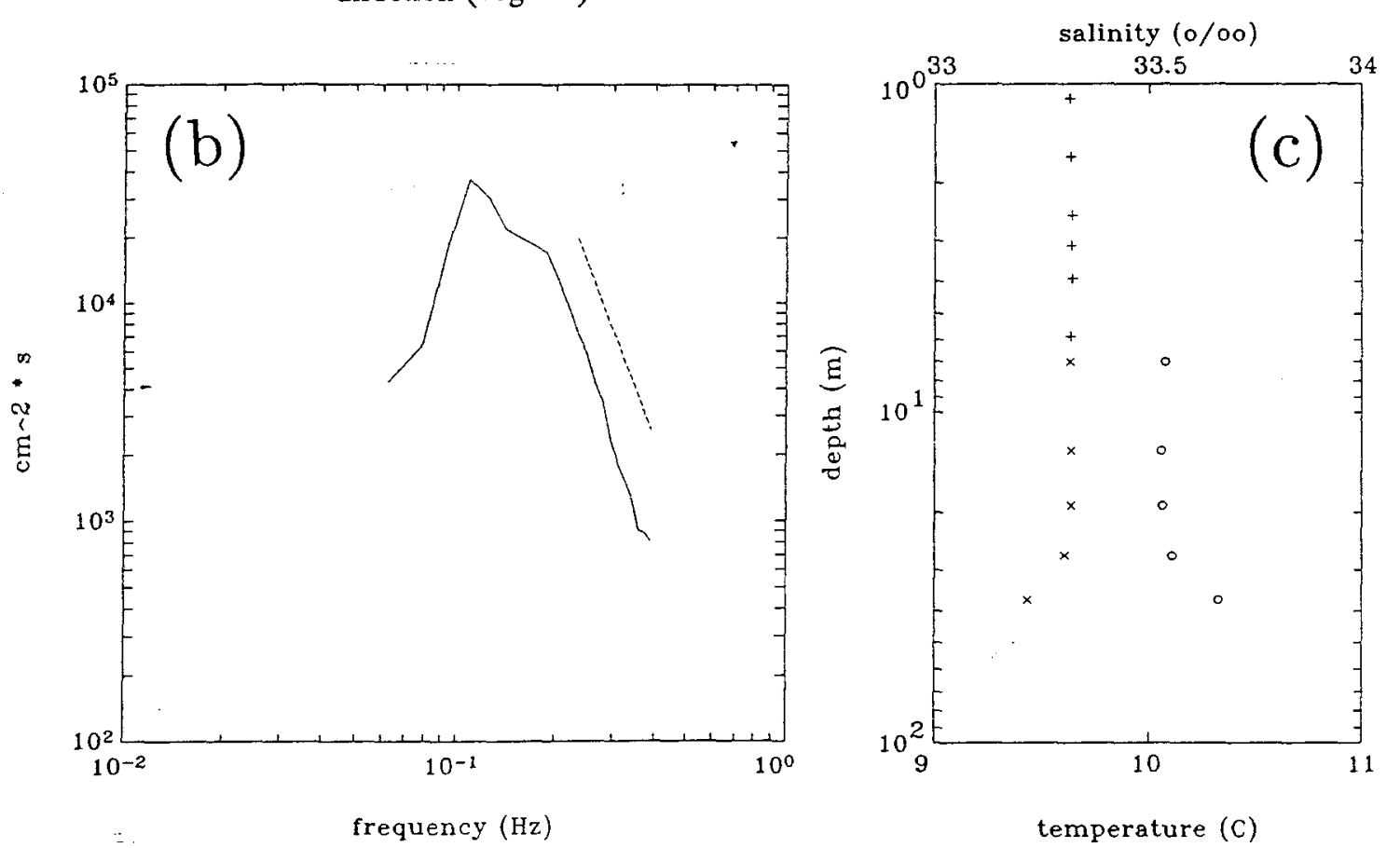


\section{file s3}

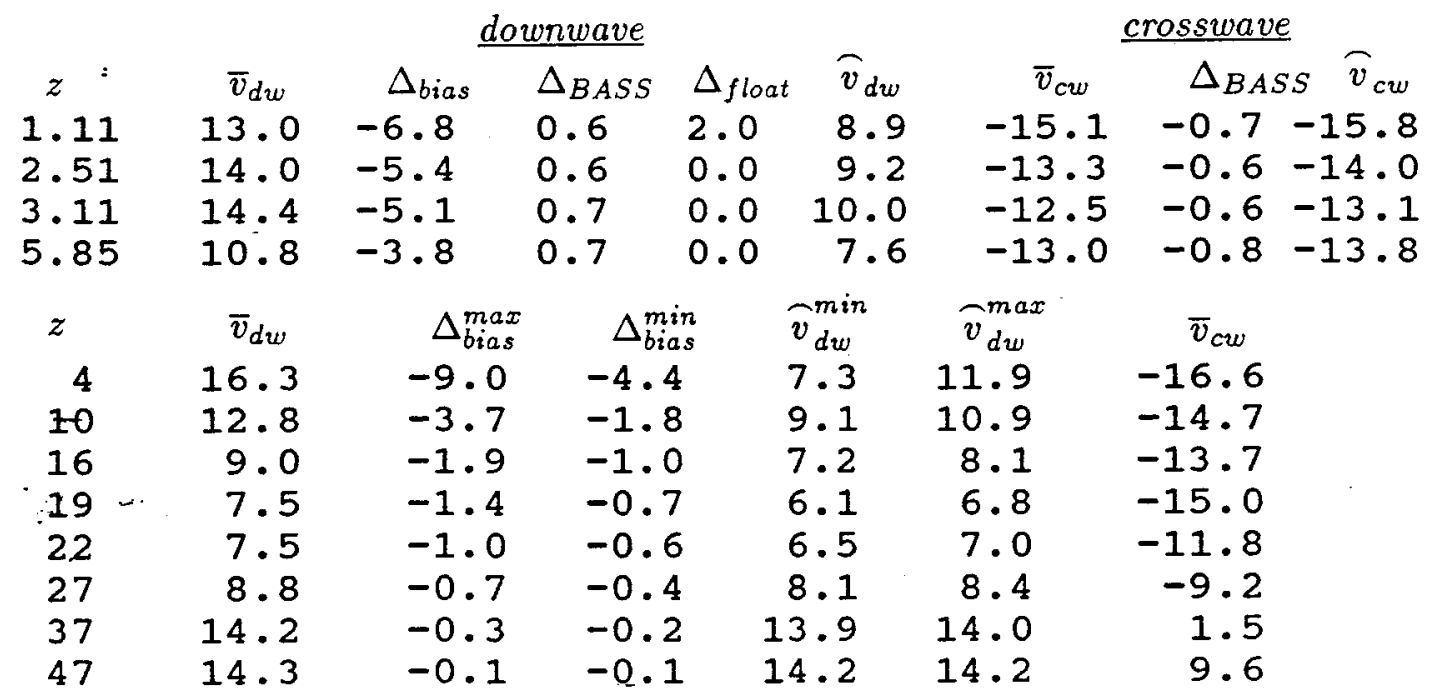

Below: SASS $=x(\mathrm{raw}) \cdots($ unbiased $), \mathrm{C} 3=0(\mathrm{raw}) \cdots($ unbiased $)$, theoretical log-law shear $=-$
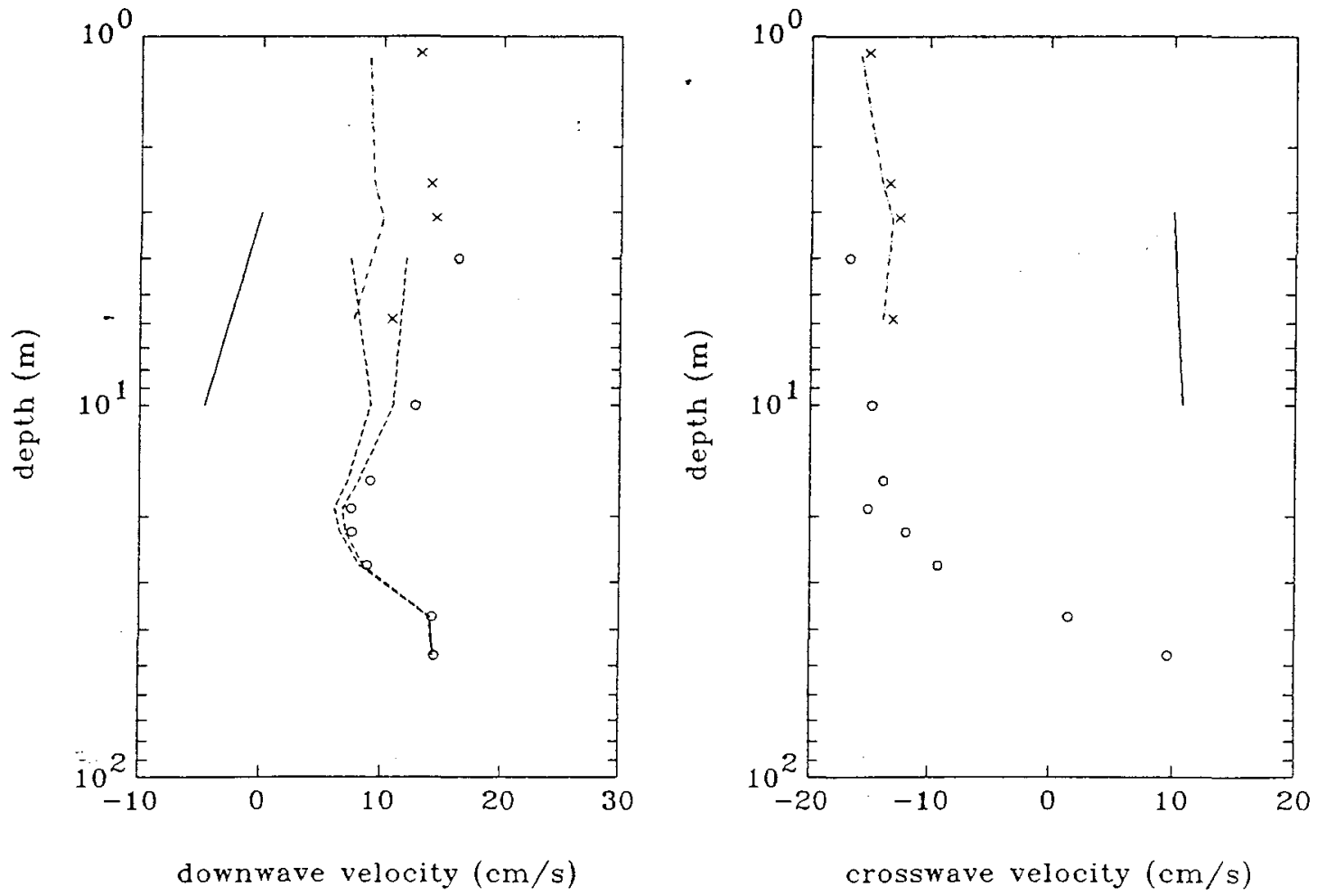


\section{file t1}

date $=$ February $28,1989 \quad$ time $=12-54-24.25 \quad$ duration $=00-38-24.50$

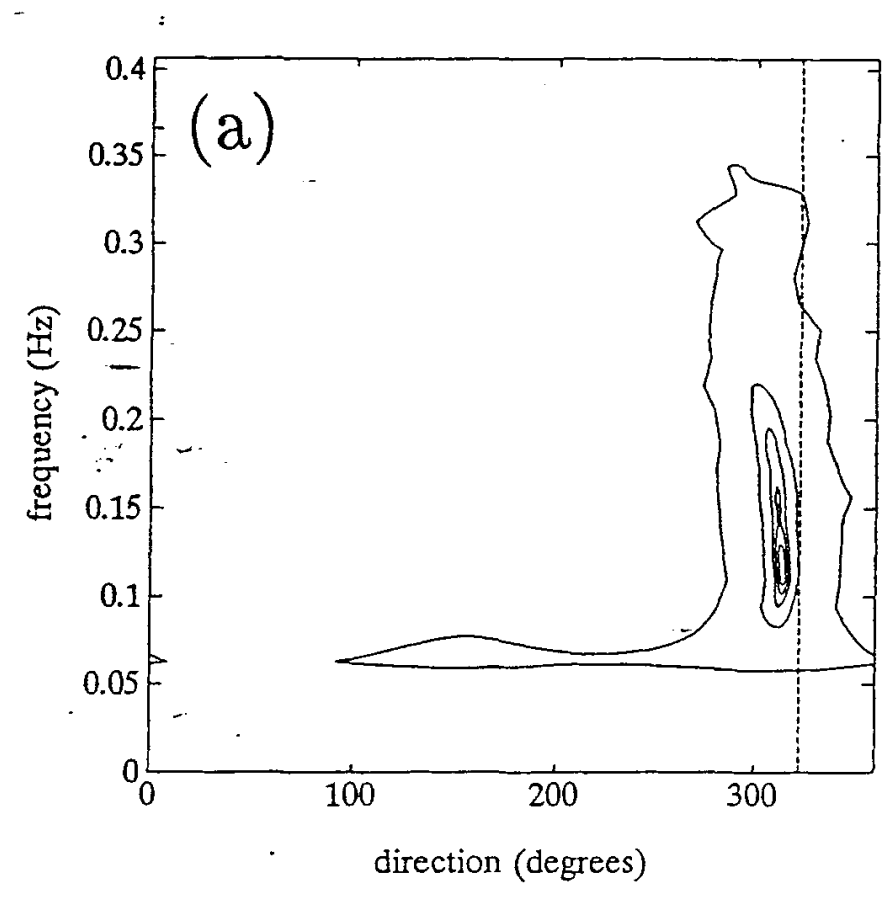

$$
\begin{array}{ll}
\underline{\text { wind }} & \multicolumn{1}{c}{\text { waves }} \\
U_{10}=9.5 \mathrm{~m} / \mathrm{s} & \bar{H}_{1 / 3}=1.9 \mathrm{~m} \\
\theta_{\tau}=322^{\circ} & \theta_{w}=305^{\circ} \\
\frac{z}{L}=-0.03 & T_{p}=8.0 \mathrm{sec} \\
u_{*_{a}}=33.1 \mathrm{~cm} / \mathrm{s} & \bar{T}=5.4 \mathrm{sec} \\
\multicolumn{2}{c}{\Delta_{\text {temp }}=-0.34 \mathrm{C}}
\end{array}
$$

(a) directional spectrum

(b) energy spectrum $\left(\cdots=f^{-4}\right)$

(c) $0=$ salinity, $x,+=$ temperature
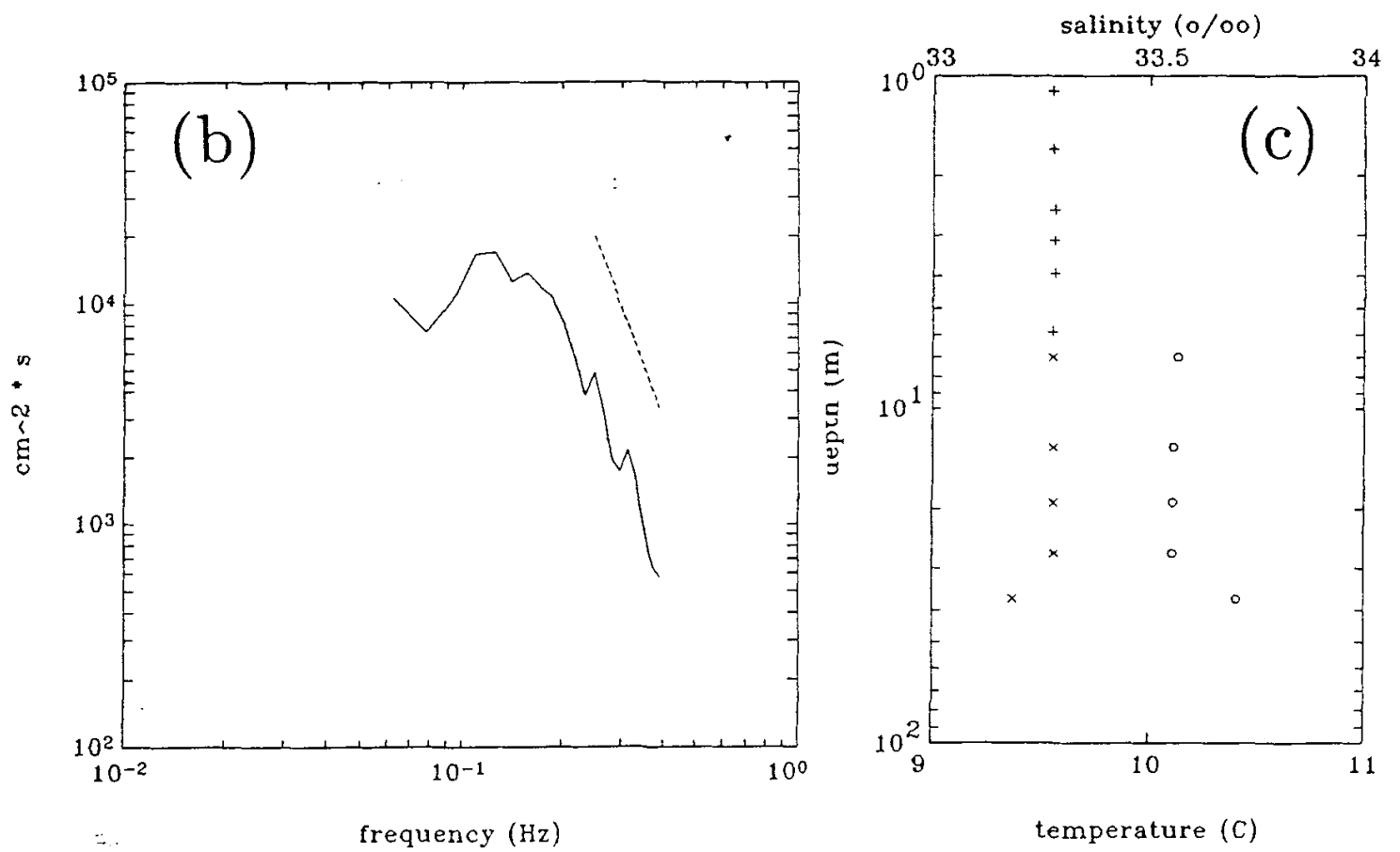


\section{file $\mathrm{t} 1$}

\begin{tabular}{|c|c|c|c|c|c|c|c|c|}
\hline \multirow[b]{2}{*}{$z$} & \multicolumn{5}{|c|}{ downwave } & \multicolumn{3}{|c|}{ crosswave } \\
\hline & $\bar{v}_{d w}$ & $\Delta_{\text {bias }}$ & $\Delta_{B A S S}$ & $\Delta_{\text {float }}$ & $\widehat{v}_{d w}$ & $\bar{v}_{c w}$ & $\Delta_{B A S}$ & $s \widehat{v}_{c w}$ \\
\hline $\begin{array}{l}1.11 \\
2.51 \\
3.11 \\
5.85\end{array}$ & $\begin{array}{l}13.0 \\
14.3 \\
14.6 \\
11.3\end{array}$ & $\begin{array}{l}-4.5 \\
-3.5 \\
-3.3 \\
-2.4\end{array}$ & $\begin{array}{l}0.7 \\
0.7 \\
0.7 \\
0.8\end{array}$ & $\begin{array}{l}1.8 \\
0.0 \\
0.0 \\
0.0\end{array}$ & $\begin{array}{r}10.9 \\
11.5 \\
12.0 \\
9.6\end{array}$ & $\begin{array}{l}-11.6 \\
-11.1 \\
-10.2 \\
-10.4\end{array}$ & $\begin{array}{l}-0.6 \\
-0.5 \\
-0.5 \\
-0.8\end{array}$ & $\begin{array}{l}-12.2 \\
-11.7 \\
-10.7 \\
-11.1\end{array}$ \\
\hline $\begin{array}{l}z \\
\frac{4}{10} \\
16 \\
19 \\
22 \\
27 \\
37 \\
47\end{array}$ & $\begin{array}{r}\bar{v}_{d w} \\
18.1 \\
15.7 \\
14.3 \\
13.2 \\
13.8 \\
11.9 \\
8.0 \\
6.9\end{array}$ & 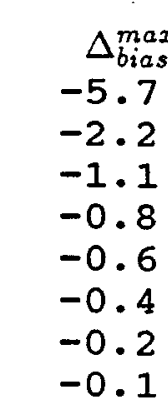 & $\begin{array}{rl} & \Delta_{b i c}^{m i} \\
-2.8 & -1.1 \\
-0.6 & 0.6 \\
-0.4 & 0.3 \\
-0.2 & 0.1 \\
-0.1 & -1\end{array}$ & & $\begin{array}{l}\widehat{v}_{d w}^{\min } \\
2.4 \\
3.5 \\
3.2 \\
2.4 \\
3.2 \\
1.6 \\
7.8 \\
6.8\end{array}$ & $\begin{array}{c}\widehat{v}_{d w}^{\max } \\
15.3 \\
14.6 \\
13.7 \\
12.8 \\
13.5 \\
11.7 \\
7.9 \\
6.8\end{array}$ & $\begin{array}{c}\bar{v}_{c w} \\
-8.1 \\
-6.2 \\
-6.1 \\
-5.1 \\
-3.5 \\
-3.8 \\
-4.9 \\
-3.7\end{array}$ & \\
\hline
\end{tabular}

Below: SASS $=x$ (raw) -... (unbiased), C3=o (raw) - - (unbiased), theoretical log-law shear $=-$
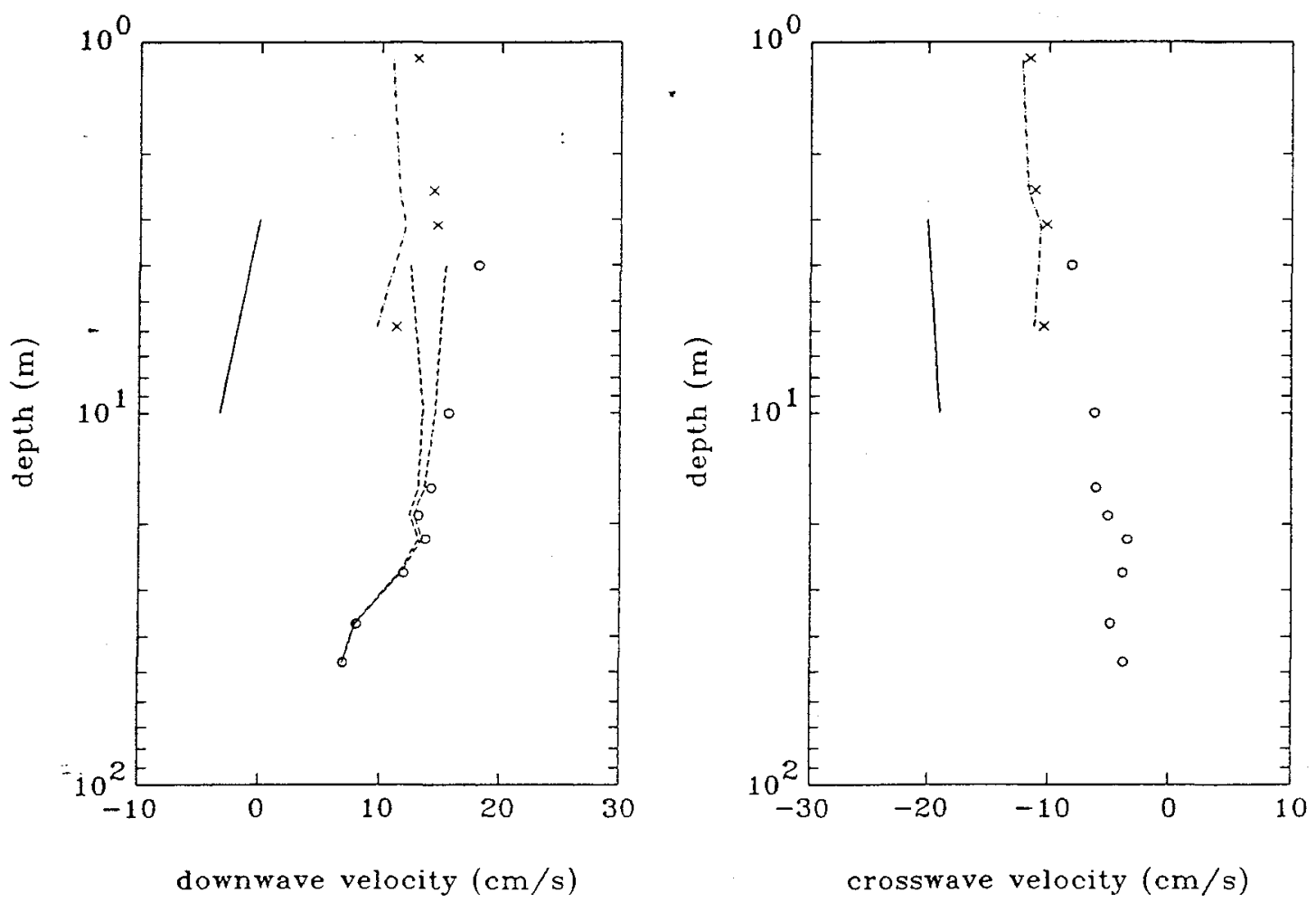


\section{file $\mathrm{u} 1$}

date $=$ February 28, $1989 \quad$ - time $=13-43-20.50 \quad$ duration $=00-52-16.75$

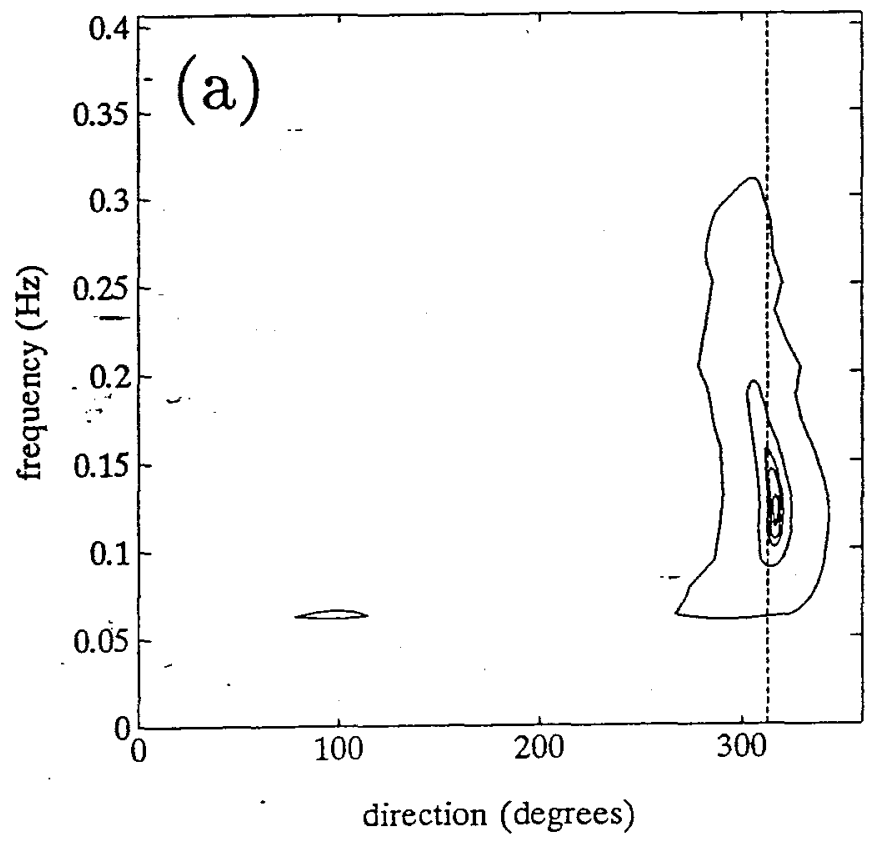

$$
\begin{array}{cc}
\underline{\text { wind }} & \underline{\text { waves }} \\
U_{10}=8.9 \mathrm{~m} / \mathrm{s} & \bar{H}_{1 / 3}=1.9 \mathrm{~m} \\
\theta_{\tau}=313^{\circ} & \theta_{w}=304^{\circ} \\
\frac{z}{L}=-0.04 & T_{p}=8.0 \mathrm{sec} \\
u_{* a}=31.1 \mathrm{~cm} / \mathrm{s} & \bar{T}=5.4 \mathrm{sec} \\
\Delta_{\text {temp }}=-0.34 \mathrm{C}
\end{array}
$$

(a) directional spectrum $\left(--=\theta_{\tau}\right)$

(b) energy spectrum $\left(-\cdots=f^{-4}\right)$

(c) $0=$ salinity, $x,+=$ temperature
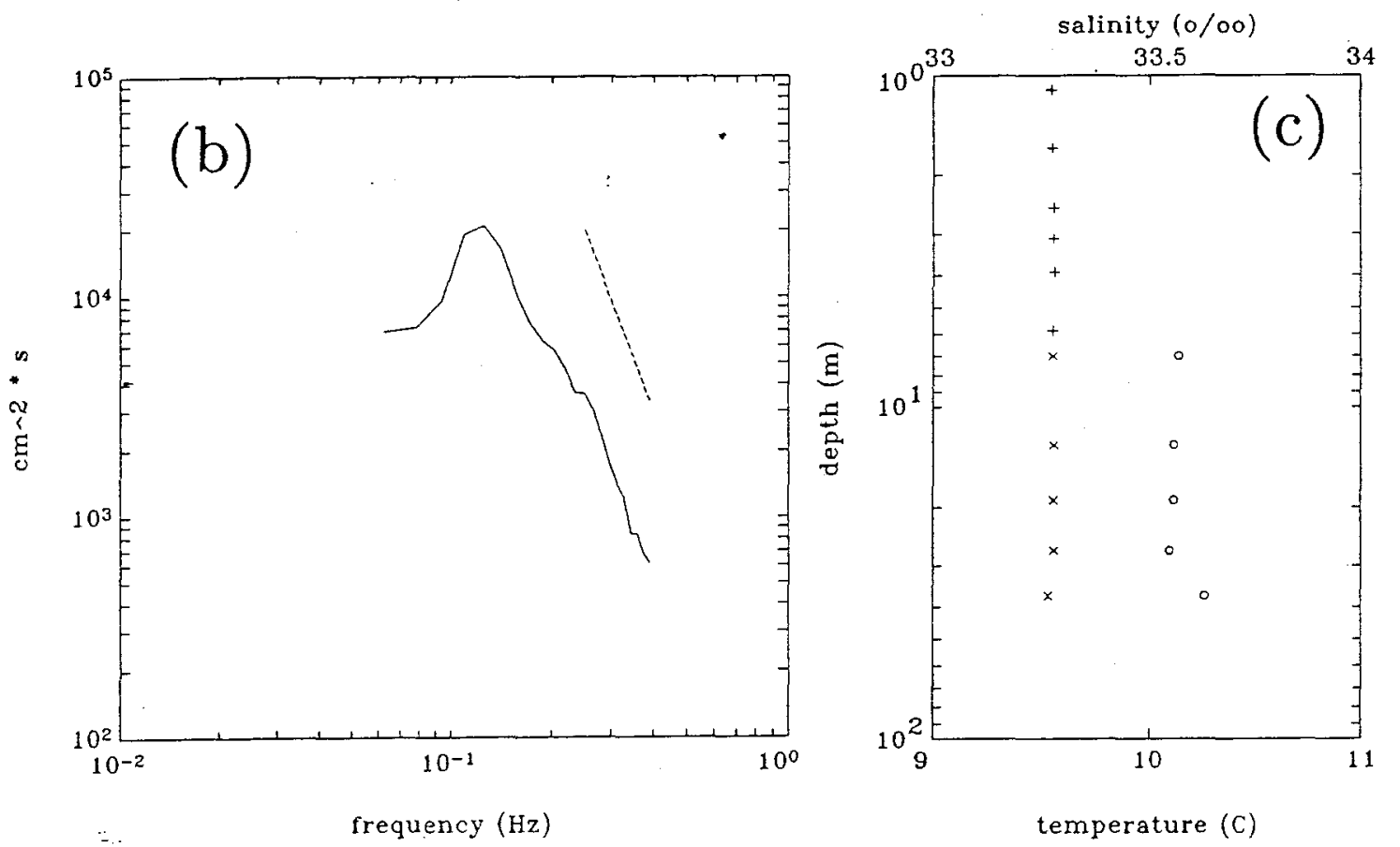


\section{file $\mathrm{u} 1$}

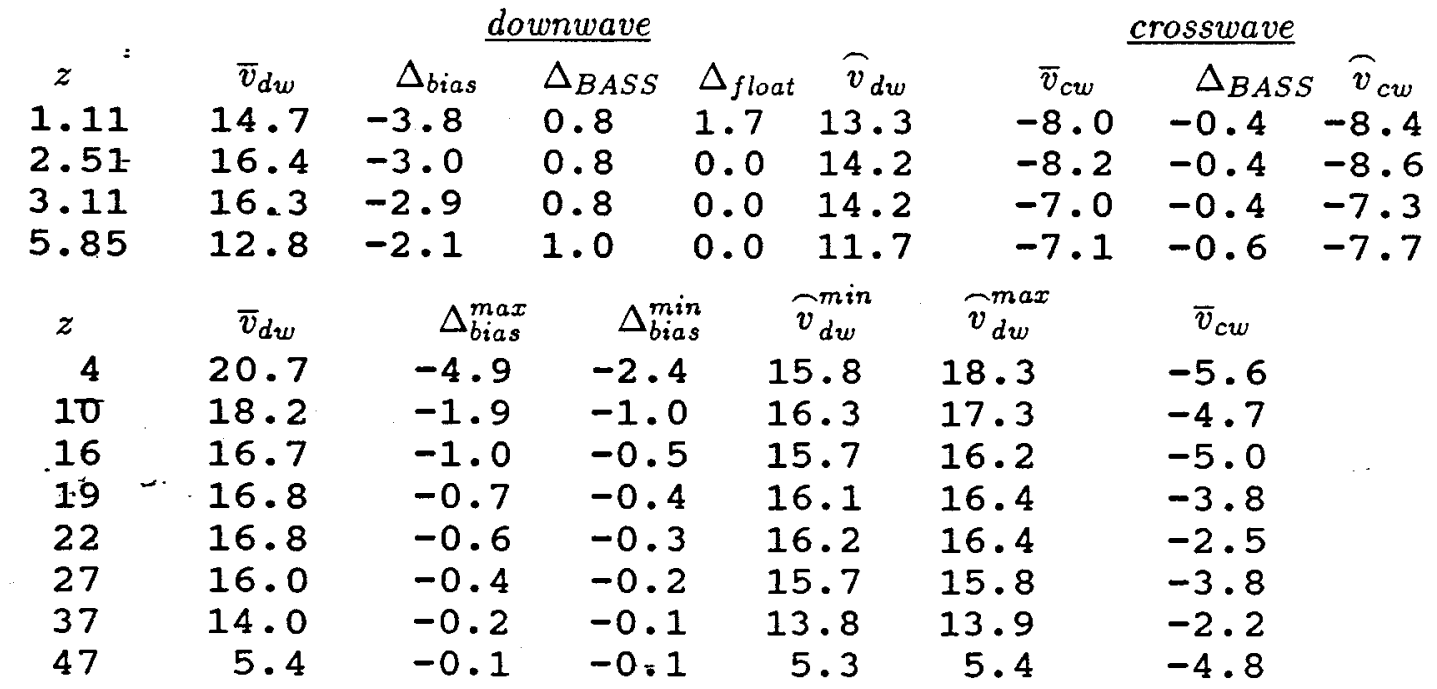

Below: $\mathrm{SASS}=x(\mathrm{raw}) \cdots($ unbiased $), \mathrm{C} 3=o(\mathrm{raw}) \cdots($ unbiased $)$, theoretical log-law shear $=-$
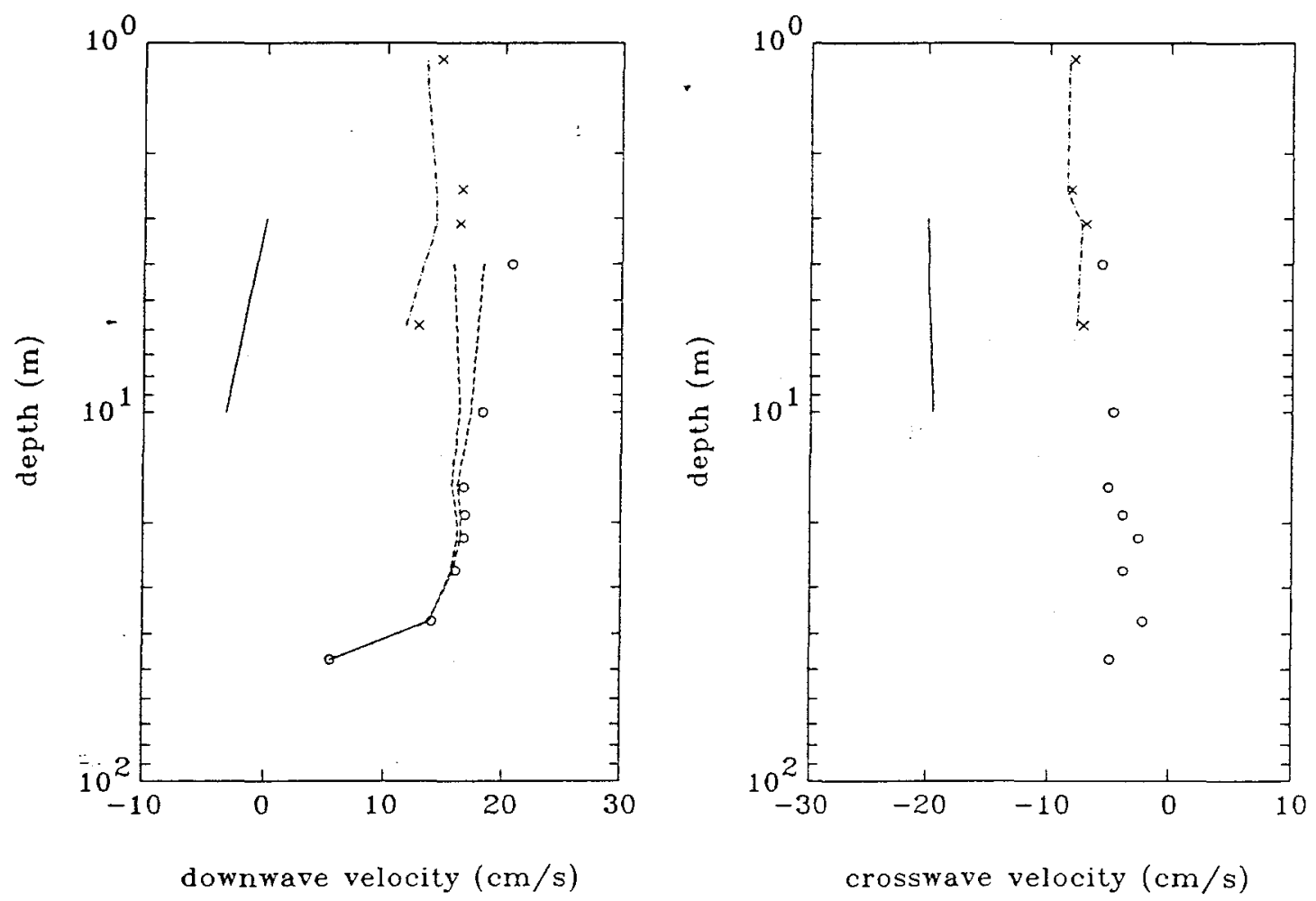


\section{file v1}

date $=$ February $28,1989 \quad$ time $=17-32-23.50 \quad$ duration $=00-26-05.50$

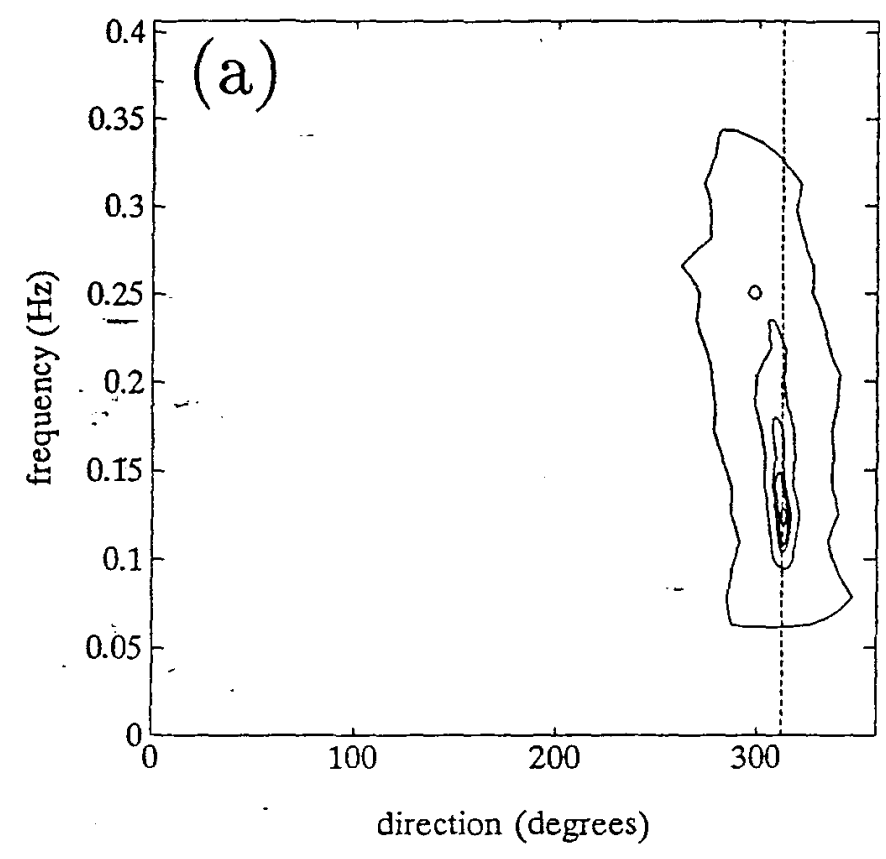

$$
\begin{array}{cc}
\text { wind } & \multicolumn{1}{c}{\text { waves }} \\
U_{10}=10.2 \mathrm{~m} / \mathrm{s} & \bar{H}_{1 / 3}=1.7 \mathrm{~m} \\
\theta_{\tau}=313^{\circ} & \theta_{w}=302^{\circ} \\
\frac{z}{L}=-0.03 & T_{p}=7.1 \mathrm{sec} \\
u_{*_{a}}=35.4 \mathrm{~cm} / \mathrm{s} & \bar{T}=4.9 \mathrm{sec} \\
\multicolumn{2}{c}{\Delta_{\text {temp }}=-0.30 \mathrm{C}}
\end{array}
$$

(a) directional spectrum

(b) energy spectrum $\left(\cdots=f^{-4}\right)$

(c) $\mathrm{o}=$ salinity; $\mathrm{x},+=$ temperature
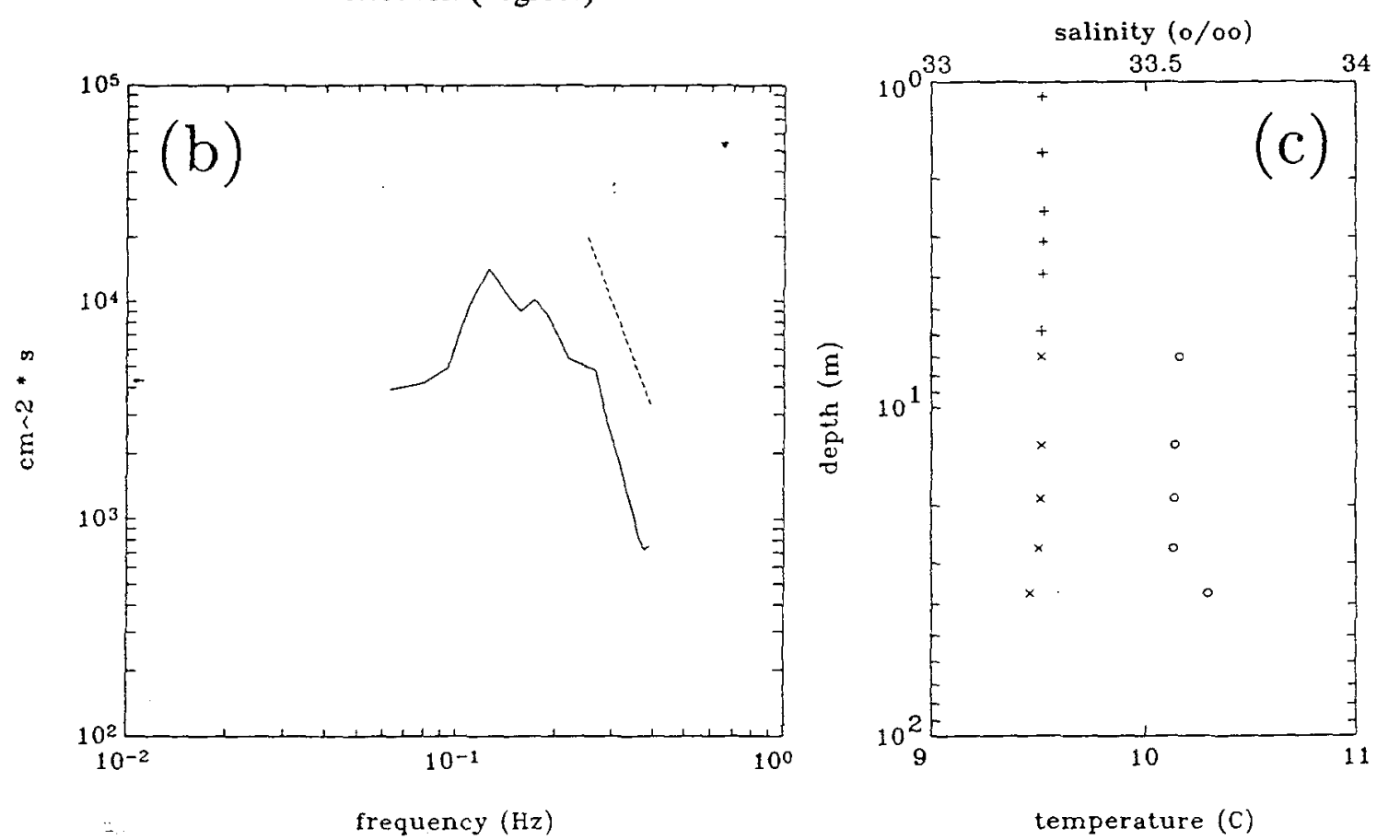


\section{file v1}

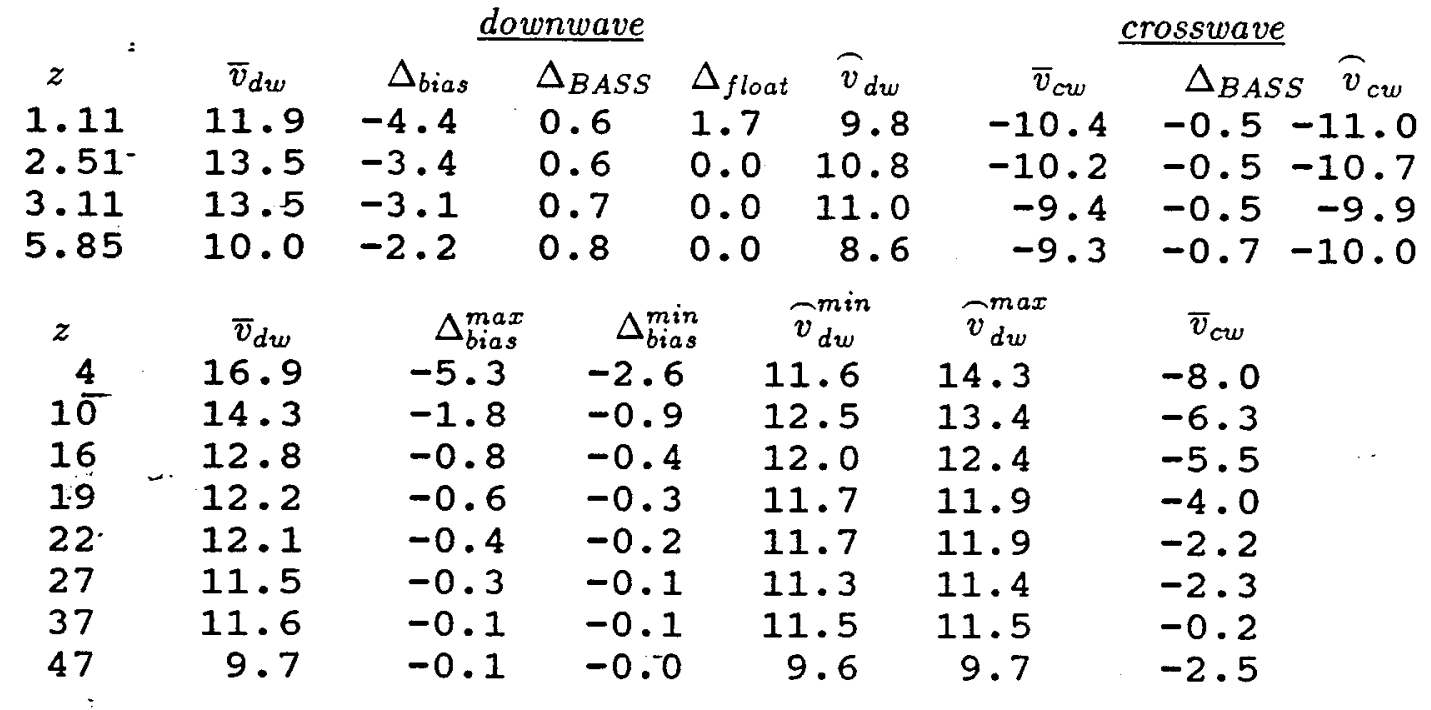

Below: SASS $=x($ raw $) \cdots($ unbiased $), C 3=0($ raw $)-\cdots($ unbiased $)$, theoretical log-law shear $=-$
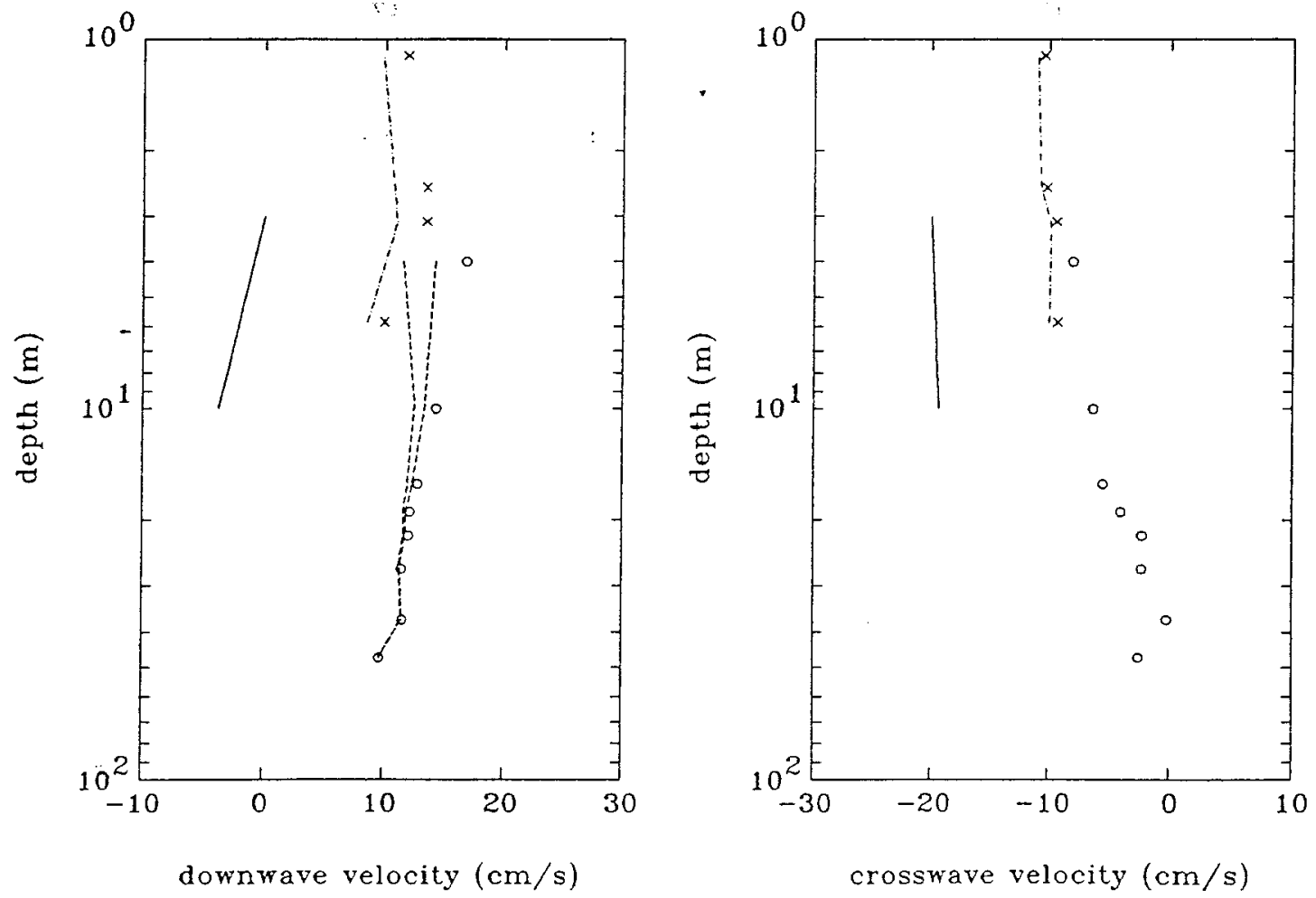


\section{file w1}

$$
\text { date }=\text { February 28, } 1989 \quad \text { time }=18-48-47.75 \quad \text { duration }=00-41-09.00
$$

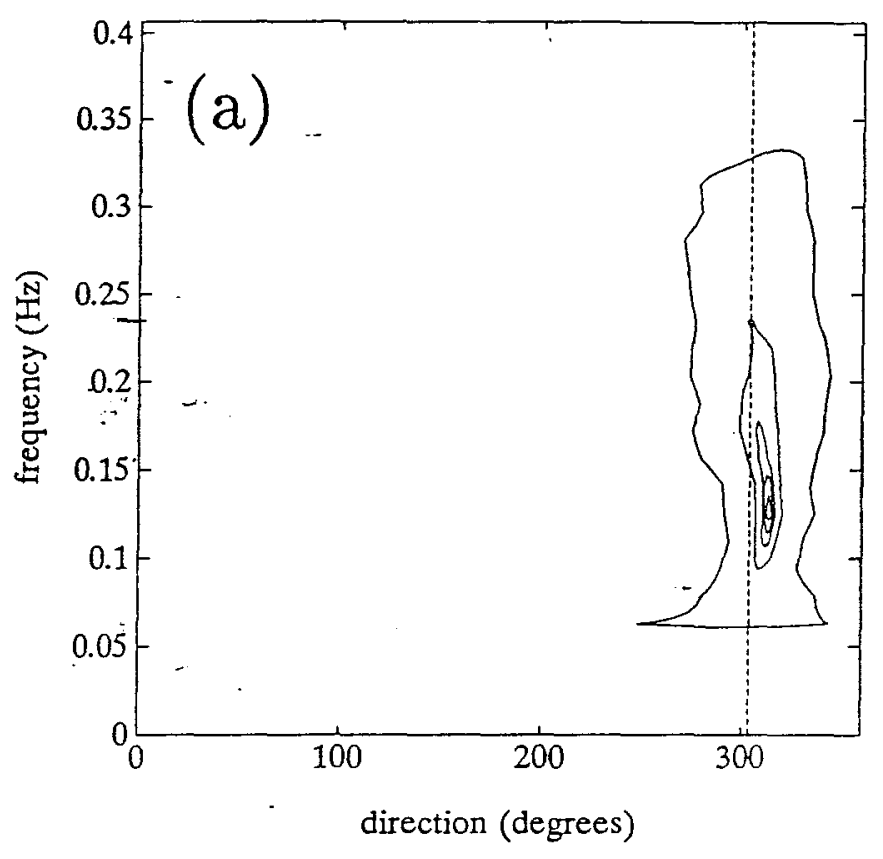

$$
\begin{array}{cc}
\underline{\text { wind }} & \multicolumn{1}{c}{\text { waves }} \\
U_{10}=11.4 \mathrm{~m} / \mathrm{s} & \bar{H}_{1 / 3}=1.9 \mathrm{~m} \\
\theta_{\tau}=304^{\circ} & \theta_{w}=306^{\circ} \\
\bar{z}=-0.02 & T_{p}=7.1 \mathrm{sec} \\
u_{*_{a}}=39.9 \mathrm{~cm} / \mathrm{s} & \bar{T}=4.9 \mathrm{sec} \\
\multicolumn{2}{c}{\Delta_{\text {temp }}=-0.30 \mathrm{C}}
\end{array}
$$

(a) directional spectrum $\left(---\theta_{\tau}\right)$

(b) energy spectrum $\left(-\cdots=f^{-4}\right)$

(c) $0=$ salinity, $x,+=$ temperature
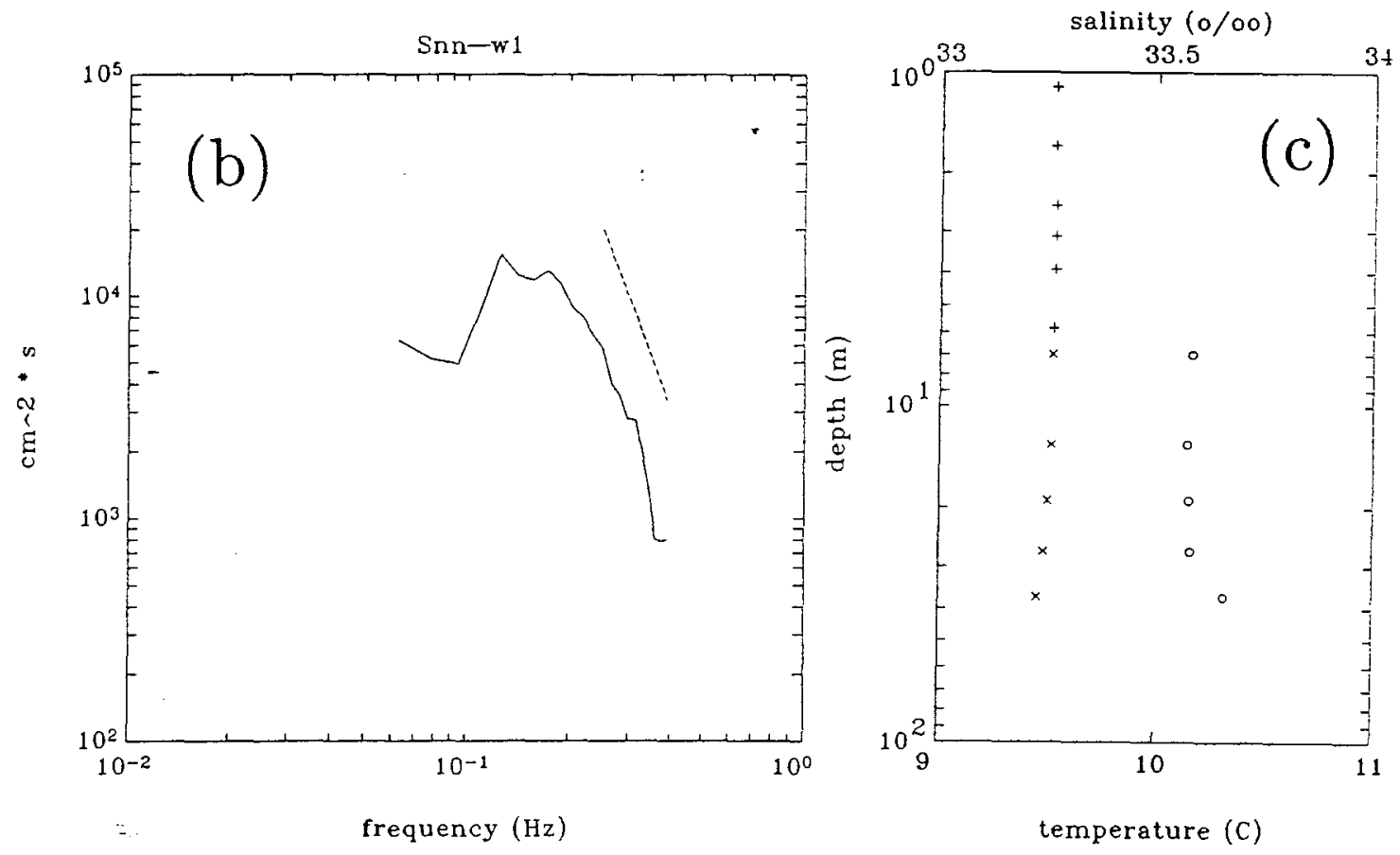


\section{file w1}

\begin{tabular}{|c|c|c|c|c|c|c|c|c|}
\hline \multirow[b]{3}{*}{$\begin{array}{c}z \\
1.11 \\
2.51 \\
3.11 \\
5.85\end{array}$} & \multicolumn{5}{|c|}{ downwave } & \multicolumn{3}{|c|}{ crosswave } \\
\hline & $\bar{v}_{d w}$ & $\Delta_{\text {bias }}$ & $\Delta_{B A S S}$ & $\Delta_{\text {float }}$ & $v_{d w}$ & $\bar{v}_{c w}$ & $\triangle_{B A S}$ & $S S \widehat{v}_{c w}$ \\
\hline & $\begin{array}{l}15.5 \\
16.5 \\
16.5 \\
12.4\end{array}$ & $\begin{array}{l}-5.4 \\
-4.1 \\
-3.8 \\
-2.6\end{array}$ & $\begin{array}{r}-0.7 \\
0.8 \\
0.8 \\
0.9\end{array}$ & $\begin{array}{l}1.9 \\
0.0 \\
0.0 \\
0.0\end{array}$ & $\begin{array}{l}11.3 \\
13.2 \\
13.4 \\
10.6\end{array}$ & $\begin{array}{r}-10.7 \\
-10.6 \\
-9.8 \\
-9.6\end{array}$ & $\begin{array}{r}0.5 \\
-0.5 \\
-0.5 \\
-0.7\end{array}$ & $\begin{array}{l}-10.2 \\
-11.1 \\
-10.2 \\
-10.3\end{array}$ \\
\hline$z$ & $\bar{v}_{d w}$ & $\Delta_{b i}^{m}$ & $\Delta_{b_{1}}^{m}$ & & $\widehat{v}_{d w}^{\min }$ & $\widehat{v}_{d w}^{\max }$ & $\bar{v}_{c w}$ & \\
\hline 4 & 18.6 & -6. & -3 & & 2.2 & 15.4 & $-12 \cdot 8$ & \\
\hline 10 & 15.3 & -2. & -1 & & 3.1 & 14.2 & -11.0 & \\
\hline 16 & 12.8 & -1. & -0. & & 1.8 & 12.2 & -8.6 & \\
\hline 19 & 10.7 & -0.7 & -0 & & 0.0 & 10.4 & -7.7 & \\
\hline $22^{\circ}$ & 12.4 & -0.5 & -0. & & 1.9 & 12.1 & -4.6 & \\
\hline 27 & 12.4 & -0.3 & -0. & & 2.1 & 12.2 & -5.2 & \\
\hline 37 & 8.6 & -0.2 & -0 & & 8.5 & 8.5 & -2.7 & \\
\hline 47 & 4.2 & -0. & -0.1 & & 4.1 & 4.1 & 0.8 & \\
\hline
\end{tabular}

Below: $\mathrm{SASS}=x(\mathrm{raw}) \cdots($ unbiased $), \mathrm{C} 3=0(\mathrm{raw})-\cdots($ unbiased $)$, theoretical log-law shear $=-$
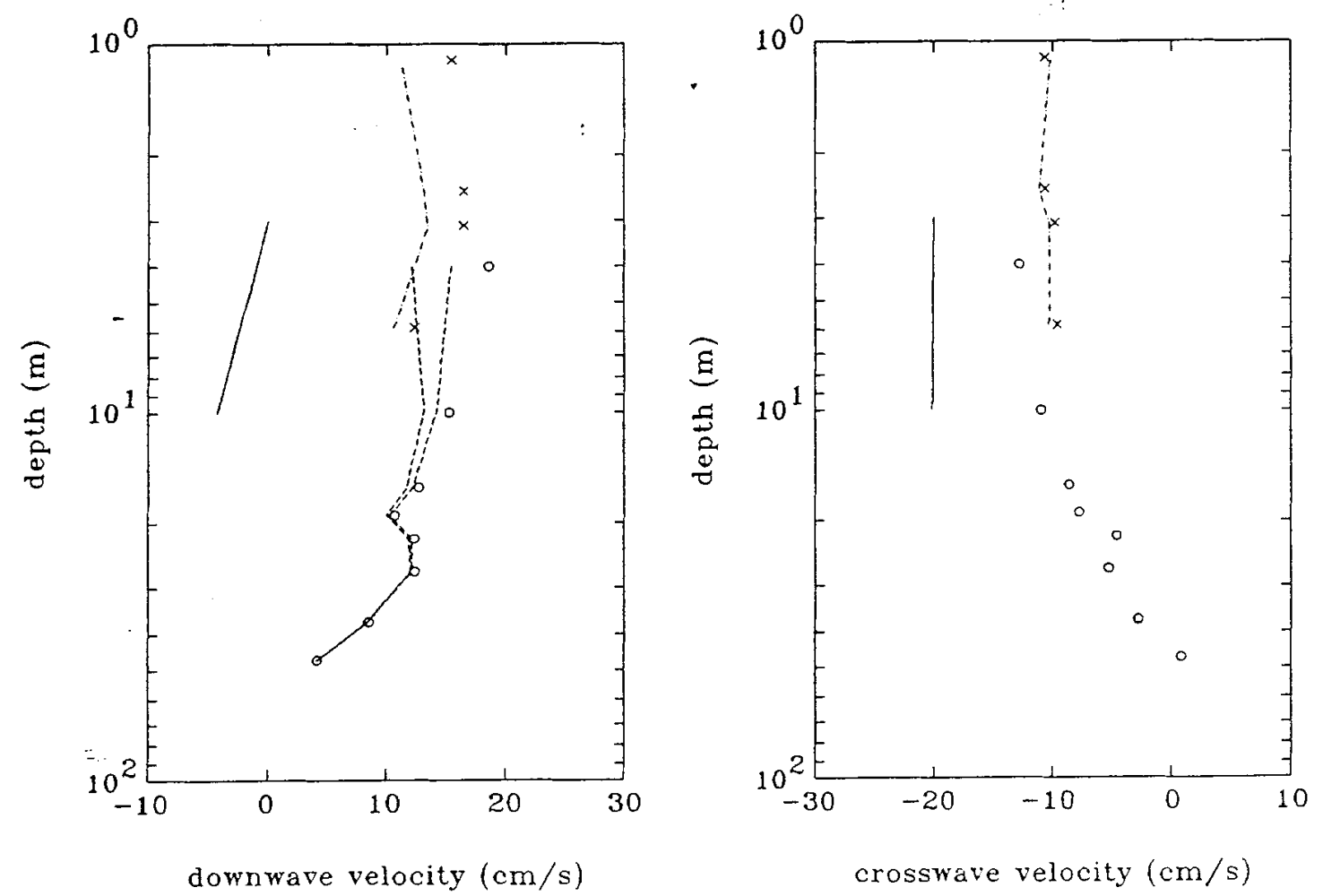


\section{Chapter 7}

\section{DISCUSSION}

\section{$-$ \\ 7.1 . Summarizing the data}

\section{the bias}

Throughout the thesis a great deal of attention has been focused on how best to make measurements of the near-surface shear currents. The surface-following reference frame was seen to provide many advantages. Primary among these was the fact that, in making a boundary layer measurement, we obtain the best resolution by having sensors move with the boundary. But, when making such a measurement, the biasing effects of the waves must also be addressed. A great deal of effort was expended in demonstrating how the wave bias could be estimated. I would like first to show that this energy was well spent.

The data plots of the last chapter show the effect of the bias on a case-by-case basis. To get an idea of how the wave bias will affect our overall interpretation of the data we need to summarize the previous results. For the moment, assume that the shear in the depths from $2.5 m$ to $13 m$ can be described by a log-law velocity deficit. If we find the linear fit of the velocity differences versus the logarithm of sampling depths and assume that von Karman's constant $\kappa=0.4$, we obtain an estimate of the water friction velocity $u_{*_{w}}$ from the slope of the fit. (Throughout this chapter I will employ two versions of $u_{*}$. The unsubscripted $u_{*}$ is based of the ratio of the wind stress applied at the sea surface and the density of the water, 
$u_{*}=\sqrt{\frac{\tau_{0}}{\rho}}$. The friction velocity $u_{*_{w}}$ is, as mentioned, based on measured velocity shears in the water.) To obtain this fit from the combined SASS and C3 data, the calibration offsets needed to be removed. This was accomplished by subtracting the offset that existed between the VMCM output at the $4 m$ depth and the SASS output (interpolated) at the $4 m$ depth. Files where the $4 m$ VMCM output was unavailable were excluded. Files where the rms deviation of the velocities from the fit curve was worse than $0.5 \mathrm{~cm} / \mathrm{s}$ were excluded. Estimates were finally obtained for the following files: o1, o2, p1, p2, p3, $11, r 1, r 2, r 3$, $s 1, s 2, s 3, t 1, u 1, v 1$ and $w 1$. Log profiles were fit to both the biased and unbiased data. The only difference in the two sets of fits was the wave bias; both sets of estimates include the SASS flow disturbance estimates. For the unbiased C3 results I used the lower limit bias correction. Resulting unbiased estimates are, therefore, upper bounds on $u_{*_{w}}$. The results (Figure 7-1) clearly show that the estimation of the wave bias is important for the interpretation of the final results.

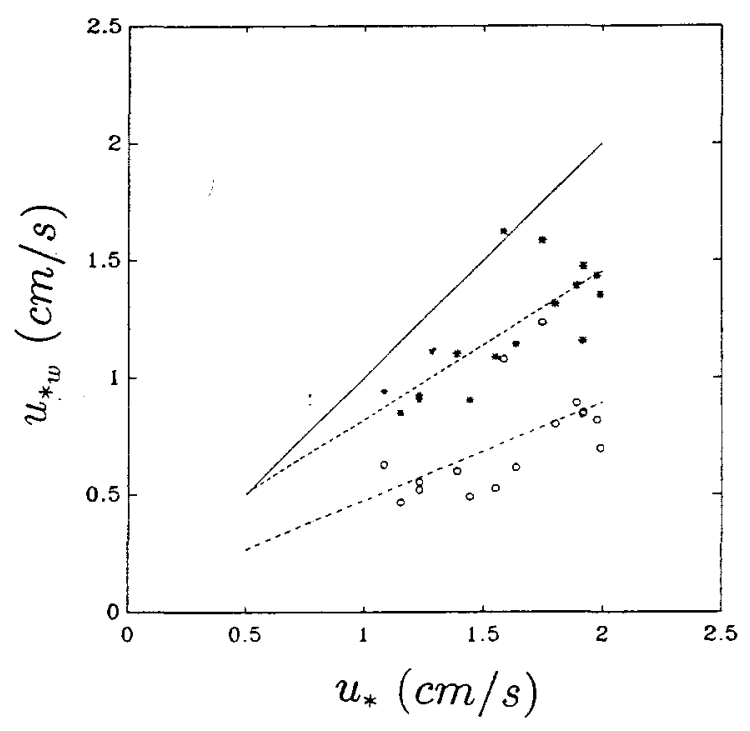

Figure 7-1: Comparison of estimated friction velocity using a biased $(*)$ and an unbiased (o) estimator. The data from the $2 m$ to $13 m$ range was forced to fit a log-profile and the fluid friction velocity was predicted from the fit. The horizontal axis is friction velocity formed from the applied wind stress. The solid line assumes all the wind stress is supported by a log-layer.

The curve fit from the raw data has a large offset at zero wind stress. The reason for this 
is that swell was always present at the SMILE site. So, even when the wind vanishes, an uncorrected time average of data will still indicate some shear due to the wave effects. The wave conditions varied, particularly from the two deployments, but a background swell with amplitude of $60 \mathrm{~cm}$ and average period of velocity variations of $9 \mathrm{sec}$ might be considered typical. For a buoy which perfectly follows the waves in vertical excursion only, the bias is estimated to be $0.34 \mathrm{~cm} / \mathrm{s}$. The $y$-intercept of the fit of the biased estimates is $0.38 \mathrm{~cm} / \mathrm{s}$. The difference between the biased and unbiased estimate increases with wind stress because the waves grow with increasing wind stress. In light of this, and previous evidence presented throughout the thesis, I claim that to understand the shear in the upper ocean the wave effect5must be eliminated. In what remains I will only consider the unbiased estimates.

\section{the large-scale dynamics}

Again, the goal of the thesis was to look at the mean shear current in the upper ten or so meters of the ocean. To study this small scale problem it is necessary to first put the problem in the proper dynamic context. As was shown in Figure 5-1, the SASS and C3 data were taken in a coastal region. The direction of the wind was generally along the coastline, as is shown in Figure 7-2. In this respect, we have a classic upwelling scenario - a long straight coastline with wind blowing alongshore.

The measurements from the SASS and C3 provide us with excellent resolution of the water column but do not give us any spatial resolution. Our first goal then is to examine to what extent the spatial variability of the flow field will need to be considered. Specifically, we'd like to know if lateral density gradients in the flowfield could lead to a sheared flow.

While the standard "thermal wind equations" represent something of an oversimplification for our coastal application, they do provide us with the appropriate dynamic balance that will occur between shear and horizontal density gradients. The coordinate system we'll use here is shown in Figure 7-3. To derive the thermal wind equations, we begin by assuming that there exists a steady flowfield where lateral variations in velocity are sufficiently 
(a)
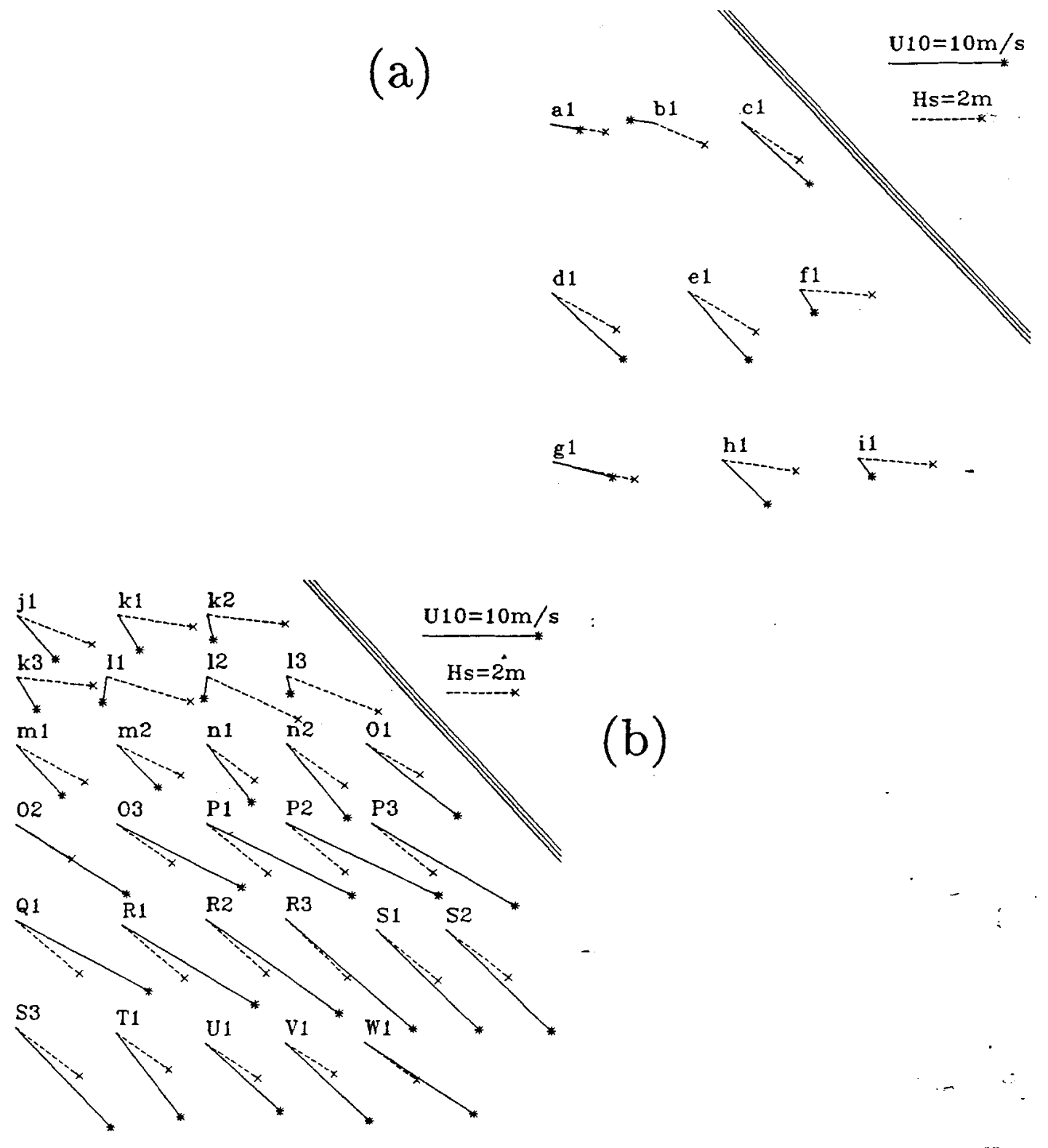

Figure 7-2: The wind and wave directions and magnitudes during the SASS files. Figure (a) presents the fall deployment data and figure (b) presents the spring deployment data. The narrow triple line represents the alongshore direction. This direction was defined by the $100 \mathrm{~m}$ depth contour from positions offshore of the Gualala river to Fort Ross. The scales for the $10 \mathrm{~m}$ wind speed and significant waveheight are given in the upper righthand corner of each plot. The files used in the final analysis have their designation typed in capital letters 
small such that the nonlinear terms in the equations of motion may be ignored,

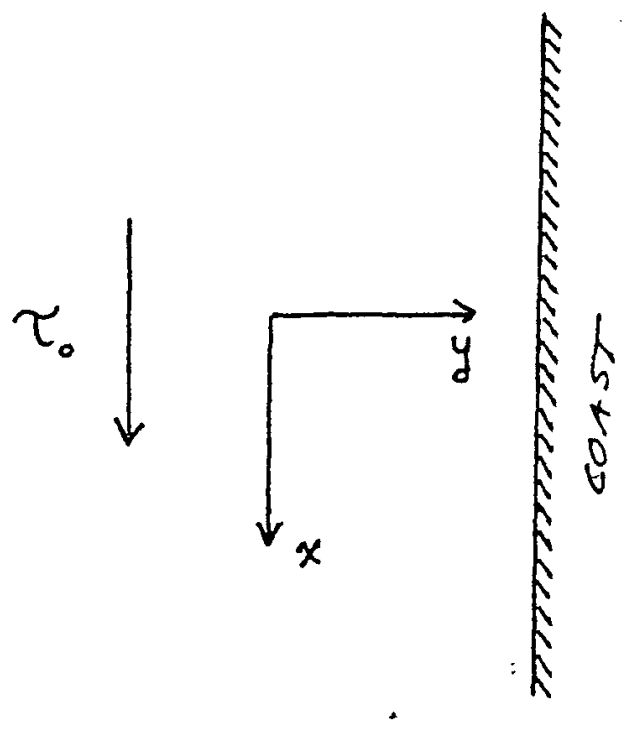

Figure 7-3: Coordinate system for consideration of large scale dynamics

$$
\begin{aligned}
-\rho f v & =-\frac{\partial p}{\partial x}+\frac{\partial \tau_{x z}}{\partial z}, \\
\rho f u & =-\frac{\partial p}{\partial y}+\frac{\partial \tau_{y z}}{\partial z} .
\end{aligned}
$$

Both the equations are then differentiated with respect to $z$. By then changing the order of differentiation on $p$ and assuming that $\frac{\partial p}{\partial z}=-\rho g$, we obtain the thermal wind equations

$$
\begin{aligned}
-\frac{\partial}{\partial z}(\rho f v) & =g \frac{\partial \rho}{\partial x}+\frac{\partial^{2} \tau_{x z}}{\partial z^{2}} \\
\frac{\partial}{\partial z}(\rho f u) & =g \frac{\partial \rho}{\partial y}+\frac{\partial^{2} \tau_{y z}}{\partial z^{2}} .
\end{aligned}
$$

If we assume that lateral variations in the density field are balanced entirely by shear currents we obtain an upper bound on the effect of spatial inhomogeneities. So as not confuse these estimates of shear with later estimates we make I will explicity label these shear estimates by the mechanism which gives rise to them as

$$
\begin{aligned}
& \left.\frac{\partial v}{\partial z}\right|_{\begin{array}{c}
\text { thermal } \\
\text { wind }
\end{array}}=-\frac{g}{\rho f} \frac{\partial \rho}{\partial x}, \\
& \left.\frac{\partial u}{\partial z}\right|_{\begin{array}{c}
\text { thermal } \\
\text { wind }
\end{array}}=\frac{g}{\rho f} \frac{\partial \rho}{\partial y} .
\end{aligned}
$$


The data which we ultimately end up analyzing were recorded on February 27 and 28 of 1989. The results of a large scale hydrographic survey of the SMILE site, performed on these two days, have been presented by Limeburner and Beardsley (1989). From the results of this survey we may attempt to estimate the effects of horizontal density gradients. In the upper $1,000 \mathrm{~m}$ of the ocean, the density $\rho$ is well approximated by $\sigma_{t}$ (Pond and Pickard, 1983). It is $\sigma_{t}$ that Limeburner and Beardsley presented. The gradient of density in the inshore direction was estimated from the survey to be

$$
\begin{array}{ll}
\frac{\partial \rho}{\partial y}=5.5 \times 10^{-6} \frac{\mathrm{kg}}{\mathrm{m}^{4}}, & \text { at the surface, } \\
\frac{\partial \rho}{\partial y}=1.3 \times 10^{-5} \frac{\mathrm{kg}}{\mathrm{m}^{4}}, & \text { at the } 50 \mathrm{~m} \mathrm{depth.}
\end{array}
$$

(The gradients were estimated on spatial scales of $9 \mathrm{~km}$ at the surface and $5 \mathrm{~km}$ at $50 \mathrm{~m}$ depth.) The larger of these values implies that we might expect an alongshore thermal wind shear on the order of

$$
\left.\frac{\partial v}{\partial z}\right|_{\substack{\text { thermal } \\ \text { wind }}} \approx-0.0014 s^{-1}
$$

Over $5 m$ depth a shear this size will give rise to an absolute velocity difference of only about $0.7 \mathrm{~cm} / \mathrm{s}$. In the alongshore direction, the density gradients were even smaller than in the inshore direction,

$$
\begin{aligned}
& \frac{\partial \rho}{\partial x}=2.7 \times 10^{-6} \frac{\mathrm{kg}}{\mathrm{m}^{4}}, \quad \text {-at the surface, } \\
& \frac{\partial \rho}{\partial x}=6.7 \times 10^{-7} \frac{\mathrm{kg}}{\mathrm{m}^{4}}, \quad \text { at the } 50 \mathrm{~m} \text { depth. }
\end{aligned}
$$

(Both of the above gradients were estimated over a spatial scale of $30 \mathrm{~km}$.) The larger estimate implies that the inshore thermal wind shear would be

$$
\left.\frac{\partial u}{\partial z}\right|_{\substack{\text { thermal } \\ \text { wind }}} \approx 0.00029 \mathrm{~s}^{-1},
$$

leading to an absolute velocity difference of only about $0.1 \mathrm{~cm} / \mathrm{s}$ over $5 \mathrm{~m}$ of depth. From these results we conclude that the coastal region at the time of the spring '89 deployment was sufficiently homogeneous that we can ignore the horizontal density gradients in theinterpretation of the near-surface shear currents. 
We are now in a position to predict the large-scale features that we should observe in the SASS and C3 data. If we again assume that the large scale flow is steady and that the nonlinear terms in the equations of momentum are negligible we may write

$$
\begin{gathered}
\frac{\partial p}{\partial x}=\rho f v+\frac{\partial \tau_{x z}}{\partial z}, \\
\frac{\partial p}{\partial y}=-\rho f u+\frac{\partial \tau_{y z}}{\partial z} .
\end{gathered}
$$

Integrating the above with respect to $z$ and assuming $\left.\left.\tau\right|_{z=-h} \ll \tau\right|_{z=0}$, we have that

$$
\begin{aligned}
& \int_{-h}^{0} \frac{\partial p}{\partial x} d z=\int_{-h}^{0} \rho f v d z+\tau_{x \eta}, \\
& \int_{-h}^{0} \frac{\partial p}{\partial y} d z=-\int_{-h}^{0} \rho f u d z, \quad\left(\tau_{y \eta}=0\right)
\end{aligned}
$$

where $\tau_{x \eta}$ is the wind stress applied at the surface in the alongshore direction (it has been assumed that there is no inshore component of wind stress). Then we define the mass transports as

$$
\begin{aligned}
& M_{x} \equiv \int_{-h}^{0} \rho u d z \\
& M_{y} \equiv \int_{-h}^{0} \rho v d z
\end{aligned}
$$

Now, to make the above equations useful for our coastal region, it will be helpful to decompose the total mass transports into wind-influenced (subscript $E$ for Ekman) and geostrophic (subscript $g$ for geostrophic) terms,

$$
\begin{aligned}
& M_{x}=M_{x E}+M_{x g} \\
& M_{y}=M_{y E}+M_{y g}
\end{aligned}
$$

The depth average equations (7.8) may the be rewritten in decomposed form as,

$$
\begin{aligned}
f M_{y E} & =f \int_{-h}^{0} \rho v_{E} d z=-\tau_{x \eta}, \\
f M_{y g} & =f \int_{-h}^{0} \rho v_{g} d z=\int_{-h}^{0} \frac{\partial p}{\partial x} d z, \\
f M_{x E} & =f \int_{-h}^{0} \rho u_{E} d z=0, \\
f M_{x g} & =f \int_{-h}^{0} \rho u_{g} d z=-\int_{-\hbar}^{0} \frac{\partial p}{\partial y} d z .
\end{aligned}
$$


From the above equations, we can deduce the general features of the flow in the coastal region.

Because equations 7.10 to 7.13 are depth averaged, we cannot deduce from them any detailed flow features. They do, however, tell us some very important things. Equation 7.10 gives us the basic Ekman layer result. The wind stress is applied in the alongshore direction and the result is a near-surface offshore transport. Equation 7.13 is the statement of geostrophic balance, the net alongshore transport gives rise to an offshore pressure gradient. As a word of caution, one should resist the temptation of reading too much from the above equations; the are admittedly gross simplifications.

Interpretation of a shear current profile in the presence or absence of a vertical density gradient would be as troublesome as the horizontal gradients that we examined earlier. One of the great advantages of having the SASS and the C3 buoy simultaneously deployed is that it allows the data from the two instruments to be compared. To allow us to utlize this capability we will try to use the C3 VMCM's down to $16 \mathrm{~m}$. To ensure that the results are not contaminated by stratification effects we will only examine files where the mixed layer depth is deeper than $20 \mathrm{~m}$.

Pollard, Rhines and Thompson (1973) have proposed a very simple relation for the maximum depth of the mixed layer depth $H$ in a steady state wind,

$$
H_{\max }=2^{3 / 4} \frac{u_{*}}{\sqrt{f N}}
$$

where $N$ is the Brunt-Väisälä frequency. The Brunt-Väisälä frequency can be estimated as the gradient of $\sigma_{t}$ as

$$
N^{2} \approx g\left[\frac{1}{\rho} \frac{\partial \sigma_{t}}{\partial z}\right]
$$

(Pond and Pickard, 1983). The gradient of $\sigma_{t}$ can be estimated graphically from our temperature and salinity plots using the simplified relation between temperature and salinityproposed by Mamaev (1964),

$$
\frac{\partial \sigma_{t}}{\partial z}=(-0.0735-0.00938 T) \frac{\partial T}{\partial z}+0.802 \frac{\partial S}{\partial z},
$$


where I have performed the partial differentiation with respect to $z$ on Mamaev's original form.

Previous to file $f 1$, the wind had been nearly constant for about ten hours. It had, however, been very light $\left(u_{*}=0.0026 \mathrm{~cm} / \mathrm{s}\right)$. The bound for the mixed layer depth $H_{\max }$ for such a situation is only about $5 \mathrm{~m}$. The actual mixed layer depth at this time was less than $2 m$ (determined using an absolute temperature difference $(0.05 \mathrm{C})$ criterion as has been done by Lentz, 1991). Though higher winds were experienced in the fall deployment, they were never persistent enough to deepen the mixed layer to $20 \mathrm{~m}$.

In the spring deployment, much greater wind speeds were encountered. The Pollard, Rhines and Thompson depth again gives us a fairly good indication that this depth does scale with the friction velocity. Previous to file $s 3$ the average friction velocity was about $1.7 \mathrm{~cm} / \mathrm{s}$. From the salinity and temperature profiles I estimated that $N^{2} \approx 0.000112$, which leads to a maximum mixed layer depth estimate of $30 \mathrm{~m}$. The actual depth was $28 \mathrm{~m}$.

Of course, while the above analysis does indicate how the mixed layer scales with the wind stress, we do much better estimating the mixed layer depths from our data (plotted out in Chapter 6) than by using the approximate relation for $H_{\max }$. In the ensuing arialysis we shall consider files $o 1, o 2, o 3, p 1, p 2, p 3, q 1, r 1, r 2, r 3, s 1, s 2, s 3, t 1, u 1, v 1, w 1$. Files not considered because the mixed layer depth was too shallow were files a1 to 13 . During files $m 1$ to $n 2$ the mixed layer was deeper than $20 m$ but the shear profiles were of an ariomalous character and thus were also not considered (these anomalies and the mismatches in the SASS and C3 data are discussed in Appendix C).

\section{the "log-law"}

At the beginning of the chapter, velocity profiles were forced to fit logarithmic profiles so wecould examine the importance of the wave bias effects. Though the log-law profile provides the standard model which is often applied to near-surface current profiles, the question 
still remains whether the logarithmic profile is appropriate. Wu (1975) found that, in the laboratory, the flow beneath the surface was well described by a constant stress (log-layer) model. The stress supported by the shear current was less than that applied by the wind. The discrepancy in momentum flux was attributed to "wave drag". Stewart(1961) estimated wave drag for open ocean conditions. From measurements of wave growth rates, he concluded that less than $20 \%$ of the stress would be supported by the waves. Our log-law estimates show that for the SMILE data only $22 \%$ of the stress was supported by the shear current. Even if we assume that $20 \%$ of the momentum flux is generating waves, we still have a grave momentum flux discontinuity from the air to the sea.

While Stewart's estimates were rather rough, it is unlikely that the wave drag would be much larger than he had estimated. This must lead us to a critical evaluation of the constant stress layer model. If a log-layer does exist, it may not hold over the large range of depths $(2.5 \mathrm{~m}$ to $13 \mathrm{~m})$ initially assumed. If the shear current were accurately described by a constant stress region, we expect that the downwind current $v_{d}$ is described by

$$
\frac{z}{u_{*}} \frac{\partial v_{d}}{\partial z}=\text { constant }
$$

This means that $z \frac{\partial v_{d}}{\partial z}$ at a fixed depth should scale linearly with the friction velocity. And for a fixed friction velocity, $z \frac{\partial v_{d}}{\partial z}$ should be constant with depth. Estimates of $z \frac{\partial v_{d}}{\partial z}$ and $z \frac{\partial v_{c}}{\partial z}$ (where $v_{c}$ is the current in the crosswind direction) were obtained by finding the discrete estimates of the shear from pairs of adjacent sensors and then multiplying by their. mean depth. For the SASS data, several of the discrete estimates were averaged to get a smoothed estimate. Though averaging sensors from the SASS gave smoother estimates, it did lead to some overlap in the estimation ranges. Overlap of the lower SASS data (bottom sensor at $5.8 m$ ) with the $\mathrm{C} 3$ data (upper sensor at $4 m$ depth) was unavoidable.

In using equation 7.17 , we are making some implicit assumptions. By looking at the data in distinct directions, we're assuming that Ekman turning is negligible over the depthrange examined. The Ekman layer thickness $\delta_{E}$ for the typical eddy viscosity we later derive 
$\left(\nu_{T} \approx 400 \mathrm{~cm}^{2} / \mathrm{s}\right)$ is

$$
\delta_{E}=\sqrt{\frac{\nu_{T}}{f}} \approx 28 m
$$

Even in the classical Ekman solution, the turning with depth is not too severe at up to $0.5 \delta_{E}$. The solution of Madsen(1977), based on a variable eddy viscosity, would -show almost negligible turning even down to the $16 \mathrm{~m}$ depth for our typical parameters. Our observations, however, do not show the near-surface log-layer that Madsen assumed to exist. Huang (1979) attempted to take a unified approach (including viscous terms, Coriolis and wave effects) to the near-surface layer. He proposes that if the Ekman number

$$
E=\frac{f}{\nu_{T} k_{o}^{2}}
$$

is much less than one, then the Coriolis term should not be important in the near-surface region ( $k_{o}$ is the wavenumber of the dominant waves). For the files we'll finally end up examining, the average wave periods are usually just under $6 s e c$; this gives $E<0.1$. Ultimately, we must determine the appropriate dynamic balance for our specific case: We will find that the viscous forces (mixing) in our problem is much greater than has usually been assumed. We expect little effect even in the upper $16 m$ of the water column.

The resulting estimates (Figure 7-4) show that while the downwind shear current does scale with wind stress, the shear stress is not constant with depth. A most notable feature is the absence of virtually any downwind shear at all in the upper $3 \mathrm{~m}$. If we estimate $u_{*_{w}}$ as

$$
u_{*_{w}}=\kappa z \frac{\partial v_{d}}{\partial z}
$$

the stress input by the atmosphere and the stress supported by the shear current agree to within $13 \%$ in the 10 to $16 \mathrm{~m}$ range.

\section{The reduced shear layer}

In both the lab (e.g., Wu, 1975) and the field (e.g., Csanady, 1984), previous investigators have found constant stress layers beneath relatively small constant shear layers. Csanady 

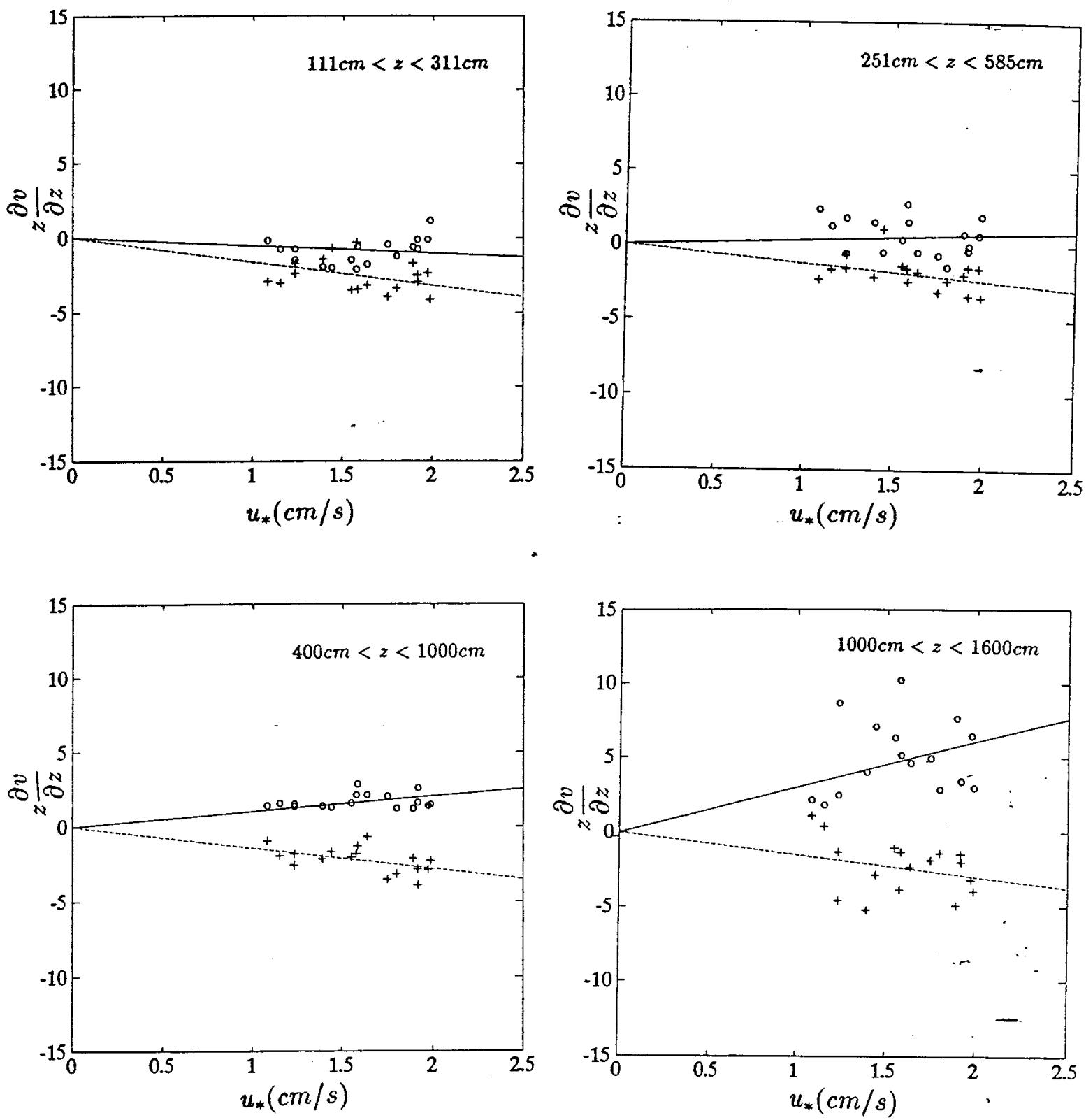

Figure 7-4: The quantity $z \frac{\partial v}{\partial z}$ ( $o=$ downwind, $+=$ crosswind) is estimated over various depth ranges (as labelled). The linear fits ( $-=$ downwind, - - =crosswind) were forced to zero at zero wind friction velocity, $u_{*}$. 
claims, on the basis of drifter results, that the constant velocity gradient layer is on the order of $5 \mathrm{~cm}$ thick when $u_{*}=\sqrt{\frac{\tau_{0}}{\rho}}$ is about $1 \mathrm{~cm} / \mathrm{s}$. For the SASS data, when $u_{*}$ varies between $1.0 \mathrm{~cm} / \mathrm{s}$ and $2.0 \mathrm{~cm} / \mathrm{s}$, we find that there is essentially no shear in the top_ $3 \mathrm{~m}$, a transition region of low shear, and a region where the stress has finally increased to agree with the level input by the atmosphere. There are some observations that are consistent with those made here.

Observations (unpublished) have been made from fixed sensors mounted on the CCIW tower in Lake Ontario (E. Terray, personal communication) which support the notion that there exists, above the constant stress region, a surface layer with little or no shear. These observations indicate a region of reduced shear approximately as large as has been measured here. Though the resolution is poor, the shear current profile presented by Santala and Terray (1991) (and found in Chapter 3), also shows a region of reduced shear beneath the air-sea interface. These, and the SMILE results, seem to be at odds with many past observations. Next, I will seek to explain the virtual absence of shear in the downwind direction. Two, not necessarily exclusive explanations emerge, wave mixing and Langmuir circulation, each will be discussed in turn.

\section{alternative 1 : wave mixing}

Intuitively, it seems natural to assume that very near the ocean's surface, the vigorous action of wave mixing will destroy any shear current. Though it is scant, some evidence does exist to support the idea of an intensely mixed wave zone, of significant thickness, which is inconsistent with a constant stress (log-layer) model. Kitaigorodskii et. al. (1983) analyzed near-surface velocity measurements by Donelan (1978) and found dissipation rates two orders of magnitude larger than those consistent with a log-layer modēl. The thickness of this extremely turbulent region was approximately ten times the rms wave amplitude. For the rms amplitudes we measured in SMILE, typically $50 \mathrm{~cm}$ or larger, this translates. to a physical dimension of $5 \mathrm{~m}$ or more. This is consistent with the SMILE observations. Scaling in this fashion might be considered a bit tenuous since I have essentially lumped 
the swell and wind-driven wave contributions together.

An alternate way to scale the depth of the reduced shear region, which would exclude the swell, would be to predict the wave action from the measured windspeeds. Representing the acceleration due to gravity as $g$, the $r m s$ wave amplitude in a fully developed wavefield scales as $u_{*}^{2} / g$ (see, for example, Longuet-Higgins, 1969). The nondimensional shear $\frac{z}{u_{*}} \frac{\partial v_{d}}{\partial z}$ is then to be plotted versus the nondimensional depth $\frac{g z}{u_{*}^{2}}$. Since the winds were generally alongshore or even slightly onshore, the wavefield was not fetch limited. The winds did tend to vary on time scales of a few hours so the waves generally were duration limited. This means that in merely scaling by the instantaneous wind friction velocity we can not hope to fully characterize the intensity of the wind-driven part of the wave spectrum. This scaling does, however, begin to reveal the trend in the reduced shear layer thickness (Figure 7-5). There are a few things which we should note. First of all, the transition between the reduced shear and constant stress region occurs between nondimensional depths of $1.24 \times 10^{5}$ and $2.88 \times 10^{5}$. A few of the SASS data points are in the transition region, and others extend into the depths of the "constant stress layer". These few points show the expected trend towards a nondimensional stress of $\frac{1}{\kappa}$. The C3 estimate of shear made from the $4 m$ and $10 \mathrm{~m}$ VMCMs tend to fall in the transition region and again seem to show the expected trend. The deeper VMCM data appears very noisy when plotted in this fashion because the absolute value of the shear $\frac{\partial v_{d}}{\partial z}$, which is very small, is scaled by the physical dimension $z$, thus amplifying any noise in the measurement (recall that the individual shear profiles were quite regular and smooth). A later plot shows this data with a more "flattering" dimensionless scaling (Figure 7-11). The Kitaigorodskii et. al. (1983) data are consistent with our observations, they show elevated dissipation to depths of $\frac{g z}{u_{*}^{2}} \approx 1 \times 10^{5}$. They attribute the elevated levels of dissipation to wave mixing.

Dynamically, I have not carefully defined how the wave action reduces the near-surface shear. I have only argued that intuitively this mechanism seems plausible and have noted the limited observations of dissipation rates that are inconsistent with a log-layer model. On the other hand, there are measurements of dissipation rates even as close to the sur- 

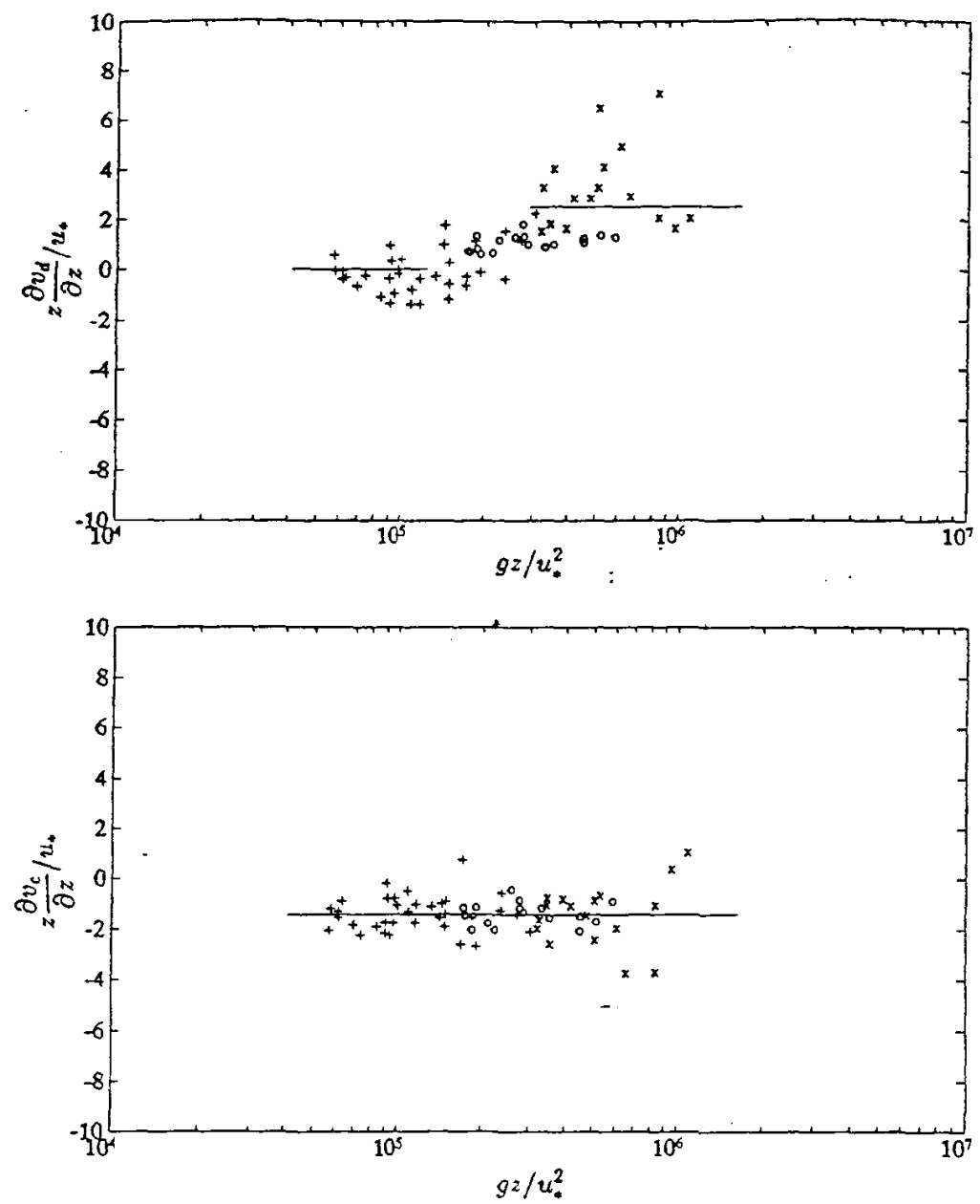

Figure 7-5: Nondimensional variation of shear with depth for the downwind (a) and crosswind (b) directions. $+=$ SASS data, $\mathrm{o}=4 \mathrm{~m}$ to $10 \mathrm{~m}$ VMCM data, $\mathrm{x}=10 \mathrm{~m}$ to $16 \mathrm{~m}$ VMCM data. 
face as $\frac{g z}{u_{*}^{2}} \approx 1 \times 10^{5}$ that support the existence of a log-layer (Soloviev et. al., 1988). Csanady(1984) notes that if larger waves were dissipative (he holds that they are not), then the log-layer could not hold at least until depths on order of their amplitude below the surface. The evidence from previous experiments simply does not seem strong enough to accept or reject the explanation of wave mixing as a mechanism for the reduced shear layer.

That the transition region is best defined when we scale with $\frac{g z}{u_{*}}$ is not necessarily indicative of wave mixing, most dynamic processes in the upper ocean have some windspeed dependence (depth scalings which included the rms wave amplitude didn't seem to work as well). The three-dimensional flow pattern referred to as Langmuir circulation is also windspeed dependent, and may be a plausible explanation for our observations.

\section{alternative 2 : Langmuir cells}

Langmuir(1938) first hypothesized that streaks observed on the water's surface, aligned with the wind direction, were evidence of large scale helical flow patterns. These motions, now called Langmuir cells, have since been studied by many investigators. Though very recent investigation continues to increase our knowledge of Langmuir circulation, the review by . Pollard (1977) provides a relatively up-to-date summary of Langmuir circulation from both an experimental and theoretical point of view. In this review, Pollard makes the following conclusion:

If, as existing observations suggest, Langmuir circulations appear rapidly after . the onset of winds greater than $3 \mathrm{~m} / \mathrm{s}$, it is likely that they, rather than smallscale turbulence generated by breaking waves, control the downward diffusion and redistribution of wind generated momentum through the surface layers.

For the observations presented in this chapter, where wind speeds varied from $9 \mathrm{~m} / \mathrm{s}$ to $15 \mathrm{~m} / \mathrm{s}$, we expect that Langmuir circulation may exist. Though Langmuir cells may be formed in winds greater than $3 \mathrm{~m} / \mathrm{s}$, their existence under these conditions is not guaranteed. There is still a great deal of controversy with regards to the theories governing Langmuir circulation. For this reason we would like to base our conjecture on observation 
(preferably our own).

Weller et. al. (1985) suggested that Langmuir circulation is best observed at depths where the wave velocities are significantly attenuated. Because the BASS velocity sensors measure a complete vector velocity, we are able to resolve the velocity in the vertical direction but, detection of Langmuir circulation will be complicated by the fact that the deepest sensor is located at a depth of only $5.85 \mathrm{~m}$. At this depth it would be hard to recognize the instantaneous downwelling velocity from the wave velocities as a function of time. Our best hope is to try to average the time series of vertical velocity and thereby remove the wave signal.

The spatially integrated vertical mass flux due to Langmuir cells must be zero. If a buoy maintains a constant position, and Langmuir cells advect by laterally at a uniform rate, the time averaged vertical velocity should be zero. If a buoy is on a slack mooring, as was the SASS, it might spend more time in the convergence (downwelling) zones and hence measure a net downward velocity. In Figure 7-6 I give a schematic which demonstrates this idea. It appears that something of this sort was occurring. In Figure 7-7 the mean unbiased vertical velocity measured by the top and bottom sensor is plotted versus the friction velocity for all . 37 files (in Appendix $\mathrm{F}$ the vertical velocity samples are plotted in the long term windspeed time series). At low wind speeds the net vertical velocity at both sensors is approximately zero. As the friction velocity increases from $0.70 \mathrm{~cm} / \mathrm{s}$ to $1.4 \mathrm{~cm} / \mathrm{s}$, the vertical velocity at the bottom sensor approaches $-2.5 \mathrm{~cm} / \mathrm{s}$. When $u_{*}$ increases above $1.4 \mathrm{~cm} / \mathrm{s}$, the vertical velocity is relatively constant at about $-2 \mathrm{~cm} / \mathrm{s}$. To examine whether such observed_means are plausible, let's make the simplifying assumption that the vertical velocities are constant across the downwelling and upwelling regions. The convergence zones of Langmuir cells have been observed to have a smaller lateral dimension and larger vertical velocity than the divergence zone. If we assume that the lateral extent of the convergence zone is one third that of the total cell width, then the downwelling velocities will be twice as great as the upwelling velocities. Now, if the buoy, due to its freedom to move laterally spends half its time in the convergence zone, then the mean velocity measured will be one half that of the 


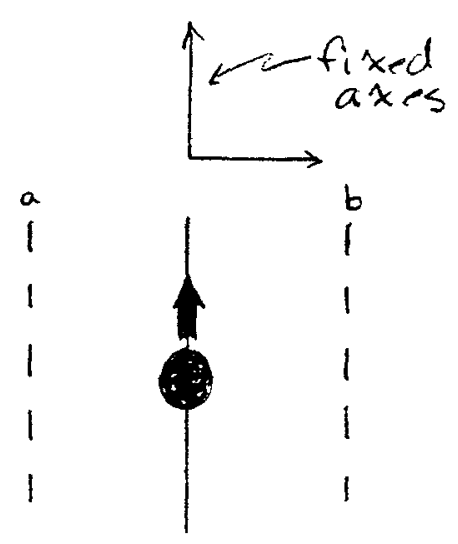

(a)

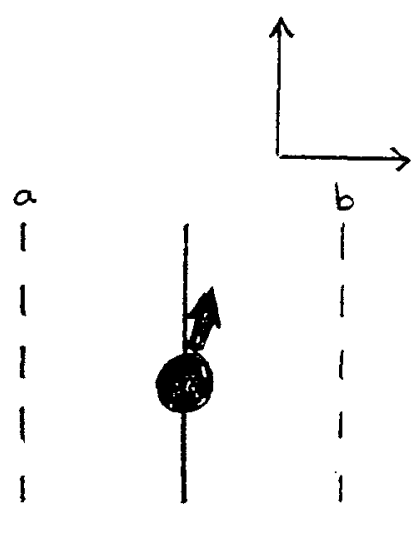

(b)

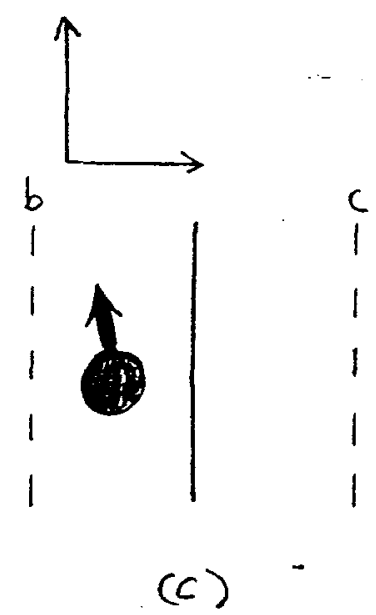

Figure 7-6: The SASS may have preferentially sought convergence zones (solid lines). As Langmuir cells advect by laterally, the SASS becomes "locked" into a convergence zone. The SASS unlocks when the lateral component of drag force (arrow) becomes to great. But then, the combination of diverging flow and mooring force quickly carry the SASS to the next convergence zone.

downwelling velocity. This is an extremely rough argument but it shows us that it would be reasonable to measure $-2 \mathrm{~cm} / \mathrm{s}$ mean velocities in a case where the typical downwelling velocity is $4 \mathrm{~cm} / \mathrm{s}$. Figure $7-8$ shows the spatial variation of vertical velocity across the Langmuir cell that we've assumed in this argument. A downwelling velocity of $4 \mathrm{~cm} / \mathrm{s}$ at a depth of almost $6 \mathrm{~m}$ is in a range that we would expect for our peak wind conditions if Langmuir circulation were active.

In spite of all the arguments in the thesis in favor of correcting mean velocity measurements for the wave bias, the reader may still have doubts about this correction. For the vertical measurements made, the wave bias is very small (never greater than $1 \mathrm{~cm} / \mathrm{s}$ ), owing to the fact that the SASS did follow the waves well (recall that existence of a vertical bias requires a phase shift in response). What bias there was tended to be a downward bias. For those who wish to doubt the bias correction, the raw time average of vertical velocity can be approximately recovered by increasing the magnitude of vertical velocities I present by $10 \%$ when $u_{*}<1 \mathrm{~cm} / \mathrm{s}$ and by $24 \%$ when $u_{*}>1 \mathrm{~cm} / \mathrm{s}$. 


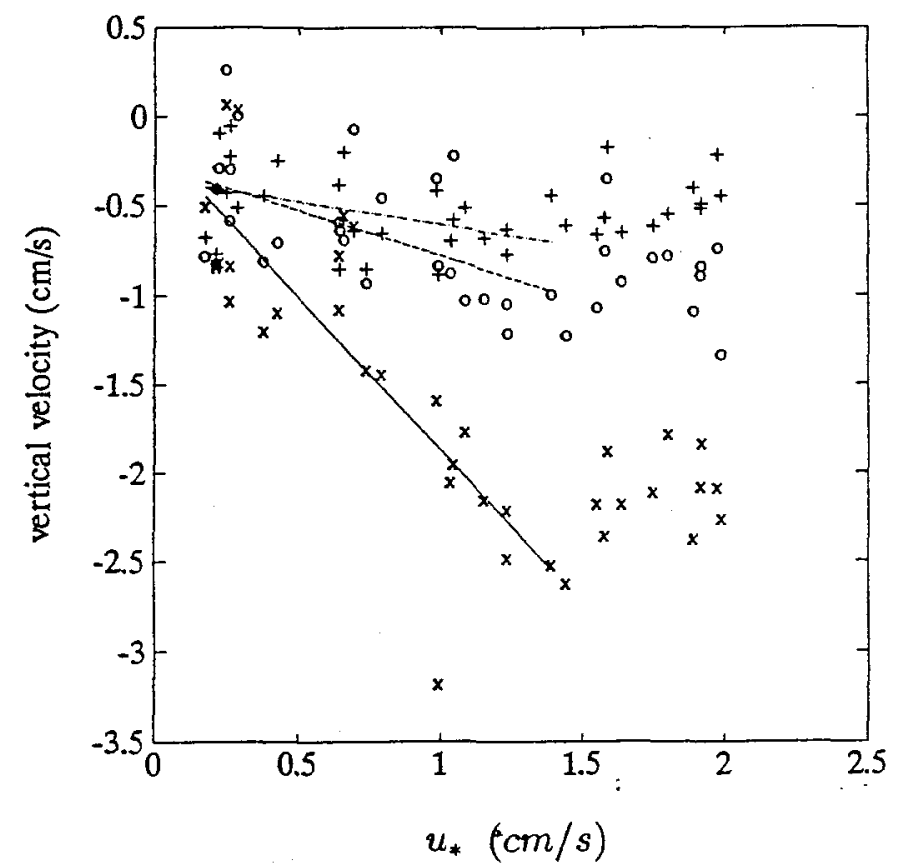

Figure 7-7: Vertical velocity measured at the $111 \mathrm{~cm}$ depth $(+)$, the $251 \mathrm{~cm}$ depth (o) and the $585 \mathrm{~cm}$ depth $(\mathrm{x})$ with linear fits over the range of $u_{*}$ where the velocity seems to be increasing.

The bottom BASS sensor was attached to the sensor array from above and open at the bottom (Figure 7-9). If there is flow disturbance due to drag on the BASS cage, then it should be that relative velocities from above should be retarded to a greater extent than those from below and the resulting error should be a net upward velocity. The preceeding argument is an intuitively based argument that would lead us to believe that while flow disturbance errors may exist, they would tend to be in the wrong direction to explain the phenomenon we observe. Next, I will also show from the data that the features we observe in the vertical velocity are not consistent with a flow disturbance explanation.

One of the interesting features of the vertical velocities observed at the bottom sensor is the break in slope at $u_{*}=1.4 \mathrm{~cm} / \mathrm{s}$. Flow disturbance should scale with the relative velocity squared. If the observed phenomenon is the result of flow disturbance, we expect to see 


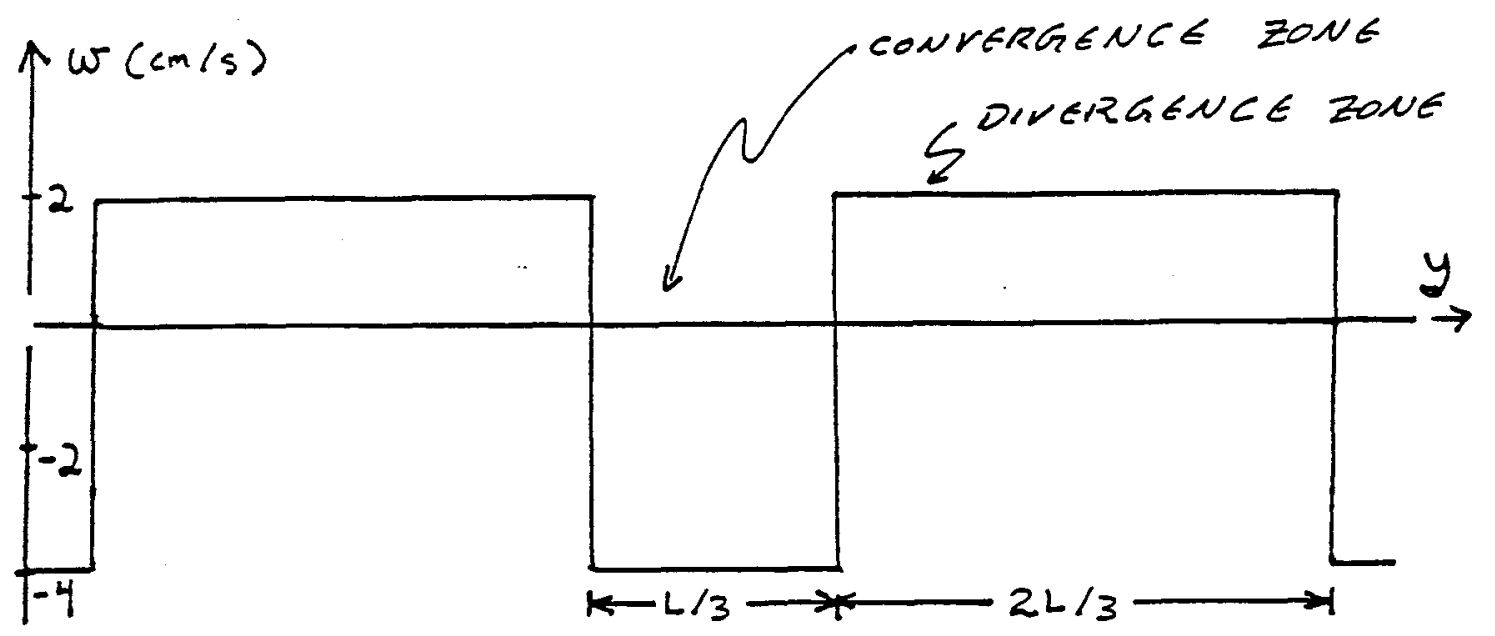

Figure 7-8: An idealization of the vertical velocity structure across the dominant scale Langmuir cells at $6 \mathrm{~m}$ depth. Only the relative size of the horizontal extent of the convergence and divergence regions are important to the arguments in the text. We expect that $L$ is on the order of $10 \mathrm{~m}$.

a change in behavior of the variance of vertical velocity relative to the instrument at this break point (Figure 7-10). This is not seen. As wind friction velocity increases, the variance of relative velocity increases linearly. Over the range $u_{*}=0.70 \mathrm{~cm} / \mathrm{s}$ to $u_{*}=1.4 \mathrm{~cm} / \mathrm{s}$ the vertical velocity at the bottom sensor also increases linearly. But beyond $u_{*}=1.4 \mathrm{~cm} / \mathrm{s}$, while the variance of vertical velocity continues to grow linearly, the vertical velocity remains approximately constant. If a flow disturbance mechanism is in some sense saturating at $\sigma_{V_{K^{-}}}^{2}=900 \mathrm{~cm}^{2} / \mathrm{s}^{2}$ and this explains the break point in vertical velocity at the bottom sensor, then we would not expect to see the same break point at the middle pod until similar relative velocities were reached. In fact, the mid-depth pod reaches the same break point at a much lower relative velocity level $\left(\sigma_{V_{K}}^{2}=300 \mathrm{~cm}^{2} / \mathrm{s}^{2}\right)$. That both sensors, in different local flow regimes, exhibit the same behavior when scaled by the same atmospheric parameter is highly suggestive that the observation is evidence of a flow forced by atmospheric conditions.

Finally, a simple BASS calibration done specifically for this thesis (Appendix C) included a test where the BASS sensor was shaken in a direction perpendicular to the mean flow. One of the goals of this test was to see if the "pumping" would cause a spurious mean 


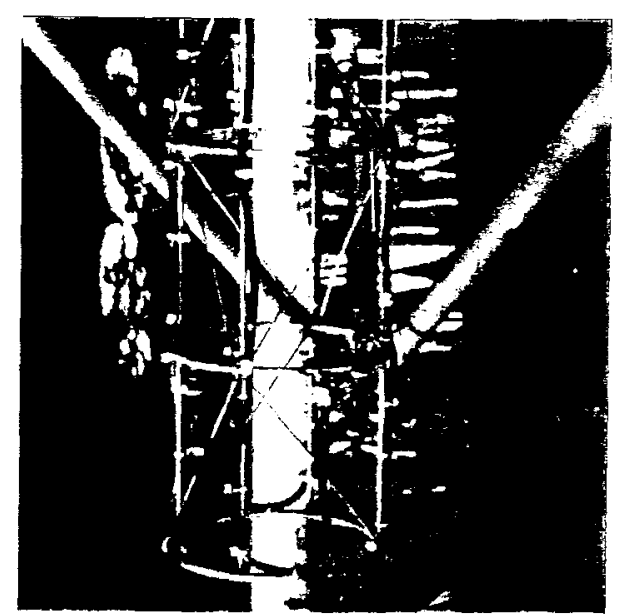

Figure 7-9: The bottom BASS sensor mounted onto the SASS while on the dock prior to deployment. The edge of the dock is vertical in the background because the picture has been rotated to show orientation of the sensor is as it would be during deployment

velocity to be measured in the vertical direction. No mean vertical velocities were measured as a result of the oscillations in the vertical direction.

On the strength of this calibration, the above argument regarding the break point in the observed vertical velocities, and the fact that flow disturbance at the bottom sensor would probably lead to flow disturbance of the wrong direction to explain the observed phenomenon, I claim that the vertical velocity records presented are evidence of Langmuir circulation. I argued earlier that we would only find evidence of Langmuir cells if we sampled them nonuniformly. Because the lateral advection rate of cells past the SASS is probably quite small, there is little hope of reconstructing a detailed picture of the three-dimensional flow which gives rise to the characteristics we observe in the mean. Let me explain. If the lateral advection rate of the cells were even as high as $5 \mathrm{~cm} / \mathrm{s}$, then for a Lagmuir cell width of $12 \mathrm{~m}$, we will find the frequency signature of the cells occurring at a period of $600 \mathrm{sec}$. Due to the low-frequency gyro stabilization errors, we have previously only attempted to resolve periods shorter than $26 \mathrm{sec}$. This precludes an approach based on the absolute velocities. In using just the relative velocities we are, as mentioned previously, hampered by the presence of waves. If, as I roughly estimated, the peak downwelling velocity is on order 


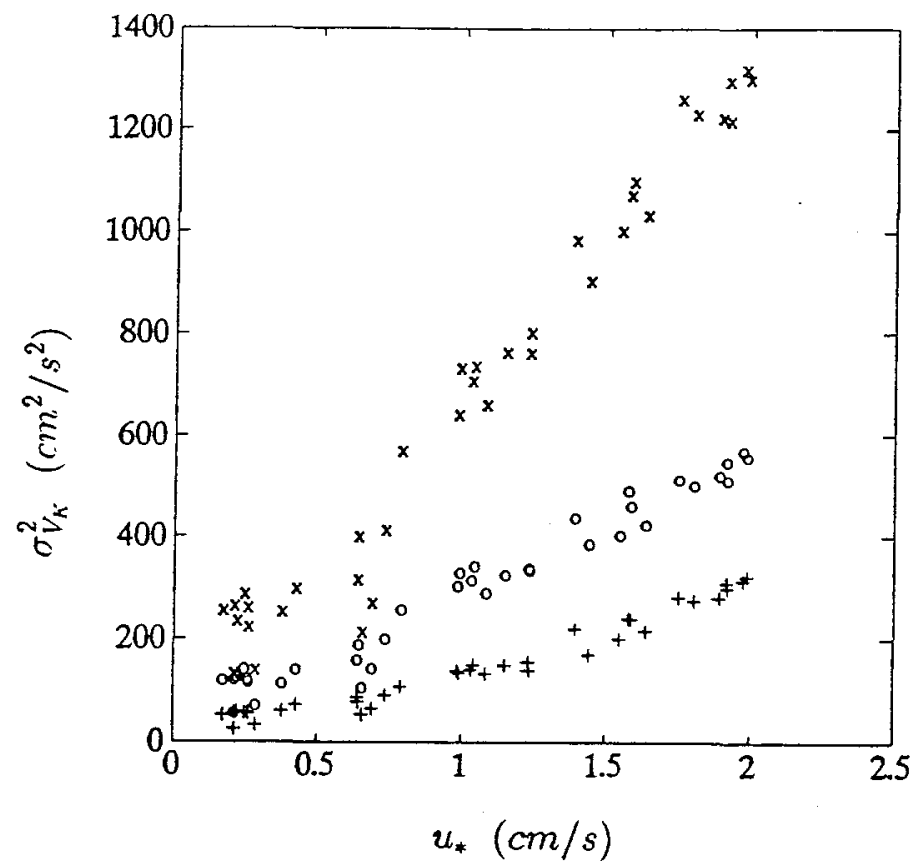

Figure 7-10: Variance of vertical velocity relative to:the sensor at the $111 \mathrm{~cm}$ depth $(+)$, the $251 \mathrm{~cm}$ depth (o) and the $585 \mathrm{~cm}$ depth (x) wersus $u_{*}$.

$4 \mathrm{~cm} / \mathrm{s}$ at the bottom pod then the strength signal we seek to observe is about $22 \mathrm{~dB}$ less (the $r m s$ is thirteen times smaller) than the signal due to the relative velocity forced by the waves.

In spite of the fact that the details of the three-dimensional flow can not be resolved, the fact that a three-dimensional flow has been identified is of extreme importance in our final interpretation of the shear profiles. The Langmuir cells will provide an efficient mechanism for the transfer of momentum from the surface to the deeper fluid layers. Gordon(1970) has previously noted this and proposed that the actual momentum transfer "path" is "from the wind to the waves to Langmuir cells to the deeper ocean layers." This is entirely consistent with the near-surface profiles that were measured during SMILE.

\section{The shear profiles and mixing}

Up to this point, I've discussed various aspects of the shear by looking at differences between sensors. It was necessary to do this because the mean velocity is not necessarily well 
correlated to the wind. With the estimates of the shear, we can now derive a wind-induced shear current profile. In Figure 7-5 we saw that the downwind shear was roughly broken up into three regions; an upper layer where there was essentially no shear, a transition-region and a constant stress layer where the stress was approximately equal to that applied by the wind. Our resolution is too poor to describe the functional dependence in the transition region. But, as the transition region is relatively narrow, we might hope to describe the overall shear profile by just assuming that the transition between the two regions is linear. If this is done, the nondimensional downwind shear can be expressed as

$$
\frac{z}{u_{*}} \frac{\partial v_{d}}{\partial z}= \begin{cases}0 & \text { if } 0<\frac{g z}{u_{*}^{2}}<1.24 \times 10^{5} \\ \frac{1}{1.64 \times 10^{5} \kappa}\left[1.24 \times 10^{5}-\frac{g z}{u_{*}^{2}}\right] & \text { if } 1.24 \times 10^{5}<\frac{g z}{u_{*}^{2}}<2.88 \times 10^{5} \\ -\frac{1}{\kappa} & \text { if } 2.88 \times 10^{5}<\frac{g z}{u_{*}^{2}}\end{cases}
$$

and using the same scaling on the crosswind shear we find

$$
\frac{z}{u_{*}} \frac{\partial v_{c}}{\partial z}=1.41 \quad \text { for } 0.20 \times 10^{5}<\frac{g z}{u_{*}^{2}}
$$

If both sides of the above equations are divided by $z$ for each depth range, we can obtain the nondimensional current by integration with respect to $z$. The only question remains is as to what the constants of integration will be. At the intersection of regions these are, of course, set to make the profile continuous. The mean downwind and crosswind velocities at the surface for the files examined were approximately $14 \mathrm{~cm} / \mathrm{s}$ and $-13 \mathrm{~cm} / \mathrm{s}$, respectively. The typical friction velocity was $u_{*}=\sqrt{\frac{\tau_{0}}{p}}=1.6 \mathrm{~cm} / \mathrm{s}$. To remove the last constant we'll use the ratio of these numbers to form the nondimensional drift velocity at the surface. I carltion that the surface velocities are not all wind-induced, if this data is used for comparison purposes, consider the offset arbitrary. The results of the integration yield

$$
\frac{v_{d}}{u_{*}}= \begin{cases}9 & \text { if } 0<\frac{g z}{u_{*}^{2}}<1.24 \times 10^{5} \\ \frac{1}{1.64 \times 10^{5} \kappa}\left[1.24 \times 10^{5} \log \left(\frac{g z}{u_{*}^{2}}\right)-\frac{g z}{u_{*}^{2}}\right]-11.3 & \text { if } 1.24 \times 10^{5}<\frac{g z}{u_{*}^{2}}<2.88 \times 10^{5} \\ -\frac{1}{\kappa} \log \left(\frac{g z}{u_{*}^{2}}\right)+39.5 & \text { if } 2.88 \times 10^{5}<\frac{g z}{u_{*}^{2}}\end{cases}
$$

and

$$
\frac{v_{c}}{u_{*}}=1.41 \log \left(\frac{g z}{u_{*}^{2}}\right)-22.0 \quad \text { for } 0.2 \times 10^{5}<\frac{g z}{u_{*}^{2}} .
$$



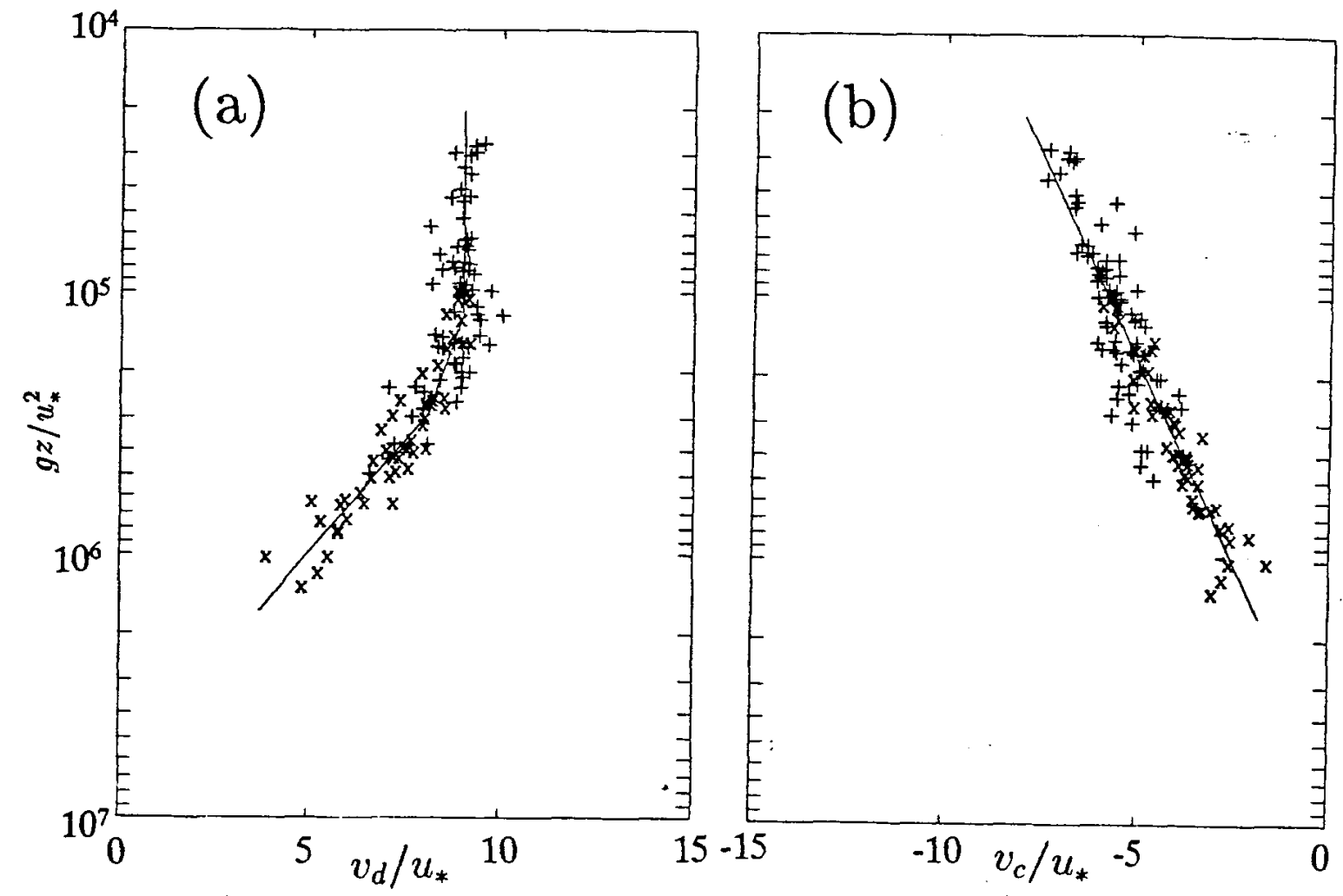

Figure 7-11: (a) The nondimensional downwind shear current profile for SMILE and the fit data. The offset from each instrument, SASS and C3, was subtracted before fitting. Note that the data from both SASS $(+)$ and C3 (x) describe the transition region. (b) The crosswind version of (a).

The model we have is quite simple but, as Figure-7-11 (a) shows, seems to describe our observations in the downwind direction quite well. The scaling doesn't seem to work so well in the crosswind direction (the SASS, physically nearer to the surface, seems to indicate near-surface shear should be less than that indicated by equation 7.24). Indee $\bar{d}$, we had little reason to expect that the crosswind shear current should scale with windspeed.

\section{testing the alternatives}

Upon discovering the reduced shear layer we discussed two alternatives to explain its presence; wave mixing and Langmuir cells. Here we will try to examine which of the alternatives is a more likely explanation. We will begin by considering wave mixing.

If wave mixing is a mechanism which dramatically alters the near-surface region, we 
expect the mixing effects to be more-or-less isotropic. Realizing that there are objections to such an approach, we might nevertheless hope to characterize the mixing by using an eddy viscosity model. If we assume that the near-surface flow is steady and that the nonlinear transport terms are negligible then the momentum equations are

$$
\begin{aligned}
-f v_{c} & =-\frac{1}{\rho} \frac{\partial p}{\partial x}+\frac{\partial}{\partial z}\left(\nu_{T} \frac{\partial v_{d}}{\partial z}\right) \\
f v_{d} & =-\frac{1}{\rho} \frac{\partial p}{\partial y}+\frac{\partial}{\partial z}\left(\nu_{T} \frac{\partial v_{c}}{\partial z}\right)
\end{aligned}
$$

where $\nu_{T}$ is the eddy viscosity. If the large scale flow is in geostrophic balance, then the alongshore current leads to an offshore pressure gradient. For the files analyzed, the alongshore and downwind directions are nearly the same and for purposes of this argument the difference will be ignored. The alongshore velocities are typically on the order of $10 \mathrm{~cm} / \mathrm{s}$, so that from equation 7.13 we have

$$
\frac{\partial p}{\partial y}=-f \rho v_{d} \approx 5 \times 10^{-4} \mathrm{~cm} / \mathrm{s}^{2}
$$

The downwind pressure gradient is assumed to be zero. By assuming different forms of the eddy viscosity $\nu_{T}$ and applying boundary conditions appropriate to our typical friction velocity of $u_{*}=1.6 \mathrm{~cm} / \mathrm{s}$, we solve the coupled equations for $v_{d}$ and $v_{c}$. Results show that reasonable agreement with the data can be obtained with a modified version of the standard wall-layer mixing-length form of $\nu_{T}$ (see Figure 7-12).

The eddy viscosity below the reduced shear region is of an expected form and size. Below depths of $400 \mathrm{~cm}$ the modelled eddy viscosity increases linearly from the surface. Standard estimates of eddy viscosity for our prevailing conditions yield value of $\nu_{T} \approx 100 \mathrm{~cm}^{2} / \mathrm{s}$ (using the estimation form of Leibovich and Radhakrishnan (1977) gives $\nu_{T}=45 \mathrm{~cm}^{2} / \mathrm{s}$, the form of Ichiye $(1967)$ gives $\nu_{T}=204 \mathrm{~cm}^{2} / \mathrm{s}$ ). This modelling approach leads to slightly larger values, at $8 m$ depth we estimate that $\nu_{T}$ is about $400 \mathrm{~cm}^{2} / \mathrm{s}$.

The striking feature of the model is the extremely large eddy viscosity needed in the top $4 m$ of the ocean. Doubtlessly, with greater experimentation, better agreement between the modelled and measured velocity profiles could have been obtained. Two features would 

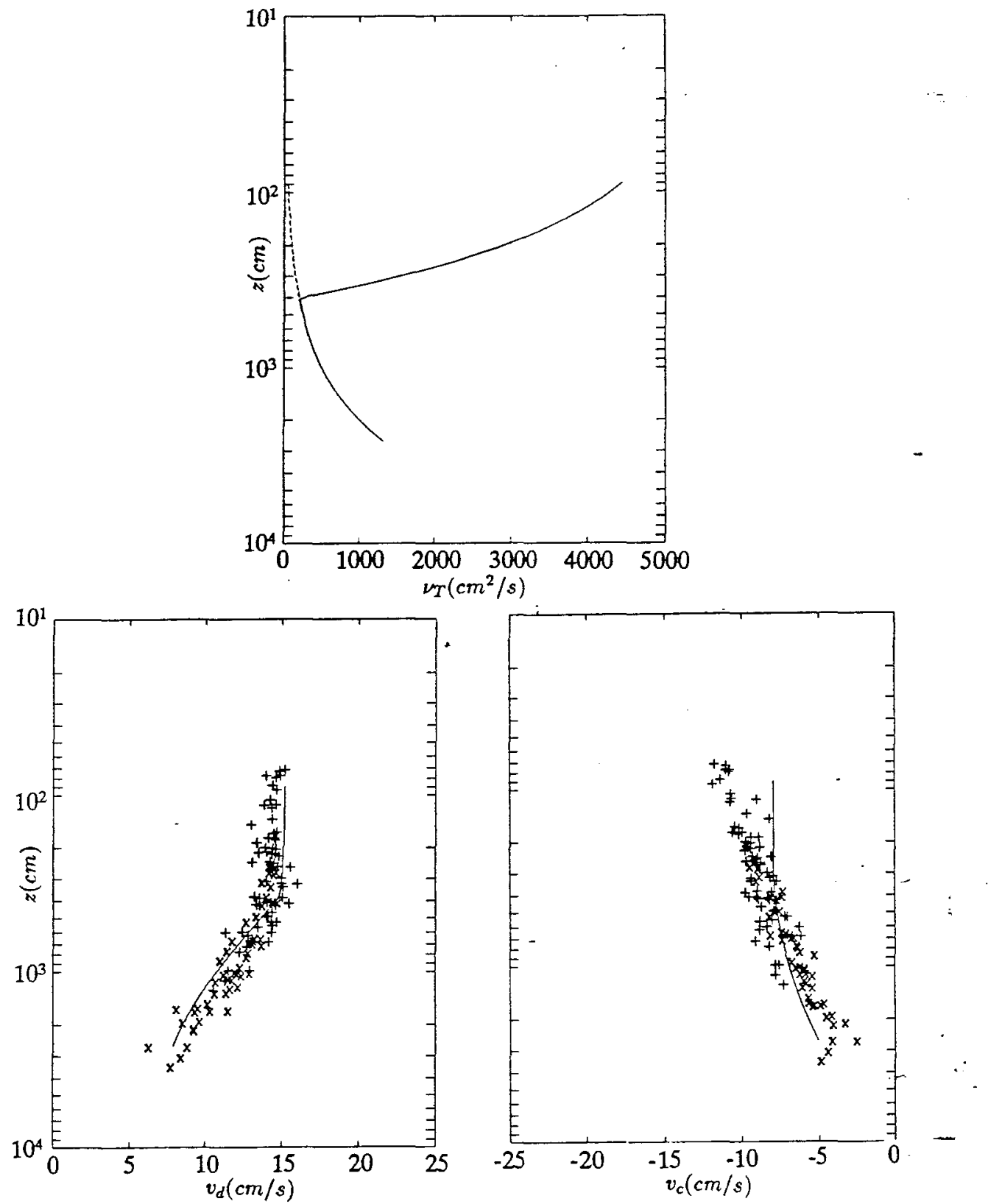

Figure 7-12: Eddy viscosity model for wave mixing hypothesis. The assumed eddy viscosity is shown at the top as a solid line, the dashed line is an eddy viscosity which increases linearly from the surface. At the bottom left is the implied current profile for the downwind direction when $u_{*}=1.6 \mathrm{~cm} / \mathrm{s}$. The SASS $(+)$ and $\mathrm{C} 3(\mathrm{x})$ data have been rescaled to physical dimensions assuming that $u_{*}=1.6 \mathrm{~cm} / \mathrm{s}$ and are superposed on our modelled curve for comparison purposes. The plot on the lower right shows the corresponding result for the crosswind direction. 
have had to, however, remain the same. First, an extremely large eddy viscosity is necessary near the surface to remove the near-surface shear in the downwind direction. Second, the transition to a much lower eddy viscosity must be relatively rapid or the sheared regions below $4 m$ in both the downwind and crosswind directions would be poorly modelled.

As is obvious from Figure 7-12 the highly anisotropic nature of the measured nearsurface profile is not entirely captured by this eddy viscosity approach. But, considering the extreme simplicity of the approach taken, the agreement is not too bad.

Often, when examining the near-surface shear current, the nonlinear terms in the momentum equation are ignored. Figure $7-7$ is highly suggestive that some coupling with a vertical velocity component may be taking place. Including the nonlinear transport terms, the momentum equations may be written as

$$
\begin{aligned}
-f v_{c}+v_{d} \frac{\partial v_{d}}{\partial x}+v_{c} \frac{\partial v_{d}}{\partial y}+w \frac{\partial v_{d}}{\partial z} & =-\frac{1}{\rho} \frac{\partial p}{\partial x}+\frac{\partial}{\partial z}\left(\tau_{x z}\right), \\
f v_{d}+v_{d} \frac{\partial v_{c}}{\partial x}+v_{c} \frac{\partial v_{c}}{\partial y}+w \frac{\partial v_{c}}{\partial z} & =-\frac{1}{\rho} \frac{\partial p}{\partial y}+\frac{\partial}{\partial z}\left(\tau_{y z}\right) .
\end{aligned}
$$

We do not possess nearly enough information to be able to estimate the nonlinear terms. The only things we can estimate are the mean downwind and crosswind current profiles $v_{d}$ and $v_{c}$ and the mean vertical velocity $w$. We can not estimate the time varying contribution to any of the correlations on the left hand side of the above equation nor do we have any way to estimte the horizontal gradients. Also, recall that our observations of vertical velocity must be a consequence of some nonuniform sampling of Langmuir cells, the spatially averaged mean vertical velocity should be equal to zero. These difficulties preclude anything but the simplest of estimates from being made. Faced with a similar sparsity of data other investigators (Gordon, 1970 and Pollard, 1977) have at least tried to estimate if the vertical velocity structure of a Langmuir cell would be important to the momentum transport. In Figure $7-8$ we tried to estimate, from our measurements and from what previous investigators have measured, what the spatial variation of the vertical velocity looked like. It is widely believed that the horizontal velocities in the convergence zone are greater than those in the upwelling zone of the Langmuir cell. Pollard and Gordon 
both assumed that the perturbation to the downstream horizontal velocity was of the same form but opposite sign as the vertical velocity profile. If we make this assumption we find that the downward stress supported by the downwind and vertical velocity correlation is $\tau=\rho \overline{v_{d} w} \approx-8 d y n e s / \mathrm{cm}^{2}$. This is greater than the applied wind stress of our typical conditions $T=-\rho u_{*}^{2} \approx-3$ dynes $/ \mathrm{cm}^{2}$ (we assume the typical $u_{*}=1.6 \mathrm{~cm} / \mathrm{s}$ ). From our measuremenrs we can't hope to find the proper balance of all the transport terms but clearly, if Langmuir cells of the strength hypothesized were present, they could have been the dominant mechanism for momentum transport from the near-surface to the deeper layers of fluid.

\subsection{Conclusions}

The wave bias is an important issue when analyzing measurements from a sensor moving through a wavefield. This effect was considered when interpreting the SMILE data. Due to the unknown but predictable response of the $\mathrm{C} 3$ buoy, we were able to estimate the bias correction for the VMCM measurements. The lower bound correction was applied for purposes of data interpretation. This means that the shears estimated in the discussion were upper bounds. Even in these bounding values, almost no shear is found in the downwind direction in the upper $4 m$.

As an explanation of the low levels of downwind shear near the surface, the mechanism of wave mixing cannot be entirely rejected. But, since the evidence from previous observations of dissipation rates could be used either to support or reject this hypothesis, it is also difficult to wholeheartedly accept this hypothesis. Observed mean vertical velocities provide strong evidence that Langmuir circulation was consistently present when measurements were made. As Gordon(1970) and Pollard(1977) have both pointed out, when Langmuir circulation is present, it is quite possible for this mechanism to transport most of the momentum from the atmosphere to the deeper layers of fluid. During the SMILE experiments, it seems quite probable that the primary mechanism for the downward flux of momentum from the atmosphere to the depths greater than $10 \mathrm{~m}$ is Langmuir circulation. 
The fact that Langmuir circulation may not be ubiquitous may explain why the observations here are not consistent with those of previous investigators. Another explanation would be that some investigators have ignored the biasing effects of the waves. Admittedly, the SASS should not have measured a net vertical velocity unless it was nonuniformly sampling the Langmuir cells. As was discussed, unravelling the details of the Langmuir circulation from the time series is probably not possible. The general feature of the transition to a reduced shear region can not, however, be discounted on this basis. The same feature is observed from the VMCM current meters mounted from the tautly moored C3 buoy. 


\section{Appendix A}

\section{$\mu$-FILTER INTEGRALS}

Recalling the forms for the $\mu$ factors that we derived as filters to remove the bias we see that it is necessary to perform many integrals of the form

$$
\int_{\theta} \cos ^{i} \theta \sin ^{j} \theta f(\sigma, \theta) d \theta
$$

where $i$ and $j$ are integers. Recall that $f(\sigma, \theta)$ are two-pole representations of the spreading. If we use residue calculus to compute the above integrals then we avoid doing the numerical integration at each frequency and instead only have to do a simple algebraic computation. This not only reduces the number of computations necessary but also gives exact answers for the integrals.

If, in the integrals above, we make the substitution (see Hildebrand, 1976, p. 583) that

$$
z=e^{i \theta}, \quad d z=i e^{i \theta} d \theta
$$

then

$$
d \theta=\frac{d z}{i z}, \quad \sin \theta=\frac{z^{2}-1}{2 i z}, \quad \cos \theta=\frac{z^{2}+1}{2 z} .
$$

Once this substitution has been made we can evaluate all the integrals using the-formula for a integral on a closed contour. Denoting the residue of $F(z)$ at $z=p_{i}$ by $\operatorname{Res}\left\{F(z) ; p_{i}\right\}$. And, if the function $F(z)$ has a poles of order $m$ at $z=p_{i}$, then

$$
\oint_{C} F(z) d z=2 \pi \imath \sum_{i} \operatorname{Res}\left(F(z), p_{i}\right)
$$


with

$$
\operatorname{Res}\left(p_{i}\right)=\frac{1}{(m-1) !}\left[\frac{d^{m-1}}{d z^{m-1}}\left\{\left(z-p_{i}\right)^{m} F(z)\right\}\right]_{z=p_{i}}
$$

where $C$ is a simple closed contour enclosing the $i$ singular points at $z=p_{i}$ and excluding all other singularities of $F(z)$. In our case, $C$ will be a unit circle in the complex $z$-plane.

This principle is illustrated with a simple example. Assume that a pitch and roll type measurement has been made and that the correlation coefficients measured are:

$$
c_{0}=\frac{1}{2 \pi}, \quad c_{1}=\frac{1}{4 \pi}, \quad c_{2}=\frac{1}{8 \pi}
$$

From equation 4.39 we have that the MEM-parameters are:

$$
a_{1}=-\frac{1}{2}, \quad a_{2}=0, \quad \vartheta^{2}=\frac{3}{8 \pi}
$$

First, the normalization of the parameters will be checked by performing the integral $\int_{\theta} f(\sigma, \theta) d \theta=1$. The spreading function described in equation 4.39 is transformed into a complex integral using equation A.3.

$$
\int_{\theta} f(\sigma, \theta) d \theta=\oint \frac{-\imath z \vartheta^{2} d z}{\left(z^{2}+a_{1} z+a_{2}\right)\left(1+a_{1}^{*} z+a_{2}^{*} z^{2}\right)}
$$

This implies that

$$
F(z)=\frac{-i z \vartheta^{2}}{\left(z-p_{1}\right)\left(z-p_{2}\right)\left(1+a_{1}^{*} z+a_{2}^{*} z^{2}\right)}
$$

where $p_{1}$ and $p_{2}$ are the two poles of the spreading function inside the unit circle and, of course, can be found from the quadratic equation as

$$
p_{1,2}=-\frac{a_{1}}{2} \pm \sqrt{\left(\frac{a_{1}}{2}\right)^{2}-a_{2}} \Rightarrow p_{1}=0, \quad p_{2}=\frac{1}{4}
$$

The integral can then be evaluated as an algebraic expression as

$$
\begin{aligned}
\oint f(\sigma, \theta) d \theta & =2 \pi \imath \sum_{i} \operatorname{Res}\left(F(z), p_{i}\right) \\
& =2 \pi \imath\left[\left.\frac{-\imath z \vartheta^{2}}{\left(z-p_{2}\right)\left(1+a_{1}^{*} z+a_{2}^{*} z^{2}\right)}\right|_{z=0}+\left.\frac{-\imath z \vartheta^{2}}{\left(z-p_{1}\right)\left(1+a_{1}^{*} z+a_{2}^{*} z^{2}\right)}\right|_{z=\frac{1}{4}} ^{:}\right]=1 .
\end{aligned}
$$


Any of the expressions needed to be evaluated to determine the $\mu$ 's can be determined in this way. For instance,

$$
\begin{aligned}
\oint \cos \theta f(\sigma, \theta) d \theta & =\oint \frac{-\imath\left(z^{2}+1\right) \vartheta^{2} d z}{2\left(z^{2}+a_{1} z+a_{2}\right)\left(1+a_{1}^{*} z+a_{2}^{*} z^{2}\right)} \\
& \Rightarrow F(z)=\frac{-\imath\left(z^{2}+1\right) \vartheta^{2}}{2\left(z^{2}+a_{1} z+a_{2}\right)\left(1+a_{1}^{*} z+a_{2}^{*} z^{2}\right)}
\end{aligned}
$$

and so,

$$
\oint \cos \theta f(\sigma, \theta) d \theta=2 \pi \imath \sum_{i} \operatorname{Res}\left(F(z), p_{i}\right)=\frac{5}{8}
$$

I add one word of caution. When taking integrals where the combined powers of $\sin$ and $\cos$ is two or greater (i.e. $i+j \geq 2$ in equation A.1) there will be a singularity of order $i+j-1$ at $z=0$. This poses no great difficulty so long as one remembers to add in the extra terms into the sum of residues! 


\section{Appendix B}

\section{PARAMETERIZATION OF}

\section{DRAG}

The parameterization of the drag force in tęrms of the free stream velocity is not entirely obvious. To show that this notion makes sense I will derive this model from first principles for a very simple two-dimensional case. The situation we'll study is shown in Figure B-1. The flow upstream of the cylinder, $V_{o}$, is assumed to be uniform and constant. The free stream is assumed to be free of pressure gradients. Downstream, the velocity is given by $v(y)$. The distance $y_{0}$ is that distance outside which the flow is not retarded on the downstream control surface (i.e. from $y_{0}$ to $y_{0}+\delta$ the flow speed is $V_{0}$ ). The upstream control volume half-width is chosen to be $y_{0}$. The upper and lower control volume surfaces are streamlines.

The centerline is a stream line so we can balance the mass flux by considering just the part of the control volume above $y=0$. The flux in at the upstream boundary must balance the flux out at the downstream boundary,

$$
\int_{0}^{y_{0}} \rho V_{0} d y=\int_{0}^{y_{0}+\delta} \rho v(y) d y=\int_{0}^{y_{0}} \rho v(y) d y+\int_{y_{0}}^{y_{0}+\delta} \rho V_{o} d y
$$

where the expression for the downstream flux has been broken into two parts. Note that we've employed the fact that $v(y)=V_{o}$ outside $y_{0}$. Since $V_{o}$ is constant, the offset in the bounds of the integral from $y_{0}$ to $y_{o}+\delta$ may be removed. If we also combine the integrals 


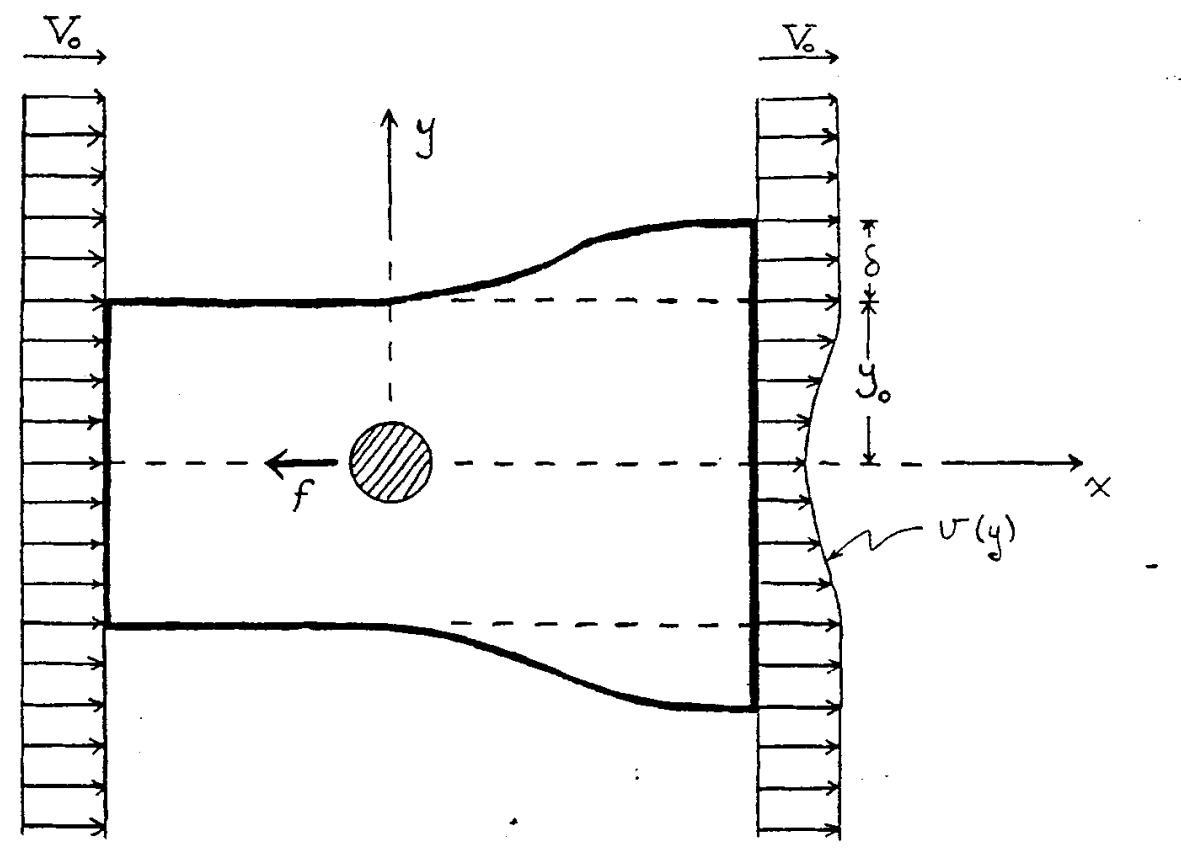

Figure B-1: A two dimensional steady flow field. The flow is obstructed by a cylinder. The cylinder applies a force per unit depth $f$ on the fluid. The heavy boundary shows the control volume.

from 0 to $y_{0}$ and multiply through the equations by the constant, $V_{o}$, we find

$$
\rho \int_{0}^{y_{0}} V_{0}\left\{V_{o}-v(y)\right\} d y=\rho \int_{0}^{\delta} V_{o}^{2} d y
$$

The momentum balance per unit depth for the $x$-direction is

$$
-f=-\int_{-y_{0}}^{y_{0}} \rho V_{o}^{2} d y+\int_{-y_{0}-\delta}^{y_{0}+\delta} \rho v(y)^{2} d y .
$$

Again, the downstream integral is broken up and simplified where possible to obtain

$$
f=\rho \int_{-y_{0}}^{y_{0}}\left\{V_{o}^{2}-v(y)^{2}\right\} d y-2 \rho \int_{0}^{\delta} v(y)^{2} d y
$$

We eliminate the integral from 0 to $\delta$ by substituting in equation B.2

$$
f=\rho \int_{-y_{0}}^{y_{0}}\left\{V_{o}^{2}-v(y)^{2}\right\} d y-\rho \int_{-y_{0}}^{y_{0}} V_{0}\left\{V_{0}-v(y)\right\} d y .
$$


Now, if we define the deficit velocity as

$$
v_{d}(y) \equiv V_{o}-v(y)
$$

and assume that $v_{d}(y) \ll V_{o}$, then upon eliminating $v(y)$ from equation $B .5$ we find that

$$
f=\rho V_{o} \int_{-y_{0}}^{y_{0}} v_{d}(y) d y=\rho V_{o} q
$$

if a two-dimensional equivalent sink term is defined as

$$
q \equiv \int_{-y_{0}}^{y_{0}} v_{d}(y) d y
$$

These results are two-dimensional equivalents to the equations we used in chapter 5 section 5 . 


\section{Appendix $\mathrm{C}$}

\section{BASS FLOW DISTURBANCE}

The BASS current meter has been previously calibrated by Trivett et al (1991). That calibration addressed many of the issues of interest for typical BASS deployments but does not totally satisfy our requirements for the SASS. Specifically, in the wave environment of the SASS we want to know whether oscillations crosswise to the mean flow will (a) reduce the measured mean flow and (b) will induce a spurious mean to the vertical velocity.

The calibrations here were performed at the Woods Hole Oceanographic Institution tow tank. The tests were accomplished by towing the current meter through just over $10 \mathrm{~m}$ of still water. Two type of tests were performed to examine these issues. First, the BASS cage was towed through the water at a fixed speed and known elevation angle (the elevation angle is defined in Figure C-1). This determines the steady calibration for the BASS as a function of elevation angle. Next, the velocimeter was towed and oscillated crosswise to the travel direction. This was done to determine the validity of the error estimation techniques used in the thesis and to see if the measurements of mean vertical velocity could have been caused by a flow disturbance. The apparatus for these tests is shown in Figure C-2.

\section{the static calibration}

The scale factor for this relatively simple test could not be as precisely tested as in the more sophisticated calibration by Trivett et al (1991). In that calibration it was found that the BASS undermeasured the velocity at zero elevation angle by $4 \%$. Here, the scale factor was 


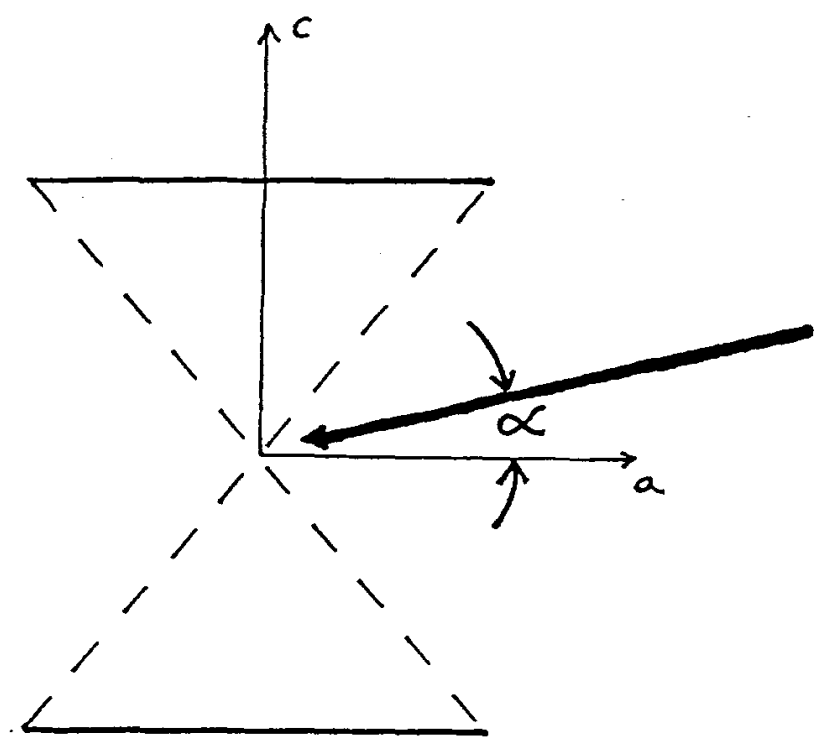

Figure C-1: The definition of the elevation angle, $\alpha$, of the BASS sensor.

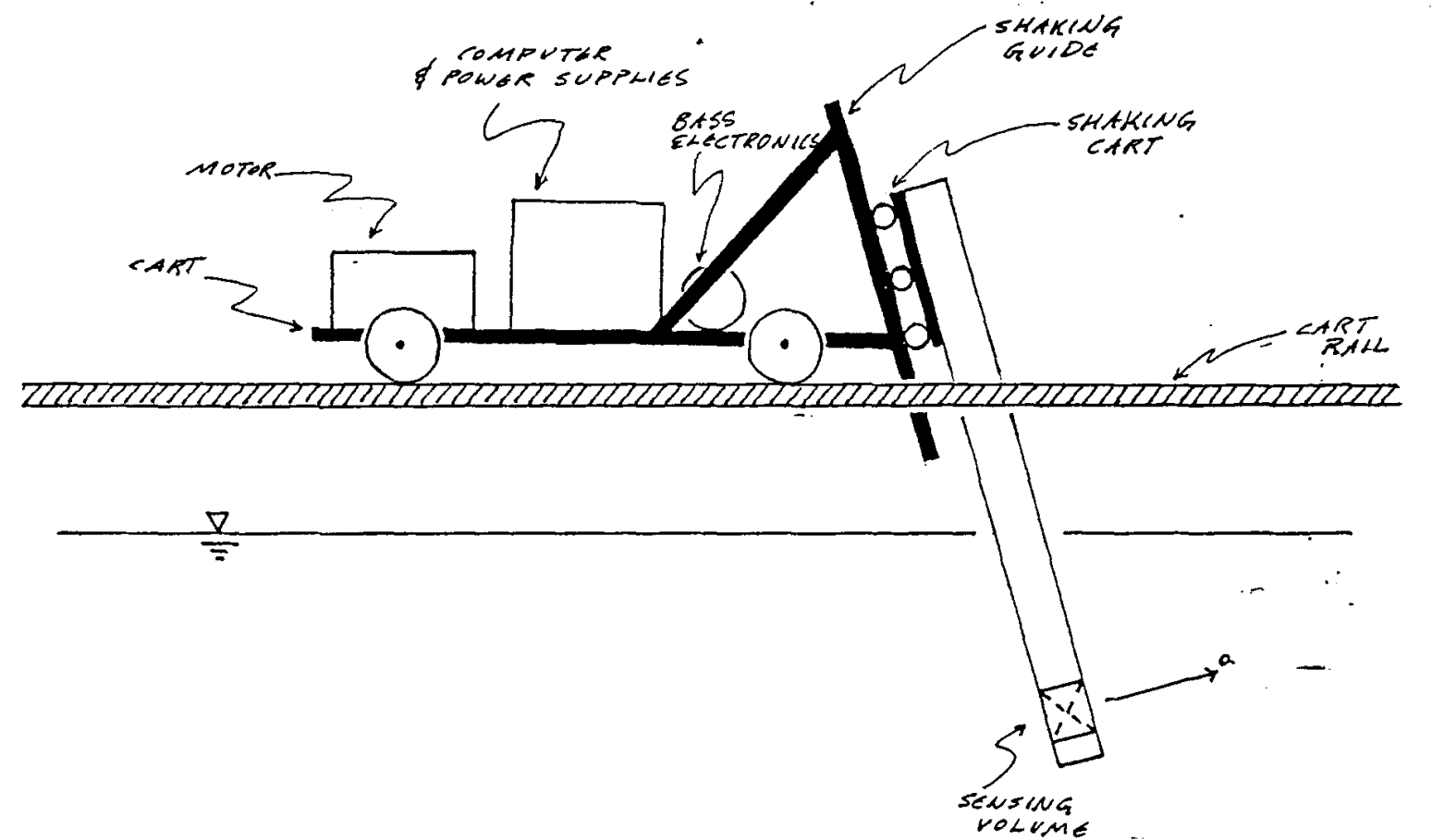

Figure C-2: The test apparatus. The BASS sensing volume was driven through $10 \mathrm{~m}$ of still water to obtain a calibration. The "shaking guide" could be tipped to varying extents to test the sensor at various elevation angles (previous figure). For the static calibration the "shaking cart" was clamped to the guide. For the dynamic tests the "shaking cart" was unclamped and oscillated manually along the guide while the cart was being driven 


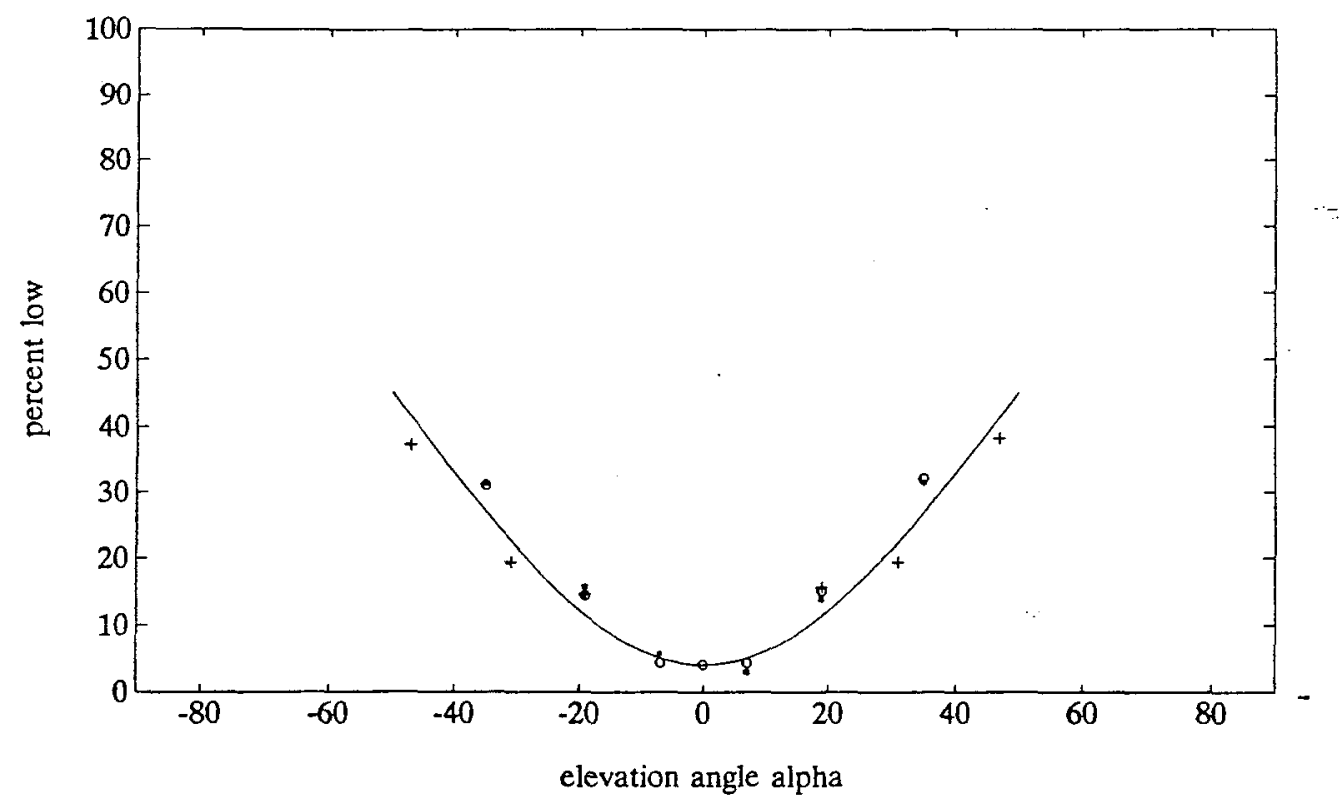

Figure C-3: The static calibration results. The different symbols indicate the various ranges of tow speeds used in the calibration; $*<15 \mathrm{~cm} / \mathrm{s}, 15 \mathrm{~cm} / \mathrm{s}<o<25 \mathrm{~cm} / \mathrm{s},+>25 \mathrm{~cm} / \mathrm{s}$. The solid line indicates the qualitatively fit curve discussed in the text.

found by forcing the results to underread by $4 \%$ at zero elevation angle. With the apparatus constructed, the elevation angle could only be varied out to about $47^{\circ}$. The results of the static calibration are shown in Figure C-3. An expression which seems to describe the static calibration results quite well is

$$
\text { percent low }=39-35 \cos 2 \alpha \text {. }
$$

As will be discussed later, these steady calibration results are of little help in interpreting the SASS data.

\section{the dynamic calibration}

For the dynamic calibration, the BASS sensor was shook up-and-down on the guide tracks as nearly sinusoidally as possible. The tracks allowed non-negligible oscillations in line with the direction of motion as well. The test was done at both zero elevation angle and with a slight tip. Because it experienced the greatest relative velocities, and measured the largest vertical means, the tests were made to conform as much as possible to the field conditions 
experienced by the lowest sensor. It was felt that the salient scaling parameter was the ratio of the rms relative velocity to the mean velocity. For the tests, this ratio varied from about 1.20 to 2.15. Among the field data (lowest sensor), the mean and standard deviation of this ratio were 1.83 and 0.43 . There was no field data where the ratio was lower than that tested in the lab and there were only five files where the ratio was higher (the field data point furthest outside the test range had a ratio of $r m s$ to mean velocity only $33 \%$ higher than the upper limit of our test range). The relative excursion of a fluid particle relative to a sensor was a difficult parameter to match in the lab. This is because in the field, the total orbital excursion was probably on order of $60 \mathrm{~cm}$. For the lab results, due to limitations on the shaking mechanism and the size of the tow tank, the excursion was probably just about half that of the field. Of course, since the rms value of the relative velocity (and therefore orbital excursion) decreases by a factor of 1.5 to 2 when looking at the top instead of bottom sensor, we see that in excursion, the lab results replicated the values at the top sensor quite well. This test did not identically replicate the field conditions but it should uncovered any gross defects in measurement capability had they existed (none were found).

The raw output of the current meter tended to indicate mean flows about ten to fifteen percent lower than those indicated by the cart speed. After processing using exactly the . same procedure that was used for the field data, the disagreement was reduced to five percent or less. The results are summarized in Figure C-4. The improvement of the processed data over the raw data is mostly (about two-thirds) due to making estimates with the best three ("unshaded") axes as opposed to all four axes. The remainder of the improvement comes from the momentum defect model presented in the thesis. The processing in the calibration results shown is exactly the same as that applied to the SASS data.

An important point made in the thesis was that applying a static calibration as a function of elevation angle to the unsteady data would be theoretically incorrect. In fact, if the calibration represented by equation C.1 is inverted and applied to the data the cart speed is over-estimated by 47 to 87 percent. The conclusion is that the steady calibration results are not applicable to the unsteady data. Further evidence of this fact is given in Appendix 


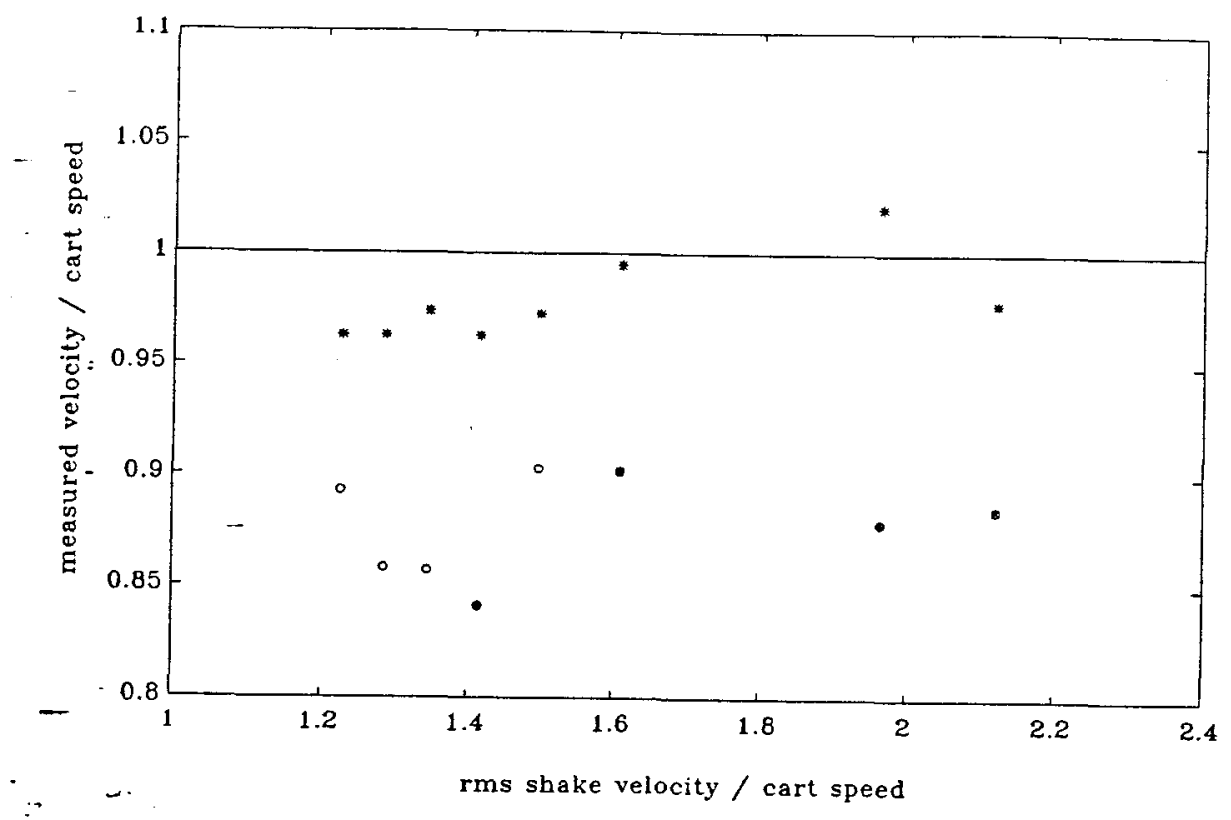

Figure C-4: The dynamic calibration results. Absolute tow speeds were between $10 \mathrm{~cm} / \mathrm{s}$ and $20 \mathrm{~cm} / \mathrm{s}$. The open circles represent data taken with the BASS at zero mean tilt. Circles with x's are at mean tilts of $\alpha=-7^{\circ}$ and circles with + 's are at mean tilts of $\alpha=+7^{\circ}$.

$\mathrm{D}$, where- the wave velocities are discussed.

Besides evaluating the momentum defect model, another major goal of this calibration was to see if oscillations of the current meter transverse to the mean flow would cause there to be a spurious mean velocity in the transverse (vertical) direction. The mean vertical velocity of all eight dynamic calibration runs was $-0.2 \mathrm{~cm} / \mathrm{s}$. The largest mean for any run was $-0.5 \mathrm{~cm} / \mathrm{s}$. Velocities of this size could easily be explained in terms of zero offset errors any and slight misalignments of the current meter. The BASS does not seem to create any spurious-velocities in the vertical direction.

\section{errors in the predicted tilt of the BASS tower}

Misalignment of the current meter or errors in pitch and roll measurements could not have lead to observations of $2.5 \mathrm{~cm} / \mathrm{s}$ downward velocities discussed in Chapter 7 . Suppose there was a stabilization error which was perfectly correlated with the waves. Let $u_{V}$ be the measured vertical velocity and $\left[u_{I}, u_{K}\right]$ be the true components of horizontal and vertical 
velocity. Then, in terms of the error in angle measurement $\phi$,

$$
u_{V}=-u_{I} \sin \phi+u_{K} \cos \phi
$$

If the true vertical mean were zero, the erroneously measured vertical mean would be (using a small angle approximation on $\phi$ )

$$
\bar{u}_{V} \approx-\overline{u_{I} \phi}
$$

The absolute largest relative horizontal velocities measured by the SASS had a standard deviation of $38 \mathrm{~cm} / \mathrm{s}$. In such a case, generating an erroneous mean of $-2.5 \mathrm{~cm} / \mathrm{s}$ would require a -perfectly correlated error in angular measurement of $5.3^{\circ}$. Such an error is absolutely implausible. Furthermore, because the BASS sensors were in a rigid array any error in angular orientation would be the same for each sensor. This means that the error would scale precisely as the $r m s$ relative velocities. The relative $r m s$ velocities varied by a factor of 1.5 to 2 from top to bottom. The measured vertical velocities varied typically by a factor of five: So, neither the form nor the size of the measured vertical velocity is consistent with a mismeasurement of angular orientation. It appears most likely then that the observation of mean vertical velocities discussed in Chapter 7 are geophysical in origin.

\section{SASS-C3 agreement}

Throughout Chapters 6 and 7 the SASS and the VMCM's mounted on the C3 buoy were compared. Several reasons for disagreements between the two sets of sensors were given in Chapter 5 and file-by-file comparisons were shown in Chapter 6 but, the results have not yet been shown in summarized form. This is done in Figure C-5. The unbiased SASS mean velocity interpolated at $4 m$ depth is compared to the midrange estimate of the unbiased $4 m$ VMCM mean velocity estimate. The errors do not seem at all correlated with the relative velocity but do seem to show some correlation with the mean velocity. The in situ scale factor of the BASS was checked by increasing or decreasing the scale factor of the BASS to see if there would be an improvement of the predicted wave orbital measurements (using a scale factor either higher or lower than that actually used leads to worse results of SASS 


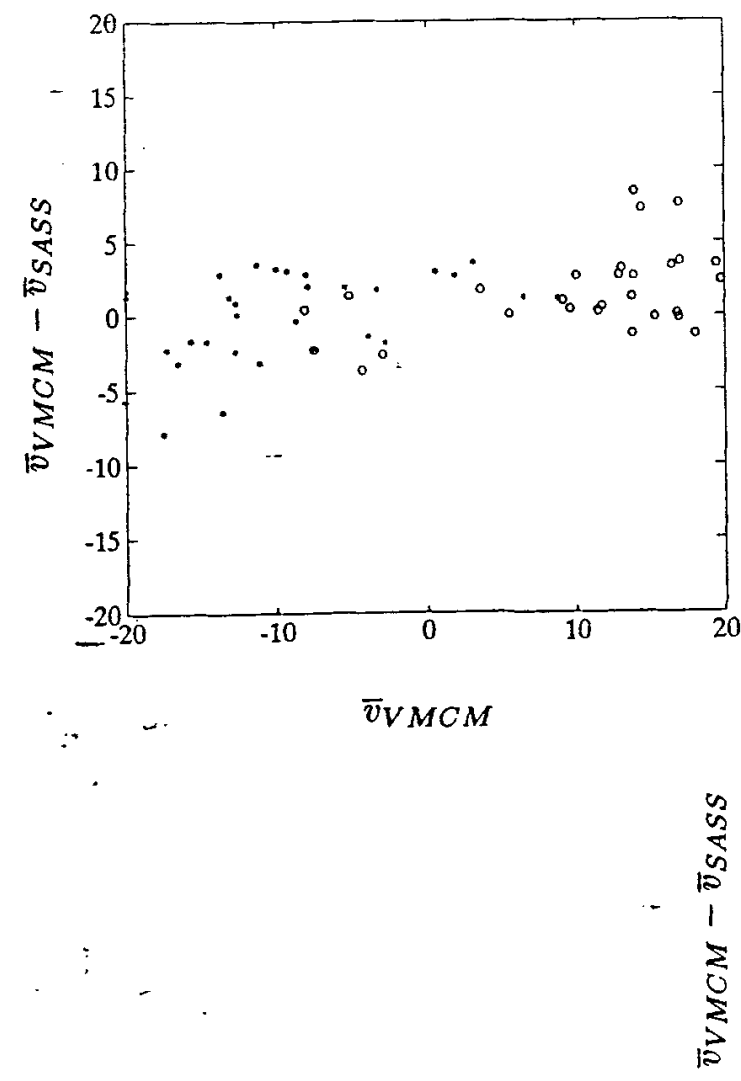

(a)

(b)

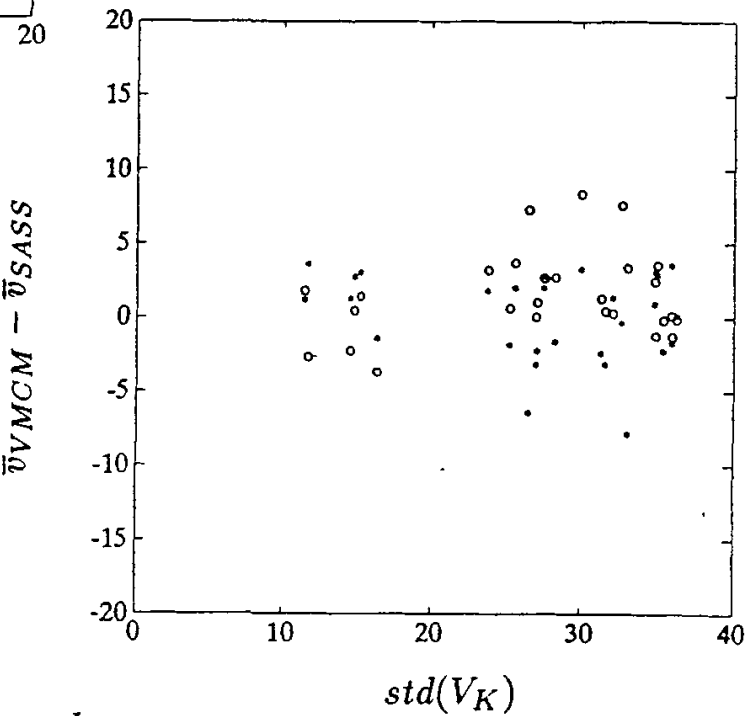

Figure C-5: The relative error in measurement of the mean velocity between the SASS and the best estimate of the VMCM velocity at $4 m$ depth. (a) Shows the error in the downwave(o) and crosswave( $x)$ mean velocity versus the mean velocity measured at the $4 \mathrm{~m}$ VMCM (files where the $4 m$ VMCM were not working could not be included). (b) Again using the same symbols, the downwave and crosswave error are plotted versus the standard deviation of vertical velocity at the lowest SASS sensor $(5.85 \mathrm{~m})$ 
wave measurements).

\section{anomalous files}

As we discussed in Chapters 5 and 6 , the were a whole host of reasons why the two instruments would not read exactly the same mean. A more distressing factor is the presence of what were referred to in Chapter 7 of "anomalous files". The files I have considered anomalous would be the following: $c 1, d 1, e 1, n 1$ and $n 2$ (also to a lesser extent $m 1$ and $m 2$ ). These files are not characterized by unusually bad agreement in the overall mean but rather, have extremely bad agreement in comparison of shear at the bottom of the SASS array and the top VMCM measurements. It seems to be an extreme low reading on the BASS sensor which is causing the mismatch (and indicating far too much shear at the bottom of the SASS array). It is perhaps significant that all the anomalous can be lumped into two consecutive time intervals (although there is a large unsampled time interval between the two $m$ files

- and the $n$ files). Files $n 1$ and $n 2$ were taken during a rather dramatic windspeed jump. The unsteady conditions may have some bearing on the results but, files $c 1$ to $e 1$, which are the most anomalous files, occurred during a relatively constant wind interval. There does not seem to be any consistent parameter which sets apart the anomalous files from the ones which are not considered anomalous. It may be that some unmeasured parameter took on extreme values during these files (e.g. there may have been unusually energetic yaw motion in the resonant null of the compass or, in the realm of the more mundane, a piece of seaweed may have caught of the bottom BASS sensor). In any case, I have been unable to determine what external parameters indicate the few anomalous files that do exist (the infrequent occurrence of these files is one factor why I have, perhaps, been unable to isolate them statistically). Having the VMCM and the SASS as cross checks on each other seems to me to be a positive rather than a negative aspect of the experiment. As such, when the cross check showed that some unknown and unobserved aspect of the experiment (recall that the buoys were left unattended) led to results that were geophysically implausible, I felt no compunction in excluding those data from the analysis. 


\section{Appendix D}

\section{WAVE DECAY}

The measurement of wave orbital velocities provides, to the extent that linearized wave theory holds, an in situ dynamic calibration of the BASS sensors. This idea has already been touched upon in the body of the thesis. Recall that in Figure 5-16 it was shown that in file: $f$ the bottom sensor experienced much larger relative velocities than the shallower sensors (especially in the frequency range of $0.3 \mathrm{~Hz}$ ). Once the sensor velocities and the relative velocities were combined to form the absolute fluid velocities, however, we found that the orbital velocities decayed with depth. This is what we would expect. In this appendix I will show that not only do the orbital velocities decay with depth, but they do so in a fashion well described by linearized potential theory. Because our measurement of the absolute velocity is a combination of the sensor and relative velocities, the decay of the orbital motions could not be correctly predicted if the calibration of either the BASS or the motion sensing package were seriously in error.

If the directional wave spectrum is known then it is possible to predict the velocity spectrum at any depth. Let the directional wave spectrum $F(\sigma, \theta)$ be expressed as a product of the waveheight spectrum $S_{\eta \eta}(\sigma)$ and a directional spreading function $f(\sigma, \theta)$,

$$
F(\sigma, \theta)=S_{\eta \eta}(\sigma) f(\sigma, \theta)
$$

The downwave, crosswave and vertical velocity spectra can the expressed at any arbitrary 
depth $z$ as

$$
\begin{aligned}
S_{v_{d w} v_{d w}}(\sigma, z) & =\sigma^{2} e^{2 k z} S_{\eta \eta}(\sigma) \int \cos ^{2} \theta f(\sigma, \theta) d \theta \\
S_{v_{c \boldsymbol{w}} v_{c w}}(\sigma, z) & =\sigma^{2} e^{2 k z} S_{\eta \eta}(\sigma) \int \sin ^{2} \theta f(\sigma, \theta) d \theta \\
S_{v_{K} v_{K}}(\sigma, z) & =\sigma^{2} e^{2 k z} S_{\eta \eta}(\sigma),
\end{aligned}
$$

respectively (where $\theta=0$ is assumed to correspond to the downwave direction). Likewise, if the velocity spectrum is measured at any depth $z_{m}$ it is possible to predict the velocity spectrum at any other depth $z_{\tau}$ as

$$
\begin{aligned}
S_{v_{d w} v_{d w}}\left(\sigma, z_{r}\right) & =e^{2 k\left(z_{r}-z_{m}\right)} S_{v_{d w} v_{d w}}\left(\sigma, z_{m}\right), \\
S_{v_{c w} v_{c w}}\left(\sigma, z_{r}\right) & =e^{2 k\left(z_{r}-z_{m}\right)} S_{v_{c w} v_{c w}}\left(\sigma, z_{m}\right), \\
S_{v_{K} v_{K}}\left(\sigma, z_{r}\right) & =e^{2 k\left(z_{r}-z_{m}\right)} S_{v_{K} v_{K}}\left(\sigma, z_{m}\right) .
\end{aligned}
$$

Using the preceeding expressions, we can predict the velocity spectrum at some reference . depth $z_{r}$ for each of the BASS sensors. If our measurements are consistent with linearized potential theory, each of the six sensors (or four for Spring 1989 files) should predict the same velocity spectrum at a given reference depth $z_{r}$. As a practical issue it is best to use a $z_{r}$ which is below the depth of the deepest sensor. In this way noise at the high frequencies will be attenuated rather than amplified. The reference depth chosen for comparison is $z_{r}=700 \mathrm{~cm}$. The files examined here will be $61, e 1, f 1,12, p 2, q 1$ and $u 1$. Files $f 1$ and $q 1$ were examined in detail in Chapter 5 . The other files represent a variety of conditions, as is discussed in Appendix E. 

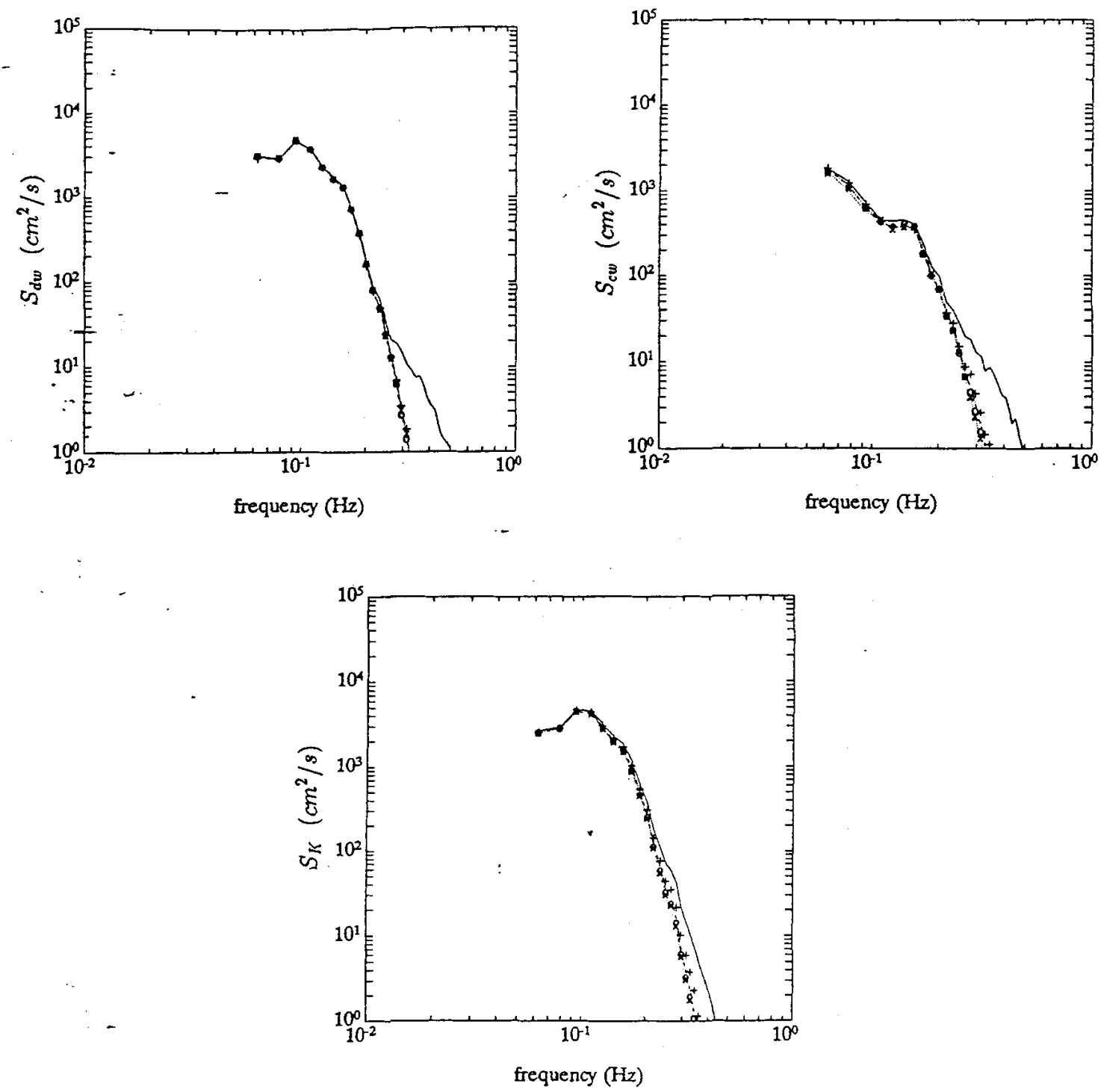

Figure D-1: Predicted wave velocity spectra during file $b 1$ at a nominal reference depth of $700 \mathrm{~cm}$. Clockwise from the upper left we have the spectra for the downwave, crosswave and vertical directions. Estimates are from sensors located at nominal depths of $586 \mathrm{~cm}(-)$, $391 \mathrm{~cm}(+++), 311 \mathrm{~cm}(-\cdot-\cdot-), 251 \mathrm{~cm}(\mathrm{o} \mathrm{o} \mathrm{o}), 166 \mathrm{~cm}(\mathrm{x} \mathrm{x} \mathrm{x})$ and $111 \mathrm{~cm}(\cdots \cdots)$. 

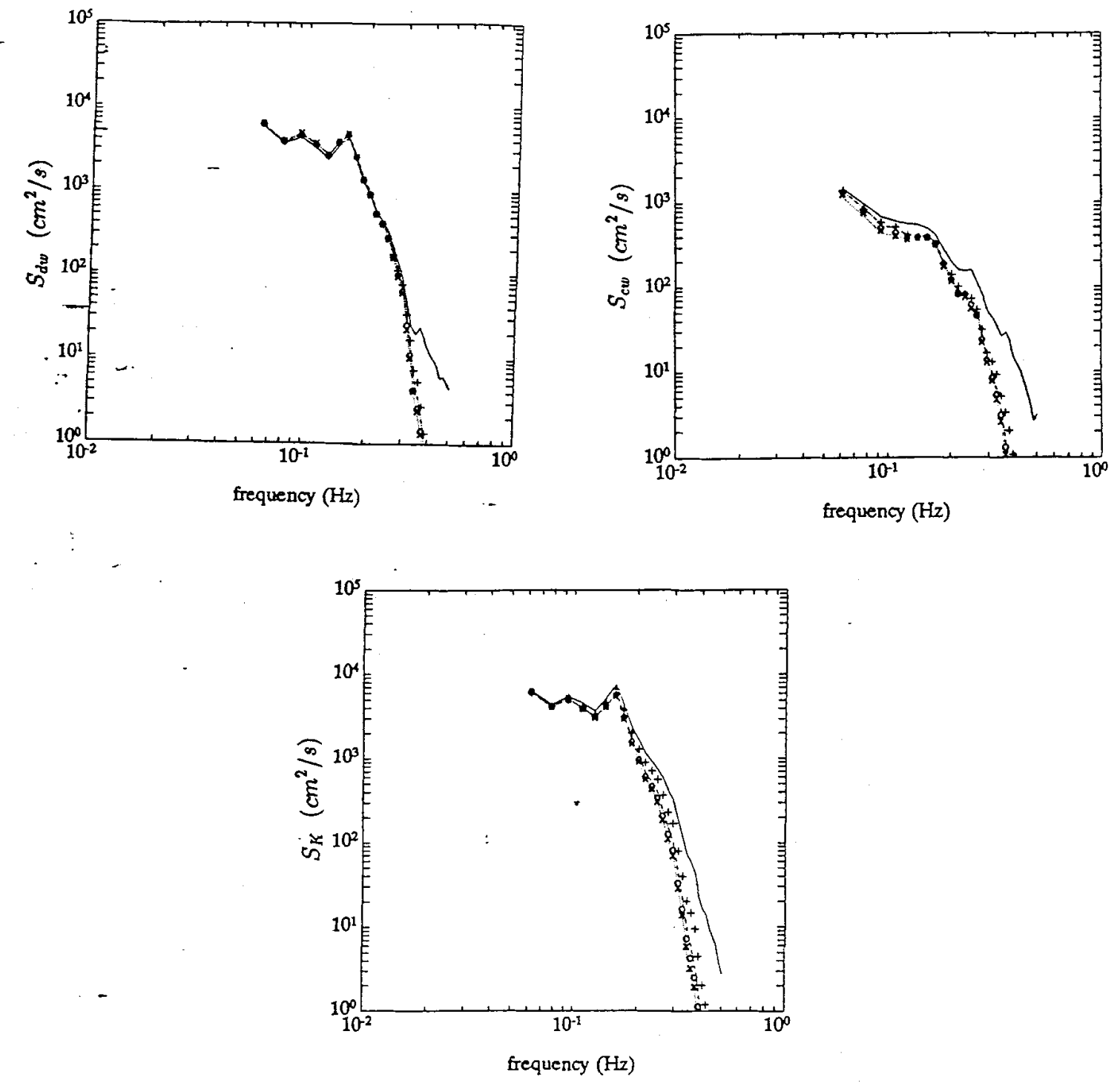

Figure D-2: Predicted wave velocity spectra during file $e 1$ at a nominal reference depth of $700 \mathrm{~cm}$. Clockwise from the upper left we have the spectra for the downwave, crosswave and vertical directions. Estimates are from sensors located at nominal depths of $586 \mathrm{~cm}(-)$, $391 \mathrm{~cm}(+++), 311 \mathrm{~cm}(-\cdot-\cdot), 251 \mathrm{~cm}(\mathrm{o} \mathrm{o} \mathrm{o}), 166 \mathrm{~cm}(\mathrm{x} \times \mathrm{x})$ and $111 \mathrm{~cm}(\cdots \cdots)$. 

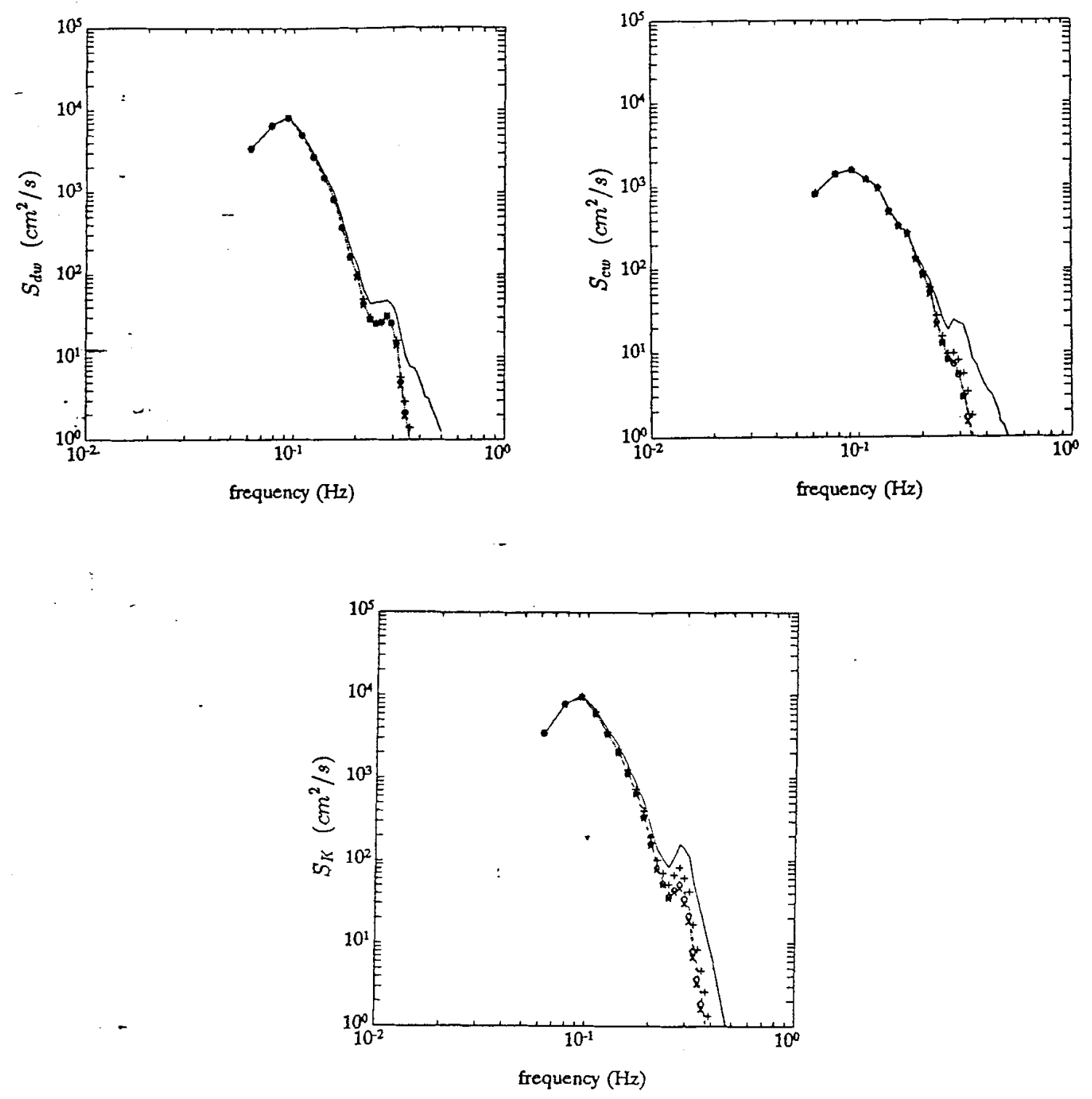

Figure D-3: Predicted wave velocity spectra during file $f 1$ at a nominal reference depth of $700 \mathrm{~cm}$. Clockwise from the upper left we have the spectra for the downwave, crosswave and vertical directions. Estimates are from sensors located at nominal depths of $586 \mathrm{~cm}(-)$, $391 \mathrm{~cm}(+++), 311 \mathrm{~cm}(-\cdot-\cdot-), 251 \mathrm{~cm}$ ( o o o), $166 \mathrm{~cm}(\mathrm{x} \mathrm{x} \mathrm{x})$ and $111 \mathrm{~cm}(\cdots \cdots)$. 

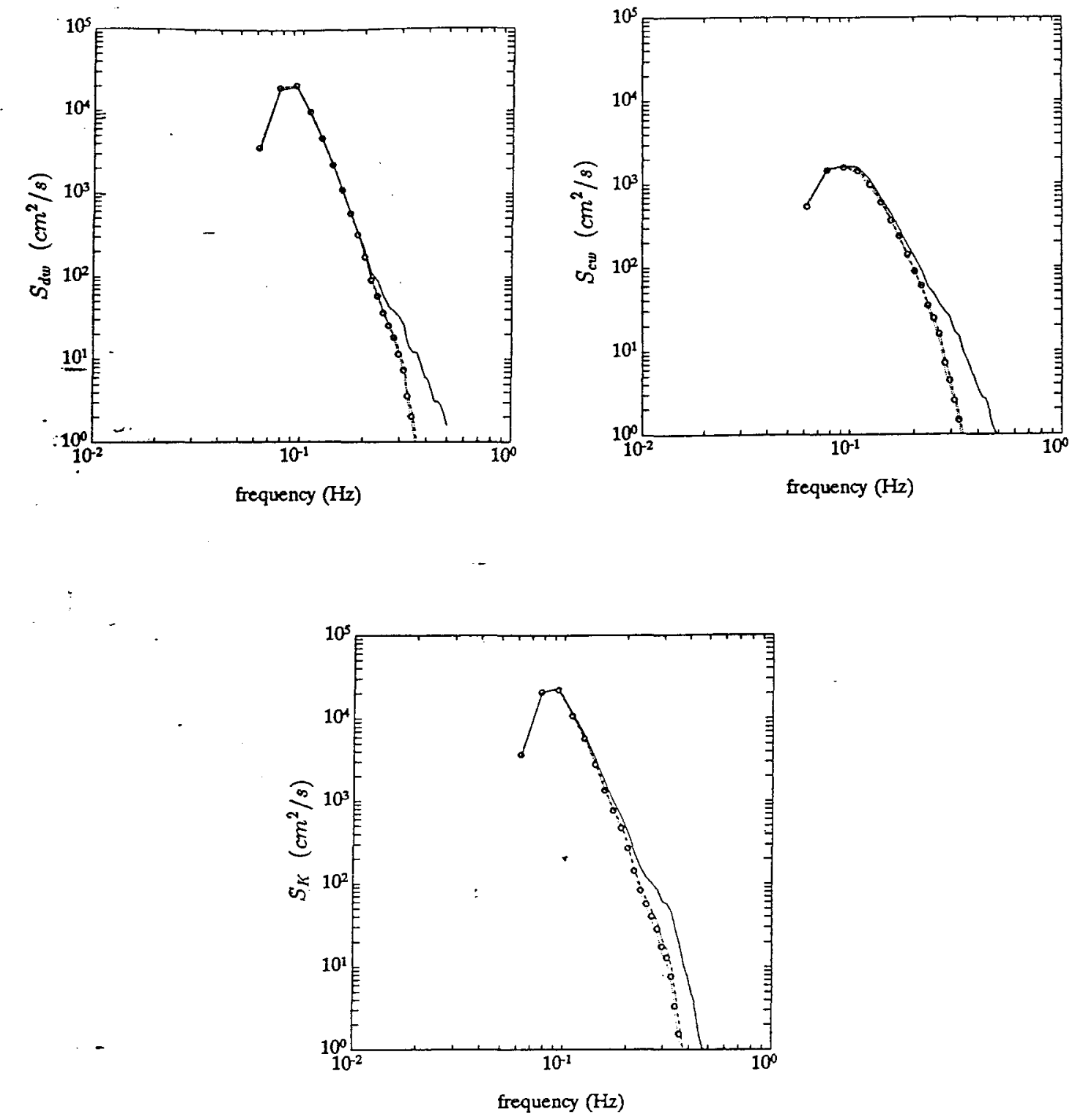

Figure D-4: Predicted wave velocity spectra during file 12 at a nominal reference depth of $700 \mathrm{~cm}$. Clockwise from the upper left we have the spectra for the downwave, crosswave and vertical directions. Estimates are from sensors located at nominal depths of $586 \mathrm{~cm}(-)$, $311 \mathrm{~cm}(-\cdot-\cdot), 251 \mathrm{~cm}(\mathrm{o} o \mathrm{o})$ and $111 \mathrm{~cm}(\cdots \cdots)$. 

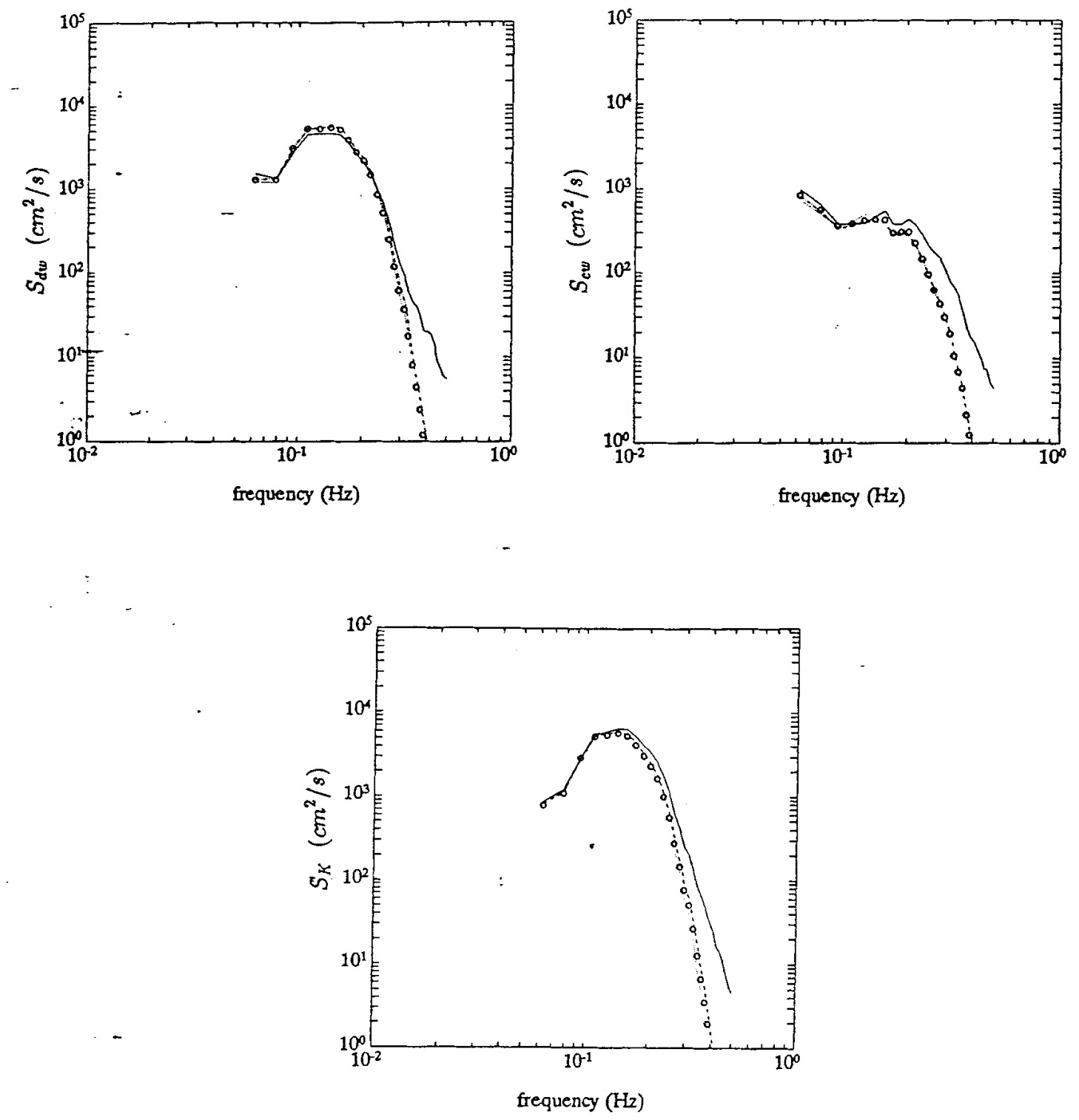

Figure D-5: Predicted wave velocity spectra during file $p 2$ at a nominal reference depth of $700 \mathrm{~cm}$. Clockwise from the upper left we have the spectra for the downwave, crosswave and vertical directions. Estimates are from sensors located at nominal depths of $586 \mathrm{~cm}(-)$, $311 \mathrm{~cm}(-\cdot-\cdot-), 251 \mathrm{~cm}(\mathrm{o} o \mathrm{o})$ and $111 \mathrm{~cm}(\cdots \cdots)$. 

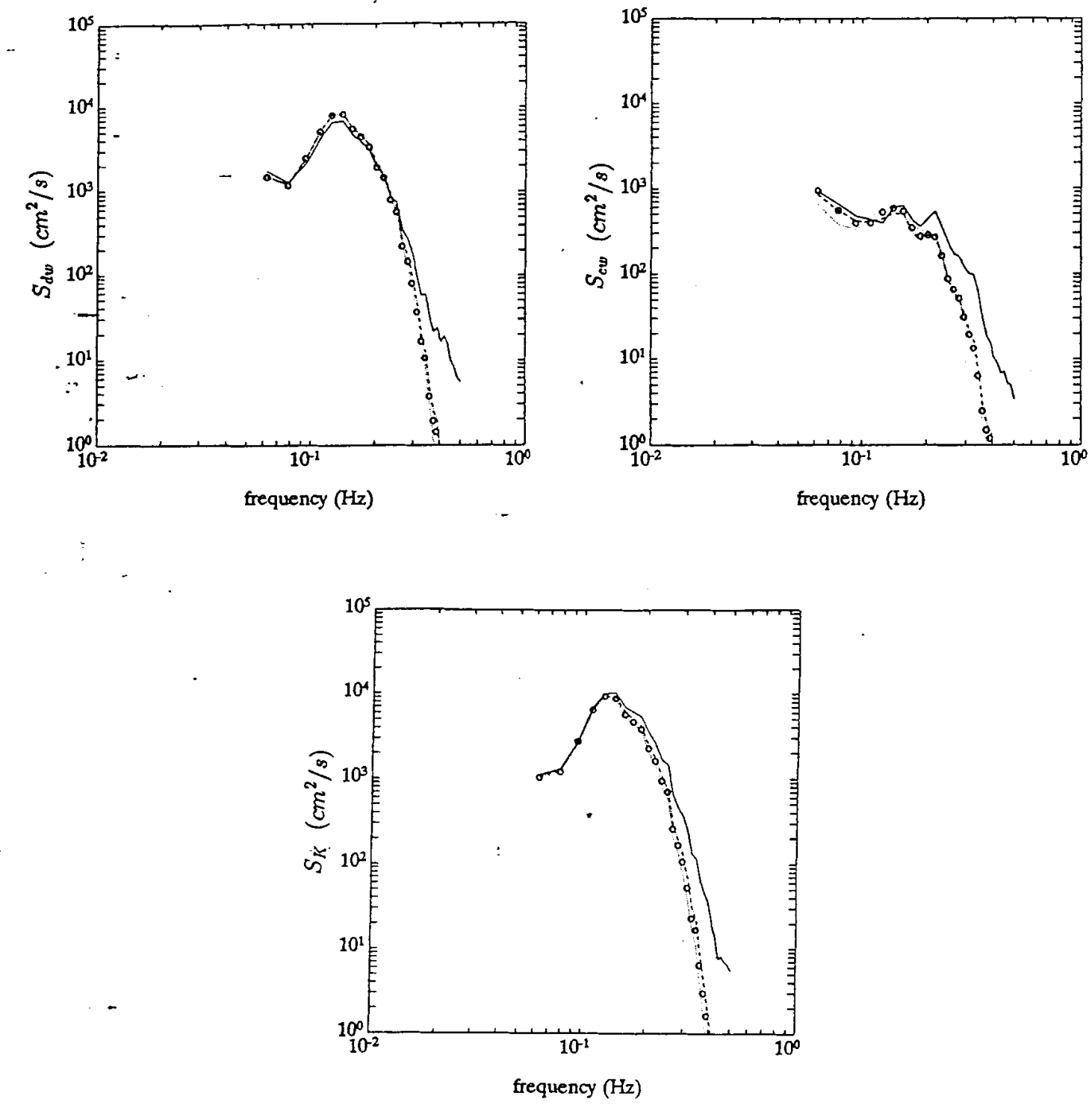

Figure D-6: Predicted wave velocity spectra during file $q 1$ at a nominal reference depth of $700 \mathrm{~cm}$. Clockwise from the upper left we have the spectra for the downwave, crosswave and vertical directions. Estimates are from sensors located at nominal depths of $586 \mathrm{~cm}(-)$, $311 \mathrm{~cm}(-\cdot-\cdot-), 251 \mathrm{~cm}$ (o o o) and $111 \mathrm{~cm}(\cdots \cdots)$. 

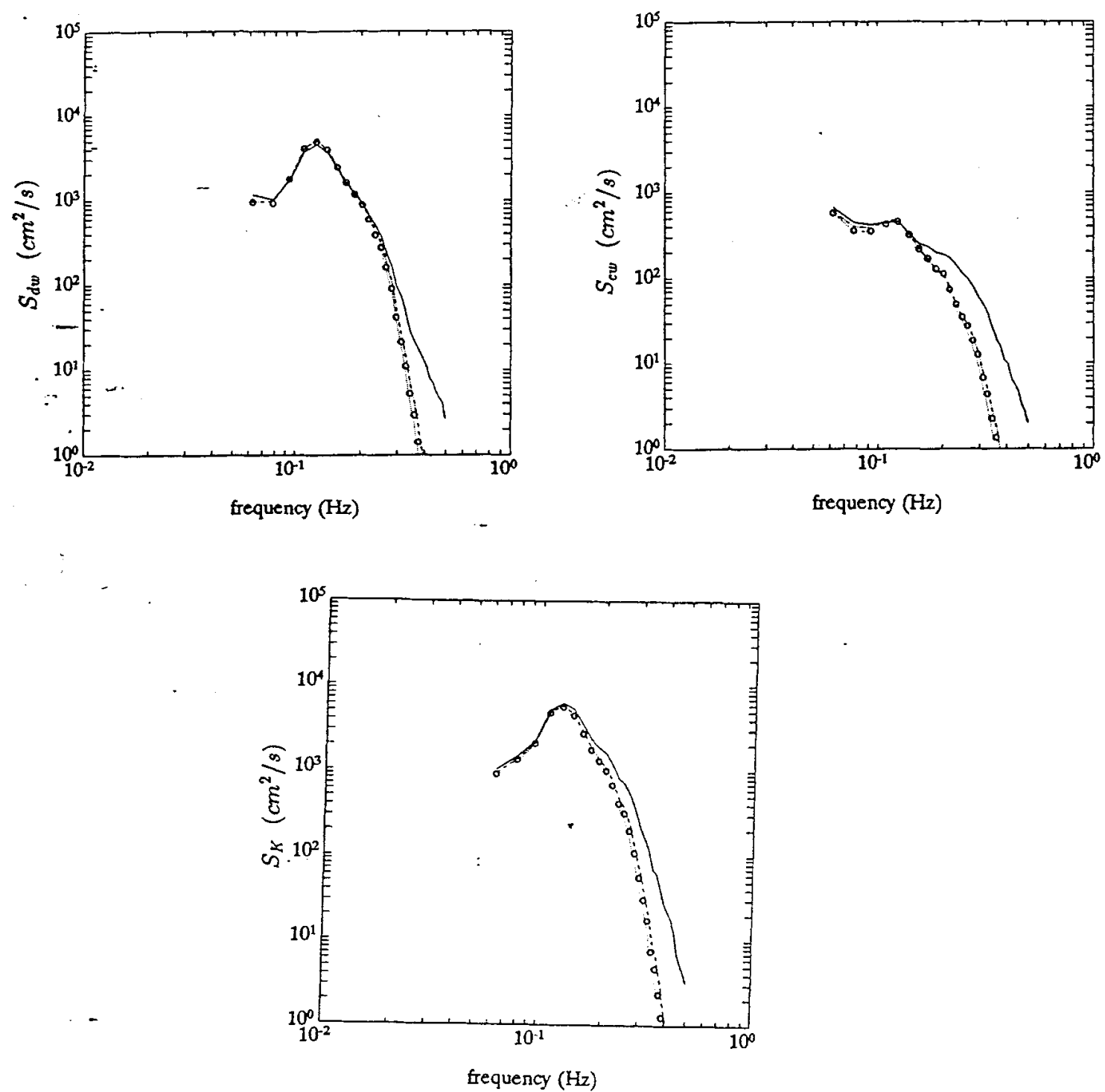

Figure D-7: Predicted wave velocity spectra during file $u 1$ at a nominal reference depth of $700 \mathrm{~cm}$. Clockwise from the upper left we have the spectra for the downwave, crosswave and vertical directions. Estimates are from sensors located at nominal depths of $586 \mathrm{~cm}(-)$, $311 \mathrm{~cm}(-\cdot-\cdot-), 251 \mathrm{~cm}$ (o o o) and $111 \mathrm{~cm}(\cdots \cdots)$. 


\section{Appendix E}

\section{COMPASS SENSITIVITY}

Of all the problems associated with the motion sensing package, the compass response seems to be most troubling. The calibration by Patch et al (1991) showed that there should be a notch in the compass response at about $0.22 \mathrm{~Hz}$. This was not observed. The conditions that the SASS compass experienced in the field were quite different than those applied in the aforementioned calibration. Specifically, the SASS was subjected to linear accelerations as well as twisting. The possible effects of these linear motions as well as the implications of Patch's calibration will be the topic of this appendix.

To determine whether or not linear accelerảtions could affect compass output the compass was placed on a small cart and the cart was oscillated in a straight line. In theory, the output of the compass should have remained constant during these tests. In actuality, it is only at low frequency that this is true. At and above the resonant frequency of the compass the linear shaking does lead to small oscillations in the compass signal. This gives us a very plausible explanation as to why there is no spectral gap in the observed compass spectrum (as would have been expected from Patch's results). The discussion here has been purposefully qualitative. The test described was very crude; its only purpose was to reconcile the observed compass data to the known compass response.

How then is the compass signal to be interpreted? At high frequencies, above $0.60 \mathrm{~Hz}$, the compass signal is dominated by digitization noise. At low frequencies, below $0.125 \mathrm{~Hz}$ 
the compass response should be good and only minimally affected by linear accelerations. In the frequency band near $0.22 \mathrm{~Hz}$ we expect the actual yaw signal to be attenuated by the mechanism described by Patch et al (1991). Furthermore, there may be significant levels of noise present due to linear accelerations of the compass. Attempts to remove the noise from the signal and to invert the attenuated signal would undoubtedly end in failure. If the compass noise is not well correlated with the other signals, its effects may be minimal. Rather than trying to "fix" the compass signal, I will test the sensitivity of the processing scheme to the compass signal.

The compass sensitivity will be tested by comparing the data as processed in the thesis (compass lowpass filters set to remove just the quantitization noise above $0.6 \mathrm{~Hz}$ ) to a version of processing where the cutoff frequency of the compass filters was set to $0.125 \mathrm{~Hz}$. In this way, the noise is for the most part removed (at the cost, perhaps, of also filtering away some of the signal). We expect that if the results are insensitive to this change in processing - that the final results obtained are not seriously affected by the poor compass performance.

Seven files where selected for "alternate processing". The files were selected to represent as wide a range of conditions as possible (see Table E-1). The directional spectra and final velocity profiles (i.e. including bias and flow disturbance corrections) are compared for each file in the plots found on the following pages. For all but the file which I had previously characterized as anomalous (file $e 1$ ) there is virtually no difference which processing scheme was used. This is not surprising. It was observed during the deployment of the SASS that the pitching-and rolling motions of the buoy were more vigorous than the yawing motions. In Chapter 5, under a more beligerent assumption on compass motions, computer simulation results showed that the SASS should be able to obtain resonable estimates of directional wave spectra and mean currents. In general, then, we do not expect that the overall results would be very sensitive to the poor compass response at high (above $0.125 \mathrm{~Hz}$ ) frequencies.

Though the alternate processing actually makes the comparison of file $e 1$ with the VMCM a bit worse (and does nothing to change the strong downwave shear at the bottom 


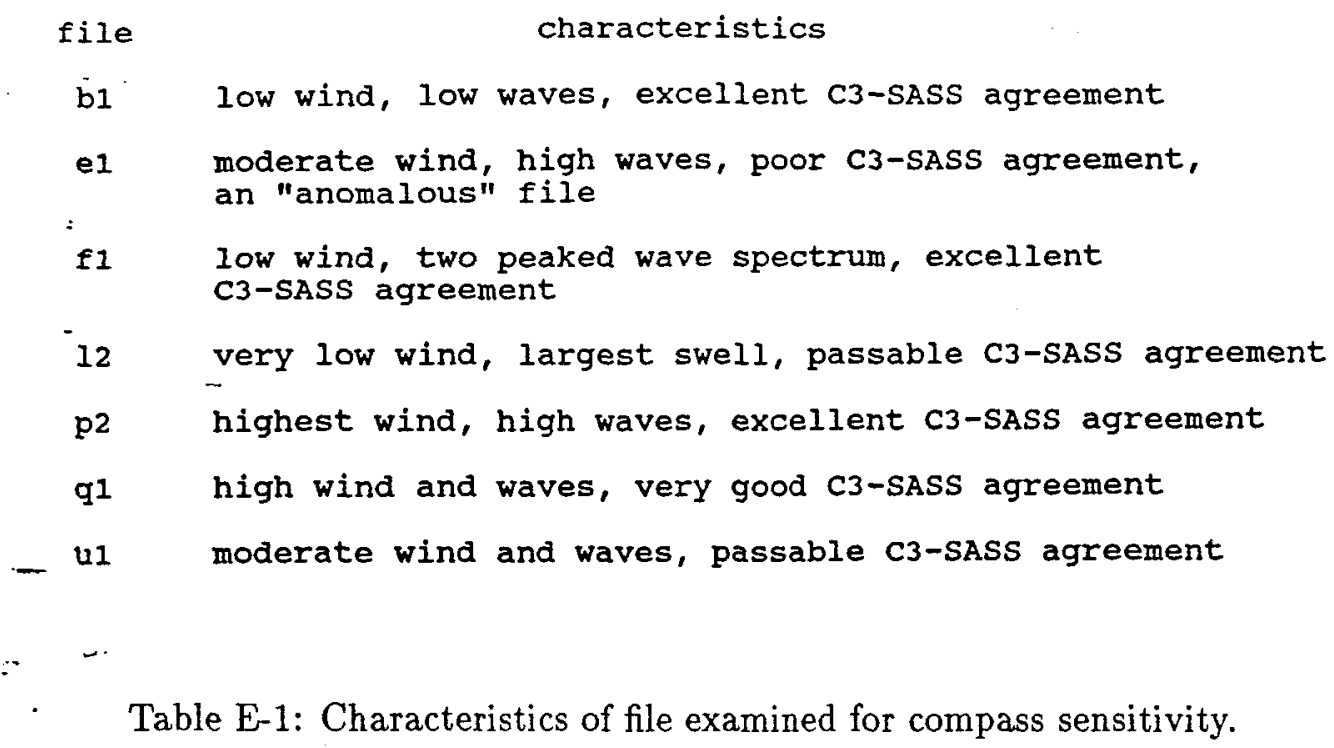

Table E-1: Characteristics of file examined for compass sensitivity.

sensor that earned the file the moniker "anomalous"), perhaps the fact that there is any change at all indicates that the SASS did experience some unusually intense yaw motions during the files where agreement was particularly bad. This was one of the conjectures of Appendix C. . 

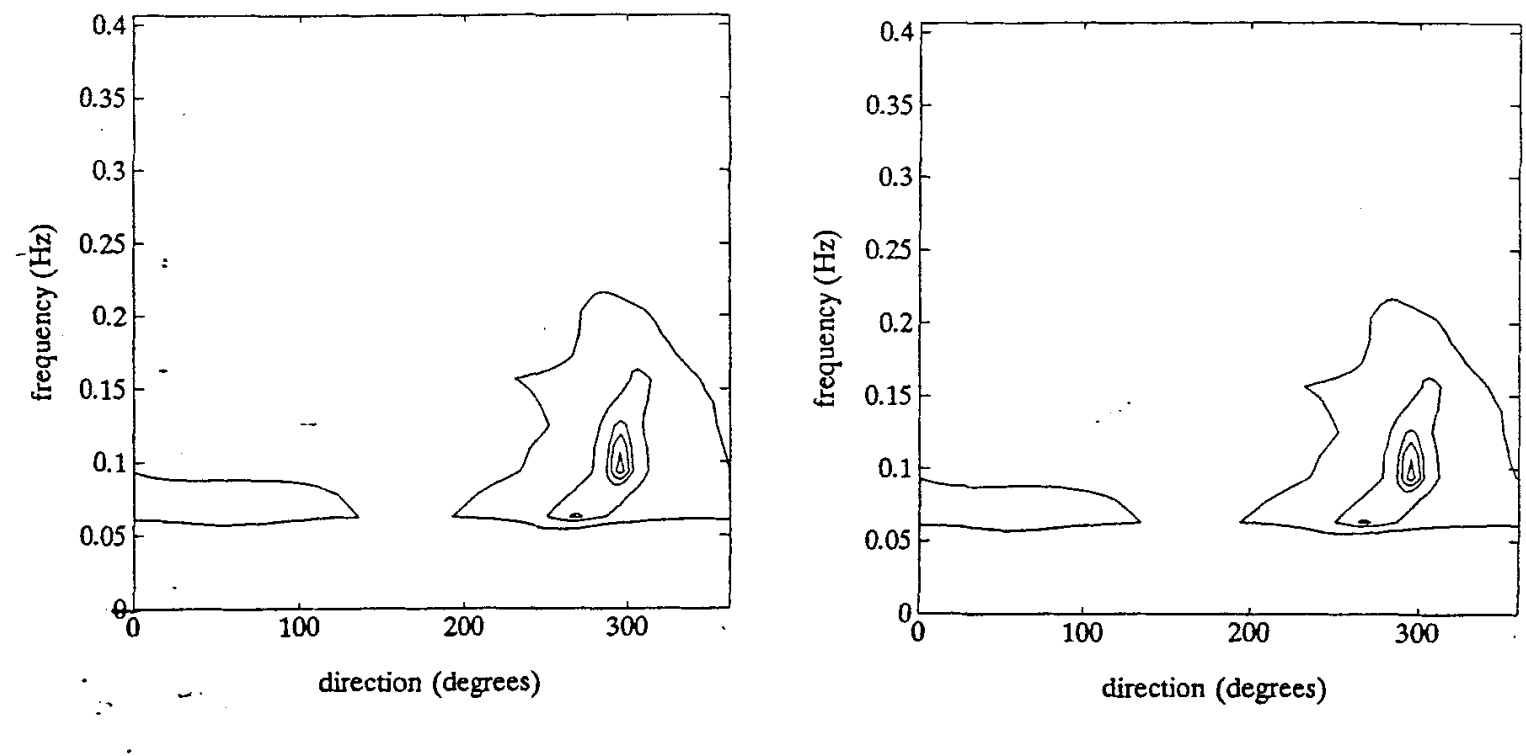

Figure E-1: Comparison of directional spectra for file b1. The plot on the left shows the estimated directional spectra when the compass has been lowpass filtered to $0.6 \mathrm{~Hz}$ (as in the thesis). The plot on the right shows the estimated directional spectra when the compass has been lowpass filtered to $0.125 \mathrm{~Hz}$.
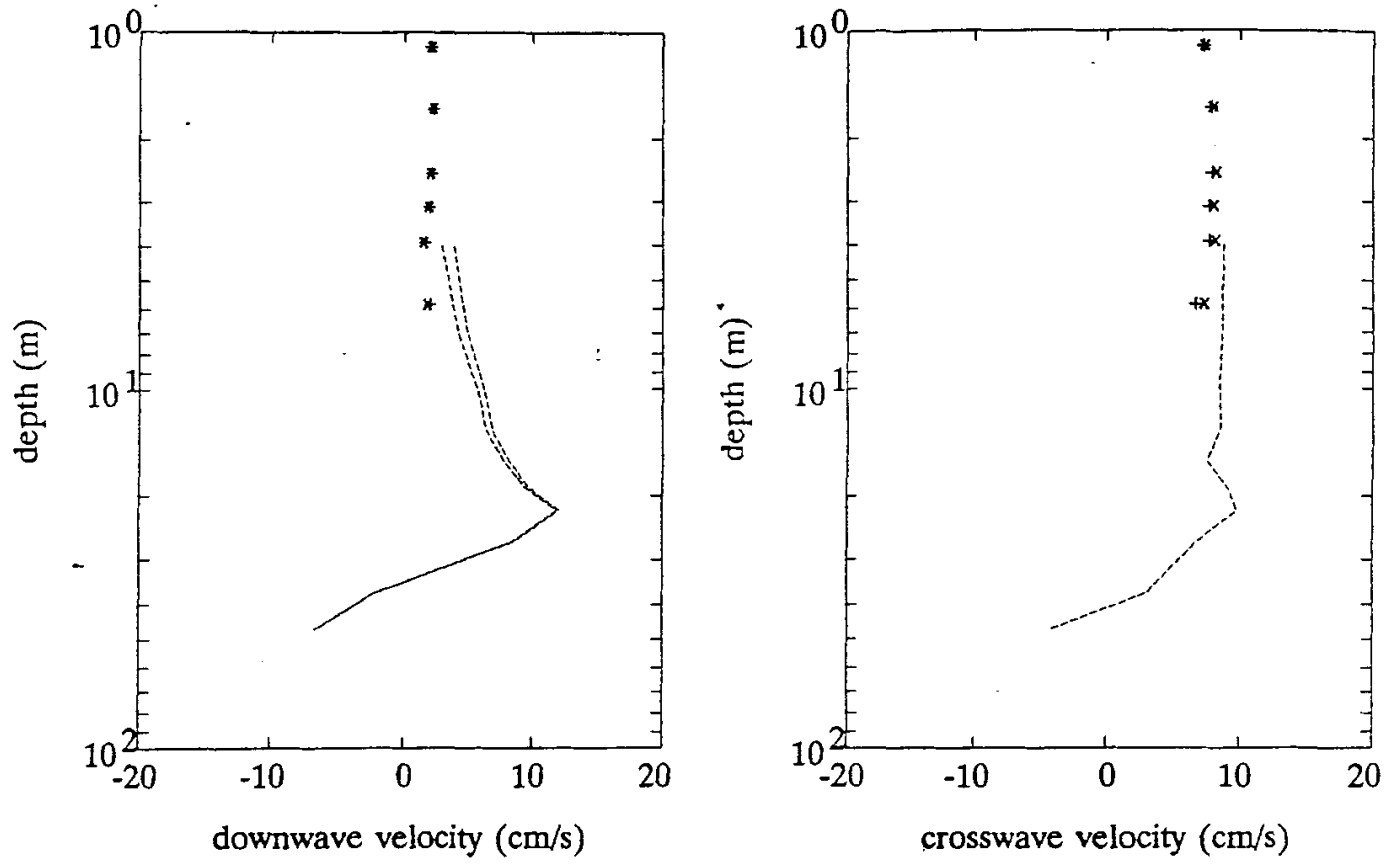

Figure E-2: Comparison of the downwave and crosswave mean velocity profiles for file $b 1$. The +'s represent the normally processed data and the $x$ 's represent the data processed with the compass filtered at $0.125 \mathrm{~Hz}$. The estimates of the unbiased $\mathrm{C} 3$ dashed line. As explained in Chapter 6, a range is estimated for the downwave direction. 

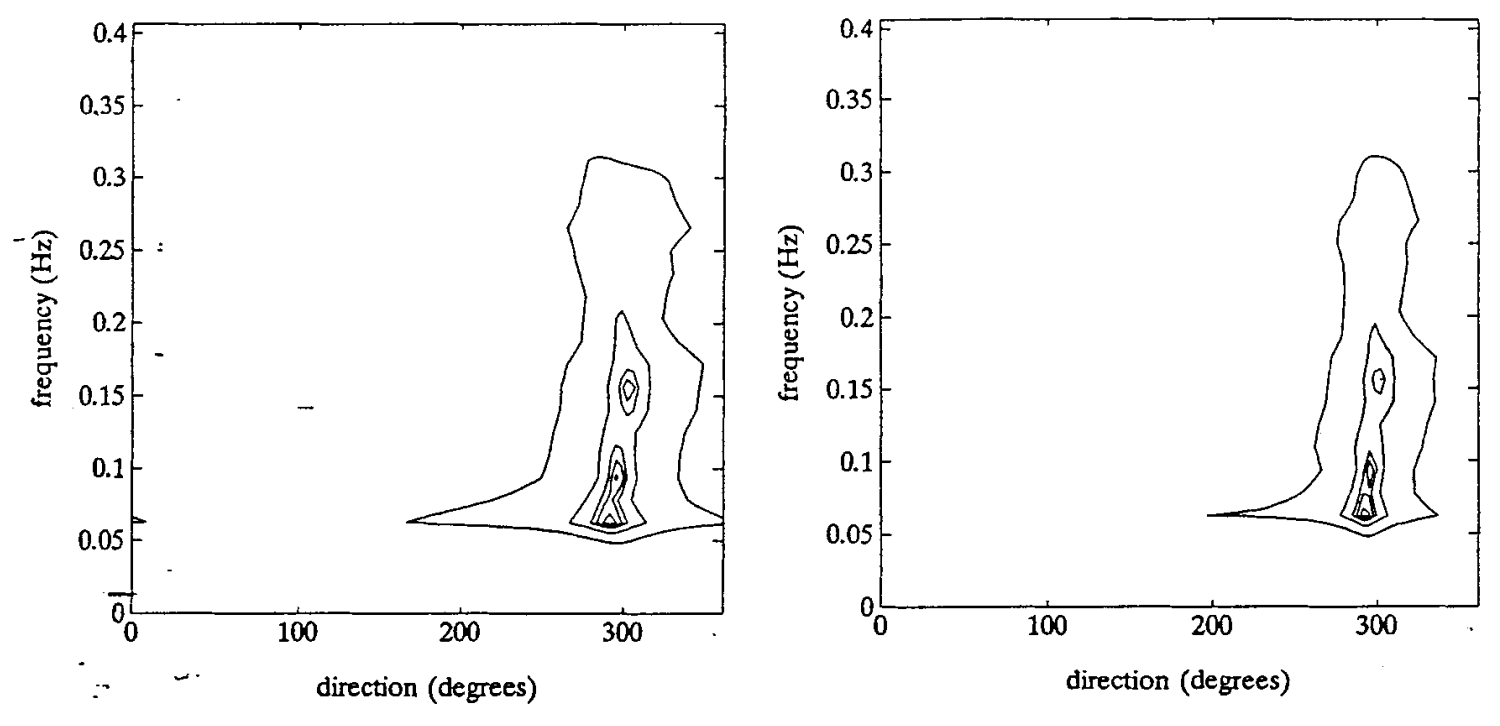

Figure E-3: Comparison of directional spectra for file $e 1$. The plot on the left shows the estimated directional spectra when the compass has been lowpass filtered to $0.6 \mathrm{~Hz}$ (as in the thesis). The plot on the right shows the estimated directional spectra when the compass has been lowpass filtered to $0.125 \mathrm{~Hz}$.
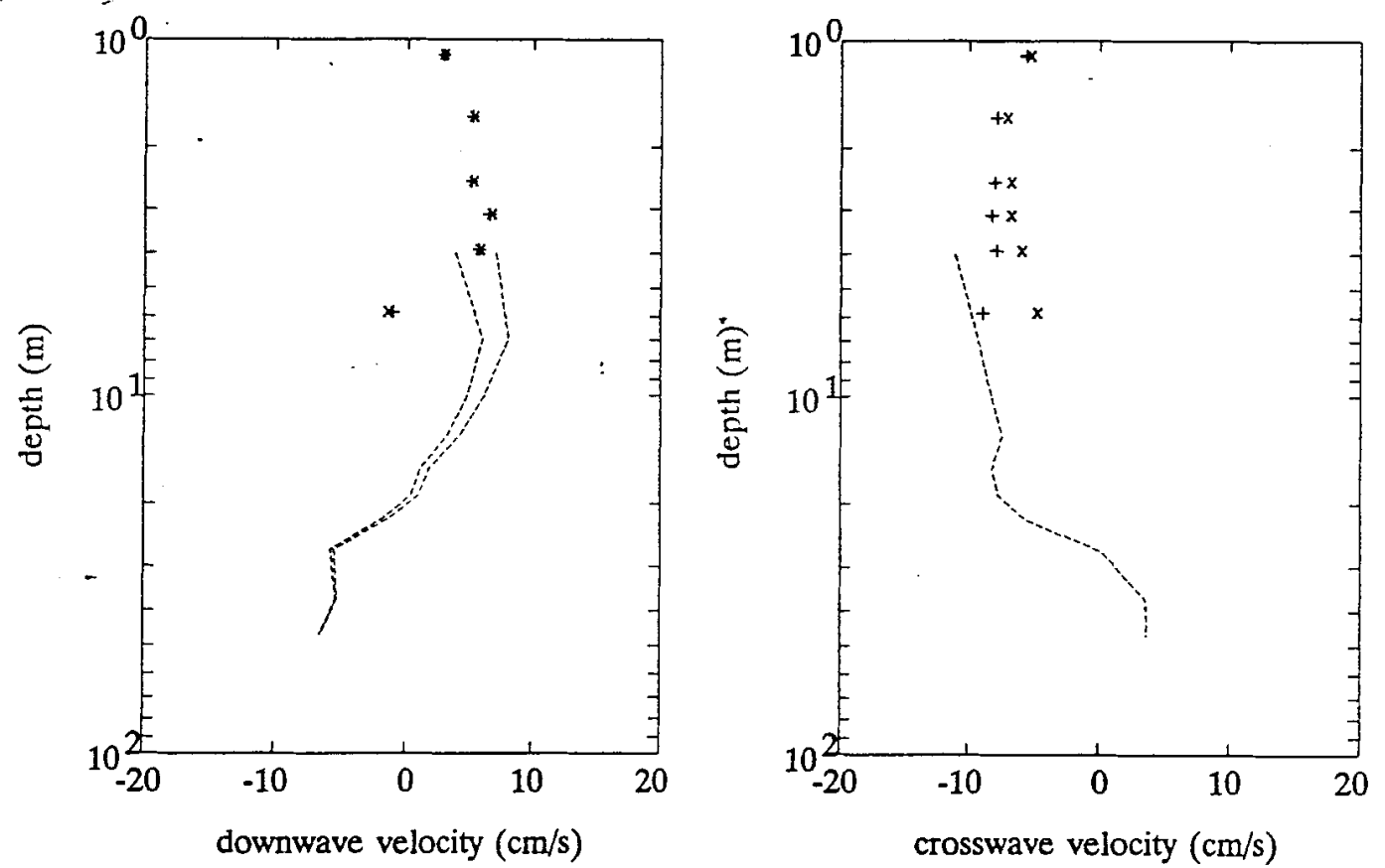

Figure E-4: Comparison of the downwave and crosswave mean velocity profiles for file $e 1$. The +'s represent the normally processed data and the $x$ 's represent the data processed with the compass filtered at $0.125 \mathrm{~Hz}$. The estimates of the unbiased $\mathrm{C} 3$ dashed line. As explained in Chapter 6 , a range is estimated for the downwave direction. 

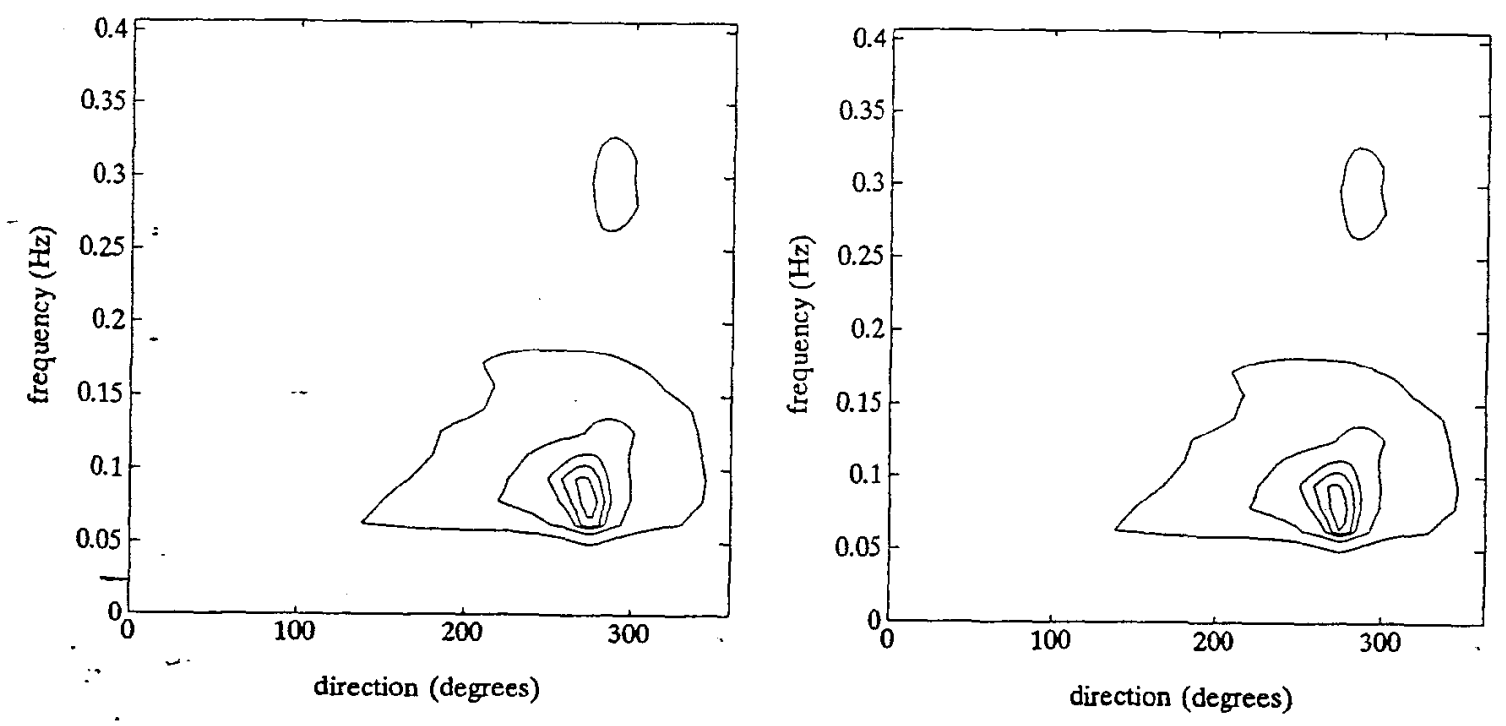

Figure E-5: Comparison of directional spectra for file $f 1$. The plot on the left shows the estimated directional spectra when the compass has been lowpass filtered to $0.6 \mathrm{~Hz}$ (as in the thesis). The plot on the right shows the estimated directional spectra when the compass has been lowpass filtered to $0.125 \mathrm{~Hz}$.
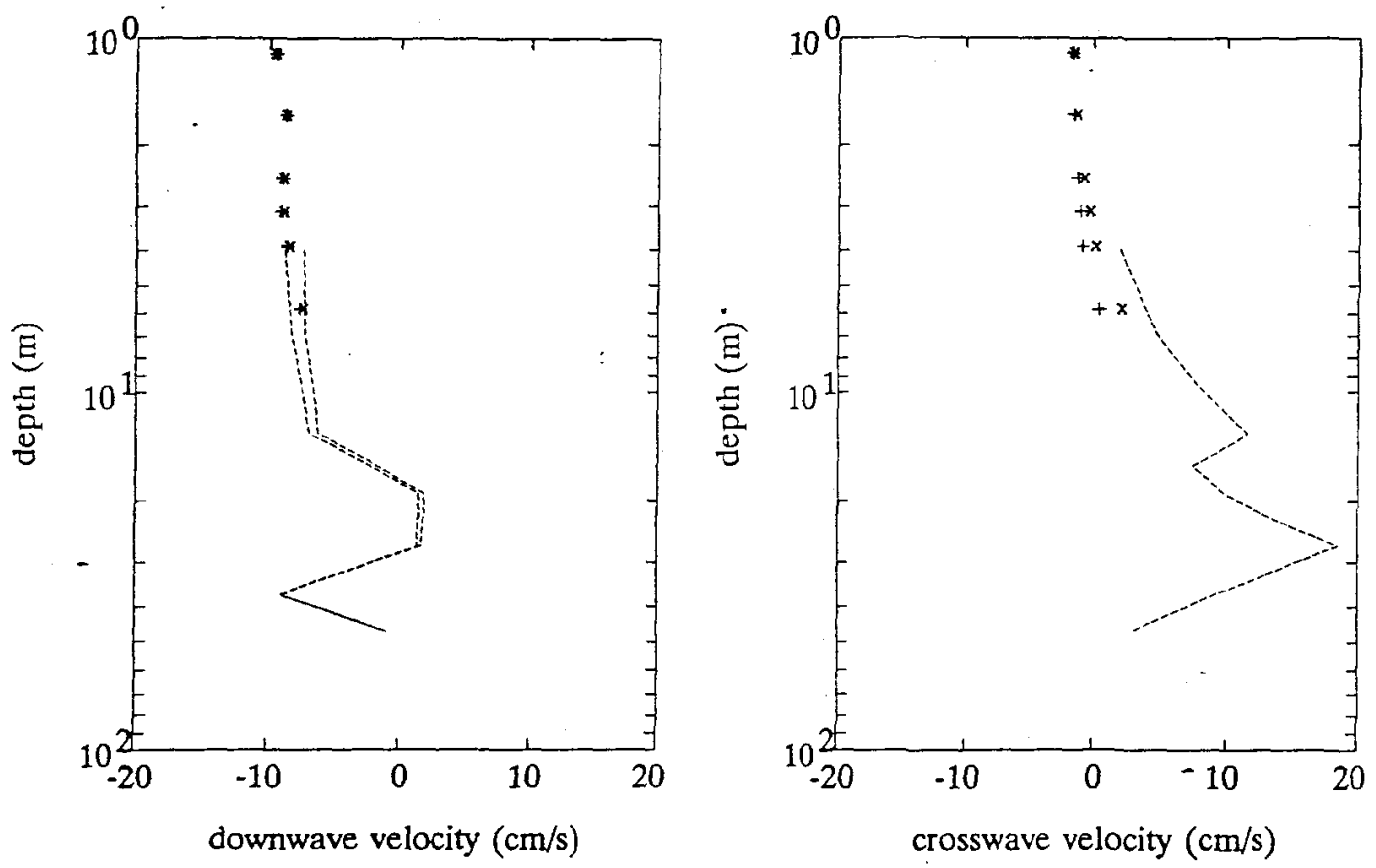

Figure E-6: Comparison of the downwave and crosswave mean velocity profiles for file $f 1$. The +'s represent the normally processed data and the $x$ 's represent the data processed with the compass filtered at $0.125 \mathrm{~Hz}$. The estimates of the unbiased C3 dashed line. As explained in Chapter 6 , a range is estimated for the downwave direction. 

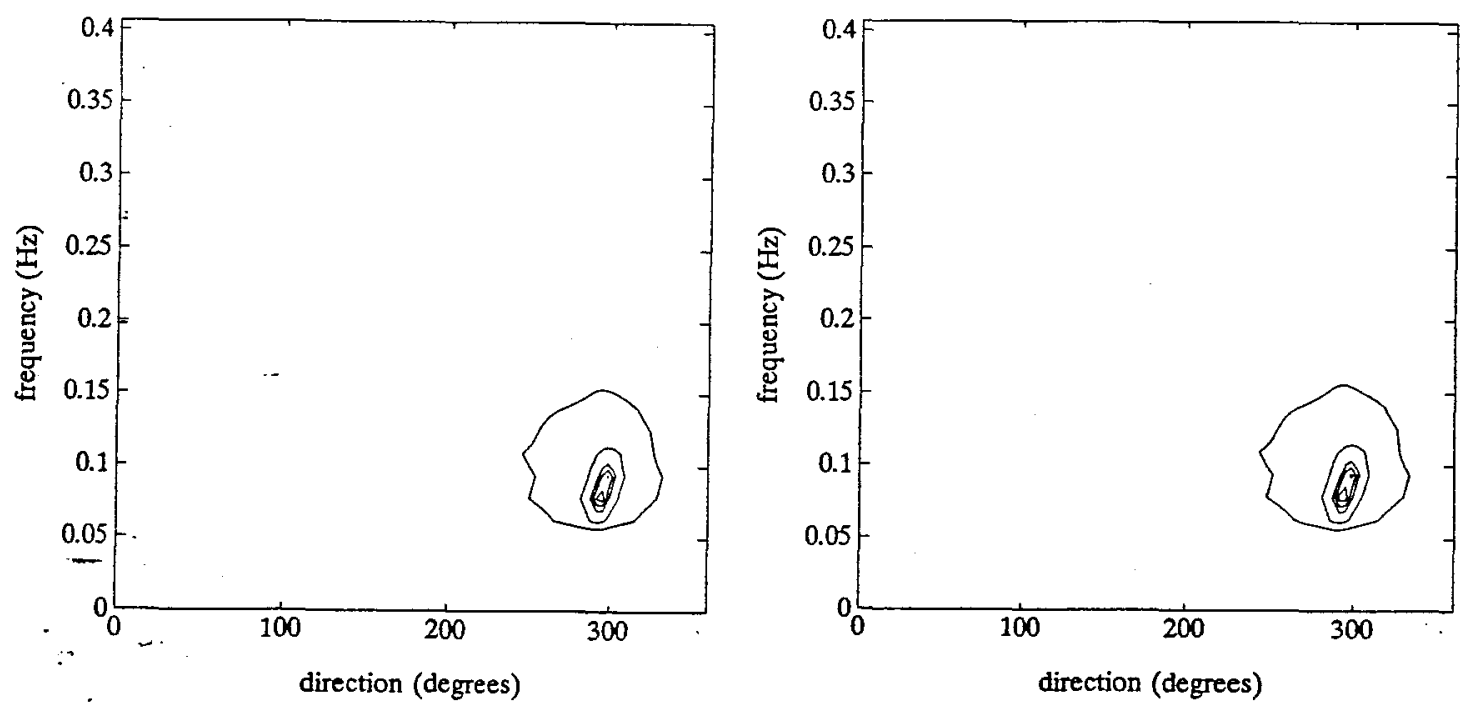

Figure E-7: Comparison of directional spectra for file 12. The plot on the left shows the estimated directional spectra when the compass has been lowpass filtered to $0.6 \mathrm{~Hz}$ (as in the thesis). The plot on the right shows the estimated directional spectra when the compass has been lowpass filtered to $0.125 \mathrm{~Hz}$.
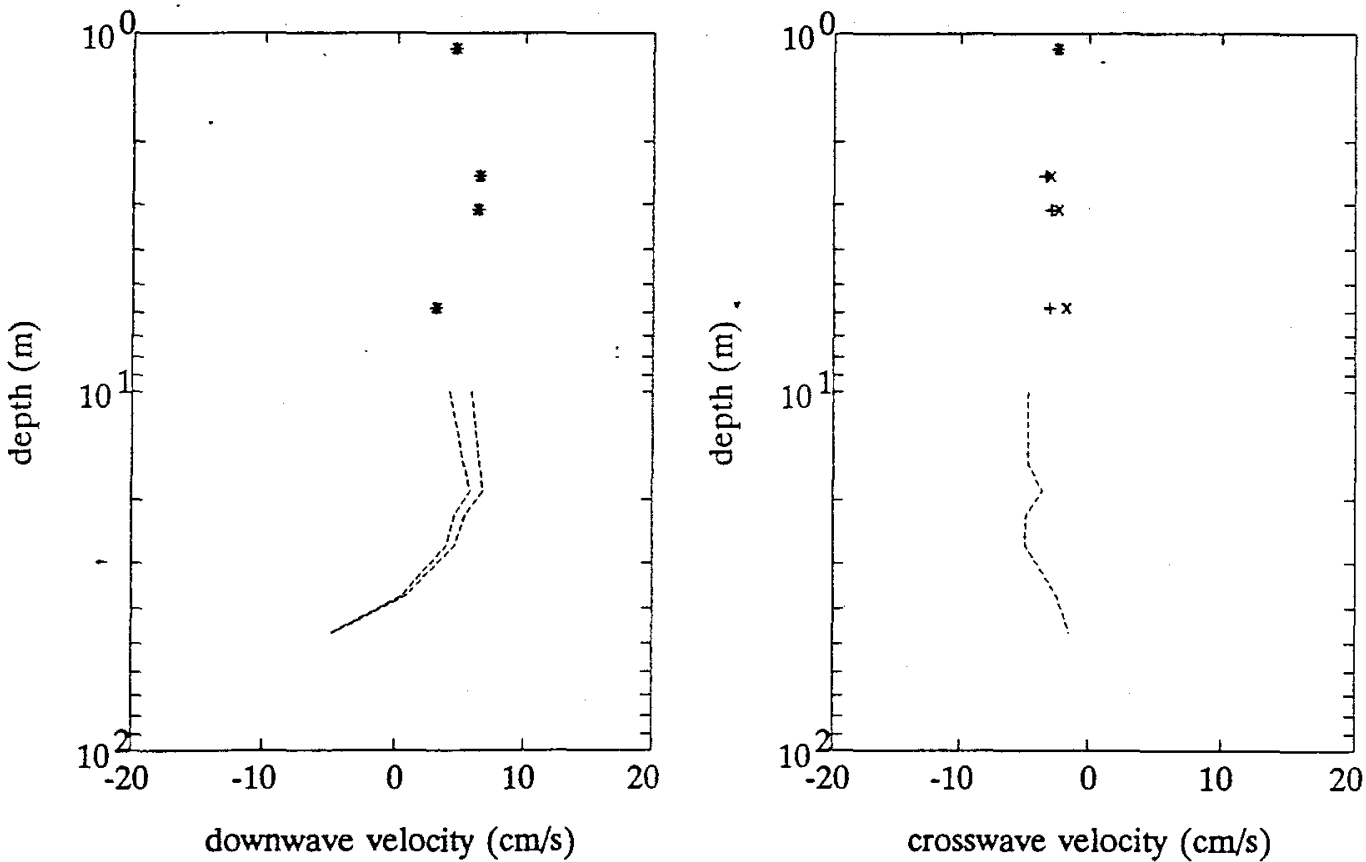

Figure E-8: Comparison of the downwave and crosswave mean velocity profiles for file 12 . The 't's represent the normally processed data and the $x$ 's represent the data processed with the compass filtered at $0.125 \mathrm{~Hz}$. The estimates of the unbiased $\mathrm{C} 3$ dashed line. As explained in Chapter 6 , a range is estimated for the downwave direction. 

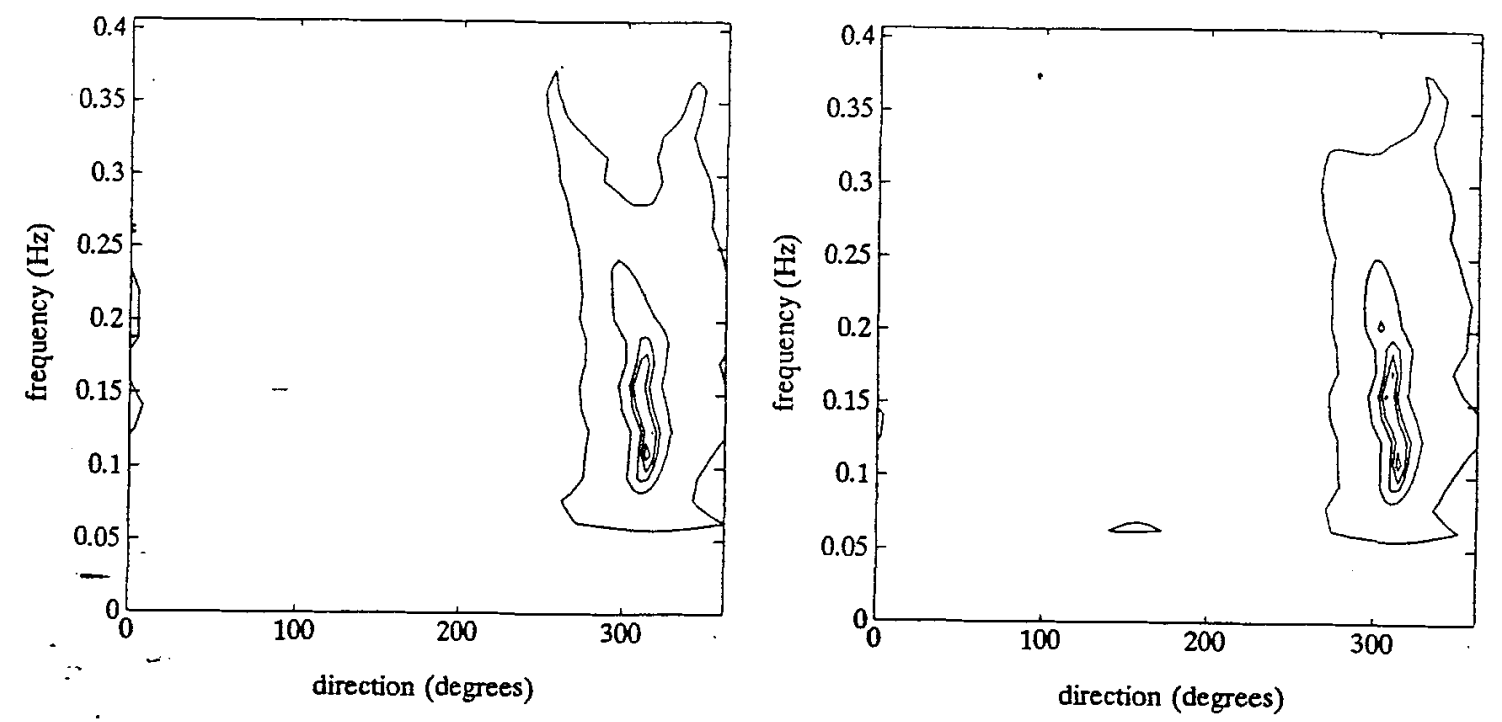

Figure E-9: Comparison of directional spectra for file $p 2$. The plot on the left shows the estimated directional spectra when the compass has been lowpass filtered to $0.6 \mathrm{~Hz}$ (as in the thesis). The plot on the right shows the estimated directional spectra when the compass has been lowpass filtered to $0.125 \mathrm{~Hz}$.
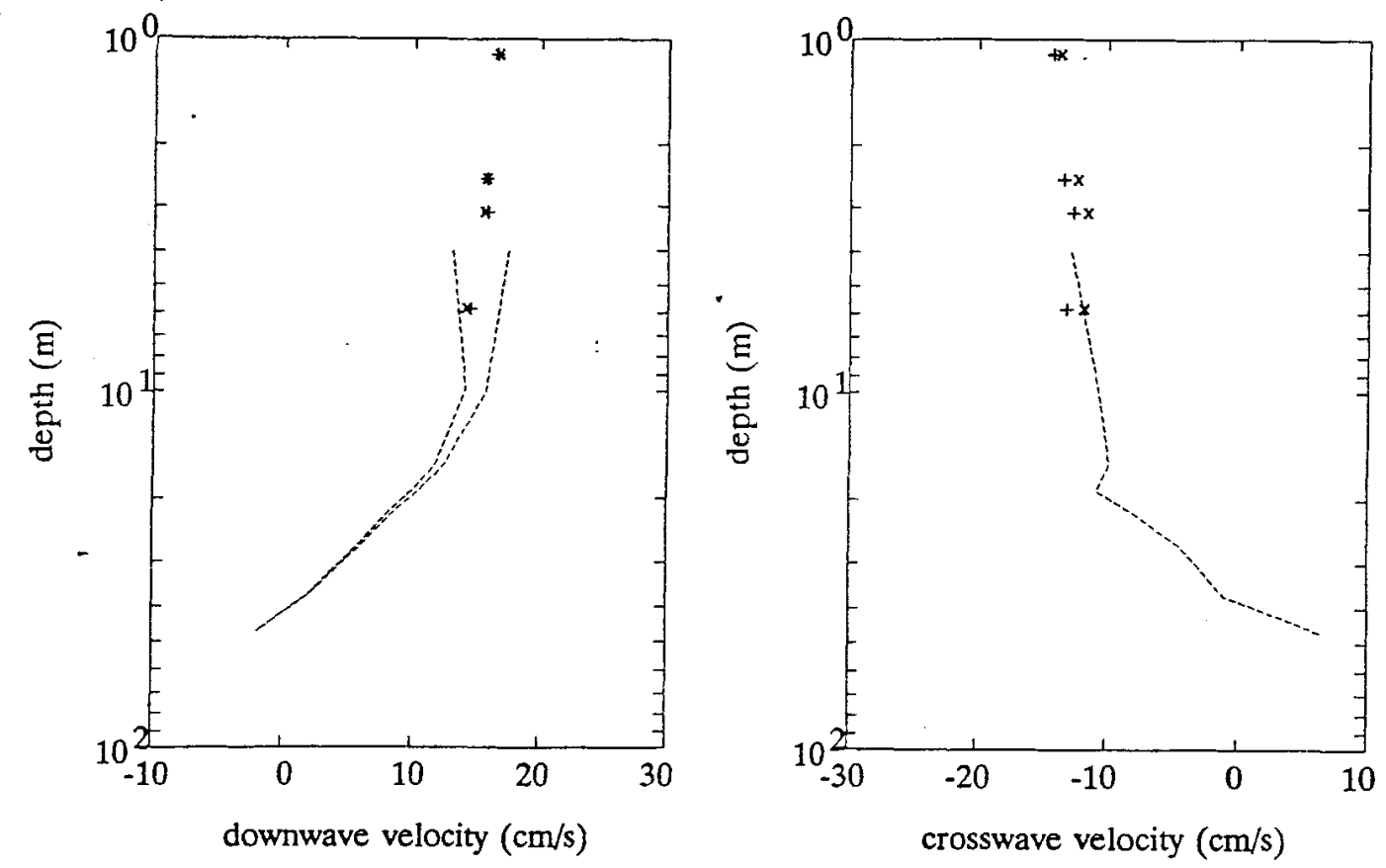

Figure E-10: Comparison of the downwave and crosswave mean velocity profiles for file $p 2$. The +'s represent the normally processed data and the $x$ 's represent the data processed with the compass filtered at $0.125 \mathrm{~Hz}$. The estimates of the unbiased C3 dashed line. As explained in Chapter 6 , a range is estimated for the downwave direction. 

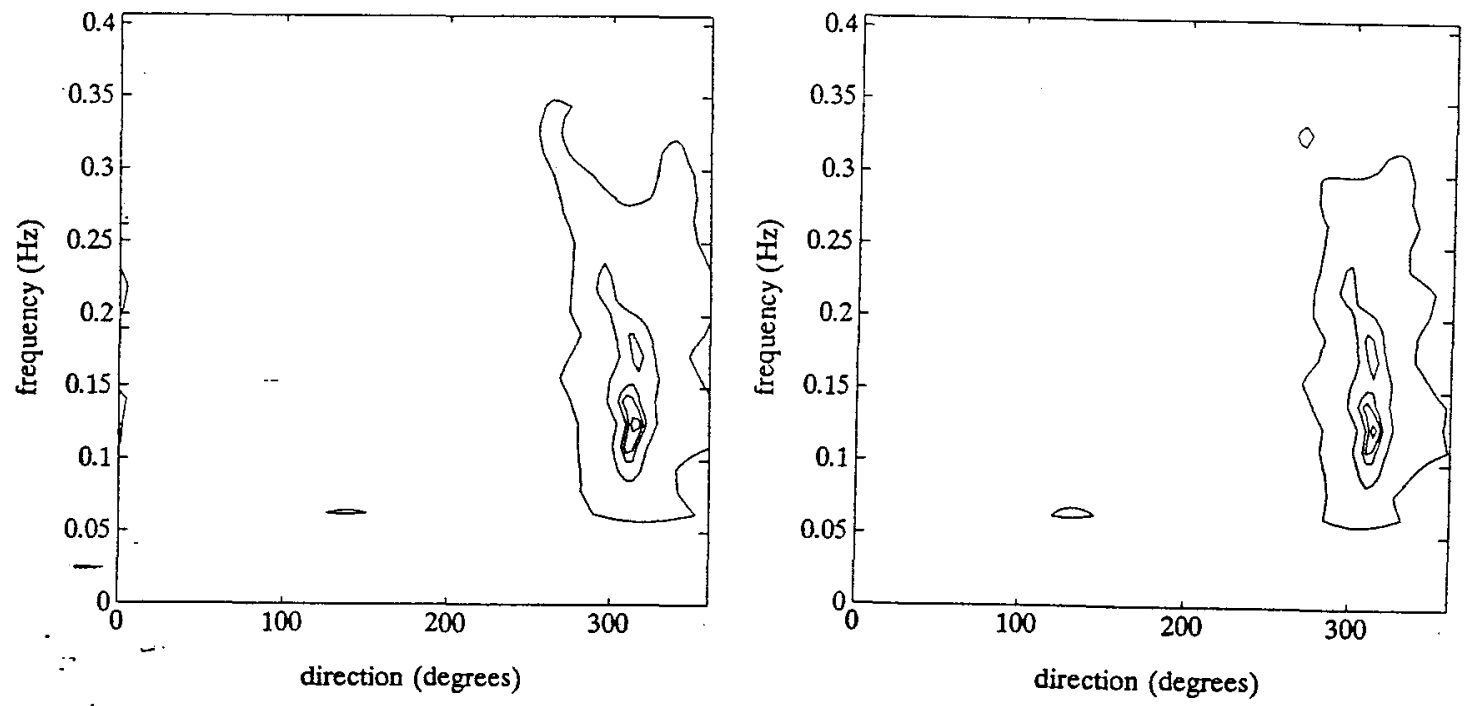

Figure E-11: Comparison of directional spectra for file $q 1$. The plot on the left shows the estimated directional spectra when the compass has been lowpass filtered to $0.6 \mathrm{~Hz}$ (as in the thesis). The plot on the right shows the estimated directional spectra when the compass has been lowpass filtered to $0.125 \mathrm{~Hz}$.
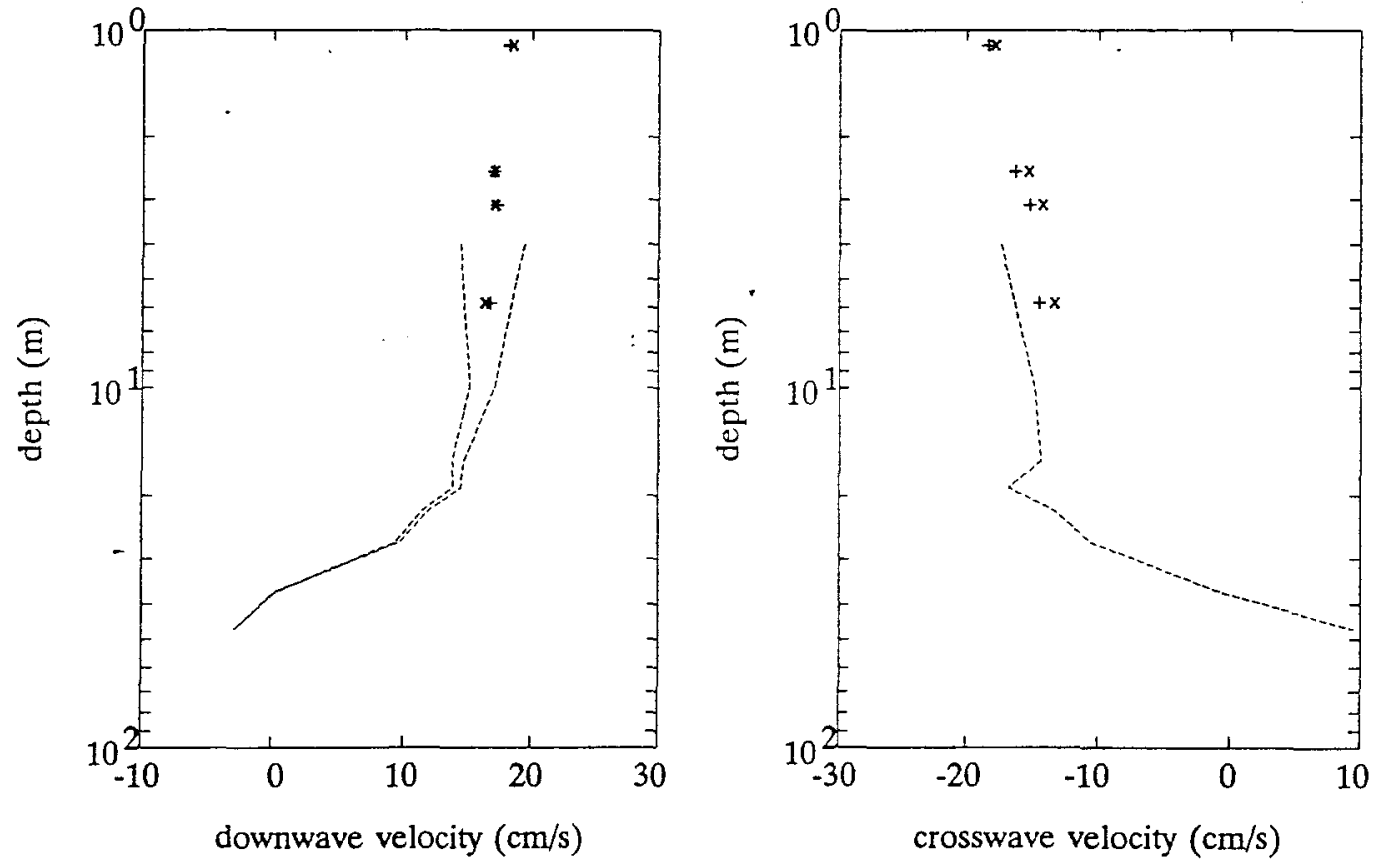

Figure E-12: Comparison of the downwave and crosswave mean velocity profiles for file $q 1$. The +'s represent the normally processed data and the $x$ 's represent the data processed with the compass filtered at $0.125 \mathrm{~Hz}$. The estimates of the unbiased $\mathrm{C} 3$ dashed line. As explained in Chapter 6 , a range is estimated for the downwave direction. 

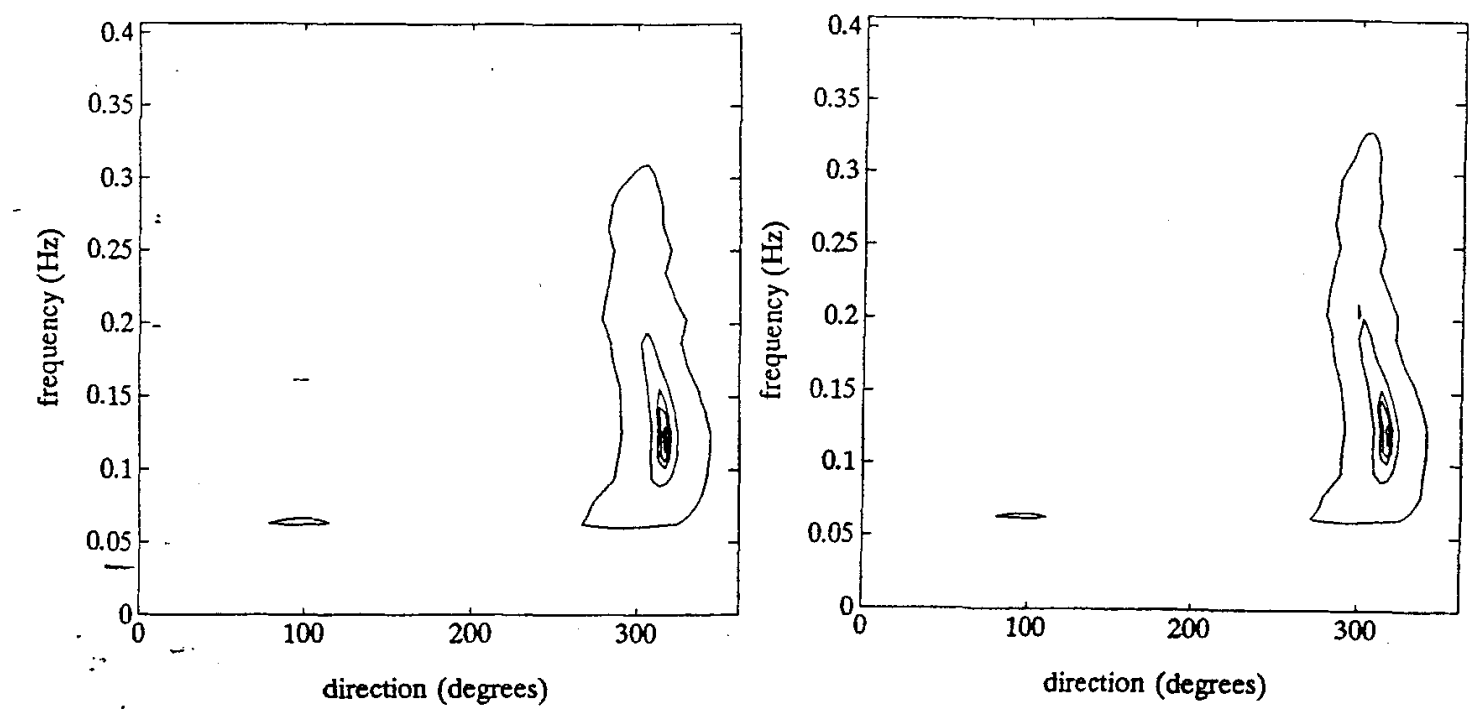

Figure E-13: Comparison of directional spectra for file $u 1$. The plot on the left shows the estimated directional spectra when the compass has been lowpass filtered to $0.6 \mathrm{~Hz}$ (as in the thesis). The plot on the right shows the estimated directional spectra when the compass has been lowpass filtered to $0.125 \mathrm{~Hz}$.
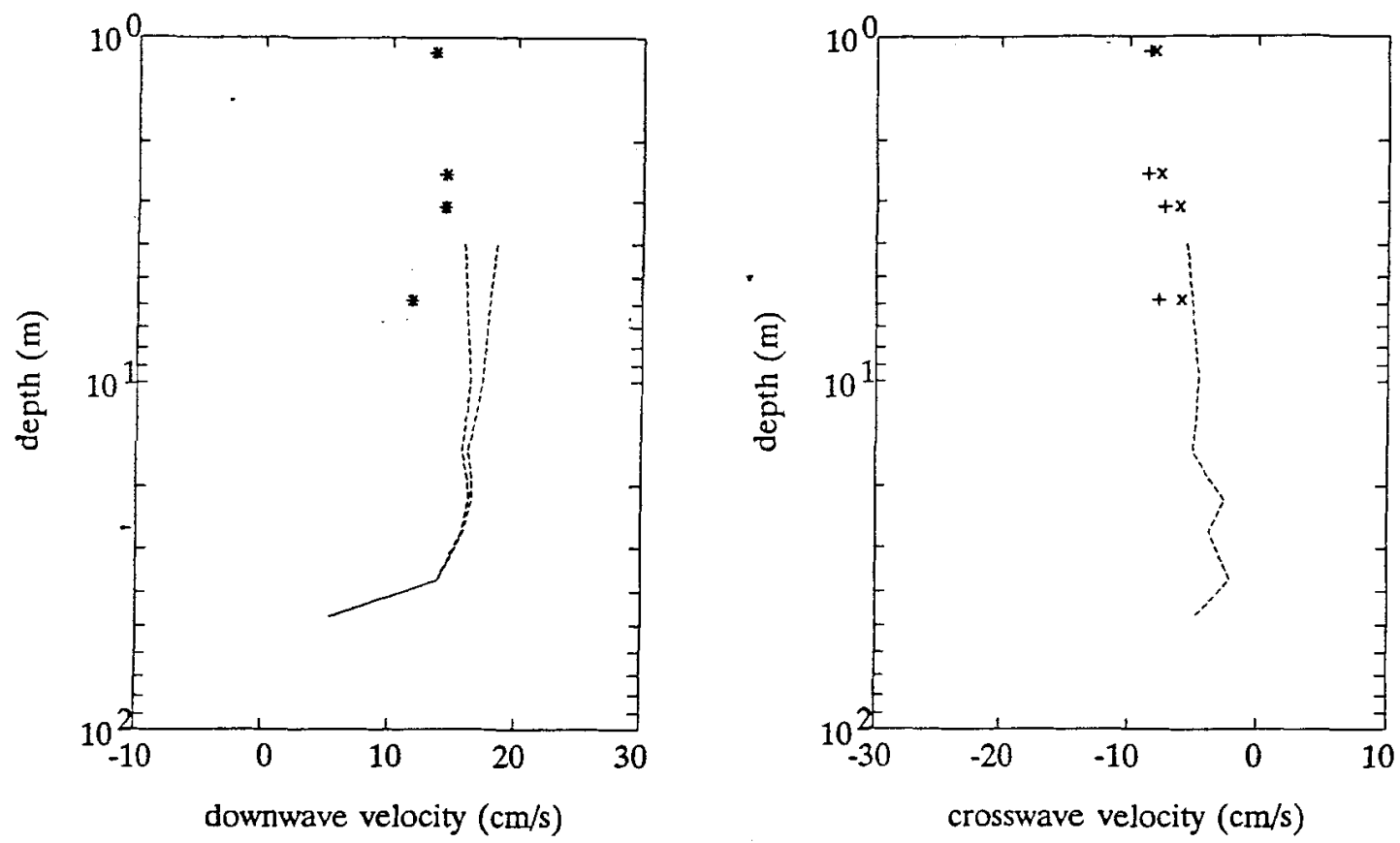

Figure E-14: Comparison of the downwave and crosswave mean velocity profiles for file $u 1$. The +'s represent the normally processed data and the $x$ 's represent the data processed with the compass filtered at $0.125 \mathrm{~Hz}$. The estimates of the unbiased C3 dashed line. As explained in Chapter 6, a range is estimated for the downwave direction. 


\section{Appendix F}

\section{VERTICAL VELOCITIES}

In Figure 7-7 we plotted the mean vertical velocities measured by the SASS versus the friction velocity at the sampling time. We might expect that, if a three-dimensional flow structure such as Langmuir circulation.were set up in the near-surface, the flowfield at any given instant might not depend just on the instantaneous wind conditions but could also be dependent on the wind history. While some of the groups of files do show interesting behaviour in vertical fluid velocity vis-à-vis the long term wind speed history, the complete set of files seemed to defy any neat statistical summary. Therefore, I have presented the data in a raw form (Figure F-1 and F-2). This format may prove useful as, in the future, we learn more about the near-surface dynamics and wish to go back and reconsider what are now our "best guesses".

I again caution that if the vertical velocities are geophsical in nature we should have only measured a mean velocity if the SASS was nonuniformly sampling the flowfield. Because of this, when we consider any trends we see in the plots presented here we must consider that not only the three-dimensional flow structures, but that the nature of the sampling could also have changed over time. 

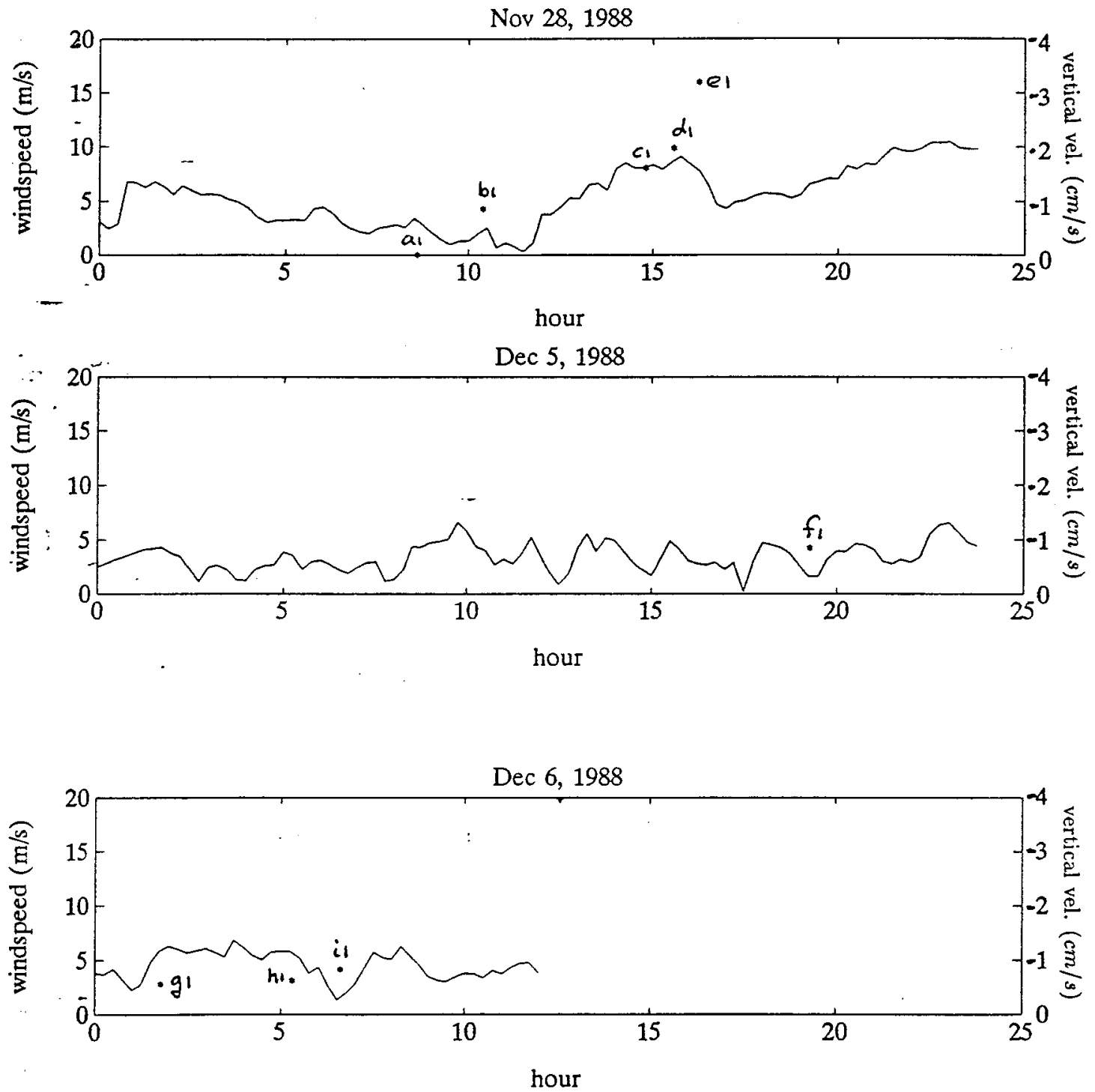

Figure F-1: The solid line represents the $7 m$ windspeed measured at the C3 buoy during the fall 1988 deployment. The mean vertical velocity velocity for each file is plotted (*) at the time corresponding to the middle of the sampled interval. 
Feb 24, 1989
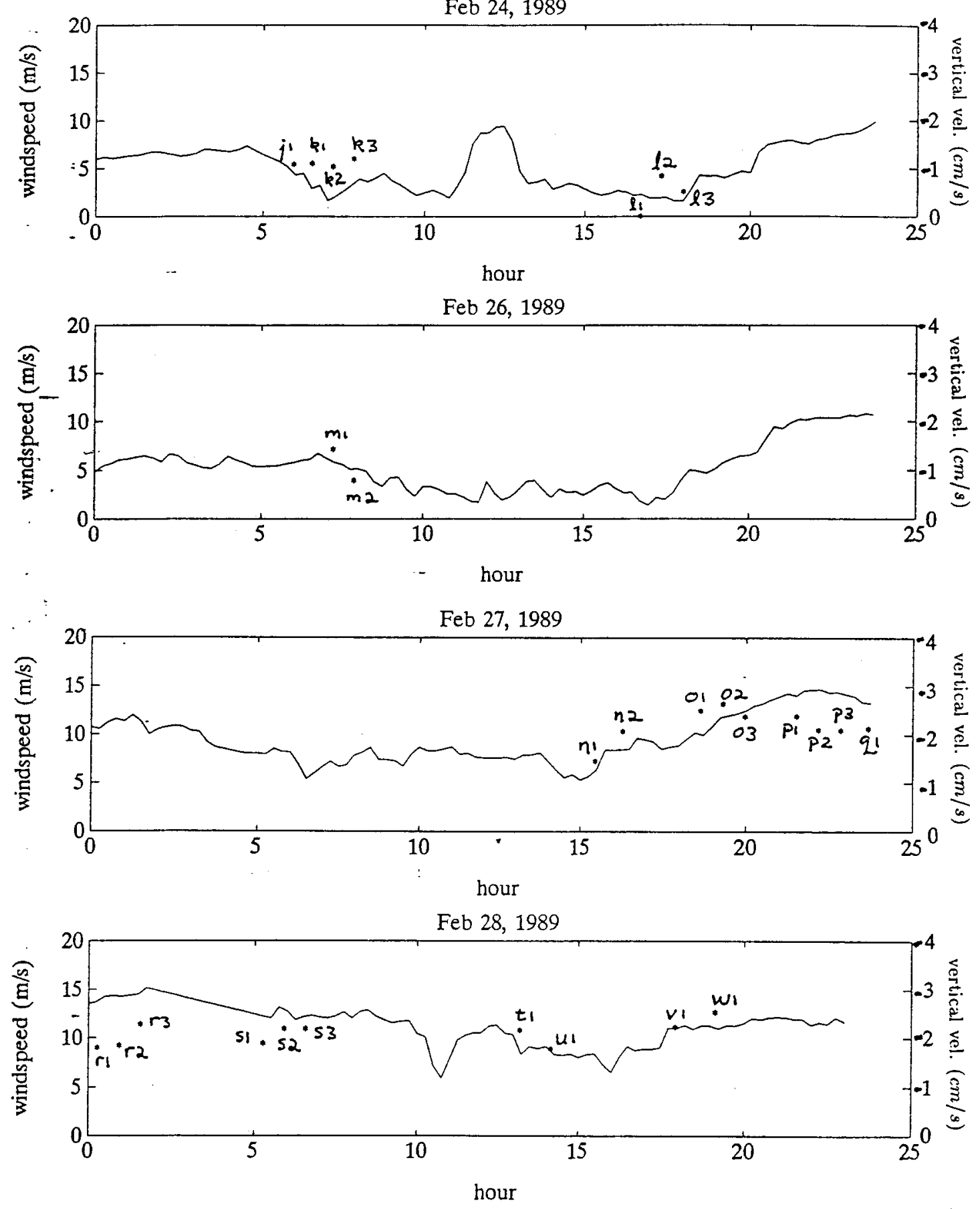

Figure F-2: The solid line represents the $7 \mathrm{~m}$ windspeed measured at the C3 buoy during the spring 1989 deployment. The mean vertical velocity velocity for each file is plotted (*) at the time corresponding to the middle of the sampled interval. 


\section{REFERENCES}

Barrick, D.E., B.J. LiPA and K.E. Steele (1989) Comments on "Theory and application of calibration techniques for an NDBC directional wave measurements buoy" : nonlinear effects. IEEE J. Oceanic Eng. 14(3) 268-272.

Batchelor, G.K. (1967) An Introduction to Fluid Dynamics. Cambridge University Press, Cambridge.

Brink, K.H. (1983) The near-surface dynamics of coastal upwelling. Progress in Oceanographȳ, 12, 223-257.

ByE, J.A.T. (1967) The wave drift current. Journal of Marine Research, 25, 95-102.

Cavaleri, L. and S. Zechetto (1987) Reynolds stresses under wind waves. Journal of Geophysical Research, 92, 3894-3904.

Churchill, J.H. and G.T. Csanady (1983) Near-surface measurements of quasi-Lagrangian velocities in open water. Journal of Physical Oceanography 13, 1669-1680.

Clay, C.S. and H. Medwin (1977), Acoustical Oceanography, John Wiley, New York.

Collar, P.G., R.M. Carson and G. Griffiths (1983) Measurement of near-surface current from a moored wave-slpoe follower. Deep-Sea Research, 30, 63-75.

Csanady, G.T. (1984) The free surface turbulent shear layer. Journal of Physical Oceanography 14, 402-411.

Csanady, G.T. (1985) Air-sea momentum transfer by means of short-crested wavelets. Journal of Physical Oceanography 15, 1486-1501. 
Donelan, M.A. (1978) Whitecaps and momentum transfer. In: Turbulent Fluxes Through the Sea Surface, Wave Dynamics and Prediction, A. FAVRE and K. Hasselman, editors, Plenum Press, pp 273-287.

Donelan, M.A., J. Hamilton and W.H. Hui (1985) Directional spectra of wind generated waves. Philosophical Transactions of the Royal Society of London, A305, 509-562.

Geyer,.W.R. (1989) Field calibration of mixed-layer drifters. Journal of Atmospheric and Oceanic Technology, 6, 333-342.

Gordon, A.L. (1970) Vertical momentum flux accomplished by Langmuir circulation. Journal of Geophysical Research, 75, 4177-4179.

HARRIS, F.J. (1978) On the use of windows for harmonic analysis with the discrete Fourier transform. Proceedings of the IEEE, 66, 51-83.

Hildebrand, F.B. (1976) Advanced Calculus for Applications, Prentice-Hall. 733p.

HuANG, N.E. (1979) On surface drift currents in the ocean. Journal of Fluid Mechanics, $\mathbf{9 1}, 191-208$.

ICHIYe, T. (1964) On a dye diffusion experiment off Long Island. Lamont Geological Observatory Technical Report CU-2663-10.

KAY, S.M. and S.L. MARPLE JR. (1981) Spectrum analysis - a modern perspective. Proceedings of the IEEE 69, 1380-1419.

KEnYon, K.E. (1969) Stokes drift for random gravity waves. Journal of Geophysical Research 74, 6991-6994.

Kitaigorodskit, S.A., M.A. Donelan, J.L. Lumely and E.A. Terray (1983) Waveturbulence interactions in the upper ocean. Part II: Statistical characteristics of wave and turbulent components of the random velocity field in the marine surface layer. Journal of Physical Oceanography 13, 1988-1999.

LANGMUir, I. (1937) Surface motion of water induced by wind. Science 87, 119-123.

LARge, W.G. and S. POND (1981) Open ocean momentum flux measurements in moderate to strong winds. Journal of Physical Oceanography 11, 324-336. 
LEIBOVICH, S. and K. RADHAKRISHNAN (1977) On the evolution of the system of wind drift çurrents and Langmuir circulations in the ocean. Part II. Structure of the Langmuir vortices. Journal of Fluid Mechanics 80, 481-507.

LENTZ, S.J. (1991) The surface boundary layer in coastal upwelling regions. Submitted to Journal of Physical Oceanography.

Limeburner, R. and R.C. Beardsley, (1989) CTD observations off Northern California during the Shelf Mixed Layer Experiment, SMILE, February/March 1989. WHOI technical report 89-41,218p.

Longuet-Higgins, M.S., D.E. Cartwright and N.D. Smith (1963) Observations of the directional spectrum of sea waves using the motions of floating buoy. Ocean Wave Spectra, Prentice-Hall, 111-136.

LONGUET-Higgins, M.S. (1969) On wave breaking and the equilibrium spectrum of windgenerated waves. Proceedings of the Royal Society of London A 310, 151-159.

LoNgUET-HIgGins, M.S. (1987) Lagrangian moments and mass transport in Stokes waves. Journal of Fluid Mechanics, 179, 547-555.

LYGRE, A. and H.E. KROGSTAD (1986) Maximum entropy estimation of the directional distribution in ocean wave spectra. Journal of Physical Oceanography, 16 2052-2060.

MADSEN, O.S. (1977) A realistic model of the wind-induced Ekman boundary layer. Journal of Physical Oceanography, 7, 248-255.

MAMAEV, O.I. (1964) Uproshchennaya zavisimost' mezhdu plotnost'yu, temperaturoi i solenost'yu morskoi vody (A simplified relation between density, temperature and salinity of sea water). Izvestiya Akademii Nauk SSSR, Seriya Geofizicheskaya 2.

Medwin, H. and N.D. Breitz (1989) Ambient and transient bubble spectral densities in quiescent seas and under spilling breakers. Journal of Geophysical Research, 94, 12751-12759.

MEI, C.C. (1989) The Applied Dynamics of Ocean Surface Waves, World Scientific. 740p.

Melville, W.K. and R.J. Rapp (1985) Momentum flux in breaking waves. Nature, 317, 514-516. 
Miles, J.W. (1957) On the generation of surface waves by shear flows. Journal of Fluid Mechanics, 3, 185-204.

Mitsuyasu, H. (1985) A note on the momentum transfer from wind to waves. Journal of Geophysical Research, 90, 3343-3345.

-

Montgomery, E.T and M.J. Santala (1989) The Surface Acoustic Shear Sensor (SASS) as used during the Shelf MIxed Layer Experiment. WHOI technical report 89-34, 19p.

Niller, P.P., R.E. DAVIS and H.J. White (1987) Water-following characteristics of a mixed layer drifter. Deep-Sea Research 34, 1867-1881.

OKUDA, K. (1982) Internal flow structure of short wind waves (Part I). Journal of the - Ocearrographical Society of Japan, 38, 28-42.

Oppenheim, A.V. and R.W. Schafer (1975) Digital Signal Processing, Prentice-Hall. $585 \mathrm{p}$.

Phillips, O.M. (1957) On the generation of waves by turbulent wind. Journal of Fluid Mechanics, 2, 417-445.

Pollard, R.T. (1973) Interpretation of near-surface current meter observations. Deep-Sea Research, 20, 261-268.

Pollard, R.T., P.B. Rhines and R.O.R.Y. Thompson (1973) The deepening of the windmixed layer. Geophysical Fluid Dynamics, 3, 381-404.

Pollard, R.T. (1977) Observations and theories of Langmuir circulations and their role in near surface mixing. In: A Voyage of Discovery: G. Deacon 70th Anniversary Volume, M. ANGEL, editor, Pergamon Press, New York, pp. 235-251.

Pond, S. and G.L. Pickard (1983) Introductory Physical Oceanography (2nd edition), Permagon Press, New York, 329p.

Richman, J.G., R.A. DeSzoeke and R.E. Davis (1987) Measurements of near-surface shear in the ocean, Journal of Geophysical Research 92, 2851-2858.

RudNick, P. (1967) Motion of a large spar buoy in sea waves, Journal of Ship Research 11, $257-267$. 
Santala, M.J. and A.J. Williams III (1990) Estimates of directional spectra from the surface acoustic shear sensor (SASS) OCEANS'90 Conference Proceedings, 41-45.

Santala, M.J. and E.A. Terray (1991) A technique for making unbiased estimates of current shear from a wave-follower, Deep-Sea Research, in press.

ShONTING, D.H. (1968) Autospectra of observed observed particle motions in wind waves, Journal of Marine Research 26, 43-65.

Shonting, D.H. (1967) Measurements of particle motions in ocean waves. Journal of Marine Research 25, 162-181.

Soloxiev, A.V., N.V. Vershinsky and V.A. Bezverchin (1988) Small-scale turbulence - measurements in the thin surface layer of the ocean. Deep-Sea Research 12, 1859-1874.

Stewart, R.W. (1961) The wave drag of wind over water. Journal of Fluid Mechanics, 10, 189-194.

Trivett, D.A., E.A. Terray and A.J. Williams, III (1991) Error analysis of an acoustic currént meter. IEEE Journal of Oceanic Engineering, in press.

Trivett, D.A: (1991) M.I.T.-Woods Hole Oceanographic Institution Ph.D. Thesis.

Patch, S.K., E.P. Dever, R.C. Beardsley, and S.J. Lentz (1991) Response characteristics of the VACM compass and vane follower. Submitted to: Journal of Atmospheric and Oceanic Technology.

VAN Dorn, W.G. (1953) Wind stress on an artificial pond. Journal of Marine Research, $12,249-276$.

Wehausen, J.V. and E.V. Laitone (1960) Surface Waves. In Encyclopedia of Physics, vol. 9. Berlin-Göttingen-Heidelberg : Springer-Verlag.

WELLER, R.A. (1985) Near-surface velocity variability at inertial and subinertial frequencies in the vicinity of the California current. Journal of Physical Research 15, 372-385.

Weller, R.A. (1985) Three-dimensional flow in the upper ocean. Science, 227, 1552-1556.

Weller, R.A. and R.E. DAvis (1980) A vector measuring current meter. Deep-Sea Research, 27, 565-581. 
Williams, A.J. III, J.S. Toch ko, R.L. Koehler, W.D. GranT, T.F. Gross, and C.V.R. DUNN (1987) Measurement of turbulence in the oceanic bottom boundary layer with an acoustic current meter array. J. Atmos. and Oceanic Tech. 4(2), 312-327.

WU, JIN (1969) An estimation of wind effects on dispersion in wide channels. Water Resources Research, 5, 1097-1104.

WU, JiN (1975) Wind-induced drift currents. Journal of Fluid Mechanics, 68, 49-70.

WU, JIN (1983) Sea-surface drift currents induced by wind and waves. Journal of Physical Oceanography, 13, 1441-1451.

Wu, Jin and P.A. HWANg (1991) Comment on "Ambient and transient bubble spectral densities in quiescent seas and under spilling breakers" by H. Medwin and N.D. Breitz. Journal Geophysical Research, 96, 865-866.

Zdravkovich, M.M., V.P. Brand, G. Mathew, and A. Weston (1989) Flow past short circułar cylinders with two free ends. Journal of Fluid Mechanics, 203, 557-575. 